\title{
Measurement of Neutrino Induced, Charged Current, Charged Pion Production
}

by

Michael Joseph Wilking

B.Ch.E., University of Minnesota, 2001

M.S., University of Colorado, 2007

A thesis submitted to the

Faculty of the Graduate School of the

University of Colorado in partial fulfillment

of the requirements for the degree of

Doctor of Philosophy

Department of Physics

2009 
This thesis entitled:

Measurement of Neutrino Induced, Charged Current, Charged Pion Production written by Michael Joseph Wilking

has been approved for the Department of Physics

Prof. Eric Zimmerman

Prof. John Cumalat

Prof. Kevin Stenson

Prof. Senarath De Alwis

Prof. Andrew Hamilton

Date

The final copy of this thesis has been examined by the signatories, and we find that both the content and the form meet acceptable presentation standards of scholarly work in the above mentioned discipline. 
Wilking, Michael Joseph (Ph.D., Physics)

Measurement of Neutrino Induced, Charged Current, Charged Pion Production

Thesis directed by Prof. Eric Zimmerman

Neutrinos are among the least understood particles in the standard model of particle physics. At neutrino energies in the $1 \mathrm{GeV}$ range, neutrino properties are typically determined by observing the outgoing charged lepton produced in a charged current quasi-elastic interactions. The largest charged current background to these measurements comes from charged current pion production interactions, for which there is very little available data.

This document presents a measurement of neutrino induced, charged current, charged pion production at neutrino energies directly relevant to many of the next generation of oscillation experiments. The interaction cross section has been measured as a function of several kinematic quantities, such as the kinetic energies and directions of the muons and pions produced in these processes, as well as the incident neutrino energy. The cross section measured on a $\mathrm{CH}_{2.06}$ target has been found to steadily increase from $(0.62 \pm 0.08) \times 10^{-38} \mathrm{~cm}^{2}$ at a neutrino energy of $0.5 \mathrm{GeV}$, to a value of $(10.7 \pm 2.1) \times 10^{-38} \mathrm{~cm}^{2}$ at the highest measured neutrino energy of $2 \mathrm{GeV}$. 


\section{Dedication}

To my wife, Jen. Despite the sacrifices we have made for science, you have always been my top priority. 


\section{Acknowledgements}

My path to graduation has been a bit more circuitous than most. I have had the benefit (or misfortune?) of working on three different experiments as a graduate student: $\mathrm{KTeV}$, CKM, and MiniBooNE. Throughout my time on each of these experiments, I've received support from more people than I can possibly hope to list here, so let me simply say thank you to all of my colleagues. With so many intelligent, creative, interesting, and kind people throughout the experiments on which I have worked, it is very easy to enjoy what I do.

In 2001, I began working on the $\mathrm{KTeV}$ experiment under the direction of Tony Barker. Tony had an intensity about the research that, I think it is safe to say, was unmatched by anyone else I have ever met. At times, this resulted in heated arguments that were often difficult to navigate. However, these interactions taught me quite a bit about thinking efficiently, maintaining academic rigor, and gaining a complete understanding of a problem before making a judgment. I firmly believe that I grew more as a physicist in those initial, somewhat turbulent years than would have otherwise been possible. When Tony passed away in the spring of 2004, I was left without an advisor, but much more importantly, I lost a good friend.

My KTeV days were spent working with the Colorado kaon group. Jason Ladue was the first person I met when I arrived at CU. As the first group member to graduate during my tenure, Jason provided the earliest exposure I had to the frenetic pace of the Ph.D. endgame. Despite his misguided love for the Chicago Bears, Jason was very 
good-natured and was always willing to devote some time to help out the new guy. I had quite a bit more overlap with the other senior graduate student in our group, Pat Toale. Pat became the de facto postdoc of the group, helping the rest of us through our analyses. Discussions with Pat greatly contributed to my understanding of what it meant to study physics, and playing tackle football games with Pat greatly contributed to my understanding of physical pain. Rune Niclasen and I began graduate school the same year. I will greatly miss wandering into Rune's office to discuss the latest quantum mechanical paradox that I had spent way too much time thinking about. If you ever find yourself in a Rubik's cube competition, you may want to avoid a match-up with the fragile Dane. The last person to join the Colorado kaon group was Jim Hirschaur. Jim and I were already friends long before his return from ski-batical. Our conversations would range from intense discussions about wave-function collapse, to which album was Ween's best, to whether Joe Borowski was even qualified to close for the Kane County Cougars. Just don't tell him that you can throw a screwball.

After spending the better part of three years working on flavor physics, I transitioned to neutrinos and the MiniBooNE experiment with a new advisor, Eric Zimmerman. Eric's managerial style was about as different as I could have imagined. He had a very laissez faire approach to his graduate students, emphasizing the importance of working through an issue rather than of simply being provided with an answer. This was a perfect fit for me at this stage in my education, as it allowed me to formulate my own methodologies from scratch and take more on responsibility for, and ownership of, my analysis. Whenever I did run into problems, however, he always provided excellent advice. Eric had a unique ability to immediately ascertain the important issues at the core of any given problem. Through these conversations, I gained a much finer appreciation for the distinction between working hard and working effectively. I have learned an incredible amount from Eric, and I am grateful for having had the opportunity to work with him. 
The majority of my time on MiniBooNE was spent with the Colorado neutrino group. On my first neutrino trip to Fermilab, I was introduced to MiniBooNE by our first postdoc, Terry Hart. Terry took the time to explain many of the design and analysis concepts of the experiment, as well as guiding me through the details of living at the lab. He also taught me that if you fit historical data to a particular combination of hyperbolic tangent functions, you can do an extremely good job of filling out an NCAA tournament bracket. Many thanks are in order for my fellow graduate student and office mate, Bob Nelson. A significant fraction of our time was spent proposing ideas to each other and working them out on the blackboard. They didn't always pan out, but I always enjoyed the discussions. Much has already been said about Bob's ability to find a good time in even the most unlikely situations, but I will always know him as a resourceful problem solver, an inquisitive mind, and a loyal friend. During the spring and summer I spent at Fermilab in 2005, my desk was occupied by Josh Spitz. One of our more enjoyable rituals was the daily hike to the engineering building with Jim and Rune to get a coffee from Lindsey at Celestial Seasonings. Josh liked it so much that he married her. While his fantasy football skills may be questionable, there was never any doubt that he was on his way to graduate school, and I was glad I had the opportunity to get to know him. Martin Tzanov didn't move to Boulder until the last couple of years of my time there, but we wasted no time ramping up to full blown physics debates. During our review of the Princeton analysis, countless hours were spent arguing about the details error evaluation and data unfolding. I'm fully aware of how that last sentence sounds, but it was one of the most enjoyable months that I spent on the experiment. Martin's understanding of statistical techniques and his grounding in advanced mathematics meant that he was terrific resource throughout those last few years, and he was always willing to spend as much time as required to discuss any issues I might encounter.

I'd like to thank all of my MiniBooNE collaborators for contributing so much 
great work to such a well executed experiment. To my fellow graduate students, whose talent and dedication established such lofty standards of achievement, it has been a privilege to work with you, and I have very much enjoyed our time together (even the 2 mile walks through Tampa, FL at 1am). Special thanks are in order for Ryan Patterson who, after leaving the experiment for a postdoc at Caltech, spent a week of his time in Pasadena guiding me through the details of his reconstruction package, and, perhaps more importantly, for introducing me to the double double, animal style. I would also like to thank both of my analysis coordinators: Steve Brice for the oscillation analysis and Sam Zeller for cross sections. To all the young physicists out there, I strongly suggest you get yourself a Steve. I still fail to understand how someone with Steve's time constraints was able to set aside 3 hours at a moment's notice to discuss unfolding bias, or flux systematics, or multisim weights, or anything else I might be curious about that particular day. For lack of a better phrase, Sam Zeller is the anchor for all MiniBooNE cross section analysis. Her enthusiasm for the physics is unmistakable. It was always comforting to know that any new insight or result that one might produce, no matter how seemingly unimportant, wouldn't go unnoticed. I very much admire the care with which she examines each new physics result to ensure that the collaboration is always well represented.

To my parents, Joe and Sue Wilking, thank you for your steady support through the full duration of my education. From a very young age I was always encouraged to question the world around me, which is perhaps the trait that I now value most. Despite discarding my undergraduate studies and making the somewhat unconventional switch from engineering to physics, you always supported my decisions, and for that I am grateful. To my sisters, Melissa and Jenny, thanks for always making an effort to keep in touch even after I decided to move a thousand miles away. I've appreciated the phone calls and the trips to Colorado; they've always been a lot of fun.

Finally, I'd like to thank my wife, Jen, for all of her support throughout this very 
long journey. The field of high energy physics demands long periods of separation that can be trying for even the most self-sufficient people. You have always been there for me during the rough and even tumultuous periods along the way, and that means more to me than you may ever know. 


\section{Contents}

\section{Chapter}

1 Introduction 1

1.1 Charged Current Interactions . . . . . . . . . . . . . . 2

1.1.1 Charged Current Quasi-Elastic Interactions . . . . . . . . . . 2

1.1.2 Charged Current Pion Production . . . . . . . . . . . . . 2

1.2 Nuclear Effects . . . . . . . . . . . . . . . . . . . 9

1.3 The $\mathrm{CC} \pi^{+}$Measurement . . . . . . . . . . . . . . . . . 11

1.4 MiniBoonE . . . . . . . . . . . . . . . . . . . . . 12

2 The Booster Neutrino Beam 13

2.1 Booster Proton Beam _. . . . . . . . . . . . . . . . . . . 13

2.2 Target and Horn . . . . . . . . . . . . . . . . . . . . 14

2.3 Meson Decay Region . . . . . . . . . . . . . . . . . . 17

2.4 Neutrino Flux . . . . . . . . . . . . . . . . . . . . 18

3 The MiniBooNE Detector $\quad 19$

3.1 Mineral Oil . . . . . . . . . . . . . . . . . . . . . . . . . 21

3.2 Photomultiplier Tubes . . . . . . . . . . . . . . . . 22

3.3 Electronics . . . . . . . . . . . . . . . . . . . . . . . . 24

3.4 Calibration Systems . . . . . . . . . . . . . . . 24

3.5 Data Acquisition . . . . . . . . . . . . . . . . 26 
4 Event Simulation 28

4.1 Neutrino Flux Simulation . . . . . . . . . . . . . . . 28

4.1 .1 Beam Monte Carlo . . . . . . . . . . . . . . . . 28

4.1 .2 Meson Decays . . . . . . . . . . . . . . . . . . . . . 37

4.1 .3 Statistics Amplification . . . . . . . . . . . . . . 38

4.1 .4 Flux Predictions .................... 41

4.1 .5 Systematic Uncertainties . . . . . . . . . . . . . . 42

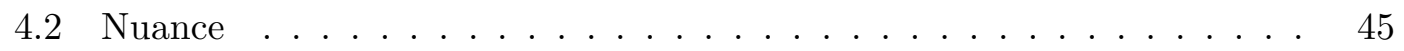

$4.2 .1 \mathrm{CCQE} \ldots \ldots \ldots \ldots \ldots \ldots \ldots$

$4.2 .2 \quad$ Nuclear Effects . . . . . . . . . . . . . . . . . . . . . . . . 48

4.2 .3 Systematic Uncertainties . . . . . . . . . . . . . . . . 49

4.3 BooNEGlob ............................... 50

4.4 Detector Simulation . . . . . . . . . . . . . . . 51

4.4 .1 Optical Model ..................... 52

4.4 .2 Systematic Uncertainties . . . . . . . . . . . . . 54

5 Event Reconstruction $\quad 57$

5.1 Straight Pion Tracks . . . . . . . . . . . . . . . . 59

5.1 .1 Charge Likelihood ................... 59

5.1 .2 Time Likelihood ................... 63

5.1 .3 Likelihood Maximization . . . . . . . . . . . . 69

5.1.4 Comparison With the Muon Fitter . . . . . . . . . . . . 72

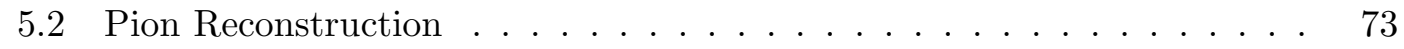

5.2 .1 Fitting Kinked Tracks . . . . . . . . . . . . . 75

$5.2 .2 \quad$ Fit Seeding . . . . . . . . . . . . . . . 78

5.2 .3 Particle Identification . . . . . . . . . . . . . 79

5.2 .4 Fit Resolution . . . . . . . . . . . . . . . 81 
5.3 The $\mathrm{CC} \pi^{+}$Fitter . . . . . . . . . . . . . . . . 82

5.3 .1 Fit Seeding ........................ 84

$5.3 .2 \quad$ Fit Results . . . . . . . . . . . . . . . . . . 87

$6 \mathrm{CC} \pi^{+}$Cross Section Analysis $\quad 94$

6.1 Cross Section Definitions . . . . . . . . . . . . . . . . 94

6.2 Defining the Signal: "Effective" CC $\pi^{+}$Events . . . . . . . . . . . . . . 97

6.3 Event Selection . . . . . . . . . . . . . . . . . . . . . 98

6.4 Cross Section Measurements . . . . . . . . . . . . . . . . . . . . 102

6.4.1 Event Rate Corrections . . . . . . . . . . . . . . . . 105

6.4 .2 Data Unfolding . . . . . . . . . . . . . . . . 105

6.4 .3 Unfolding Bias . . . . . . . . . . . . . . . 108

6.4.4 Efficiency Correction . . . . . . . . . . . . . . . . 113

6.4.5 Interaction Targets . . . . . . . . . . . . . . . 114

6.4.6 Flux Factor . . . . . . . . . . . . . . . . . 115

6.4 .7 Systematic Uncertainties . . . . . . . . . . . . . . . . 116

6.4 .8 Results . . . . . . . . . . . . . . . . . . 122

7 Conclusion 152

$\begin{array}{ll}\text { Bibliography } & 155\end{array}$

\section{Appendix}

$\begin{array}{ll}\text { A Event Displays } & 160\end{array}$

A.1 Truth Seeds . . . . . . . . . . . . . . . . . . . . . 161

A.2 A CC $\pi^{+}$Fit Example . . . . . . . . . . . . . . . 175 
$\begin{array}{ll}\text { B } \text { Measurement Details } & 187\end{array}$

B.1 Neutrino Energy . . . . . . . . . . . . . . . . . . . . . . 188

B.2 Q Squared .......................... 192

B.3 Muon Kinetic Energy _ . . . . . . . . . . . . . . . . . . . 196

B.4 Muon Direction . . . . . . . . . . . . . . . . 200

B.5 Pion Kinetic Energy . . . . . . . . . . . . . . . . . . . 204

B.6 Pion Direction . . . . . . . . . . . . . . . 208

B.7 Pion/Nucleon Mass . . . . . . . . . . . . . . . . . . . . . . 212

B.8 Q Squared vs Neutrino Energy . . . . . . . . . . . . . . . 213

B.9 Muon Kinetic Energy vs Neutrino Energy . . . . . . . . . . . . . . . 219

B.10 Muon Direction vs Neutrino Energy . . . . . . . . . . . . . . 225

B.11 Pion Kinetic Energy vs Neutrino Energy . . . . . . . . . . . . . . 231

B.12 Pion Direction vs Neutrino Energy . . . . . . . . . . . . . . 237

B.13 Muon Direction vs Kinetic Energy . . . . . . . . . . . . . . . . 243

B.14 Pion Direction vs Kinetic Energy . . . . . . . . . . . . . . . . . . 249

B.15 Cross Section Values . . . . . . . . . . . . . . . 255 


\section{Tables}

\section{Table}

3.1 The optical parameters of the beam are listed just upstream of the target. 27

4.1 The beam optics parameters are listed just upstream of the target. . . . 29

4.2 A list of Sanford-Wang parameters for the $\pi^{+}, \pi^{-}$, and $K_{L}$, fits is shown. 36

4.3 The result for each of the Feynman scaling parameters in the $K^{+}$fit is

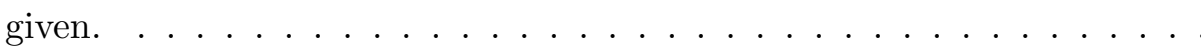

4.4 The branching fractions used in the beam Monte Carlo simulation are given for $\pi^{ \pm}, K^{ \pm}$, and $K_{L}$. The values that have been modified from their Particle Data Group values are labeled as "mod." For the negatively charged mesons, the same values are used for the charge conjugate

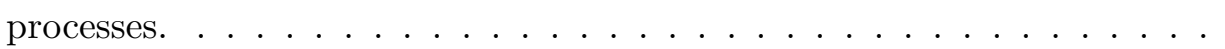

4.5 The parameters for the exponential weighting function in Equation 4.5 are given for each modified particle species. . . . . . . . . . . . .

4.6 Event rate fractions produced by Nuance using the MiniBooNE flux from Figure 4.8. The $\mathrm{CC} \pi^{+}$and $\mathrm{NC} \pi^{0}$ fractions include coherent scattering off of the entire nucleus. . . . . . . . . . . . . .

4.7 The axial mass values for CCQE and CCmulti $\pi$ interactions are listed with associated systematic uncertainties. . . . . . . . . . .

6.1 Signal efficiency, purity, and composition . . . . . . . . . . . . . 102 
6.2 Background acceptance and composition . . . . . . . . . . . 103

6.3 Uncertainties in the integrated flux from each of the flux error sources . 122

B.1 The $\sigma\left(E_{\nu}\right)$ results from Figure 6.11 are given with the total uncertainty. 255

B.2 The $\partial \sigma / \partial\left(Q^{2}\right)$ results from Figure 6.12 are given with the total uncertainty.256

B.3 The $\partial \sigma / \partial\left(K E_{\mu}\right)$ results from Figure 6.13 are given with the total uncertainty. . . . . . . . . . . . . . . . . 257

B.4 The $\partial \sigma / \partial\left(\cos \left(\theta_{\mu, \nu}\right)\right)$ results from Figure 6.14 are given with the total uncertainty. . . . . . . . . . . . . . . . 258

B.5 The $\partial \sigma / \partial\left(K E_{\pi}\right)$ results from Figure 6.15 are given with the total uncertainty. . . . . . . . . . . . . . . . . . 259

B.6 The $\partial \sigma / \partial\left(\cos \left(\theta_{\pi, \nu}\right)\right)$ results from Figure 6.16 are given with the total uncertainty. . . . . . . . . . . . . . . . . 260

B.7 The first of three tables detailing the $\partial \sigma\left(E_{\nu}\right) / \partial\left(Q^{2}\right)$ results from Figure $6.17\left(10^{-44} \mathrm{~cm}^{2} / \mathrm{MeV}^{2}\right) \ldots \ldots \ldots \ldots \ldots 26 \ldots \ldots$

B.8 The second of three tables detailing the $\partial \sigma\left(E_{\nu}\right) / \partial\left(Q^{2}\right)$ results from Figure $6.17\left(10^{-44} \mathrm{~cm}^{2} / \mathrm{MeV}^{2}\right) \ldots \ldots \ldots \ldots \ldots 26 \ldots \ldots \ldots$

B.9 The third of three tables detailing the $\partial \sigma\left(E_{\nu}\right) / \partial\left(Q^{2}\right)$ results from Figure $6.17\left(10^{-44} \mathrm{~cm}^{2} / \mathrm{MeV}^{2}\right) \ldots \ldots \ldots \ldots \ldots \ldots$

B.10 The first of three tables detailing the percent uncertainty of the $\partial \sigma\left(E_{\nu}\right) / \partial\left(Q^{2}\right)$ results from Figure $6.17 . \quad \ldots \ldots \ldots$. . . . . . . . . . . 264

B.11 The second of three tables detailing the percent uncertainty of the $\partial \sigma\left(E_{\nu}\right) / \partial\left(Q^{2}\right)$ results from Figure $6.17 . \ldots \ldots \ldots \ldots$

B.12 The third of three tables detailing the percent uncertainty of the $\partial \sigma\left(E_{\nu}\right) / \partial\left(Q^{2}\right)$ results from Figure $6.17 . \ldots \ldots \ldots 266$

B.13 The first of three tables detailing the $\partial \sigma\left(E_{\nu}\right) / \partial\left(K E_{\mu}\right)$ results from Figure $6.20\left(10^{-41} \mathrm{~cm}^{2} / \mathrm{MeV}\right) . \ldots \ldots \ldots \ldots \ldots 26 \ldots \ldots \ldots$ 
B.14 The second of three tables detailing the $\partial \sigma\left(E_{\nu}\right) / \partial\left(K E_{\mu}\right)$ results from Figure $6.20\left(10^{-41} \mathrm{~cm}^{2} / \mathrm{MeV}\right) . \ldots \ldots \ldots \ldots$

B.15 The third of three tables detailing the $\partial \sigma\left(E_{\nu}\right) / \partial\left(K E_{\mu}\right)$ results from Figure $6.20\left(10^{-41} \mathrm{~cm}^{2} / \mathrm{MeV}\right) . \ldots \ldots \ldots \ldots \ldots$

B.16 The first of three tables detailing the percent uncertainty of the $\partial \sigma\left(E_{\nu}\right) / \partial\left(K E_{\mu}\right)$ results from Figure $6.20 . \quad \ldots \ldots \ldots$. . . . . . . . . 270

B.17 The second of three tables detailing the percent uncertainty of the $\partial \sigma\left(E_{\nu}\right) / \partial\left(K E_{\mu}\right)$ results from Figure $6.20 . \quad \ldots \ldots \ldots . \ldots . \ldots . \ldots 271$

B.18 The third of three tables detailing the percent uncertainty of the $\partial \sigma\left(E_{\nu}\right) / \partial\left(K E_{\mu}\right)$ results from Figure $6.20 . \quad \ldots \ldots \ldots$. . . . . . . . . . 272

B.19 The first of three tables detailing the $\partial \sigma\left(E_{\nu}\right) / \partial\left(\cos \left(\theta_{\mu, \nu}\right)\right)$ results from Figure $6.23\left(10^{-38} \mathrm{~cm}^{2}\right) \ldots \ldots \ldots \ldots \ldots . \ldots \ldots . \ldots \ldots$

B.20 The second of three tables detailing the $\partial \sigma\left(E_{\nu}\right) / \partial\left(\cos \left(\theta_{\mu, \nu}\right)\right)$ results from Figure $6.23\left(10^{-38} \mathrm{~cm}^{2}\right) . \ldots \ldots \ldots \ldots \ldots . \ldots \ldots . \ldots \ldots$

B.21 The third of three tables detailing the $\partial \sigma\left(E_{\nu}\right) / \partial\left(\cos \left(\theta_{\mu, \nu}\right)\right)$ results from Figure $6.23\left(10^{-38} \mathrm{~cm}^{2}\right) \ldots \ldots \ldots \ldots \ldots \ldots . \ldots \ldots \ldots$

B.22 The first of three tables detailing the percent uncertainty of the $\partial \sigma\left(E_{\nu}\right) / \partial\left(\cos \left(\theta_{\mu, \nu}\right)\right)$ results from Figure $6.23 . \quad \ldots \ldots \ldots$. . . . . . . . . . 276

B.23 The second of three tables detailing the percent uncertainty of the $\partial \sigma\left(E_{\nu}\right) / \partial\left(\cos \left(\theta_{\mu, \nu}\right)\right)$ results from Figure $6.23 . \quad \ldots \ldots \ldots$. . . . . . . . . . . 277

B.24 The third of three tables detailing the percent uncertainty of the $\partial \sigma\left(E_{\nu}\right) / \partial\left(\cos \left(\theta_{\mu, \nu}\right)\right)$ results from Figure $6.23 . \quad \ldots \ldots \ldots$. . . . . . . . . . . . 278

B.25 The first of three tables detailing the $\partial \sigma\left(E_{\nu}\right) / \partial\left(K E_{\pi}\right)$ results from Figure $6.26\left(10^{-41} \mathrm{~cm}^{2} / \mathrm{MeV}\right) . \ldots \ldots \ldots \ldots \ldots \ldots$

B.26 The second of three tables detailing the $\partial \sigma\left(E_{\nu}\right) / \partial\left(K E_{\pi}\right)$ results from Figure $6.26\left(10^{-41} \mathrm{~cm}^{2} / \mathrm{MeV}\right) . \ldots \ldots \ldots \ldots . \ldots \ldots$ 
B.27 The third of three tables detailing the $\partial \sigma\left(E_{\nu}\right) / \partial\left(K E_{\pi}\right)$ results from Figure $6.26\left(10^{-41} \mathrm{~cm}^{2} / \mathrm{MeV}\right) . \ldots \ldots \ldots \ldots \ldots \ldots$

B.28 The first of three tables detailing the percent uncertainty of the $\partial \sigma\left(E_{\nu}\right) / \partial\left(K E_{\pi}\right)$ results from Figure $6.26 . \quad \ldots \ldots \ldots \ldots$. . . . . . . . . 281

B.29 The second of three tables detailing the percent uncertainty of the $\partial \sigma\left(E_{\nu}\right) / \partial\left(K E_{\pi}\right)$ results from Figure $6.26 . \quad \ldots \ldots \ldots \ldots$

B.30 The third of three tables detailing the percent uncertainty of the $\partial \sigma\left(E_{\nu}\right) / \partial\left(K E_{\pi}\right)$ results from Figure $6.26 . \quad \ldots \ldots \ldots$. . . . . . . . . . . . . 282

B.31 The first of three tables detailing the $\partial \sigma\left(E_{\nu}\right) / \partial\left(\cos \left(\theta_{\pi, \nu}\right)\right)$ results from Figure $6.29\left(10^{-38} \mathrm{~cm}^{2}\right) \ldots \ldots \ldots \ldots \ldots \ldots$

B.32 The second of three tables detailing the $\partial \sigma\left(E_{\nu}\right) / \partial\left(\cos \left(\theta_{\pi, \nu}\right)\right)$ results from Figure $6.29\left(10^{-38} \mathrm{~cm}^{2}\right) . \ldots \ldots \ldots \ldots . \ldots \ldots \ldots$

B.33 The third of three tables detailing the $\partial \sigma\left(E_{\nu}\right) / \partial\left(\cos \left(\theta_{\pi, \nu}\right)\right)$ results from Figure $6.29\left(10^{-38} \mathrm{~cm}^{2}\right) . \ldots \ldots \ldots \ldots \ldots . \ldots \ldots 285$

B.34 The first of three tables detailing the percent uncertainty of the $\partial \sigma\left(E_{\nu}\right) / \partial\left(\cos \left(\theta_{\pi, \nu}\right)\right)$ results from Figure $6.29 . \quad \ldots \ldots \ldots$. . . . . . . . . . . 286

B.35 The second of three tables detailing the percent uncertainty of the $\partial \sigma\left(E_{\nu}\right) / \partial\left(\cos \left(\theta_{\pi, \nu}\right)\right)$ results from Figure $6.29 . \quad \ldots \ldots \ldots$. . . . . . . . . . . 287

B.36 The third of three tables detailing the percent uncertainty of the $\partial \sigma\left(E_{\nu}\right) / \partial\left(\cos \left(\theta_{\pi, \nu}\right)\right)$ results from Figure $6.29 . \quad \ldots \ldots \ldots$. . . . . . . . . . . . 288

B.37 The first of three tables detailing the $\partial^{2} \sigma / \partial\left(K E_{\mu}\right) \partial\left(\cos \left(\theta_{\mu, \nu}\right)\right)$ results from Figure $6.32\left(10^{-42} \mathrm{~cm}^{2} / \mathrm{MeV}\right) . \quad \ldots \ldots \ldots$. . . . . . . . . 289

B.38 The second of three tables detailing the $\partial^{2} \sigma / \partial\left(K E_{\mu}\right) \partial\left(\cos \left(\theta_{\mu, \nu}\right)\right)$ results from Figure $6.32\left(10^{-42} \mathrm{~cm}^{2} / \mathrm{MeV}\right) . \quad \ldots \ldots \ldots . \ldots . \ldots . . \ldots 290$

B.39 The third of three tables detailing the $\partial^{2} \sigma / \partial\left(K E_{\mu}\right) \partial\left(\cos \left(\theta_{\mu, \nu}\right)\right)$ results from Figure $6.32\left(10^{-42} \mathrm{~cm}^{2} / \mathrm{MeV}\right)$. 
B.40 The first of three tables detailing the percent uncertainty of the $\partial^{2} \sigma / \partial\left(K E_{\mu}\right) \partial\left(\cos \left(\theta_{\mu, \nu}\right)\right)$

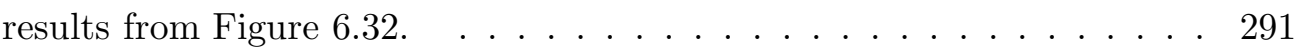

B.41 The second of three tables detailing the percent uncertainty of the $\partial^{2} \sigma / \partial\left(K E_{\mu}\right) \partial\left(\cos \left(\theta_{\mu, \nu}\right)\right)$ results from Figure $6.32 . \quad \ldots \ldots \ldots 292$

B.42 The third of three tables detailing the percent uncertainty of the $\partial^{2} \sigma / \partial\left(K E_{\mu}\right) \partial\left(\cos \left(\theta_{\mu, \nu}\right)\right)$

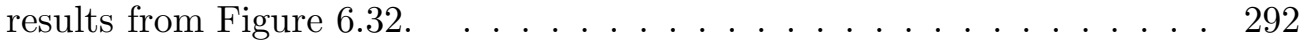

B.43 The first of three tables detailing the $\partial^{2} \sigma / \partial\left(K E_{\pi}\right) \partial\left(\cos \left(\theta_{\pi, \nu}\right)\right)$ results from Figure $6.35\left(10^{-41} \mathrm{~cm}^{2} / \mathrm{MeV}\right) . \quad \ldots \ldots \ldots . \ldots . \ldots . \ldots 293$

B.44 The second of three tables detailing the $\partial^{2} \sigma / \partial\left(K E_{\pi}\right) \partial\left(\cos \left(\theta_{\pi, \nu}\right)\right)$ results from Figure $6.35\left(10^{-41} \mathrm{~cm}^{2} / \mathrm{MeV}\right) . \quad \ldots \ldots \ldots . \ldots . \ldots . \ldots 294$

B.45 The third of three tables detailing the $\partial^{2} \sigma / \partial\left(K E_{\pi}\right) \partial\left(\cos \left(\theta_{\pi, \nu}\right)\right)$ results from Figure $6.35\left(10^{-41} \mathrm{~cm}^{2} / \mathrm{MeV}\right) . \quad \ldots \ldots \ldots . \ldots . \ldots . . \ldots 295$

B.46 The first of three tables detailing the percent uncertainty of the $\partial^{2} \sigma / \partial\left(K E_{\pi}\right) \partial\left(\cos \left(\theta_{\pi, \nu}\right)\right)$ results from Figure $6.35 . \quad \ldots \ldots \ldots . \ldots . \ldots . \ldots 296$

B.47 The second of three tables detailing the percent uncertainty of the $\partial^{2} \sigma / \partial\left(K E_{\pi}\right) \partial\left(\cos \left(\theta_{\pi, \nu}\right)\right)$ results from Figure $6.35 . \quad \ldots \ldots \ldots$. . . . . . . . . . . 297

B.48 The third of three tables detailing the percent uncertainty of the $\partial^{2} \sigma / \partial\left(K E_{\pi}\right) \partial\left(\cos \left(\theta_{\pi, \nu}\right)\right)$

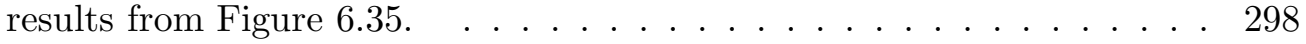




\section{Figures}

\section{Figure}

1.1 A Feynman diagram is shown for CCQE interactions. There is a lepton and a nucleon in both the initial and final states, which accounts for the (quasi) elastic character of the interaction. . . . . . . . . . . .

1.2 Charged current and Neutral current Feynman diagrams are shown for the resonant $\nu N$ interactions considered by the Rein-Sehgal model. . . .

2.1 A cartoon of the neutrino flux production is shown. Protons from the Booster interact in the target, which is located inside the horn. The secondary particles created in the interactions are focused by the horn and decay within the decay region. The resulting neutrinos then travel through half a kilometer of earth before reaching the MiniBooNE Detec-

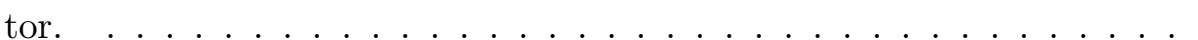

2.2 The layout of the Booster Neutrino Beam [25] is shown. The extraction point from the Main Injector $8 \mathrm{GeV}$ transfer line is shown in the bottom right. The beam then passes to the MI-12 service building, which houses the target and horn. The secondary beam created in MI-12 passes 
2.3 The weekly and integrated protons on target delivered to MiniBooNE are given from November 2002 through August 2008. The periods of neutrino $(\nu)$ and anti-neutrino $(\bar{\nu})$ horn configurations have been separated for clarity. Only the neutrino configuration is used for the present analysis.

2.4 An elevation view of the horn and water cooling system [26]. The outer conductor is transparent to show the inner horn structure. . . . . . .

3.1 A schematic of the MiniBooNE detector. Half of the front face has been removed to reveal the internal structure. The tank photomultiplier tubes are shown along the inside surface of the main fiducial region. The white outer shell houses the veto tubes $[27] \ldots \ldots \ldots \ldots$

3.2 An illustration of the vault and surface building that house the MiniBooNE detector. The earth overburden is shown covering the enclosure $[27] \ldots \ldots \ldots \ldots \ldots \ldots \ldots \ldots \ldots \ldots \ldots \ldots \ldots \ldots \ldots \ldots \ldots \ldots \ldots \ldots$

3.3 The distribution of "old" Hamamatsu R1480 (clear) and "new" Hamamatsu R5912 (solid) photomultiplier tubes over the inside surface of the

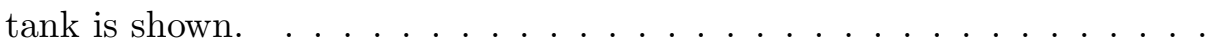

3.4 The PMT configurations for both the main tank and veto region are shown. The tank PMTs face the center of the fiducial volume, while the veto PMTs are arranged to view the directions perpendicular to the tank

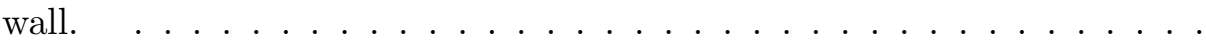

3.5 A cartoon of the PMT electronic is shown. A PMT hit triggers the asynchronous discriminator, which triggers a voltage ramp, $V_{t}$. The integrated charge, $V_{q}$, and $V_{t}$ are recorded at the 4 times labeled (t-1) through $(\mathrm{t}+2)$ from which the charge and time is deduced. $\ldots \ldots \ldots$ 
3.6 The energy determined by the muon range in the tracker/cube system is compared with the visible energy in the tank. The data show the expected linear correlation and agree well with the Monte Carlo simulation. . . .

4.1 The horn geometry in the beam Monte Carlo simulation (top) is compared with the engineering draw (bottom). The critical feature is the shape of the inner (low R) conductor, which is well modeled. . . . . . .

4.2 The nucleon total (top), inelastic (middle), and quasi-elastic (bottom) parametrizations for interactions with beryllium (left) and aluminum (right) are shown. The systematic errors assumed for each are also given and compared with data where possible $[36] . \ldots \ldots \ldots \ldots$

4.3 The pion total (top), inelastic (middle), and quasi-elastic (bottom) parametrizations for interactions with beryllium (left) and aluminum (right) are shown. The systematic errors assumed for each are also given and compared with data where possible $[36] . \ldots \ldots 33$

4.4 The result of the $\pi^{+}$Sanford-Wang fit to HARP and E910 data is shown with the HARP data (red points) overlayed. The uncertainties from both the initial fit (dashed line) and the fit with scaled data errors (dotted line)

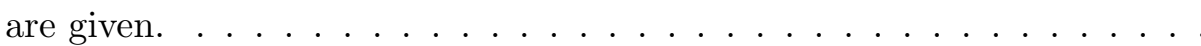

4.5 The number of neutrinos that hit the detector is plotted for each neutrino parent. Note that this plot has been made after beam Monte Carlo cross section weighting has been implemented, which exponentially increases the high multiplicity events. Even with this amplification, $75 \%$ of neutrino parents put less than 10 neutrinos in the detector. . . . . . 
4.6 The effect of the exponential weighting function of Equation 4.5 is shown on the $\pi^{+} p_{z}$ distribution after the beam Monte Carlo (left) and the parent re-decay simulation stages. The increased statistics at high $p_{z}$ improve the precision of the high energy neutrino spectrum. . . . .

4.7 The neutrino energy spectrum for each of the four neutrino species is shown for neutrinos that hit the MiniBooNE detector. . . . . . . .

4.8 The neutrino energy spectrum for $\nu_{\mu}$ particles that hit the MiniBooNE detector. The contributions from each of the neutrino parent particle types are shown separately. . . . . . . . . . . . .

4.9 The result of the spline fit (black points) is compared to the HARP data (red points) and the Sanford Wang function (blue line) as a function of pion momentum in six different pion angle bins. The uncertainties on the spline fit, shown as black error bars, grow as expected as the function moves further from the data (figure taken from Ref. [53]). . . . . . . .

4.10 The effect of the largest beam unisim excursions are shown. Any unisim that did not vary more than $2 \%$ from the central value has been excluded for clarity. The largest integrated effects are from the nucleon and pion quasi-elastic scattering uncertainties. Along the high energy tail of the neutrino flux, the skin depth variation causes changes of almost $20 \%$.

4.11 The charged current interaction processes are shown as a function of neutrino energy. The peak neutrino energy at MiniBooNE is $\sim 700 \mathrm{MeV}$. This region is dominated by $\mathrm{CCQE}$ and $\mathrm{CC} \pi^{+}$. . . . . . . . . . . 46

4.12 The pion absorption and charge exchange cross sections are shown as a function of pion kinetic energy. The absorption systematic uncertainty is $25 \%$, and the charge exchange cross section is assigned a $30 \%$ uncertainty. 49 
4.13 The GFLUKA and GCALOR predictions for pion absorption and charge exchange cross sections in oil are compared with data as a function of pion kinetic energy. The GCALOR model is used for hadronic interactions in the detector Monte Carlo simulation. . . . . . . . . . . . . . . 52

4.14 The extinction rates are given for several light sources as a function of wavelength. The total extinction curve (black) was measured using a $10 \mathrm{~cm}$ cell at Fermilab. The rates due to the 4 fluors and the effect of Rayleigh scattering are also shown. . . . . . . . . . . .

4.15 The detector Monte Carlo simulation of new and old PMTs is compared with data taken by the $397 \mathrm{~nm}$ laser. The importance of modeling scattering and reflections is evident. . . . . . . . . . .

5.1 An event display of a typical $300 \mathrm{MeV}$ muon track is shown (for a full description of the "event displays" that are shown throughout this chapter, see Appendix A). The top plot shows the Monte Carlo emission point of every optical photon created in the event, and the bottom plot gives the identity of each particle. The display is cumulative over the entire duration of the event, including the muon propagation, muon stop point, and the electron produced from the muon decay at rest. . . . . . . .

5.2 An event display of a typical $300 \mathrm{MeV}$ electron track is shown. The Monte Carlo emission point is given for every optical photon created in the event. The initial track produces photons (which produce no light) that create $e^{+} / e^{-}$pairs further downstream. . . . . . . . . . .

5.3 The scintillation light emission PDF, $\rho\left(E_{0}, s\right)$, is shown for $300 \mathrm{MeV}$ "non-hadronic" pions. . . . . . . . . . . . . . . . .

5.4 The Cherenkov light emission PDF, $\rho\left(E_{0}, s\right)$, is shown for $300 \mathrm{MeV}$ "nonhadronic" pions. . . . . . . . . . . . . . . . . 
5.5 The angular light emission PDF, $g(\cos \theta, s)$, for Cherenkov light along the particle path length is shown for $300 \mathrm{MeV}$ "non-hadronic" pions. .

5.6 The means from the Gaussian fits to the corrected time distributions (performed in bins of predicted charge and energy) have been fit to a 6 th order polynomial function of $\log (\mu)$ (still in bins of energy). The fits in four such energy bins are shown. . . . . . . . . . . . . . .

5.7 The 7 parameters that describe the 6 th order polynomials produced in the fits shown in Figure 5.6 are, themselves, fit as a function of energy. The fit to each of these 7 parameters is shown. . . . . . . . .

5.8 The Cherenkov corrected time distributions are compared with the primitive Cherenkov time parametrization for a variety of energies and pre-

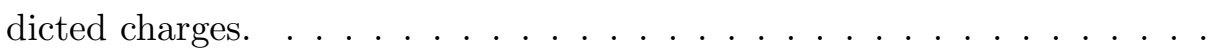

5.9 The scintillation corrected time distributions are compared with the primitive scintillation time parametrization for a variety of energies and pre-

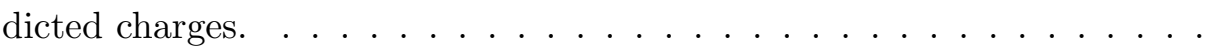

5.10 The reconstructed kinetic energies from both the muon and straight pion hypothesis fits are shown for $600 \mathrm{MeV}$ pions (left) and $600 \mathrm{MeV}$ muons (right). Hadronic interactions and decays have been turned off to correspond with conditions used to create the likelihoods. The $30 \mathrm{MeV}$ gap in the reconstructed energies of the two fit hypotheses corresponds to the $30 \mathrm{MeV}$ shift in both peaks when the generated pions are replaced by muons. (Note: All tracks in these plots have been produced at the center of the tank and point along the z-axis; track energies in this particular configuration reconstruct about $5 \%$ low overall.) . . . . . . . . 
5.11 A comparison of the muon and straight pion hypothesis fit likelihoods is shown. The plotted variable is the logarithm of the muon/pion hypothesis fit likelihood ratio. Muons (red) and pions (black) without hadronic interactions were generated at 300 (left) and $600 \mathrm{MeV}$ (right). For $300 \mathrm{MeV}$ pions, which are more indicative of a typical $\mathrm{CC} \pi^{+}$pion energy, there is no muon/pion separation. At $600 \mathrm{MeV}$, the fits to pions are shifted slightly higher than the fits to muons. . . . . . . . . .

5.12 An event display for a typical pion track is shown. The top plots show the emission point of every optical photon in the event. The bottom plots give the identity of each particle emerging from a hadronic interaction or decay. A hadronic interaction occurs (at $\mathrm{z}=100 \mathrm{~cm}$ ) that emits a sub-Cherenkov proton and causes a sharp "kink" in the pion trajectory.

5.13 The composition of a kinked track is shown. The base track provides the charge prediction for the upstream portion of the track. The anti-track charge prediction is subtracted from each PMT to remove the downstream portion of the base track. A third track that begins at the kink point provides the charge prediction for the downstream portion of the

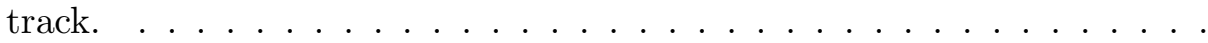

5.14 The event in Figure 5.12 has been fit with a muon hypothesis (red line), a straight pion hypothesis (magenta line), and a kinked pion hypothesis (black line). The straight fitters underestimate the track energy by more than $30 \%$. The kinked fitter provides an energy estimate that is about $10 \%$ low, which is near the edge of the energy resolution. . . . . . . 
5.15 The straight muon and kinked pion likelihood ratios are shown for muons (red) and pions (black) with full hadronic interactions and decays. The particles were generated from a flat kinetic energy distribution ranging from 50 to $450 \mathrm{MeV}$ to more closely represent the true pion energy spectrum of $\mathrm{CC} \pi^{+}$events. Unlike the $300 \mathrm{MeV}$ straight fitter comparison in Figure 5.11, there is now clear separation in the muon and pion peaks, and a large excess of pion events is seen along the high side tail. . . . .

5.16 The reconstructed pion energy resolution is shown for both the straight and kinked pion fitters (left). The kinked fitter resolution is also plotted versus the true pion energy (right). The two-dimensional plots indicate that the low fit energy "shoulder" is caused by higher energy pions. The low energy shoulder is significantly reduced in the kinked fitter, and rather than being $10 \%$ low, as is the case with the straight fitter, the peak of the kinked fitter resolution function is centered at zero. . . . . . . . .

5.17 The angle between the reconstructed and true pion directions is shown for both the straight and kinked pion fitters. The population in the lowest few bins where the properly reconstructed events lie is nearly twice as large for the kinked fitter. $\ldots \ldots \ldots \ldots \ldots$

5.18 The muon energy resolution is shown for the default Monte Carlo, and the Monte Carlo absent the extra energy fit (EFit) stages of the fit seeding process. The lower plot shows the ratio of the two distributions. The EFit Monte Carlo has a smaller width, and the peak is shifted closer to zero. 
5.19 The kinetic energy resolution is shown for muons (left) and pions (right) for all signal events and correctly paired signal events. Most of the low fit energy muon tail is due to mispaired events. The pion distribution shows the same reconstructed low energy shoulder seen in the pion-only fit in Figure $5.16(\mathrm{a}) . \ldots \ldots \ldots \ldots \ldots$

5.20 The reconstructed angle between the muon and pion directions is shown versus the larger (i.e. worse reconstructed) of the two reconstructed/true angles, $\theta\left(\mu_{\text {rec }}, \mu_{\text {true }}\right)$ and $\theta\left(\pi_{\text {rec }}, \pi_{\text {true }}\right)$. The events in the left-most columns represent events where both tracks have been properly reconstructed. The events where the tracks have been misidentified appear along the

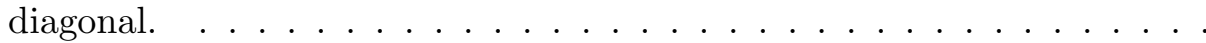

5.21 The comparison of reconstructed and true neutrino energy is shown (left) along with the Gaussian widths of the energy resolution distribution as a function of true neutrino energy (right). The resolution is about $15 \%$ over most of the energy range to which the analysis is sensitive. . . . .

5.22 The reconstructed Monte Carlo $\pi^{+}+N$ distribution is shown in the left plot for signal and background events. On the right, the background distribution is divided into CCQE background events, and all other backgrounds. Since the CCQE events do not contain a pion, they are peaked near threshold $\left(m_{\pi}+m_{N}\right)$. The definition of signal events is given in Section $6.2 \ldots \ldots \ldots \ldots \ldots \ldots \ldots$

5.23 The fractional error is plotted versus true $Q^{2}$ (left) where each true column has been normalized to one to highlight the population of misidentified events at high true $Q^{2}$, and low fit $Q^{2}$. The Gaussian width of the resolution distribution for events with correctly identified tracks is also

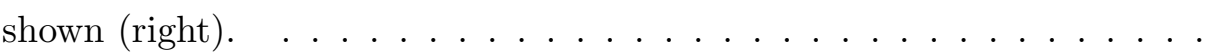


6.1 The plot shows the average hit time distribution for the first subevent (i.e. the first arrival of PMT hits) with no cuts (black), after requiring more than 200 hits in the tank region (red), after requiring less than 6 hits from PMTs in the veto region (green), and with both the tank and veto hits applied simultaneously (blue). The veto hits cut removes all events that contain particles entering or exiting the tank, and the tank hits cut removes the exponentially falling background due to decays of stopped cosmic muons that entered the tank prior to the event time window. These two cuts remove essentially all of the beam-unrelated background. Note that in the present analysis, very few Michels satisfy the three subevent cut, so the tank hits cut has been relaxed to 175 . . .

6.2 The tank hits distribution is shown for the second subevent before and after requiring less than 6 hits in the veto. The Michel electron peak is mostly unaffected, while the large tail from entering comic rays is mostly removed. ........................... 101

6.3 The data/Monte Carlo comparisons of the muon and pion trajectory tank wall distances are presented. The bottom panels show the data/Monte Carlo ratio. A cut is placed on both distributions to remove events below $150 \mathrm{~cm} \ldots \ldots \ldots \ldots \ldots \ldots \ldots$

6.4 The reconstructed versus true (top), bin migration (middle), and unfolding (bottom) matrices are shown for muon kinetic energy. The bin migration matrix is formed by normalizing the true columns of the reconstructed vs true matrix to unity, while the unfolding matrix is formed by normalizing the reconstructed rows. . . . . . . . . . . . 107 
6.5 The reconstructed versus true distribution for the two-dimensional muon kinetic energy versus cos(muon-neutrino angle) histogram is shown. Each bin of the histogram has been assigned a number between 1 and 775 and plotted in the appropriate $2 \mathrm{D}$ bin of the $775 \times 775$ reconstructed versus true histogram. . . . . . . . . . . . . . . . . . 109

6.6 The ratios of the iterated inferred true data distributions (i.e. $\sum_{j} M_{i j}^{i t e r, n}\left(D_{j}-\right.$ $\left.B_{j}\right)$ ) to the uniterated distribution are shown. The number of iterations for the numerator of each ratio are given in the legend. Note the oscillatory behavior of successive iterations, and that the largest excursion from the uniterated distribution occurs in the first iteration. . . . . . . . 111

6.7 The ratios of the iterated inferred true data distributions (i.e. $\sum_{j} M_{i j}^{i t e r, n}\left(D_{j}-\right.$ $\left.B_{j}\right)$ ) to the uniterated distribution are shown for the two-dimensional case. Note the oscillatory behavior of successive iterations, and that the largest excursion from the uniterated distribution occurs in the first iter-

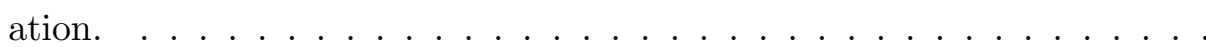

6.8 The signal efficiency is shown in bins of muon kinetic energy with Monte Carlo statistical errors. Low energy events pass the tank hits cut less frequently, and high energy tracks tend to exit the tank and trigger the

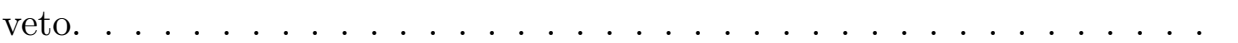

6.9 The reconstructed muon kinetic energy is plotted in each of the 67 optical model multisims. The central value Monte Carlo distribution (red) is overlayed for comparison. . . . . . . . . . . . . . . . . . 118

6.10 The $\nu_{\mu}$ flux is shown (top) along with the fractional diagonal flux errors

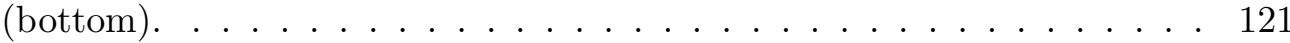


6.11 The $\sigma\left(E_{\nu}\right)$ measurement is shown with cumulative systematic errors. The absolutely normalized Monte Carlo prediction is shown for comparison. The bottom plot shows the fractional uncertainties and the ratio to the Monte Carlo prediction. . . . . . . . . . . . . . . .

6.12 The $\partial \sigma / \partial\left(Q^{2}\right)$ measurement is shown with cumulative systematic errors. The absolutely normalized Monte Carlo prediction is shown for comparison. The bottom plot shows the fractional uncertainties and the ratio to the Monte Carlo prediction. . . . . . . . . . . . . . . .

6.13 The $\partial \sigma / \partial\left(K E_{\mu}\right)$ measurement is shown with cumulative systematic errors. The absolutely normalized Monte Carlo prediction is shown for comparison. The bottom plot shows the fractional uncertainties and the ratio to the Monte Carlo prediction. . . . . . . . . . . .

6.14 The $\partial \sigma / \partial \cos \left(\theta_{\mu, \nu}\right)$ measurement is shown with cumulative systematic errors. The absolutely normalized Monte Carlo prediction is shown for comparison. The bottom plot shows the fractional uncertainties and the ratio to the Monte Carlo prediction. . . . . . . . . . . . .

6.15 The $\partial \sigma / \partial\left(K E_{\pi}\right)$ measurement is shown with cumulative systematic errors. The absolutely normalized Monte Carlo prediction is shown for comparison. The bottom plot shows the fractional uncertainties and the ratio to the Monte Carlo prediction. . . . . . . . . . . . .

6.16 The $\partial \sigma / \partial \cos \left(\theta_{\pi, \nu}\right)$ measurement is shown with cumulative systematic errors. The absolutely normalized Monte Carlo prediction is shown for comparison. The bottom plot shows the fractional uncertainties and the ratio to the Monte Carlo prediction. . . . . . . . . . . . .

6.17 The measured $\partial \sigma\left(E_{\nu}\right) / \partial\left(Q^{2}\right)$ values are shown along with the total fractional uncertainties. The Monte Carlo predicted cross section is shown for comparison. .................... 
6.18 The fractional systematic uncertainties on the measured $\partial \sigma\left(E_{\nu}\right) / \partial\left(Q^{2}\right)$ values are shown for four of the error sources. The central value measurement is shown for reference. Note that the color scales differ for each systematic error source. An explanation of each error is given in Section $6.4 .7 \ldots \ldots \ldots \ldots \ldots \ldots \ldots$

6.19 The fractional systematic uncertainties on the measured $\partial \sigma\left(E_{\nu}\right) / \partial\left(Q^{2}\right)$ values are shown for four of the error sources. The central value measurement is shown for reference. Note that the color scales differ for each systematic error source. An explanation of each error is given in Section $6.4 .7 \ldots \ldots \ldots \ldots \ldots \ldots \ldots$

6.20 The measured $\partial \sigma\left(E_{\nu}\right) / \partial\left(K E_{\mu}\right)$ values are shown along with the total fractional uncertainties. The Monte Carlo predicted cross section is shown for comparison. . . . . . . . . . . . . . . .

6.21 The fractional systematic uncertainties on the measured $\partial \sigma\left(E_{\nu}\right) / \partial\left(K E_{\mu}\right)$ values are shown for four of the error sources. The central value measurement is shown for reference. Note that the color scales differ for each systematic error source. An explanation of each error is given in Section $6.4 .7 \ldots \ldots \ldots \ldots \ldots \ldots$

6.22 The fractional systematic uncertainties on the measured $\partial \sigma\left(E_{\nu}\right) / \partial\left(K E_{\mu}\right)$ values are shown for four of the error sources. The central value measurement is shown for reference. Note that the color scales differ for each systematic error source. An explanation of each error is given in Section $6.4 .7 \ldots \ldots \ldots \ldots \ldots \ldots \ldots$

6.23 The measured $\partial \sigma\left(E_{\nu}\right) / \partial\left(\cos \left(\theta_{\mu, \nu}\right)\right)$ values are shown along with the total fractional uncertainties. The Monte Carlo predicted cross section is shown for comparison. . . . . . . . . . . . . . . . . 137 
6.24 The fractional systematic uncertainties on the measured $\partial \sigma\left(E_{\nu}\right) / \partial\left(\cos \left(\theta_{\mu, \nu}\right)\right)$ values are shown for four of the error sources. The central value measurement is shown for reference. Note that the color scales differ for each systematic error source. An explanation of each error is given in

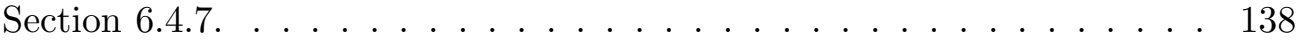

6.25 The fractional systematic uncertainties on the measured $\partial \sigma\left(E_{\nu}\right) / \partial\left(\cos \left(\theta_{\mu, \nu}\right)\right)$ values are shown for four of the error sources. The central value measurement is shown for reference. Note that the color scales differ for each systematic error source. An explanation of each error is given in

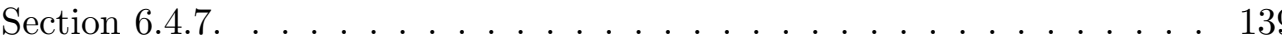

6.26 The measured $\partial \sigma\left(E_{\nu}\right) / \partial\left(K E_{\pi}\right)$ values are shown along with the total fractional uncertainties. The Monte Carlo predicted cross section is shown for comparison. . . . . . . . . . . . . . . . . . . . . 140

6.27 The fractional systematic uncertainties on the measured $\partial \sigma\left(E_{\nu}\right) / \partial\left(K E_{\pi}\right)$ values are shown for four of the error sources. The central value measurement is shown for reference. Note that the color scales differ for each systematic error source. An explanation of each error is given in Section $6.4 .7 \ldots \ldots \ldots \ldots \ldots \ldots$

6.28 The fractional systematic uncertainties on the measured $\partial \sigma\left(E_{\nu}\right) / \partial\left(K E_{\pi}\right)$ values are shown for four of the error sources. The central value measurement is shown for reference. Note that the color scales differ for each systematic error source. An explanation of each error is given in Section 6.4.7. . . . . . . . . . . . . . . . . . . 142

6.29 The measured $\partial \sigma\left(E_{\nu}\right) / \partial\left(\cos \left(\theta_{\pi, \nu}\right)\right)$ values are shown along with the total fractional uncertainties. The Monte Carlo predicted cross section is shown for comparison. . . . . . . . . . . . . . . . . . . 143 
6.30 The fractional systematic uncertainties on the measured $\partial \sigma\left(E_{\nu}\right) / \partial\left(\cos \left(\theta_{\pi, \nu}\right)\right)$ values are shown for four of the error sources. The central value measurement is shown for reference. Note that the color scales differ for each systematic error source. An explanation of each error is given in

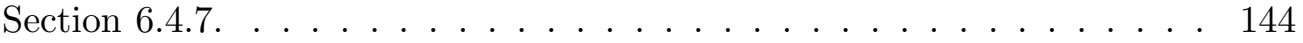

6.31 The fractional systematic uncertainties on the measured $\partial \sigma\left(E_{\nu}\right) / \partial\left(\cos \left(\theta_{\pi, \nu}\right)\right)$ values are shown for four of the error sources. The central value measurement is shown for reference. Note that the color scales differ for each systematic error source. An explanation of each error is given in

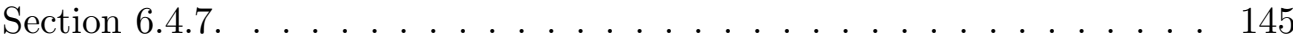

6.32 The measured $\partial^{2} \sigma / \partial\left(\cos \left(\theta_{\mu, \nu}\right)\right) \partial\left(K E_{\mu}\right)$ values are shown along with the total fractional uncertainties. The Monte Carlo predicted cross section is shown for comparison. . . . . . . . . . . . . . . 146

6.33 The fractional systematic uncertainties on the measured $\partial^{2} \sigma / \partial\left(\cos \left(\theta_{\mu, \nu}\right)\right) \partial\left(K E_{\mu}\right)$ values are shown for four of the error sources. The central value measurement is shown for reference. Note that the color scales differ for each systematic error source. An explanation of each error is given in Section $6.4 .7 \ldots \ldots \ldots \ldots \ldots \ldots \ldots \ldots$

6.34 The fractional systematic uncertainties on the measured $\partial^{2} \sigma / \partial\left(\cos \left(\theta_{\mu, \nu}\right)\right) \partial\left(K E_{\mu}\right)$ values are shown for four of the error sources. The central value measurement is shown for reference. Note that the color scales differ for each systematic error source. An explanation of each error is given in

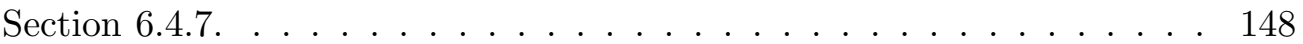

6.35 The measured $\partial^{2} \sigma / \partial\left(\cos \left(\theta_{\pi, \nu}\right)\right) \partial\left(K E_{\pi}\right)$ values are shown along with the total fractional uncertainties. The Monte Carlo predicted cross section is shown for comparison. . . . . . . . . . . . . . . . . . 149 
6.36 The fractional systematic uncertainties on the measured $\partial^{2} \sigma / \partial\left(\cos \left(\theta_{\pi, \nu}\right)\right) \partial\left(K E_{\pi}\right)$ values are shown for four of the error sources. The central value measurement is shown for reference. Note that the color scales differ for each systematic error source. An explanation of each error is given in Section $6.4 .7 \ldots \ldots \ldots \ldots \ldots \ldots$

6.37 The fractional systematic uncertainties on the measured $\partial^{2} \sigma / \partial\left(\cos \left(\theta_{\pi, \nu}\right)\right) \partial\left(K E_{\pi}\right)$ values are shown for four of the error sources. The central value measurement is shown for reference. Note that the color scales differ for each systematic error source. An explanation of each error is given in Section $6.4 .7 \ldots \ldots \ldots \ldots \ldots \ldots \ldots \ldots$

7.1 Previous measurements of $\nu_{\mu}$ induced $\mathrm{CC} \pi^{+}$measurements are shown for proton and neutron targets $[69,70,71,72,73,74,75,76]$. Those experiments not measured on $\mathrm{H}_{2}$ have been extrapolated to single nucleon cross sections. The line indicating the Nuance prediction represents the Monte Carlo predicted cross section in Figure 6.11 prior to the modeling of nuclear effects. . . . . . . . . . . . . . . . . 153

A.1 The true seed for an event with a large pion energy. About half of the pion energy is lost in the upstream track segment, with about a quarter of the energy lost in both the kink and the downstream segment. The downstream segment is well above Cherenkov threshold. . . . . . . 163

A.2 A likelihood maximum clearly exists for this event. Almost no change has occurred in the track directions. A small amount of energy from the pion track (mostly the energy lost in the hadronic interaction) has been transferred to the muon track. . . . . . . . . . . . . . . 164 
A.3 A fit seed for a low energy, unkinked pion track that creates a Cherenkov ring that overlaps with the ring from a high energy muon track is shown. The Cherenkov emission angle at the upstream portion of the muon track is $\sim 50$ degrees, and the angle between the two tracks is $\sim 15$ degrees. . .

A.4 Despite overlapping rings, there is still a likelihood maximum where both tracks are properly reconstructed. . . . . . . . . . . . . 166

A.5 A seed for an event with multiple hadronic interactions is shown. The "true" seed algorithm chooses the two longest tracks segments. The middle segment has been treated as part of the kink, which is why the "true" energy lost in the kink is so large. . . . . . . . . . . . . . . . . 167

A.6 The likelihood maximum was able to be properly reconstructed, in the sense that the physically interesting quantities are the initial energies and directions of each track. (Note: the reconstruction is less sensitive to the event vertex location.) _.................. 168

A.7 More than half of the initial pion energy is lost in the hadronic interaction in this event, and the downstream segment emerges from the interaction below Cherenkov threshold. . . . . . . . . . . . . . .

A.8 The upstream pion segment is lengthened slightly, and the energy lost in the kink is increased somewhat, but the energy separation between the pion and the muon remains mostly unchanged. . . . . . . . . . .

A.9 This seed underestimates the energy lost in the upstream pion segment, and instead puts all of the excess energy into the hadronic interaction.

A.10 The fitter has added the extra $50 \mathrm{MeV}$ required for the upstream track segment, but only $17 \mathrm{MeV}$ of that total has been taken from the hadronic energy loss. The downstream segment has also increased, largely due to a lack of constraints due to the close proximity of the tank wall, which then forces the total pion energy to be increased. 
A.11 This seed is for an event in which the pion does not experience a hadronic

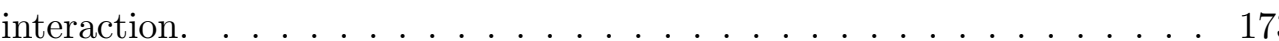

A.12 A kink has been introduced to the pion trajectory, but it occurs after the track has crossed below Cherenkov threshold where there are no constraints on the track direction. . . . . . . . . . . . .

A.13 $\mathrm{CC} \pi^{+}$fit/seeding step 1: the one track fitter has found the ring belonging to the upstream segment of the kinked pion track. The fit energy overestimates the pion energy in an attempt to account for the additional light created by the other two tracks in the event. . . . . . . . . . . . 176

A.14 $\mathrm{CC} \pi^{+}$fit/seeding step 2: the likelihood scan for the second track (keeping the result of the one track fit fixed) has found the muon. The scan was performed with a track energy of $150 \mathrm{MeV}$, although $200 \mathrm{MeV}$ is used in the final version of the fitter. Although the scan found a ring, the track direction isn't perfect. The underestimate for the track energy means that the predicted ring diameter is smaller than the actual ring diameter, so the scan has some freedom in choosing the actual track direction. . . 177

A.15 $\mathrm{CC} \pi^{+}$fit/seeding step 3: the two track fit has corrected the directions of both tracks to point at the center of their respective rings. The energy overestimate from the one track fit has been greatly reduced, although the second track energy has only been slightly increased so far. . . . . .

A.16 CC $\pi^{+}$fit/seeding step 4: the three track likelihood scan has landed near the most downstream portion of the downstream pion track. Some portion of the upstream pion ring may have pulled the scan slightly toward the upstream pion track. . . . . . . . . . . . . . . . 179 
A.17 CC $\pi^{+}$fit/seeding step 5: the three track fit has corrected the final scan track to point in the initial direction of the downstream pion segment. The energy has also been redistributed among the three tracks to more accurately represent the size of the Cherenkov rings. The first two tracks now only slightly underestimate the true muon and pion energies. . . . 180

A.18 $\mathrm{CC} \pi^{+}$fit/seeding step 6: the first of the three seed configurations has picked the incorrect tracks for the muon and upstream pion segments. Since the upstream pion segment does not actually point at a pion track segment, the simultaneous $\Delta E_{u p} / \Delta E_{k i n k}$ scan has incorrectly placed the energy lost in the kink at $300 \mathrm{MeV} . \ldots \ldots \ldots$

A.19 $\mathrm{CC} \pi^{+}$fit/seeding step 7: the fit result for the incorrect seed in Figure A.18 is still incorrect, as expected. The fit minimum is: $-\log (\mathcal{L})=$

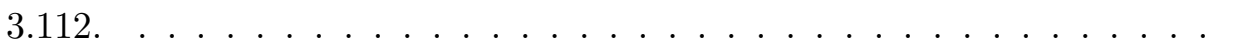

A.20 CC $\pi^{+}$fit/seeding step 8: the second of the three seed configurations has picked the incorrect tracks for the muon and downstream pion segments. 183

A.21 $\mathrm{CC} \pi^{+}$fit/seeding step 9: the fit result for the incorrect seed in Figure A.20 is still incorrect, as expected. The fit minimum is: $-\log (\mathcal{L})=$

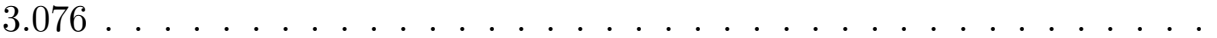

A.22 $\mathrm{CC} \pi^{+}$fit/seeding step 10: the last $\mathrm{CC} \pi^{+}$seed configuration correctly assigns all three tracks. . . . . . . . . . . . . .

A.23 $\mathrm{CC} \pi^{+}$fit/seeding step 10: the $\mathrm{CC} \pi^{+}$fit from the correct seed gives the best fit, $-\log (\mathcal{L})=2.982$.

B.1 A Data/Monte Carlo comparison of reconstructed neutrino energy is shown with cumulative systematic errors. The bottom plot shows the fractional errors compared with the Data/Monte Carlo ratio. . . . . . . 
B.2 The reconstructed vs true distribution for neutrino energy is shown, along with the unfolding matrix used for the central value cross section measurement. . . . . . . . . . . . . . . . . . . . . 190

B.3 The signal efficiency and purity is given in terms of neutrino energy. The error bars represent the Monte Carlo statistical uncertainty. . . . . . . 191

B.4 A Data/Monte Carlo comparison of reconstructed $Q^{2}$ is shown with cumulative systematic errors. The bottom plot shows the fractional errors compared with the Data/Monte Carlo ratio. . . . . . . . . . . .

B.5 The reconstructed vs true distribution for $Q^{2}$ is shown, along with the unfolding matrix used for the central value cross section measurement.

B.6 The signal efficiency and purity is given in terms of $Q^{2}$. The error bars represent the Monte Carlo statistical uncertainty. . . . . . . . . . . 195

B.7 A Data/Monte Carlo comparison of reconstructed muon kinetic energy is shown with cumulative systematic errors. The bottom plot shows the fractional errors compared with the Data/Monte Carlo ratio. . . . . . .

B.8 The reconstructed vs true distribution for muon kinetic energy is shown, along with the unfolding matrix used for the central value cross section measurement. ..................... . . . .

B.9 The signal efficiency and purity is given in terms of muon kinetic energy. The error bars represent the Monte Carlo statistical uncertainty. . . . .

B.10 A Data/Monte Carlo comparison of reconstructed cos(muon, neutrino angle) is shown with cumulative systematic errors. The bottom plot shows the fractional errors compared with the Data/Monte Carlo ratio.

B.11 The reconstructed vs true distribution for $\cos ($ muon, neutrino angle) is shown, along with the unfolding matrix used for the central value cross section measurement. . . . . . . . . . . . . . . . 202 
B.12 The signal efficiency and purity is given in terms of $\cos ($ muon, neutrino angle). The error bars represent the Monte Carlo statistical uncertainty.

B.13 A Data/Monte Carlo comparison of reconstructed pion kinetic energy is shown with cumulative systematic errors. The bottom plot shows the fractional errors compared with the Data/Monte Carlo ratio. . . . . . . 205

B.14 The reconstructed vs true distribution for pion kinetic energy is shown, along with the unfolding matrix used for the central value cross section

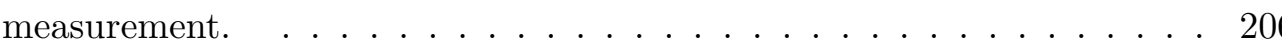

B.15 The signal efficiency and purity is given in terms of pion kinetic energy. The error bars represent the Monte Carlo statistical uncertainty. . . . . 207

B.16 A Data/Monte Carlo comparison of reconstructed cos(pion, neutrino angle) is shown with cumulative systematic errors. The bottom plot shows the fractional errors compared with the Data/Monte Carlo ratio. . . . 209

B.17 The reconstructed vs true distribution for $\cos ($ pion, neutrino angle) is shown, along with the unfolding matrix used for the central value cross section measurement. . . . . . . . . . . . . . . . . . . . . 210

B.18 The signal efficiency and purity is given in terms of $\cos ($ pion, neutrino angle). The error bars represent the Monte Carlo statistical uncertainty.

B.19 A Data/Monte Carlo comparison of reconstructed $N+\pi^{+}$mass is shown with cumulative systematic errors. The bottom plot shows the fractional errors compared with the Data/Monte Carlo ratio.

B.20 The reconstructed $Q^{2}$ vs neutrino energy distribution is shown for both the data (top) and Monte Carlo (bottom). . . . . . . . . . . . . . 214

B.21 The Monte Carlo reconstructed $Q^{2}$ vs energy distribution is shown (top) along with the total fractional uncertainties (bottom). . . . . . . . 215 
B.22 The fractional systematic uncertainties on the reconstructed Monte Carlo distribution are shown for four of the error sources. The central value reconstructed Monte Carlo distribution is also shown for reference. Note that the color scales differ for each systematic error source. An explanation of each error is given in Section 6.4.7 . . . . . . . . . . . . 216

B.23 The fractional systematic uncertainties on the reconstructed Monte Carlo distribution are shown for four of the error sources. The central value reconstructed Monte Carlo distribution is also shown for reference. Note that the color scales differ for each systematic error source. An explanation of each error is given in Section 6.4.7. . . . . . . . . . . . . . 217

B.24 The signal efficiency and purity are shown for the $Q^{2}$ vs neutrino energy distribution. The fractional errors due to Monte Carlo statistics for each of these distributions is also given for all bins with at least 10 events in the numerator. . . . . . . . . . . . . . . . . . 218

B.25 The reconstructed muon kinetic energy vs neutrino energy distribution is shown for both the data (top) and Monte Carlo (bottom). . . . . . . 220

B.26 The Monte Carlo reconstructed muon kinetic energy vs neutrino energy distribution is shown (top) along with the total fractional uncertainties

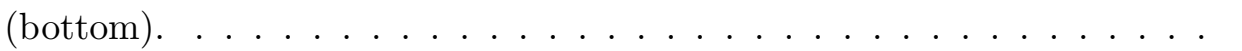

B.27 The fractional systematic uncertainties on the reconstructed Monte Carlo distribution are shown for four of the error sources. The central value reconstructed Monte Carlo distribution is shown for reference. Note that the color scales differ for each systematic error source. An explanation of each error is given in Section 6.4.7. . . . . . . . . . . . . . . . 222 
B.28 The fractional systematic uncertainties on the reconstructed Monte Carlo distribution are shown for four of the error sources. The central value reconstructed Monte Carlo distribution is shown for reference. Note that the color scales differ for each systematic error source. An explanation of each error is given in Section 6.4.7. . . . . . . . . . . . . . 223

B.29 The signal efficiency and purity are shown for the muon kinetic energy vs neutrino energy distribution. The fractional errors due to Monte Carlo statistics for each of these distributions is also given for all bins with at least 10 events in the numerator. . . . . . . . . . . . . . . . 224

B.30 The reconstructed $\cos ($ muon, neutrino angle) vs neutrino energy distribution is shown for both the data (top) and Monte Carlo (bottom). . . 226

B.31 The Monte Carlo reconstructed muon cos(muon, neutrino angle) vs neutrino energy distribution is shown (top) along with the total fractional

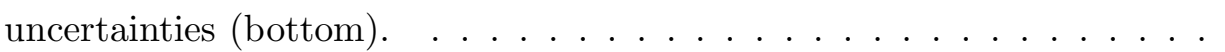

B.32 The fractional systematic uncertainties on the reconstructed Monte Carlo distribution are shown for four of the error sources. The central value reconstructed Monte Carlo distribution is shown for reference. Note that the color scales differ for each systematic error source. An explanation of each error is given in Section 6.4.7. . . . . . . . . . . . . . . 228

B.33 The fractional systematic uncertainties on the reconstructed Monte Carlo distribution are shown for four of the error sources. The central value reconstructed Monte Carlo distribution is shown for reference. Note that the color scales differ for each systematic error source. An explanation of each error is given in Section 6.4.7. . . . . . . . . . . . . . . . 229 
B.34 The signal efficiency and purity are shown for the muon $\cos ($ muon, neutrino angle) vs neutrino energy distribution. The fractional errors due to Monte Carlo statistics for each of these distributions is also given for all bins with at least 10 events in the numerator. . . . . . . . . . . 230

B.35 The reconstructed pion kinetic energy vs neutrino energy distribution is shown for both the data (top) and Monte Carlo (bottom). . . . . . .

B.36 The Monte Carlo reconstructed pion kinetic energy vs neutrino energy distribution is shown (top) along with the total fractional uncertainties

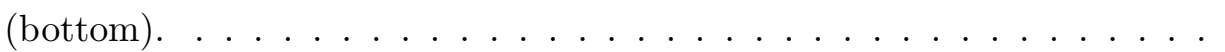

B.37 The fractional systematic uncertainties on the reconstructed Monte Carlo distribution are shown for four of the error sources. The central value reconstructed Monte Carlo distribution is shown for reference. Note that the color scales differ for each systematic error source. An explanation of each error is given in Section 6.4.7. . . . . . . . . . . . . . . . 234

B.38 The fractional systematic uncertainties on the reconstructed Monte Carlo distribution are shown for four of the error sources. The central value reconstructed Monte Carlo distribution is shown for reference. Note that the color scales differ for each systematic error source. An explanation of each error is given in Section 6.4.7. . . . . . . . . . . . . 235

B.39 The signal efficiency and purity are shown for the pion kinetic energy vs neutrino energy distribution. The fractional errors due to Monte Carlo statistics for each of these distributions is also given for all bins with at least 10 events in the numerator. . . . . . . . . . . . . . 236

B.40 The reconstructed $\cos ($ pion, neutrino angle) vs neutrino energy distribution is shown for both the data (top) and Monte Carlo (bottom). . . . 238 
B.41 The Monte Carlo reconstructed pion cos(pion, neutrino angle) vs neutrino energy distribution is shown (top) along with the total fractional uncertainties (bottom). . . . . . . . . . . . . 239

B.42 The fractional systematic uncertainties on the reconstructed Monte Carlo distribution are shown for four of the error sources. The central value reconstructed Monte Carlo distribution is shown for reference. Note that the color scales differ for each systematic error source. An explanation of each error is given in Section 6.4.7. . . . . . . . . . . . . . . 240

B.43 The fractional systematic uncertainties on the reconstructed Monte Carlo distribution are shown for four of the error sources. The central value reconstructed Monte Carlo distribution is shown for reference. Note that the color scales differ for each systematic error source. An explanation of each error is given in Section 6.4.7. . . . . . . . . . . . . . 241

B.44 The signal efficiency and purity are shown for the pion $\cos ($ pion, neutrino angle) vs neutrino energy distribution. The fractional errors due to Monte Carlo statistics for each of these distributions is also given for all bins with at least 10 events in the numerator. . . . . . . . . . . . . . . . 242

B.45 The reconstructed $\cos ($ muon, neutrino angle) vs muon kinetic energy distribution is shown for both the data (top) and Monte Carlo (bottom). .

B.46 The Monte Carlo reconstructed cos(muon, neutrino angle) vs muon kinetic energy distribution is shown (top) along with the total fractional uncertainties (bottom). . . . . . . . . . . . . . 245

B.47 The fractional systematic uncertainties on the reconstructed Monte Carlo distribution are shown for four of the error sources. The central value reconstructed Monte Carlo distribution is shown for reference. Note that the color scales differ for each systematic error source. An explanation of each error is given in Section 6.4.7. . . . . . . . . . . . . . 246 
B.48 The fractional systematic uncertainties on the reconstructed Monte Carlo distribution are shown for four of the error sources. The central value reconstructed Monte Carlo distribution is shown for reference. Note that the color scales differ for each systematic error source. An explanation of each error is given in Section 6.4.7. . . . . . . . . . . . . . . . 247

B.49 The signal efficiency and purity are shown for the cos(muon, neutrino angle) vs muon kinetic energy distribution. The fractional errors due to Monte Carlo statistics for each of these distributions is also given for all bins with at least 10 events in the numerator. . . . . . . . . . . . 248

B.50 The reconstructed cos(pion, neutrino angle) vs pion kinetic energy distribution is shown for both the data (top) and Monte Carlo (bottom). . 250

B.51 The Monte Carlo reconstructed cos(pion, neutrino angle) vs pion kinetic energy distribution is shown (top) along with the total fractional uncertainties (bottom). . . . . . . . . . . . . . . 251

B.52 The fractional systematic uncertainties on the reconstructed Monte Carlo distribution are shown for four of the error sources. The central value reconstructed Monte Carlo distribution is also shown for reference. Note that the color scales differ for each systematic error source. An explanation of each error is given in Section 6.4.7. . . . . . . . . . . . 252

B.53 The fractional systematic uncertainties on the reconstructed Monte Carlo distribution are shown for four of the error sources. The central value reconstructed Monte Carlo distribution is also shown for reference. Note that the color scales differ for each systematic error source. An explanation of each error is given in Section 6.4.7. . . . . . . . . . . . 253 
B.54 The signal efficiency and purity are shown for the pion $\cos ($ pion, neutrino angle) vs kinetic energy distribution. The fractional errors due to Monte Carlo statistics for each of these distributions is also given for all bins with at least 10 events in the numerator. . . . . . . . . . . . 254 


\section{Chapter 1}

\section{Introduction}

Neutrinos are unique among all matter particles in that they participate in only two of the four fundamental interactions. In addition to gravity, which is very weak and affects all particles, neutrinos interact only through the weak nuclear force. Since neutrino interactions are free of interfering electromagnetic and strong contributions, they are very clean probes of the structure of atomic nuclei. In addition, neutrinos are uniquely able to probe both the vector and axial-vector character of interactions with target nuclei due to the parity violating nature of the weak force.

Weak interactions can proceed via charged current (CC) or neutral current (NC) channels. In charged current interactions, a $W^{ \pm}$boson is emitted as the neutrino converts into its charged lepton partner. Neutral current interactions are facilitated by the exchange of a $Z^{0}$ boson that leaves the neutrino flavor unchanged. The specific type of charged or neutral current interaction is classified by the composition of the hadronic final state.

This introductory chapter describes the properties of charged current neutrino scattering on baryon targets. The theory of these interactions is introduced, in addition to complications that arise when these processes take place in a nuclear medium. The main topic of this document is discussed, a measurement of charged current $\pi^{+}$ production, as well as the experiment in which the measurement is made: MiniBooNE. 


\subsection{Charged Current Interactions}

At neutrino energies below a few $\mathrm{GeV}$, the most common neutrino interactions are those that minimally affect the interaction target. For charged current interactions with baryon targets, the baryon must, at a minimum, undergo a change in its electric charge to accommodate the exchange of the charged $W$ boson; these are called charged current quasi-elastic interactions. If, instead of simply altering the charge of the target baryon, the $W^{ \pm}$transfers enough momentum to promote the target into a low-mass resonance state, the decay of the resonance will typically produce a nucleon and a pion. Such processes are referred to as charged current pion production.

\subsubsection{Charged Current Quasi-Elastic Interactions}

The simplest type of charged current interaction occurs when the neutrino interacts with a nucleon and produces the requisite charged lepton along with a single nucleon in the final state. These are referred to as charged current quasi-elastic (CCQE) interactions. Since a $W^{+}$is exchanged, the hadronic current must convert from a neutron to a proton as shown in Fig. 1.1.

CCQE interactions are the dominant reaction channel for neutrino energies below $\sim 1.5 \mathrm{GeV}$. Due to its clean experimental signature, and because the neutrino type is provided by the flavor of the outgoing lepton, the CCQE channel is the preferred signal mode in neutrino oscillation searches.

\subsubsection{Charged Current Pion Production}

When a neutrino interacts with a target nucleon, the hadronic current can be excited into a resonance state. At low neutrino energies, these resonance states are composed of isospin $1 / 2\left(N^{*}\right)$ and $3 / 2(\Delta)$ states, which generally decay into a nucleon and a pion as shown in Fig. 1.2 [1]. In charged current interactions, the most common 


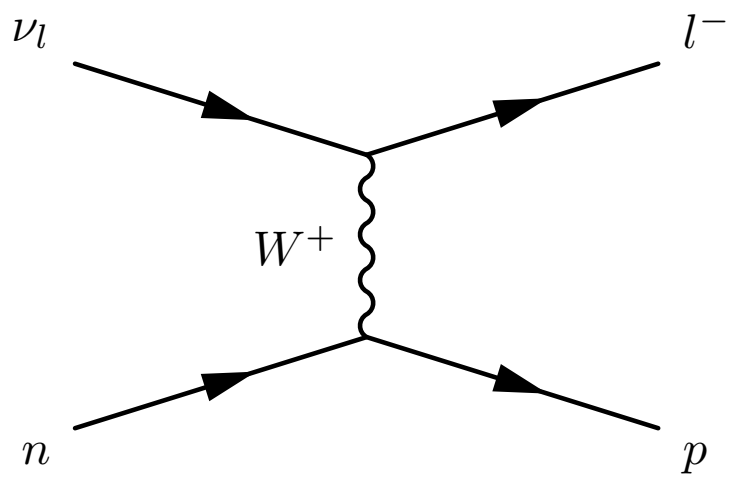

Figure 1.1: A Feynman diagram is shown for CCQE interactions. There is a lepton and a nucleon in both the initial and final states, which accounts for the (quasi) elastic character of the interaction.

of these processes produces a $\pi^{+}$particle and the original target nucleon type. This channel, $\nu_{\mu} N \rightarrow \mu^{-} \pi^{+} N$, is referred to as charged current $\pi^{+}$production $\left(\mathrm{CC} \pi^{+}\right)$.

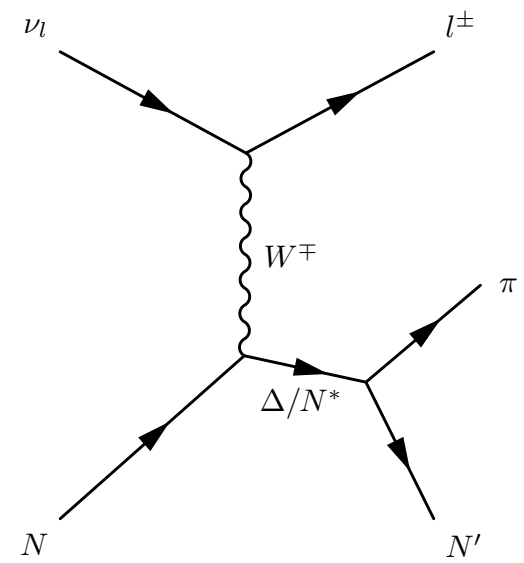

(a) Charged current resonant interaction

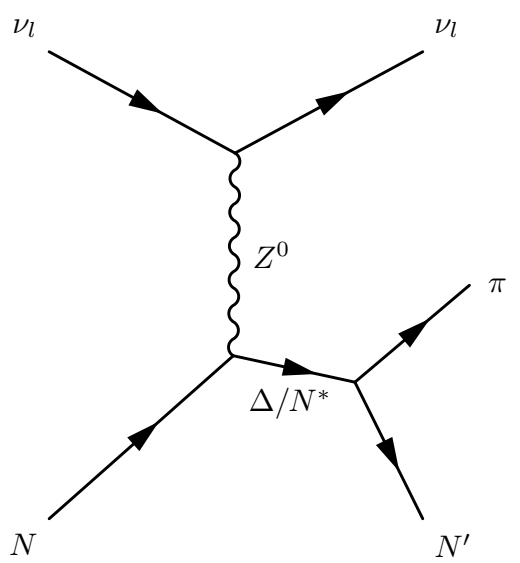

(b) Neutral current resonant interaction

Figure 1.2: Charged current and Neutral current Feynman diagrams are shown for the resonant $\nu N$ interactions considered by the Rein-Sehgal model. 


\subsubsection{Rein-Sehgal Model of $\nu$-induced Pion Production}

The Rein-Sehgal (RS) model describes all neutrino- and anti-neutrino-induced pion production processes using one uniform framework [2]. All non-strange resonances below $2 \mathrm{GeV}$ are combined, including all interference terms, to produce the amplitudes of the 14 neutrino induced pion production channels. In addition, a small amount of isospin $1 / 2$ non-resonant background is added incoherently to improve the agreement with data.

Using the weak interaction Feynman rules and leaving the hadronic current in a general form for now, the invariant amplitude is written in the following way,

$$
\begin{gathered}
T_{C C}\left(\nu N \rightarrow l N^{*}\right)=\frac{g^{2} \cos \theta_{C}}{8}\left[\bar{u}_{l} \gamma^{\mu}\left(1-\gamma^{5}\right) u_{\nu}\right]\left(\frac{g_{\mu \nu}-\frac{q_{\mu} q_{\nu}}{M_{W}^{2}}}{q^{2}-M_{W}^{2}}\right)\left\langle N^{*}\left|J^{\mu}\right| N\right\rangle \\
T_{N C}\left(\nu N \rightarrow \nu N^{*}\right)=\frac{g^{2} \cos \theta_{C}}{8 \cos ^{2} \theta_{W}}\left[\bar{u}_{l} \gamma^{\mu}\left(1-\gamma^{5}\right) u_{\nu}\right]\left(\frac{g_{\mu \nu}-\frac{q_{\mu} q_{\nu}}{M_{Z}^{2}}}{q^{2}-M_{Z}^{2}}\right)\left\langle N^{*}\left|J^{\mu}\right| N\right\rangle
\end{gathered}
$$

where $\theta_{W}$ is the weak mixing angle, and $\theta_{C}$ is the Cabibbo angle. The $W$ and $Z 4$ momenta are given by $q=\left(E_{q}, \mathbf{q}\right)$, with on-shell masses of $M_{Z}$ and $M_{W}$, respectively.

Since the model is concerned with $\left|q^{2}\right|<2 \mathrm{GeV}$, all terms proportional to $\left|q^{2}\right| / M_{W, Z}$ can be neglected. By making the identifications

$$
\frac{G_{F}}{\sqrt{2}}=\frac{g^{2}}{8 M_{W}^{2}}, \quad G=G_{F} \cos \theta_{C} \approx G_{F}, \quad \frac{M_{W}^{2}}{M_{Z}^{2} \cos ^{2} \theta_{W}} \approx 1,
$$

the expressions in Equations 1.1 and 1.2 can be combined in a single form,

$$
T\left(\nu N \rightarrow l N^{*}\right)=\frac{G}{\sqrt{2}}\left[\bar{u}_{l} \gamma^{\mu}\left(1-\gamma_{5}\right) u_{\nu}\right]\left\langle N^{*}\left|J_{\mu}\right| N\right\rangle
$$

The leptonic current matrix element gives the polarization of the exchanged $\mathrm{W}$ or $\mathrm{Z}$ boson in the interaction, and can be expressed in terms of its right-handed, left- 
handed, and scalar components, which are defined by the following unit vectors.

$$
\begin{aligned}
e_{L}^{\mu} & =\frac{1}{\sqrt{2}}(0,1,-i, 0) \\
e_{R}^{\mu} & =\frac{1}{\sqrt{2}}(0,-1,-i, 0) \\
e_{0}^{\mu} & =(1,0,0,0)
\end{aligned}
$$

The leptonic matrix element is most easily evaluated in the lepton Breit frame (LBF) where the neutrino momentum is pointed along the z-axis, and is equal and opposite to the outgoing lepton momentum. Neglecting lepton masses,

$$
\left.\bar{u}_{l} \gamma^{\mu}\left(1-\gamma_{5}\right) u_{\nu}\right|_{L B F}=-2 \sqrt{-2 q^{2}} e_{L}^{\mu}
$$

The hadronic current matrix element, however, is most conveniently expressed in the resonance rest frame $(\mathrm{RRF})$ with the incoming nucleon traveling along the -zaxis. This can be accomplished by two Lorentz transformations. The first connects the lepton Breit frame with the nucleon Breit frame (NBF) in which the incoming nucleon and outgoing resonance momenta are equal and opposite. This transformation yields

$$
\left.\bar{u}_{l} \gamma^{\mu}\left(1-\gamma_{5}\right) u_{\nu}\right|_{N B F}=-\sqrt{-2 q^{2}}\left[(1-\cosh \xi) e_{L}^{\mu}+(1-\cosh \xi) e_{R}^{\mu}+2 \sinh \xi e_{0}^{\mu}\right],
$$

where $\cosh \xi=\left(E_{\nu}^{l a b}+E_{l}^{l a b}\right) /\left|\mathbf{q}^{l a b}\right|$. To reach the RRF, an additional boost is needed along the z-axis to bring the resonance momentum to zero. Both $e_{L}^{\mu}$ and $e_{R}^{\mu}$ are unaffected by this transformation, while the scalar component can be transformed by replacing $e_{0}^{\mu}$ with $e_{s}^{\mu}$, which is defined as

$$
e_{s}^{\mu}=\frac{1}{\sqrt{-q^{2}}}\left(\left|\mathbf{q}^{R R F}\right|, 0,0, E_{q}^{R R F}\right)
$$

To simply the notation, define

$$
u=\frac{E_{\nu}^{l a b}+E_{l}^{l a b}+\left|\mathbf{q}^{l a b}\right|}{2 E_{\nu}}, \quad v=\frac{E_{\nu}^{l a b}+E_{l}^{l a b}-\left|\mathbf{q}^{l a b}\right|}{2 E_{\nu}}, \quad Q^{2}=-q^{2} .
$$


The final expression for the leptonic matrix element in the RRS is then

$$
\left.\bar{u}_{l} \gamma^{\mu}\left(1-\gamma_{5}\right) u_{\nu}\right|_{R R F}=-2 E_{\nu}^{l a b} \sqrt{\frac{-2 q^{2}}{\left|\mathbf{q}^{l a b}\right|^{2}}}\left[u e_{L}^{\mu}-v e_{R}^{\mu}+\sqrt{2 u v} e_{s}^{\mu}\right] .
$$

The energy dimension of the hadronic current, $J_{\mu}$, is removed by factoring out the resonance mass,

$$
F_{\mu}=J_{\mu} / 2 M
$$

The components of the remaining portion of the current are extracted in the same basis as the leptonic current using the same polarization unit vectors.

$$
\begin{array}{ll}
F_{+}= & e_{R}^{\mu} F_{\mu}=\frac{-1}{\sqrt{2}}\left(F_{x}+i F_{y}\right) \\
F_{-}= & e_{L}^{\mu} F_{\mu}=\frac{1}{\sqrt{2}}\left(F_{x}-i F_{y}\right) \\
F_{0}=\sqrt{\frac{Q^{2}}{\left|\mathbf{q}^{R R F}\right|^{2}}} e_{s}^{\mu} F_{\mu}=F_{t}+\frac{E_{q}}{\left|\mathbf{q}^{R R F}\right|} F_{z}
\end{array}
$$

Combining the appropriate terms of the hadronic and leptonic currents according to helicities gives

$$
T\left(\nu N \rightarrow l N^{*}\right)=-4 G M E\left[\sqrt{\frac{Q^{2}}{|\mathbf{q}|^{2}}}\left\langle N^{*}\left|u F_{-}-v F_{+}\right| N\right\rangle+\frac{m_{N}}{M} \sqrt{2 u v}\left\langle N^{*}\left|F_{0}\right| N\right\rangle\right]
$$

The invariant amplitude can be combined with the usual phase space and flux factors to produce a cross section,

$$
\frac{\partial \sigma}{\partial Q^{2} \partial E_{q}}=\frac{1}{64 \pi m_{N} E_{\nu}^{2}} \sum_{\text {spins }}\left|T\left(\nu N \rightarrow l N^{*}\right)\right|^{2} \frac{1}{2 \pi} \cdot \frac{\Gamma}{(W-M)^{2}+\Gamma^{2} / 4} .
$$

The last factor in Equation 1.17 is the Breit-Wigner function for a resonance of mass $M$, width $\Gamma$, and observed mass $W$.

To properly determine each cross section, all resonances that contribute to the interaction process must be included in the calculation. The relevant resonances for each process are determined by isospin conservation. $\mathrm{CC} \pi^{+}$events occur with two different final states, $\left(\mu^{-}, \pi^{+}\right.$,neutron) and $\left(\mu^{-}, \pi^{+}\right.$,proton $)$, depending on the target nucleon 
with which the neutrino interacts. The Clebsch-Gordan coefficients for each final state, $\left|I, I_{3}\right\rangle$, give

$$
\begin{aligned}
& \left|\pi^{+} p\right\rangle=|1,1\rangle \otimes\left|\frac{1}{2}, \frac{1}{2}\right\rangle=\left|\frac{3}{2}, \frac{3}{2}\right\rangle \\
& \left|\pi^{+} n\right\rangle=|1,1\rangle \otimes\left|\frac{1}{2},-\frac{1}{2}\right\rangle=\sqrt{\frac{1}{3}}\left|\frac{3}{2}, \frac{1}{2}\right\rangle+\sqrt{\frac{2}{3}}\left|\frac{1}{2}, \frac{1}{2}\right\rangle
\end{aligned}
$$

Isospin $3 / 2$ states are referred to as $\Delta$ resonances, whereas isospin $1 / 2$ states, having the same isospin as protons and neutrons, are simply referred to as $N$ resonances.

The final ingredient needed to determine the cross sections are the helicity amplitudes from Equation 1.16,

$$
\begin{gathered}
f_{ \pm}=\left\langle N, j_{z} \pm 1\left|F_{ \pm}\right| N^{*}, j_{z}\right\rangle \\
f_{0}=\left\langle N, j_{z}\left|F_{0}\right| N^{*}, j_{z}\right\rangle
\end{gathered}
$$

These are provided by the Feynman-Kislinger-Ravndal (FKR) relativistic quark model of hadronic states [3]. The FKR model represents hadrons as relativistic harmonic oscillators of their component quarks. For baryons, the Hamiltonian is given by

$$
\mathcal{H}=3\left(p_{a}^{2}+p_{b}^{2}+p_{c}^{2}\right)+\frac{\Omega^{2}}{36}\left(\left(u_{a}-u_{b}\right)^{2}+\left(u_{b}-u_{c}\right)^{2}+\left(u_{c}-u_{a}\right)^{2}\right)+C,
$$

where $p_{a}$ and $u_{a}$ are the 4-momentum and 4-position of quark $a$. Using only 3 adjustable constants (the spacing of energy levels per unit angular momentum, $\Omega$, the pseudoscalarmeson coupling to hadrons, and a scaling factor as a function of energy) and the particle masses, 75 different transition amplitudes have been calculated.

The FKR model has been extended by Ravndal to calculate production cross sections for all nuclear resonances below $1.75 \mathrm{GeV}$ [4]. This formulation makes use of separate vector and axial vector form factors, each with its own free mass parameter, $m_{V}$ and $m_{A}$, respectively.

$$
\begin{aligned}
& G^{V}\left(Q^{2}\right)=\left(1+\frac{Q^{2}}{4 m_{N}^{2}}\right)^{1 / 2-n}\left(\frac{1}{1+Q^{2} / m_{V}^{2}}\right)^{2} \\
& G^{A}\left(Q^{2}\right)=\left(1+\frac{Q^{2}}{4 m_{N}^{2}}\right)^{1 / 2-n}\left(\frac{1}{1+Q^{2} / m_{A}^{2}}\right)^{2}
\end{aligned}
$$


where $n$ is the number of oscillator quanta present in the final resonance. The vector mass, $m_{V}$, is well measured in electron scattering experiments to be $0.84 \mathrm{GeV}$, under the conserved vector current hypothesis [5]. The only remaining uncertain quantity in the determination of the $\mathrm{CC} \pi^{+}$cross section is the axial mass, $m_{A}$, which can only be measured in neutrino interactions.

\subsubsection{Corrections to the Rein-Sehgal Model}

The RS model, in its original form, is insufficient to fully describe the kinematics of $\mathrm{CC} \pi^{+}$interactions. There exist experimental data that significantly disagree with the model, especially at low $Q^{2}$ [6]. The data show a significant deficit of events with forward going muons relative to the prediction. Several modifications have been proposed in an attempt to explain this discrepancy [7].

In the calculation presented in the previous section, the muon mass has been neglected. Several methods for reinstating the muon mass have been proposed $[8,9,10$, 11]. The size of the correction varies depending on the method, but each method has the effect of reducing the low $Q^{2}$ prediction [7].

Alternatives to the vector and axial vector form factors used in the RS model have also been suggested. As a common example, several of these new models employ the Rarita-Schwinger formalism, in which a spin-3/2 field is introduced to describe the $\Delta^{++}$resonance $[12,13,14]$. Instead of incorporating the results of the FKR model, the vector and axial vector components of the $\Delta$ transition amplitude are written more generally in terms of several form factors, $C_{i}^{V, A}$, 


$$
\begin{aligned}
\left\langle\Delta^{++}\left(p^{\prime}\right)\left|J_{\mu}^{V}\right| N(p)\right\rangle= & \sqrt{3} \Psi_{\lambda}\left(p^{\prime}\right)\left[g_{\mu}^{\lambda} T_{\nu} q^{\nu}-q^{\lambda} T_{\mu}+g_{\mu}^{\lambda} C_{6}^{V}\right] \gamma_{5} u(p) \\
& \times \frac{1}{(2 \pi)^{3}} \sqrt{\frac{M m_{N}}{E_{p} E_{p^{\prime}}}} \\
\left\langle\Delta^{++}\left(p^{\prime}\right)\left|J_{\mu}^{A}\right| N(p)\right\rangle= & \sqrt{3} \Psi_{\lambda}\left(p^{\prime}\right)\left[g_{\mu}^{\lambda} B_{\nu} q^{\nu}-q^{\lambda} B_{\mu}+g_{\mu}^{\lambda} C_{5}^{A}+\frac{q^{\lambda} q_{\mu}}{M^{2}} C_{6}^{A}\right] \gamma_{5} u(p) \\
& \times \frac{1}{(2 \pi)^{3}} \sqrt{\frac{M m_{N}}{E_{p} E_{p^{\prime}}}}
\end{aligned}
$$

where $\Psi_{A}$ is the Rarita-Schwinger field, and

$$
\begin{gathered}
T_{\mu}=\frac{C_{3}^{V}}{m_{N}} \gamma_{\mu}+\frac{C_{4}^{V}}{m_{N}^{2}} p_{\mu}^{\prime}+\frac{C_{5}^{V}}{m_{N}^{2}} p_{\mu}, \\
B_{\mu}=\frac{C_{3}^{A}}{m_{N}} \gamma_{\mu}+\frac{C_{4}^{A}}{m_{N}^{2}} p_{\mu}^{\prime} .
\end{gathered}
$$

\subsubsection{Coherent $\mathrm{CC} \pi^{+}$Interactions}

In addition to the resonant interactions just described, neutrinos can interact with the entire target nucleus coherently. These coherent processes can occur in both neutral current interactions, $\nu+A \rightarrow \nu+\pi^{0}+A$, and charged current interactions, $\nu+A \rightarrow l^{-} \pi^{+} A$. In either case, the target nucleus is unchanged.

Despite the relative simplicity of the final states they produce, coherent interactions are not well understood when compared to the analogous resonant processes. Several models exist that describe coherent pion production $[15,16,17]$, but the absolute cross sections predicted by these models can vary by an order of magnitude. In addition, both the K2K and SciBooNE experiments have measured an absence of coherent $\mathrm{CC} \pi^{+}$events, well below predicted levels $[18,19]$, while similar deficits are not observed in neutral current coherent $\pi^{0}$ production [20].

\subsection{Nuclear Effects}

Neutrino scattering experiments are often conducted in nuclear media. The properties of the free nucleon interactions considered by the RS model are modified by sev- 
eral multi-nucleon effects, such as nuclear binding, particle motion, and the screening of internal nucleons by particles on the surface of the nucleus. In addition, once an interaction takes place, the final state particles must traverse the nuclear medium before they can be detected, which can alter the meaning of a measured cross section.

Most neutrino interaction simulations model the dynamics of the protons and neutrons in the nucleus as a relativistic Fermi gas. The nucleons are treated with a uniform momentum density below the cutoff Fermi momentum, $p_{F}$. This indeterminate contribution to the target momentum produces an irreducible reduction in the precision with which the kinematics of the event can be determined. Further, since all energy levels in the Fermi gas are filled, only interactions in which the outgoing nucleon has a momentum higher than $p_{F}$ are allowed. Forbidden interactions below this threshold are said to be Pauli blocked. Finally, as the target nucleons are in a bound state, some energy is consumed in liberating the interacting nucleon. Both Pauli blocking and nuclear binding have the effect of reducing the number of interactions at low $Q^{2}$.

When neutrino interactions take place in the nucleus, the particles that are produced can interact with the nuclear medium, thus modifying the observed characteristics of the interaction. Pions are particularly susceptible to the effects of the nuclear medium, since they interact via the strong nuclear force. Charged pions can either be absorbed or converted into neutral pions via $n \pi^{+} \rightarrow p \pi^{0}$. The nuclear medium can also influence whether a pion is even created. When a nucleon is excited into a $\Delta(1232)$ resonance, the $\Delta$ travels on the order of $1 \mathrm{fm}$ before it decays, which is a significant fraction of the size of the nucleus. The $\Delta$ can then interact via $\Delta N \rightarrow N N$, which reduces the number of observed pions. The sizes of these effects are discussed in Section 4.2.2.

Finally, the validity of the Rein-Sehgal model predictions for interactions with bound nucleons is unclear. The FKR predictions for transitions to higher resonances are not obviously valid for protons and neutrons bound in a nucleus, which can be significantly off-shell. Additionally, the modification to the masses and widths of the 
resonances due to the nuclear medium are not accounted for in the model.

\subsection{The $\mathrm{CC} \pi^{+}$Measurement}

In addition to furthering the theoretical understanding of neutrino-nuclear interactions, there exist more pragmatic reasons for measuring the $\mathrm{CC} \pi^{+}$cross section. Two of the largest next generation neutrino experiments, $\mathrm{T} 2 \mathrm{~K}$ and $\mathrm{NO} \nu \mathrm{A}$, will search for neutrino oscillations at peak energies of $\sim 0.7 \mathrm{GeV}$ and $\sim 2 \mathrm{GeV}$, respectively [21, 22]. The oscillation parameters are measured via CCQE interactions and, at these energies, the largest charged current neutrino background is from $\mathrm{CC} \pi^{+}$events. In addition, the $\mathrm{T} 2 \mathrm{~K}$ and $\mathrm{NO} \nu \mathrm{A}$ detectors employ nuclear targets, whereas most previous cross section measurements were on hydrogen and deuterium targets, and therefore do not probe the nuclear effects described in section 1.2.

Any measurement of the $\mathrm{CC} \pi^{+}$cross section on a nuclear target will contain contributions from many weakly constrained physical effects. Several different models attempt to describe the nucleon form factors of these interactions, and none of them are particularly well constrained. Off shell effects of the nuclear medium have not been accounted for, there are large uncertainties in the final state interactions that occur in the nucleus, and finally, as recently described by one group of theorists, "There is a general consensus among the theorists that a simple Fermi Gas (FG) model, widely used in the analysis of neutrino oscillation experiments, fails to provide a satisfactory description of the measured cross sections, and inclusion of further nuclear effects is needed." [23].

There is a clear demand for more data to provide a means by which the current theoretical picture can be improved. The current levels of theoretical uncertainty suggest that a model-independent accounting of the observed, post-nucleus cross section would be the most fruitful result to report, rather than a measurement of the parameters of a small subset of available models. The results presented in subsequent chapters are 
guided by this philosophy.

\section{$1.4 \quad$ MiniBooNE}

MiniBooNE (Mini Booster Neutrino Experiment; E898) is a neutrino oscillation experiment located at the Fermi National Accelerator Laboratory in Batavia, Illinois. Neutrinos produced by the Booster neutrino beam travel half a kilometer before reaching the 807 ton MiniBooNE detector. The Cherenkov and scintillation light from the particles produced in a neutrino interaction is used to reconstruct the kinematics of each event.

Although originally designed to search for the appearance of $\nu_{e}$ in a high purity $\nu_{\mu}$ beam, MiniBooNE is well suited to measure neutrino cross sections. The experiment has collected the world's largest sample of accelerator-based $\nu_{\mu}$ events below $2 \mathrm{GeV}$, with very little contamination from $\nu_{e}$ and $\bar{\nu}$ interactions. MiniBooNE has a unique opportunity to provide high statistics measurements of weakly constrained neutrino cross sections on a nuclear target, and at neutrino energies that are directly applicable to the next generation of accelerator based neutrino oscillation experiments. The remainder of this document describes a measurement of the largest charged current background to these oscillation searches: $\mathrm{CC} \pi^{+}$interactions. 


\section{Chapter 2}

\section{The Booster Neutrino Beam}

The purpose of the Booster neutrino beam (BNB) is to produce a high purity $\nu_{\mu}$ beam with very little intrinsic $\nu_{e}$ contamination as required by the oscillation analysis. The main source of $\nu_{\mu}$ 's are charged pions, which decay via $\pi^{+} \rightarrow \mu^{+} \nu_{\mu} 99.9870(4) \%$ of the time [1]. To maximize the $\pi^{+}$flux, the Booster proton beam is focused onto a very long, thin beryllium target to ensure a high beam reaction rate, while minimizing the absorption of the resulting large-angle pions. The target is placed inside of a magnetic horn that produces a toroidal field to reduce the transverse component of the $\pi^{+}$particles and create a forward-focused neutrino beam. A cartoon of the entire process is shown in Figure 2.1.

\section{$2.1 \quad$ Booster Proton Beam}

The Booster is the third in a series of five accelerator stages at Fermilab. Protons emerging from the Linear Accelerator with a kinetic energy of $400 \mathrm{MeV}$ are accelerated to $8 \mathrm{GeV}$ after $\sim 20,000$ laps around the $472 \mathrm{~m}$ accelerator ring. The beam is then sent along the Main Injector $8 \mathrm{GeV}$ beam line before being diverted toward the MI-12 enclosure (as shown in Fig. 2.2), which houses the MiniBooNE target and horn assembly.

Beam spills to MiniBooNE delivered $\sim 4 \times 10^{12}$ protons at $3-5 \mathrm{~Hz}$. Each spill was composed of 84 buckets arriving at a rate of $53 \mathrm{MHz}$, which corresponded to a total spill duration of $1.6 \mu \mathrm{s}$. The weekly accumulation of protons on target (POT) over 


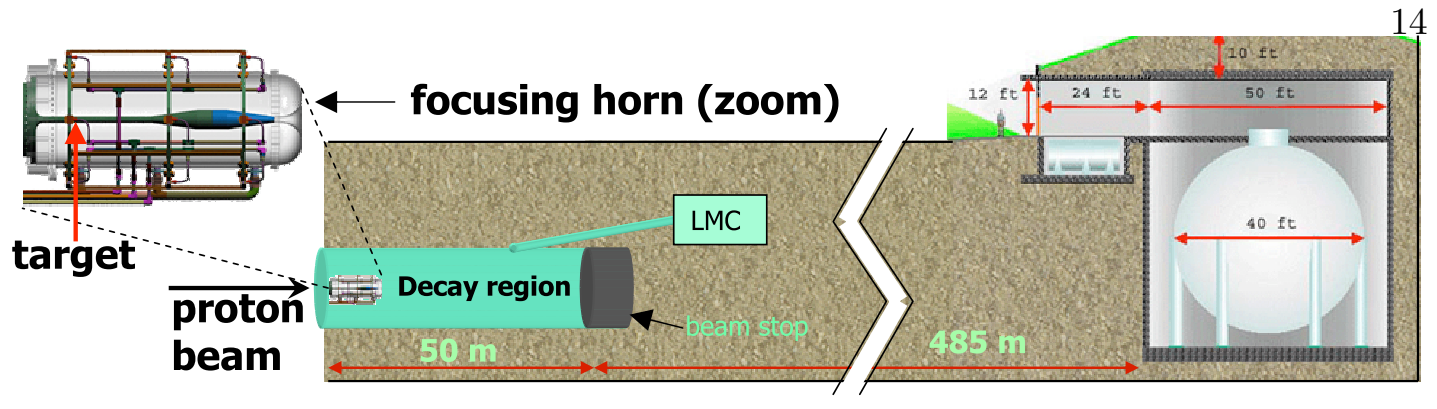

Figure 2.1: A cartoon of the neutrino flux production is shown. Protons from the Booster interact in the target, which is located inside the horn. The secondary particles created in the interactions are focused by the horn and decay within the decay region. The resulting neutrinos then travel through half a kilometer of earth before reaching the MiniBooNE Detector.

the duration of the run is shown in Fig. 2.3. The POT was measured by two toroids placed 5 and $50 \mathrm{~m}$ upstream of the target, and provided a measurement uncertainty of $\sim 1 \%[24]$. The results in this thesis are based on the entire MiniBooNE neutrino dataset of $6.461 \times 10^{20}$ POT.

The $8 \mathrm{GeV}$ Booster proton beam was directed toward the center of the MiniBooNE target. Just upstream of the target, the root mean square (RMS) beam size was $1.51 \mathrm{~mm}$ horizontally and $0.75 \mathrm{~mm}$ vertically. The beam was focused such that the horizontal and vertical angular distributions (RMS values of $0.66 \mathrm{mrad}$ and $0.40 \mathrm{mrad}$, respectively) were highly anti-correlated with the corresponding position distributions, which produced a beam waist at the center of the target.

\section{$2.2 \quad$ Target and Horn}

The target was composed of 7 cylindrical beryllium slugs, each 4 inches long and $3 / 8$ inches in diameter. The target was attached to a cylindrical beryllium sleeve by 21 thin beryllium fins spaced at regular intervals around the target. The beam deposited a significant amount of heat, which was removed using a stream of forced air. 


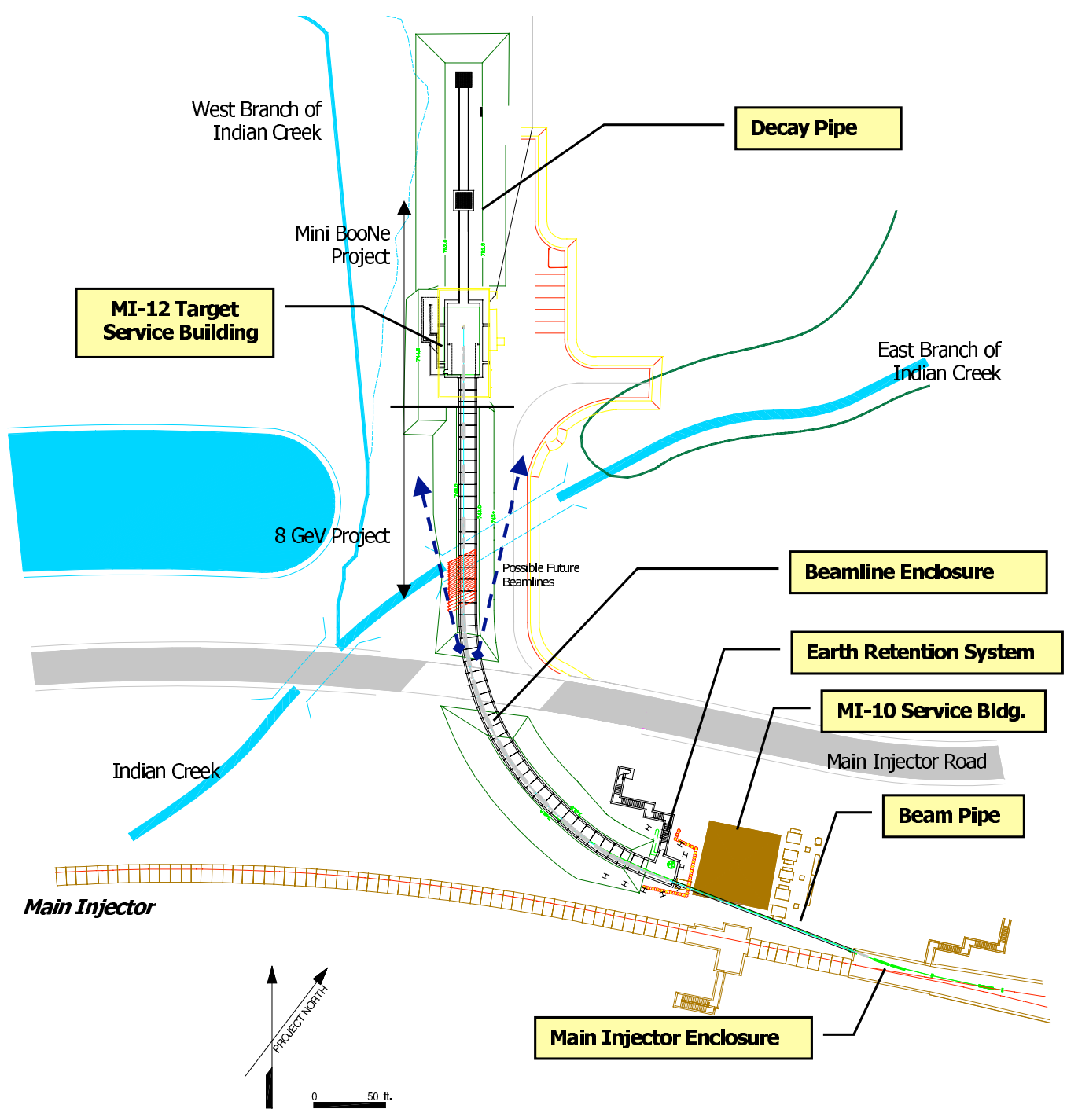

Figure 2.2: The layout of the Booster Neutrino Beam [25] is shown. The extraction point from the Main Injector $8 \mathrm{GeV}$ transfer line is shown in the bottom right. The beam then passes to the MI-12 service building, which houses the target and horn. The secondary beam created in MI-12 passes through the $50 \mathrm{~m}$ decay pipe before reaching the steel beam stop. 


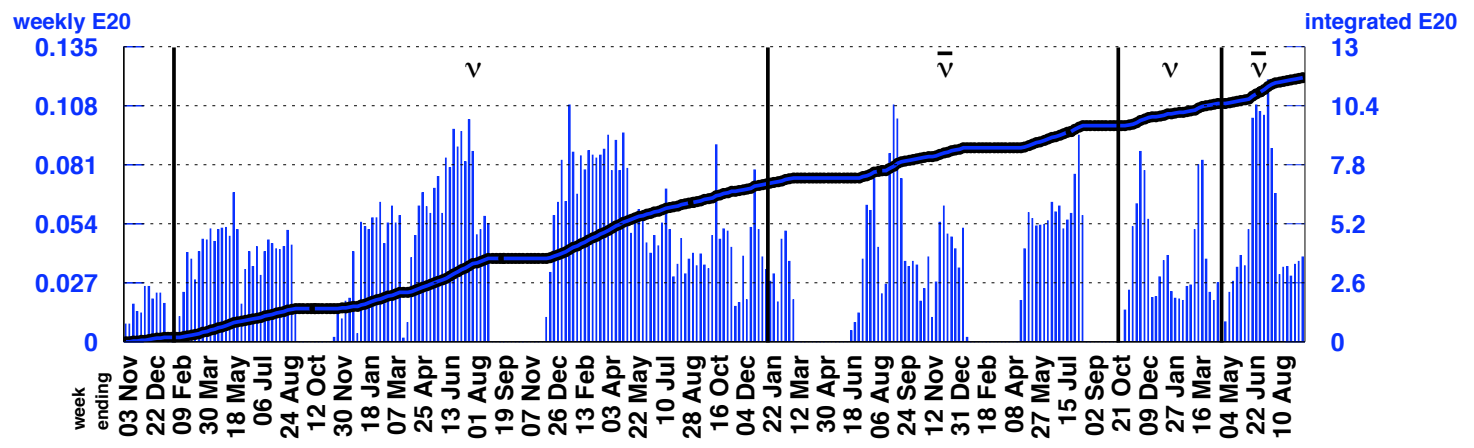

Figure 2.3: The weekly and integrated protons on target delivered to MiniBooNE are given from November 2002 through August 2008. The periods of neutrino $(\nu)$ and antineutrino $(\bar{\nu})$ horn configurations have been separated for clarity. Only the neutrino configuration is used for the present analysis.

The target was positioned in the center of the two coaxial aluminum cylinders that constituted the horn. The horn was $185.4 \mathrm{~cm}$ in length with a diameter of $60 \mathrm{~cm}$. The inner conductor radius varied between $2.2 \mathrm{~cm}$ and $6.54 \mathrm{~cm}$. An electric current was run along the outer conductor toward the interface with the inner conductor at the downstream end of the horn. The current then traveled back up the inner conductor, producing an azimuthal magnetic field between the conductors and a negligible field elsewhere. The azimuthal symmetry of the horn produced an easily understood magnetic field given by Ampere's Law,

$$
B=\frac{\mu_{0} I}{2 \pi r}
$$

where $I$ is the current and $r$ is the distance from the horn axis. The current was sinusoidally ramped to a peak current of $174 \mathrm{kA}$ and lowered back to zero over a period of $143 \mu \mathrm{s}$. The peak of the current pulse was timed to correspond with the arrival of each beam spill.

Several nozzles were inset along the outer conductor to spray cooling water into the horn. The nozzles were designed to direct most of the water toward the outer surface of the inner conductor. A schematic drawing of the horn and water cooling system is 
shown in figure 2.4.

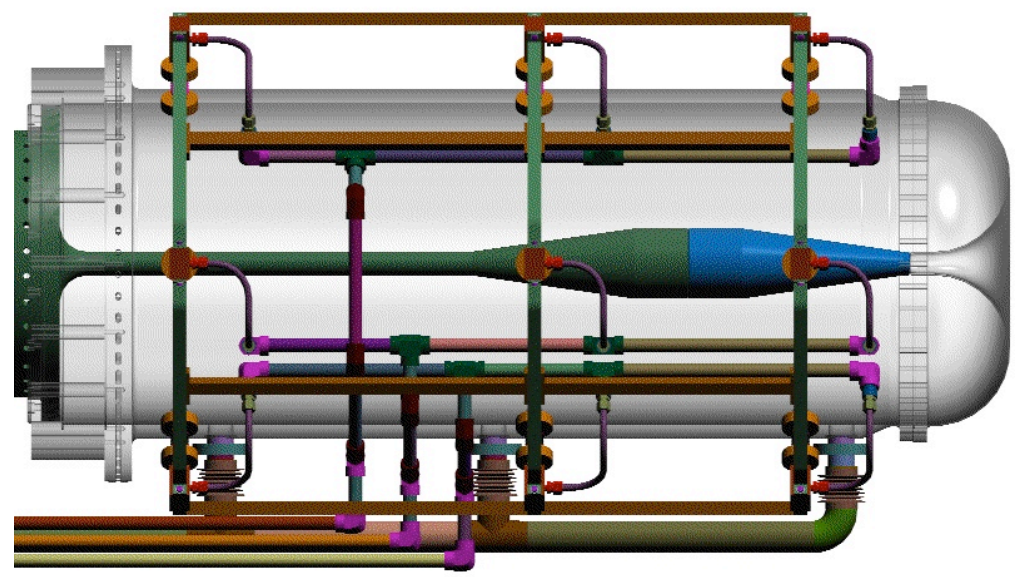

Figure 2.4: An elevation view of the horn and water cooling system [26]. The outer conductor is transparent to show the inner horn structure.

The target and horn were placed inside a small cavity, which was separated from the accessible portion of the target hall by nearly 2000 tons of concrete. Just downstream of the horn was a steel collimator that shaped the secondary beam before it entered the meson decay region. The collimator began $259 \mathrm{~cm}$ downstream of the target and extended for $214 \mathrm{~cm}$; the upstream radius was $30 \mathrm{~cm}$ and expanded linearly to $35.5 \mathrm{~cm}$.

\subsection{Meson Decay Region}

Mesons that passed through the collimator entered the $50 \mathrm{~m}$ decay pipe. The pipe was $6 \mathrm{ft}$ in diameter and surrounded by dolomite pebbles. The remaining neutrinos not created upstream of the collimator were produced by decays of mesons and muons inside the air volume contained in the pipe.

The content of the neutrino beam produced by BNB could be discretely varied by changing the direction of the horn current. In " $\nu$-mode" running, the current (in the positive sense) traveled from the outer conductor to the inner conductor, which produced a counter-clockwise pointing magnetic field along the direction of the beam. 
This caused positively charged particles with momentum components transverse to the beam direction to deflect forward toward the detector, while negatively charged particles were bent radially outward and removed from the secondary beam. By reversing the current direction, the horn could also be run in " $\bar{\nu}$-mode", which focused negatively charged particles to produce a $\bar{\nu}$ beam via $\pi^{-} \rightarrow \mu^{-} \bar{\nu}_{\mu}$ decay.

At the end of the pipe was a beam stop composed of $10 \mathrm{ft}$ of steel and $3 \mathrm{ft}$ of concrete. The beam stop terminated the portion of the decay pipe in which particles could decay in flight, however a significant number of neutrinos were produced from particle decays at rest. All decay-at-rest neutrino energies are too low to contribute to $\mathrm{CC} \pi^{+}$production. In the center of the pipe, about $25 \mathrm{~m}$ downstream of the target, a duplicate beam stop was suspended above the decay region and could be lowered to shorten the effective length of the pipe. The $25 \mathrm{~m}$ absorber was not implemented for the data used in this analysis.

\subsection{Neutrino Flux}

The properties of the neutrino flux produced by the Booster Neutrino Beam (BNB) must be known in order to extract useful information from the data. The center of the MiniBooNE detector is located 541 meters downstream of the center of the target and only 1.896 meters above the center of the neutrino beam. Neutrinos that intersect the detector, therefore, are almost uniformly traveling in the same direction. This means that the flux can be characterized almost completely by the neutrino energy distributions for each neutrino type.

The neutrino energy spectrum is not measured at the point of production. As such, MiniBooNE relies on a detailed simulation of the aforementioned components of the BNB to predict the properties of the flux. The results of this simulation are described in Section 4.1. 


\section{Chapter 3}

\section{The MiniBooNE Detector}

The MiniBooNE detector is a $610.6 \mathrm{~cm}$ radius, spherical tank filled with 818 tons of mineral oil. Inside the tank is an additional $574.6 \mathrm{~cm}$ radius concentric sphere that divides the volume into an inner "main" region and an outer "veto" region. The design of the detector was driven by the $\nu_{\mu} \rightarrow \nu_{e}$ oscillation search, which required that $\nu_{\mu}$ and $\nu_{e}$ events be distinguishable with sufficiently well determined energies. In a charged current interaction, the species and energy of a neutrino are derived from the kinematics of the outgoing charged lepton (described in Section 5.3.2.1). As charged particles propagate through the oil, a large amount of Cherenkov radiation is produced along with some additional scintillation light. An array of 1280 photomultiplier tubes is mounted on the inside surface of the inner volume region to detect the generated light. The properties of each charged particle can then be deduced from the distribution of light in the event, and, in particular, from the rings produced by the cone shaped emission of Cherenkov light. The inner surface of the main tank volume is painted black to reduce reflections that can degrade the performance of event reconstruction algorithms.

The outer veto region of the tank is a spherical shell with a $35 \mathrm{~cm}$ thickness that surrounds the inner fiducial volume. The purpose of the veto is to detect particles entering or exiting the tank. The neutrino interactions of interest occur inside the main tank volume, and do not produce particles that exit the tank. Conversely, background events from cosmic rays and neutrino interactions just outside the tank can be rejected 


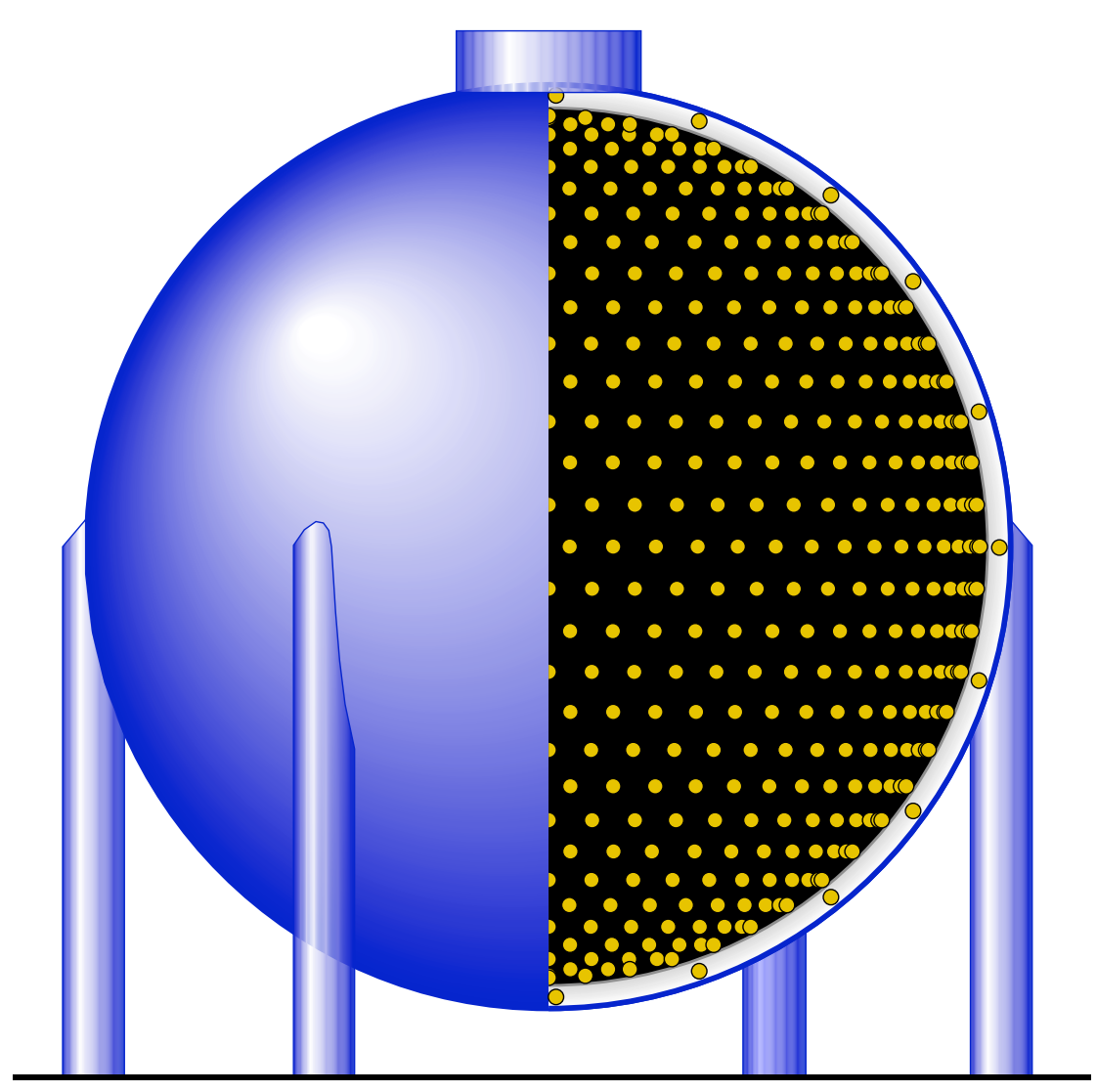

Figure 3.1: A schematic of the MiniBooNE detector. Half of the front face has been removed to reveal the internal structure. The tank photomultiplier tubes are shown along the inside surface of the main fiducial region. The white outer shell houses the veto tubes [27].

based on the presence of light in the outer veto region. The veto is instrumented with 240 photomultiplier tubes, and the walls are painted white to maximize the chances of photon detection.

The tank is located in a 1.5 foot thick, concrete cylindrical vault. The vault is 43 feet high with a 45 foot diameter, the bulk of which lies below ground level. Directly above the vault is the MiniBooNE surface building, which houses most of the detector electronics. The surface building is covered with an earth overburden rising to a maximum of 24 feet above the ground that provides a minimum of $3 \mathrm{~m}$ earth 
equivalent that reduces the rate of cosmic rays entering the detector.

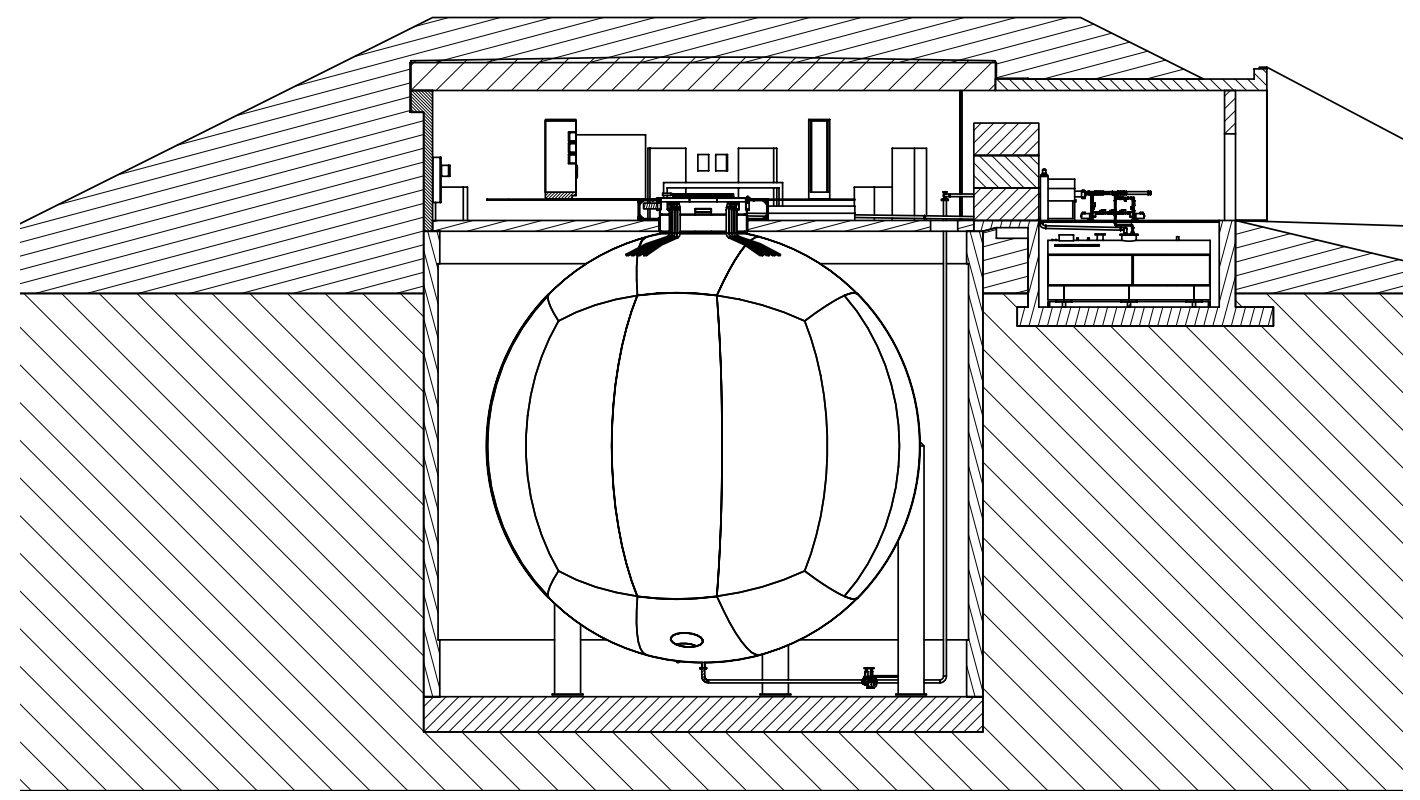

Figure 3.2: An illustration of the vault and surface building that house the MiniBooNE detector. The earth overburden is shown covering the enclosure [27].

\subsection{Mineral Oil}

The oil used in MiniBooNE was Exxon/Mobile Marcol 7 Light Mineral Oil. It was chosen among 10 oils tested based on its superior optical properties. Since the faces of the photo-tubes are $\sim 550 \mathrm{~cm}$ from the center of the tank, photons often must travel a substantial distance before they are detected. An extinction length $>20 \mathrm{~m}$ for $420 \mathrm{~nm}$ light was required to ensure that no more than $25 \%$ of the light would be lost in a neutrino interaction at the center of the tank [28]. Other desirable properties of the oil, such as a high refraction index, low dispersion, and low levels of scintillation light, also contributed to the selection process.

Despite providing fewer nuclear targets due to its somewhat lower density of $0.845(1) \mathrm{g} / \mathrm{cm}^{3}$, mineral oil provides several advantages over water. Light travels slower in mineral oil, which results in more Cherenkov light and improved particle recon- 
struction. In addition, a lower energy Cherenkov threshold extends the reach of the MiniBooNE detector to lower neutrino energies. One of the most important event identification tools in MiniBooNE is the presence of delayed light from stopped particle decays (see Section 6.3), and mineral oil has a muon capture rate of only $8 \%$ compared to $20 \%$ in water [28]. Further details on the optical properties of the oil can be found in the detector simulation discussion in Section 4.4.

\subsection{Photomultiplier Tubes}

The MiniBooNE detector was instrumented with 1,520 eight inch diameter photomultiplier tubes (PMTs). Of these, 1,198 were Hamamatsu R1480 PMTs reused from the LSND experiment, and 322 Hamamatsu R5912 tubes ordered specifically for MiniBooNE. Because of their superior detection capabilities, the "new" (R5912) PMTs were all deployed inside the main tank to be used in event reconstruction. From the 1,198 "old" (R1480) PMTs, the 240 with the lowest measured dark rates were selected for the veto region to minimize the number of false vetoes. The remaining 958 old tubes were randomly distributed in the main tank volume. Figure 3.3 shows a map of old and new photo-tube placement throughout the tank.

The PMTs were distributed over the inside surface of the tank as uniformly as practical. For logistical purposes, the PMTs were separated into evenly-spaced horizontal rows. The reduction in the circumference of each latitudinal slice from equator to pole makes a full staggering of adjacent PMT rows impossible, however each row was clocked by half a PMT spacing relative to the PMT start point of the previous row. The final tube positions were surveyed by the Fermilab Alignment Group. The veto PMTs were mounted back-to-back, facing perpendicular to the center of the tank to view as much of the veto shell as possible. A schematic of the main and veto tube mounting is shown in Figure 3.4. 


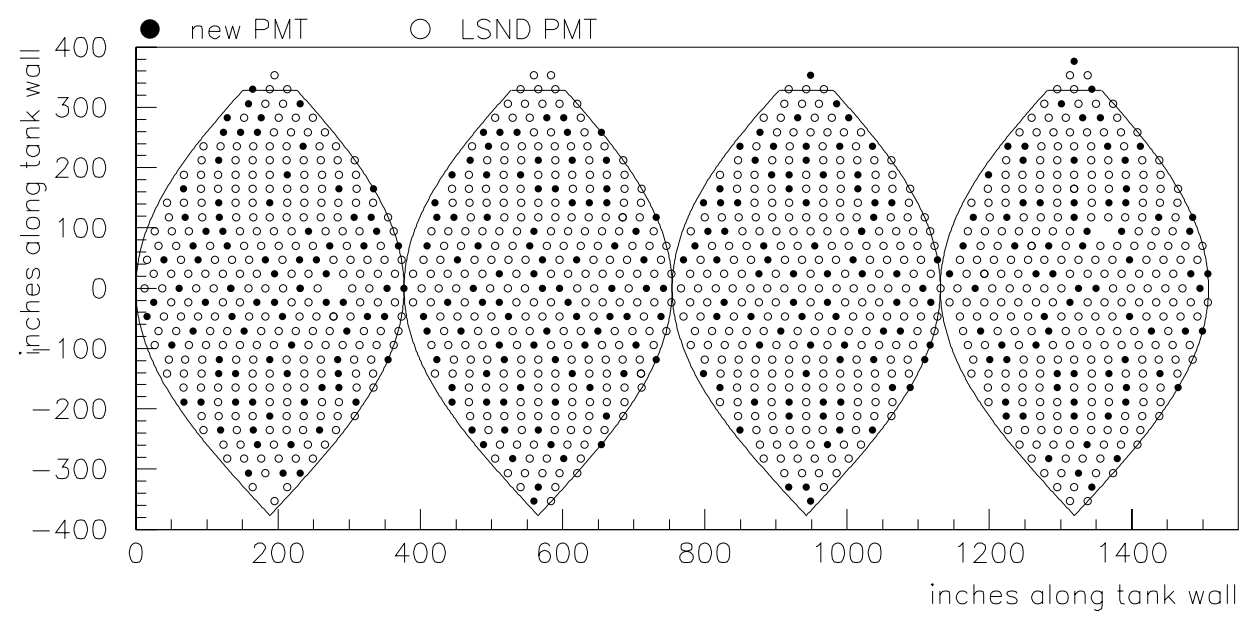

Figure 3.3: The distribution of "old" Hamamatsu R1480 (clear) and "new" Hamamatsu R5912 (solid) photomultiplier tubes over the inside surface of the tank is shown.

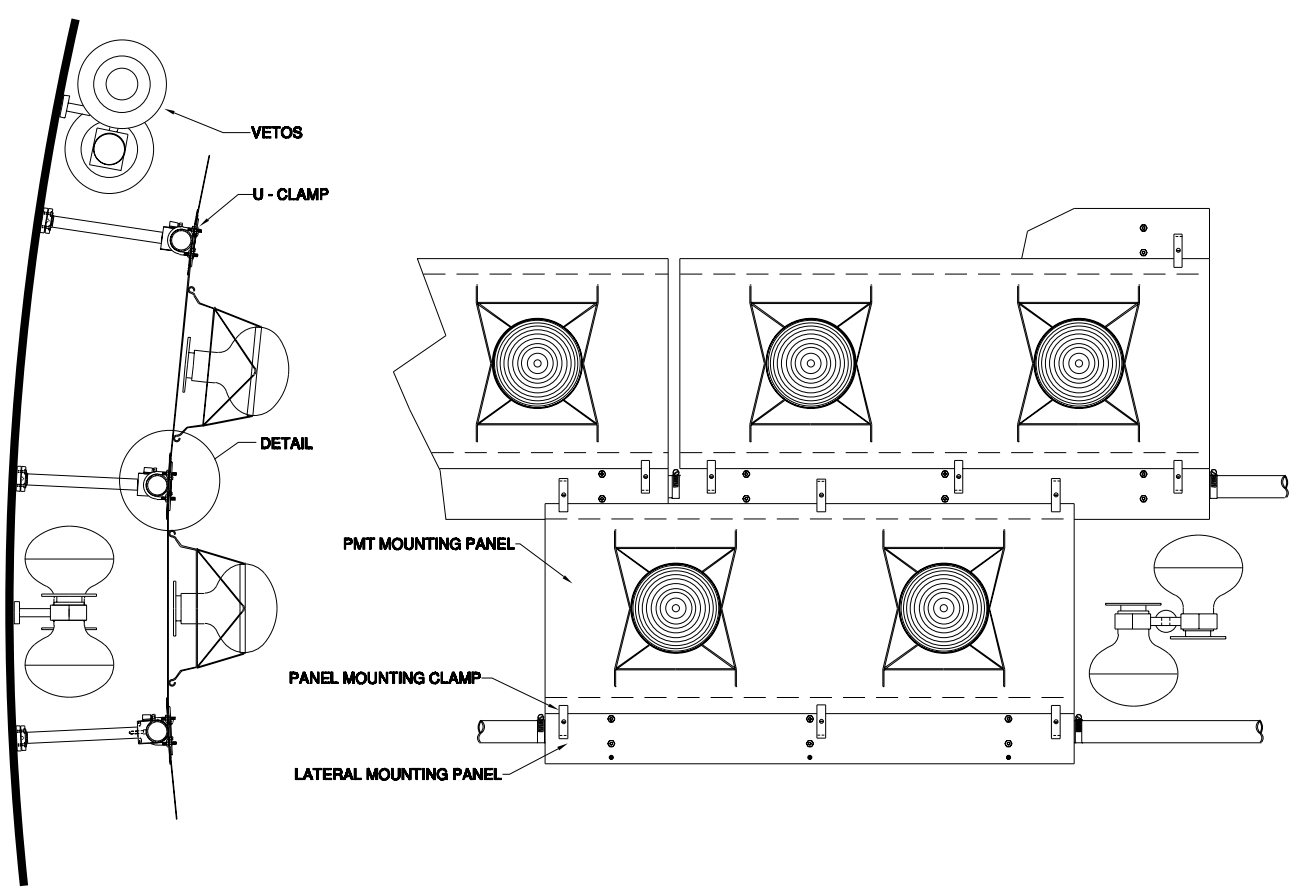

Figure 3.4: The PMT configurations for both the main tank and veto region are shown. The tank PMTs face the center of the fiducial volume, while the veto PMTs are arranged to view the directions perpendicular to the tank wall. 


\subsection{Electronics}

The measurable quantities recorded by the PMTs are the number of photoelectrons that hit the tube, given by the measured charge, and the time of the hit. The determination of these values is illustrated in Figure 3.5. Charge and time information is recorded at intervals determined by the $10 \mathrm{MHz}$ clock. When a tube is hit, the resulting pulse, $V_{p m t}$, is integrated and convolved with a slowly decaying exponential with a time constant of $\sim 1.2 \mu \mathrm{s}$. This integrated charge signal, $V_{q}$, is sampled at four consecutive clock times, beginning with the time immediately preceding the hit. Using the empirically measured shapes of the integrated charge bleed-off curves, the charged deposited by the hit can be extracted. If the initial pulse, $V_{p m t}$, crosses a threshold of roughly 0.25 photo-electrons, the asynchronous discriminator is fired, which triggers a voltage ramp, $V_{t}$, at the time of the crossing. The ramp voltage is sampled at the same 4 clock ticks during which $V_{q}$ is recorded to determine the initial hit time.

\subsection{Calibration Systems}

Cosmic muons, which enter the tank at a rate of $10 \mathrm{kHz}$, are used to understand the detector energy reconstruction. A muon hodoscope composed of four planes of Bicron BC-408 plastic scintillator is positioned directly above the detector to measure the transverse position of entering muon tracks at two vertical locations separated by one meter. Inside the detector are six $5 \mathrm{~cm}$ scintillator cubes deployed at various depths ranging from 30 to $400 \mathrm{~cm}$ (the deepest cube is $7.6 \mathrm{~cm}$ on a side). Muons that are detected by the tracker and stop in a cube have a well defined initial energy given by the muon range in mineral oil. With these data, a variety of detector properties can be studied such as the time offsets, gains, and time slewing of the PMTs, as well as the light attenuation properties of the oil. The direction and energy also allow for tests of higher level reconstructed quantities. Figure 3.6 shows a comparison between the muon 


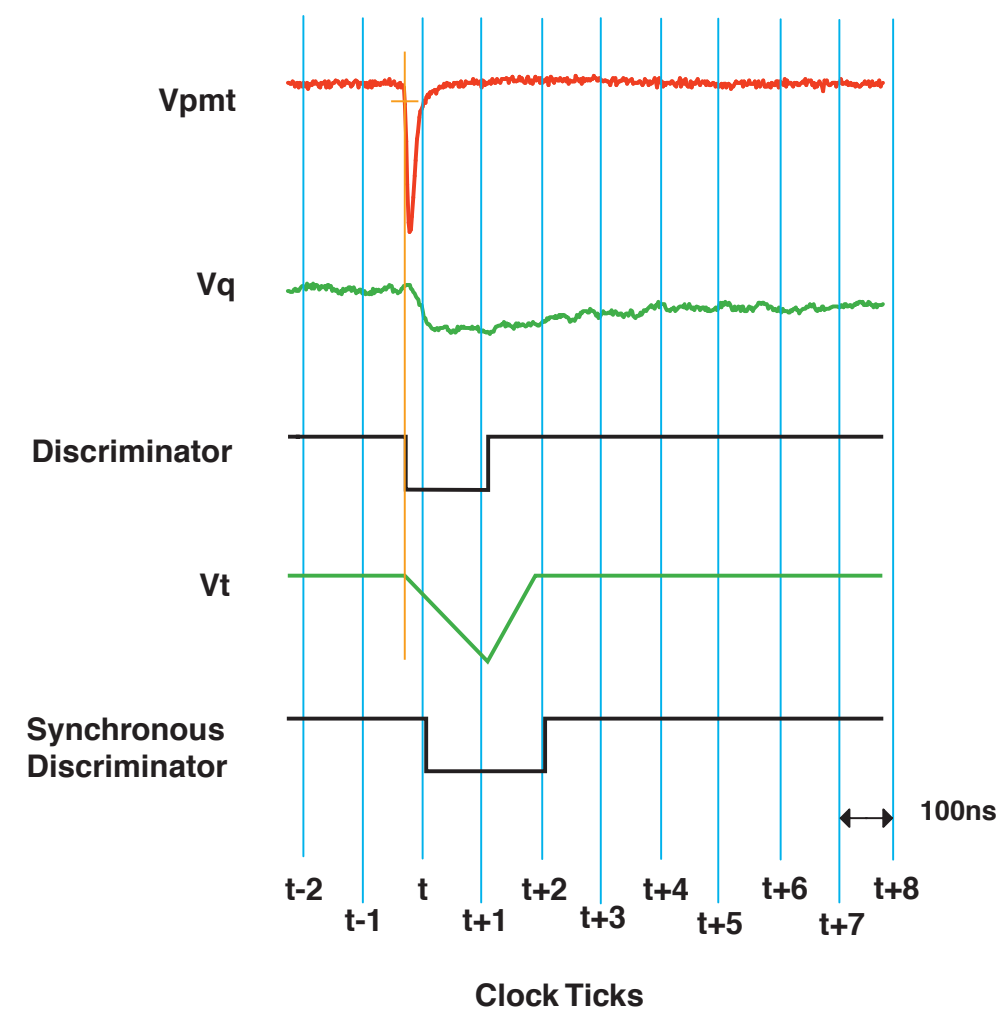

Figure 3.5: A cartoon of the PMT electronic is shown. A PMT hit triggers the asynchronous discriminator, which triggers a voltage ramp, $V_{t}$. The integrated charge, $V_{q}$, and $V_{t}$ are recorded at the 4 times labeled (t-1) through $(\mathrm{t}+2)$ from which the charge and time is deduced.

range energy and the measured tank energy.

To produce controlled bursts of light of known intensity and wavelength, four calibration laser sources are deployed throughout the tank. Each source is a $10 \mathrm{~cm}$ glass bulb filled with LUDOX to disperse the light isotropically. Under normal running conditions, a laser flask at the center of the tank produces $100 \mathrm{ps}$ pulses of $397 \mathrm{~nm}$ light at a rate of $3.33 \mathrm{~Hz}$. The laser data are used to calibrate the PMT gains, as well as tube-to-tube time measurement discrepancies. 


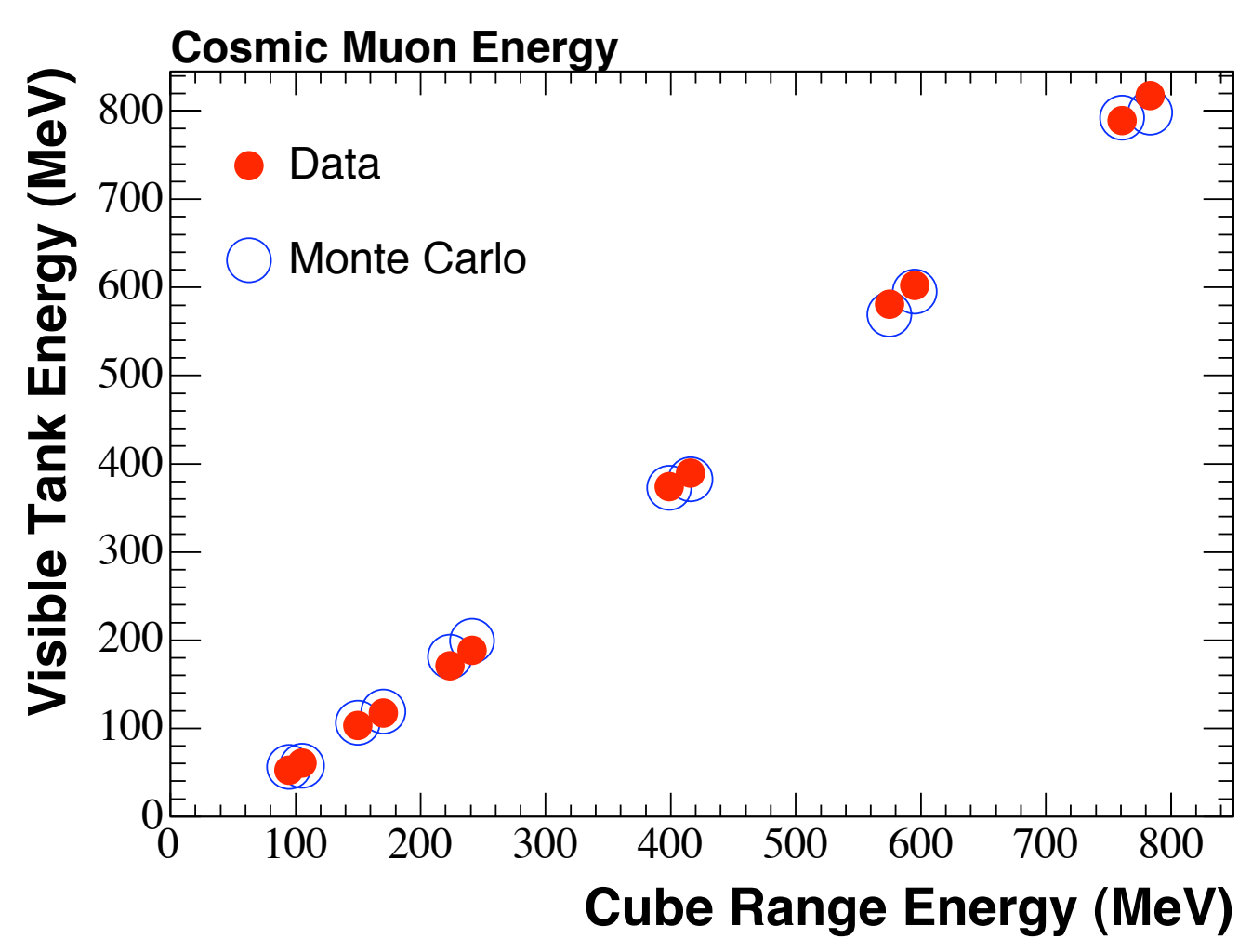

Figure 3.6: The energy determined by the muon range in the tracker/cube system is compared with the visible energy in the tank. The data show the expected linear correlation and agree well with the Monte Carlo simulation.

\subsection{Data Acquisition}

The data collection triggers are based on PMT hit multiplicity information, as well as external signals from the accelerator and calibration systems. The primitive PMT signals formed by the trigger logic indicate tank activity exceeding 10, 24, 60, 100, and 200 hits, as well as the presence of at least 4 or 6 veto PMT hits. The presence of beam is determined from the accelerator clock signals.

The main physics trigger for the experiment was satisfied whenever beam was present, regardless of the activity in the tank. The data stream was initialized $5 \mu \mathrm{s}$ prior to the arrival of the beam spill and remained active for a total of $19.2 \mu \mathrm{s}$. The presence of beam prohibited the firing of any of the other triggers. 
Additional triggers were defined to perform a variety of other functions. Calibration triggers for the laser and cube data were used for the aforementioned detector studies. A random trigger was implemented to collect beam off data to provide a sample of cosmic activity that could be overlayed on simulated events to better reproduce the features of the data. Events were also recorded during beam spills to the adjacent NuMI beam line. Other triggers based on PMT multiplicities were implemented to collect samples of events with $\geq 60$ tank and $\geq 6$ veto hits for detector monitoring, cosmic muon decays (i.e. "Michel" events; $\leq 200$ tank hits and $\leq 6$ veto hits), and to search for cosmic and supernova neutrinos. A list of MiniBooNE triggers with average rates is given in Table 3.1.

Table 3.1: The optical parameters of the beam are listed just upstream of the target.

\begin{tabular}{|c|c|c|c|}
\hline Trigger & Rate $(\mathrm{Hz})$ & Prescale & Time Window $(\mu \mathrm{s})$ \\
\hline \hline Booster Beam & $2-5$ & 1 & 19.2 \\
\hline NuMI Beam & 0.5 & 1 & 19.2 \\
\hline random & 2 & 1 & 19.2 \\
\hline cosmic $\mu$ & 0.66 & 1 & 19.2 \\
\hline Michel & 1.2 & 600 & 19.2 \\
\hline supernova & 9.9 & 1 & 3.2 \\
\hline tank & 0.4 & 90000 & 19.2 \\
\hline veto & 0.4 & 5000 & 19.2 \\
\hline laser & 3.33 & 1 & 9.6 \\
\hline cube & 1.1 & 1 & 12.8 \\
\hline tracker & 0.7 & 170 & 12.8 \\
\hline \hline total & $\sim 26$ & & \\
\hline
\end{tabular}




\section{Chapter 4}

\section{Event Simulation}

To produce a cross section measurement, the entire data production process must be simulated, including the booster proton beam, the properties of the neutrino flux, neutrino interaction processes, and the propagation of final state particles through the detector. To accomplish this, several different software packages are run in succession. In this chapter, each element of the simulation chain is described along with any associated systematic uncertainties.

\subsection{Neutrino Flux Simulation}

Protons that interact inelastically produce a variety of secondary particles. At the proton energy produced by the Booster, $8 \mathrm{GeV}$, the dominant particle species produced in the target is $\pi^{+}$, which is primarily responsible for the $<2 \mathrm{GeV}$ neutrino flux. The goal of the flux simulation is to predict the number of neutrinos that hit the detector per proton on target, and to determine the neutrino energy spectrum.

\subsubsection{Beam Monte Carlo}

The majority of the flux prediction is produced in the GEANT4-based [29] beam Monte Carlo simulation. Beam protons are generated $4.5 \mathrm{~cm}$ upstream of the front face of the target with an initial position and direction randomly selected according to the beam parameters given in Table 4.1. The protons then interact with the target to 
produce a variety of secondary particles. Many of these particles enter the horn where their trajectories are modified by the magnetic field. Any neutrino produced in the decay of a secondary particle is recorded in the beam Monte Carlo output steam.

Table 4.1: The beam optics parameters are listed just upstream of the target.

\begin{tabular}{|c|c|}
\hline mean $x$ & 0 \\
\hline mean $y$ & 0 \\
\hline$\sigma_{x}$ & $1.51 \mathrm{~mm}$ \\
\hline$\sigma_{y}$ & $0.75 \mathrm{~mm}$ \\
\hline mean $\theta_{x}$ & 0 \\
\hline mean $\theta_{y}$ & 0 \\
\hline$\sigma_{\theta_{x}}$ & $0.66 \mathrm{mr}$ \\
\hline$\sigma_{\theta_{y}}$ & $0.40 \mathrm{mr}$ \\
\hline
\end{tabular}

\subsubsection{Geometry}

The full geometry of the Booster neutrino beam, described in Chapter 2, is modeled in the simulation. The most critical elements of the geometry are the target and the inner conductor of the horn. The target is composed of pure beryllium with a length of 28 in and a diameter of $3 / 8$ in. The target is attached to a thin cylinder of beryllium (inner radius of $13.7779 \mathrm{~mm}$, outer radius of $14.6177 \mathrm{~mm}$ ) via twenty-one beryllium fins, three for each slug, positioned at intervals of 120 degrees around the target. Each flange had a cross sectional area of $2.1545 \mathrm{~mm}$ and a length of 3.5 inches.

The horn is defined by 14 vertices in length and radius. A plot of the length versus radius is compared to the horn engineering drawing in Figure 4.1. The aluminum inner conductor has a thickness of $3 \mathrm{~mm}$ and is cylindrically symmetric. Between the inner and outer conductor the magnetic field is given by Ampere's law, and inside the inner conductor the field is zero. The field within the inner conductor aluminum is determined by the "skin depth" of the horn current, and is approximated as an exponential function of the radius. 


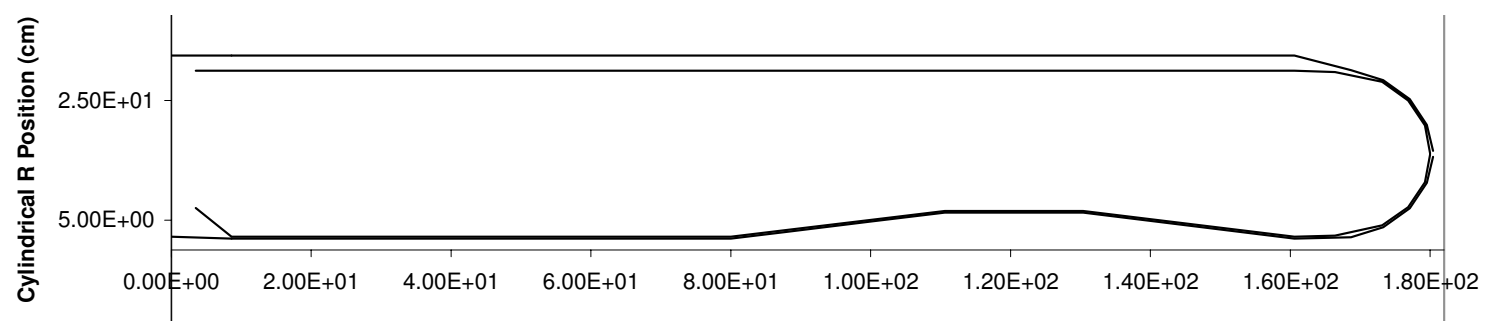

(a) The radial cross section of the horn as modeled in the beam Monte Carlo simulation

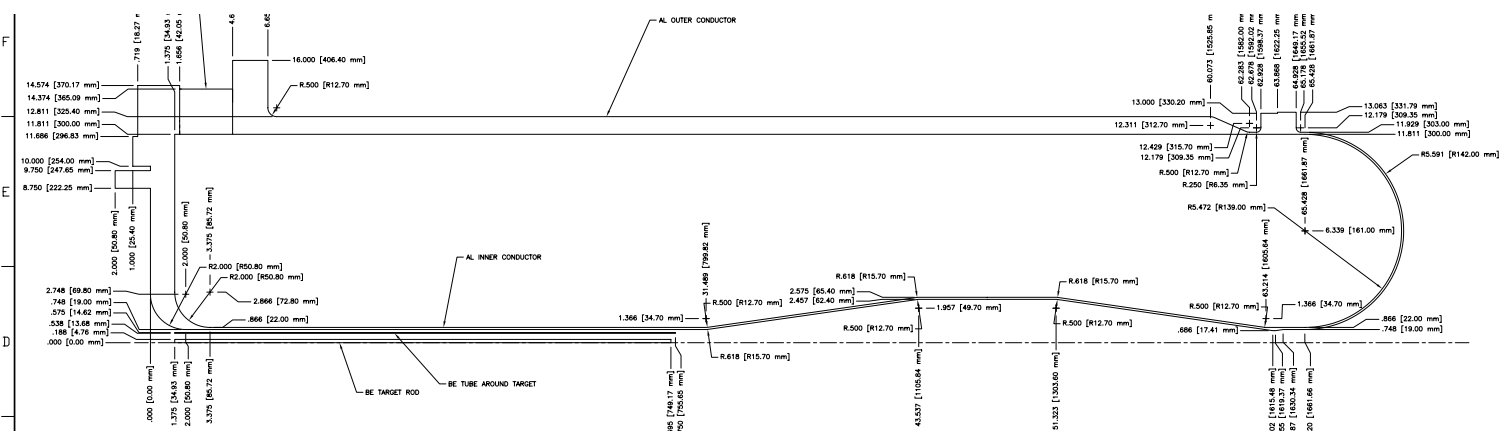

(b) The engineering drawing of the horn radial cross section

Figure 4.1: The horn geometry in the beam Monte Carlo simulation (top) is compared with the engineering draw (bottom). The critical feature is the shape of the inner (low R) conductor, which is well modeled.

The target and horn assembly are located within a $1.3 \mathrm{~m}$ thick box of 1030 steel, which is positioned inside a $60 \mathrm{~cm}$ thick concrete enclosure. The beam line enters the horn enclosure through a $14 \mathrm{~cm}$ diameter steel tube, and the steel collimator through which the secondary beam exits has a $60 \mathrm{~cm}$ diameter that grows to $71 \mathrm{~cm}$ at the terminal end, $7 \mathrm{ft}$ downstream.

The $50 \mathrm{~m}$ decay region is composed of two $6 \mathrm{ft}$ inner-diameter concrete tubes that surround the $25 \mathrm{~m}$ absorber enclosure. At the end of the decay region is the steel and concrete beam stop. The entire beam Monte Carlo structure is surrounded by dolomite, which is simulated with a density of $2.28 \mathrm{~g} / \mathrm{cm}^{3}$ to account for the actual dolomite density of $2.84 \mathrm{~g} / \mathrm{cm}^{3}$ and a packing fraction of 0.79 . 


\subsubsection{Interaction Cross Sections}

The beam Monte Carlo simulation employs a custom model for proton, neutron, and pion interactions with both beryllium and aluminum [30]. The total cross section is divided into (coherent) elastic and inelastic components. Interactions between the incident hadron and individual nucleons that do not produce or absorb initial state particles are referred to as quasi-elastic interactions, and are separated from the remaining "reaction" portion of the inelastic cross section. Each of these cross sections are related by the following expression,

$$
\sigma_{T O T}=\sigma_{E L A}+\sigma_{I N E}=\sigma_{E L A}+\sigma_{Q E}+\sigma_{R E A}
$$

Theoretical models are used to parametrize many of the cross sections in Equation 4.1. Wherever possible, these parametrizations are fit to the existing data. The forward elastic cross section amplitude is calculated using the Glauber model [31], which combines known hadron-nucleon scattering amplitudes into one coherent target. From the elastic scattering amplitude, the total cross section can be derived using the optical theorem. The quasi-elastic interactions are mostly described by the corresponding hadron-nucleon cross sections with an additional correction for the attenuation of the hadron wave function as it penetrates the nucleus. This effect is treated using the shadowed multiple scattering expansion [31].

The inelastic cross sections are well measured, and parametrizations are used to fit the existing data. The proton-Be/Al interactions have been measured over the entire momentum range of interest $[32,33]$. Data exist for nearly all of the $\pi^{ \pm}$momentum range as well, except at low momentum where the data are extrapolated from higher nuclei $[32,33,34,35]$. These parametrizations of the total, quasi-elastic, and inelastic cross sections fully specify the relation given in Equation 4.1. Plots of the pion and nucleon total, inelastic, and quasi-elastic parametrizations are given in Figures 4.2 and 4.3. Additional details can be found in Refs. [36, 37]. 

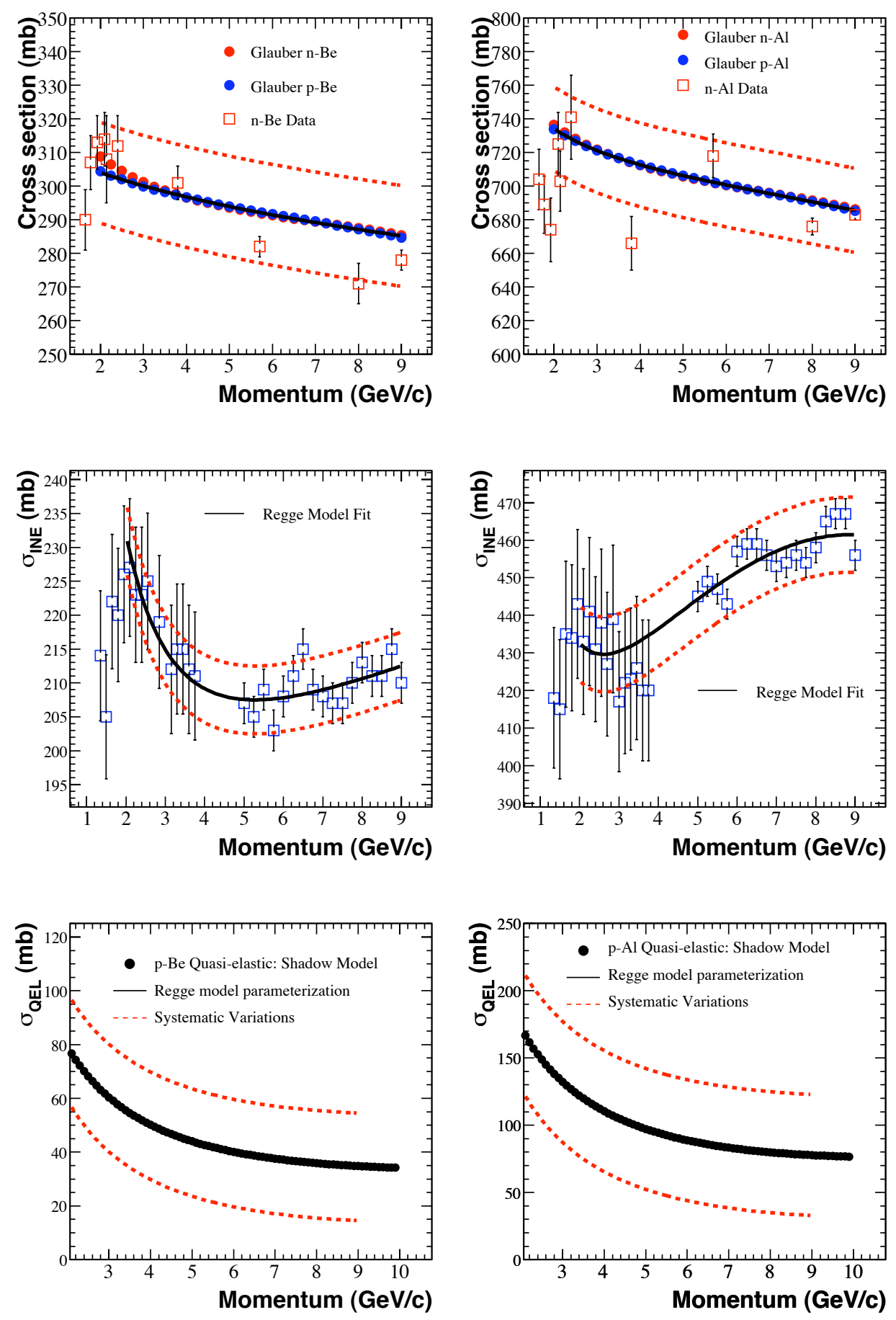

Figure 4.2: The nucleon total (top), inelastic (middle), and quasi-elastic (bottom) parametrizations for interactions with beryllium (left) and aluminum (right) are shown. The systematic errors assumed for each are also given and compared with data where possible [36]. 

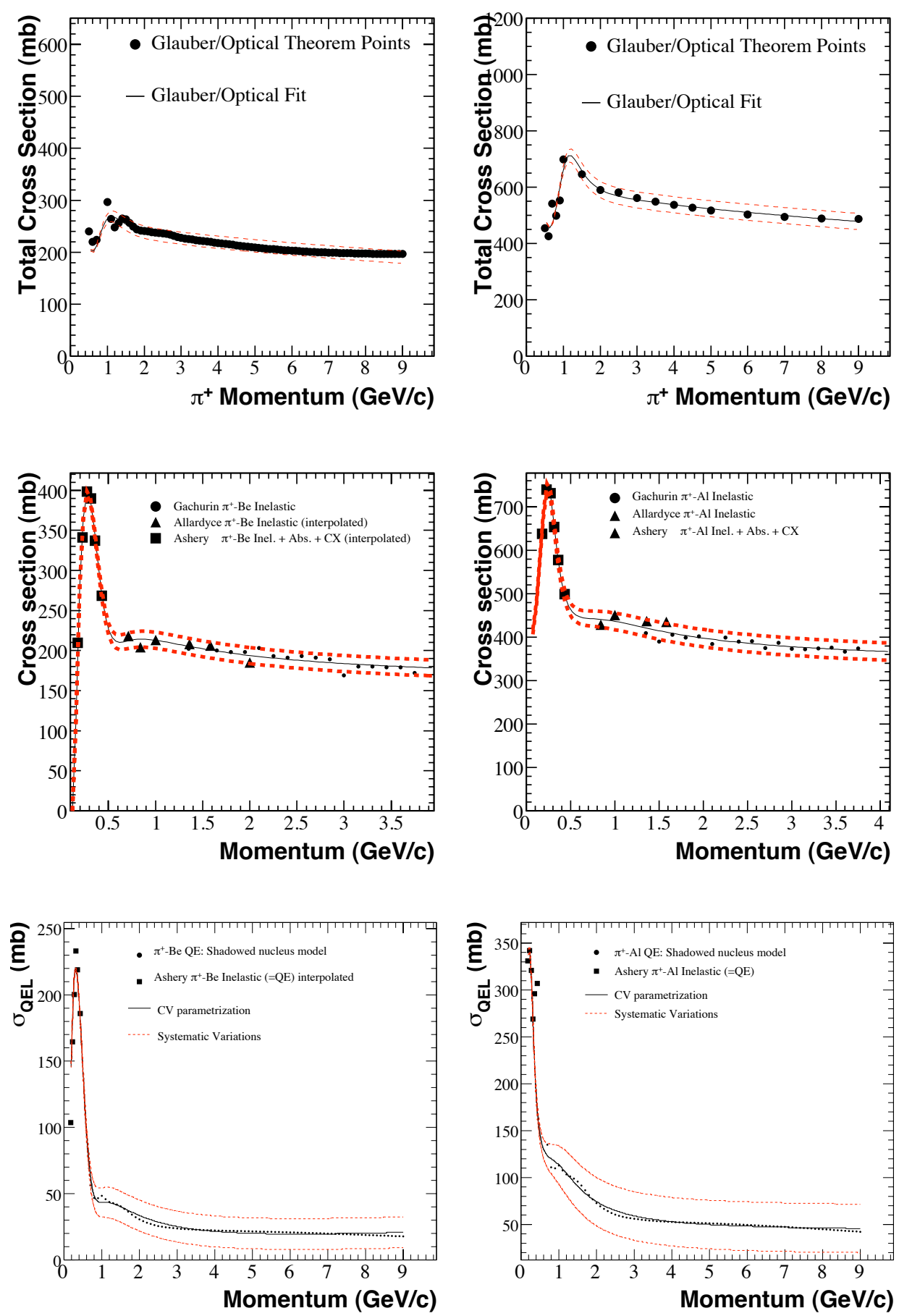

Figure 4.3: The pion total (top), inelastic (middle), and quasi-elastic (bottom) parametrizations for interactions with beryllium (left) and aluminum (right) are shown. The systematic errors assumed for each are also given and compared with data where possible [36]. 


\subsubsection{Particle Production Cross Sections}

For each inelastic proton-beryllium interaction that takes place, a collection of secondary particles are produced. Interactions between $8 \mathrm{GeV}$ kinetic energy protons and nucleons at rest can produce any flavorless or strange hadron. The center of momentum energy for such collisions is $4.2 \mathrm{GeV}$, which is insufficient to create a $\mathrm{J} / \psi, N, N$ final state $\left(E_{\min }=3.10+0.94+0.94=4.98 \mathrm{GeV}\right)$, or for the reaction $p+N \rightarrow N+D+\Lambda_{c}^{+}$ to occur $\left(E_{\min }=0.94+1.86+2.29=5.09 \mathrm{GeV}\right)$; therefore, the booster neutrino beam contains no charm decay products.

The kinematics and multiplicity of each secondary particle species are determined from double differential production cross sections, $\partial^{2} \sigma / \partial p \partial \Omega$. Since the simulation is only concerned with generating the proper secondary particle spectra on average, no attempt is made to conserve energy or momentum in individual proton-beryllium interactions. Instead, the multiplicity of each secondary particle type is drawn from a Poisson distribution with a mean given by the ratio of the integrated production cross section to the total inelastic cross section. To determine the momentum and angle of a given secondary particle, the double differential cross section is normalized to unity, and the particle kinematics are drawn from the resulting probability distribution function. For the secondaries most important to the neutrino flux, pions and kaons, the double differential cross sections are determined from fits to external data $[38,39]$. Production cross sections for $\pi^{+}, \pi^{-}$, and $K_{L}$ particles are parametrized by SanfordWang function [40]. For $K^{+}$particles, a Feynman scaling parametrization is used [39].

The remaining simulated secondary particles, $p, n$, and $K^{-}$, have very little impact on the neutrino flux, and are generated according to the MARS14 hadron production program [41].

The Sanford-Wang parametrization of double differential production cross sec- 
tions takes the following form,

$$
\frac{\partial^{2} \sigma}{\partial p \partial \Omega}=c_{1}\left(1-\frac{p}{p_{B}-c_{9}}\right) \exp \left(-c_{3} \frac{p^{c_{4}}}{p_{B}^{c_{5}}}-c_{6} \theta\left(p-c_{7} p_{B} \cos ^{c_{8}} \theta\right)\right)
$$

where $p$ and $\theta$ are the direction and angle of the outgoing pion, and $p_{B}$ is the momentum of the incident beam protons. The parameter $c_{9}$ is set to $1 \mathrm{GeV}$ in fits to pion data [38]. The HARP experiment at CERN was run at the same $8 \mathrm{GeV}$ proton beam energy produced by the Fermilab booster, and measures a range in the pion phase space $\left(0.75 \mathrm{GeV} / \mathrm{c}<p_{\pi}<6.5 \mathrm{GeV} / \mathrm{c} ; 30 \mathrm{mr}<\theta_{\pi}<210 \mathrm{mr}\right)$ that covers more than $80 \%$ of the pion phase space that produces neutrinos that reach the MiniBooNE detector [42]. Data from the E910 experiment at the Brookhaven National Laboratory also constrain a large portion of the pion phase space $\left(0.4 \mathrm{GeV} / \mathrm{c}<p_{\pi}<5.6 \mathrm{GeV} / \mathrm{c} ; 18 \mathrm{mr}<\theta_{\pi}<\right.$ $400 \mathrm{mr}$ ) at beam energies of $6.4,12.3$, and $17.5 \mathrm{GeV} / \mathrm{c}$, and has been extrapolated to $8 \mathrm{GeV}$ to be included in the fit as well.

The simultaneous fit to HARP and E910 data minimizes the following $\chi^{2}$,

$$
\chi^{2}=\sum_{i, j, k}\left(D_{i k}-N_{k} T_{i k}\right) V_{i j k}^{-1}\left(D_{j k}-N_{k} T_{j k}\right)+\sum_{k} \frac{\left(N_{k}-1\right)^{2}}{\sigma_{k}^{2}},
$$

where $V_{i j k}$ is the covariance matrix for experiment $k$ over measured bins $i$ and $j, D_{i k}$ and $T_{i k}$ are the measured and SW function values in bin $i$ of experiment $k$, and $N_{k}$ is the normalization of experiment $k$, which is constrained by the reported normalization uncertainty, $\sigma_{k}$. The fit produces a minimum $\chi^{2} / N_{\text {dof }}$ value of 1.8 , indicating that the fit uncertainty does not sufficiently cover the spread of the data. To compensate for this deficiency, the error on each data point is scaled by $\sqrt{1.8}$ and the fit is performed again. The final fit result gives normalization values of 0.973 and 1.039 for HARP and E910, respectively, which are slightly smaller than the $4 \%$ and $5 \%$ normalization uncertainties reported by each experiment. The best fit Sanford-Wang function is compared to the HARP data in Figure 4.4 .

The Sanford-Wang parametrization is used to fit the $\pi^{-}$and $K_{L}$ production cross sections as well. Data from both HARP and E910 are once again used to fit the $\pi^{-}$ 


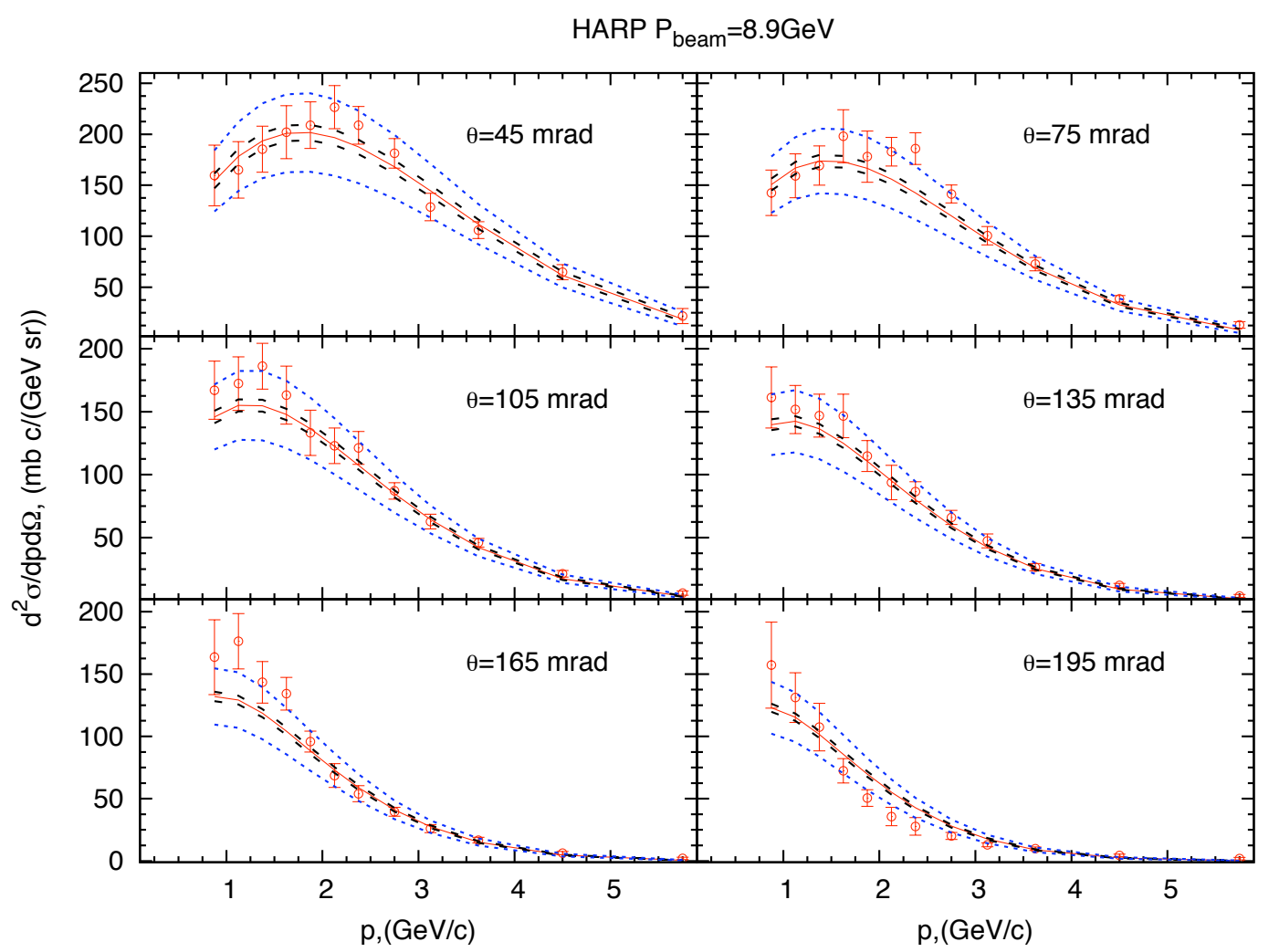

Figure 4.4: The result of the $\pi^{+}$Sanford-Wang fit to HARP and E910 data is shown with the HARP data (red points) overlayed. The uncertainties from both the initial fit (dashed line) and the fit with scaled data errors (dotted line) are given.

cross section. The $K_{L}$ fit is performed on data from E910 and Abe et. al. [43]. Unlike the pion fits, the $K_{L}$ fit allows $c_{9}$ to float [44]. The results from each fit are given in Table 4.2.

Table 4.2: A list of Sanford-Wang parameters for the $\pi^{+}, \pi^{-}$, and $K_{L}$, fits is shown.

\begin{tabular}{|c|c|c|c|c|c|c|c|c|c|}
\hline Fit & $c_{1}$ & $c_{2}$ & $c_{3}$ & $c_{4}$ & $c_{5}$ & $c_{6}$ & $c_{7}$ & $c_{8}$ & $c_{9}$ \\
\hline \hline$\pi^{+}$ & 220.7 & 1.080 & 1.000 & 1.978 & 1.32 & 5.572 & 0.08678 & 9.686 & 1 \\
\hline$\pi^{-}$ & 213.66 & 0.93785 & 5.4537 & 1.2096 & 1.2836 & 4.7807 & 0.073383 & 8.3294 & 1 \\
\hline$K_{L}$ & 15.13 & 1.975 & 4.084 & 0.9277 & 0.7306 & 4.362 & 0.04789 & 13.3 & 1.278 \\
\hline
\end{tabular}

The $K^{+}$production cross section is fit using a Feynman scaling parametriza- 
tion [39]. The cross section is written in terms of the transverse momentum of the secondary, $p_{t}$, and a scaling factor, $x_{F}=p_{\|}^{c m} / p_{\| \max }^{c m}$, where $p_{\|}^{c m}$ is the longitudinal momentum in the center of momentum frame of the proton-beryllium interaction. The cross section is given by

$$
\frac{\partial^{2} \sigma}{\partial p \partial \Omega}=\left(\frac{p}{E}\right) c_{1}\left(1-\left|x_{F}\right|\right)^{c_{8}} \exp \left[-c_{3}\left|x_{F}\right|^{c_{4}}-c_{7}\left|p_{t} x_{F}\right|^{c_{6}}-c^{2} p_{t}-c_{5} p_{t}^{2}\right]
$$

The parametrization is fit to seven different data sets $[45,46,47,48,49,50,51]$. In the fit minimization, $c_{8}$ takes on negative values with large uncertainties, so for the final fit it has been fixed to zero. Just as in the pion fit, the minimum $\sqrt{\chi^{2} / N_{d o f}}$ of $\sqrt{2.28}$ is used to scale the size of the data errors. The result of the fit is given in Table 4.3.

Table 4.3: The result for each of the Feynman scaling parameters in the $K^{+}$fit is given.

\begin{tabular}{|c|c|c|c|c|c|c|c|c|}
\hline Fit & $c_{1}$ & $c_{2}$ & $c_{3}$ & $c_{4}$ & $c_{5}$ & $c_{6}$ & $c_{7}$ & $c_{8}$ \\
\hline \hline$K^{+}$ & 11.70 & 0.88 & 4.77 & 1.51 & 2.21 & 2.17 & 1.51 & 0.0 \\
\hline
\end{tabular}

\subsubsection{Meson Decays}

The decay branching fractions for kaons and pions were last updated to correspond to the 2006 Particle Data Group averages [52], the values of which have not changed significantly to date. Of particular importance were the decay channels in which neutrinos are produced. Each such decay mode was faithfully reproduced in the simulation, and the sum of modes was set to unity by adjusting the branching fraction of the smallest neutrino-less process. The effect of $\mathrm{CP}$ violation has been accounted for in neutral kaon decays using the measured charge asymmetries [52]. The values used in the simulation are given in Table 4.4 .

The default GEANT4 routines are used to conduct all particle decays. In the case of muon decays, the G4MuonDecayChannelWithSpin.cc class properly simulates the V-A coupling with first order radiative corrections, however the neutrino energy 
Table 4.4: The branching fractions used in the beam Monte Carlo simulation are given for $\pi^{ \pm}, K^{ \pm}$, and $K_{L}$. The values that have been modified from their Particle Data Group values are labeled as "mod." For the negatively charged mesons, the same values are used for the charge conjugate processes.

\begin{tabular}{|c|l|}
\hline Decay Mode & $\begin{array}{l}\text { Branching Fraction } \\
\text { (measured uncertainty) }\end{array}$ \\
\hline \hline$\pi^{+} \rightarrow \mu^{+} \nu_{\mu}$ & $0.9998770(4)$ \\
\hline$\pi^{+} \rightarrow e^{+} \nu_{e}$ & $0.0001230(4)$ \\
\hline \hline$K^{+} \rightarrow \mu^{+} \nu_{\mu}$ & $0.6344(14)$ \\
\hline$K^{+} \rightarrow \pi^{+} \pi^{0}$ & $0.2092(12)$ \\
\hline$K^{+} \rightarrow \pi^{+} \pi^{+} \pi^{-}$ & $0.05590(31)$ \\
\hline$K^{+} \rightarrow \pi^{0} e^{+} \nu_{e}$ & $0.0498(7)$ \\
\hline$K^{+} \rightarrow \pi^{0} \mu^{+} \nu_{\mu}$ & $0.0332(6)$ \\
\hline$(\mathrm{mod}) K^{+} \rightarrow \pi^{+} \pi^{0} \pi^{0}$ & 0.0175 \\
\hline$(\mathrm{PDG}) K^{+} \rightarrow \pi^{+} \pi^{0} \pi^{0}$ & $0.01757(24)$ \\
\hline \hline$K_{L} \rightarrow \pi^{0} \pi^{0} \pi^{0}$ & $0.1956(14)$ \\
\hline$K_{L} \rightarrow \pi^{+} \pi^{-} \pi^{0}$ & $0.1256(5)$ \\
\hline$K_{L} \rightarrow \pi^{-} e^{+} \nu_{e}$ & $0.20333(75)$ \\
\hline$K_{L} \rightarrow \pi^{+} e^{-} \bar{\nu}_{e}$ & $0.20197(75)$ \\
\hline$K_{L} \rightarrow \pi^{-} \mu^{+} \nu_{\mu}$ & $0.13551(35)$ \\
\hline$K_{L} \rightarrow \pi^{+} \mu^{-} \bar{\nu}_{\mu}$ & $0.13469(35)$ \\
\hline$K_{L} \rightarrow \pi^{+} \pi^{-}$ & $0.001976(8)$ \\
\hline$K_{L} \rightarrow \pi^{0} \pi^{0}$ & $0.000869(4)$ \\
\hline$(\mathrm{mod}) K_{L} \rightarrow \gamma \gamma$ & 0.000455 \\
\hline$(\mathrm{PDG}) K_{L} \rightarrow \gamma \gamma$ & $0.000548(5)$ \\
\hline
\end{tabular}

spectra are not generated correctly. This deficiency is corrected in the following stage of the Monte Carlo generation process described next.

\subsubsection{Statistics Amplification}

Most neutrinos produced in meson decays in the beam Monte Carlo simulation do not intersect the detector. To increase the number of usable neutrinos per generated proton on target, the beam Monte Carlo output is passed to a Fortran program that, for each neutrino produced, simulates the decay of the parent meson (or muon) multiple times (typically 1000), and records the neutrinos that hit the detector. This technique effectively boosts the number of protons on target of the simulation. In addi- 
tion, since the particle decay final states produced by the beam Monte Carlo simulation are discarded, any deficiencies in the GEANT4 decay generators are corrected at this stage.

For most events, this parent "re-decay" process works quite well; however, for certain regions of neutrino parent phase space, the additional neutrinos become problematic. In particular, parents with a large longitudinal momentum component, $p_{z}$, tend to produce neutrinos that hit the tank at a very high frequency. These events contribute a large number of mono-energetic neutrinos at high energies where the neutrino energy spectrum is already sparsely populated. The result is a collection of non-statistical bumps in the high energy tail of the distribution.

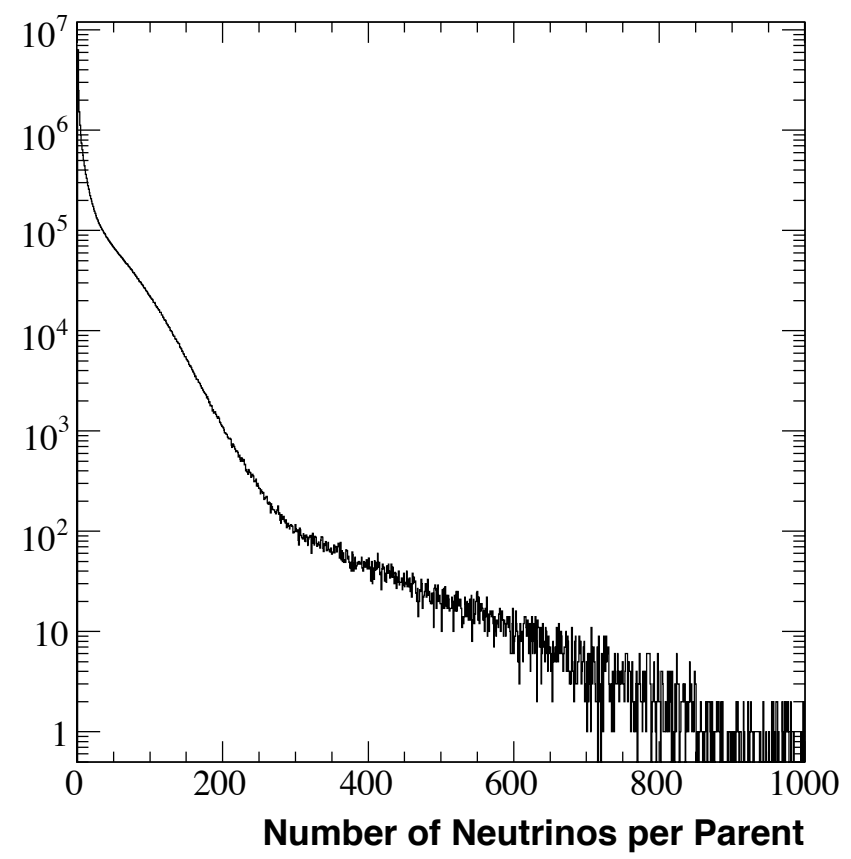

Figure 4.5: The number of neutrinos that hit the detector is plotted for each neutrino parent. Note that this plot has been made after beam Monte Carlo cross section weighting has been implemented, which exponentially increases the high multiplicity events. Even with this amplification, $75 \%$ of neutrino parents put less than 10 neutrinos in the detector. 
To mitigate the effect of these high- $p_{z}$ neutrino parents, the double differential particle production cross sections are weighted by an exponential function of $p_{z}$,

$$
w\left(p_{z}\right)=c_{1} \exp \left(c_{2} * p_{z}\right)
$$

Each event that is produced is given a weight of $1 / w$ so that the shape of the neutrino energy spectrum is restored. The effect of this weighting procedure is shown in Figure 4.6. Since the cross section weighted distributions are shifted to higher neutrino energies, the reduced the statistical uncertainty in the high energy neutrino tail occurs in the regions of phase space that are relatively more important for the simulated event rate. The values of the weighting function parameters for each modified neutrino parent species is given in Table 4.5.

Table 4.5: The parameters for the exponential weighting function in Equation 4.5 are given for each modified particle species.

\begin{tabular}{|c|c|c|c|}
\hline Secondary Particle & $c_{1}$ & $c_{2}$ & $w\left(p_{z}=10 \mathrm{GeV}\right)$ \\
\hline \hline$\pi^{+}$ & 1 & 0.852 & 5000 \\
\hline$\pi^{-}$ & 1 & 0.852 & 5000 \\
\hline$K^{+}$ & 1.1 & 0.801 & 3300 \\
\hline$K^{-}$ & 1.1 & 0.801 & 3300 \\
\hline$K_{L}$ & 3 & 0.801 & 9000 \\
\hline
\end{tabular}

For the oscillation analysis, one additional weighting technique is needed to reliably produce the neutrino spectrum from muon decays. Muons are produced through the $\pi^{+} \rightarrow \mu^{+} \nu_{\mu}$ decay channel responsible for the majority of the neutrino flux. Since the muon lifetime is two orders of magnitude larger than the pion lifetime, $\mu^{+}$particles rarely decay in flight in a typical Monte Carlo run. To estimate this important contribution to the $\nu_{e}$ flux, for each muon that is produced, nineteen identical copies are created to improve the probability of observing a decay in flight; the corresponding events are weighted by a factor of $1 / 20$. 


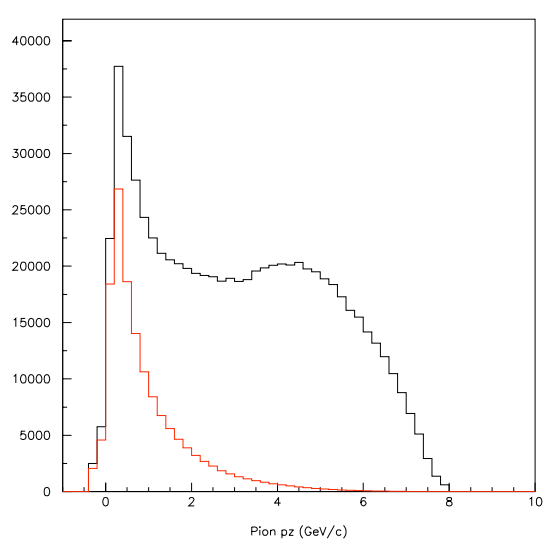

(a) The generated (black) and actual (red) $\pi^{+} p_{z}$ in the beam Monte Carlo simulation (in $\mathrm{GeV} / \mathrm{c}$ )

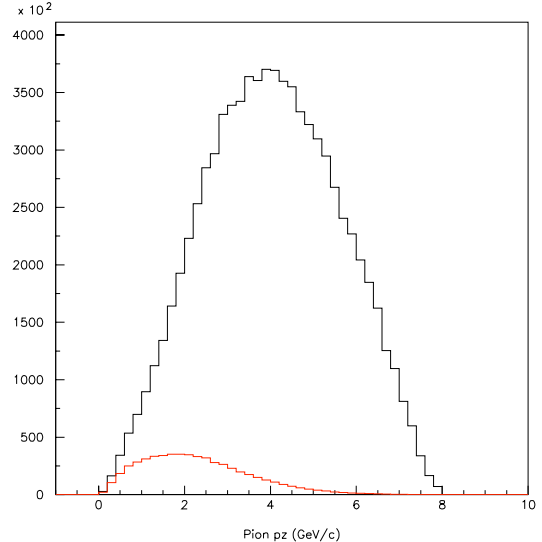

(b) The generated (black) and actual (red) $\pi^{+} p_{z}$ after the re-decaying of the neutrino parents (in $\mathrm{GeV} / \mathrm{c}$ )

Figure 4.6: The effect of the exponential weighting function of Equation 4.5 is shown on the $\pi^{+} p_{z}$ distribution after the beam Monte Carlo (left) and the parent re-decay simulation stages. The increased statistics at high $p_{z}$ improve the precision of the high energy neutrino spectrum.

\subsubsection{Flux Predictions}

The energy spectra for neutrinos that hit the detector are given in Figures 4.7 and 4.8. Figure 4.7 gives the flux for each of the four neutrino species produced by the Booster neutrino beam. In Figure 4.8, the $\nu_{\mu}$-only flux, needed to perform the $\mathrm{CC} \pi^{+}$cross section measurement, is plotted for each of the contributing neutrino parent species.

The neutrino flux is dominated by $\nu_{\mu}$, especially at energies greater than $0.3 \mathrm{GeV}$ where the $\mathrm{CC} \pi^{+}$cross section turns on. At low neutrino energies, the $\nu_{\mu}$ flux comes mostly from $\pi^{+} \rightarrow \mu^{+} \nu_{\mu}$ decays. At $\sim 2.3 \mathrm{GeV}$, kaon decays become the dominant component of the $\nu_{\mu}$ flux, however the neutrino population at that point is only $\sim 1 \%$ of the peak value. 


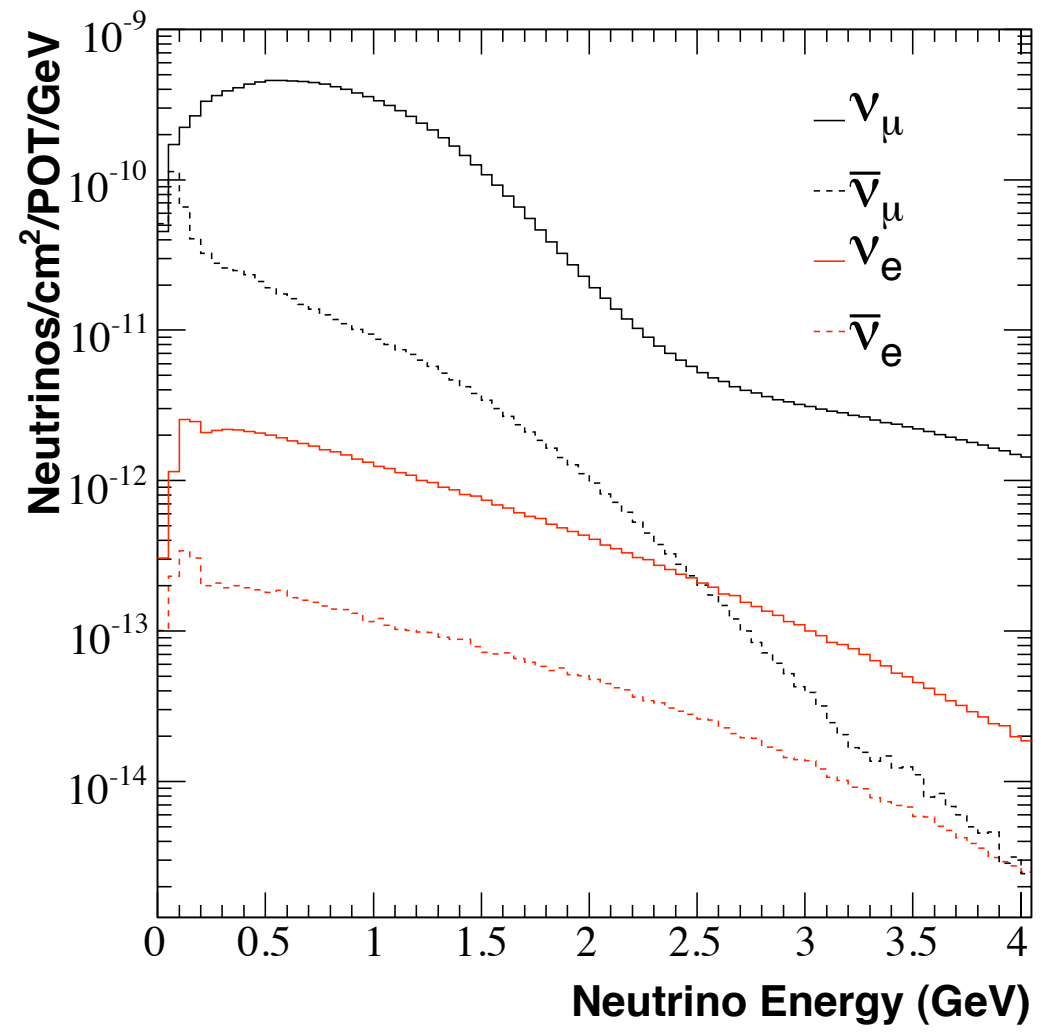

Figure 4.7: The neutrino energy spectrum for each of the four neutrino species is shown for neutrinos that hit the MiniBooNE detector.

\subsubsection{Systematic Uncertainties}

As described in Section 4.1.1.3, the $\pi^{+}$Sanford-Wang parametrization produces a poor fit to the HARP and E910 data. To account for the disagreement, the errors on the data are increased until they become compatible with the shape dictated by the parametrization. The resulting covariance matrix from such a fit is dominated by the normalization uncertainty. The failure of the parametrization to fit the data is due to its inability to reproduce the data shape for any choice of parameters. The cross section measurement data have reached a level of precision such that it can no longer be described by the model.

The systematic uncertainties in the knowledge of the neutrino flux need not absorb 


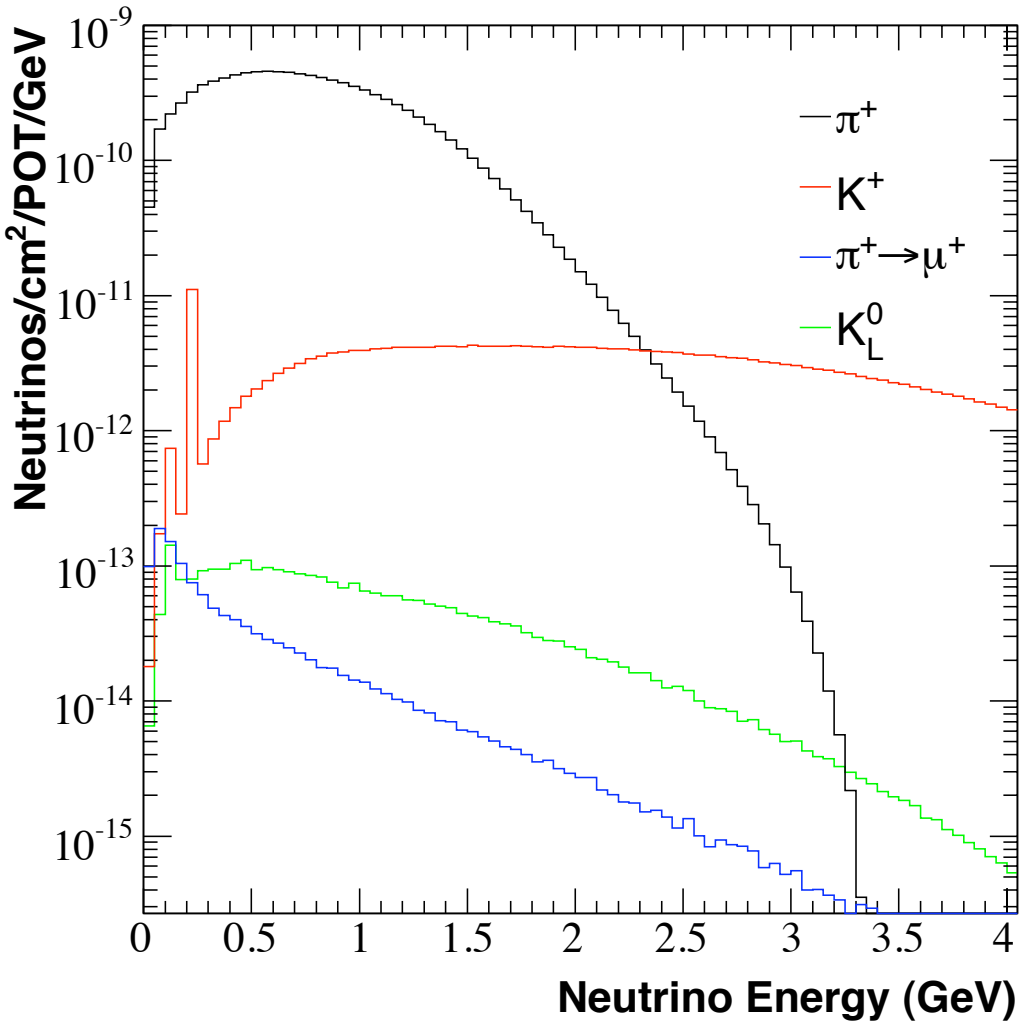

Figure 4.8: The neutrino energy spectrum for $\nu_{\mu}$ particles that hit the MiniBooNE detector. The contributions from each of the neutrino parent particle types are shown separately.

this additional contribution from the failure of the model. Instead, the data have been fit with a spline function to faithfully capture all the features of the data [53]. The spline function is a collection of 3rd-order polynomials, each fit over a small interval of data, that are required to have common first and second derivatives at the interval boundaries. The results of the spline fit are compared with the HARP data in Figure 4.9.

Rather than abandon the Sanford-Wang shape completely the systematic error is calculated from the variation of the spline covariance matrix about the Sanford-Wang central value. In this way, the uncertainty acquires an additional component in regions where the parametrization has trouble accommodating the data. Further details are 

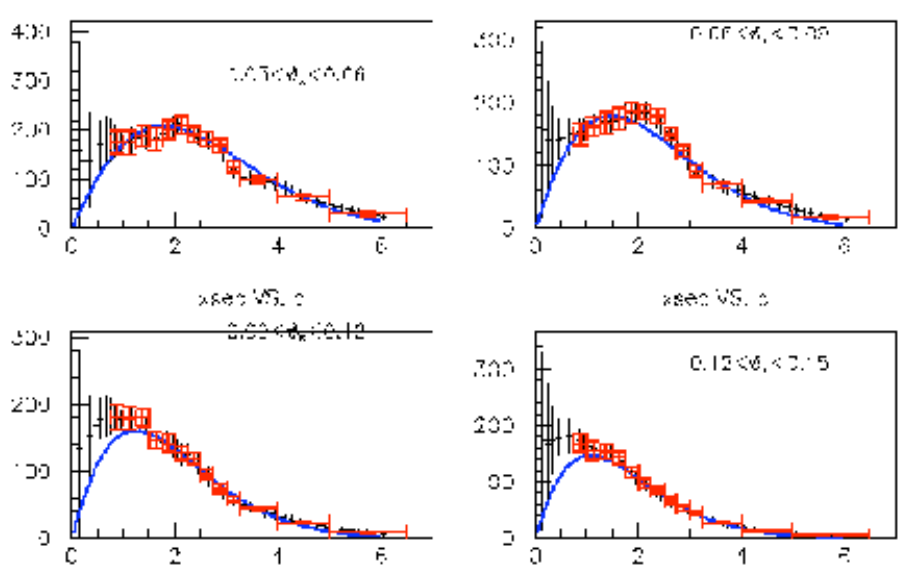

$\therefore \operatorname{sen} \sin$

$\sin :=$
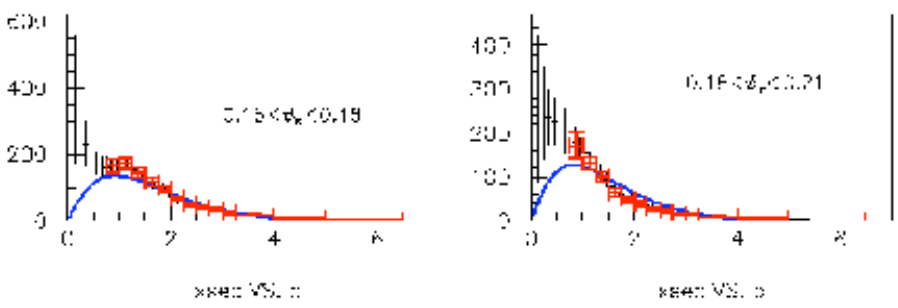

Figure 4.9: The result of the spline fit (black points) is compared to the HARP data (red points) and the Sanford Wang function (blue line) as a function of pion momentum in six different pion angle bins. The uncertainties on the spline fit, shown as black error bars, grow as expected as the function moves further from the data (figure taken from Ref. [53]).

given in Section 6.4.7.1. The flux contributions from the other neutrino parent particles are small enough that the full uncertainty from fits to either Sanford-Wang or Feynman scaling are used with little impact on the total error.

The remaining uncertainties in the neutrino flux are calculated from additional Monte Carlo simulations in which the uncertain parameters are varied by their uncertainties. Each of these altered Monte Carlo sets is referred to as a "unisim." The uncertainties in the nucleon and pion interaction cross sections are shown in Figures 4.2 and 4.3. Without data to which it can be compared, the quasi-elastic cross section uncertainties have been estimated to be very large and dominate the uncertainties from hadron production. 
The remaining beam unisims vary the properties of the horn. The horn current has been varied by $1 \mathrm{kA}$, significantly outside of its measured variation. The uncertainty in the extent to which the current penetrates the inner conductor (i.e. the skin depth) has been estimated by removing the effect completely and treating the result as a variation of one standard deviation. The effect of the largest beam unisim excursions is shown in Figure 4.10.

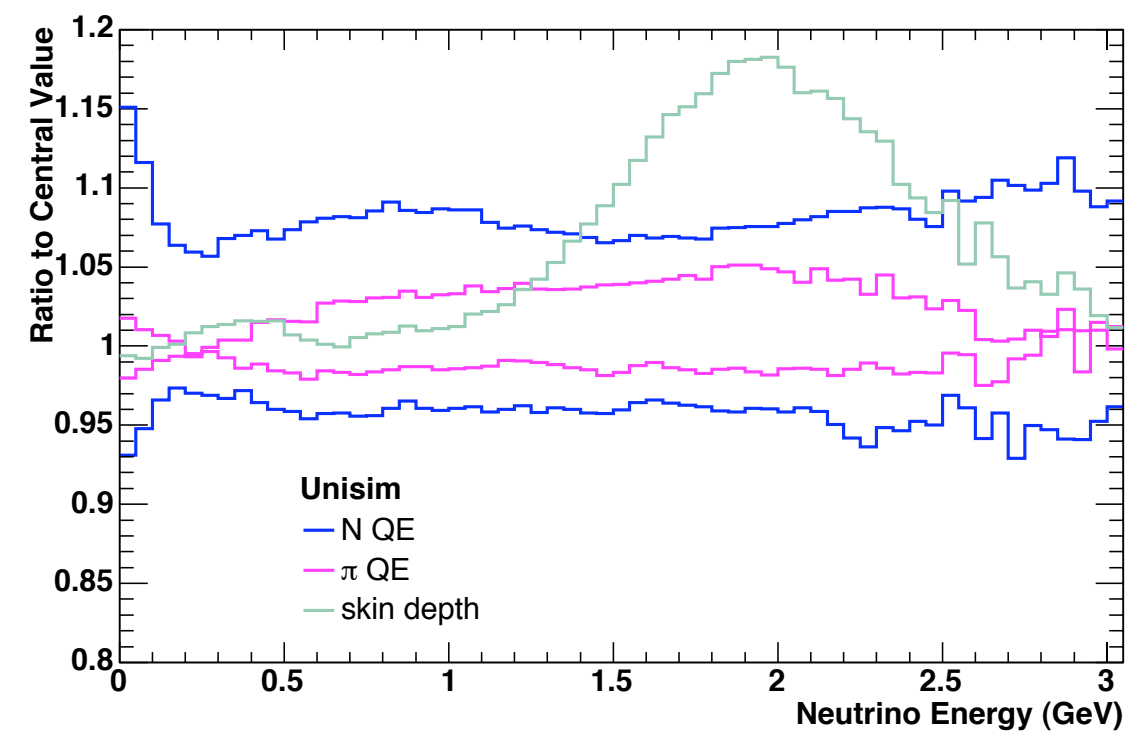

Figure 4.10: The effect of the largest beam unisim excursions are shown. Any unisim that did not vary more than $2 \%$ from the central value has been excluded for clarity. The largest integrated effects are from the nucleon and pion quasi-elastic scattering uncertainties. Along the high energy tail of the neutrino flux, the skin depth variation causes changes of almost $20 \%$.

\subsection{Nuance}

MiniBooNE uses the Nuance event generator to simulate neutrino interactions [54]. Nuance is a Fortran-based software package that takes as input the generated fluxes for each neutrino species and produces a cross section weighted spectrum for each type of neutrino interaction. These event rate distributions are then used to simulate neutrino 
interactions and propagate the particles created in the interaction through the nuclear medium. Each event is then written out as a list of particle types and momenta emerging from the nucleus.

Nuance simulates essentially all neutrino interaction processes relevant at MiniBooNE energies. Ninety-nine charged and neutral current processes for both neutrinos and anti-neutrinos are simulated. A summary of the relevant charged current processes as a function of neutrino energy is given in Figure 4.11. At MiniBooNE flux energies $(<2 \mathrm{GeV})$, neutrino interactions are dominated by CCQE and $\mathrm{CC} \pi^{+}$interactions.

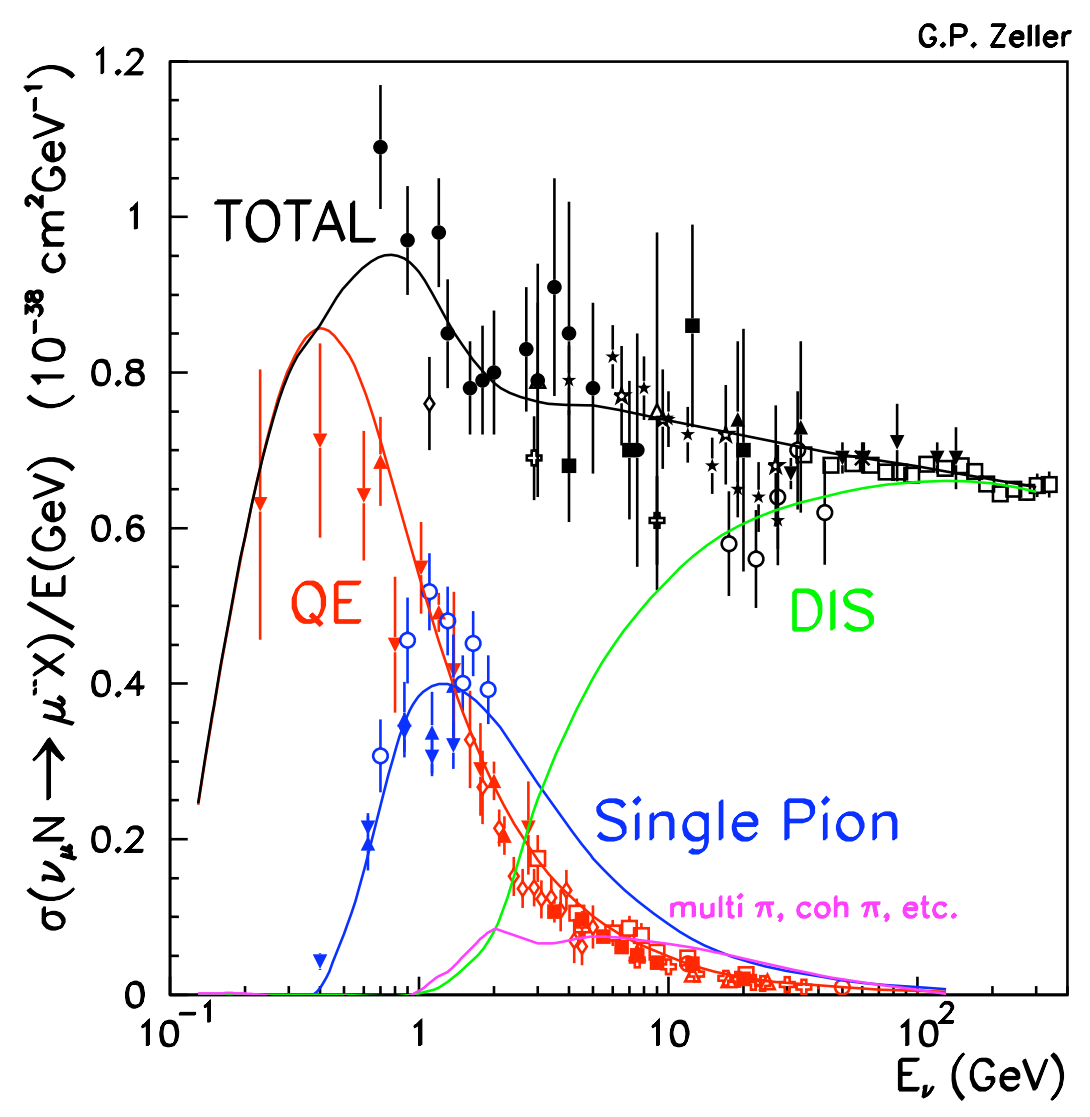

Figure 4.11: The charged current interaction processes are shown as a function of neutrino energy. The peak neutrino energy at MiniBooNE is $\sim 700 \mathrm{MeV}$. This region is dominated by CCQE and $\mathrm{CC} \pi^{+}$. 
Nuance classifies each cross section based on the particles produced in the initial interaction, before they traverse the nucleus. The event rates derived from the MiniBooNE flux are given in Table 4.6.

Table 4.6: Event rate fractions produced by Nuance using the MiniBooNE flux from Figure 4.8. The $\mathrm{CC} \pi^{+}$and $\mathrm{NC} \pi^{0}$ fractions include coherent scattering off of the entire nucleus.

\begin{tabular}{|c|l|c|}
\hline Name & \multicolumn{1}{|c|}{ Interaction Process } & Rate Fraction \\
\hline \hline $\mathrm{CCQE}$ & $\nu_{\mu} n \rightarrow \mu^{-} p$ & 0.400 \\
\hline $\mathrm{CC} \pi^{+}$ & $\nu_{\mu} N \rightarrow \mu^{-} N \pi^{+}$ & 0.237 \\
\hline $\mathrm{NCEL}$ & $\nu_{\mu} N \rightarrow \nu_{\mu} N$ & 0.170 \\
\hline $\mathrm{NC} \pi^{0}$ & $\nu_{\mu} N \rightarrow \nu_{\mu} N \pi^{0}$ & 0.066 \\
\hline $\mathrm{CC} \pi^{0}$ & $\nu_{\mu} n \rightarrow \mu^{-} p \pi^{0}$ & 0.040 \\
\hline $\mathrm{NC} \pi^{+}$ & $\nu_{\mu} p \rightarrow \nu_{\mu} n \pi^{+}$ & 0.021 \\
\hline $\mathrm{CCmulti} \pi$ & $\nu_{\mu} N \rightarrow \mu^{-} \Delta \pi$ & 0.015 \\
\hline $\mathrm{NC} \pi^{-}$ & $\nu_{\mu} n \rightarrow \nu_{\mu} p \pi^{-}$ & 0.016 \\
\hline $\mathrm{CCDIS}$ & $\nu_{\mu} N \rightarrow \mu^{-}+$hadrons & 0.010 \\
\hline $\mathrm{CCmeson} B$ & $\nu_{\mu} N \rightarrow \mu^{-}+(\rho, K, \eta)+$ baryon & 0.006 \\
\hline $\bar{\nu}$ & $\bar{\nu} N \rightarrow$ anything & 0.004 \\
\hline other & each $<0.01$ & 0.015 \\
\hline
\end{tabular}

\subsubsection{CCQE}

Charged current quasi-elastic interactions (CCQE) are simulated according to the model of Llewellyn Smith [55]. Just as in the derivation of $\mathrm{CC} \pi^{+}$interactions described in Section 1.1.2.1, the leptonic current is easily calculated, and the crux of the model lies in the parametrization of the hadronic current. The cross section for neutrino scattering is given by

$$
\frac{\partial \sigma}{\partial Q^{2}}=\frac{G_{F}^{2} m_{N}^{2} V_{u d}^{2}}{8 \pi E_{\nu}^{2}}\left[A\left(Q^{2}\right)+B\left(Q^{2}\right) \frac{s-u}{m_{N}^{2}}+C\left(Q^{2}\right) \frac{(s-u)^{2}}{m_{N}^{4}}\right]
$$


where $s$ and $u$ are the Mandelstam variables such that $(s-u)=4 m_{N} E_{\nu}-Q^{2}-m_{l}^{2}$, and

$$
\begin{aligned}
A\left(Q^{2}\right)= & \frac{m_{l}^{2}+Q^{2}}{m_{N}^{2}}\left[(1+\tau) F_{A}^{2}-(1-\tau) F_{1}^{2}+\tau(1+\tau) F_{2}^{2}+4 \tau F_{1} F_{2}\right. \\
& -\frac{m_{l}^{2}}{4 m_{N}^{2}}\left[F_{1}^{2}+\left(F_{A}+2 F_{P}-4(1+\tau) F_{P}^{2}\right]\right] \\
B\left(Q^{2}\right)= & 4 \tau F_{A}\left(F_{1}+F_{2}\right) \\
C\left(Q^{2}\right)= & \frac{1}{4}\left(F_{A}^{2}+F_{1}^{2}+\tau F_{2}^{2}\right)
\end{aligned}
$$

for $\tau=\frac{Q^{2}}{4 m_{N}^{2}}$. The hadronic form factors, $\left(F_{1}, F_{2}, F_{P}, F_{A}\right)$, are once again parametrized with a dipole form given by,

$$
\begin{aligned}
& F_{1}\left(Q^{2}\right)=\frac{1+\tau\left(1+\mu_{p}-\mu_{n}\right)}{(1+\tau)\left(1+\frac{Q^{2}}{m_{V}^{2}}\right)^{2}}, \\
& F_{2}\left(Q^{2}\right)=\frac{\left(\mu_{p}-\mu_{n}\right)}{(1+\tau)\left(1+\frac{Q^{2}}{m_{V}^{2}}\right)}, \\
& F_{A}\left(Q^{2}\right)=\frac{F_{A}(0)}{\left(1+\frac{Q^{2}}{m_{A}^{2}}\right)^{2}}, \\
& F_{P}\left(Q^{2}\right)=\frac{2 m_{N}^{2}}{m_{\pi}^{2}+Q^{2}},
\end{aligned}
$$

where $m_{V}$ and $m_{A}$ are the vector and axial masses described in Section 1.1.2.1, and $\mu_{p}$ and $\mu_{n}$ are the proton and neutron magnetic moments. The parameter $F_{A}(0)=-1.27$ is determined from neutron beta decay [55].

\subsubsection{Nuclear Effects}

The motion of the protons and neutrons in the nucleus are described by the Fermi gas model of Smith and Moniz [56]. The observed cross sections are modified by the motion of the nucleons as well as the strength to which they are bound to the rest of the nucleus. The binding energy of $34 \mathrm{MeV}$ and cutoff momentum of $220 \mathrm{MeV} / \mathrm{c}$ are determined using electron scattering data [57]. 
Once a neutrino interaction occurs, the final state particles must traverse the nucleus before they can be observed. This effect is particularly important for final state pions, as they interact strongly with the nucleons. Events created with $\pi^{+}$will not be observed if the pion is either absorbed or takes part in a charge exchange interaction, $\pi^{+}+n \rightarrow \pi^{0}+p$. The cross section for both of these processes is given as a function of pion energy in Figure 4.12.

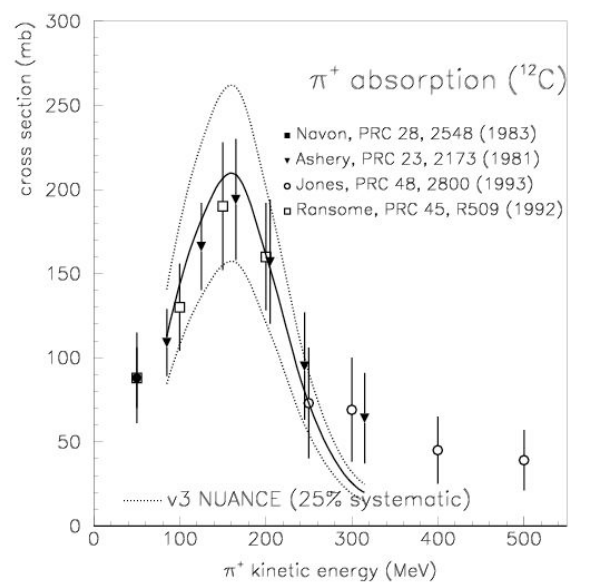

(a) pion absorption vs pion kinetic energy

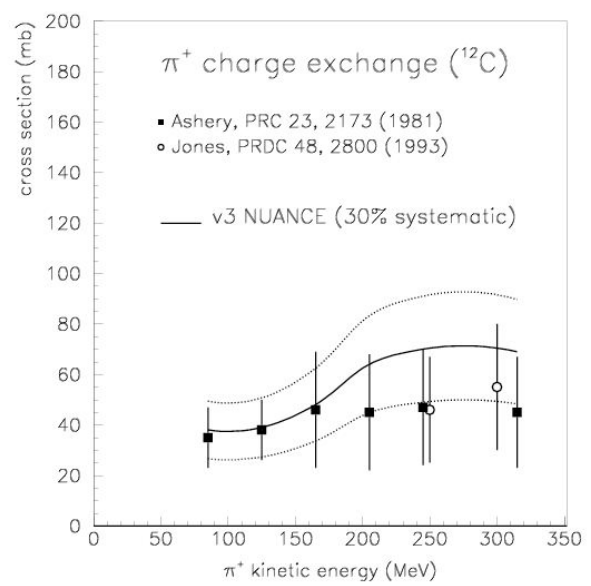

(b) pion charge exchange vs pion kinetic energy

Figure 4.12: The pion absorption and charge exchange cross sections are shown as a function of pion kinetic energy. The absorption systematic uncertainty is $25 \%$, and the charge exchange cross section is assigned a $30 \%$ uncertainty.

\subsubsection{Systematic Uncertainties}

The common feature of all exclusive charged current interactions is that the axialvector portion of each interaction is parametrized as a dipole with an axial mass, $m_{A}$. The two background processes most relevant to the $\mathrm{CC} \pi^{+}$cross section measurement described in Chapter 6 are CCQE and CCmulti $\pi$. The main uncertainty in each of these processes is the value of the axial mass, and the variations for each are given in 
Table 4.7. The CCDIS cross section normalization is varied by $25 \%$.

Table 4.7: The axial mass values for CCQE and CCmulti $\pi$ interactions are listed with associated systematic uncertainties.

\begin{tabular}{|l|l|l|}
\hline parameter & central value & systematic uncertainty \\
\hline \hline$m_{A}(\mathrm{CCQE})$ & $1.234 \mathrm{GeV}$ & $0.077 \mathrm{GeV}$ \\
\hline$m_{A}(\mathrm{CCmulti} \pi)$ & $1.30 \mathrm{GeV}$ & $0.52 \mathrm{GeV}$ \\
\hline
\end{tabular}

The pion absorption and charge exchange uncertainties are given in Figure 4.12. Pions can also be effectively absorbed if the $\Delta$ resonance interacts with a nucleon via $\Delta N \rightarrow N N$. The effect of eliminating this process completely is taken as a one sigma variation. For historical reasons, the pion interaction uncertainties in the nucleus are included in the detector optical model uncertainties, while the pion interaction uncertainties for processes that occur outside the nucleus (discussed in Section 4.2.3) are included here as part of the cross section model uncertainties.

Finally, systematic uncertainties are assigned to the properties of the Fermi gas model. The binding energy is varied by $9 \mathrm{MeV}(26 \%)$ and the Fermi momentum is assigned an uncertainty of $30 \mathrm{MeV} / \mathrm{c}(14 \%)$.

\subsection{BooNEGlob}

Nuance can generate neutrino events only from a neutrino energy histogram for each neutrino species. All other information from the beam Monte Carlo simulation is discarded. In particular, the particle type and generation kinematics of the neutrino parent is lost. Additionally, several intra-event correlations are not preserved, such as the neutrino angle and interaction vertex, the event time and neutrino parent type, and the neutrino parent type and the neutrino interaction process.

To reinstate these correlations, each Nuance event is paired with a beam event in a Fortran-based program known as "BooNEGlob." In any given Nuance event, the only variables that are shared with the incident beam neutrino are the neutrino energy 
and species. BooNEGlob loops over the beam Monte Carlo output used to generate the neutrino flux and tabulates the events with the same neutrino type that are within a small energy window surrounding the Nuance event. For $\nu_{\mu}$ events, this energy tolerance is set to $1 \mathrm{MeV}$. An event is then randomly selected from the list of pairing candidates according to its beam Monte Carlo event weight.

Once a Nuance event is paired, the event can be manipulated in a variety of useful ways. Most importantly, the directions of the Nuance final state particles are all rotated to correspond to the direction of the incident neutrino. The Nuance events can also be weighted and filtered to amplify interesting regions of phase space. Finally, the beam Monte Carlo also assigns a neutrino parent type to each event so that it can be reweighted according to its parent production cross section function (SanfordWang, Feynman scaling, or the spline fit) when evaluating the systematic errors due to uncertainties in the fit parameters.

\subsection{Detector Simulation}

After Nuance produces a list of final state particles that exit the nucleus, the remainder of the particle propagation is handled by the detector Monte Carlo simulation. The detector Monte Carlo is GEANT3-based simulation package [58] that tracks each particle through the oil, including each optical photon produced via Cherenkov or scintillation radiation. As these photons constitute all of the data recorded by the detector, a detailed optical model has been developed that simulates effects such as absorption, scattering, and reflections. This section presents an overview of the features of the simulation. A detailed description of each component can be found elsewhere [59].

Several modifications have been made to the default GEANT3 algorithms. Additional processes have been added to model $\pi^{0}$ Dalitz decays $\left(\pi^{0} \rightarrow e^{+} e^{-} \gamma\right)$ and muon decay $(\mu \rightarrow e \nu \nu)$. The observed stopped $\mu^{-}$capture rate on carbon of 7.77 simulated $[60]$. 
The default GEANT3 hadronic interaction model, GFLUKA, has been replaced with GCALOR [61]. This switch was motivated by the handling of the pion absorption and charge exchange interactions that occur in the tank, analogous to the nuclear interactions described in Section 4.2.2. The cross sections predicted by each model as a function of pion kinetic energy are compared with data from Ashery et. al. in Figure 4.13 [34]. The FLUKA model over-predicts the charge exchange and under-predicts absorption by significant margins, whereas the GCALOR model mostly agrees with the data.

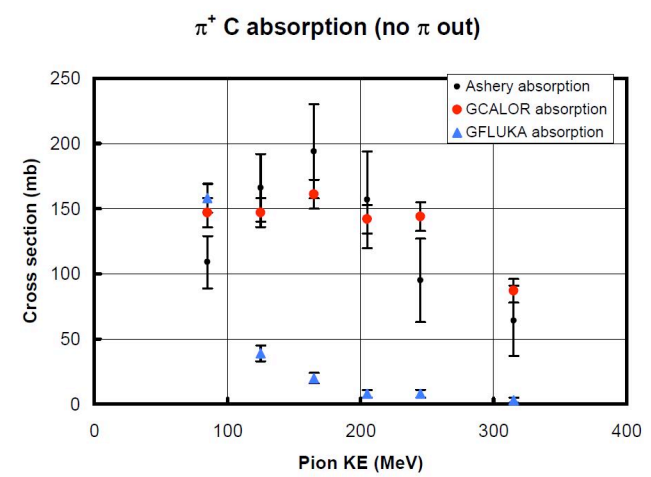

(a) pion absorption vs pion kinetic energy

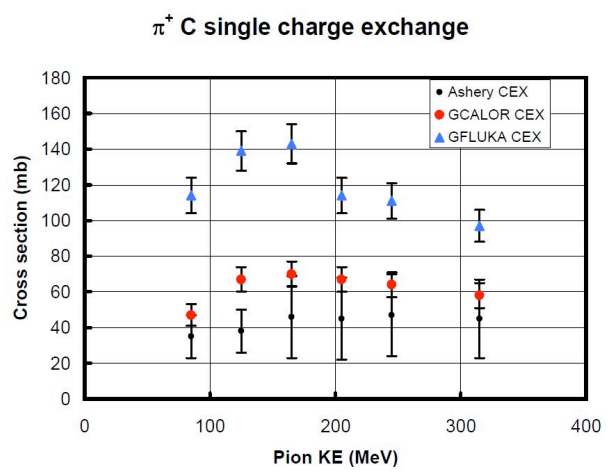

(b) pion charge exchange vs pion kinetic energy

Figure 4.13: The GFLUKA and GCALOR predictions for pion absorption and charge exchange cross sections in oil are compared with data as a function of pion kinetic energy. The GCALOR model is used for hadronic interactions in the detector Monte Carlo simulation.

\subsubsection{Optical Model}

The creation, propagation, and detection of optical photons produced in neutrino interactions are handled by the optical model. Photons are propagated until they are either absorbed or detected by a PMT. The photons are created through Cherenkov and scintillation emission from charged particle tracks, or through fluorescence from the 
molecules in the mineral oil. After the photons are created, they can undergo Rayleigh or Raman scattering, which has also been incorporated into the model.

Cherenkov radiation is created when a charged particle moves through a medium faster than light can propagate in the medium. The angular emission profile relative to the path of the particle is given by the ratio of these velocities,

$$
\cos \theta_{\text {Cher }}=\frac{v_{\text {light }}}{v_{\text {particle }}}
$$

where $v_{\text {light }}$ and $v_{\text {particle }}$ are the velocities of the light and the charged particle, respectively. Cherenkov light is produced instantaneously, and as the particle slows down due to energy losses in the medium, the Cherenkov "cone" is reduced until the particle drops below Cherenkov threshold as its velocity slows below $v_{\text {light }}$.

As charged particles travel through the oil, carbon and hydrogen atoms are ionized. The subsequent de-excitation of these states produces scintillation light. The flux of scintillation light produced per unit of energy deposited is given by Birks law [62],

$$
\frac{d N_{s c i}}{d E}=\frac{31.64 \mathrm{MeV}^{-1}}{1+B_{1}\left(\frac{1}{\rho_{\text {oil }}} \frac{d E}{d x}\right)+B_{2}\left(\frac{1}{\rho_{\text {oil }}} \frac{d E}{d x}\right)^{2}} .
$$

Unlike Cherenkov radiation, scintillation light is delayed exponentially relative to the track from which it is produced with a time constant of $34 \mathrm{~ns}$.

Molecules in the mineral oil can also become excited through interactions with higher energy photons and decay to produce optical photons in a process known as fluorescence. The characteristics of the fluoresced light depend on the properties of the medium. An analysis of the mineral oil has identified 4 independent fluors, each with its own time constant and emission profile, all of which are included in the simulation.

As optical photons propagate through the detector, Figure 4.14 shows the extinction rates for various processes that can affect the photon propagation path. The absorption used in the simulation is determined by the difference of total extinction rate and the sum of the exclusively measured components. A comparison of hit time 
distributions between laser calibration data and the simulation is shown in Figure 4.15. The modeling of photon reflections and scattering account for a significant portion of the timing structure, especially at late times.

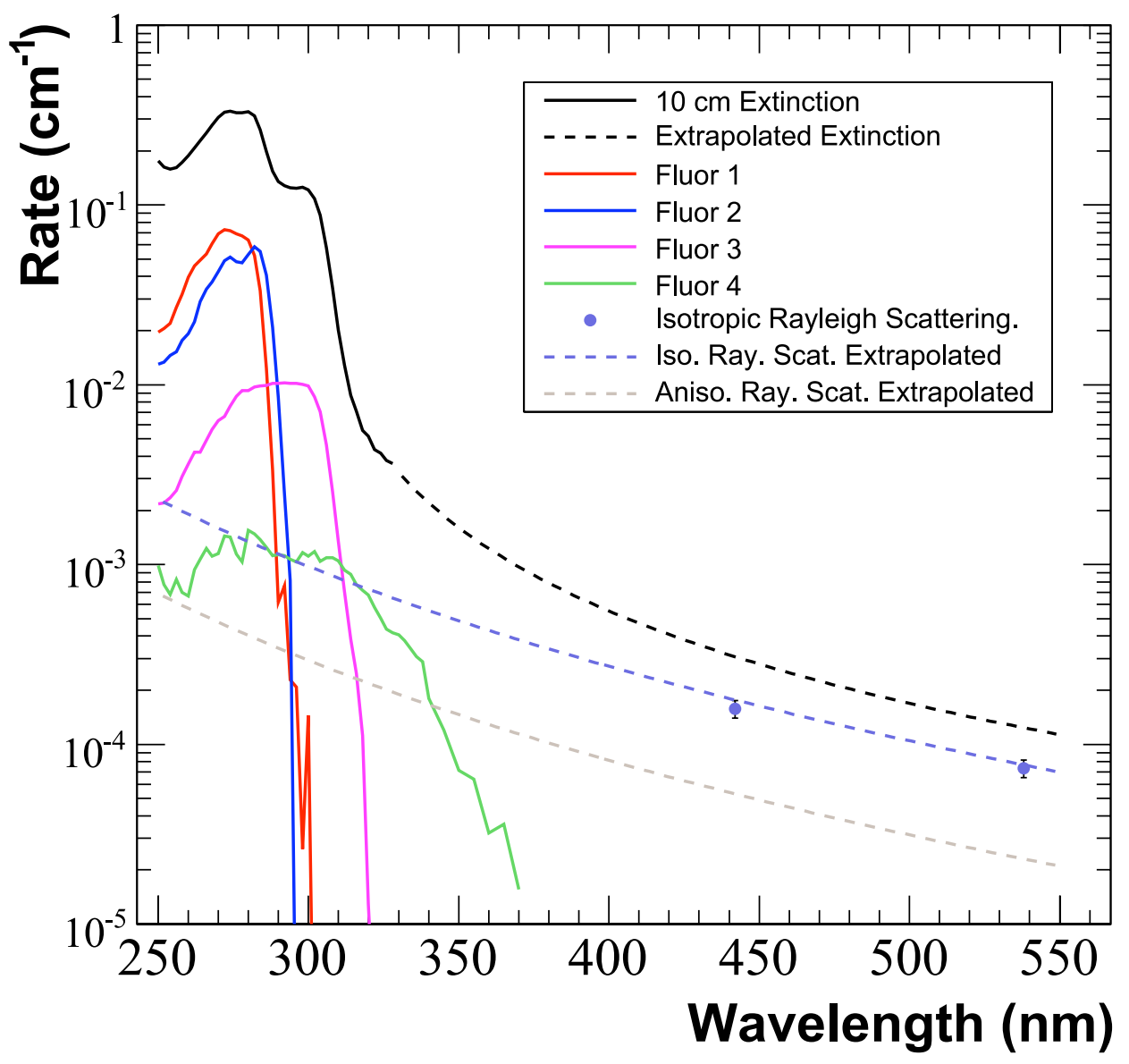

Figure 4.14: The extinction rates are given for several light sources as a function of wavelength. The total extinction curve (black) was measured using a $10 \mathrm{~cm}$ cell at Fermilab. The rates due to the 4 fluors and the effect of Rayleigh scattering are also shown.

\subsubsection{Systematic Uncertainties}

The optical model is a conglomeration of several physical quantities used to describe the production and propagation of optical photons. Of these quantities, 35 parameters describing phenomena such as the light yield from each of the sources, the 

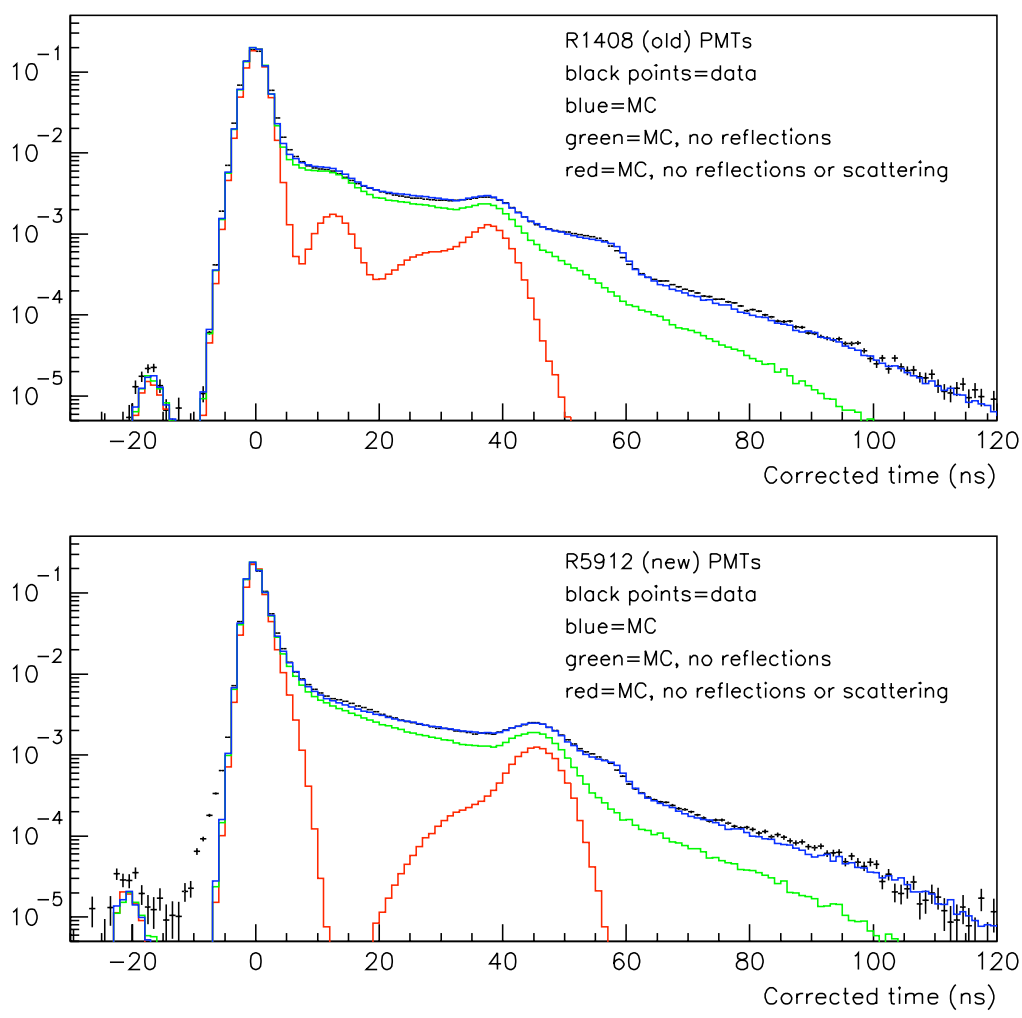

Figure 4.15: The detector Monte Carlo simulation of new and old PMTs is compared with data taken by the $397 \mathrm{~nm}$ laser. The importance of modeling scattering and reflections is evident.

Birks law coefficients, the extinction length, scattering, reflections, and the quantum efficiency of the PMTs have been varied to determine the systematic uncertainties of the model. Several thousand random draws from this parameter set were used in an attempt to quantify, not only the uncertainties in the parameters, but some amount of the correlation between them. The resulting covariance matrix is used to produce systematic variations to assess the uncertainties in the measured distributions (see Section 6.4.7.1).

The uncertainties in the pion absorption and charge exchange cross sections in the tank are slightly higher than their nuclear analogs. The discrepancy between the GCALOR description and the data requires a $35 \%$ variation in pion absorption and a $50 \%$ uncertainty in the charge exchange cross section. As mentioned in Section 4.2.3, 
the uncertainties on pion interactions in the tank are counter-intuitively included in the cross section errors, while the analogous nuclear processes are included in the optical model uncertainties. 


\section{Chapter 5}

\section{Event Reconstruction}

The MiniBooNE data acquisition system records charge and time information from each photomultiplier tube (PMT) over a $20 \mu$ s time window surrounding the arrival of the beam pulse. When a neutrino interaction occurs, the particles produced in the interaction propagate through the tank for tens of centimeters and come to rest on the order of $10 \mathrm{~ns}$ after the interaction takes place. Any promptly produced photons in the interaction can take up to $40 \mathrm{~ns}$ to reach a PMT if they traverse the entire tank, and some amount of delayed light is produced with an exponential decay constant of about 30 ns. Since the discriminator for each PMT can fire only once every $200 \mathrm{~ns}$, each neutrino interaction produces no more than one charge and one time in each PMT from which the event must be reconstructed. Only the PMTs inside the main tank can be used for event reconstruction, so the kinematics of all particles in the event must be deduced from, at most, 2,560 pieces of information.

The initial state of a particle track is fully specified by the particle type and the following seven parameters:

- $\operatorname{energy}\left(E_{0}\right)$

- direction $\left(\theta_{0}\right.$ and $\left.\phi_{0}\right)$

- 4-vertex $\left(X_{0}, Y_{0}, Z_{0}\right.$, and $\left.T_{0}\right)$

To reconstruct a track, an operator must be produced that converts a given set of track 
parameters, $\mathbf{x}$, into probability distribution functions (PDFs) of the charge, $q$, and time, $t$, for each hit PMT in the event. The PDFs from each hit tube can then be combined with the measured information from each tube to form a likelihood function,

$$
\mathcal{L}(\mathbf{x})=\prod_{i=1}^{N_{\text {unhit }}} \mathcal{P}_{i}(\text { unhit } ; \mathbf{x}) \prod_{j=1}^{N_{h i t}} \mathcal{P}_{j}(\text { hit } ; \mathbf{x}) f\left(q_{j} ; \mathbf{x}\right) f\left(t_{j} ; \mathbf{x}\right),
$$

where $N_{\text {hit }}\left(N_{\text {unhit }}\right)$ is the number of hit(unhit) PMTs in the event and $\mathcal{P}_{i}($ hit; $\mathbf{x})\left(\mathcal{P}_{i}(\right.$ unhit; $\left.\mathbf{x})\right)$ is the probability that PMT $i$ will be hit(unhit) for a track specified by $\mathbf{x}$. The charge and time PDFs for $\mathbf{x}\left(f\left(q_{j} ; \mathbf{x}\right)\right.$ and $f\left(t_{j} ; \mathbf{x}\right)$, respectively) are evaluated at the measured charge, $q_{j}$, and time, $t_{j}$, in PMT $j$. The task, then, is to find the set of parameters, $\mathbf{x}$, that maximize the likelihood.

Rather than work with $\mathcal{L}$ directly, it is often more convenient to use $-\log (\mathcal{L})$. This transforms the product in Equation 5.1 into a sum over each PMT. The charge and time components become additive and can be treated independently,

$$
-\log (\mathcal{L})(\mathbf{x})=F_{q}(\mathbf{x})+F_{t}(\mathbf{x})
$$

where

$$
\begin{aligned}
& F_{q}(\mathbf{x})=-\sum_{i=1}^{N_{\text {unhit }}} \log \left(\mathcal{P}_{i}(\text { unhit } ; \mathbf{x})\right)-\sum_{j=1}^{N_{\text {hit }}} \log \left(\mathcal{P}_{j}(\text { hit } ; \mathbf{x}) f\left(q_{j} ; \mathbf{x}\right)\right), \\
& F_{t}(\mathbf{x})=-\sum_{j=1}^{N_{\text {hit }}} \log \left(f\left(t_{j} ; \mathbf{x}\right)\right) .
\end{aligned}
$$

The hit probabilities are naturally grouped with the charge PDFs, since an unhit PMT is just a measurement of zero charge. The functions $F_{q}(\mathbf{x})$ and $F_{t}(\mathbf{x})$ are referred to as the charge and time likelihoods despite technically being the negative logarithm of their respective likelihoods. To determine the track parameters, $\mathbf{x}$, that best fit the event, the sum $F_{q}(\mathbf{x})+F_{t}(\mathbf{x})$ must be minimized.

MiniBooNE employs a detailed description of the light production along an extended track to derive the time and charge likelihoods in Equation 5.2. A complete description of this process in terms of the muon and electron track hypotheses can be 
found in Ref. [59]. The next section will present the important features of the likelihood calculation in terms of the new pion particle hypothesis.

\subsection{Straight Pion Tracks}

As particles propagate through the tank, they experience a variety of processes including elastic scattering, inelastic nuclear interactions, and decay. To convert a set of track parameters into a likelihood function, one must decide which features of the particle track to attempt to measure, and which features to average over. For charged leptons, the reconstruction assumes that the particle travels in a straight line. Typical trajectories for a muon and a pion are illustrated in Figures 5.1 and 5.2, respectively. The straight line hypothesis is quite accurate for muons, which propagate in long, smooth paths with very little scattering. Electrons, on the other hand, travel relatively short distances and produce energetic photons that convert into $e^{+} / e^{-}$pairs some time later. The charge and time likelihoods for electrons are averages over all of these interactions.

In building the pion likelihood functions, the $\pi^{+}$trajectories are characterized by the same straight track characterization of the particle propagation as was used for the charged leptons. However, for reasons that will be explained in Section 5.2, the likelihoods are calculated for pions that do not decay or interact hadronically.

\subsubsection{Charge Likelihood}

The charge recorded by a PMT is only a function of the number of photoelectrons produced in the tube. If an average number of photoelectrons (called the predicted charge, $\mu$ ) can be found for each PMT as a function of the track parameters, $\mathbf{x}$, the probability for a tube to be hit is given by the Poisson distribution,

$$
\mathcal{P}(\text { hit } ; \mu(\mathbf{x}))=1-\mathcal{P}(\text { unhit } ; \mu(\mathbf{x}))=1-e^{-\mu} \text {. }
$$

The task, then, is to calculate the predicted charge on each tube. 

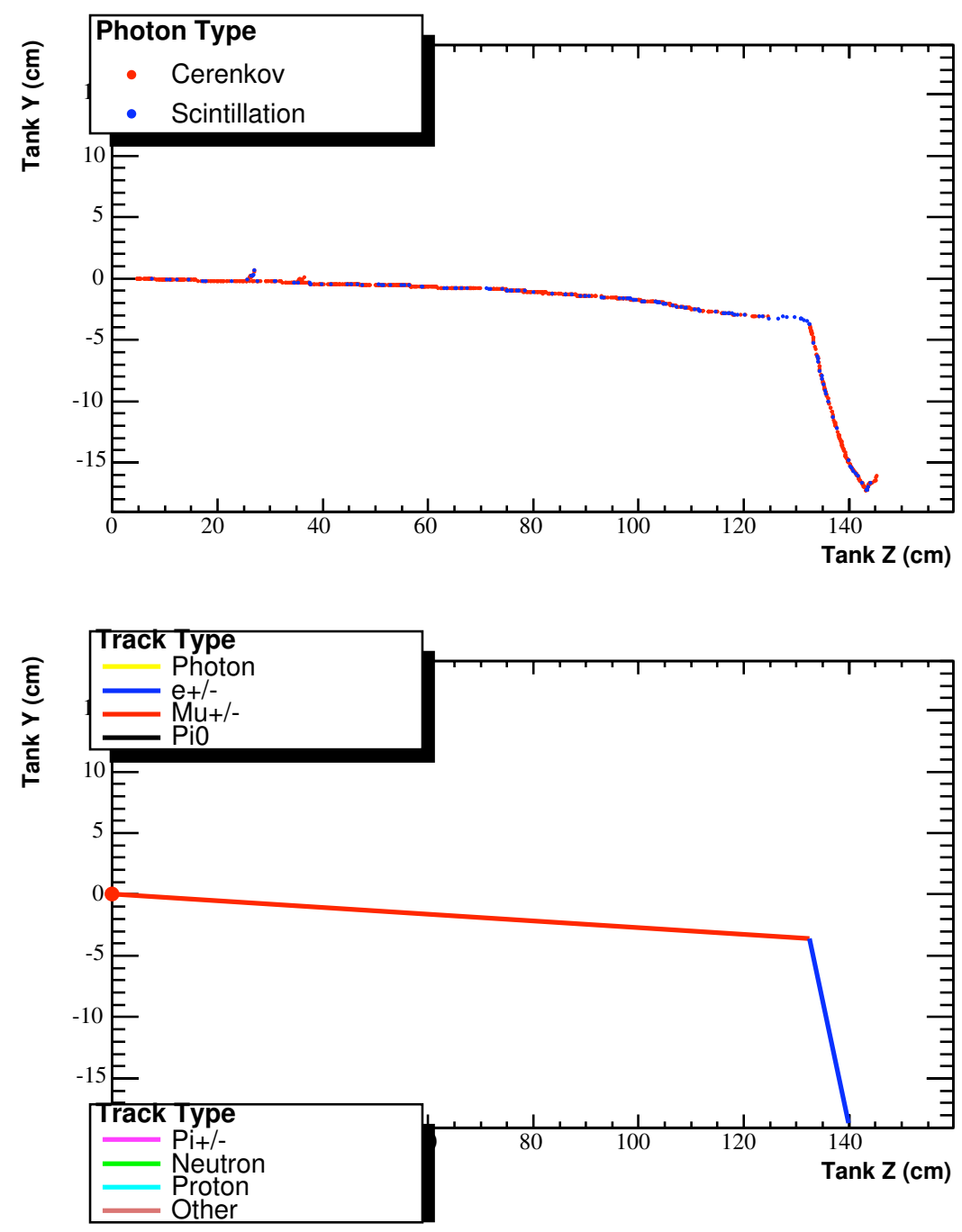

Figure 5.1: An event display of a typical $300 \mathrm{MeV}$ muon track is shown (for a full description of the "event displays" that are shown throughout this chapter, see Appendix A). The top plot shows the Monte Carlo emission point of every optical photon created in the event, and the bottom plot gives the identity of each particle. The display is cumulative over the entire duration of the event, including the muon propagation, muon stop point, and the electron produced from the muon decay at rest.

The predicted charge depends on the amount of light produced by the track, $\Phi$, the transmission of the light through the oil, $T$, the solid angle of the PMT as seen by the track, and the angular acceptance of the PMTs, $\epsilon$. Each of these properties are functions of the position along the track, $s$. If the light produced by the track is 


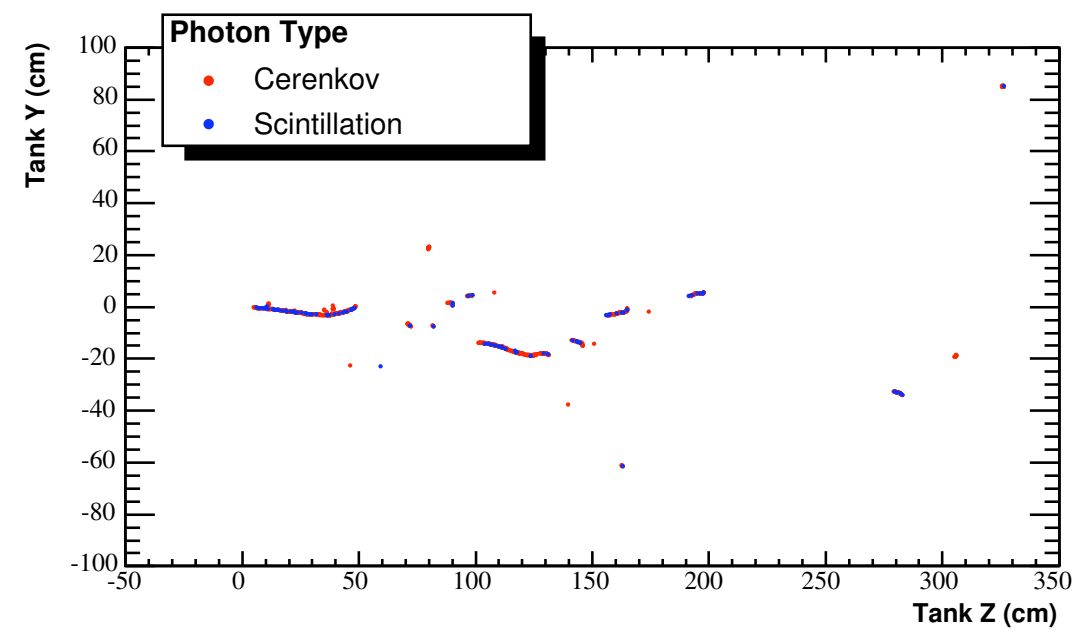

Figure 5.2: An event display of a typical $300 \mathrm{MeV}$ electron track is shown. The Monte Carlo emission point is given for every optical photon created in the event. The initial track produces photons (which produce no light) that create $e^{+} / e^{-}$pairs further downstream.

isotropic (i.e. scintillation light), the predicted charge is given by

$$
\mu_{s c i}=\Phi\left(E_{0}\right) \int_{s} \rho\left(E_{0}, s\right) \Omega(s) T(s) \epsilon(s) d s
$$

where $\Phi\left(E_{0}, s\right)$ has been separated into the total light produced, $\Phi\left(E_{0}\right)$, and the light emission probability along the track, $\rho\left(E_{0}, s\right)$. An example of $\rho\left(E_{0}, s\right)$ for scintillation light is given in Figure 5.3.

Cherenkov light is a bit more complicated since it is not emitted isotropically. In addition to integrating over the probability for light emission along the track path, a new factor, $g(\cos \theta, s)$, must be introduced that gives the angular distribution of the emitted light as a function of $s$ and satisfies

$$
\int_{\theta} g(\cos \theta, s) d(\cos \theta)=1
$$

for all $s$. The predicted charge for Cherenkov emission can then be written as

$$
\mu_{C h e r}=\Phi\left(E_{0}\right) \int_{s} \rho\left(E_{0}, s\right) g(\cos \theta, s) \Omega(s) T(s) \epsilon(s) d s
$$




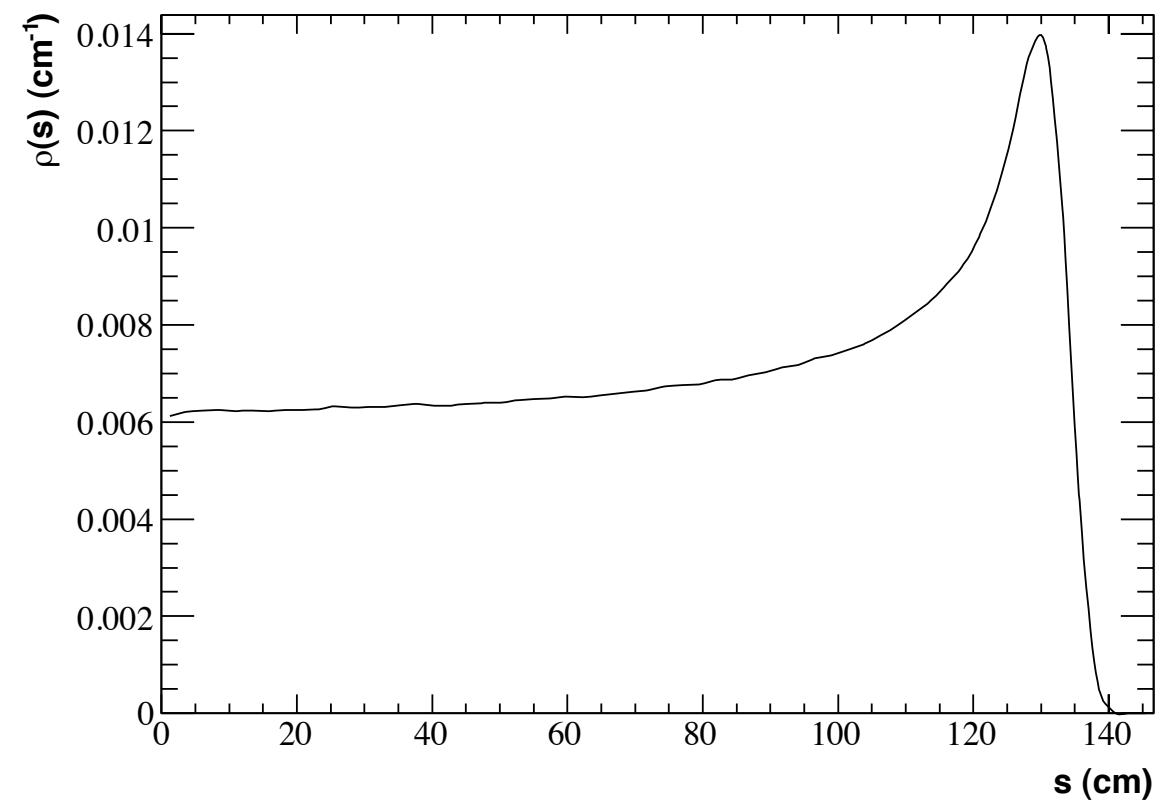

Figure 5.3: The scintillation light emission PDF, $\rho\left(E_{0}, s\right)$, is shown for $300 \mathrm{MeV}$ "nonhadronic" pions.

The $\rho\left(E_{0}, s\right)$ and $g(\cos \theta, s)$ PDFs for $300 \mathrm{MeV}$ pions are given in Figures 5.4 and 5.5, respectively.

The remaining contribution to the predicted charge comes from photon scattering. Much of the generated light will experience Rayleigh and Raman scattering before reaching a PMT. To account for this effect, the detector Monte Carlo is used to generate scattering tables that map out the scintillation and Cherenkov photons that reach a PMT after scattering out of their initial trajectories. The scattering tables are a function of the position and direction of each track segment, $d s$, and are included in the scintillation and Cherenkov integrals given in Equations 5.6 and 5.8.

The final piece of Equation 5.3 needed to calculate the charge likelihood is the charge PDF for a hit tube as a function of the predicted charge. All of the details of the track geometry, optical model properties, and photo-tube acceptance have been folded into the predicted charge. Hence, the charge PDF is only meant to characterize effects 


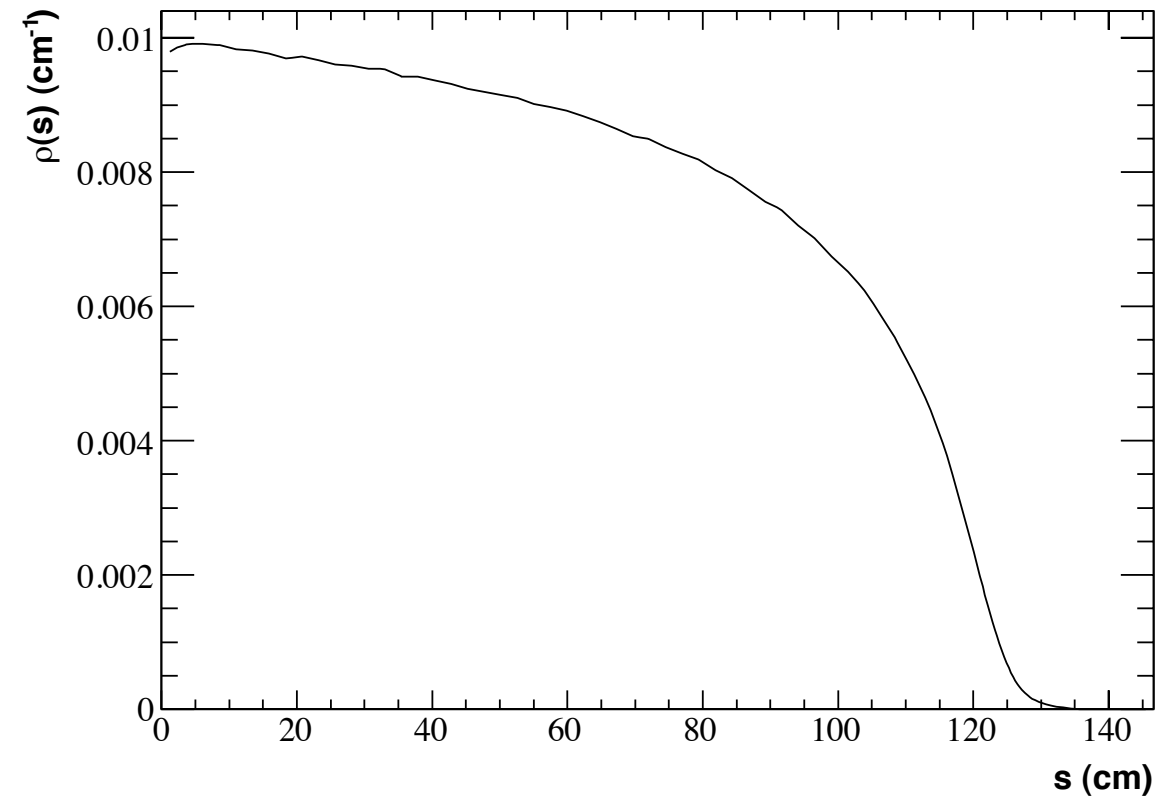

Figure 5.4: The Cherenkov light emission PDF, $\rho\left(E_{0}, s\right)$, is shown for $300 \mathrm{MeV}$ "nonhadronic" pions.

downstream of the light propagation, such as the PMT efficiencies, the electronics, the data readout, etc., for the number of photoelectrons given by $\mu$. Such PDFs can be empirically determined, and have been measured using the laser data [63].

\subsubsection{Time Likelihood}

Unlike the charge likelihood, the time likelihood cannot be simplified in terms of a "predicted time." The reason is that the charge measured by a PMT is an integrated quantity whereas the time is an instantaneous quantity. For example, if a PMT lies near the edge of a Cherenkov ring, the flux of photons it sees as a function of time might have an early peak from the prompt Cherenkov light and a later peak from a coherent contribution of scintillation light along the particle track. The predicted charge for such a configuration is just the integral of this bimodal photon arrival distribution. To determine the time PDF, the detailed shape of the bimodal distribution is 


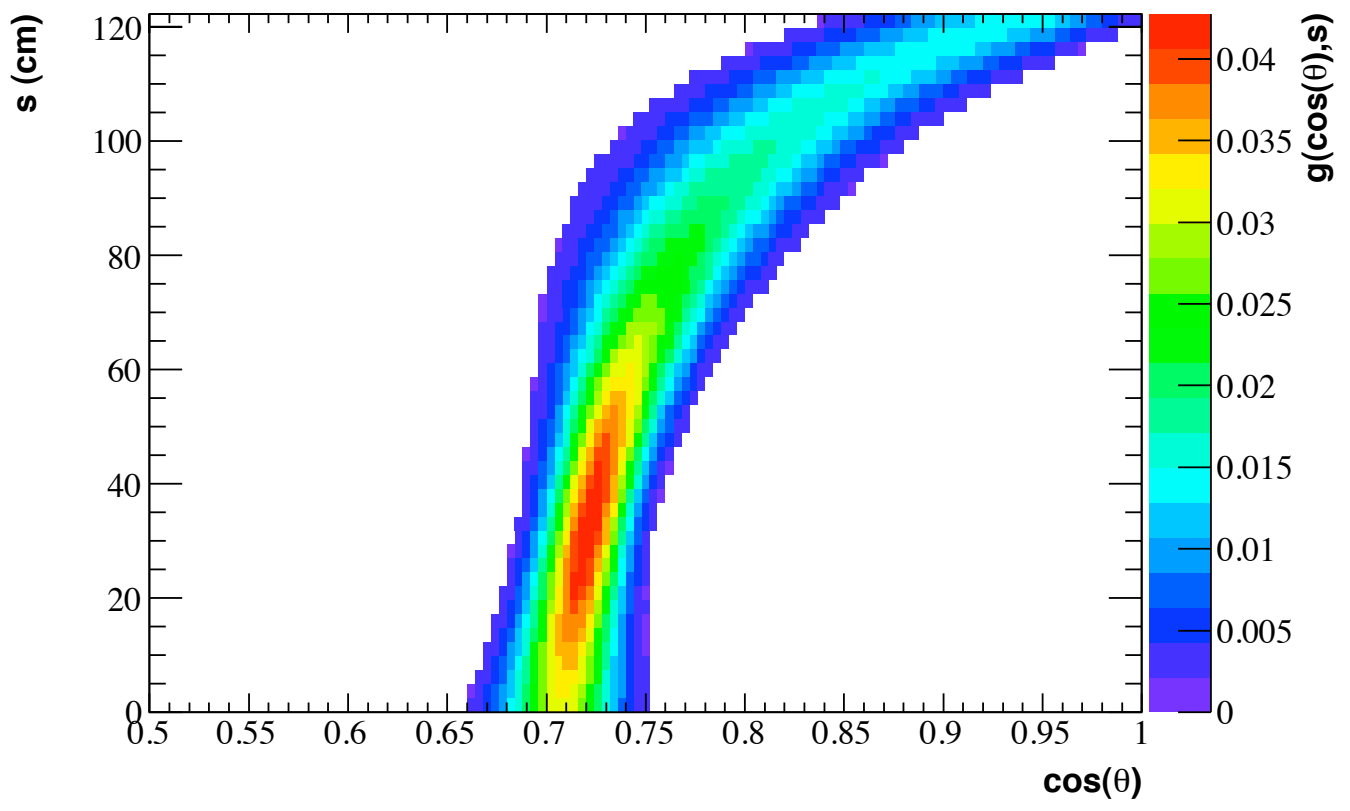

Figure 5.5: The angular light emission PDF, $g(\cos \theta, s)$, for Cherenkov light along the particle path length is shown for $300 \mathrm{MeV}$ "non-hadronic" pions.

required. Therefore, a rigorously correct treatment of the time likelihood would require a tabulation of time PDFs as a function of the full seven dimensional input parameter space (defined relative to the PMT). The generation, implementation, and use of such a tabulation is beyond the capabilities of our computing resources.

In order to calculate the time PDFs, several approximations are made. Both the event time and the PMT/track distance can be removed from the problem by defining the corrected time,

$$
t^{c}=t-T_{0}-\frac{r\left(\Delta s_{m i d}\left(E_{0}\right)\right)}{c_{n}}-\frac{\Delta s_{m i d}\left(E_{0}\right)}{c}
$$

where $t$ is the measured hit time, $T_{0}$ is the event time (i.e. when the neutrino interaction took place), $\Delta s_{\text {mid }}\left(E_{0}\right)$ is the mean of the Cherenkov emission PDF, $r\left(\Delta s_{\text {mid }}\left(E_{0}\right)\right)$ is the distance from the mean emission point to the PMT, and $c_{n}$ and $c$ are the speed of light in the tank and in vacuum, respectively. The corrected time is the elapsed time 
between the earliest moment a photon from the mean emission point could reach the PMT, and the actual recorded hit. This reduces the time PDF tabulation to a five dimensional input space.

The remaining parameters that affect the time PDF are the energy and direction of the track, and the angular position of the track with respect to the PMT. The most important feature characterized by the relative values of these four angles is the location of the PMT with respect to the Cherenkov ring. Fortunately, this information is already well characterized by the Cherenkov-only predicted charge. The critical observation to be made is that the time PDF for either Cherenkov or scintillation light can be characterized almost completely by the Cherenkov and scintillation predicted charge in the PMT. In addition, this simplification also partially takes into account several other minor effects, such as photon attenuation and the variation of the Cherenkov flux with the photon emission angle.

To determine the primitive Cherenkov and scintillation time PDFs, $G_{C h e r}\left(t^{c} ; E_{0}, \mu_{C h e r}\right)$ and $G_{s c i}\left(t^{c} ; E_{0}, \mu_{s c i}\right)$, the detector Monte Carlo is run at several pion energies ranging from 100 to $2000 \mathrm{MeV}$, allowing only the production of either direct Cherenkov light or direct scintillation light. In each simulation, pions are generated isotropically and uniformly distributed throughout the tank (with hadronic interactions and particle decays turned off). The corrected times for each PMT hit are recorded in bins of the logarithm of the corresponding predicted charge. Each histogram is then fit to a selected parametrization based on the characteristics of the light source being considered. The direct Cherenkov corrected times are well approximated by Gaussian distributions, and the scintillation light is parametrized by the sum of two exponential functions, with fixed decay constants of 5 and 30 ns, and a Gaussian smearing width.

To perform track fits, the likelihood function must be smoothly varying to avoid the presence of false local minima; therefore, the Gaussian and exponential fits of the corrected times must be extrapolated across both predicted charge and energy in a 
non-discrete fashion. To accomplish this, the fit parameters from the corrected time fits are, themselves, fit to a 6th order polynomial function of the predicted charges for each energy. An example of these fits is given in Figure 5.6. At this point, the time primitives are smooth functions of time and predicted charge at discrete energies. The same procedure is used again to smooth over energies. Each of the 7 parameters of the 6 th order polynomial predicted charged fits are, themselves, fit to 4th order polynomial functions of energy, as shown in Figure 5.7.
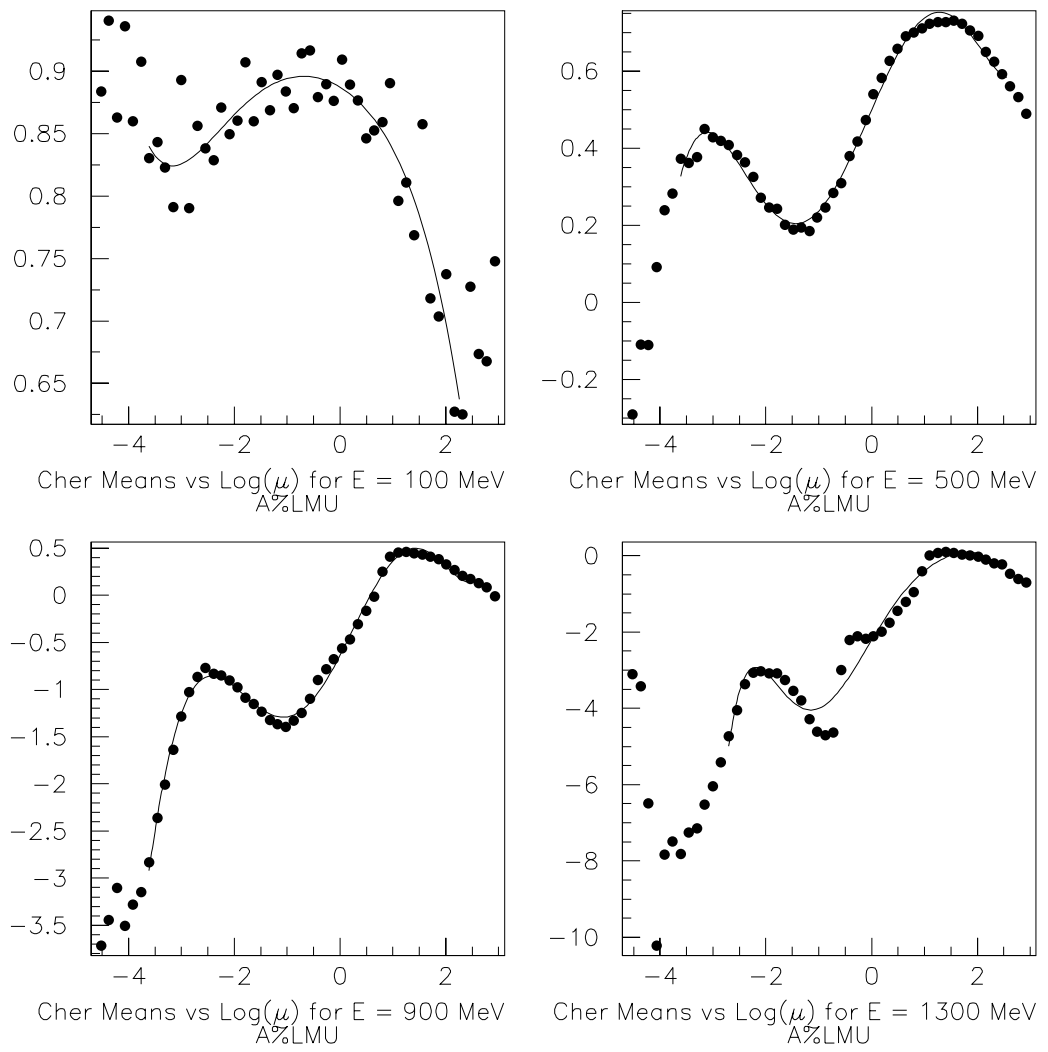

Figure 5.6: The means from the Gaussian fits to the corrected time distributions (performed in bins of predicted charge and energy) have been fit to a 6th order polynomial function of $\log (\mu)$ (still in bins of energy). The fits in four such energy bins are shown.

In order to use the primitive time PDFs to describe all the light in the event, the predicted charge is divided into two classes: prompt and late. The prompt charge is 

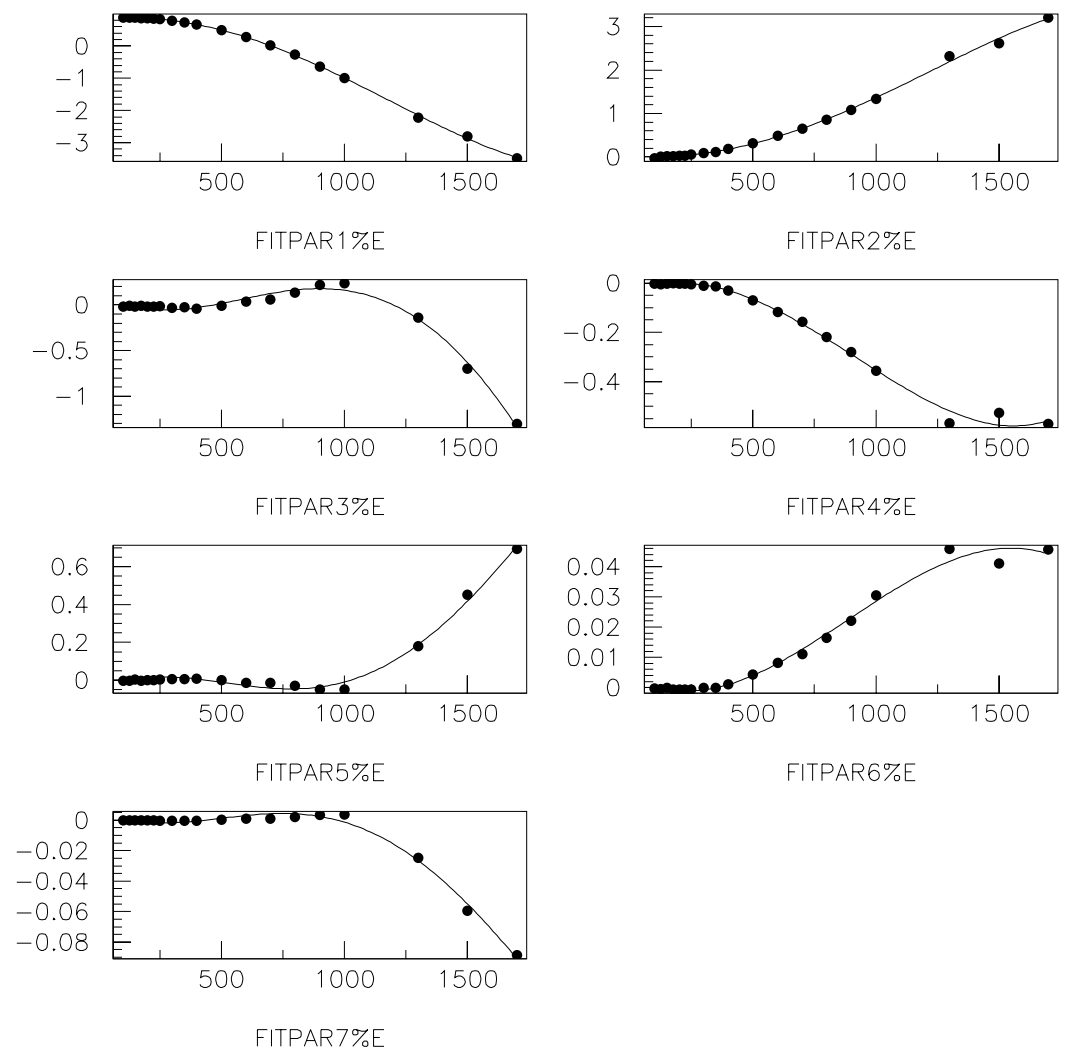

Figure 5.7: The 7 parameters that describe the 6 th order polynomials produced in the fits shown in Figure 5.6 are, themselves, fit as a function of energy. The fit to each of these 7 parameters is shown.

just (most of) the direct Cherenkov predicted charge. The late charge is everything else with $5 \%$ of the Cherenkov charge included to account for PMT late pulsing.

$$
\begin{aligned}
\mu_{\text {prompt }} & =0.95 \mu_{\text {Cher }}^{\text {direct }} \\
\mu_{\text {late }} & =0.05 \mu_{\text {Cher }}^{\text {direct }}+\mu_{\text {sci }}^{\text {direct }}+\mu_{\text {Cher }}^{\text {indirect }}+\mu_{\text {sci }}^{\text {indirect }}
\end{aligned}
$$

Since all light sources other than direct Cherenkov light have been combined into one predicted charge, the shape derived from the scintillation only distribution is implicitly used for all late light. This is a reasonable approximation since the dominant source of late light is UV florescence, which shares the same time structure as scintillation light.

To calculate the time PDF for a given set of track parameters, the following 
prescription is used.

- Evaluate all 14 of the 6th order polynomials at the given track energy.

- Build the five 4th order polynomials (Cherenkov means, Cherenkov sigmas, scintillation start time, scintillation relative exponential weights, and scintillation Gaussian smearing) from the parameters extracted in the previous step, and evaluate them at the appropriate (prompt or late) predicted charge.

- Use the parameters extracted in the previous step to produce the prompt and late primitive time PDFs

- Combine the Cherenkov and scintillation PDFs to form the full corrected time PDF.

The prompt and late primitive PDFs are combined using the probabilities that the PMT was hit by at least one prompt or late photon, both of which are given by the Poisson distribution,

$$
\begin{aligned}
& \mathcal{P}\left(\text { no prompt hit; } \mu_{\text {prompt }}\right)=1-\mathcal{P}\left(\text { prompt hit } ; \mu_{\text {prompt }}\right)=e^{-\mu_{\text {prompt }}} \\
& \mathcal{P}\left(\text { no late hit } ; \mu_{\text {late }}\right)=1-\mathcal{P}\left(\text { late hit } ; \mu_{\text {late }}\right) \quad=e^{-\mu_{\text {late }}}
\end{aligned}
$$

Any PMT that was hit by at least one prompt photon follows the prompt primitive PDF since the measured time is given by the earliest detected photon; therefore, the probability for one prompt hit sets the weight, $w_{p}$, for the prompt time PDF, and its complement sets the weight, $w_{l}$, for the late time PDF. Since the time PDF has already been multiplied by the probability that the PMT was hit by any photon (prompt or late) in Equation 5.1, only the additional probability of a prompt hit given the presence 
of any hit is needed to set the weights, $w_{p}$ and $w_{l}$,

$$
\begin{aligned}
w_{p} & =\mathcal{P}\left(\text { prompt hit } \mid \text { hit } ; \mu_{\text {prompt }}(\mathbf{x})\right) \\
& =\frac{1-\mathcal{P}\left(\text { no prompt hit } ; \mu_{\text {prompt }}(\mathbf{x})\right)}{1-\mathcal{P}\left(\text { no prompt hit } ; \mu_{\text {prompt }}(\mathbf{x})\right) \mathcal{P}\left(\text { no late hit } ; \mu_{\text {late }}(\mathbf{x})\right)} \\
& =\frac{1-e^{-\mu_{\text {prompt }}}}{1-e^{-\mu_{\text {prompt }}} e^{-\mu_{\text {late }}}}, \\
w_{l} & =1-w_{p} .
\end{aligned}
$$

These weights, together with the Cherenkov and scintillation primitive distributions, $G_{C h e r}$ and $G_{s c i}$, give the full expression for the time PDF,

$$
f(t ; \mathbf{x})=w_{p} G_{C h e r}\left(t^{c} ; E_{0}, \mu_{\text {prompt }}\right)+w_{l} G_{s c i}\left(t^{c} ; E_{0}, \mu_{\text {late }}\right) .
$$

To verify that reasonable results are produced by this method of "parametrizing the parametrization of the fit parameters," the resulting PDFs can be compared with the raw corrected time distributions from which they were produced. These comparisons are shown for a variety of energies and predicted charges in Figures 5.8 and 5.9.

\subsubsection{Likelihood Maximization}

The maximization of the likelihood is accomplished by minimizing Equation 5.2. The minimization is performed by MINUIT [64]. MINUIT takes as input any multidimensional function, and returns a set of best fit parameters. MINUIT is typically run using the "MIGRAD" minimization method, which uses derivatives of the input function with respect to the parameters to find the minimum. Unfortunately, the discrete nature of the PMTs causes the derivatives of the likelihood function to behave unreliably, and often traps the fit in false minima. Instead, the "SIMPLEX" sampling method is used, which provides much more robust results.

Before MINUIT can begin the fit minimization, an initial set of track parameters must be provided about which the fit will explore the parameter space. The values chosen for these seeding parameters can be very important for the more complicated 

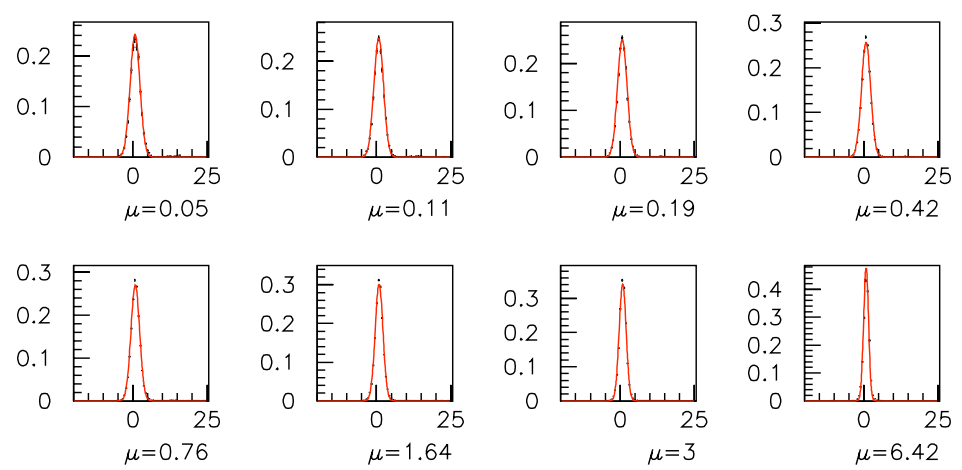

(a) Corrected time (ns) for $250 \mathrm{MeV}$ "non-hadronic" pions.
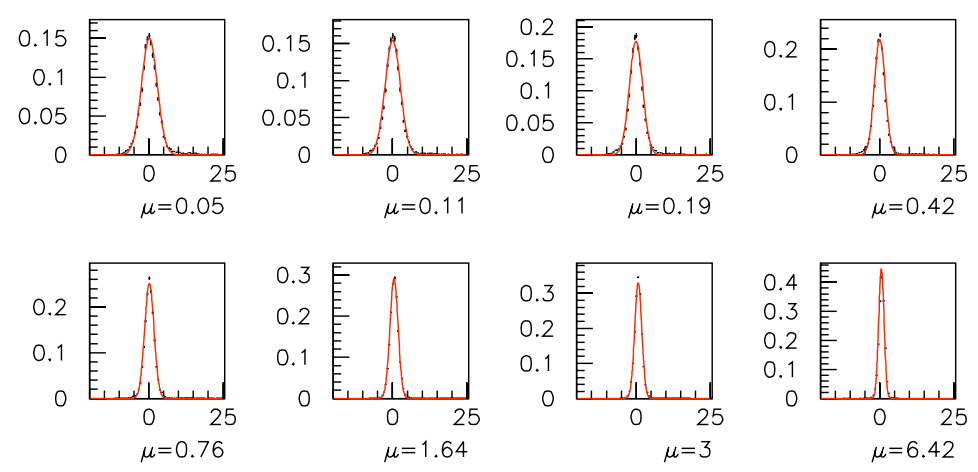

(b) Corrected time (ns) for $600 \mathrm{MeV}$ "non-hadronic" pions.
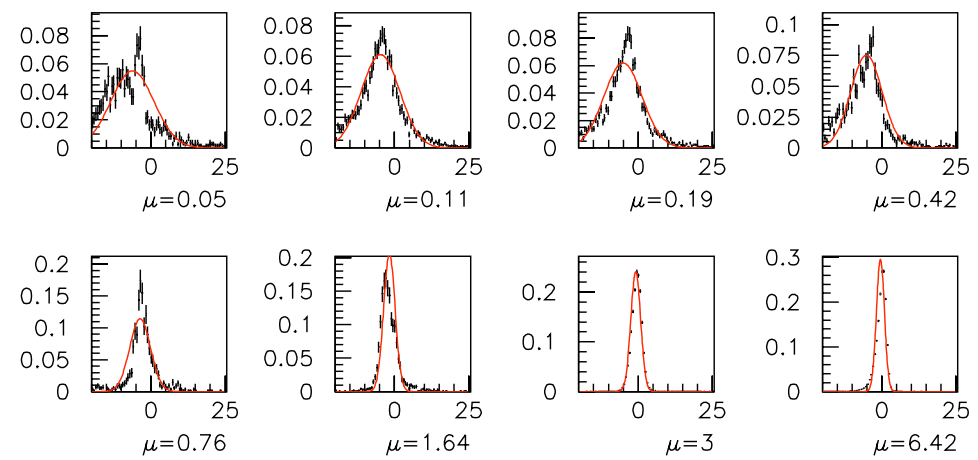

(c) Corrected time (ns) for $1500 \mathrm{MeV}$ "non-hadronic" pions.

Figure 5.8: The Cherenkov corrected time distributions are compared with the primitive Cherenkov time parametrization for a variety of energies and predicted charges. 

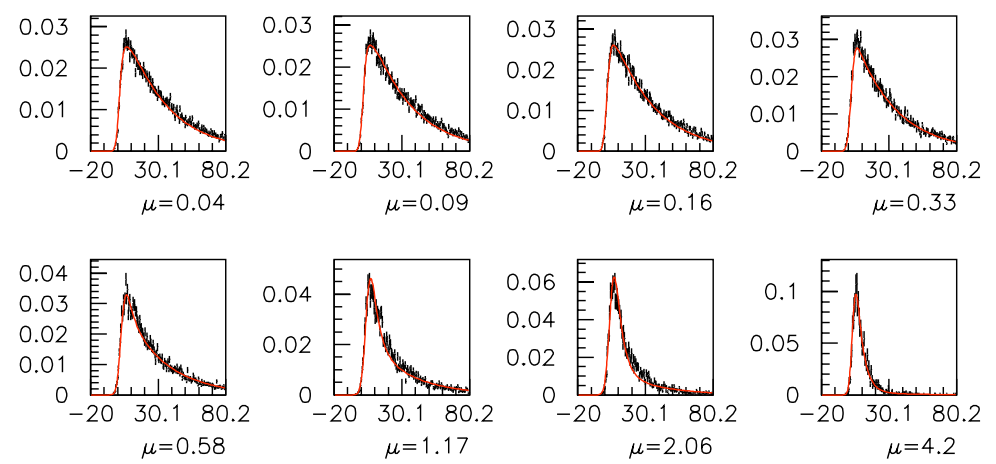

(a) Corrected time (ns) for $250 \mathrm{MeV}$ "non-hadronic" pions.
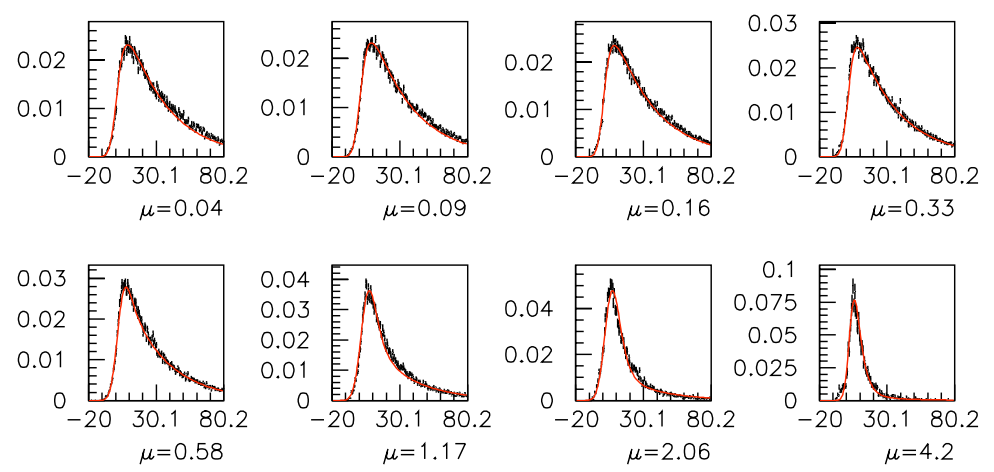

(b) Corrected time (ns) for $600 \mathrm{MeV}$ "non-hadronic" pions.
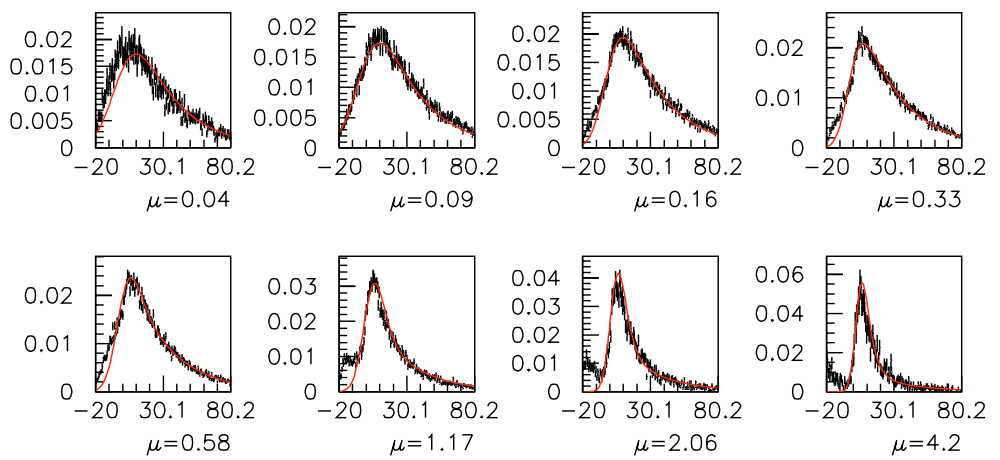

(c) Corrected time (ns) for $1500 \mathrm{MeV}$ "non-hadronic" pions.

Figure 5.9: The scintillation corrected time distributions are compared with the primitive scintillation time parametrization for a variety of energies and predicted charges. 
fitters discussed throughout the remainder of this chapter, but for single track events, a fit seed that generally points in the direction of the Cherenkov cone is sufficient. Fortunately, there exists a fast fitter that interprets the observed light as though it were generated by a point source. The fast fitter provides a reasonable estimate of the track parameters and is used to seed the fit [63].

\subsubsection{Comparison With the Muon Fitter}

The charge and time likelihood components have been calculated for muons and electrons as well [59]. To reconstruct an event, one of the three particle hypotheses must be chosen. The fitter will interpret the measured light distribution as having been produced by the hypothesized particle and return corresponding fit parameters.

With hadronic interactions removed, which is how the likelihood tables were calculated, muons and pions propagate in a similar manner. As such, the muon and pion hypothesis fits produce very similar results. The most notable exception is the kinetic energy reconstruction. Figure 5.10 shows $600 \mathrm{MeV}$ muons and pions reconstructed with the muon and straight pion hypothesis fits. For either set of particles, the reconstructed muon hypothesis energy is shifted $30 \mathrm{MeV}$, or $5 \%$, lower than the pion hypothesis energy. For each event, the energy derived from the same tank light distribution varies by $30 \mathrm{MeV}$ when interpreted as either the result of a muon or a pion. Since the size of this variation is the same as the change in the reconstructed energy of both fitters when the generated particles are switched from pions to muons, the relative energy reconstruction from the two fit hypotheses exhibits the correct behavior.

Perhaps the most important reason for creating different fit hypotheses is to provide a method for particle identification. This is accomplished by fitting each event with two (or more) different particle hypotheses and comparing the likelihoods (i.e. the goodness of fit) returned by each fitter. In fits to electrons and muons, the likelihoods from the electron and muon fit hypotheses differ significantly due to the differences in 


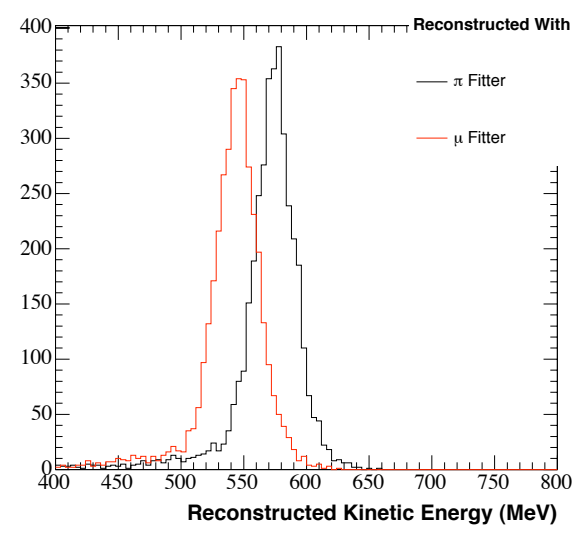

(a) $600 \mathrm{MeV}$ pions with hadronic interactions and decays turned off

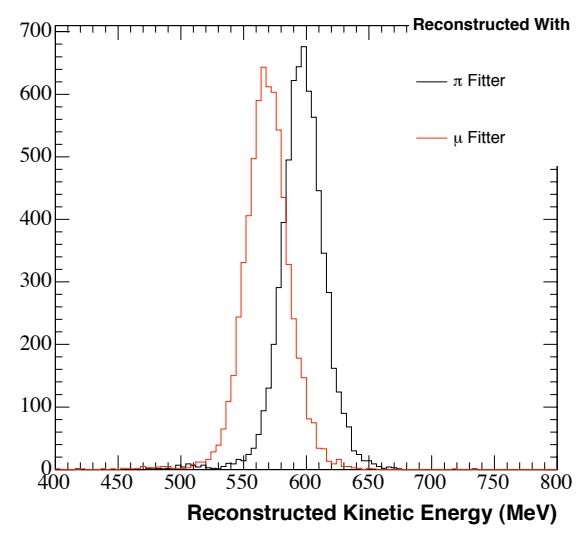

(b) $600 \mathrm{MeV}$ muons with hadronic interactions and decays turned off

Figure 5.10: The reconstructed kinetic energies from both the muon and straight pion hypothesis fits are shown for $600 \mathrm{MeV}$ pions (left) and $600 \mathrm{MeV}$ muons (right). Hadronic interactions and decays have been turned off to correspond with conditions used to create the likelihoods. The $30 \mathrm{MeV}$ gap in the reconstructed energies of the two fit hypotheses corresponds to the $30 \mathrm{MeV}$ shift in both peaks when the generated pions are replaced by muons. (Note: All tracks in these plots have been produced at the center of the tank and point along the z-axis; track energies in this particular configuration reconstruct about $5 \%$ low overall.)

the manner in which each track propagates through the detector as shown in Figures 5.1 and 5.12 [59]. Conversely, pions without hadronic interactions and muons produce very similar trajectories through the tank. Figure 5.11 shows the logarithm of the likelihood ratio for fits to muons and non-hadronic pions. The average likelihood ratio for fits to non-hadronic pions is shifted relative to the ratio for muons, but the size of the shift is too small to provide any meaningful particle separation. The improvement in particle identification is the topic of the next section.

\subsection{Pion Reconstruction}

In the absence of hadronic interactions, pions and muons are nearly indistinguishable, as demonstrated in Figure 5.11. Fortunately, pions do interact hadronically, and 


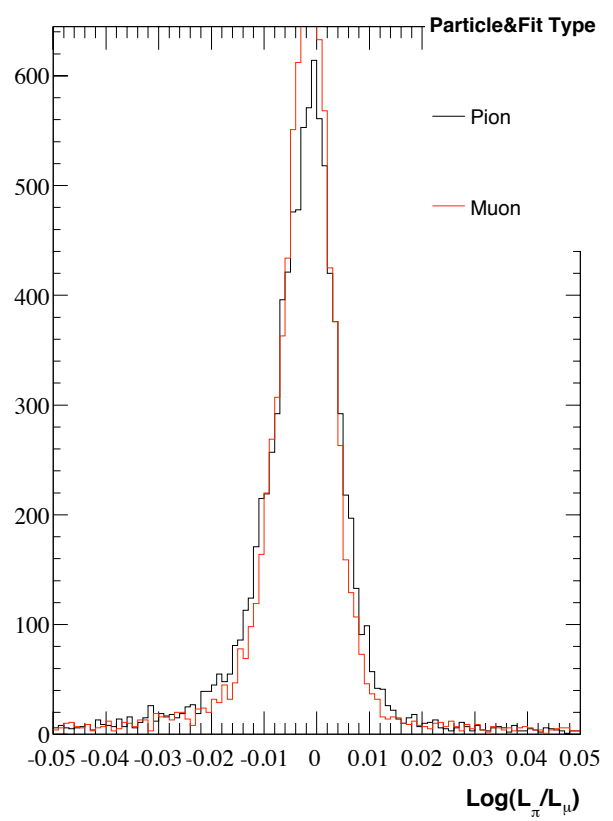

(a) $300 \mathrm{MeV}$ muons and pions

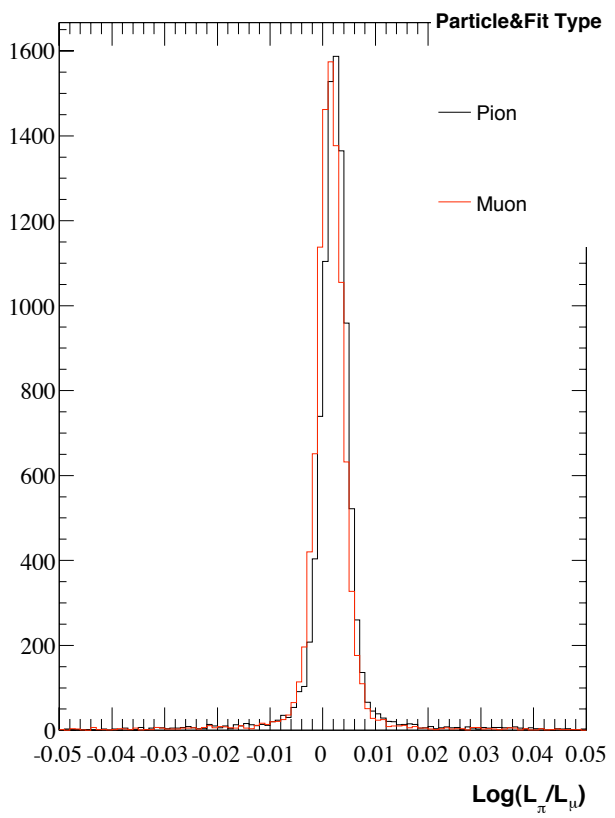

(b) $600 \mathrm{MeV}$ muons and pions

Figure 5.11: A comparison of the muon and straight pion hypothesis fit likelihoods is shown. The plotted variable is the logarithm of the muon/pion hypothesis fit likelihood ratio. Muons (red) and pions (black) without hadronic interactions were generated at 300 (left) and $600 \mathrm{MeV}$ (right). For $300 \mathrm{MeV}$ pions, which are more indicative of a typical $\mathrm{CC} \pi^{+}$pion energy, there is no muon/pion separation. At $600 \mathrm{MeV}$, the fits to pions are shifted slightly higher than the fits to muons.

when such an interaction occurs, it produces a very distinct signature. The characteristic straight pion trajectory experiences a sharp change in direction at the interaction point. These "kinked" particle tracks offer a measurable characteristic of pion propagation to exploit with the appropriate particle identification algorithm. The hadronic debris produced in the interaction is almost always below Cherenkov threshold, and thus contributes only a slight increase to the late time charge distribution. An example of a typical pion trajectory is given in Figure 5.12. 

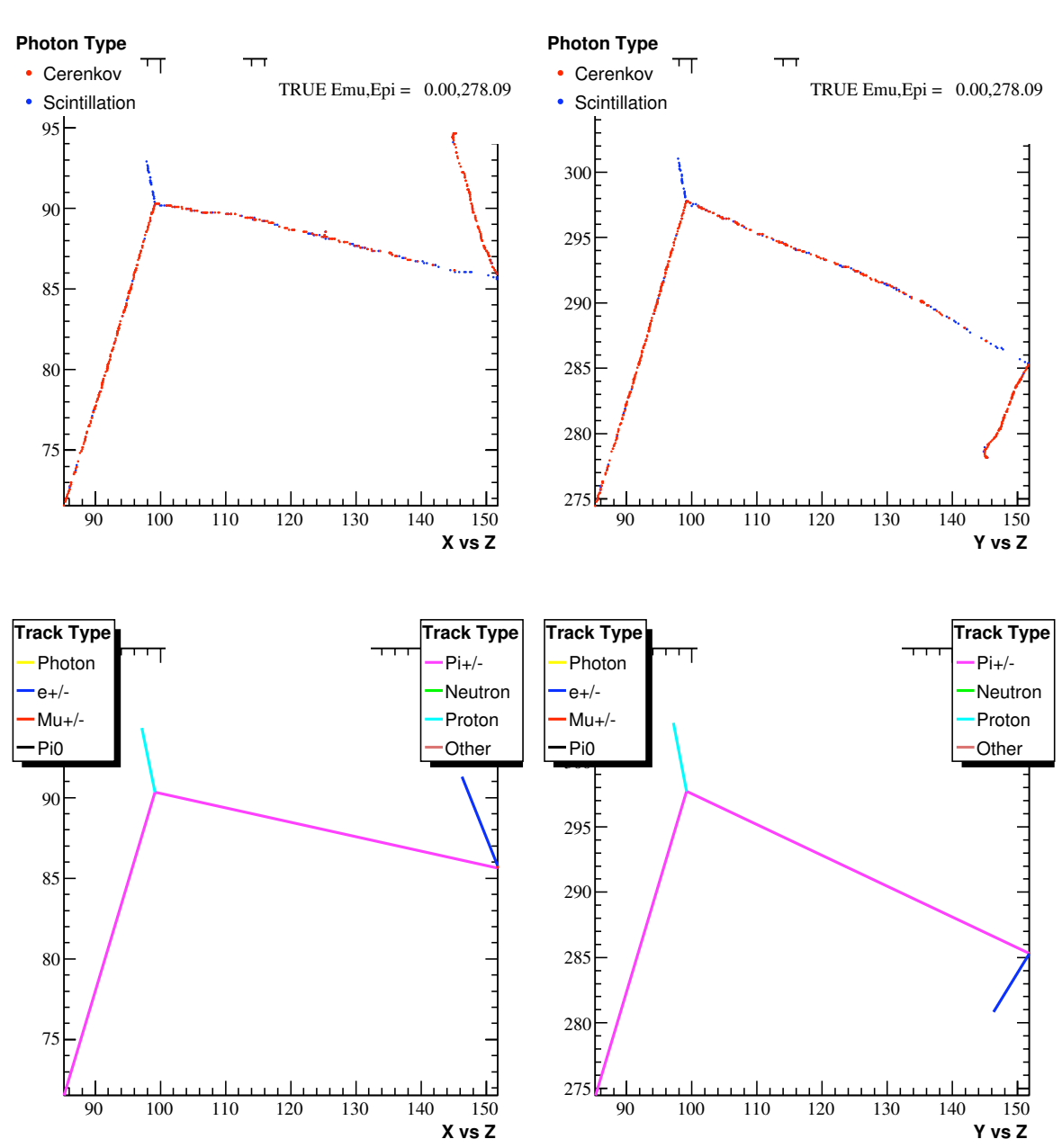

Figure 5.12: An event display for a typical pion track is shown. The top plots show the emission point of every optical photon in the event. The bottom plots give the identity of each particle emerging from a hadronic interaction or decay. A hadronic interaction occurs (at $\mathrm{z}=100 \mathrm{~cm}$ ) that emits a sub-Cherenkov proton and causes a sharp "kink" in the pion trajectory.

\subsubsection{Fitting Kinked Tracks}

To properly reconstruct pions, the likelihood method described in Section 5.1.1 must be slightly altered. One way to account for the hadronic interactions would be to regenerate the charge and time likelihood tables with hadronic interactions turned on. This method, however, would essentially average over the entire space of kinked trajectories and force the resulting light distributions into a straight line track hypoth- 
esis, thus worsening the resolution on the track energy and direction. Instead, the track hypothesis is altered to allow for the reconstruction of a kinked track trajectory.

To fit a kinked track trajectory, the initial set of seven track parameters must be expanded to include four new parameters.

- $\Delta E_{u p}$ is the amount of energy lost in the track from the moment it is created until the hadronic interaction takes place. This quantity characterizes the length of the upstream portion of the kinked track.

- $\Delta E_{k i n k}$ is the amount of energy lost by the pion in the hadronic interaction. This parameter allows the fitter to start the downstream portion of the track at an energy lower than $E_{0}-\Delta E_{u p}$.

- $\theta_{\text {down }}$ and $\phi_{\text {down }}$ specify the direction of the downstream (i.e. post-kink) portion of the track in tank coordinates.

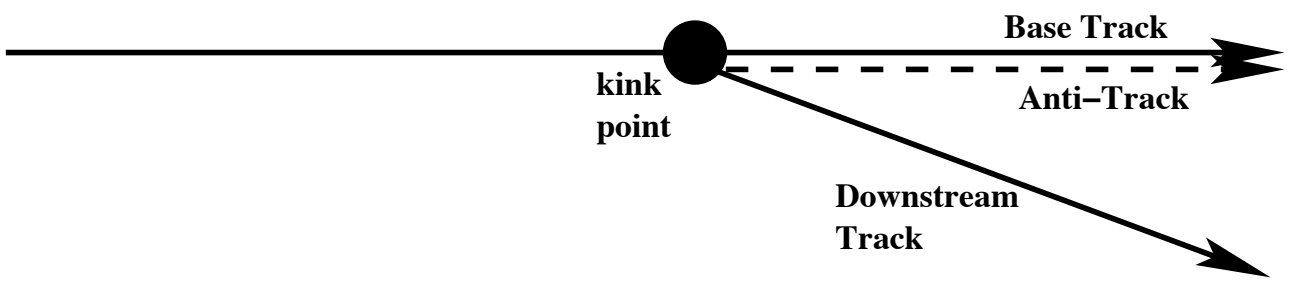

Figure 5.13: The composition of a kinked track is shown. The base track provides the charge prediction for the upstream portion of the track. The anti-track charge prediction is subtracted from each PMT to remove the downstream portion of the base track. A third track that begins at the kink point provides the charge prediction for the downstream portion of the track.

A kinked track is built from a set of three standard straight tracks as shown in Figure 5.13. The "base track" is created at the event vertex, $\left(X_{0}, Y_{0}, Z_{0}, T_{0}\right)$, with an energy and direction given by $E_{0}, \theta_{0}$, and $\phi_{0}$. Above Cherenkov threshold, the distance traveled as a function of kinetic energy lost is well approximated as a linear relationship. ${ }^{1}$ 
The value of $\Delta E_{u p}$, therefore, gives the distance along the base track between the event vertex and the kink point. The time difference is found by integrating the inverse of the velocity from the kink point to the vertex,

$$
\Delta t=\frac{1}{c} \int_{x_{1}}^{x_{2}} \frac{d x}{\beta(x)}=\frac{1}{c} \int_{x_{1}}^{x_{2}} \frac{d x}{\sqrt{1-1 / \gamma(x)^{2}}} .
$$

Assuming, once again, a linear relationship between the change in kinetic energy and the distance traveled, $x(K)=s K+x_{0}$, the integral over distance can be shifted to an integral over kinetic energy,

$$
\Delta t=\frac{1}{c} \int_{K_{1}}^{K_{2}} \frac{s \cdot d K}{\sqrt{1-\frac{m^{2}}{(K+m)^{2}}}}=\frac{s m}{c}\left[\sqrt{\left(\frac{K+m}{m}\right)^{2}-1}\right]_{K_{1}}^{K_{2}} .
$$

At the kink point, a second track, known as the "anti-track," is created with a direction given by $\theta_{0}$ and $\phi_{0}$, and an energy of $E_{0}-\Delta E_{u p}$. The predicted charges calculated from the anti-track are subtracted from the base track predicted charges to remove contributions to the light distribution from the downstream portion of the base track. Finally, the downstream track is produced at the kink point 4-vertex with an energy given by, $E_{0}-\Delta E_{u p}-\Delta E_{k i n k}$, and a direction determined by the fit parameters, $\theta_{\text {down }}$ and $\phi_{\text {down }}$.

The primitive time distributions for the upstream and downstream portions of the track are combined in a similar manner to the method used for prompt and late distributions in the straight track fitter. Each light source (prompt and late from both tracks) must again be prioritized according to the time of flight of its parent track to a given PMT. In this case, there are two prompt sources of light, one from each track segment. Priority is given to the segment with the shortest time of flight to the PMT. For the downstream track, the time of flight is calculated with respect to its center, as is done in a standard straight track fit. The upstream segment time of flight is calculated

\footnotetext{
1 To determine the linear relationship between kinetic energy and propagation distance, $x(K)=$ $s K+x_{0}$, a Monte Carlo sample of pions without hadronic interactions was produced, and the scatter plot of stopping distance versus kinetic energy was fit to a straight line between 100 and $800 \mathrm{MeV}$.
} 
from the kink point. ${ }^{2}$ In this way, the downstream prompt time distribution is given priority in all PMTs located in the half sphere pointed to by the downstream track. The late time light sources for both tracks are always prioritized last, and are combined by averaging the respective PDFs.

Using these criteria to define the "near" $(n)$, "far" $(f)$, and "late" $(l)$ no-hit probabilities,

$$
\begin{aligned}
& \overline{\mathcal{P}}_{n} \equiv \mathcal{P}_{n}\left(\text { no hit } ; \mu_{\text {prompt }, n}(\mathbf{x})\right)=e^{-\mu_{\text {prompt }, n}} \\
& \overline{\mathcal{P}}_{f} \equiv \mathcal{P}_{f}\left(\text { no hit } ; \mu_{\text {prompt }, f}(\mathbf{x})\right)=e^{-\mu_{\text {prompt }, f}} \\
& \overline{\mathcal{P}}_{l} \equiv \mathcal{P}_{l}\left(\text { no hit; } \mu_{\text {late }}(\mathbf{x})\right) \quad=e^{-\mu_{\text {late }}},
\end{aligned}
$$

the weights for each of the PDFs are given by

$$
\begin{aligned}
w_{n} & =\frac{1-\overline{\mathcal{P}}_{n}}{1-\overline{\mathcal{P}}_{n} \overline{\mathcal{P}}_{f} \overline{\mathcal{P}}_{l}} \\
w_{f} & =\frac{1-\overline{\mathcal{P}}_{f}}{1-\overline{\mathcal{P}}_{f} \overline{\mathcal{P}}_{l}}\left(1-w_{n}\right) \\
w_{l} & =1-w_{n}-w_{f},
\end{aligned}
$$

and the full expression for the time PDF is

$f(t ; \mathbf{x})=w_{n} G_{C h e r}\left(t_{n}^{c} ; E_{n}, \mu_{\text {prompt }, n}\right)+w_{f} G_{C h e r}\left(t_{f}^{c} ; E_{f}, \mu_{\text {prompt }, f}\right)+w_{l} \hat{G}_{\text {sci }}\left(\hat{t}^{c} ; E_{0}, \mu_{\text {late }, \text { tot }}\right)$.

\subsubsection{Fit Seeding}

The seeding of the kinked fitter begins with a straight track fit. The result of the straight fit is assumed to point at either the filled-in ring of the downstream track or the open ring formed by the upstream track segment. To determine the direction

\footnotetext{
${ }^{2}$ The corrected time for the upstream track is still calculated with respect to the center of the base track. The predicted prompt charge on a PMT depends only on the portion of the track from which it was created, so although the predicted charge contributions from the post-kink section of the base track have been removed in the anti-track subtraction, the time PDFs calculated in the non-subtracted PMTs still require the same definition of corrected time used to build the charge dependent time likelihood tables.
} 
of the other ring, a downstream track segment is attached to the straight track fit result, and a scan of the downstream directions is performed over 100 equally spaced directions. At each point in the scan, the likelihood function is evaluated at several energies, ranging from one to two times the one track fit energy, and each energy is divided between the upstream and downstream portions of the track in several different fractions. This process is repeated under the assumption that the straight track fit instead found the downstream track segment. The best likelihood returned by both the upstream and downstream scans determines the seed point for the fit. A sample fit is shown in Figure 5.14.

A scan of the angular phase space is conducted to avoid starting the fit near a local, but not global, minimum. The shape of the likelihood surface gets significantly altered in the presence of another ring, so there is much more potential for the fitter to get trapped in the wrong part of phase space. A scan coarsely surveys the phase space in its entirety, and provides the fitter with the best opportunity to correctly reconstruct the event.

\subsubsection{Particle Identification}

The separation ability of the kinked pion fitter is displayed in Figure 5.15. The reconstructed pion peak is shifted relative to the reconstructed muons. More significantly, the pion distribution has a much larger tail of events that extend away from the muon portion of the likelihood ratio. These are events where kinked trajectories occurred and were successfully found by the fitter. The peak region is mostly composed of lower energy pions that may not have developed a kink, or produced downstream portions that are near or below the Cherenkov energy threshold. A small population of muon events extend into the pion region as well. Although muons do not interact hadronically, it is possible to produce kink-like trajectories over small distances via hard Coulomb scattering. The kinked hypothesis will fit these events much better as well. 

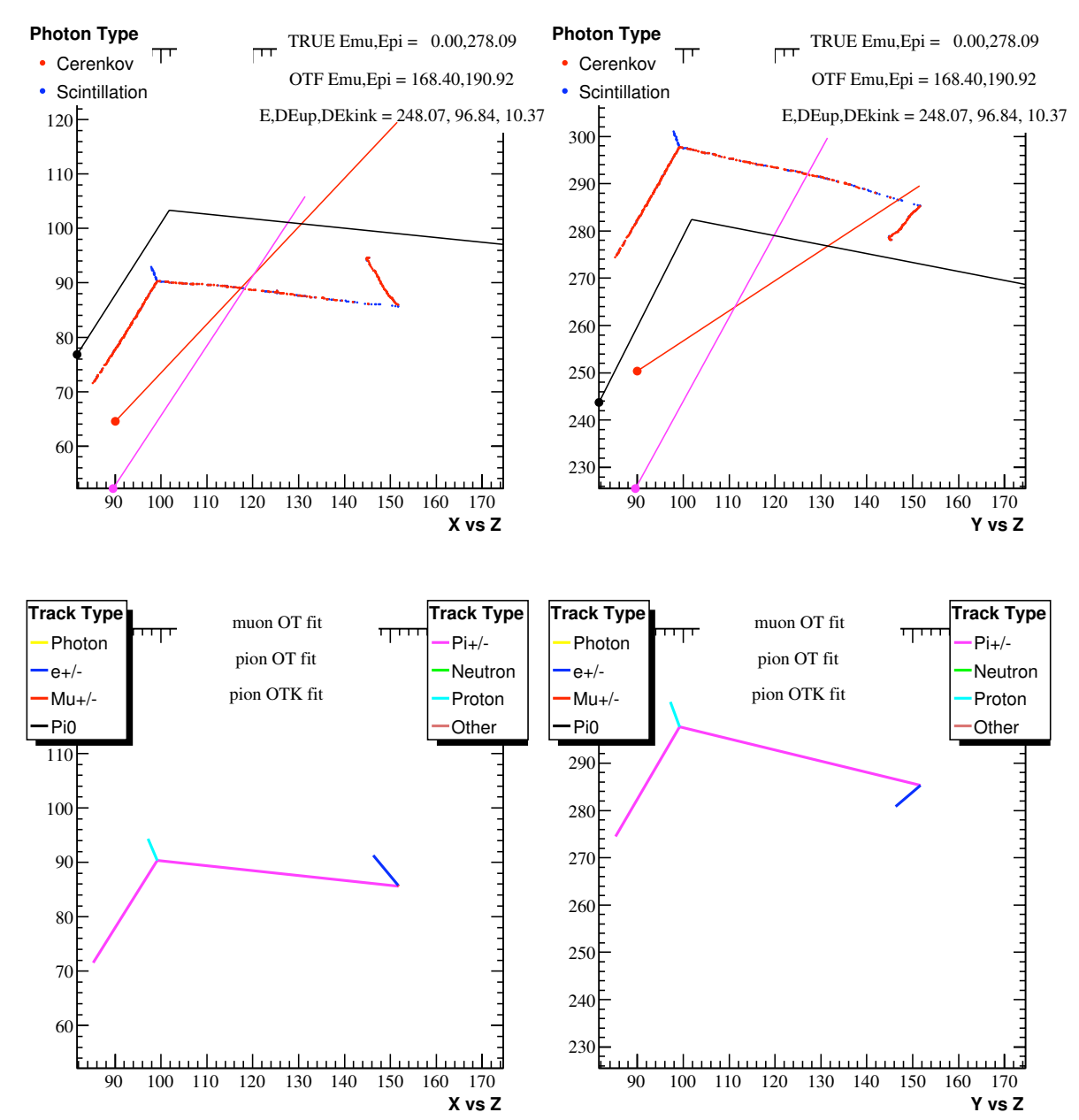

Figure 5.14: The event in Figure 5.12 has been fit with a muon hypothesis (red line), a straight pion hypothesis (magenta line), and a kinked pion hypothesis (black line). The straight fitters underestimate the track energy by more than $30 \%$. The kinked fitter provides an energy estimate that is about $10 \%$ low, which is near the edge of the energy resolution.

The $\mu / \pi$ separation provided by the kinked pion fitter is not perfect. There is no ideal location to place a cut that would reject a large population of muons while retaining a significant fraction of pions. The goal of this analysis, however, is to reconstruct events with both a muon and a pion present, and to determine the identity of each track. In that case, the separation power indicated by Figure 5.15 is doubled, and the probability that at least one track travels a significant distance, and thus increases the discriminating power of the fitters, is increased. 


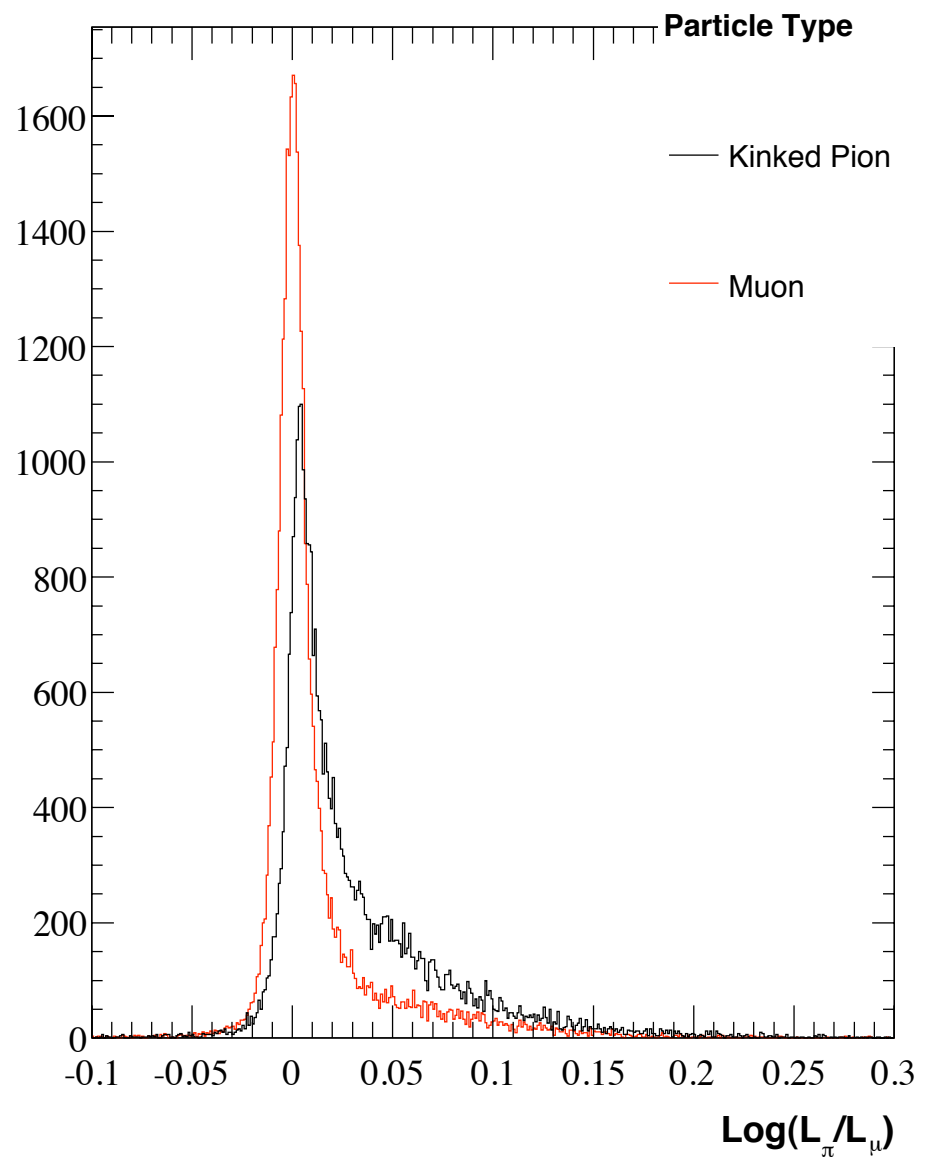

Figure 5.15: The straight muon and kinked pion likelihood ratios are shown for muons (red) and pions (black) with full hadronic interactions and decays. The particles were generated from a flat kinetic energy distribution ranging from 50 to $450 \mathrm{MeV}$ to more closely represent the true pion energy spectrum of $\mathrm{CC} \pi^{+}$events. Unlike the $300 \mathrm{MeV}$ straight fitter comparison in Figure 5.11, there is now clear separation in the muon and pion peaks, and a large excess of pion events is seen along the high side tail.

\subsubsection{Fit Resolution}

Although the main motivation for developing a kinked track fitter was to provide a means for separating muons from pions, the improved modeling of pion trajectories results in superior event reconstruction. The energy resolutions from both the straight and kinked pion fitters are shown in Figure 5.16(a). The straight pion fitter recon- 
structs pion energies $10 \%$ low, whereas the kinked fitter resolution function peaks at zero. In addition, the "shoulder" feature just below the peak of the resolution function, where the reconstructed energy underestimates the true pion energy, is reduced by the kinked pion fitter. The two-dimensional plot of the resolution versus the true energy in Figure 5.16(b) shows that the shoulder comes from higher energy pions that can produce multi-kink events and cause larger pion energy losses at each kink. The pion direction reconstruction is also significantly improved with the kinked fitter, as shown in Figure 5.17. The event populations in each of the first few bins of the angle between the reconstructed and true directions are nearly doubled in the kinked fitter relative to the straight fitter.

\subsection{The $\mathrm{CC} \pi^{+}$Fitter}

With the ability to reconstruct charged pions, a full $\mathrm{CC} \pi^{+}$fitter is formed by simultaneously fitting for a straight muon and a kinked pion. A $\mathrm{CC} \pi^{+}$fit has 14 parameters:

- $\left(X_{0}, Y_{0}, Z_{0}, T_{0}\right)$ : the event 4 -vertex

- $E_{\mu}$ : the initial energy of the muon track

- $\left(\theta_{\mu}, \phi_{\mu}\right)$ : the initial direction of the muon track

- $E_{\pi}$ : the initial energy of the pion track

- $\left(\theta_{\pi}, \phi_{\mu}\right)$ : the initial direction of the pion track

- $\Delta E_{\text {up }}$ : the energy lost by the pion track before the kink

- $\Delta E_{k i n k}$ : the energy lost by the pion track in the kink

- $\left(\theta_{\text {down }}, \phi_{\text {down }}\right)$ : the direction of the downstream segment of the pion track 


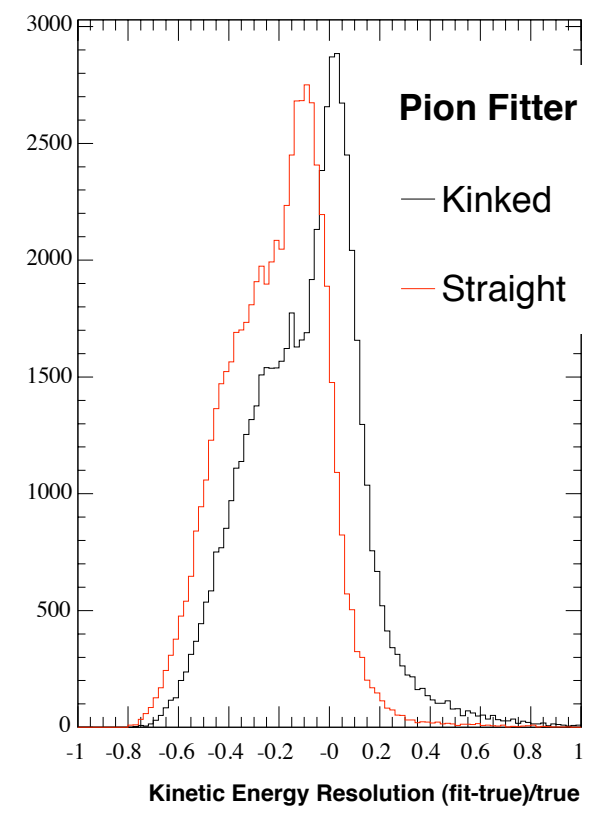

(a) Pion kinetic energy resolution from the straight and kinked pion fits

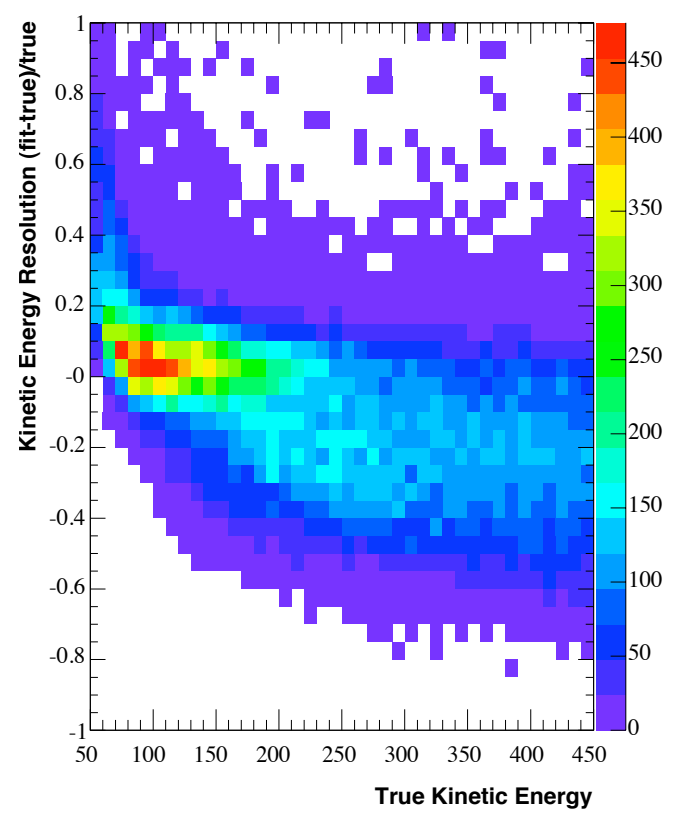

(b) The pion kinetic energy resolution as a function of true pion kinetic energy from the kinked pion fit

Figure 5.16: The reconstructed pion energy resolution is shown for both the straight and kinked pion fitters (left). The kinked fitter resolution is also plotted versus the true pion energy (right). The two-dimensional plots indicate that the low fit energy "shoulder" is caused by higher energy pions. The low energy shoulder is significantly reduced in the kinked fitter, and rather than being $10 \%$ low, as is the case with the straight fitter, the peak of the kinked fitter resolution function is centered at zero.

Just as in the kinked pion fitter, the predicted charges from all track segments (upstream pion, downstream pion, and muon) are summed to get the total predicted charge for each PMT. The time PDF follows the same prescription given in Equations 5.21-5.24, with an additional prompt source from the muon that is prioritized according to the photon time of flight from the center of the track to the PMT. 


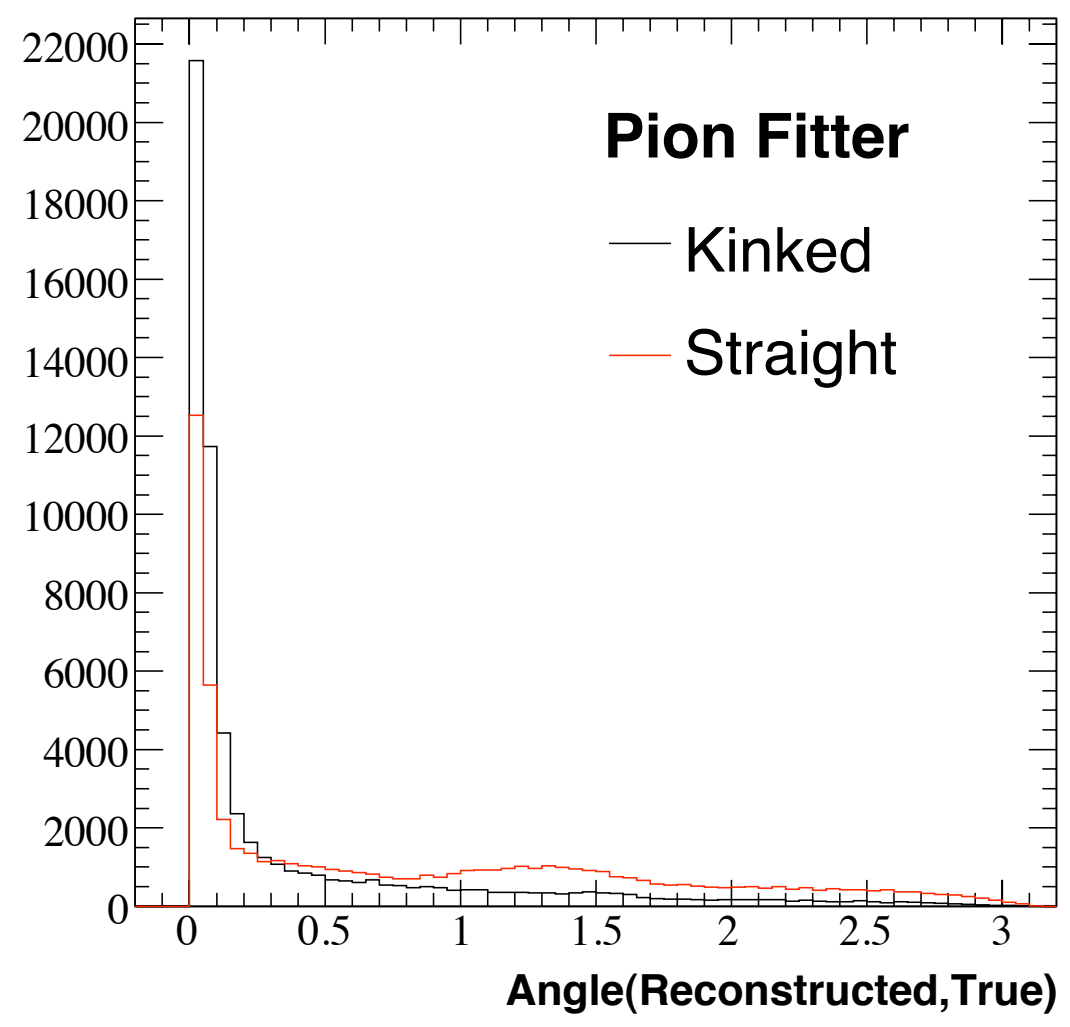

Figure 5.17: The angle between the reconstructed and true pion directions is shown for both the straight and kinked pion fitters. The population in the lowest few bins where the properly reconstructed events lie is nearly twice as large for the kinked fitter.

\subsubsection{Fit Seeding}

Each $\mathrm{CC} \pi^{+}$event is assumed to have three Cherenkov rings from the three track segments in the event: the upstream pion segment, the downstream pion segment, and the muon track. Each ring is found in succession using intermediate two and three track likelihood functions. The three tracks are then pieced together in several different pairings to create the kinked pion track and the straight muon track. The pairing that produces the best likelihood is used to seed the $\mathrm{CC} \pi^{+}$fitter.

The first of the three rings is found by performing a one track fit. The results of the fit are frozen in place, and a scan for a second track is performed over 100 equally 
spaced directions. At each scan point, a two track likelihood function is evaluated, and the configuration that gives the best likelihood value sets the direction of the second track. The one track fit result is held in place during the likelihood scan to effectively cancel out the prompt predicted charge from the first Cherenkov ring. This minimizes the effect of the first ring on the shape of the likelihood surface during the scan for the second ring. The two track scan point that returns the best likelihood value is used to seed a full two track fit. The two track fit adjusts both directions and redistributes the amount of energy in each track. This process is repeated by freezing the result of the two track fit and scanning for a third track using a three track likelihood function. The result of the scan seeds a full three track fit.

The likelihood scans are not very sensitive to the energy chosen for the new track. The energy mainly just sets the prediction for the diameter of the Cherenkov ring, so the scan will usually locate a ring even if the predicted diameter is incorrect. The excess light generated by an energy overestimate, for example, results in a worse likelihood than the proper energy would have produced, but this extra energy is present at all scan points so the relative likelihoods are mostly unaffected. For the two track scan, an energy of $200 \mathrm{MeV}$ has been chosen, which is near the average pion energy in $\mathrm{CC} \pi^{+}$ events. In the three track scan, the remaining unidentified track typically has a lower energy than the tracks returned by the two track fit, so the scan energy placed midway between the lower of the two found tracks and $60 \mathrm{MeV}$, which is slightly above the pion Cherenkov energy threshold.

Although the choice of the scan track energy has little effect on the ability of the scans to find the proper track directions, it can affect the energy reconstruction. At each intermediate fit stage, the seed track directions and event vertex have been determined much more precisely than the track energies. The energies from previous fits are usually overestimates of the true ring energies to account for some of the additional unfit light in the event, and the scan energy is an ad hoc choice. Since there is no convenient 
way to specify to MINUIT which variables have better seed values than others, a fit is performed where only the track energies are varied before the full two or three track fit is performed. The final muon resolution from the full $\mathrm{CC} \pi^{+}$fitter is shown with and without an extra energy fit stage in Figure 5.18. The extra energy fits improve the correlation between fit and true energies, and the peak is shifted closer to zero.
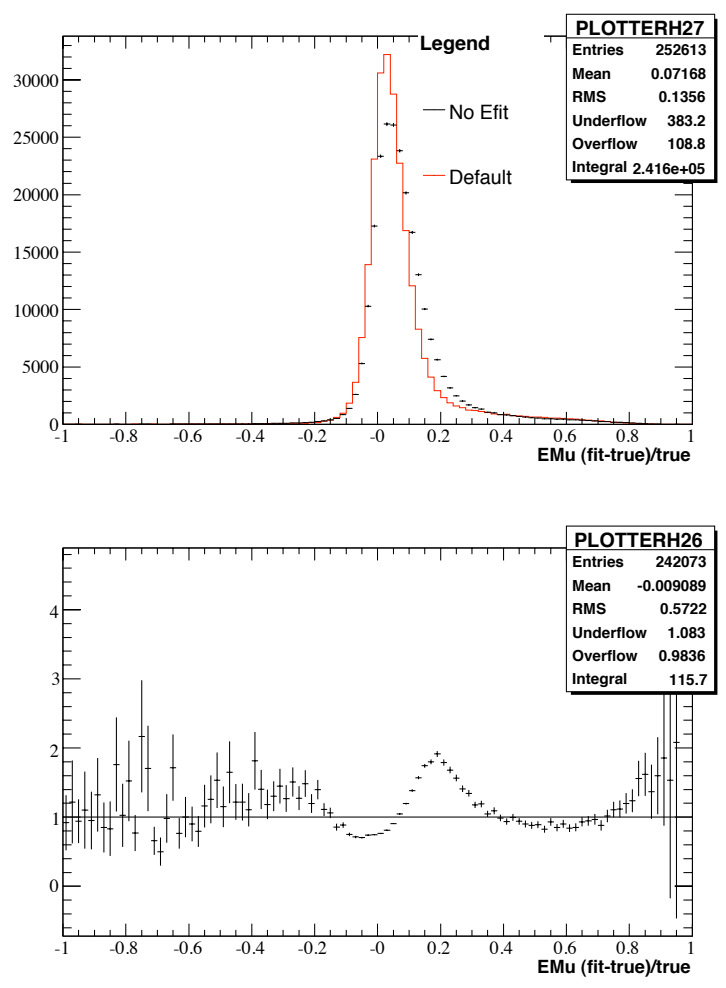

Figure 5.18: The muon energy resolution is shown for the default Monte Carlo, and the Monte Carlo absent the extra energy fit (EFit) stages of the fit seeding process. The lower plot shows the ratio of the two distributions. The EFit Monte Carlo has a smaller width, and the peak is shifted closer to zero.

Once three tracks are found that characterize the Cherenkov rings in the event, they are combined to form a straight muon and a kinked pion. In a $\mathrm{CC} \pi^{+}$event, two of the tracks found in the three track fit point to rings that are properly characterized by the likelihood tables, the muon ring and the downstream pion ring. The other track points to the upstream pion ring, which is the result of a track segment, and 
therefore not a filled in ring. Since the reconstructed track energy is mostly driven by the outer diameter of the ring, the straight track approximation of this ring only slightly underestimates the total pion energy to compensate for the missing charge at the center of the ring. Therefore, as the three tracks are combined, only pairings where the downstream pion track has a lower energy than the upstream pion track are allowed. This reduces the number of possible groupings to three. Each of these three seeds is passed to the full $\mathrm{CC} \pi^{+}$fitter, and the fit that produces the best likelihood is chosen. A series of event displays for each step of the seeding and fit procedure are shown in Appendix A.

\subsubsection{Fit Results}

The kinetic energy resolutions for the muon and pion tracks are given in Figure 5.19. The muon kinetic energy has a small tail at low reconstructed energy due to $\mu / \pi$ mispairing. ${ }^{3} \quad$ The reconstructed pion kinetic energy has the same low energy shoulder from high energy particles seen in the pion-only fit in Figure 5.16(a). In addition, the fitter tends to place about $5 \%$ too much energy in the muon track at the expense of the pion track.

Although the pion energy fit is more accurate at low track energies, the opposite is true for the reconstructed pion direction. The track direction reconstruction relies on the existence of a well defined Cherenkov ring from the upstream pion track segment. $20 \%$ of the generated pion kinetic energy spectrum lies below $70 \mathrm{MeV}$. This corresponds to an above-Cherenkov propagation distance of less than $10 \mathrm{~cm}$, which is typically insufficient to determine the direction. For comparison, only $1 \%$ of muons are generated below $70 \mathrm{MeV}$. In addition, the pion interaction length in mineral oil is approximately $1 \mathrm{~m}$ [1], which means $10 \%$ of all pions will have upstream segments shorter than $10 \mathrm{~cm}$. The

\footnotetext{
3 The pairing is determined by forming the angle between reconstructed and true track directions for all four such pairings $\left(\mu_{r e c} / \mu_{\text {true }}, \pi_{r e c} / \pi_{\text {true }}, \mu_{\text {rec }} / \pi_{\text {true }}\right.$, and $\left.\pi_{r e c} / \mu_{\text {true }}\right)$. If the smallest angle is between like particles $\left(\mu_{\text {rec }} / \mu_{\text {true }}\right.$ or $\left.\pi_{r e c} / \pi_{\text {true }}\right)$, then the event is said to be paired correctly.
} 


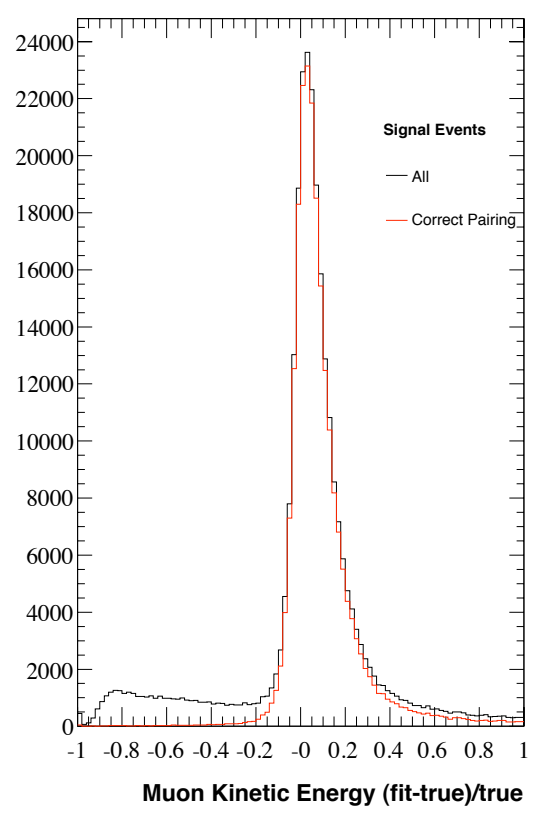

(a) Muon kinetic energy resolution

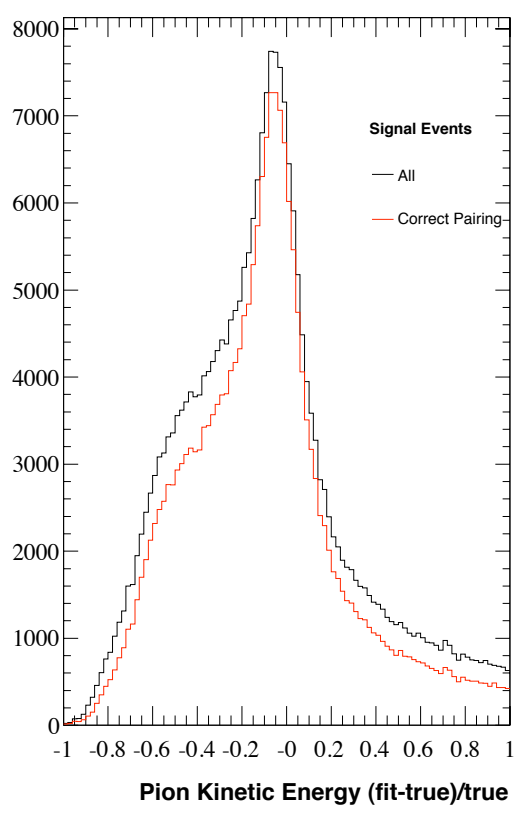

(b) Pion kinetic energy resolution

Figure 5.19: The kinetic energy resolution is shown for muons (left) and pions (right) for all signal events and correctly paired signal events. Most of the low fit energy muon tail is due to mispaired events. The pion distribution shows the same reconstructed low energy shoulder seen in the pion-only fit in Figure 5.16(a).

fitter reconstructs pions to within $300 \mathrm{mr}$ of the true direction $50 \%$ of the time, and the $\mu / \pi$ misidentification rate is $20 \%$.

The ability of the fitter to correctly reconstruct both the muon and the pion directions is shown in Figure 5.20. The reconstructed angle between the muon and pion is plotted against the larger of the two reconstructed/true angles. A perfect fitter would place all events in the lowest column where both reconstructed/true angles are zero, which has the largest population of events in Figure 5.20. The other significant event population is along the diagonal of the plot. These are events where the fitter has misidentified the muon as a pion and vice versa. In such cases, the angle between both the true and reconstructed muon and the true and reconstructed pion will be roughly 
equivalent to the reconstructed $\mu / \pi$ angle.

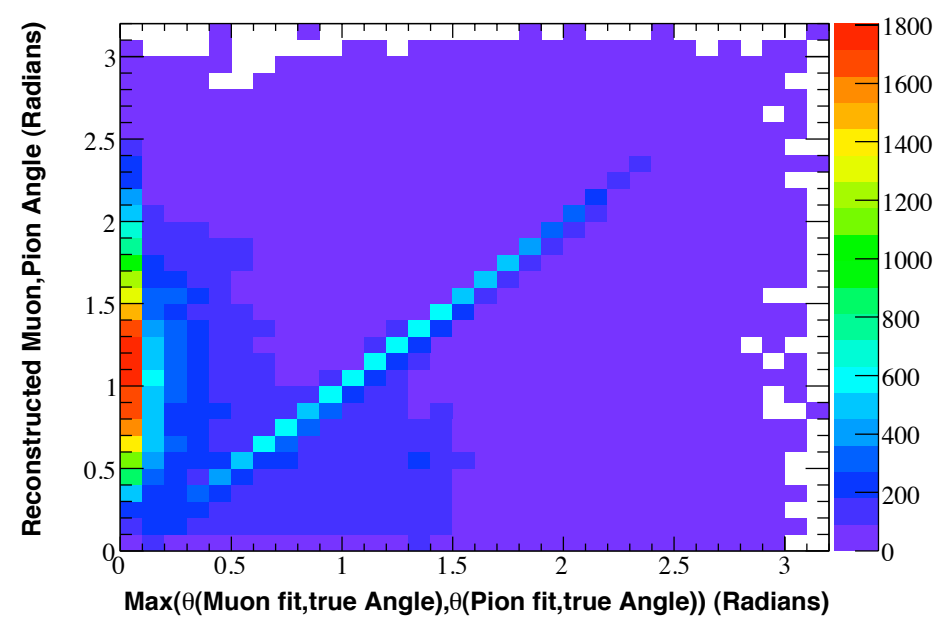

Figure 5.20: The reconstructed angle between the muon and pion directions is shown versus the larger (i.e. worse reconstructed) of the two reconstructed/true angles, $\theta\left(\mu_{\text {rec }}, \mu_{\text {true }}\right)$ and $\theta\left(\pi_{r e c}, \pi_{\text {true }}\right)$. The events in the left-most columns represent events where both tracks have been properly reconstructed. The events where the tracks have been misidentified appear along the diagonal.

\subsubsection{Neutrino Energy}

With reconstructed energies and directions for the both the muon and the pion, the energy of the incident neutrino can be determined. Assuming the target nucleon is at rest and the remaining, unmeasured final state particle is a nucleon, the neutrino energy is specified by 4-momentum conservation,

$$
E_{\nu}=\frac{m_{\mu}^{2}+m_{\pi}^{2}-2 m_{N}\left(E_{\mu}+E_{\pi}\right)+2 p_{\mu} \cdot p_{\pi}}{2\left(E_{\mu}+E_{\pi}-\left|\mathbf{p}_{\mu}\right| \cos \theta_{\nu, \mu}-\left|\mathbf{p}_{\pi}\right| \cos \theta_{\nu, \pi}-m_{N}\right)},
$$

where $m_{p}, E_{p}, p_{p}$, and $\left|\mathbf{p}_{p}\right|$ are the mass, energy, 4-momentum, and 3-momentum magnitude of particle $p$ in the detector frame, and $\theta_{\nu, \mu}\left(\theta_{\nu, \pi}\right)$ is the angle between the directions of the muon(pion) and the neutrino. The neutrino direction is determined by the event vertex location and the mean neutrino emission point from the beam Monte Carlo prediction, although the large distance between the beam and the detector means this 
angle is never larger than one degree. The comparison between reconstructed and true neutrino energy is given in Figure 5.21. The resolution is $15 \%$ over most of the sensitive range, with a slight increase at the highest energies.

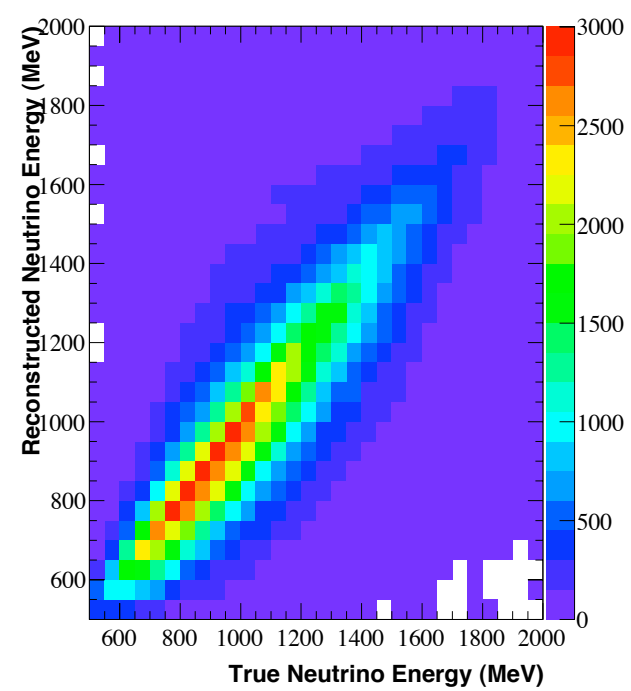

(a) Reconstructed versus true neutrino energy

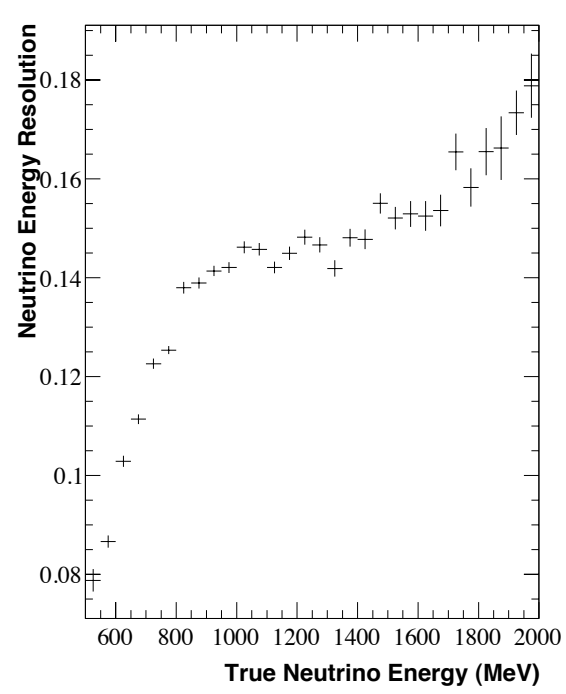

(b) Neutrino energy resolution versus true neutrino energy

Figure 5.21: The comparison of reconstructed and true neutrino energy is shown (left) along with the Gaussian widths of the energy resolution distribution as a function of true neutrino energy (right). The resolution is about $15 \%$ over most of the energy range to which the analysis is sensitive.

The inability of the fitter to properly reconstruct all pion directions has little impact on the neutrino energy reconstruction. These misreconstructed events are dominated by low energy pions and events where the muon and pion tracks were misidentifed. The neutrino energy calculation becomes less sensitive to the reconstructed pion direction as the pion energy is reduced. Events with misidentified tracks that are otherwise well reconstructed will produce nearly the same neutrino energy, since muons and pions have similar masses. 


\subsubsection{2 $\Delta$ Mass}

By making the aforementioned assumptions required to calculate the neutrino energy, the kinematics of the interaction are fully specified. Previous attempts to measure $\mathrm{CC} \pi^{+}$interactions by reconstructing only the muon required the additional assumption that the recoiling nucleon was an on-shell $\Delta$ baryon [65]. Since the width of the $\Delta$ resonance is about $10 \%$ of its mass, this assumption results an irreducible contribution neutrino energy resolution. By measuring the pion as well, the $\Delta$ mass constraint has been removed in the present analysis.

The absence of a $\Delta$ mass constraint means that the $\pi^{+}+N$ invariant mass, which is dominated by the $\Delta$ resonance mass, can be measured. Figure 5.22 shows the reconstructed $\pi^{+}+N$ mass, as well as a breakdown of the background composition. The fact that the CCQE background events peak sharply near threshold lends credence to the quality of the reconstruction since those events typically do not produce a pion. A comparison of reconstructed data and Monte Carlo with full systematic errors is given in Figure B.19.

\subsubsection{3 $Q^{2}$}

The final variable measured in this analysis is the 4-momentum transfer, $q$, from the leptonic current to the hadronic portion of the decay, which is characterized by its relativistic invariant, $Q^{2}=-q^{2}$. Since $Q^{2}$ is a property of the exchanged $W$ boson, it is completely specified by the change in the leptonic current. However, this also means that, unlike the neutrino energy calculation, the reconstructed $Q^{2}$ distribution is quite sensitive to $\mu / \pi$ misidentification. Figure 5.23(a) shows the fractional error in the reconstructed $Q^{2}$ distribution, normalized in columns of true $Q^{2}$. Most of the columns peak near zero, but at high $Q^{2}$, a second population of events appears in which the fit underestimates the true $Q^{2}$. These are events that are dominated by a high 


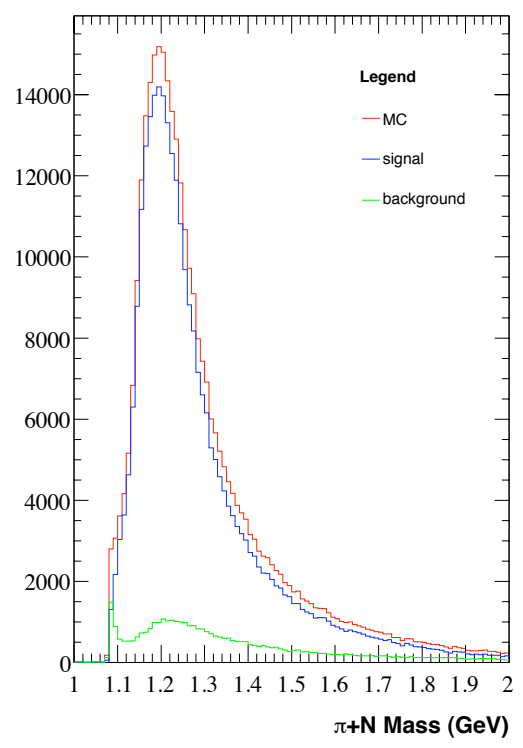

(a) The total Monte Carlo prediction (red) is divided into signal (blue) and background (green) components.

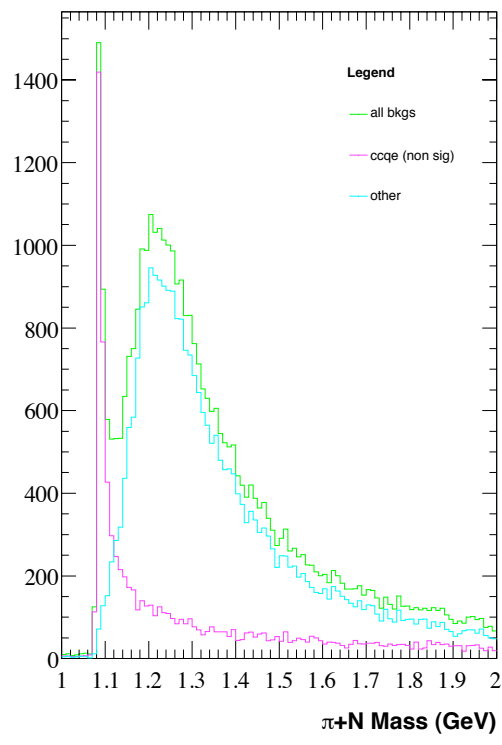

(b) The background distribution (green) is divided into contributions from CCQE (magenta) and all other backgrounds (cyan).

Figure 5.22: The reconstructed Monte Carlo $\pi^{+}+N$ distribution is shown in the left plot for signal and background events. On the right, the background distribution is divided into CCQE background events, and all other backgrounds. Since the CCQE events do not contain a pion, they are peaked near threshold $\left(m_{\pi}+m_{N}\right)$. The definition of signal events is given in Section 6.2.

energy muon that has been misidentified as a pion, giving the impression that most of the neutrino momentum was transferred to the hadronic system. The resolution for correctly identified events is given as a function of true $Q^{2}$ in Figure 5.23(b). 


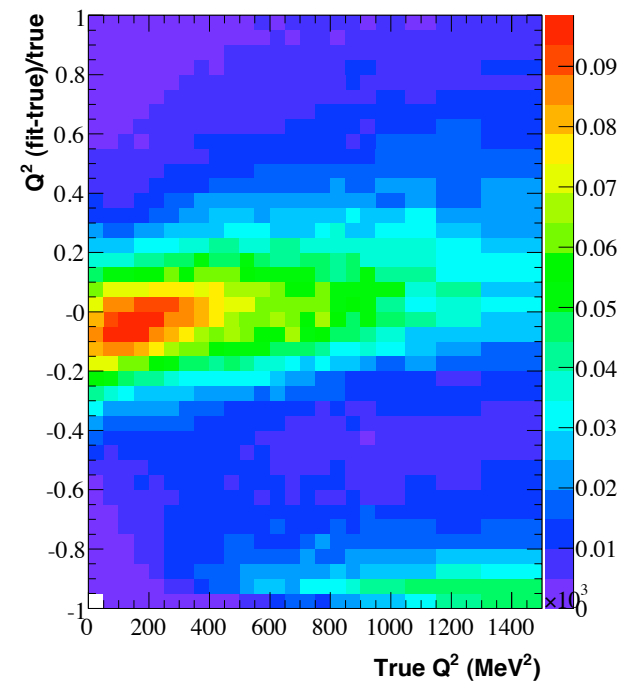

(a) $Q^{2}$ resolution versus true $Q^{2}$ (column normalized)

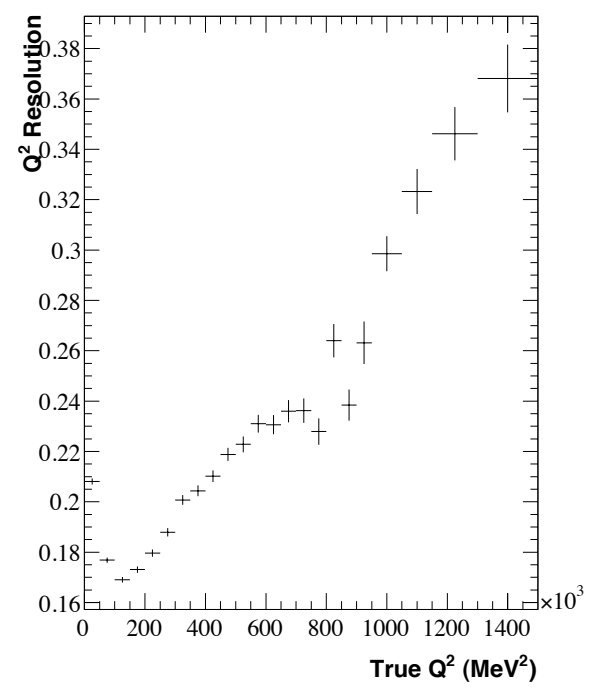

(b) $Q^{2}$ resolution versus true $Q^{2}$

Figure 5.23: The fractional error is plotted versus true $Q^{2}$ (left) where each true column has been normalized to one to highlight the population of misidentified events at high true $Q^{2}$, and low fit $Q^{2}$. The Gaussian width of the resolution distribution for events with correctly identified tracks is also shown (right). 


\section{Chapter 6}

\section{$\mathrm{CC} \pi^{+}$Cross Section Analysis}

The most fundamental quantities that can be measured in a particle interaction are the frequency with which the interaction takes place and the kinematics of the initial and final state particles. The results of these measurements are most conveniently expressed as cross sections. The cross section, $\sigma$, is defined as the ratio of the number of interactions, $N^{\text {int }}$, to the product of the number of interaction targets, $N_{\text {targ }}$, and the flux, $\Phi$, which is the number of incident neutrinos per unit area,

$$
\sigma=\frac{N^{i n t}}{N_{\text {targ }} \Phi}
$$

\subsection{Cross Section Definitions}

A cross section can be measured as a function of any variable in the interaction. There is, however, an important distinction to be made between initial and final state variables. A cross section measured in terms of an initial state variable, such as neutrino energy, still gives the probability that an interaction takes place; it just expresses that probability separately for each value of the initial state variable. A cross section measured as a function of a final state variable, such as muon kinetic energy, is a fundamentally different quantity that encapsulates not only the probability that the interaction takes place, but also the additional probability that the resulting muon has a particular energy. By definition, the integral over all possible muon energies restores 
the original interaction probability, and, therefore, cross sections expressed in terms of a final state variable, $v$, are derivatives with respect to $v$ : $\partial \sigma / \partial v$.

To perform a cross section measurement, the initial and final state dependencies of the other quantities in Equation 6.1 must be taken into account as well. The flux cannot be meaningfully expressed in terms of final state variables. For example, it is not possible to separate the number of incident neutrinos per unit area into bins of outgoing muon direction. With respect to initial state variables, the flux is an aggregate quantity (that is, the flux over an infinitesimal interval, $d u$, of an initial state variable, $u$, is half as large as the flux over twice the interval, $2 d u$ ), and so, to recover the flux, the differential flux must be integrated over the desired interval,

$$
\Phi\left(u_{\min } ; u_{\max }\right)=\int_{u_{\min }}^{u_{\max }} \frac{\partial \Phi}{\partial u} d u
$$

The number of interactions is an aggregate quantity with respect to both initial and final state variables. Put another way, the fraction of events in, for example, a given range of both neutrino energy and muon kinetic energy is well defined, and all such fractions must sum to the total number of interactions. After implementing these dependencies, Equation 6.1 can be written in its most general form in terms of $M$ initial state variables, $u_{1} \ldots u_{M}$, and $N$ final state variables, $v_{1} \ldots v_{N}$,

$$
\begin{aligned}
N^{\text {int }} & =N_{\text {targ }} \int_{u_{1}} \ldots \int_{u_{M}} \int_{v_{1}} \ldots \int_{v_{N}} \frac{\partial \sigma\left(u_{1}, \ldots, u_{M}\right)}{\partial v_{1} \ldots \partial v_{N}} d v_{1} \ldots d v_{N} \frac{\partial \Phi}{\partial u_{1} \ldots \partial u_{M}} d u_{1} \ldots d u_{M} \\
& =\int_{u_{1}} \ldots \int_{u_{M}} \int_{v_{1}} \ldots \int_{v_{N}} \frac{\partial N^{i n t}}{\partial v_{1} \ldots \partial v_{N} \partial u_{1} \ldots \partial u_{M}} d v_{1} \ldots d v_{N} d u_{1} \ldots d u_{M} .
\end{aligned}
$$

Cross section measurements are, by necessity, performed over finite intervals of the initial and final state variables. In order to measure a cross section in a bin, $i$, of an initial state variable, $u$, all integrals other than the integral over $u$ in Equation 6.3 must first be performed. The final state variable integrations are straightforward. They simply return the initial state $N^{\text {int }}$ derivatives on the second line of Equation 6.3, and 
the total cross section on the first line, since the flux dependence can be factored out of all final state integrals. The initial state integrals must be treated more carefully, since both the cross section and the flux must be integrated together. This results in a cross section that is averaged over the flux shape in the initial state variables, as will be described momentarily via Equation 6.5. The remaining integral over $u$ is performed over each bin. The number of events in bin $i, N_{i}^{i n t}$, can then be expressed as

$$
N_{i}^{i n t}=N_{\operatorname{targ}} \int_{u_{i, \min }}^{u_{i, \max }} \sigma(u) \frac{\partial \Phi}{\partial u} d u=N_{\operatorname{targ}}\left\langle\sigma \frac{\partial \Phi}{\partial u}\right\rangle_{i} \Delta u_{i}
$$

where $\left\langle\sigma \frac{\partial \Phi}{\partial u}\right\rangle_{i}$ is the average value of the integrand over bin $i$, and $\Delta u_{i}$ is the bin width (i.e. $\left.u_{i, \max }-u_{i, \min }\right)$. The average value of the differential flux within the bin my be factored out of $\left\langle\sigma \frac{\partial \Phi}{\partial u}\right\rangle_{i}$, leaving only the flux shape in the average,

$$
\left\langle\sigma \frac{\partial \Phi}{\partial u}\right\rangle_{i} \Delta u_{i}=\left(\left\langle\frac{\partial \Phi}{\partial u}\right\rangle_{i} \Delta u_{i}\right)\left\langle\sigma \frac{\partial \Phi}{\partial u} /\left\langle\frac{\partial \Phi}{\partial u}\right\rangle_{i}\right\rangle_{i} \equiv \Phi_{i}\langle\sigma\rangle_{\Phi_{i}} .
$$

$\Phi_{i}$ is the integrated flux over bin $i$, and $\langle\sigma\rangle_{\Phi_{i}}$ is called the "flux averaged" cross section in bin $i$. Any cross section measurement is necessarily averaged over the shape of the flux in each measured bin; therefore, in the following sections we will drop the cumbersome notation $\langle\sigma\rangle_{\Phi_{i}}$, and instead use $\sigma\left(u_{i}\right)$. Substituting Equation 6.5 into Equation 6.4 gives the final expression for the flux averaged cross section in bin $i$ of an initial state variable,

$$
\sigma\left(u_{i}\right) \equiv\langle\sigma\rangle_{\Phi_{i}}=\frac{N_{i}^{\text {int }}}{N_{\text {targ }} \Phi_{i}}
$$

As mentioned previously, $\sigma\left(u_{i}\right)$ is also implicitly flux averaged over the full range of all other initial state variables.

To perform a cross section measurement in a bin, $i$, of a final state variable, $v$, all integrals in Equation 6.3 are performed except for the integral over $v$, which is performed separately in each bin,

$$
N_{i}^{i n t}=N_{t a r g} \Phi \int_{v_{i, \min }}^{v_{i, \max }}\left\langle\frac{\partial \sigma}{\partial v}\right\rangle_{\Phi} d v=N_{t a r g} \Phi\left\langle\frac{\partial \sigma}{\partial v}\right\rangle_{i, \Phi} \Delta v_{i}
$$


Once again, the integrals over the initial state variables yield a flux averaged cross section times the integrated flux, and the right side has been rewritten in terms of the average integrand over the bin. The final expression for the differential cross section is then

$$
\frac{\partial \sigma}{\partial v}\left(v_{i}\right) \equiv\left\langle\frac{\partial \sigma}{\partial v}\right\rangle_{i, \Phi}=\frac{N_{i}^{i n t}}{\Delta v_{i} N_{\text {targ }} \Phi},
$$

where the explicit flux and bin averaging notation has been dropped, as was done in Equation 6.6.

In general, the cross section can be measured in bins of any number of initial and final state variables. The generalization of the expressions in Equations 6.6 and 6.8 to multidimensional cross section measurements is achieved by separating one additional integral in Equation 6.3 into bins. The explicit form for each of the three two-dimensional cases are as follows:

$$
\begin{aligned}
\frac{\partial^{2} \sigma}{\partial v_{1} \partial v_{2}}\left(v_{1 i}, v_{2 j}\right) & =\frac{N_{i j}^{i n t}}{\Delta v_{i} \Delta v_{j} N_{\text {targ }} \Phi} \\
\frac{\partial \sigma}{\partial v}\left(u_{i}, v_{j}\right) & =\frac{N_{i j}^{i n t}}{\Delta v_{j} N_{\text {targ }} \Phi_{i}} \\
\sigma\left(u_{1 i}, u_{2 j}\right) & =\frac{N_{i j}^{\text {int }}}{N_{\text {targ }} \Phi_{i j}}
\end{aligned}
$$

\subsection{Defining the Signal: "Effective" $\mathrm{CC} \pi^{+}$Events}

In nuclear media, interaction processes must be carefully defined. A neutrino can interact with a quark, a nucleon, or a collection of several nucleons. In addition, as the particles created in the initial interaction travel through the nucleus, other types of interactions can take place such as pion absorption and charge exchange $\left(\pi^{+} n \rightarrow \pi^{0} p\right)$. Regardless of the details of the goings on inside the nucleus, only the characteristics of particles that exit the nucleus are accessible to experiment. Of course, it is possible to use a simulation of the various types of targets and nuclear effects to attempt to extrapolate the measured quantities back to the initial interaction, but such a result 
would fold in quite a bit of model dependence, and an experimental measurement is typically not the place for such an extrapolation.

In the results that follow, an "effective" $\mathrm{CC} \pi^{+}$event is defined as any $\nu_{\mu}$ interaction that produces a set of particles exiting the nucleus with the following composition:

- one and only one $\mu^{-}$

- one and only one $\pi^{+}$

- any number of protons and neutrons (including zero)

- any number of multi-nucleon states (including zero)

- any number of photons (including zero)

The presence of both the $\mu^{-}$and $\pi^{+}$particles is a necessary requirement for $\mathrm{CC} \pi^{+}$ interactions. In coherent interactions, it is possible for the final state to contain no additional particles. Resonant interactions eject either a proton or neutron from the nucleus, and in some cases the remainder of the nucleus may be broken apart as well, which can produce other multi-nucleon fragments in the final state. Photons were allowed in the final state to avoid setting an arbitrary energy cut-off to differentiate between radiative and non-radiative events, and because radiative corrections were not implemented in the Monte Carlo simulation.

\subsection{Event Selection}

To perform cross section measurements, a sample of $\mathrm{CC} \pi^{+}$events must be isolated from the data. As described in section 3.5, data from the photomultiplier tubes (PMTs) are collected in a $19.2 \mu$ s time window, which begins $4.6 \mu$ s prior to the arrival of the $1.6 \mu$ s beam pulse. These data contain two main sources of background: cosmic rays and electrons from stopped muon decays (called "Michel" electrons). By requiring less than 6 hits from PMTs in the veto region, $99.987 \%$ of cosmic rays that enter the detector in 
the beam time window are rejected [28]. The Michel electron contamination comes from cosmic ray muons that enter the tank before the beginning of the beam time window and decay while data are being recorded. The maximum energy for an electron from a stopped muon decay is $52.8 \mathrm{MeV}$, which results in many less PMT hits than are created in a typical neutrino interaction. Requiring more than 175 tank hits mostly removes the Michel background. Figure 6.1 shows that after imposing the veto hits and tanks hits cuts, essentially all non-beam-related background is eliminated.

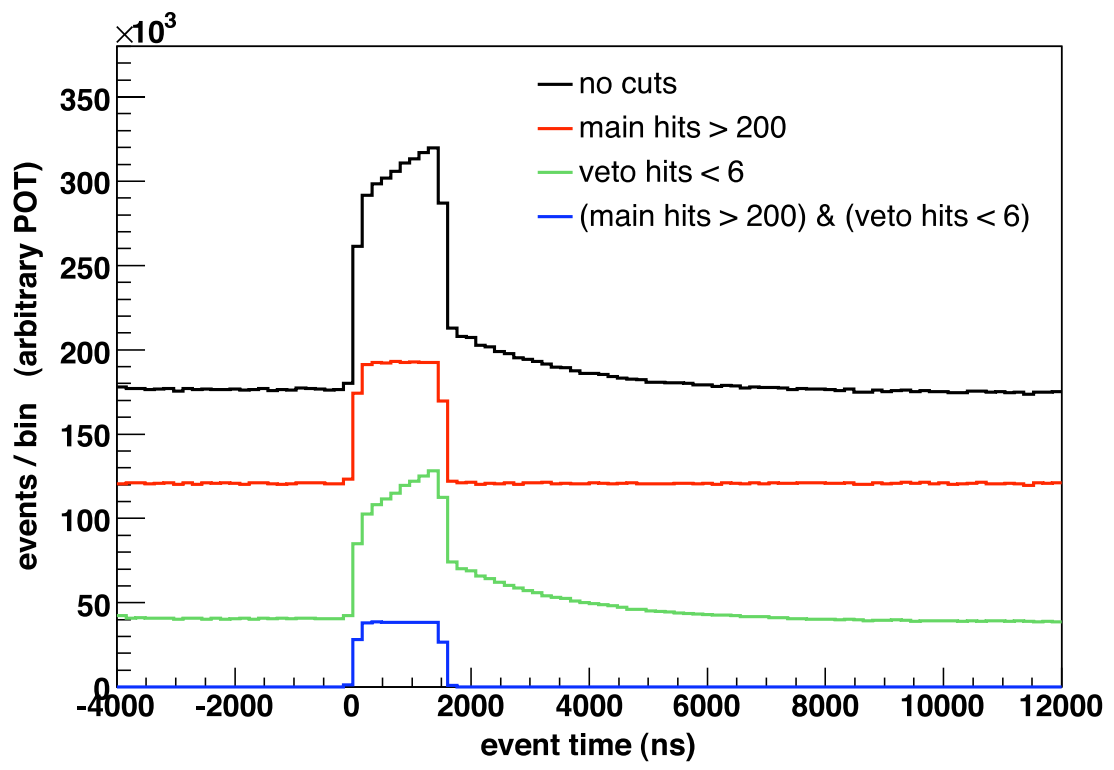

Figure 6.1: The plot shows the average hit time distribution for the first subevent (i.e. the first arrival of PMT hits) with no cuts (black), after requiring more than 200 hits in the tank region (red), after requiring less than 6 hits from PMTs in the veto region (green), and with both the tank and veto hits applied simultaneously (blue). The veto hits cut removes all events that contain particles entering or exiting the tank, and the tank hits cut removes the exponentially falling background due to decays of stopped cosmic muons that entered the tank prior to the event time window. These two cuts remove essentially all of the beam-unrelated background. Note that in the present analysis, very few Michels satisfy the three subevent cut, so the tank hits cut has been relaxed to 175 .

Isolating effective $\mathrm{CC} \pi^{+}$events from other neutrino induced backgrounds can also be accomplished simply and effectively. The PMT hits in each event are separated into time slices called subevents. A subevent is created for any group of 10 or more hits 
that have no more than two 20 ns gaps between them, and spacings no larger than 10 ns otherwise. The effective $\mathrm{CC} \pi^{+}$analysis requires exactly three subevents: one for the simultaneous muon and pion created in the initial neutrino interaction, one for the Michel electron from the decay of the stopped muon, and one for the Michel electron from the $\pi^{+} \rightarrow \mu^{+} \rightarrow e^{+}$decay chain. Muons created in stopped pion decays have a kinetic energy of only $4.1 \mathrm{MeV}$ and therefore rarely produce any light.

Most event types with comparable event rates to $\mathrm{CC} \pi^{+}$, as described in Table 4.6, are greatly reduced by the three subevents requirement. The vast majority of CCQE events are eliminated, since they typically produce only one Michel electron, which corresponds to two subevents. NCEL events produce one subevent, $\mathrm{CC} \pi^{0}$ events produce two subevents, and high multiplicity interactions such as CCmulti $\pi$ and CCDIS usually produce more than three subevents. The efficiencies for signal and background events for various combinations of cuts are given in Tables 6.1 and 6.2, respectively.

In addition to the aforementioned tank and veto hits cuts used for the initial neutrino interaction, cuts are imposed on the number of tank and veto hits in the second and third subevents as well. The same veto hits cut imposed in the first subevent is used for subevents 2 and 3 to ensure that neither subevent was caused by a cosmic ray. In effective $\mathrm{CC} \pi^{+}$events, both subsequent subevents are from Michel electrons, so each is required to have less than 200 tank hits. The effect of these cuts on the second subevent is shown in Figure 6.2.

The final set of cuts are designed to eliminate events that occur too close to the edge of the detector. Rather than define a fixed fiducial volume, events are cut based on the distance between the tank wall and the event vertex along the trajectory of either of the two reconstructed particles. Particles that are pointed at the tank wall are poorly reconstructed. They deposit Cherenkov light in very few tubes, which allows the fitter to freely vary the energy without incurring much of a penalty in the likelihood function. Conversely, events that occur very close to the tank wall with inward pointing 


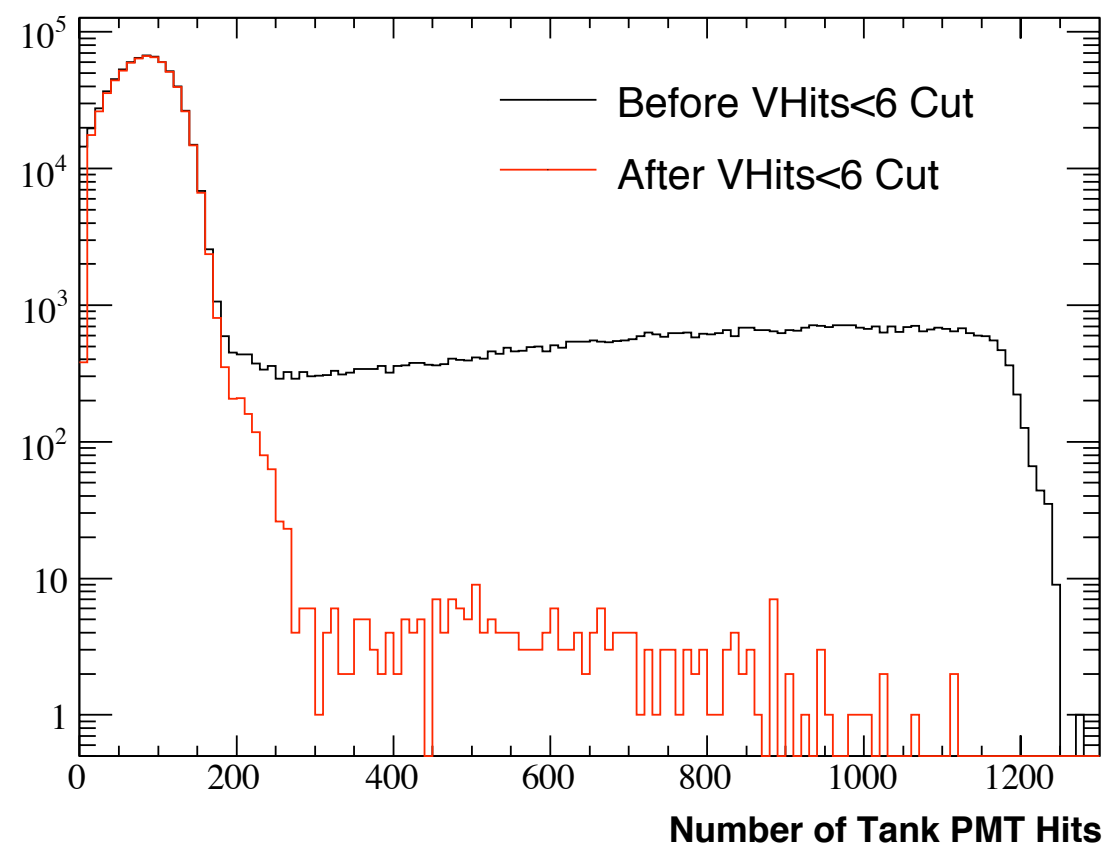

Figure 6.2: The tank hits distribution is shown for the second subevent before and after requiring less than 6 hits in the veto. The Michel electron peak is mostly unaffected, while the large tail from entering comic rays is mostly removed.

particles can be reconstructed quite well since light in the backward direction is not very important in determining particle energies and directions. The distance between the event vertex and tank wall is required to be at least $150 \mathrm{~cm}$ along both the muon and pion trajectories. The effect of this cut is shown in Figure 6.3.

After all cuts, 67,318 events are seen in the data with a signal efficiency of $16.9 \%$. The sample has a purity of $87.2 \%$, and the largest backgrounds (listed by percentage of the total sample) are from Nuance CCmulti $\pi$ events (4.0\%), Nuance CCQE events $(3.3 \%)$, and Nuance $\mathrm{CC} \pi^{+}$events $(1.7 \%)$. The complete list of signal and background compositions by Nuance process are given in Tables 6.1 and 6.2, respectively. 
Table 6.1: The signal efficiency and purity are shown after each successive analysis cut. The additional rows give the distribution of signal events according to generated Nuance process (see Section 4.2). The final three rows list the three components of the $\mathrm{CC} \pi^{+}$ population separately. Note that Nuance $\mathrm{CC} \pi^{+}$events are not equivalent to effective $\mathrm{CC} \pi^{+}$events as defined in the cross section measurement. The cuts below are defined as follows: veto hits $<6$ and tank hits $>175$ in the first subevent (H1), exactly 3 subevents (S3), veto hits $<6$ and tank hits $<200$ in the second and third subevents (H23), and vertex/wall distance along the muon and pion trajectories $>150 \mathrm{~cm}$ (WD).

\begin{tabular}{|l|c|c|c|c|c|}
\hline & No Cuts & H1 & H1+S3 & H1+S3+H23 & H1+S3+H23+WD \\
\hline \hline Signal Efficiency & 1.000 & 0.475 & 0.232 & 0.196 & 0.169 \\
\hline Signal Purity & 0.187 & 0.236 & 0.605 & 0.863 & 0.872 \\
\hline \hline Signal Composition & No Cuts & H1 & H1+S3 & H1+S3+H23 & H1+S3+H23+WD \\
\hline \hline $\mathrm{CC} \pi^{+}$ & 0.959 & 0.962 & 0.959 & 0.959 & 0.961 \\
\hline $\mathrm{CCQE}$ & 0.019 & 0.020 & 0.023 & 0.023 & 0.023 \\
\hline $\mathrm{CC} \pi^{0}$ & 0.008 & 0.009 & 0.009 & 0.009 & 0.009 \\
\hline $\mathrm{CCmulti} \pi$ & 0.004 & 0.004 & 0.004 & 0.004 & 0.004 \\
\hline $\mathrm{CCDIS}$ & 0.009 & 0.005 & 0.004 & 0.004 & 0.003 \\
\hline other & 0.001 & 0.000 & 0.001 & 0.001 & 0.000 \\
\hline \hline $\mathrm{CC} \pi^{+}$Components & No Cuts & $\mathrm{H} 1$ & $\mathrm{H} 1+\mathrm{S} 3$ & $\mathrm{H} 1+\mathrm{S} 3+\mathrm{H} 23$ & $\mathrm{H} 1+\mathrm{S} 3+\mathrm{H} 23+\mathrm{WD}$ \\
\hline \hline$\nu p \rightarrow \mu^{-} p \pi^{+}$ & 0.750 & 0.761 & 0.766 & 0.767 & 0.764 \\
\hline$\nu n \rightarrow \mu^{-} n \pi^{+}$ & 0.136 & 0.134 & 0.126 & 0.124 & 0.124 \\
\hline$\nu A \rightarrow \mu^{-} A \pi^{+}$ & 0.073 & 0.066 & 0.068 & 0.068 & 0.072 \\
\hline
\end{tabular}

\subsection{Cross Section Measurements}

The effective $\mathrm{CC} \pi^{+}$analysis includes measurements of the cross section in terms of several variables. Since the incident neutrino flux can be almost completely characterized by its energy spectrum, the cross section has been measured as a function of neutrino energy. The other one dimensional measurements are differential cross sections as a function of various final state variables that describe the muon and pion energies and directions and $Q^{2}$. Since these one dimensional measurements are necessarily averaged over the full neutrino energy spectrum, a corresponding two dimensional measurement of each variable is made in bins of neutrino energy. Finally, the energy and direction are measured together for both the muon and pion in two double differential cross section measurements. The full list of measured cross sections is as follows: 
Table 6.2: The background acceptance and the level of background contamination of the signal sample are given after each successive analysis cut. The additional rows give the distribution of background events according to generated Nuance process (see Section 4.2). Note that Nuance $\mathrm{CC} \pi^{+}$events are not equivalent to effective $\mathrm{CC} \pi^{+}$events as defined in the cross section measurement. The cuts below are defined as follows: veto hits $<6$ and tank hits $>175$ in the first subevent (H1), exactly 3 subevents (S3), veto hits $<6$ and tank hits $<200$ in the second and third subevents (H23), and vertex/wall distance along the muon and pion trajectories $>150 \mathrm{~cm}(\mathrm{WD})$.

\begin{tabular}{|l|c|c|c|c|c|}
\hline & No Cuts & H1 & H1+S3 & H1+S3+H23 & H1+S3+H23+WD \\
\hline \hline Background Acceptance & 1.000 & 0.354 & 0.035 & 0.007 & 0.006 \\
\hline Background Contamination & 0.813 & 0.764 & 0.395 & 0.137 & 0.128 \\
\hline \hline Background Composition & No Cuts & H1 & H1+S3 & H1+S3+H23 & H1+S3+H23+WD \\
\hline \hline CCmulti $\pi$ & 0.017 & 0.020 & 0.076 & 0.295 & 0.309 \\
\hline CCQE & 0.488 & 0.611 & 0.597 & 0.244 & 0.254 \\
\hline $\mathrm{CC} \pi^{+}$ & 0.071 & 0.094 & 0.110 & 0.128 & 0.131 \\
\hline $\mathrm{CCmeson} B$ & 0.008 & 0.007 & 0.025 & 0.089 & 0.090 \\
\hline $\mathrm{CCDIS}$ & 0.010 & 0.007 & 0.027 & 0.106 & 0.080 \\
\hline $\mathrm{CC} \pi^{0}$ & 0.048 & 0.059 & 0.066 & 0.059 & 0.061 \\
\hline $\bar{\nu}$ & 0.005 & 0.006 & 0.006 & 0.009 & 0.010 \\
\hline other & 0.353 & 0.196 & 0.093 & 0.070 & 0.065 \\
\hline
\end{tabular}

- the average cross section in bins of neutrino energy, $\sigma\left(E_{\nu}\right)$

- the differential cross section in $Q^{2}, \partial \sigma / \partial\left(Q^{2}\right)$

- the differential cross section in muon kinetic energy, $\partial \sigma / \partial\left(K E_{\mu}\right)$

- the differential cross section in the cosine of the muon-neutrino angle, $\partial \sigma / \partial\left(\cos \left(\theta_{\mu, \nu}\right)\right)$

- the differential cross section in pion kinetic energy, $\partial \sigma / \partial\left(K E_{\pi}\right)$

- the differential cross section in the cosine of the pion-neutrino angle, $\partial \sigma / \partial\left(\cos \left(\theta_{\pi, \nu}\right)\right)$

- the differential cross section in $Q^{2}$, measured in bins of neutrino energy, $\partial \sigma\left(E_{\nu}\right) / \partial\left(Q^{2}\right)$

- the differential cross section in muon kinetic energy, measured in bins of neutrino energy, $\partial \sigma\left(E_{\nu}\right) / \partial\left(K E_{\mu}\right)$ 

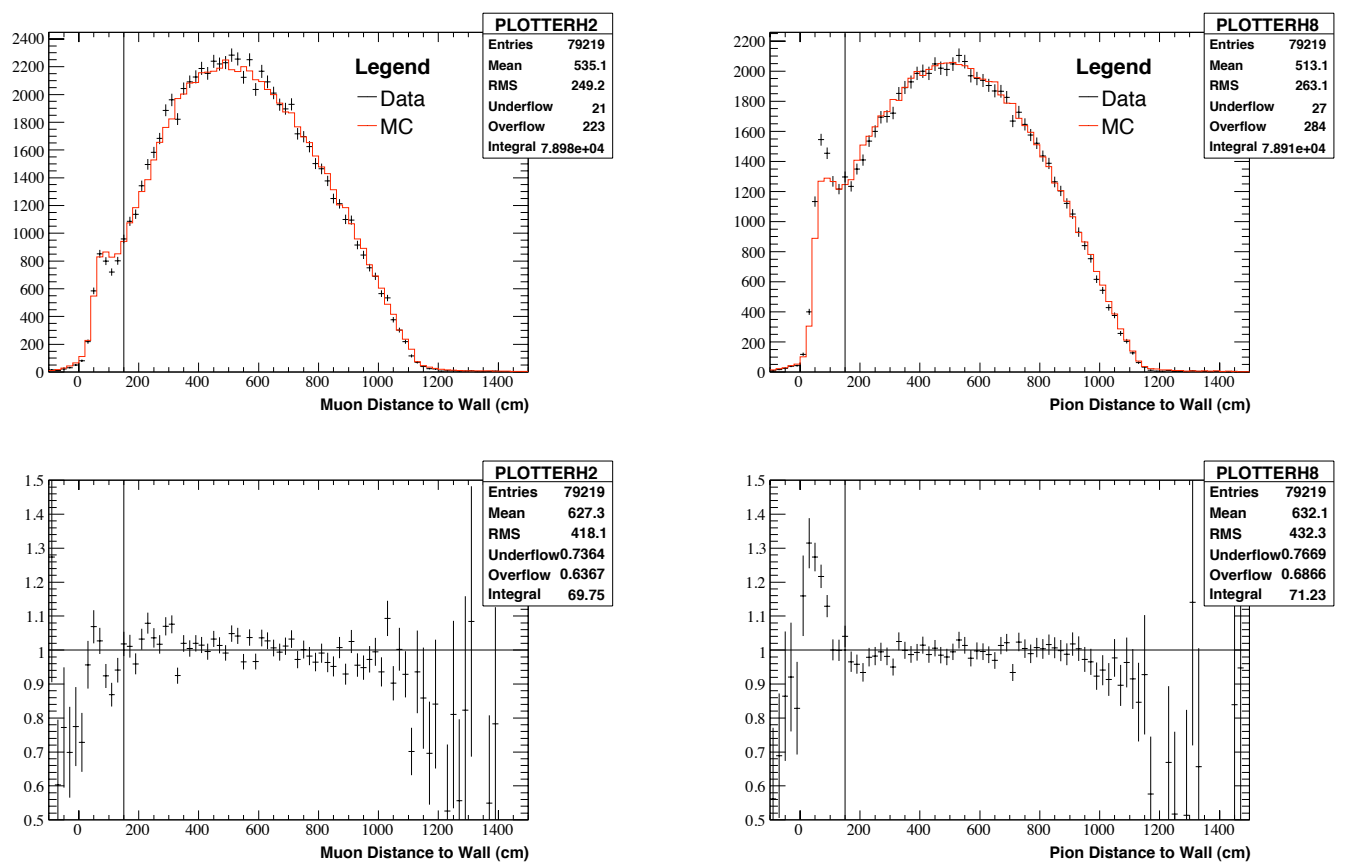

(a) Distance between the tank wall and event vertex along the muon trajectory.

(b) Distance between the tank wall and event vertex along the pion trajectory.

Figure 6.3: The data/Monte Carlo comparisons of the muon and pion trajectory tank wall distances are presented. The bottom panels show the data/Monte Carlo ratio. A cut is placed on both distributions to remove events below $150 \mathrm{~cm}$.

- the differential cross section in the cosine of the muon-neutrino angle, measured in bins of neutrino energy, $\partial \sigma\left(E_{\nu}\right) / \partial\left(\cos \left(\theta_{\mu, \nu}\right)\right)$

- the differential cross section in pion kinetic energy, measured in bins of neutrino energy, $\partial \sigma\left(E_{\nu}\right) / \partial\left(K E_{\pi}\right)$

- the differential cross section in the cosine of the pion-neutrino angle, measured in bins of neutrino energy, $\partial \sigma\left(E_{\nu}\right) / \partial\left(\cos \left(\theta_{\pi, \nu}\right)\right)$

- the double differential cross section in the cosine of the muon-neutrino angle and the muon kinetic energy, $\partial^{2} \sigma / \partial\left(\cos \left(\theta_{\mu, \nu}\right)\right) \partial\left(K E_{\mu}\right)$

- the double differential cross section in the cosine of the pion-neutrino angle and 
the pion kinetic energy, $\partial^{2} \sigma / \partial\left(\cos \left(\theta_{\pi, \nu}\right)\right) \partial\left(K E_{\pi}\right)$

The following discussion will present the measurement technique used for all cross section measurements in terms of the muon kinetic energy measurement. In certain sections, the treatment of the bivariate cross section measurements may require additional discussion, and the $\cos \left(\theta_{\mu, \nu}\right)$ versus $K E_{\mu}$ distributions will be used to illuminate the discussion as required. For the sake of brevity and to clearly describe the analysis procedure, the full details of all measurements are relegated to the appendices.

\subsubsection{Event Rate Corrections}

The expression for the differential cross section in Equation 6.8 is written in terms of the number of interactions in a final state bin, $i$. In practice, the true number of interactions is not directly accessible to experiment. The histogram measured in the data, $D_{i}$, will typically contain backgrounds, as well as inefficiencies due to detector performance and analysis cuts. The signal efficiency, $\epsilon_{i}$, and the background distribution, $B_{i}$, are both estimated from the Monte Carlo simulation. Assuming perfect detector resolution, the number of observed interactions, $N_{i}^{\text {int }}$, can be replaced by these experimental quantities,

$$
\frac{\partial \sigma}{\partial v}\left(v_{i}\right)^{\text {perfect resolution }}=\frac{\left(D_{i}-B_{i}\right) / \epsilon_{i}}{\Delta v_{i} N_{\text {targ }} \Phi}
$$

Of course, the detector does not actually have perfect resolution. The complete expression for the differential cross section requires one additional ingredient: data unfolding.

\subsubsection{Data Unfolding}

Due to biases and imperfect resolution in the event reconstruction, the event distributions measured in the data do not accurately represent the underlying "true" distributions. For example, as shown in Figure 5.16(b), the pion energy reconstructed by the $\mathrm{CC} \pi^{+}$fitter is systematically high at low true energy, and falls below the true 
energy as the energy increases. Since these biases are modeled in the Monte Carlo, it is possible to "unfold" any bin migration effects.

The Monte Carlo bin migration matrix for a given variable, $v$, is constructed by forming a two dimensional histogram of the reconstructed value of $v$ versus the true value, and normalizing each true column to unity as illustrated in Figure 6.4. Each element, $B_{i j}$, in the bin migration matrix represents the probability that an event generated with a value of $v$ in bin $j$ will be reconstructed in bin $i$. By definition,

$$
N_{j}^{i n t}=\sum_{i} B_{j i} T_{i}
$$

where $N_{i}^{i n t}$ is the reconstructed distribution and $T_{j}$ is the true distribution.

The goal of unfolding is to perform the opposite task of Equation 6.13. An operator, $M_{i j}$, must be obtained that transforms the background subtracted data distribution into the inferred true distribution $\left(I_{i}\right)$,

$$
I_{i}=\sum_{j} M_{i j}\left(D_{j}-B_{j}\right)
$$

The most obvious choice for the unfolding matrix is the inverse of the bin migration matrix. Unfortunately, this unfolding technique is highly unstable, because the bin migration matrix is not very well behaved, and is often singular. In addition, large statistical fluctuations in the off-diagonal elements of $B_{i j}$ get incorporated in the diagonal elements of $B_{i j}^{-1}$, which produce large fluctuations in the results [66].

To avoid these issues, the unfolding matrix is formed from the same reconstructed versus true histogram used to construct the bin migration matrix, except that instead of normalizing the true columns of the histogram, each reconstructed row is normalized to unity. The result is a matrix, $M_{i j}$, where each element gives the probability that an event in reconstructed bin $j$ was produced in true bin $i$, which satisfies the condition of Equation 6.14. An example of the unfolding, bin migration, and reconstructed versus true matrices used in the muon kinetic energy measurement are shown together in Figure 6.4. 

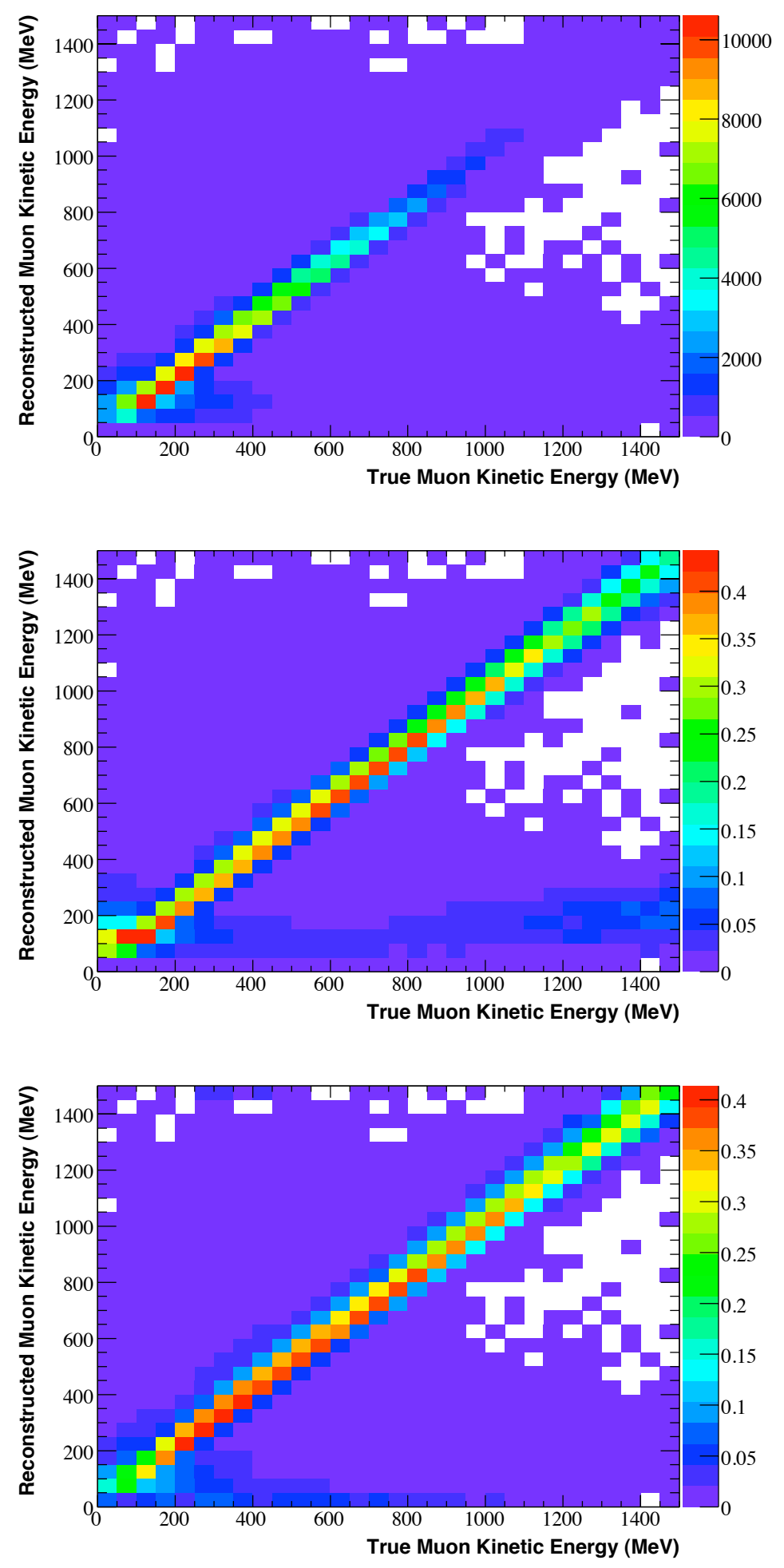

Figure 6.4: The reconstructed versus true (top), bin migration (middle), and unfolding (bottom) matrices are shown for muon kinetic energy. The bin migration matrix is formed by normalizing the true columns of the reconstructed vs true matrix to unity, while the unfolding matrix is formed by normalizing the reconstructed rows. 
Unlike $B_{i j}^{-1}$, which satisfies the unfolding condition of Equation 6.14 by definition (the product $\sum_{j} B_{i j}^{-1} B_{j k}$ gives the identity matrix) the matrix $M_{i j}$ is designed such that the true distribution, $T_{i}$, is an eigenvector of the product $\sum_{j} M_{i j} B_{j k}$ with a corresponding eigenvalue of one,

$$
T_{i}=\sum_{j} \sum_{k} M_{i j} B_{j k} T_{k}
$$

Introducing the unfolding matrix into Equation 6.12 gives the most complete expression for the differential cross section,

$$
\frac{\partial \sigma}{\partial v}\left(v_{i}\right)=\frac{\sum_{j} M_{i j}\left(D_{j}-B_{j}\right)}{\epsilon_{i} \Delta v_{i} N_{\text {targ }} \Phi} .
$$

In order to apply the same unfolding formula in Equation 6.14 to the bivariate measurements, each two-dimensional bin is arbitrarily assigned one universal bin number according to the following prescription,

$$
\operatorname{bin}=\operatorname{bin}_{x}+\operatorname{bin}_{y} \cdot N \operatorname{bins}_{x}
$$

where bin is the universal bin number and Nbins $s_{x}$ is the number of $x$ bins in the measurement. A reconstructed versus true histogram can then be created, and an unfolding matrix can be calculated using the same row normalization procedure. The central value reconstructed versus true histogram is given in Figure 6.5.

\subsubsection{Unfolding Bias}

Although the use of $M_{i j}$ rather than $B_{i j}^{-1}$ avoids the variance issues involved with matrix inversion, it does introduce some bias. In general, unfolding procedures often require the introduction of some amount of bias in order to reduce the statistical variances associated with matrix inversion such that the overall uncertainty is reduced [67]. Since the bin migration matrix, $B_{i j}$, is normalized in columns of the true distribution, $B_{i j}$ and $B_{i j}^{-1}$ are fully independent of the true Monte Carlo distribution, and are therefore unbiased transformations. The $M_{i j}$ matrix is normalized in reconstructed rows, which 


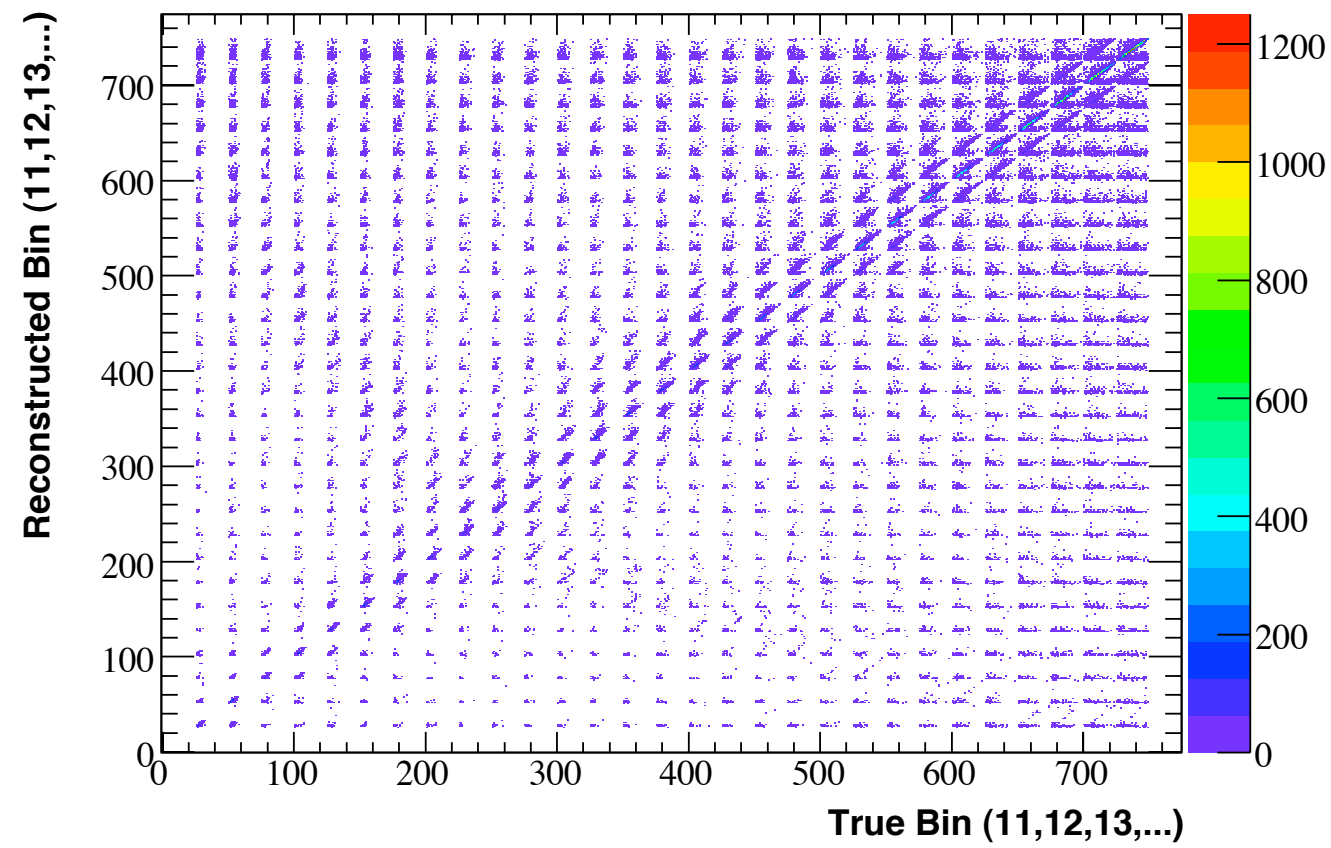

Figure 6.5: The reconstructed versus true distribution for the two-dimensional muon kinetic energy versus cos(muon-neutrino angle) histogram is shown. Each bin of the histogram has been assigned a number between 1 and 775 and plotted in the appropriate $2 \mathrm{D}$ bin of the $775 \times 775$ reconstructed versus true histogram. 
means any change to the shape of the true distribution slice within a reconstructed bin will result in the reconstructed events in that bin being assigned to the true bins in different proportions.

It is important to note that changes to the true Monte Carlo distribution do not necessarily result in an increase in unfolding bias. The normalization of each true slice may be arbitrarily varied, and as long as the shape is unchanged, the unfolding result is also unchanged, despite any changes to the total true distribution. For example, if each Monte Carlo event were reweighted (according to its reconstructed value) by an arbitrary histogram that shares the same binning as the reconstructed histogram, the unfolding result is unchanged.

In this way, the size of the unfolding bias is closely linked with the precision (but not necessarily the accuracy) of the event reconstruction. If events could be reconstructed with perfect resolution, the unfolding bias would be zero by definition, independent of any inaccuracies in the true Monte Carlo distribution. More generally, if the Monte Carlo inaccurately models the true distribution, the unfolding bias will be small as long as that inaccuracy does not change how the event reconstruction places events from true bins into reconstructed bins, even if it places them into the wrong reconstructed bins.

To quantify the size of the unfolding bias, an iterative technique is used. The background subtracted, unfolded data provide an inferred true distribution as described in Section 6.4.2. Each Monte Carlo event is then assigned a weight given by the binned ratio of inferred true data to the true Monte Carlo. Using these weights, a new reconstructed versus true histogram is created from which a new $M_{i j}$ unfolding matrix is formed. The data distribution is unfolded again using the new unfolding matrix and the processes is repeated. The result of the first 9 iterations is given in Figure 6.6. Figure 6.7 shows a two-dimensional iterative example.

Successive iterations of the inferred data distribution have two distinct features. 


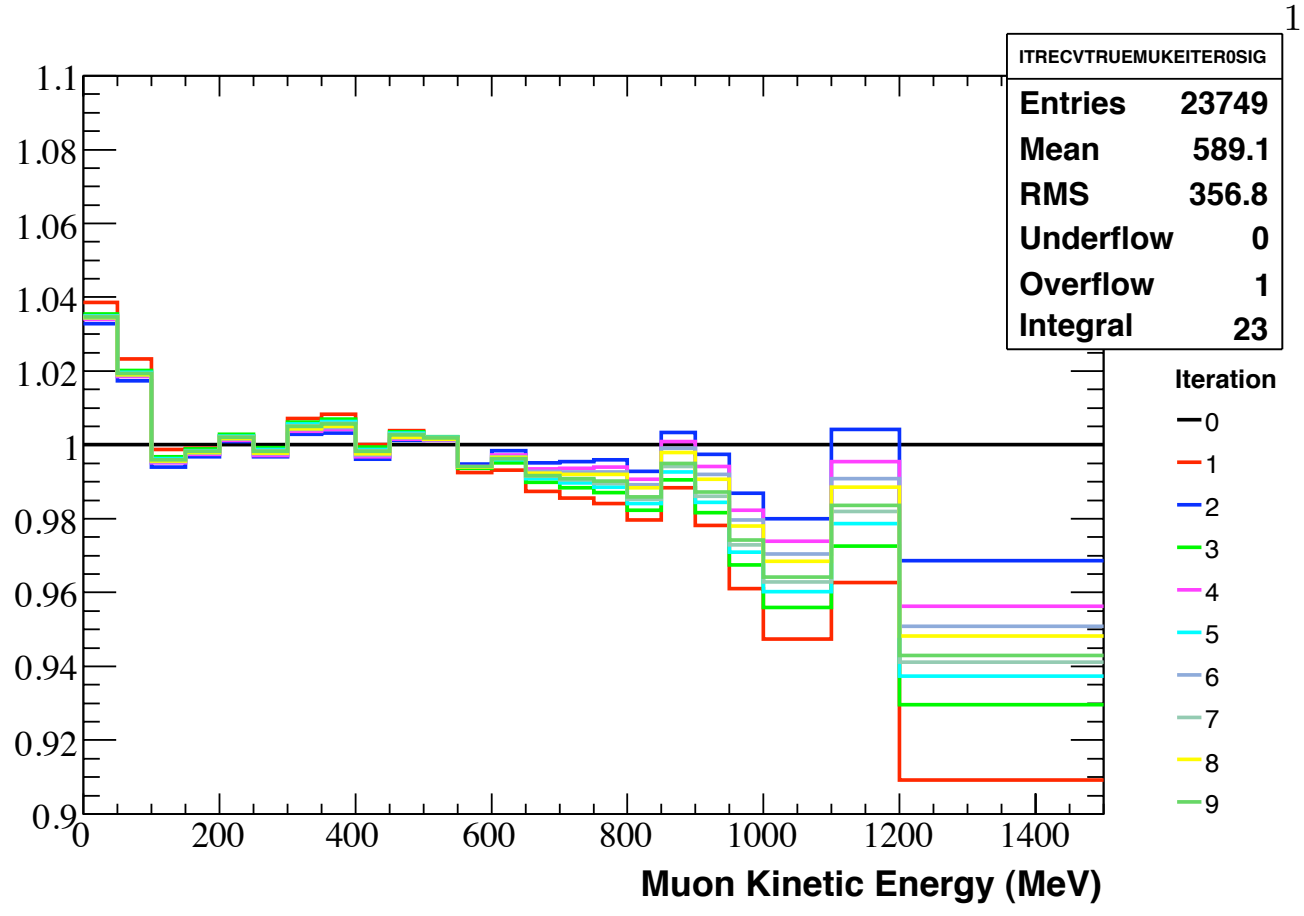

Figure 6.6: The ratios of the iterated inferred true data distributions (i.e. $\left.\sum_{j} M_{i j}^{i t e r, n}\left(D_{j}-B_{j}\right)\right)$ to the uniterated distribution are shown. The number of iterations for the numerator of each ratio are given in the legend. Note the oscillatory behavior of successive iterations, and that the largest excursion from the uniterated distribution occurs in the first iteration.

The first is that the largest excursion relative to the uniterated inferred distribution is in the first iteration. The other is that each successive iteration oscillates about an intermediate preferred value, and the amplitude of those oscillations decreases as the number of iterations increases.

It is tempting to continue iterating the result until the answer converges; however, the correction to the true Monte Carlo distribution that is taking place at each step is a convolution of the desired data informed corrections, and the inadequacies of the Monte Carlo bin migration matrix. Of the infinite possible ways to transform a reconstructed distribution into true distribution, it is unlikely that iterating the unfolding procedure along one particular dimension gives the correct true distribution. Instead, the strategy 


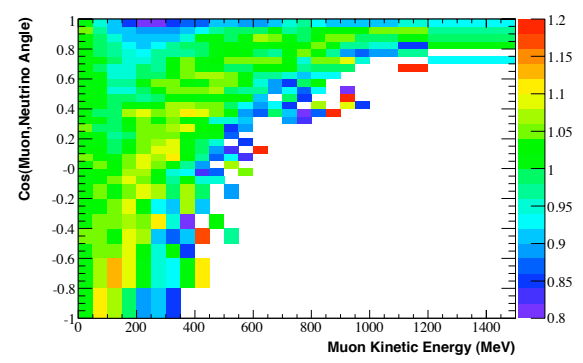

(a) The ratio of the first iteration to the uniterated distribution.

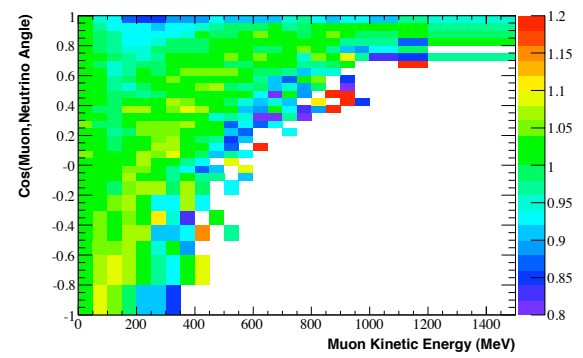

(c) The ratio of the third iteration to the uniterated distribution.

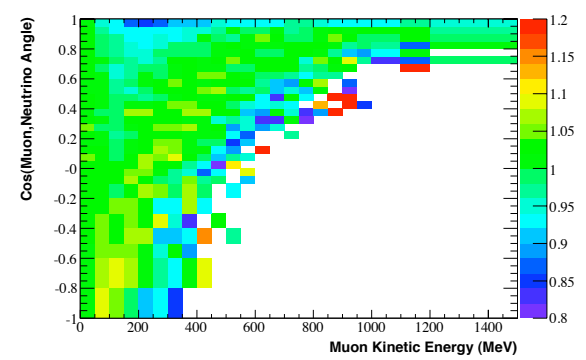

(e) The ratio of the fifth iteration to the uniterated distribution.

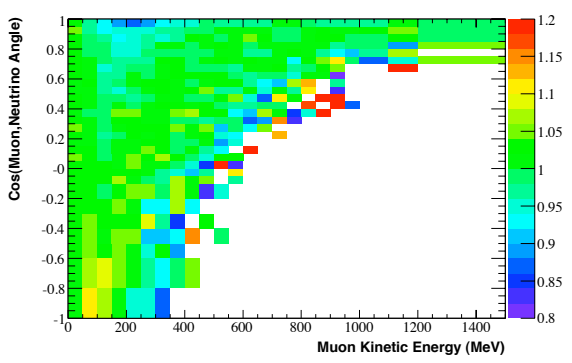

(b) The ratio of the second iteration to the uniterated distribution.

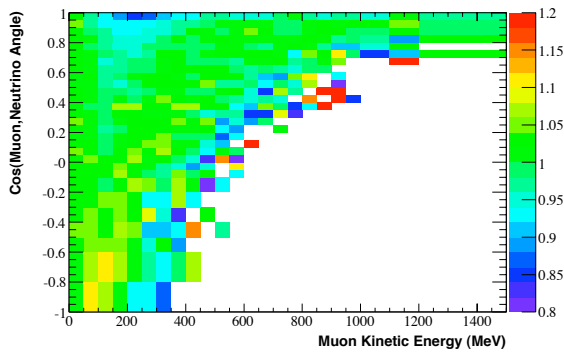

(d) The ratio of the fourth iteration to the uniterated distribution.

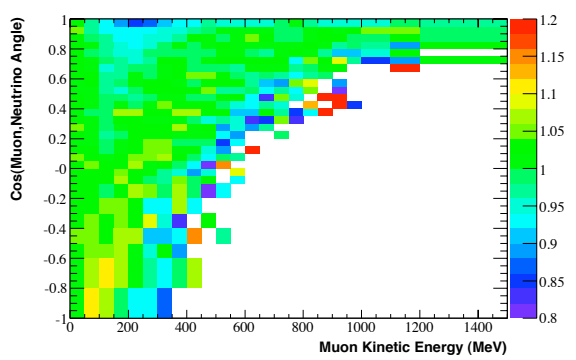

(f) The ratio of the sixth iteration to the uniterated distribution.

Figure 6.7: The ratios of the iterated inferred true data distributions (i.e. $\left.\sum_{j} M_{i j}^{i t e r, n}\left(D_{j}-B_{j}\right)\right)$ to the uniterated distribution are shown for the two-dimensional case. Note the oscillatory behavior of successive iterations, and that the largest excursion from the uniterated distribution occurs in the first iteration.

is to take the most conservative variation in each distribution as the systematic error, and show that it is not the dominant error in almost all regions of phase space. The 
uniterated inferred data distribution is taken to be the central value, and the most conservative estimate of the unfolding bias, the excursion in first iteration, is taken as a two-sided systematic uncertainty.

\subsubsection{Efficiency Correction}

After the data are unfolded, the inferred true data distribution is corrected for events lost due to data selection cuts and detector inefficiency. The numerator of the efficiency correction is the true distribution of all Monte Carlo events that pass the cuts. The denominator is the "generated" Monte Carlo distribution, formed before any cuts are imposed. The ratio of these two distributions gives the fraction of events in a particular bin that survive the analysis cuts,

$$
\epsilon_{i}=\frac{N_{i}^{\text {true after cuts }}}{N_{i}^{\text {generated }}} .
$$

The efficiency should be fairly insensitive to changes in the underlying physical parameters used to produce the generated distribution. If any portion of the generated distribution is incorrectly enhanced, a proportional effect should be seen in the true distribution, and thus cancel in the efficiency.

Monte Carlo events are generated out to a radius of $610.6 \mathrm{~cm}$ to include all neutrino interactions in the main tank, the veto region, and the tank wall. Since the measurement being performed is a neutrino cross section in mineral oil, all other materials must be excluded in forming the generated Monte Carlo distribution. To avoid the PMTs and, in particular, the material voids inside the PMTs, the efficiency denominator is formed from a subset of these events generated within a radius of $550 \mathrm{~cm}$.

Nearly all of the events generated outside of $575 \mathrm{~cm}$ are removed by the veto hits cut; however, there will be a population of events generated between $550 \mathrm{~cm}$ and $575 \mathrm{~cm}$ that pass all cuts, particularly in the upstream portion of the tank. This means that $\epsilon_{i}$ is not technically an efficiency in the strict definition of the term. However, since the 
properties of the incident neutrinos and the oil in the $550-575 \mathrm{~cm}$ shell are the same as the properties within $550 \mathrm{~cm}$, this definition of the efficiency should have no effect on the result. Any extra contributions from events in the $550-575 \mathrm{~cm}$ shell should be present in both the inferred true data and the true Monte Carlo distributions. The signal efficiency in bins of muon kinetic energy is given in Figure 6.8.

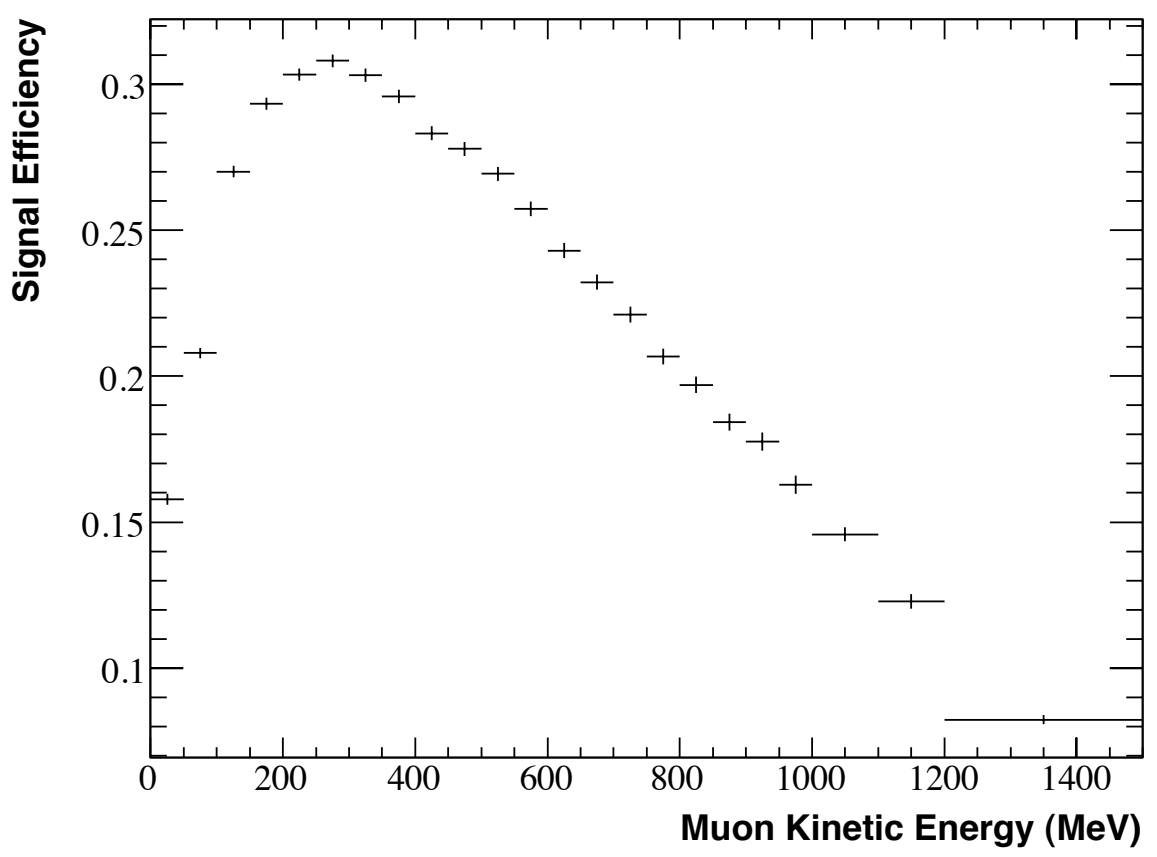

Figure 6.8: The signal efficiency is shown in bins of muon kinetic energy with Monte Carlo statistical errors. Low energy events pass the tank hits cut less frequently, and high energy tracks tend to exit the tank and trigger the veto.

\subsubsection{Interaction Targets}

To measure a cross section, an interaction target must be well defined. Mineral oil is composed of long, single-bonded chains of carbon atoms. In its atomic form, carbon possesses electrons in four of its eight outer shell states. Molecular carbon, therefore, forms four covariant bonds. Within a long chain, carbon atoms form two bonds with neighboring carbon atoms, which leaves two bonding sites available for hydrogen atom 
bonds. The exceptions are carbon atoms located at the end of a chain, which are bonded to three hydrogen atoms. The molecular formula for a mineral oil molecule is, therefore, $\mathrm{C}_{n} \mathrm{H}_{2 n+2}$, where $n$ is the length of the carbon chain. On average, the carbon chains in the mineral oil have a length of $\sim 33$, so the molecular formula can be rewritten as $n\left(\mathrm{CH}_{2.06}\right)$. Since the cross section will only depend on the relative amount of each atomic species, the interaction is chosen to be an "average" single unit on the hydrocarbon chain, $\mathrm{CH}_{2.06}$.

The number of interaction targets in the cross section formula, $N_{\text {targ }}$, must correspond with the definition of the generated volume used in the efficiency calculation. To extract the number of targets from the volume, the only experiment dependent quantity needed is the oil density, which has been measured to be $0.841 \pm 0.001 \mathrm{~g} / \mathrm{cm}^{3}$ [28]. The full expression for the number of interaction targets is

$$
N_{\text {targ }}=\frac{4}{3} \pi R_{\text {gen }}^{3} \cdot \rho_{\text {oil }} \cdot \frac{N_{A}}{W\left(\mathrm{CH}_{2.06}\right)}
$$

where $R_{g e n}$ is the radius of generated events used in the efficiency denominator $(550 \mathrm{~cm})$, $\rho_{\text {oil }}$ is the density of the oil, $N_{A}$ is Avogadro's number (number of targets per mol), and $W\left(\mathrm{CH}_{2.06}\right)$ is the molecular weight of $\mathrm{CH}_{2.06}(\mathrm{~g} / \mathrm{mol})$.

\subsubsection{Flux Factor}

The implementation of the flux factor varies significantly depending on the type of cross section measurement being performed. For the measurements binned in the lone initial state variable, $\sigma\left(E_{\nu}\right)$, the flux factor a binned quantity used normalized each measured bin to the rate per incident neutrino. While the measured cross section is still flux averaged, the averaging only takes place over the width of each bin.

In the differential and double differential cross section measurements, the flux factor is the fully integrated neutrino flux, and the cross section is flux averaged over the entire neutrino energy spectrum. Such a measurement is certainly experiment depen- 
dent in that the particular incident neutrino flux shape is folded into the cross section. However, the fundamental physics of the interaction are fully characterized if the neutrino flux prediction is reported along with the measured cross sections. The reason for providing each of the differential cross sections in bins of neutrino energy was to remove this dependence on the experiment-dependent flux shape. This topic is revisited in Chapter 7.

\subsubsection{Systematic Uncertainties}

All systematic uncertainties are from one of seven possible error sources. Each source is a set of correlated uncertainties from a particular stage of the simulation. The following is a complete list of error sources applicable to effective $\mathrm{CC} \pi^{+}$production, including the sections in which they are described:

- " $\pi^{+}$": Beam $\pi^{+}$production cross section uncertainties in proton-beryllium interactions (Section 4.1.5).

- " $K^{+}$": Beam $K^{+}$production cross section uncertainties in proton-beryllium interactions (Section 4.1.5).

- "Beam": includes each of the following (Section 4.1.5):

* total, inelastic, and quasi-elastic cross section uncertainties for proton and pion interactions with beryllium and aluminum

* horn current uncertainties

* horn current skin depth uncertainties

- "XSec": Nuance cross section model uncertainties (Section 4.2.3).

- "OM": optical model uncertainties (Section 4.4.2).

- "ITER": iteration uncertainty to assess unfolding bias (Section 6.4.3) 
- "DISC": discriminator threshold uncertainty (Section 3.3).

- "QTCORR": a conservatively chosen variation in the correlation between the integrated charged and the recorded hit time in each PMT (Section 3.3).

A handful of other uncorrelated uncertainties have been excluded due to their negligible size, such as the number of protons on target $(\sim 1 \%)$ and the oil density $(<0.1 \%)$.

\subsubsection{Multisims}

The effect of each error source on any particular measured distribution is determined by producing several systematically varied distributions called "multisims." The parameters of each error source are related by an error matrix from which a set of correlated, Gaussian distributed parameter values can be drawn. Each new set of parameters produces a systematically varied version of any reconstructed distribution. The spread in the normalization and shapes of the multisims are used to calculate the total systematic uncertainty as described in the next section.

There are two distinct types of systematic variations. Some systematics, such as the flux and cross section uncertainties, only affect the probability with which an event will occur. For this type of uncertainty, a systematically varied distribution can be produced by reweighting the central value Monte Carlo distribution. Each event is multiplied by the ratio of the event probability calculated with the systematically varied set of parameters to the central value event probability.

The other type of systematic variation affects the measured properties of the event after it is produced, such as the amount of light generated as a function of wavelength and the propagation of the light through the oil. In general, these variations cannot be accomplished via reweighting. Instead, these errors were determined using 67 data-sized Monte Carlo simulations that were generated using parameter draws from the optical model error matrix. A plot of the optical model multisims is shown in Figure 6.9. 


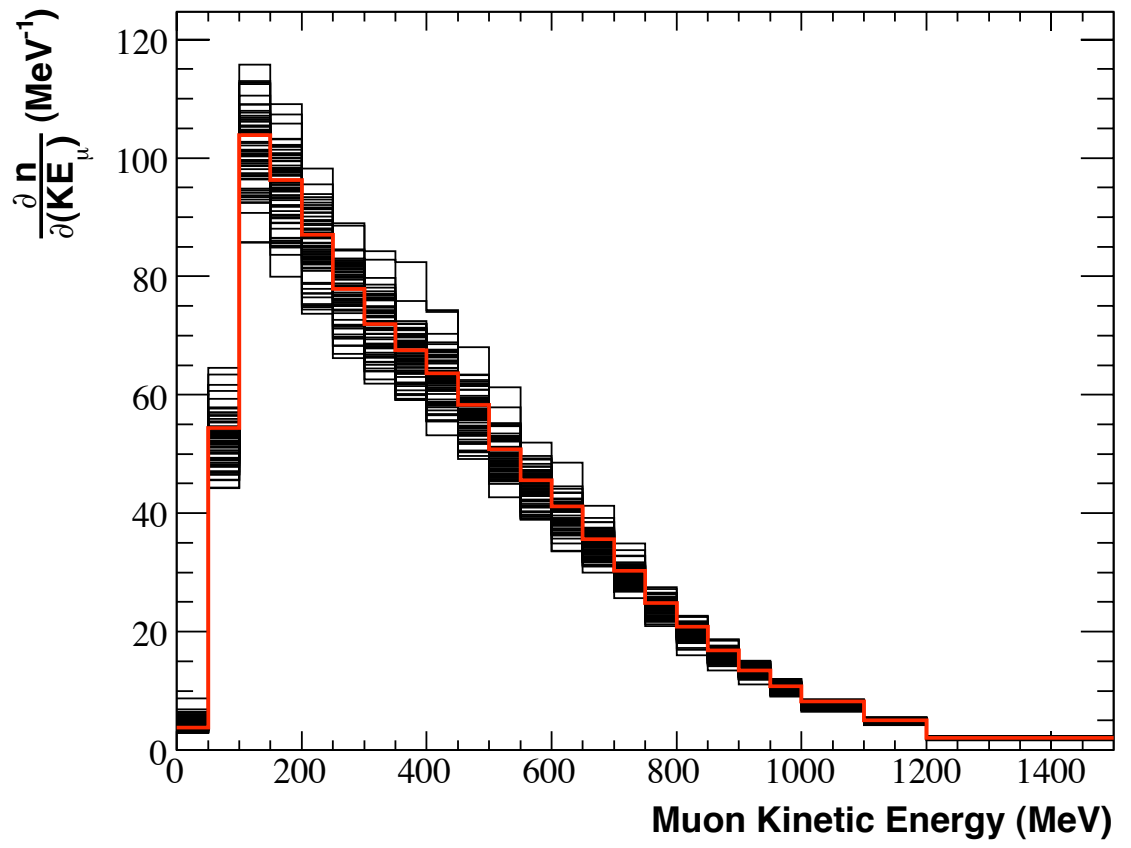

Figure 6.9: The reconstructed muon kinetic energy is plotted in each of the 67 optical model multisims. The central value Monte Carlo distribution (red) is overlayed for comparison.

\subsubsection{Error Matrices}

Just as each systematic error source was described by an error matrix that characterized the correlated uncertainties in the source parameters, the uncertainties in the measured cross sections are described by an error matrix that characterizes the correlated uncertainties in the measured values in each bin. For each error source, an error matrix is calculated from the bin population differences in the multisims compared to the central value,

$$
M_{i j}^{\text {source }}=\frac{1}{N} \sum_{m=1}^{N}\left(n_{m, i}-n_{C V, i}\right)\left(n_{m, j}-n_{C V, j}\right),
$$

where $N$ is the number of multisims, $n_{m, i}$ is the number of events in bin $i$ of multisim $m$, and $n_{C V, i}$ is the number of events in bin $i$ in the central value Monte Carlo simulation. 
Once an error matrix has been calculated for each source, the total error matrix is given by summing each component matrix. For the two-dimensional measurements, the bins are ordered according to Equation 6.17 in order to calculate the full error matrix.

Since the statistical fluctuations in a particular bin are unrelated to the fluctuations in any other bin, statistical error matrices are always diagonal. By design, these uncertainties are built into the optical model error matrix since each optical model multisim was constructed to have the same number of events as the data. Unfortunately, this also has the effect of adding statistical fluctuations to the off-diagonal terms as well. As more optical model multisims are incorporated into the calculation of the error matrix, the size of these spurious fluctuations is decreased. The fluctuations are also smaller for bins with significant event populations. For this reason, cross section results will only be reported for bins with at least 25 unfolded data events. In the onedimensional cross section measurements, the event populations are large enough that this effect is negligible. The reweighting multisims do not suffer from this effect.

To evaluate the systematic uncertainties in the cross section, the full cross section calculation procedure outlined in Equation 6.16 is performed in each multisim. The multisim distributions replace the corresponding central value distributions in the calculation. The reconstructed data distribution remains the same, but multisim distributions are used for the unfolding matrix, the background prediction, and the signal efficiency. The formula for the differential cross section with multisim dependent quantities explicitly identified is

$$
\frac{\partial \sigma^{m}}{\partial v}\left(v_{i}\right)=\frac{\sum_{j} M_{i j}^{m}\left(D_{j}-B_{j}^{m}\right)}{\epsilon_{i}^{m} \Delta v_{i} N_{\text {targ }} \Phi^{m}}
$$

where $m$ is the multisim index.

The results presented throughout the remainder of this document will only list the diagonal errors on each bin. The full error matrices for each distribution are quite large, and in the case of the two-dimensional measurements, they can contain over one million 
elements. The main uses for the full error matrix are hypothesis testing and fitting models. These matrices will be made available to the community via the MiniBooNE website [68].

\subsubsection{Flux Uncertainties}

In addition to producing separate cross section weighted distributions, the flux must also be modified to correspond to the systematically varied parameters in each multisim. Only three of the seven systematic sources affect the flux. The diagonal uncertainties of the flux variations are shown in Figure 6.10.

The largest of the flux errors is the uncertainty in the beam $\pi^{+}$production in proton-beryllium interactions. The portion of the flux relevant to effective $\mathrm{CC} \pi^{+}$interactions occurs at neutrino energies larger than about $400 \mathrm{MeV}$. For the peak neutrino energies in this range $(<1 \mathrm{GeV})$, the beam $\pi^{+}$flux uncertainties are around $8 \%$. At higher neutrino energies, the beam $\pi^{+}$uncertainty grows to as large as $25 \%$. The beam $\pi^{+}$fluctuations also exhibit very distinct features in shape. The residual effect of the wiggling behavior of the spline fits to the HARP data, discussed in Section 4.1.5, are apparent. In particular, the low energy flux exhibits very large systematic excursions since there are no HARP data to constrain the fits in that region.

The other two flux related error sources have a smaller effect on the total uncertainty. The beam unisim uncertainties are generally around the $5 \%$ level below $1 \mathrm{GeV}$ and then expand at higher neutrino energies. The main contributor at high energies is the skin depth excursion, which causes a $\sim 15 \%$ effect between 1.5 and $2.5 \mathrm{GeV}$. The $K^{+}$ uncertainties are mostly irrelevant for this analysis. $K^{+}$mesons become the dominant source of $\nu_{\mu}$ production at $2.3 \mathrm{GeV}$, and the uncertainties become dominant at neutrino energies greater than $2.5 \mathrm{GeV}$, where the flux is very small.

For the measurements not involving neutrino energy, the cross section calculation is only affected by the uncertainty in the integrated flux. The size of these variations 

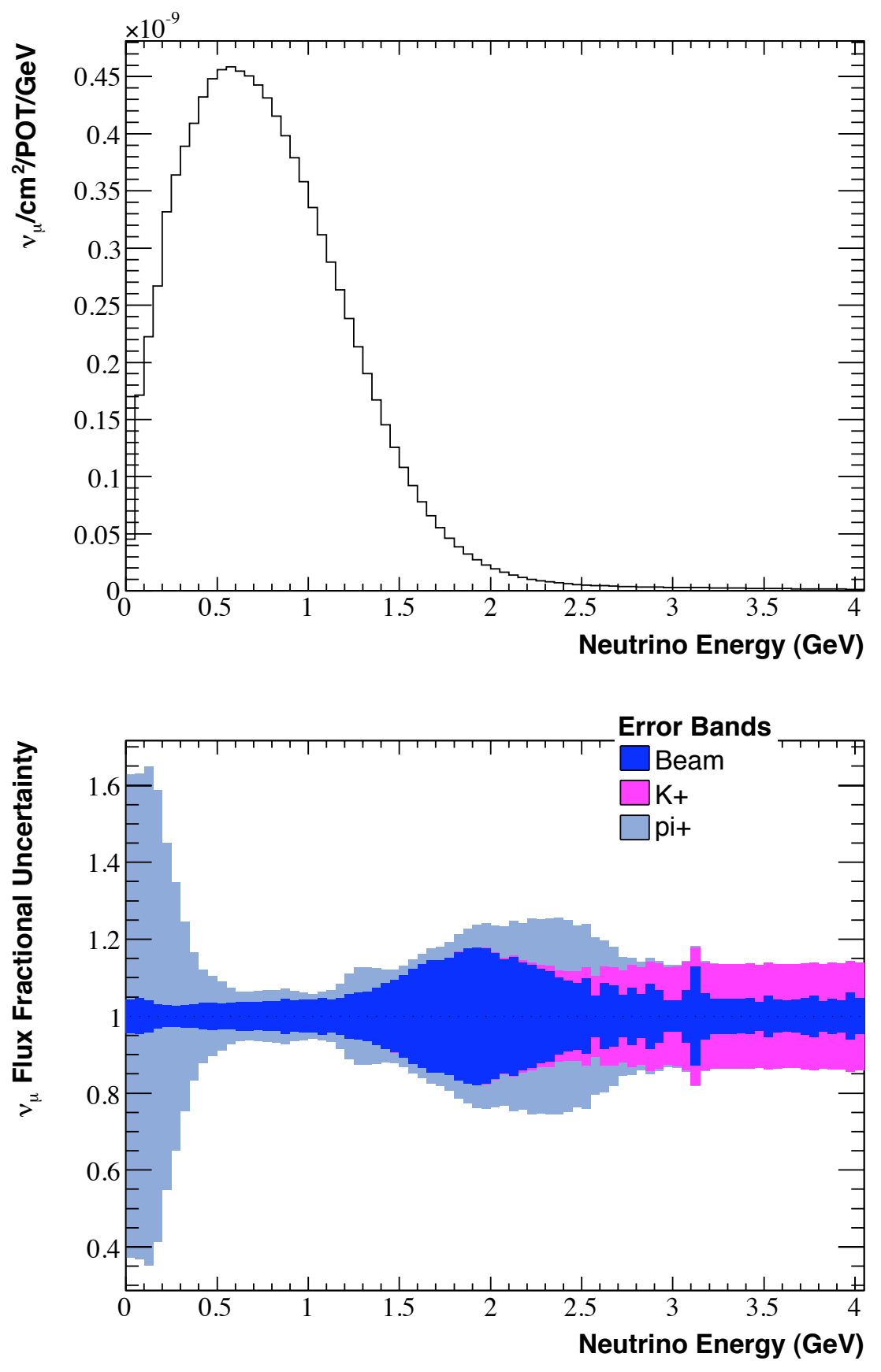

Figure 6.10: The $\nu_{\mu}$ flux is shown (top) along with the fractional diagonal flux errors (bottom).

for each of the flux error sources is given in Table 6.3. 
Table 6.3: The uncertainties in the integrated flux are given for each of the flux error sources.

\begin{tabular}{|c|c|}
\hline Error Source & Integrated Flux Uncertainty \\
\hline$\pi^{+}$ & $10.4 \%$ \\
\hline Beam & $4.1 \%$ \\
\hline$K^{+}$ & $0.4 \%$ \\
\hline
\end{tabular}

\subsubsection{Results}

The results of each of the cross section measurements are presented here.

- $\sigma\left(E_{\nu}\right)$ : Figure 6.11

- $\frac{\partial \sigma}{\partial\left(Q^{2}\right)}$ : Figure 6.12

- $\frac{\partial \sigma}{\partial\left(K E_{\mu}\right)}:$ Figure 6.13

- $\frac{\partial \sigma}{\partial\left(\cos \left(\theta_{\mu, \nu}\right)\right)}:$ Figure 6.14

- $\frac{\partial \sigma}{\partial\left(K E_{\pi}\right)}:$ Figure 6.15

- $\frac{\partial \sigma}{\partial\left(\cos \left(\theta_{\pi, \nu}\right)\right)}$ : Figure 6.16

- $\frac{\partial^{2} \sigma}{\partial\left(K E_{\mu}\right) \partial\left(\cos \left(\theta_{\mu, \nu}\right)\right)}$ : Figure 6.32 (uncertainty components are given in Figures 6.33 and 6.34)

- $\frac{\partial^{2} \sigma}{\partial\left(K E_{\pi}\right) \partial\left(\cos \left(\theta_{\pi, \nu}\right)\right)}$ : Figure 6.35 (uncertainty components are given in Figures 6.36 and 6.37)

- $\frac{\partial \sigma\left(E_{\nu}\right)}{\partial\left(Q^{2}\right)}$ : Figure 6.17 (uncertainty components are given in Figures 6.18 and 6.19)

Additional details for each measurement, including tables of the central values and uncertainties in each bin, are given in the appendices. The uncertainties in the onedimensional measurements are shown in a stacked format such that each successive error band shows the additional contribution of that error source when combined with all 
smaller error bands. The outer-most error band is the total systematic error. The twodimensional histograms are presented with their total uncertainties, and the contribution from each error source is plotted separately in subsequent figures.

The binning for each of the one dimensional distributions has been chosen such that the true Monte Carlo prediction in each bin exceeds 300 events after all cuts. The one dimensional bin sizes are used for the two dimensional measurements as well to retain sufficient precision in the most interesting regions of phase space. This also creates several bins with very small numbers of predicted events. As described in Section 6.4.7.1, the data-sized optical model multisims produce unreliable uncertainties in bins with small event populations. Hence, results will only be reported for bins that contain at least 25 inferred true data events.

In each of the one-dimensional differential cross section measurements, the two largest sources of uncertainty are the $\pi^{+}$production and the cross sections. The $12-15 \%$ $\pi^{+}$uncertainty is dominated by the variation in the integrated flux, which uniformly accounts for $10.4 \%$ of the variation across all bins. The remaining few percent variation is mostly due to changes in the background prediction. Any systematic change in a flux bin results in a proportional change to the observed Monte Carlo distribution, which directly affects the size and shape of the background subtraction. In addition, variations in the background subtraction is almost completely correlated with variations in the flux, since both an increased flux prediction and an increased background prediction result in a lower measured cross section and vice versa. Finally, any bias in the unfolding procedure that is sensitive to the flux shape can also give a small contribution to the overall $\pi^{+}$uncertainty.

The largest effect in the cross section multisims is the variation in pion absorption and charge exchange interactions in the oil. If the pion is absorbed, it will not produce a Michel electron and the event will fail the three subevent requirement; therefore, pion absorption and charge exchange will directly affect the cut efficiencies. As described 
in Section 4.4.2, a $50 \%$ uncertainty is assigned to pion charge exchange and a $35 \%$ uncertainty is assigned to pion absorption, which is the dominant source of cross section error. The remainder of the cross section uncertainty is due to variations in the form factors of each background process.

Since the neutrino energy cross section measurement uses the flux prediction in each measured bin rather than the integrated flux factor, the $\pi^{+}$uncertainty is significantly lower, and generally remains below $10 \%$ except at the highest neutrino energies. The cross section uncertainty is roughly the same size as seen in the differential cross section measurements.

For most of the measured phase space, the unfolding iteration uncertainty is negligible; however, it becomes significant at low $Q^{2}$ in both the one- and two-dimensional measurements. This particular region has two features that generally make unfolding difficult. The first is that the shape is rapidly changing, which strongly affects bin migration. Also, this is a region where the data and Monte Carlo shapes significantly disagree, which increases the probability that the shape of the true slices in each reconstructed bin are incorrect. Despite these features, the unfolding uncertainty is still not the dominant systematic effect in most bins, and is of comparable size in the few bins susceptible to these effects. 

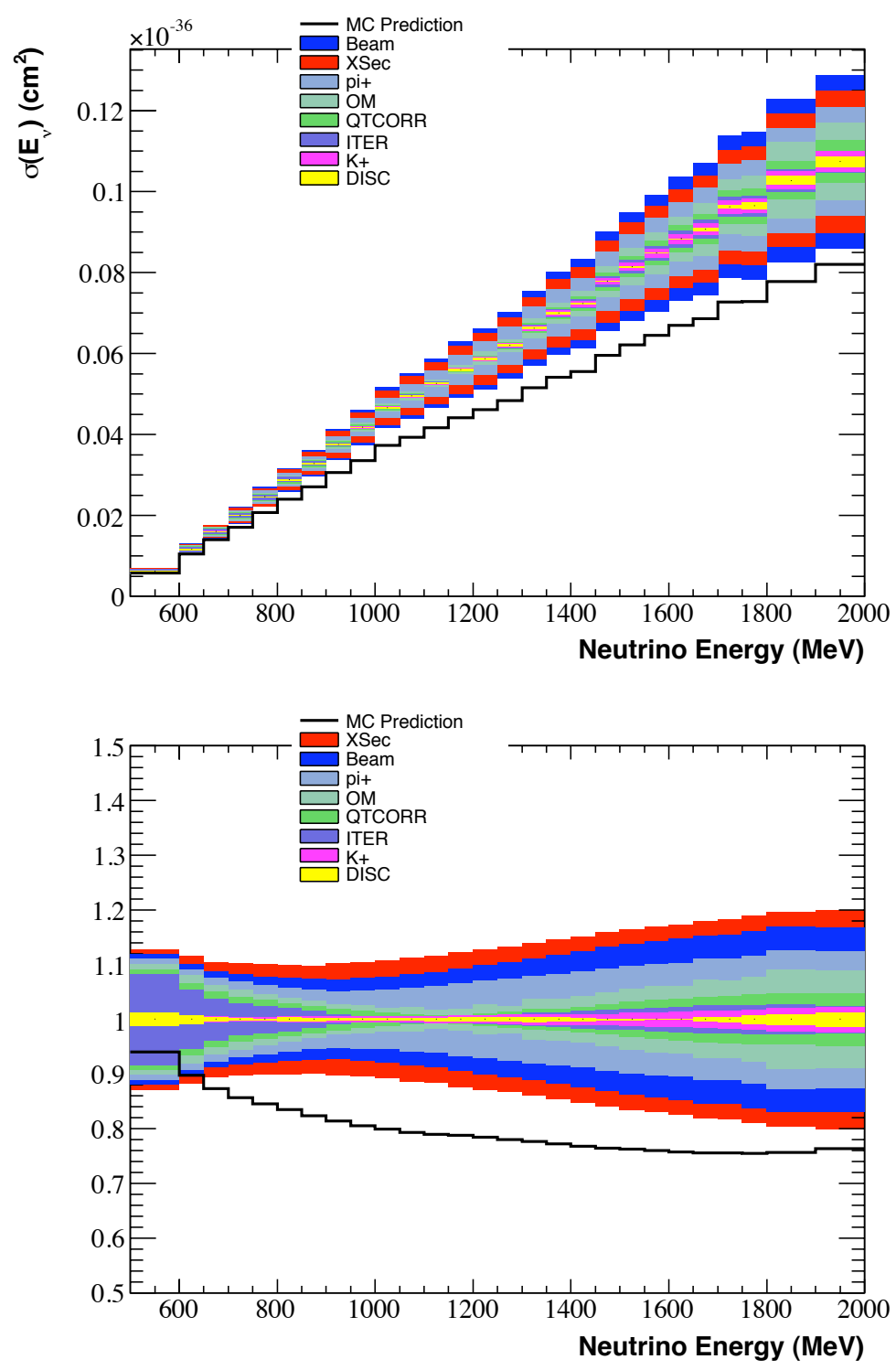

Figure 6.11: The $\sigma\left(E_{\nu}\right)$ measurement is shown with cumulative systematic errors. The absolutely normalized Monte Carlo prediction is shown for comparison. The bottom plot shows the fractional uncertainties and the ratio to the Monte Carlo prediction. 

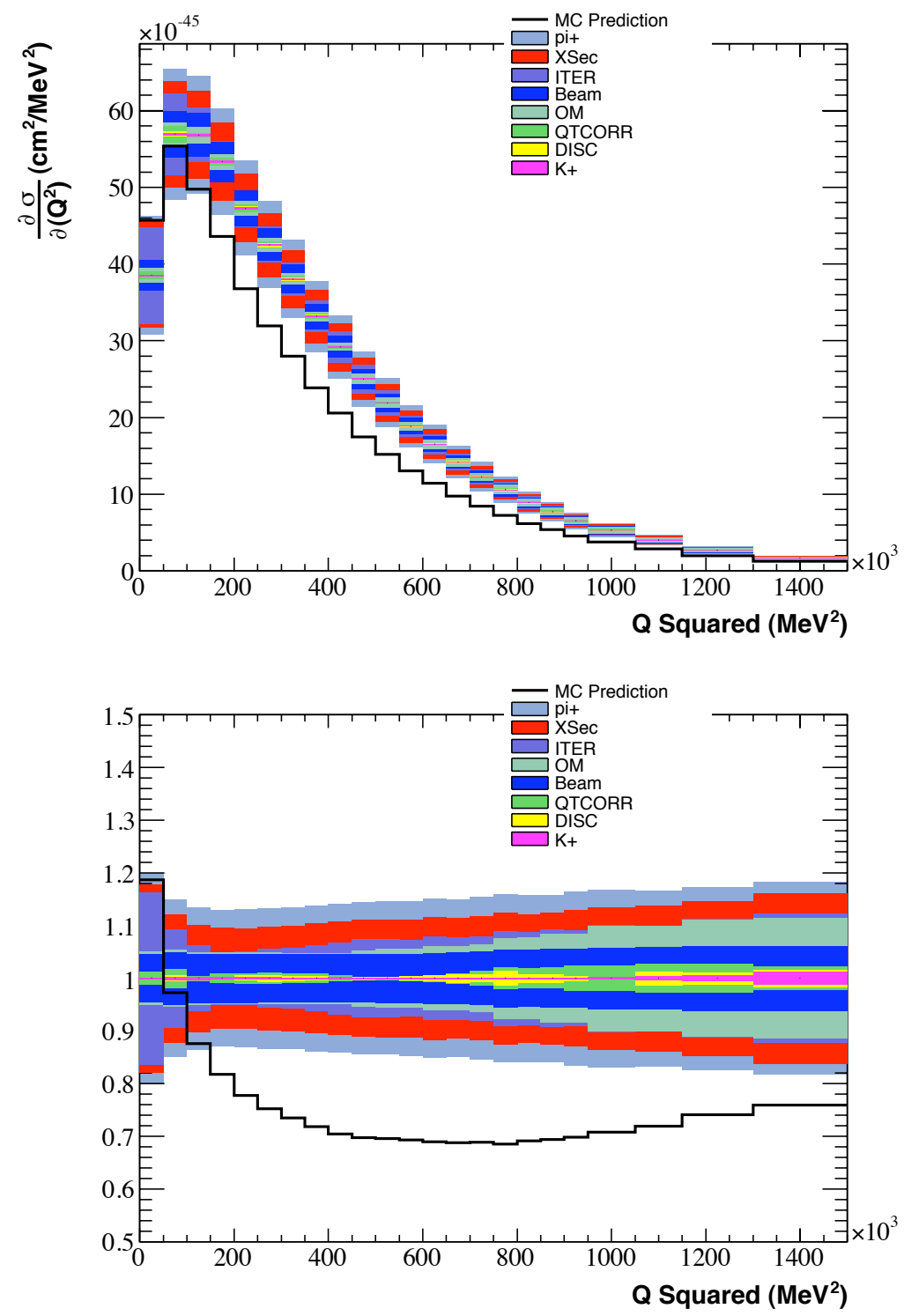

Figure 6.12: The $\partial \sigma / \partial\left(Q^{2}\right)$ measurement is shown with cumulative systematic errors. The absolutely normalized Monte Carlo prediction is shown for comparison. The bottom plot shows the fractional uncertainties and the ratio to the Monte Carlo prediction. 

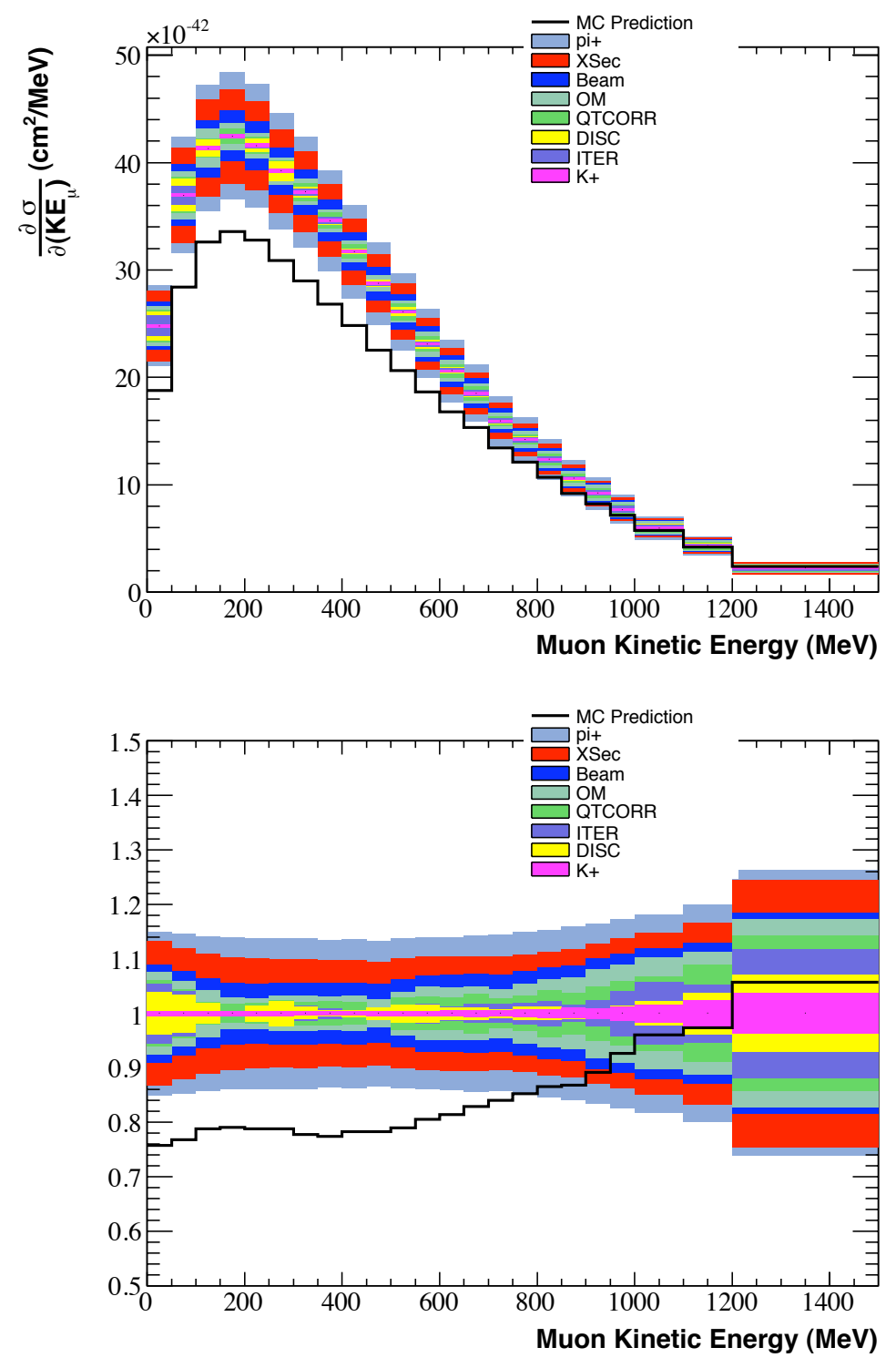

Figure 6.13: The $\partial \sigma / \partial\left(K E_{\mu}\right)$ measurement is shown with cumulative systematic errors. The absolutely normalized Monte Carlo prediction is shown for comparison. The bottom plot shows the fractional uncertainties and the ratio to the Monte Carlo prediction. 

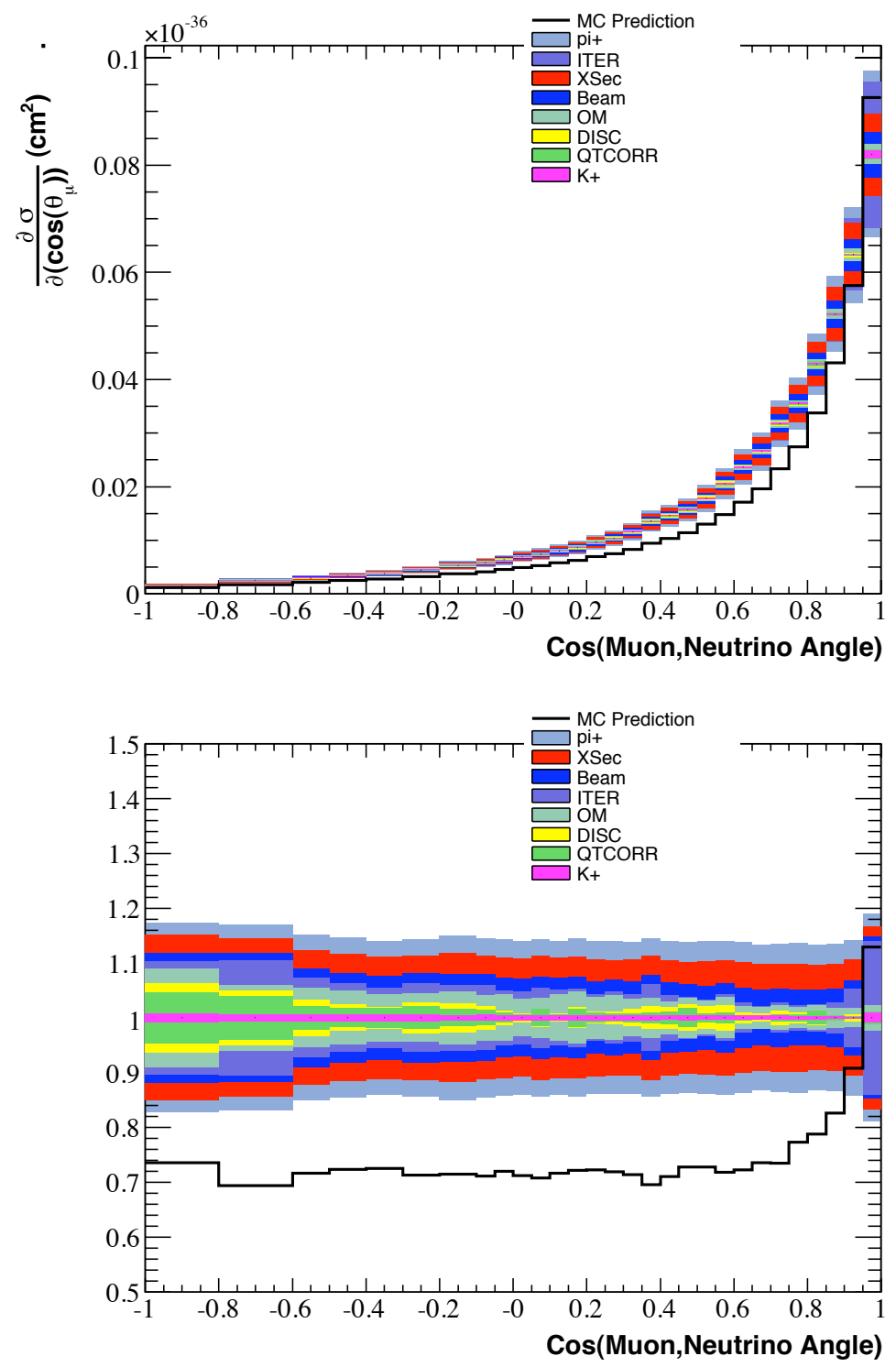

Figure 6.14: The $\partial \sigma / \partial \cos \left(\theta_{\mu, \nu}\right)$ measurement is shown with cumulative systematic errors. The absolutely normalized Monte Carlo prediction is shown for comparison. The bottom plot shows the fractional uncertainties and the ratio to the Monte Carlo prediction. 

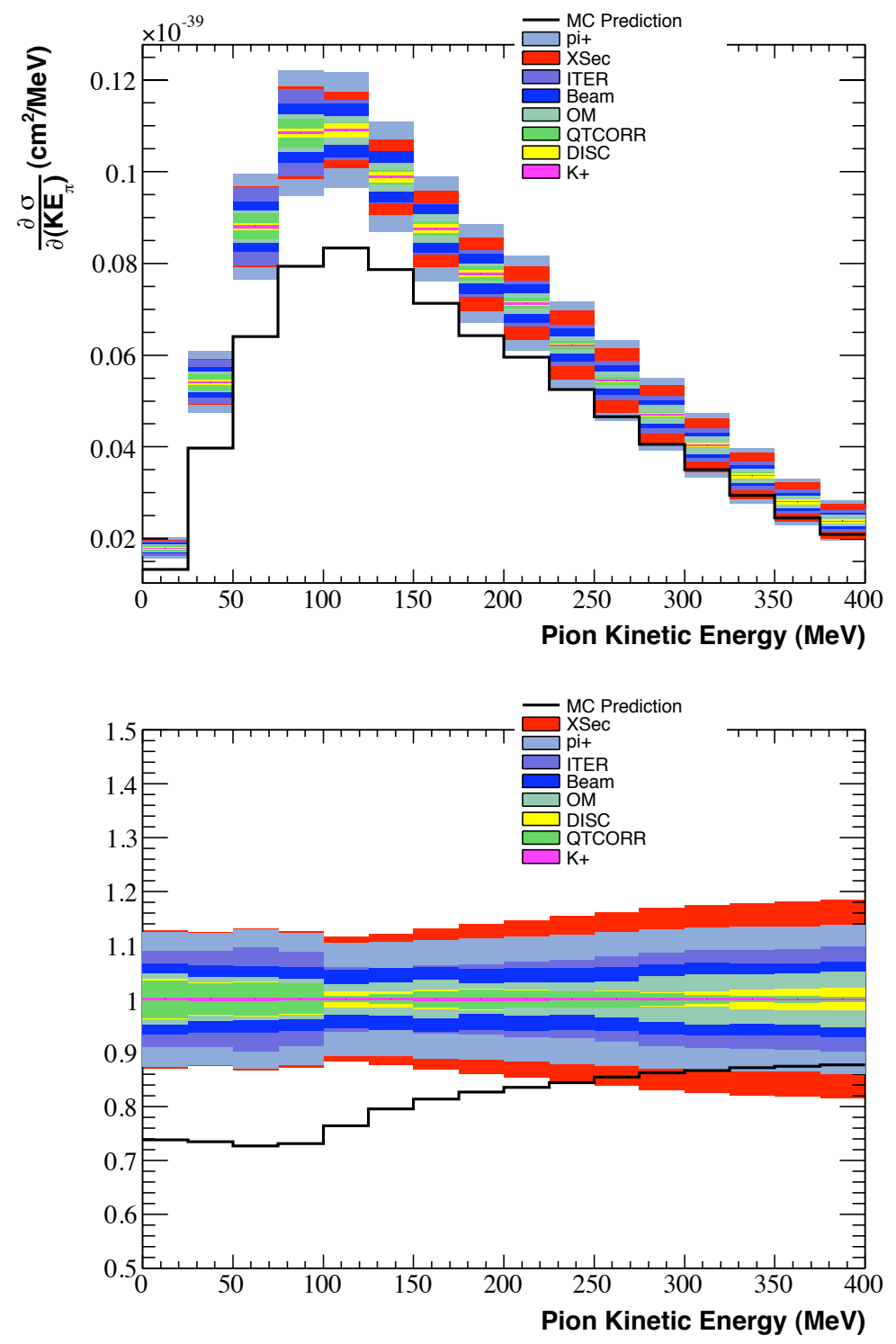

Figure 6.15: The $\partial \sigma / \partial\left(K E_{\pi}\right)$ measurement is shown with cumulative systematic errors. The absolutely normalized Monte Carlo prediction is shown for comparison. The bottom plot shows the fractional uncertainties and the ratio to the Monte Carlo prediction. 

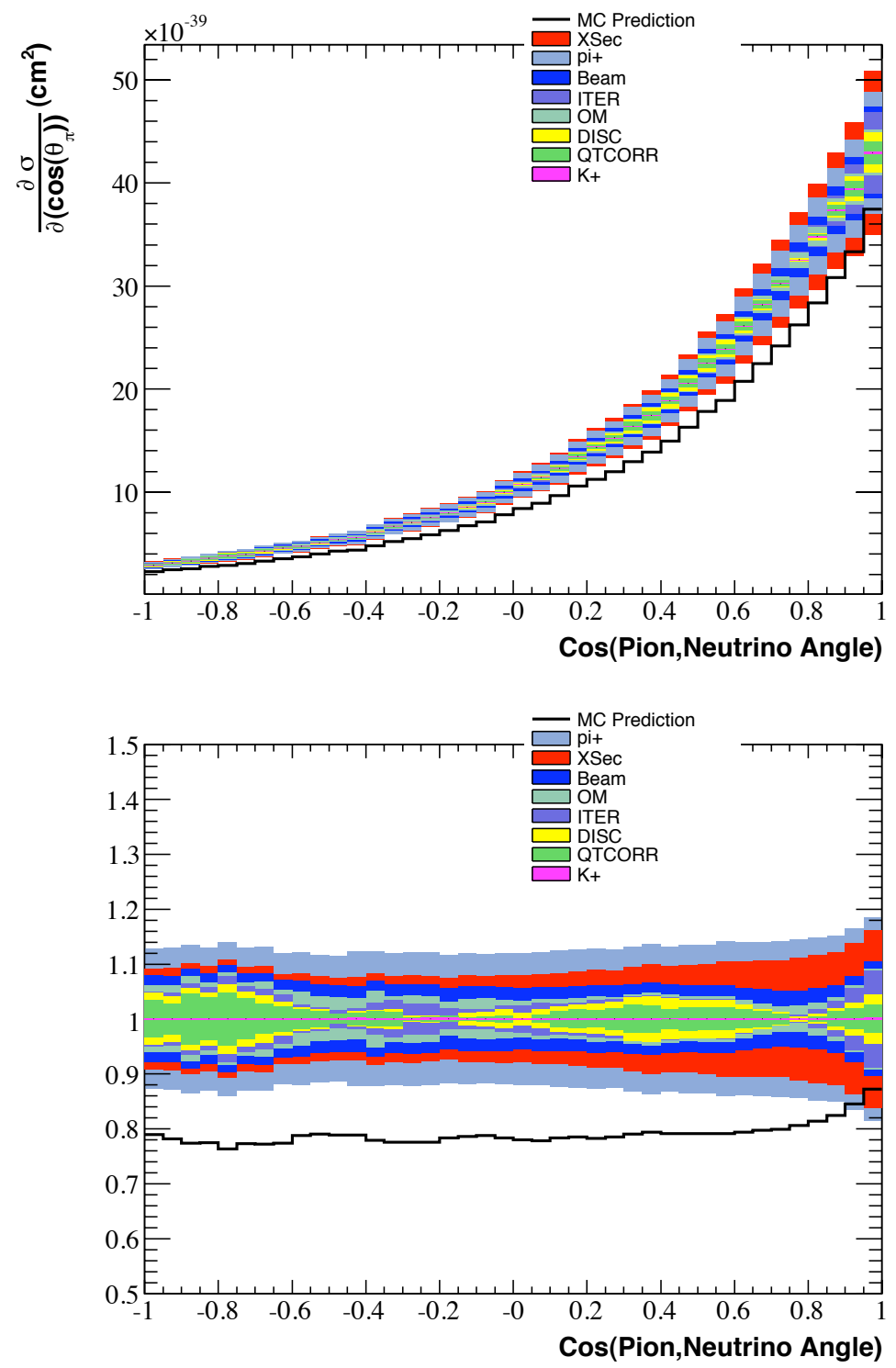

Figure 6.16: The $\partial \sigma / \partial \cos \left(\theta_{\pi, \nu}\right)$ measurement is shown with cumulative systematic errors. The absolutely normalized Monte Carlo prediction is shown for comparison. The bottom plot shows the fractional uncertainties and the ratio to the Monte Carlo prediction. 


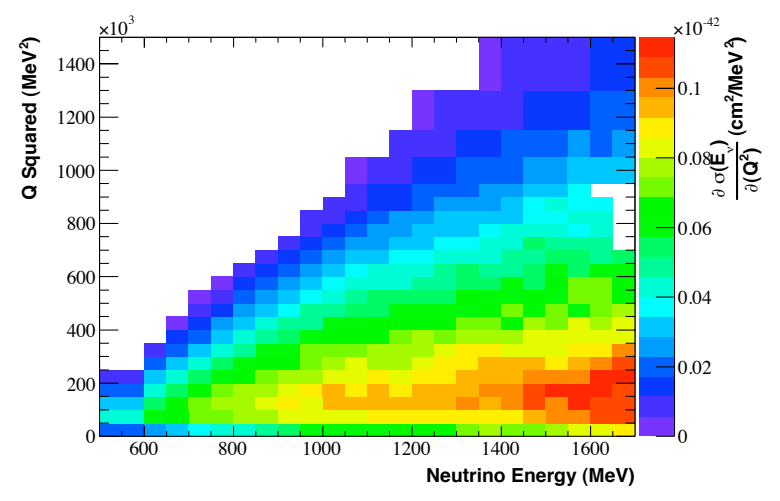

(a) Measured cross section

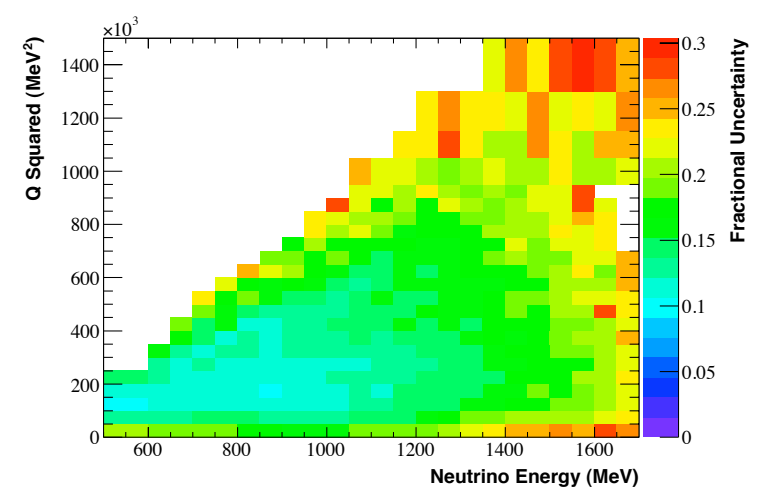

(b) Total fractional error on the measured cross section

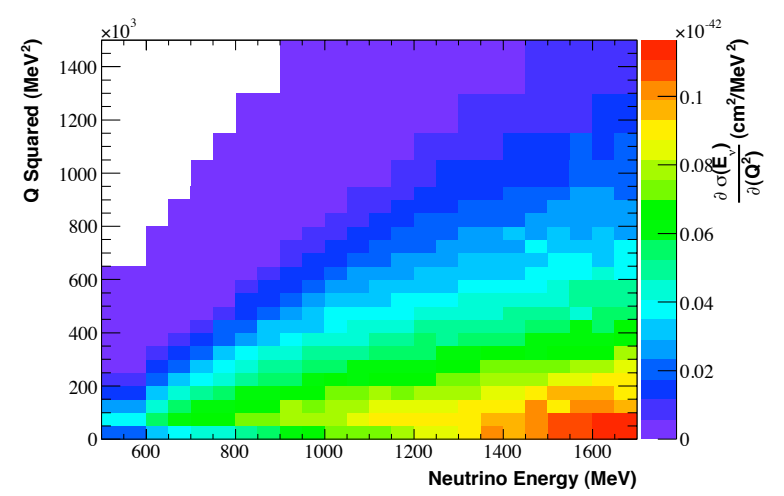

(c) Monte Carlo predicted cross section

Figure 6.17: The measured $\partial \sigma\left(E_{\nu}\right) / \partial\left(Q^{2}\right)$ values are shown along with the total fractional uncertainties. The Monte Carlo predicted cross section is shown for comparison. 


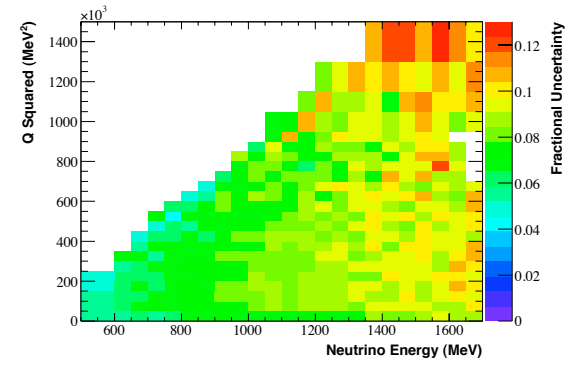

(a) The fractional systematic uncertainty from the "XSec" error source.

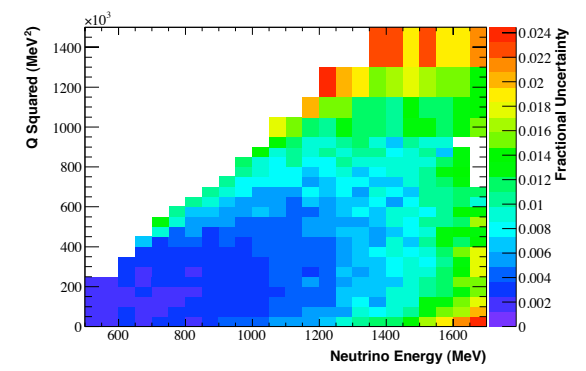

(c) The fractional systematic uncertainty from the " $K^{+}$" error source.

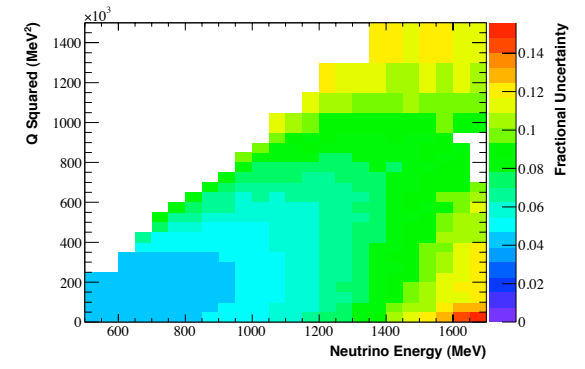

(b) The fractional systematic uncertainty from the "Beam" error source.

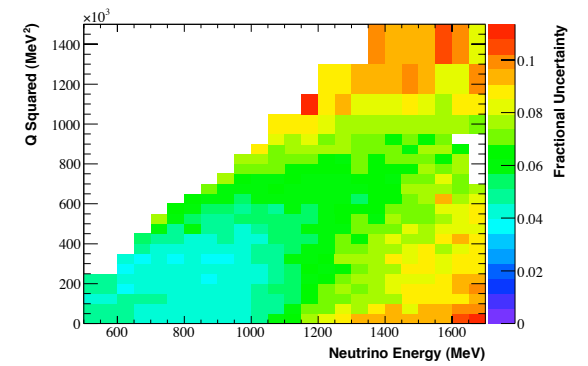

(d) The fractional systematic uncertainty from the " $\pi^{+}$" error source.

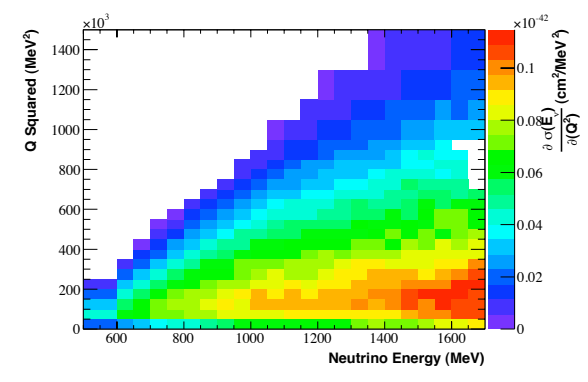

(e) Central value $\partial \sigma\left(E_{\nu}\right) / \partial\left(Q^{2}\right)$ measurement.

Figure 6.18: The fractional systematic uncertainties on the measured $\partial \sigma\left(E_{\nu}\right) / \partial\left(Q^{2}\right)$ values are shown for four of the error sources. The central value measurement is shown for reference. Note that the color scales differ for each systematic error source. An explanation of each error is given in Section 6.4.7. 


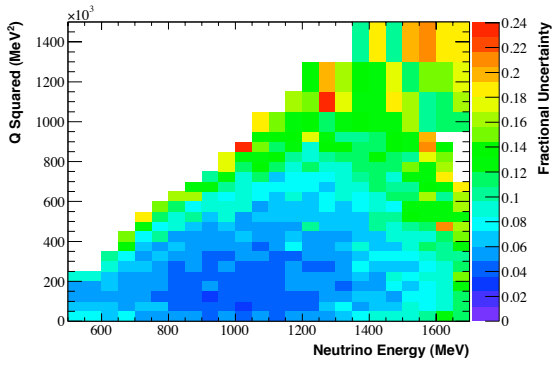

(a) The fractional systematic uncertainty from the "OM" error source.

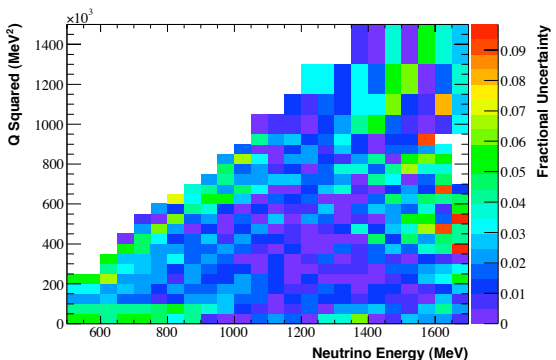

(c) The fractional systematic uncertainty from the "QTCORR" error source.

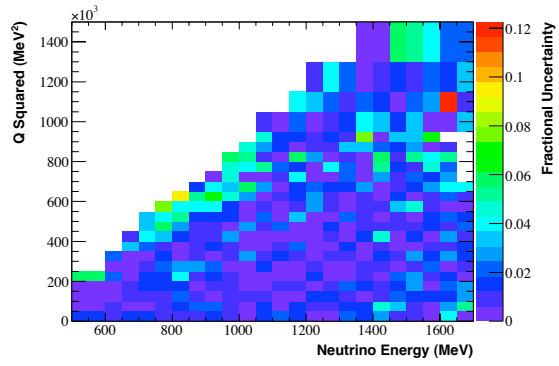

(b) The fractional systematic uncertainty from the "DISC" error source.

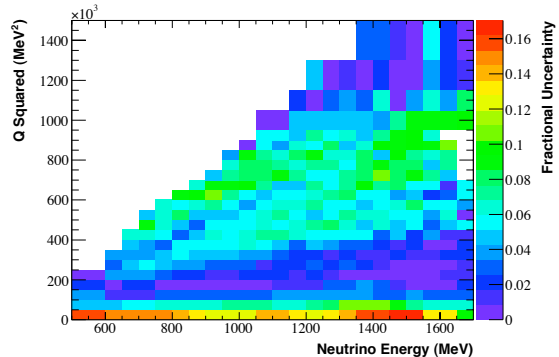

(d) The fractional systematic uncertainty from the "ITER" error source.

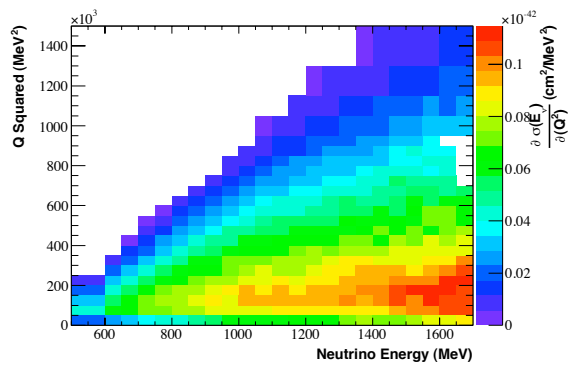

(e) Central value $\partial \sigma\left(E_{\nu}\right) / \partial\left(Q^{2}\right)$ measurement.

Figure 6.19: The fractional systematic uncertainties on the measured $\partial \sigma\left(E_{\nu}\right) / \partial\left(Q^{2}\right)$ values are shown for four of the error sources. The central value measurement is shown for reference. Note that the color scales differ for each systematic error source. An explanation of each error is given in Section 6.4.7. 


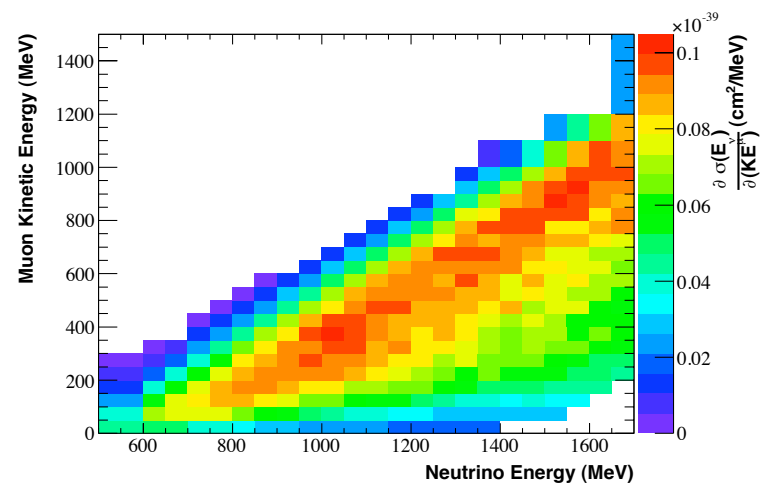

(a) Measured cross section

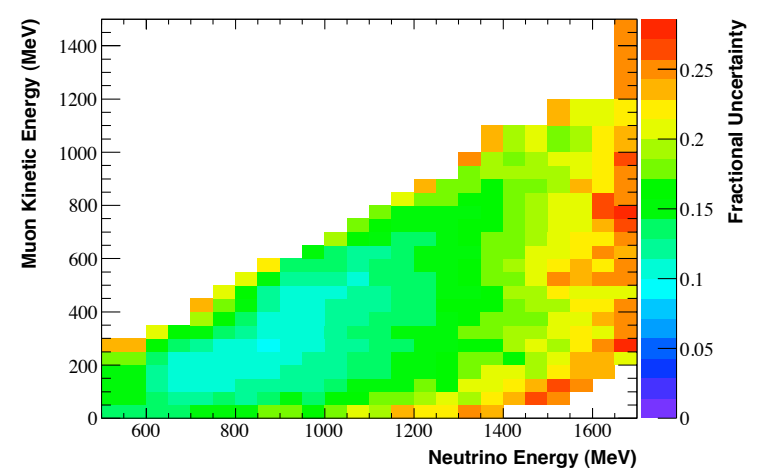

(b) Total fractional error on the measured cross section

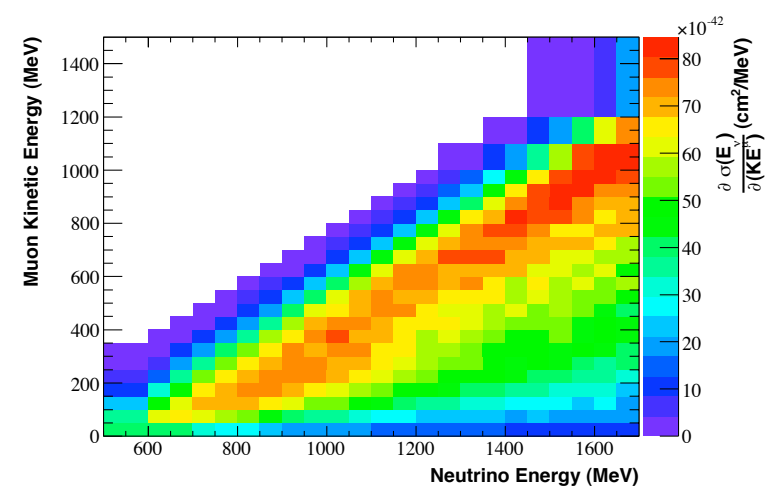

(c) Monte Carlo predicted cross section

Figure 6.20: The measured $\partial \sigma\left(E_{\nu}\right) / \partial\left(K E_{\mu}\right)$ values are shown along with the total fractional uncertainties. The Monte Carlo predicted cross section is shown for comparison. 


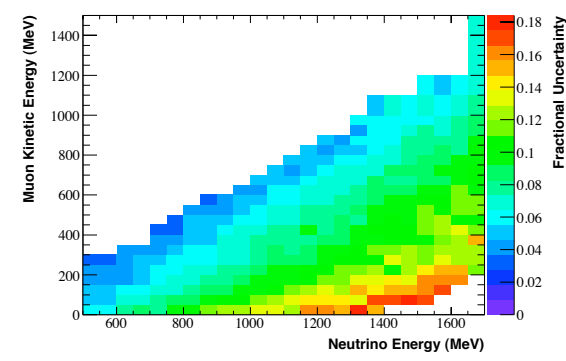

(a) The fractional systematic uncertainty from the "XSec" error source.

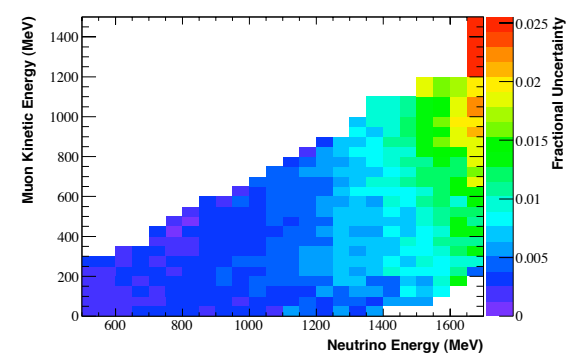

(c) The fractional systematic uncertainty from the " $K^{+}$" error source.

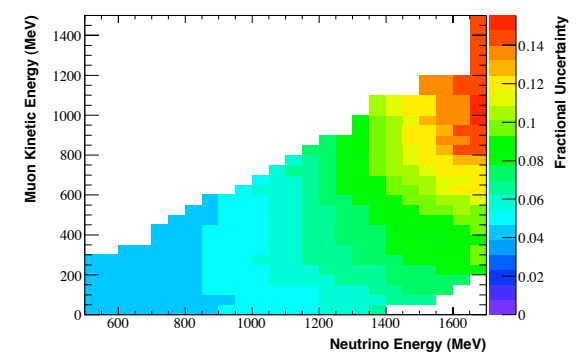

(b) The fractional systematic uncertainty from the "Beam" error source.

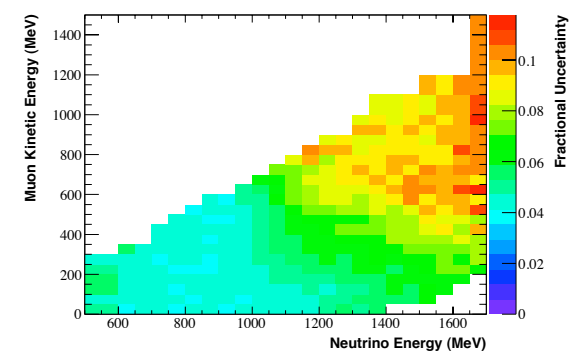

(d) The fractional systematic uncertainty from the " $\pi^{+}$" error source.

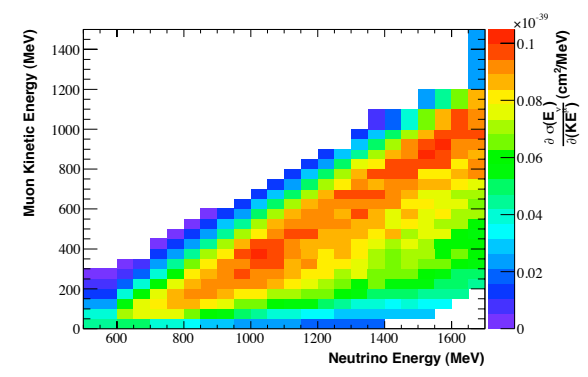

(e) Central value $\partial \sigma\left(E_{\nu}\right) / \partial\left(K E_{\mu}\right)$ measurement.

Figure 6.21: The fractional systematic uncertainties on the measured $\partial \sigma\left(E_{\nu}\right) / \partial\left(K E_{\mu}\right)$ values are shown for four of the error sources. The central value measurement is shown for reference. Note that the color scales differ for each systematic error source. An explanation of each error is given in Section 6.4.7. 


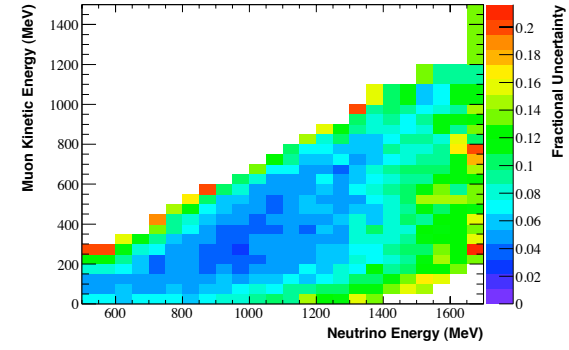

(a) The fractional systematic uncertainty from the "OM" error source.

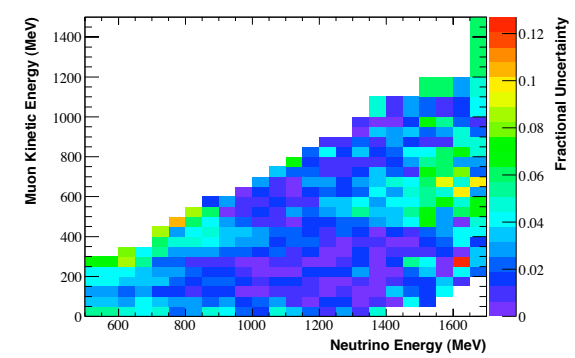

(c) The fractional systematic uncertainty from the "QTCORR" error source.

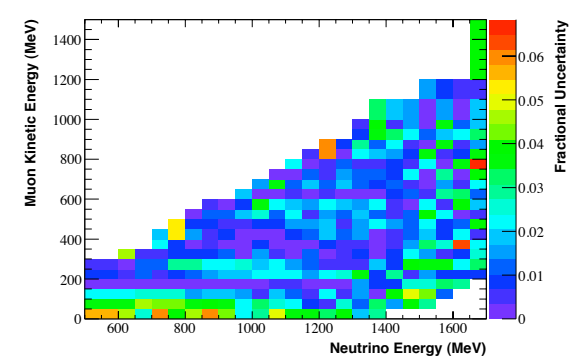

(b) The fractional systematic uncertainty from the "DISC" error source.

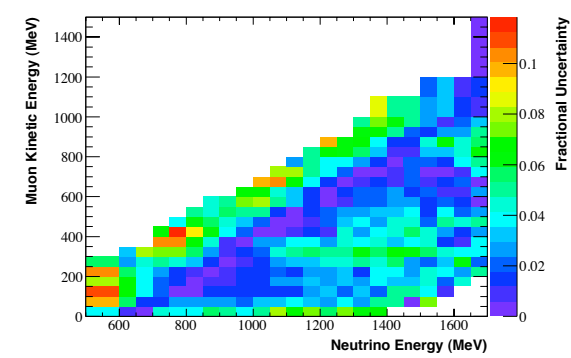

(d) The fractional systematic uncertainty from the "ITER" error source.

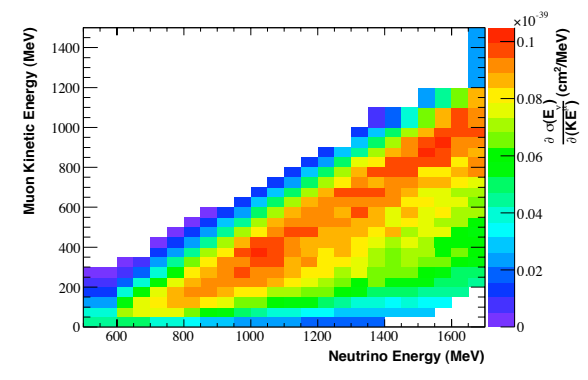

(e) Central value $\partial \sigma\left(E_{\nu}\right) / \partial\left(K E_{\mu}\right)$ measurement.

Figure 6.22: The fractional systematic uncertainties on the measured $\partial \sigma\left(E_{\nu}\right) / \partial\left(K E_{\mu}\right)$ values are shown for four of the error sources. The central value measurement is shown for reference. Note that the color scales differ for each systematic error source. An explanation of each error is given in Section 6.4.7. 


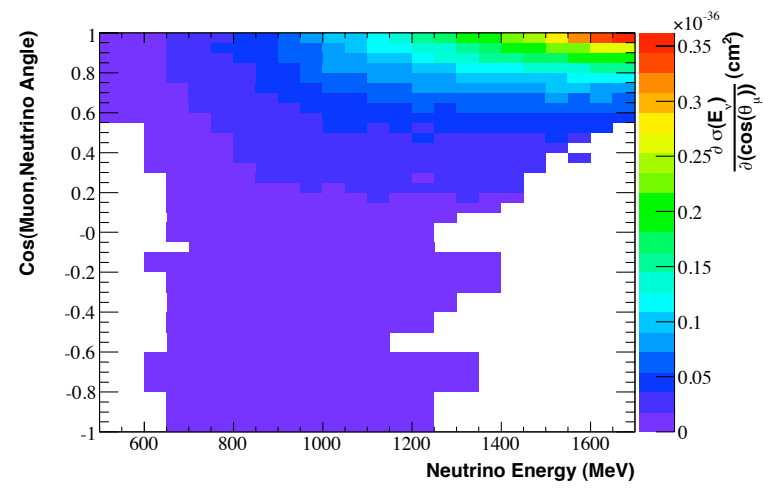

(a) Measured cross section

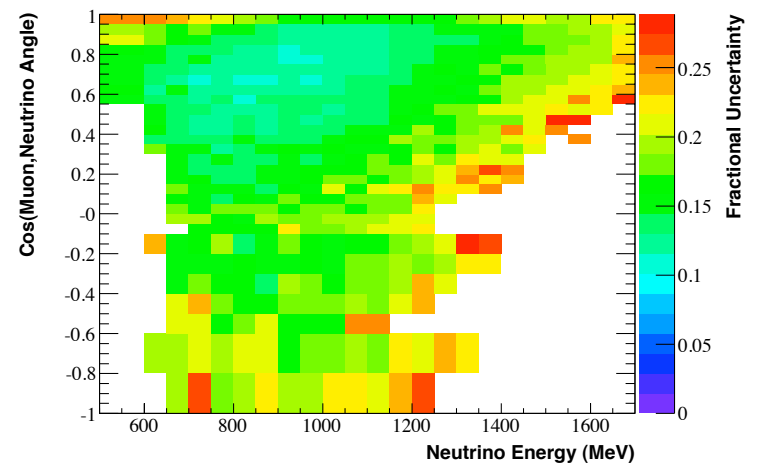

(b) Total fractional error on the measured cross section

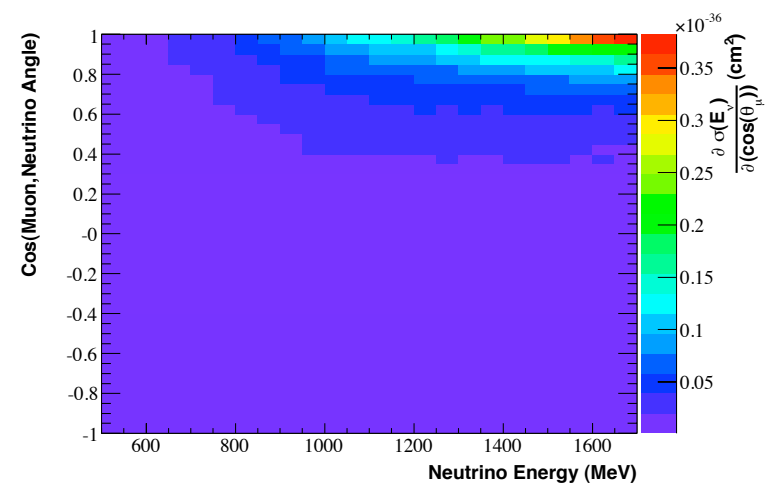

(c) Monte Carlo predicted cross section

Figure 6.23: The measured $\partial \sigma\left(E_{\nu}\right) / \partial\left(\cos \left(\theta_{\mu, \nu}\right)\right)$ values are shown along with the total fractional uncertainties. The Monte Carlo predicted cross section is shown for comparison. 


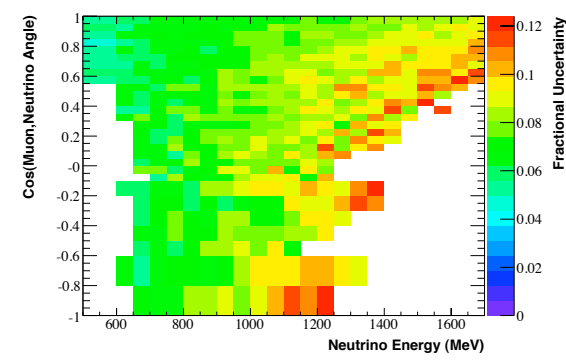

(a) The fractional systematic uncertainty from the "XSec" error source.

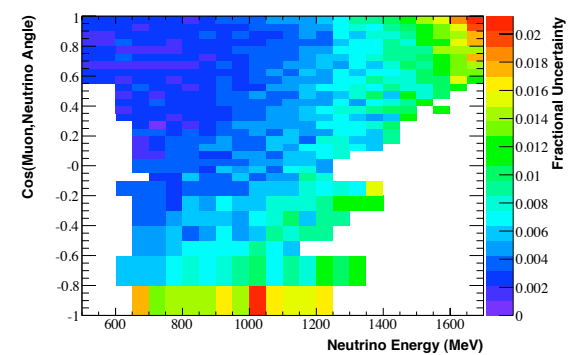

(c) The fractional systematic uncertainty from the " $K^{+}$" error source.

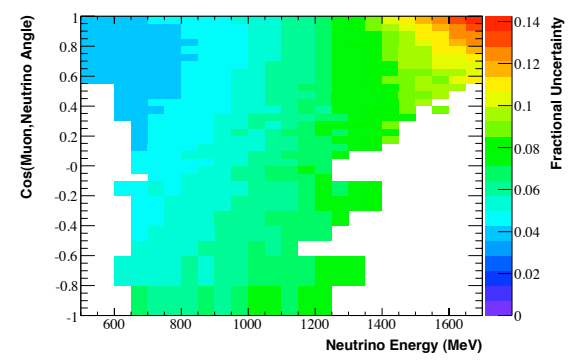

(b) The fractional systematic uncertainty from the "Beam" error source.

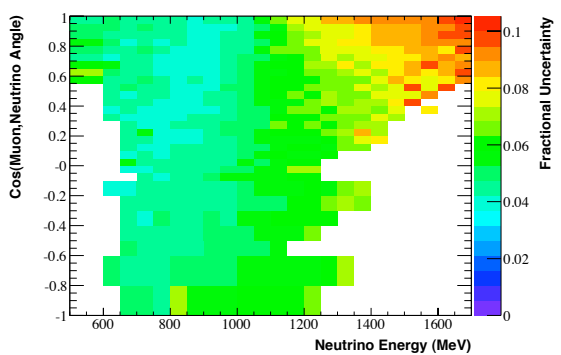

(d) The fractional systematic uncertainty from the " $\pi^{+}$" error source.

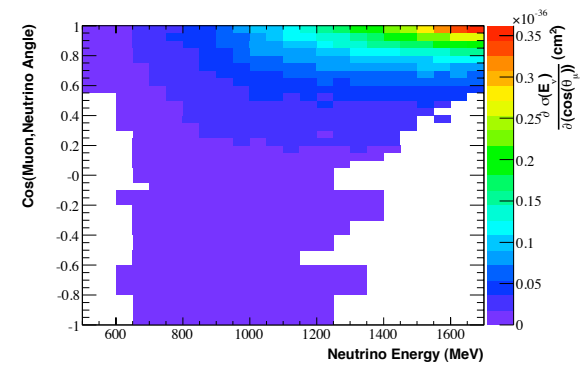

(e) Central value $\partial \sigma\left(E_{\nu}\right) / \partial\left(\cos \left(\theta_{\mu, \nu}\right)\right)$ measurement.

Figure 6.24: The fractional systematic uncertainties on the measured $\partial \sigma\left(E_{\nu}\right) / \partial\left(\cos \left(\theta_{\mu, \nu}\right)\right)$ values are shown for four of the error sources. The central value measurement is shown for reference. Note that the color scales differ for each systematic error source. An explanation of each error is given in Section 6.4.7. 


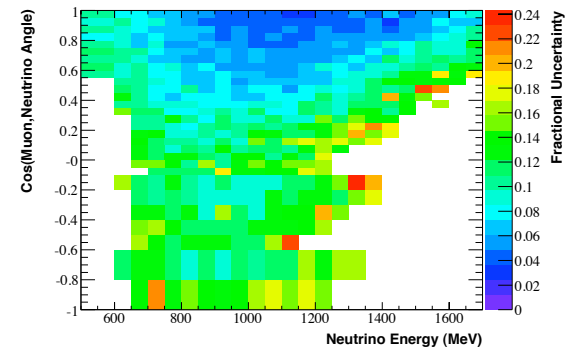

(a) The fractional systematic uncertainty from the "OM" error source.

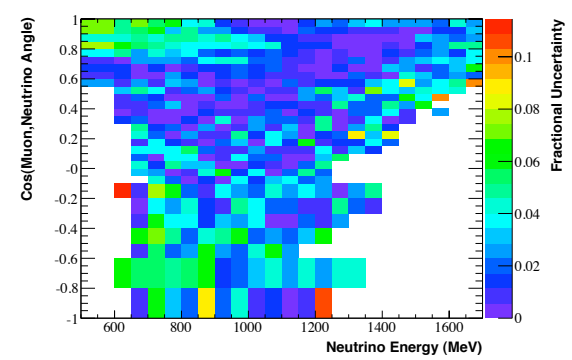

(c) The fractional systematic uncertainty from the "QTCORR" error source.

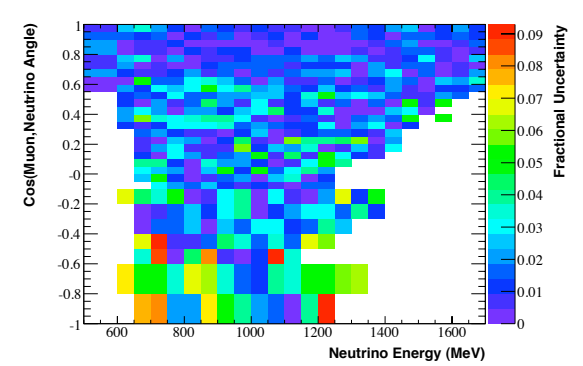

(b) The fractional systematic uncertainty from the "DISC" error source.

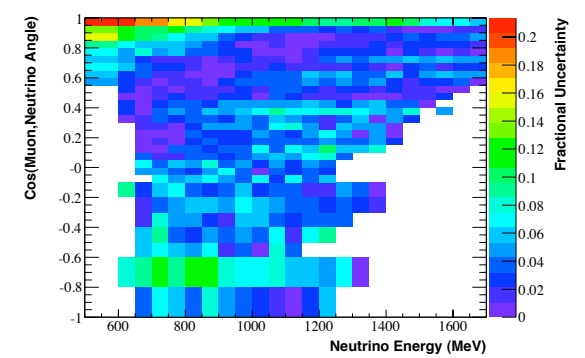

(d) The fractional systematic uncertainty from the "ITER" error source.

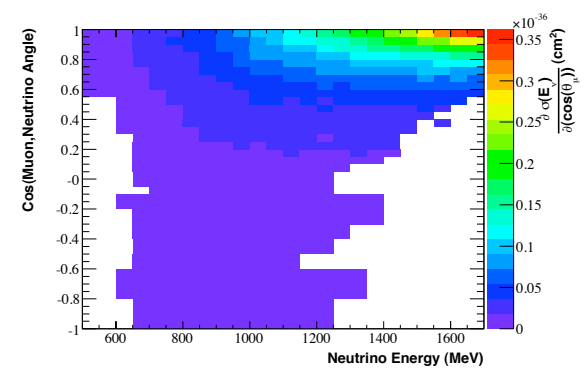

(e) Central value $\partial \sigma\left(E_{\nu}\right) / \partial\left(\cos \left(\theta_{\mu, \nu}\right)\right)$ measurement.

Figure 6.25: The fractional systematic uncertainties on the measured $\partial \sigma\left(E_{\nu}\right) / \partial\left(\cos \left(\theta_{\mu, \nu}\right)\right)$ values are shown for four of the error sources. The central value measurement is shown for reference. Note that the color scales differ for each systematic error source. An explanation of each error is given in Section 6.4.7. 


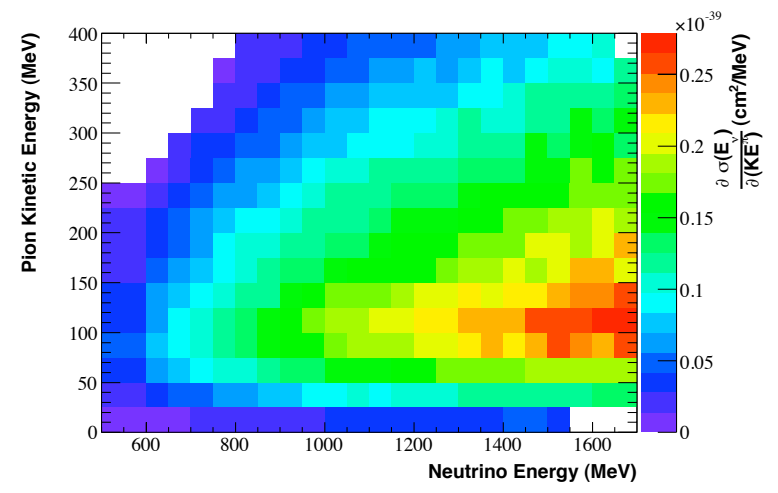

(a) Measured cross section

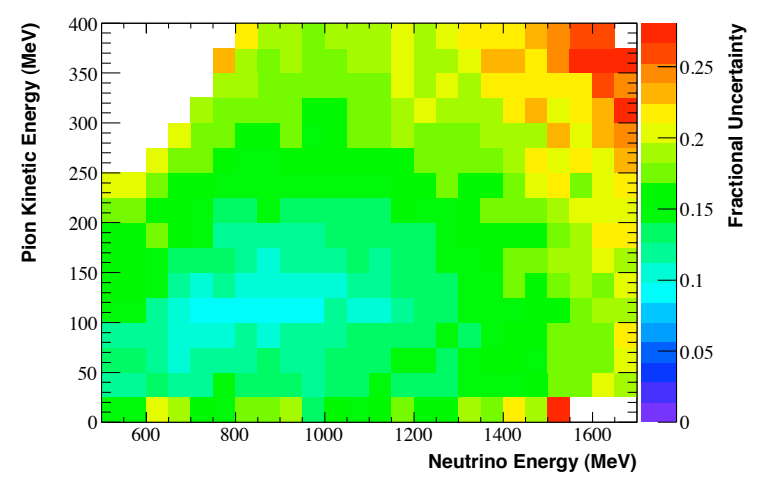

(b) Total fractional error on the measured cross section

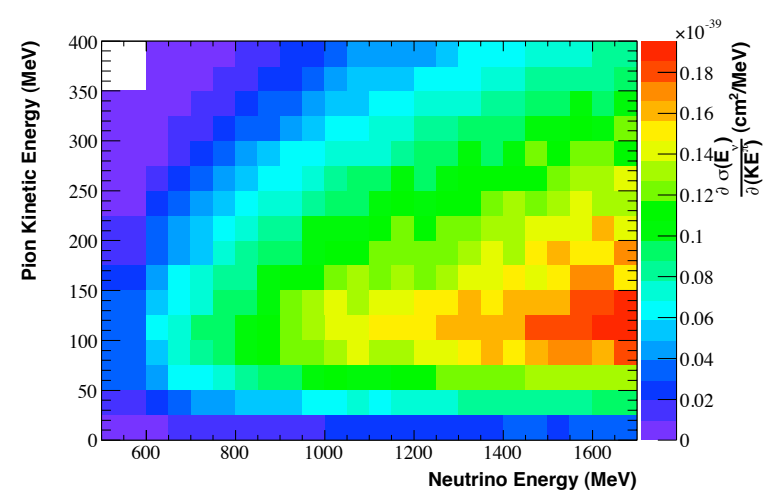

(c) Monte Carlo predicted cross section

Figure 6.26: The measured $\partial \sigma\left(E_{\nu}\right) / \partial\left(K E_{\pi}\right)$ values are shown along with the total fractional uncertainties. The Monte Carlo predicted cross section is shown for comparison. 


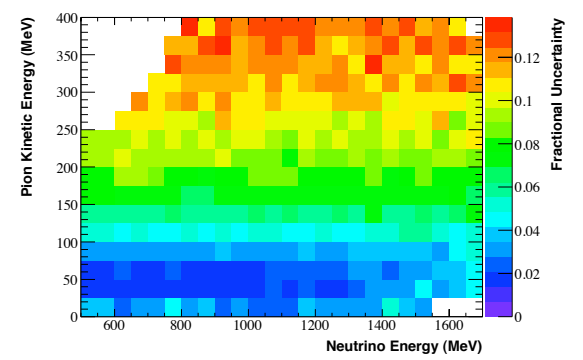

(a) The fractional systematic uncertainty from the "XSec" error source.

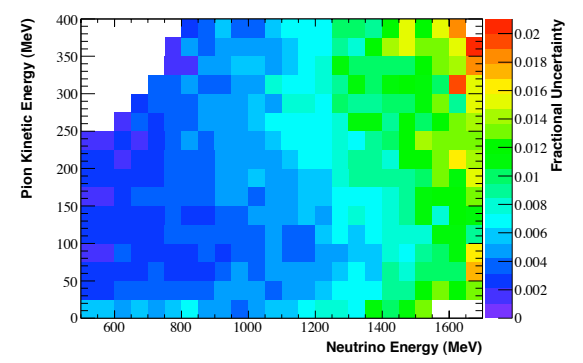

(c) The fractional systematic uncertainty from the " $K^{+}$" error source.

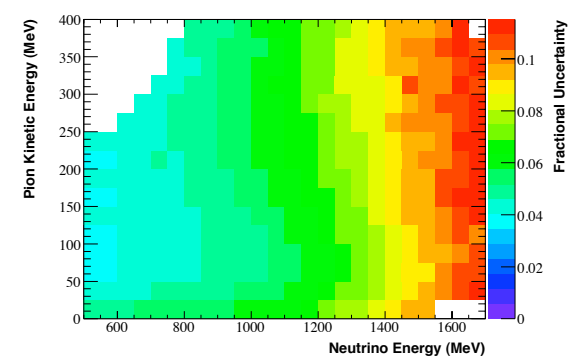

(b) The fractional systematic uncertainty from the "Beam" error source.

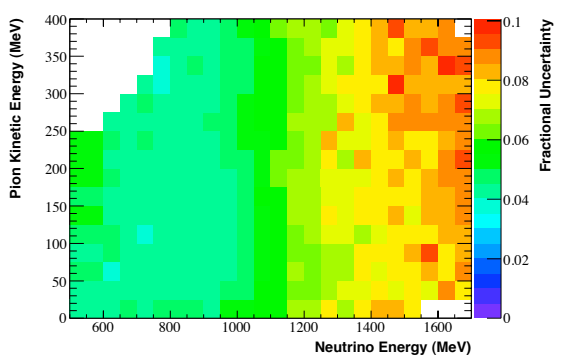

(d) The fractional systematic uncertainty from the " $\pi^{+}$" error source.

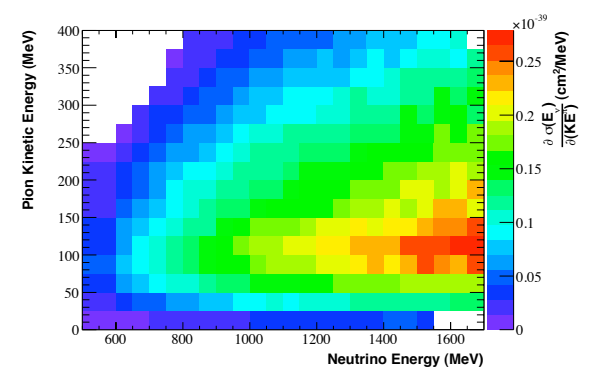

(e) Central value $\partial \sigma\left(E_{\nu}\right) / \partial\left(K E_{\pi}\right)$ measurement.

Figure 6.27: The fractional systematic uncertainties on the measured $\partial \sigma\left(E_{\nu}\right) / \partial\left(K E_{\pi}\right)$ values are shown for four of the error sources. The central value measurement is shown for reference. Note that the color scales differ for each systematic error source. An explanation of each error is given in Section 6.4.7. 


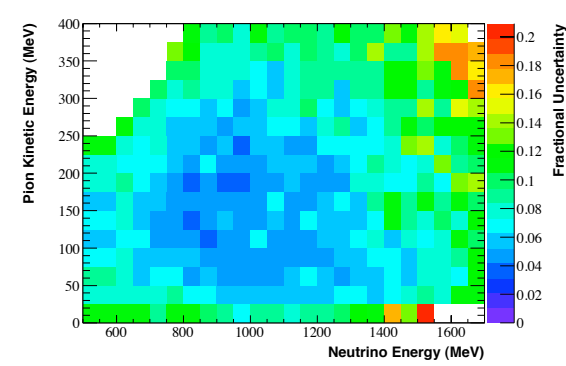

(a) The fractional systematic uncertainty from the "OM" error source.

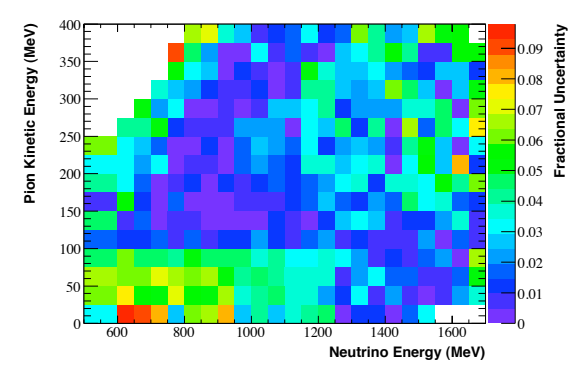

(c) The fractional systematic uncertainty from the "QTCORR" error source.

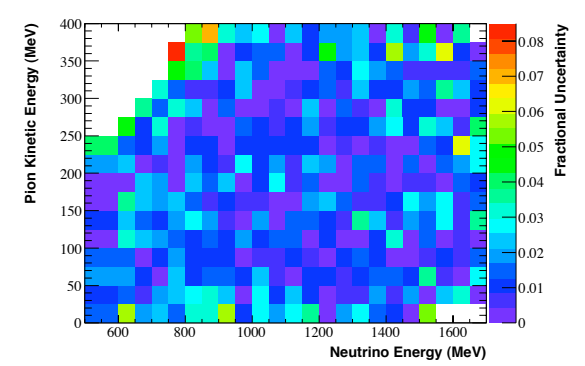

(b) The fractional systematic uncertainty from the "DISC" error source.

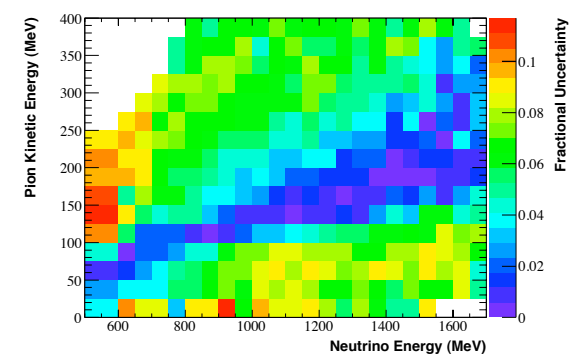

(d) The fractional systematic uncertainty from the "ITER" error source.

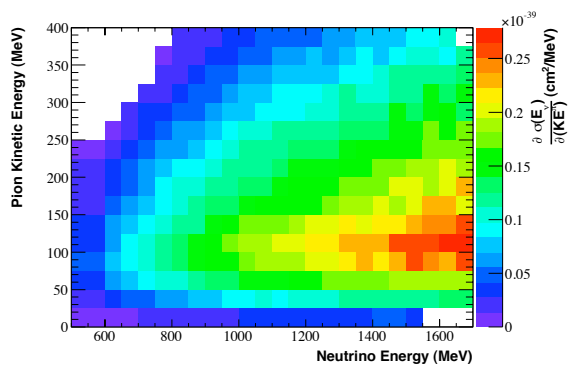

(e) Central value $\partial \sigma\left(E_{\nu}\right) / \partial\left(K E_{\pi}\right)$ measurement.

Figure 6.28: The fractional systematic uncertainties on the measured $\partial \sigma\left(E_{\nu}\right) / \partial\left(K E_{\pi}\right)$ values are shown for four of the error sources. The central value measurement is shown for reference. Note that the color scales differ for each systematic error source. An explanation of each error is given in Section 6.4.7. 


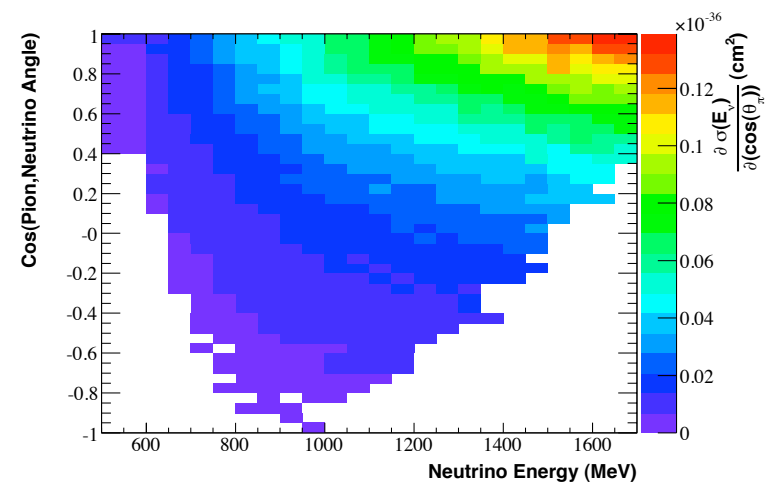

(a) Measured cross section

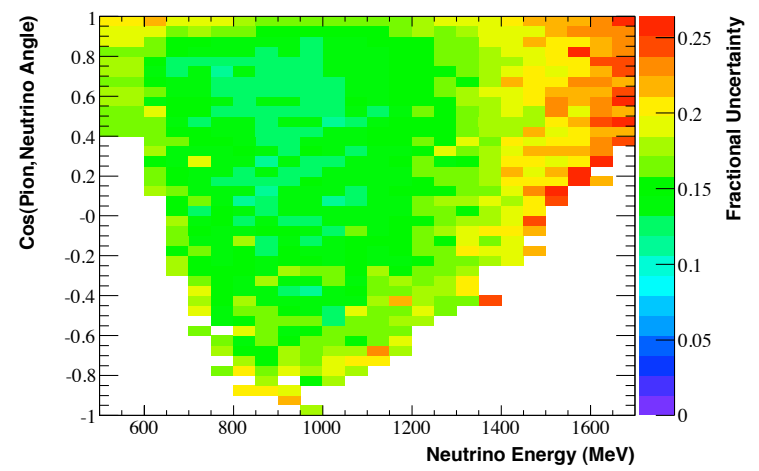

(b) Total fractional error on the measured cross section

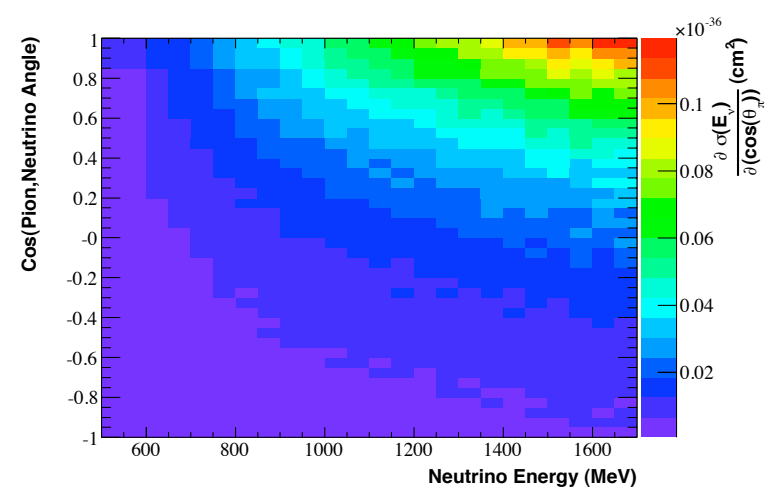

(c) Monte Carlo predicted cross section

Figure 6.29: The measured $\partial \sigma\left(E_{\nu}\right) / \partial\left(\cos \left(\theta_{\pi, \nu}\right)\right)$ values are shown along with the total fractional uncertainties. The Monte Carlo predicted cross section is shown for comparison. 


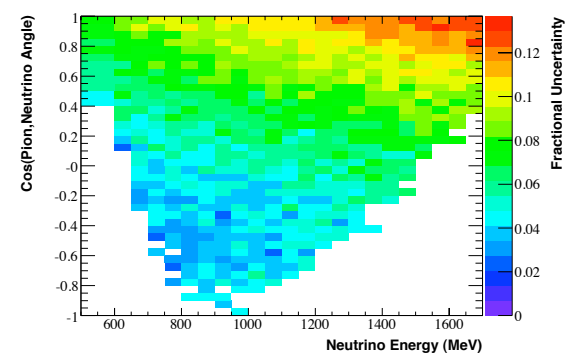

(a) The fractional systematic uncertainty from the "XSec" error source.

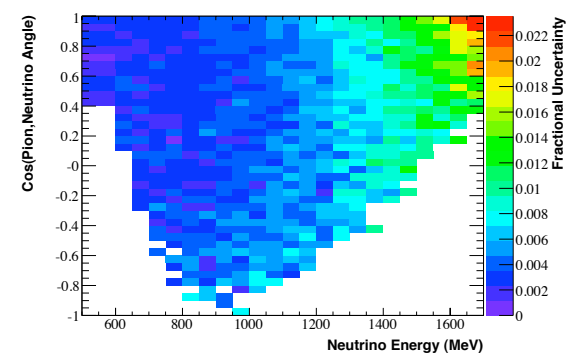

(c) The fractional systematic uncertainty from the " $K^{+}$" error source.

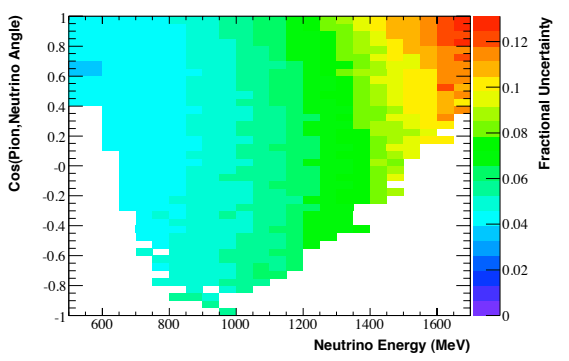

(b) The fractional systematic uncertainty from the "Beam" error source.

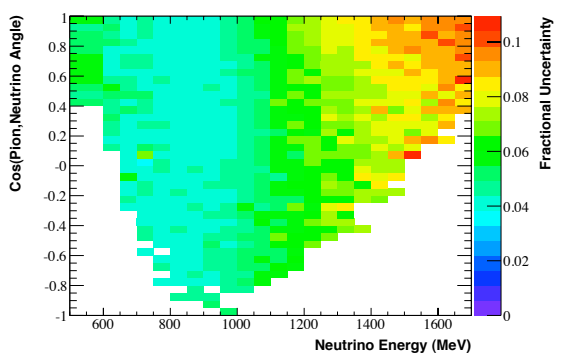

(d) The fractional systematic uncertainty from the " $\pi^{+}$" error source.

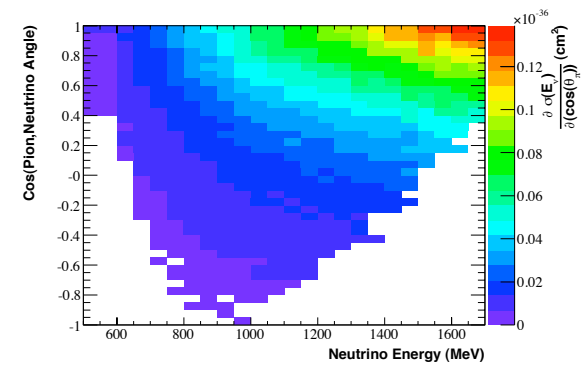

(e) Central value $\partial \sigma\left(E_{\nu}\right) / \partial\left(\cos \left(\theta_{\pi, \nu}\right)\right)$ measurement.

Figure 6.30: The fractional systematic uncertainties on the measured $\partial \sigma\left(E_{\nu}\right) / \partial\left(\cos \left(\theta_{\pi, \nu}\right)\right)$ values are shown for four of the error sources. The central value measurement is shown for reference. Note that the color scales differ for each systematic error source. An explanation of each error is given in Section 6.4.7. 


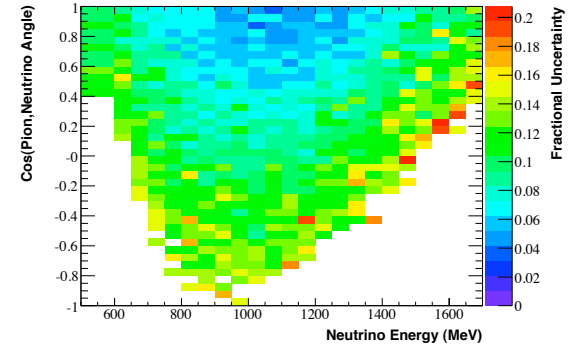

(a) The fractional systematic uncertainty from the "OM" error source.

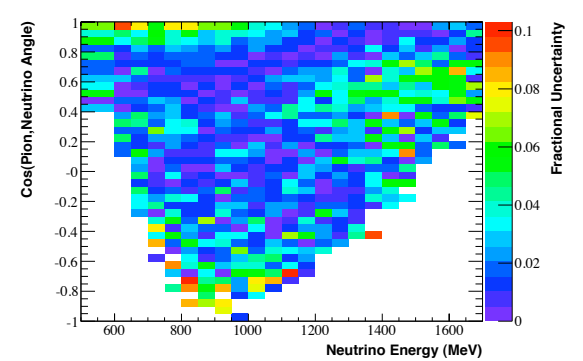

(c) The fractional systematic uncertainty from the "QTCORR" error source.

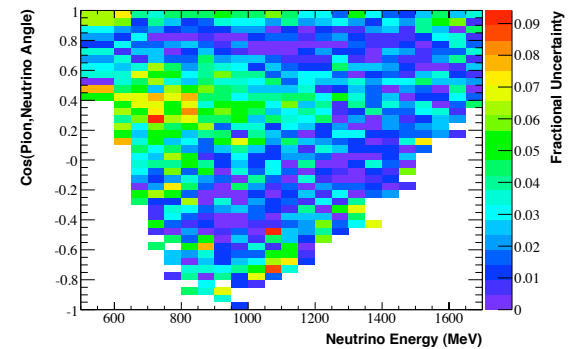

(b) The fractional systematic uncertainty from the "DISC" error source.

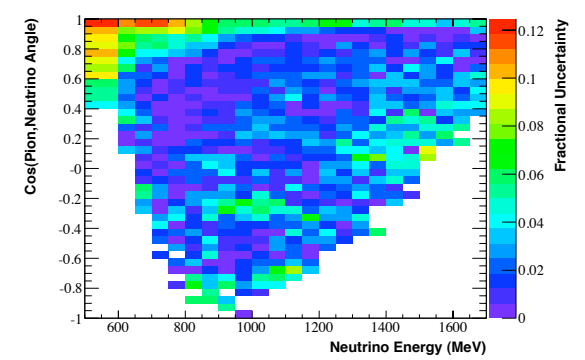

(d) The fractional systematic uncertainty from the "ITER" error source.

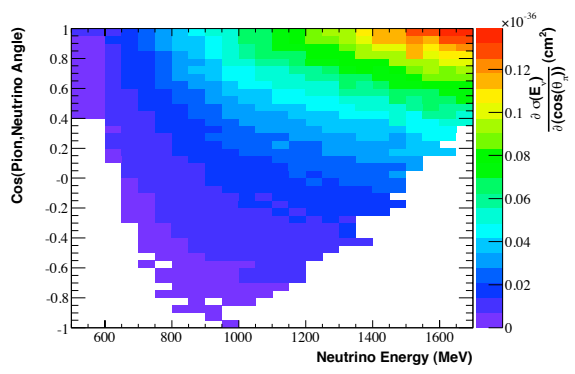

(e) Central value $\partial \sigma\left(E_{\nu}\right) / \partial\left(\cos \left(\theta_{\pi, \nu}\right)\right)$ measurement.

Figure 6.31: The fractional systematic uncertainties on the measured $\partial \sigma\left(E_{\nu}\right) / \partial\left(\cos \left(\theta_{\pi, \nu}\right)\right)$ values are shown for four of the error sources. The central value measurement is shown for reference. Note that the color scales differ for each systematic error source. An explanation of each error is given in Section 6.4.7. 


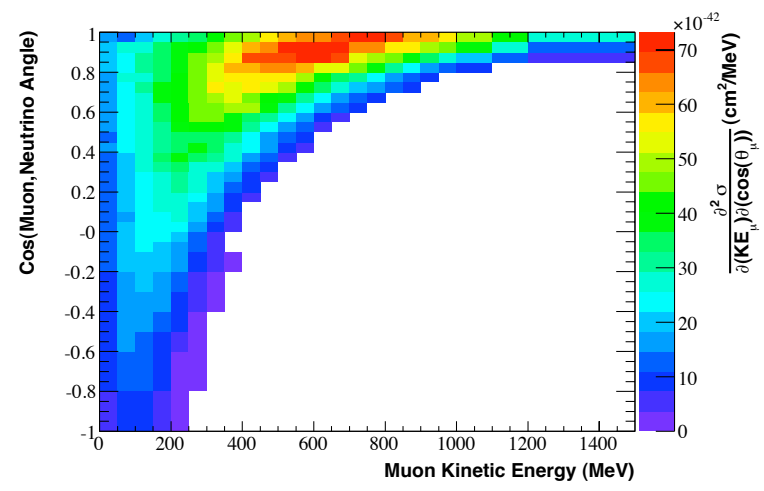

(a) Measured cross section

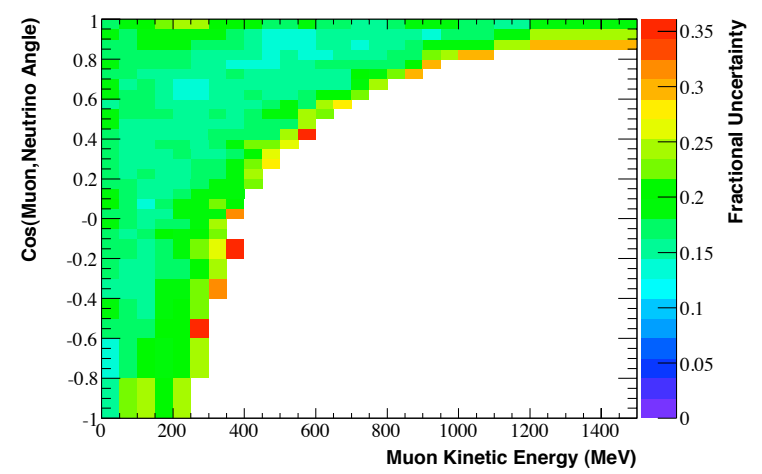

(b) Total fractional error on the measured cross section

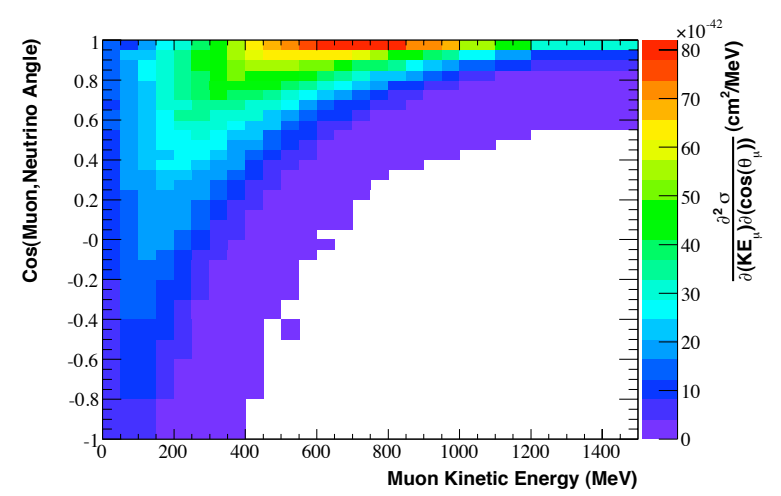

(c) Monte Carlo predicted cross section

Figure 6.32: The measured $\partial^{2} \sigma / \partial\left(\cos \left(\theta_{\mu, \nu}\right)\right) \partial\left(K E_{\mu}\right)$ values are shown along with the total fractional uncertainties. The Monte Carlo predicted cross section is shown for comparison. 


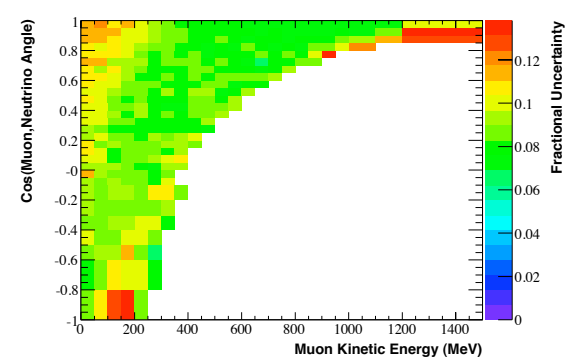

(a) The fractional systematic uncertainty from the "XSec" error source.

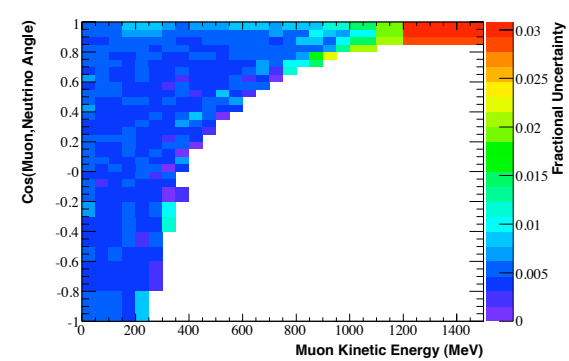

(c) The fractional systematic uncertainty from the " $K^{+}$" error source.

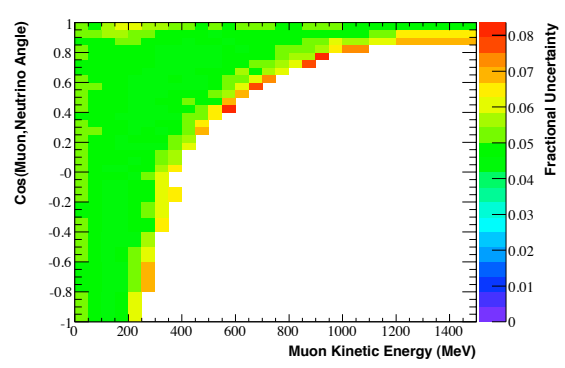

(b) The fractional systematic uncertainty from the "Beam" error source.

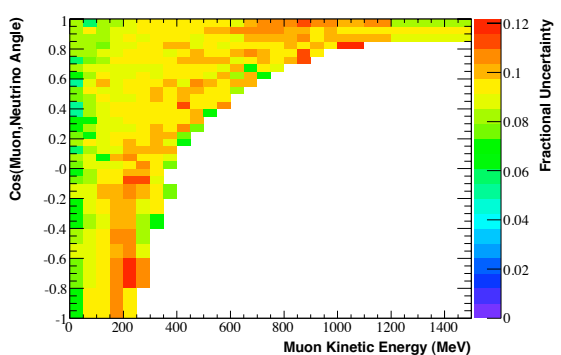

(d) The fractional systematic uncertainty from the " $\pi^{+}$" error source.

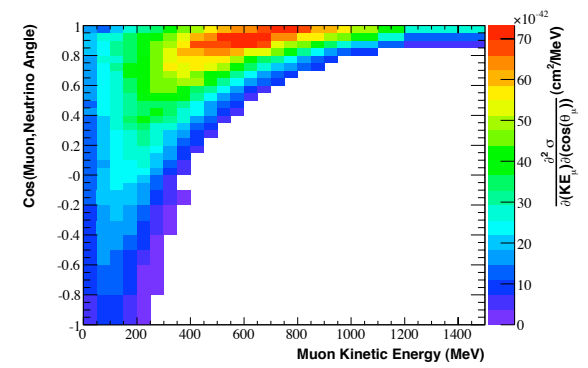

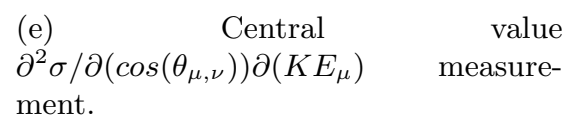

Figure 6.33: The fractional systematic uncertainties on the measured $\partial^{2} \sigma / \partial\left(\cos \left(\theta_{\mu, \nu}\right)\right) \partial\left(K E_{\mu}\right)$ values are shown for four of the error sources. The central value measurement is shown for reference. Note that the color scales differ for each systematic error source. An explanation of each error is given in Section 6.4.7. 


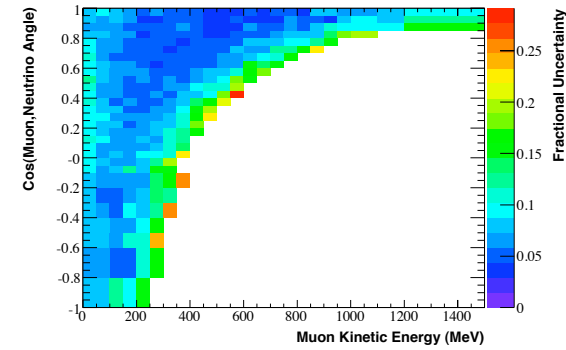

(a) The fractional systematic uncertainty from the "OM" error source.

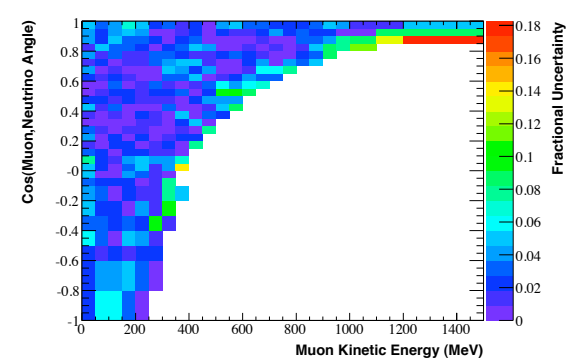

(c) The fractional systematic uncertainty from the "QTCORR" error source.

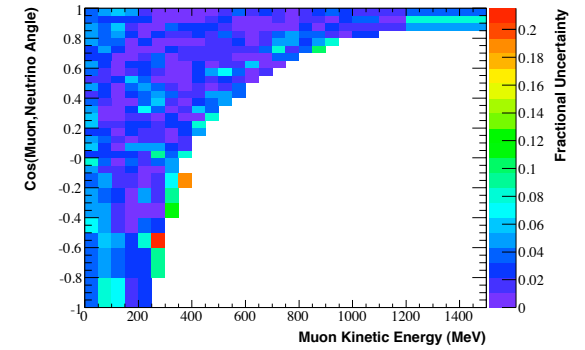

(b) The fractional systematic uncertainty from the "DISC" error source.

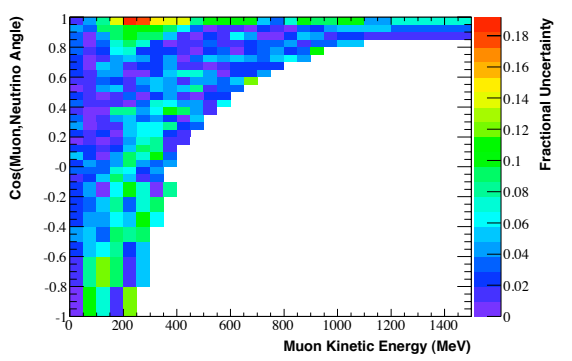

(d) The fractional systematic uncertainty from the "ITER" error source.

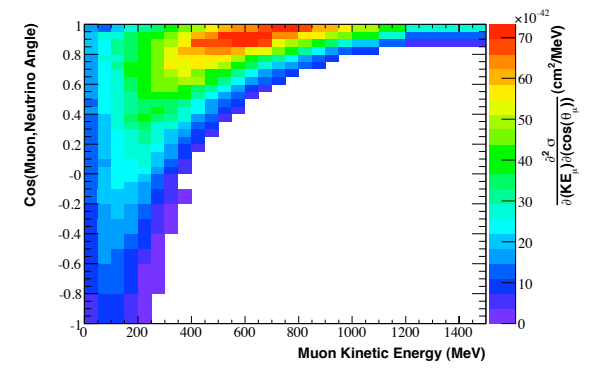

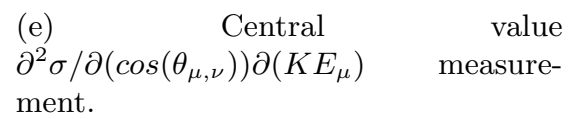

Figure 6.34: The fractional systematic uncertainties on the measured $\partial^{2} \sigma / \partial\left(\cos \left(\theta_{\mu, \nu}\right)\right) \partial\left(K E_{\mu}\right)$ values are shown for four of the error sources. The central value measurement is shown for reference. Note that the color scales differ for each systematic error source. An explanation of each error is given in Section 6.4.7. 


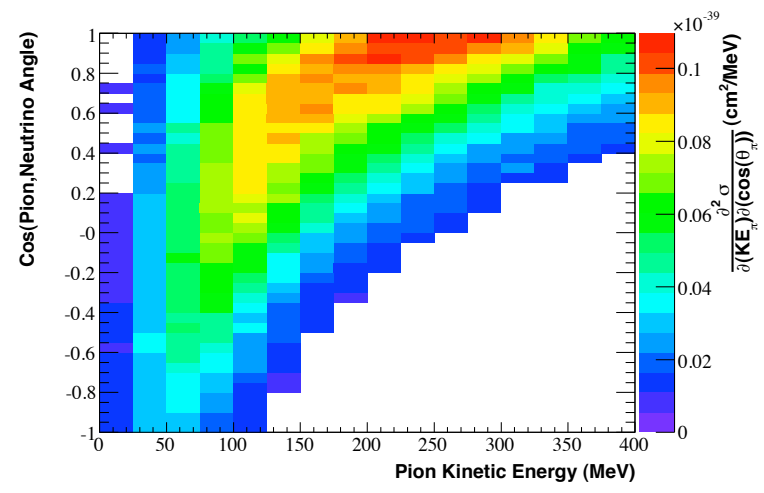

(a) Measured cross section

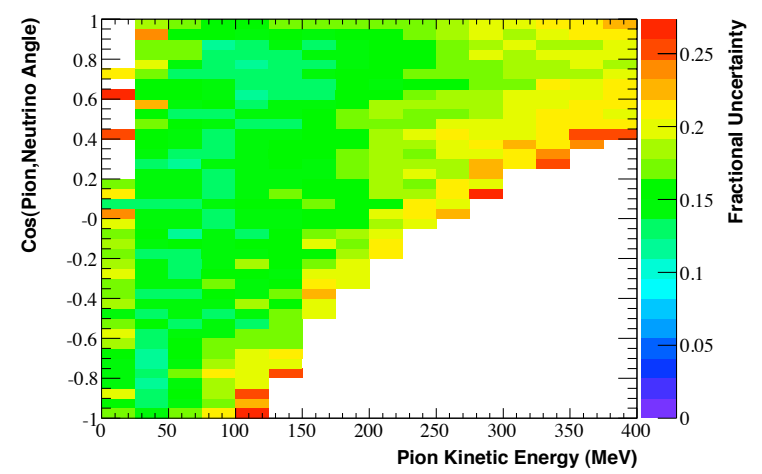

(b) Total fractional error on the measured cross section

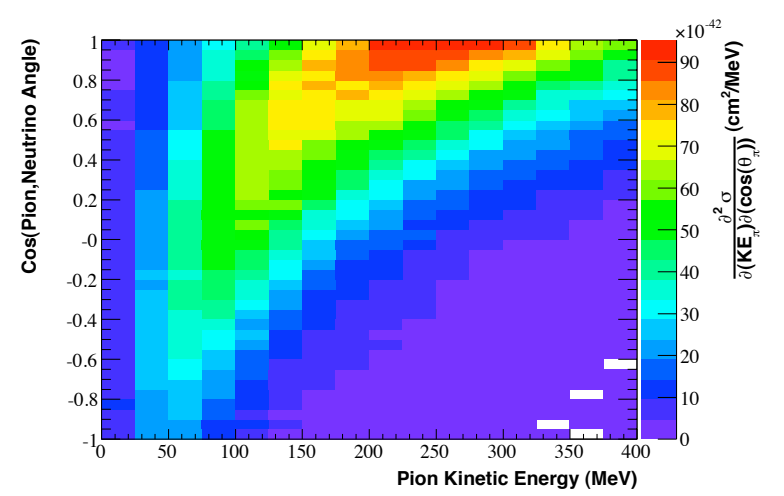

(c) Monte Carlo predicted cross section

Figure 6.35: The measured $\partial^{2} \sigma / \partial\left(\cos \left(\theta_{\pi, \nu}\right)\right) \partial\left(K E_{\pi}\right)$ values are shown along with the total fractional uncertainties. The Monte Carlo predicted cross section is shown for comparison. 


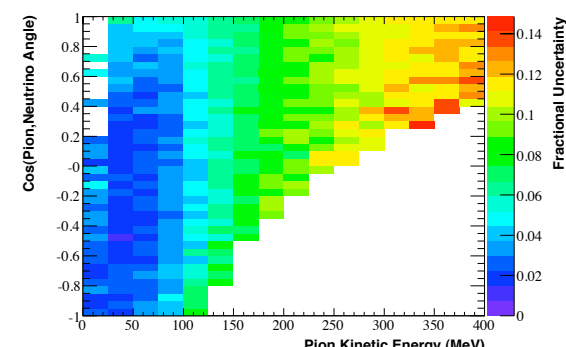

(a) The fractional systematic uncertainty from the "XSec" error source.

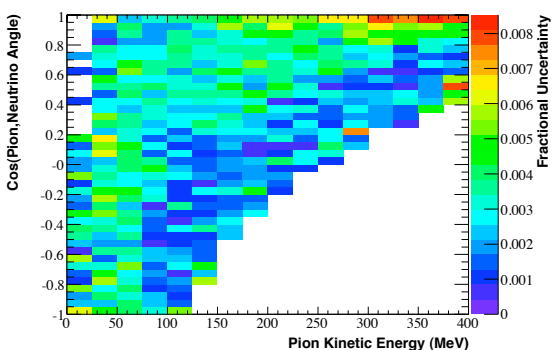

(c) The fractional systematic uncertainty from the " $K^{+}$" error source.

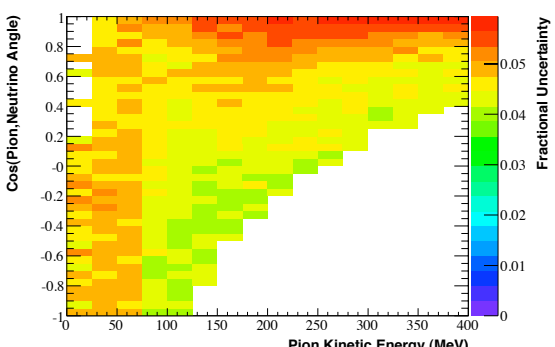

(b) The fractional systematic uncertainty from the "Beam" error source.

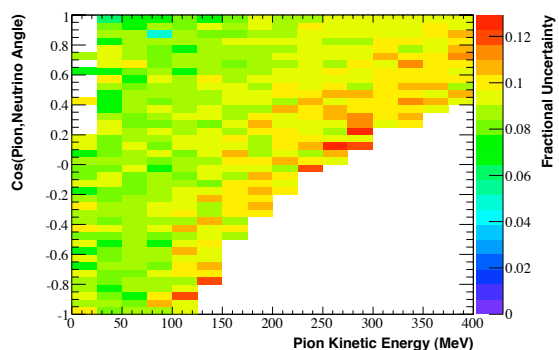

(d) The fractional systematic uncertainty from the " $\pi^{+}$" error source.

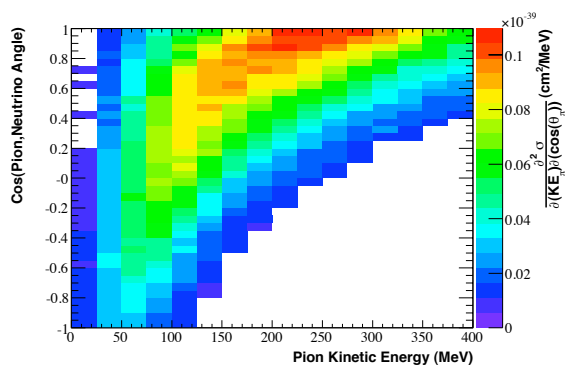

(e) Central value $\partial^{2} \sigma / \partial\left(\cos \left(\theta_{\pi, \nu}\right)\right) \partial\left(K E_{\pi}\right)$ Measurement

Figure 6.36: The fractional systematic uncertainties on the measured $\partial^{2} \sigma / \partial\left(\cos \left(\theta_{\pi, \nu}\right)\right) \partial\left(K E_{\pi}\right)$ values are shown for four of the error sources. The central value measurement is shown for reference. Note that the color scales differ for each systematic error source. An explanation of each error is given in Section 6.4.7. 


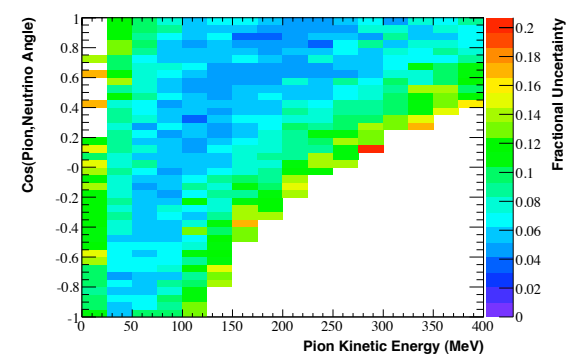

(a) The fractional systematic uncertainty from the "OM" error source.

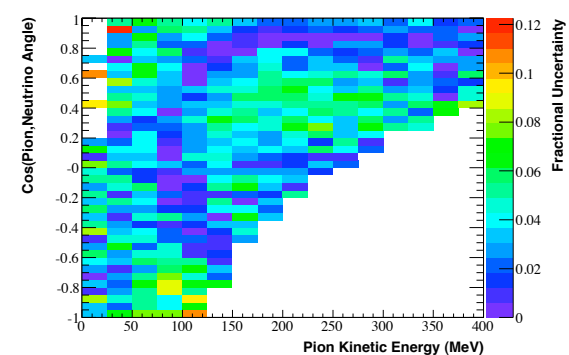

(c) The fractional systematic uncertainty from the "QTCORR" error source.

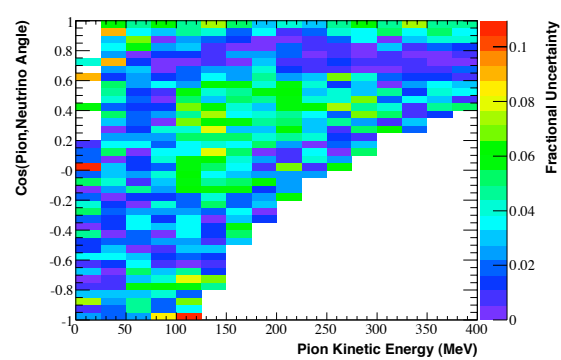

(b) The fractional systematic uncertainty from the "DISC" error source.

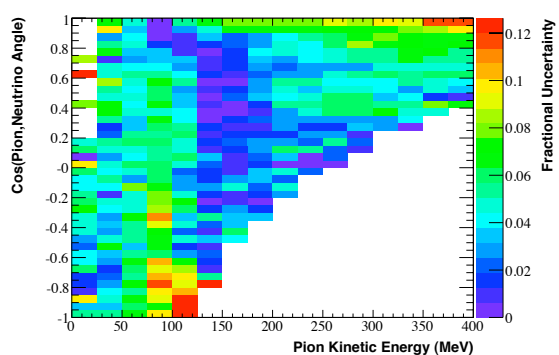

(d) The fractional systematic uncertainty from the "ITER" error source.

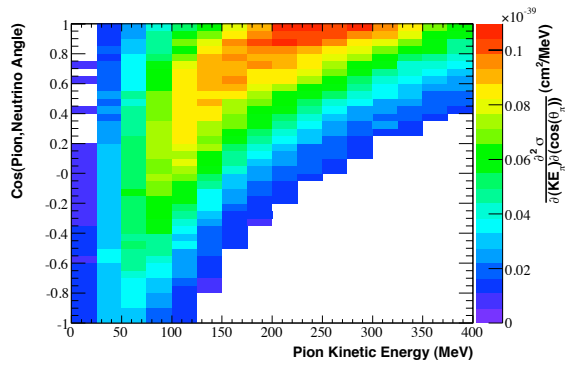

$\begin{array}{lrr}(\mathrm{e}) & \text { Central } & \text { value } \\ \partial^{2} \sigma / \partial\left(\cos \left(\theta_{\pi, \nu}\right)\right) \partial\left(K E_{\pi}\right) & \text { Measurement }\end{array}$

Figure 6.37: The fractional systematic uncertainties on the measured $\partial^{2} \sigma / \partial\left(\cos \left(\theta_{\pi, \nu}\right)\right) \partial\left(K E_{\pi}\right)$ values are shown for four of the error sources. The central value measurement is shown for reference. Note that the color scales differ for each systematic error source. An explanation of each error is given in Section 6.4.7. 


\section{Chapter 7}

\section{Conclusion}

Results have been presented for the effective $\mathrm{CC} \pi^{+}$cross section as a function of several fundamental kinematic variables. Of these results, the cross sections measured as a function of the neutrino energy are the least experiment dependent, since the predicted neutrino flux has been removed separately from each bin. Previous measurements of the neutrino energy cross section are shown in Figure 7.1. All comparable measurements below $2 \mathrm{GeV}$ have uncertainties larger than $20 \%$, upon which the present analysis provides a significant improvement. Each of these measurements has either been performed on, or extrapolated to, a single nucleon target, which has been explicitly avoided in this measurement. As discussed in Section 1.1.2.2, the current theoretical understanding of the vector and axial-vector nucleon form factors for both hydrogen and carbon, as well as nuclear effects such as the Fermi momentum distribution and nuclear screening, motivate measurements of model independent cross section quantities from which theoretical models can be derived.

To get a sense of how this measurement compares to previous measurements, the Nuance prediction in Figure 7.1 can be compared to the Monte Carlo prediction in Figure 6.11 after being convolved with the nuclear effects described in Section 1.2. The present measurement is on average about $20 \%$ higher than the Nuance prediction for the effective cross section. The Brookhaven experiment that provides the most proton and neutron data in the MiniBooNE neutrino energy range [72] is also roughly $20 \%$ higher 


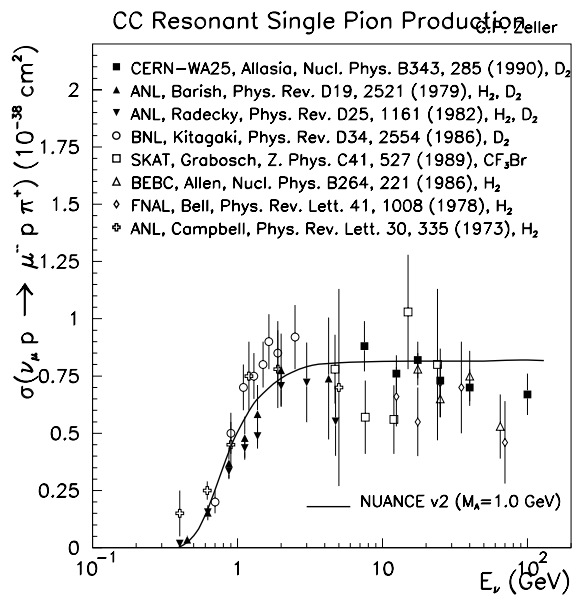

(a) Previous $\mathrm{CC} \pi^{+}$measurements extrapolated to single proton targets.

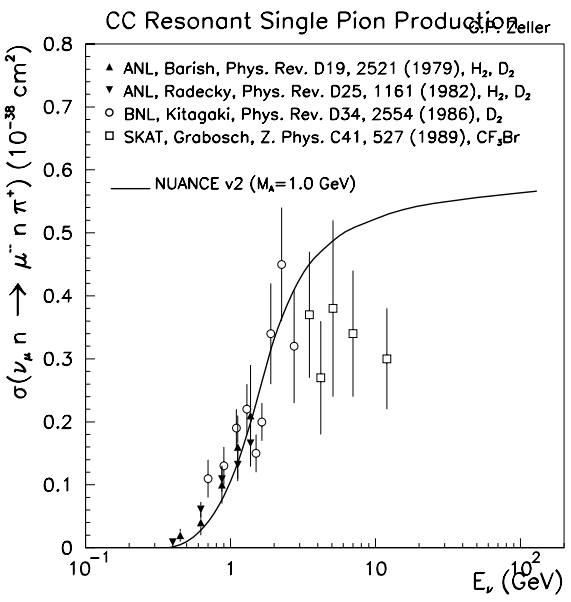

(b) Previous $\mathrm{CC} \pi^{+}$measurements extrapolated to single proton targets.

Figure 7.1: Previous measurements of $\nu_{\mu}$ induced $\mathrm{CC} \pi^{+}$measurements are shown for proton and neutron targets $[69,70,71,72,73,74,75,76]$. Those experiments not measured on $\mathrm{H}_{2}$ have been extrapolated to single nucleon cross sections. The line indicating the Nuance prediction represents the Monte Carlo predicted cross section in Figure 6.11 prior to the modeling of nuclear effects.

than the Nuance prediction for the resonant $\Delta^{++}$interactions (i.e. proton targets) that dominate the present result. ${ }^{1}$

The remaining kinematic $\mathrm{CC} \pi^{+}$cross sections have never been measured before. ${ }^{2}$

These measurements are also dependent on the flux shape prediction given in Figure 6.10. For this reason, each measurement of a final state kinematic quantity has also been measured in bins of neutrino energy. The one-dimensional have also been provided due to the familiarity of many in the field with flux-averaged results. Indeed, this is a commonly requested form for such measurements, as many theorists have been producing results according to the MiniBooNE flux for some time [77, 78, 79, 80].

\footnotetext{
${ }^{1}$ It should be mentioned that the Brookhaven experiment derived its flux prediction from the measured rate of CCQE interactions, effectively measuring a cross section ratio to CCQE.

2 Although these differential cross sections have not been measured, previous experiments have performed fits to reconstructed $Q^{2}$ and $\pi^{+}$angular distributions to extract values for parameters such as the axial mass.
} 
The cross section measurements produced by this analysis provide a detailed description of the $\mathrm{CC} \pi^{+}$interaction in terms of many of the interesting kinematic quantities. Hopefully these results will aid the effort to fully understand the properties of these interactions. In the immediate future, the cross sections provided by this analysis will help to constrain the largest charged current background in the next generation of neutrino oscillation experiments. 


\section{Bibliography}

[1] C. Amsler et al. The Review of Particle Physics. Physics Letters, B667(1), 2008.

[2] D. Rein and L. H. Sehgal. Neutrino-Excitation of Baryon Resonances and Single Pion Production. Annals Physics, 133(79), 1981.

[3] M. Kislinger R. P. Feynman and F. Ravndal. Current Matrix Elements from a Relativistic Quark Model. Phys. Rev., D3(2706), 1971.

[4] F. Ravndal. Weak Production of Nuclear Resonances in Relativistic Quark Model. Nuovo Cimento, A18(385), 1973.

[5] E. T. Osypowski M. G. Olsson and E. H. Monsay. Electroproduction of Low Hadronic Masses. Phys. Rev., D17(2938), 1978.

[6] M. Hasegawa et al. Search for Coherent Charged Pion Production in Neutrino Carbon Interactions. Phys. Rev. Lett., 95(79), 1981.

[7] J. Nowak and W. Metcalf. MiniBooNE Technical Note. 260, 2008.

[8] V. V. Lynbushkin K. S. Kuzmin and V. A. Naumov. Lepton Polarization in Neutrino Nucleon Interactions. Mod. Phys. Lett., A19(2815), 2004.

[9] C. Berger and L. M. Sehgal. Lepton Mass Effects in Single Pion Production by Neutrinos. Phys. Rev., D76(113004), 2007.

[10] C. Berger and L. M. Sehgal. Erratum: Lepton Mass Effects in Single Pion Production by Neutrinos. Phys. Rev., D77(059901), 2007.

[11] K. M. Graczyk and J. T. Sobczyk. Lepton Mass Effects in Weak CC Single Pion Production. Phys. Rev., D77(053003), 2008.

[12] K. M. Graczyk and J. T. Sobczyk. Form Factors in the Quark Resonance Model. Phys. Rev., D77(053001), 2008.

[13] S. K. Singh L. Alvarez-Ruso and M. F. Vincente-Vascas. Charged Current Weak Electroproduction of the $\Delta$ Resonance. Phys. Rev., C57(2693), 1998.

[14] O. Lalakulich and E. A. Paschos. Resonance Production By Neutrinos: J=3/2 Resonances. Phys. Rev., D71(074003), 2005. 
[15] A. Kartavtsev E. A. Paschos and G. J. Gounaris. Coherent pion Production by Neutrino Scattering Off Nuclei. Phys. Rev., D74(054007), 2006.

[16] S. Hirenzaki L. Alvarez-Ruso, L. S. Geng and M. J. Vicente Vacas. Charged Current Neutrino-Induced Coherent Pion Production. Phys. Rev., C75(055501), 2007.

[17] J. Nieves J. E. Amaro, E. Hernandez and M. Valverde. Theoretical Study of Neutrino-Induced Coherent Pion Production Off Nuclei at T2K and MiniBooNE Energies. Phys. Rev., D79(013002), 2009.

[18] K. Hiraide et al. Search for Charged Current Coherent Pion Production on Carbon in a Few-GeV Neutrino Beam. Phys. Rev., D78(112004), 2008.

[19] M. Hasegawa et al. Search for Coherent Charged Pion Production in NeutrinoCarbon Interactions. Phys. Rev. Lett., 95(252301), 2005.

[20] A. A. Aguilar-Arevalo et al. First observation of coherent $\pi^{0}$ production in neutrinonucleus interactions with $E_{\nu}<2 \mathrm{GeV}$. Phys. Lett., B664(41), 2008.

[21] T2K Collaboration. Tokai-to-Kamioka (T2K) Long Baseline Neutrino Oscillation Experiment Proposal. http://j-parc.jp/NuclPart/pac_0606/pdf/p11Nishikawa.pdf, 2006.

[22] $\mathrm{NO} \nu \mathrm{A}$ Collaboration. NO $\nu$ A Proposal to Build a 30 Kiloton Off-Axis Detector to Study Neutrino Oscillations in the Fermilab NuMI Beamline. arXiv:hepex/0503053, 2005.

[23] J. E. Amaro M. Valverde J. Nieves, M. J. Vicente-Vacas and E. Hernandez. Theoretical Update on Low Energy Neutrino-Nucleus Reactions. arXiv:0809.5219v2, 2008 .

[24] T. Kobilarcik J. Monroe, D. Finley and H-O. Meyer. MiniBooNE Technical Note. 87, 2003.

[25] The BooNE Collaboration. Technical Design Report for the $8 \mathrm{GeV}$ Beam, 2001.

[26] The BooNE Collaboration. Technical Design Report for the MiniBooNE Neutrino Beam, 2001.

[27] The BooNE Collaboration. The MiniBooNE Detector Technical Design Report, 2001.

[28] A. A. Aguilar-Arevalo et al. The MiniBooNE Detector. arXiv:0806.4201, 2008.

[29] S. Agostinelli et al. GEANT 4-A Simulation Toolkit. Nucl. Instrum. Meth., A506(250), 2003.

[30] H. A. Tanaka. MiniBooNE Technical Note. 186, 2006.

[31] R. J. Glauber. Lectures in Theoretical Physics, volume I. Interscience, New York, 1959. 
[32] V. V. Gachurin et al. Measurement of Total Inelastic Cross-Sections of the Interaction of Protons and pi+ Mesons on Nuclei in the Momentum Range from 1.35-GeV/c to 3.75-GeV/c. ITEP-59-1985, 1985.

[33] B. M. Bobchenko et al. Measurement of Total Inelastic Cross-Sections from Proton Interactions with Nuclei in the Momentum Range from 5-GeV/c to $9-\mathrm{GeV} / \mathrm{c}$ and pi- Mesons with Nuclei in the Momentum Range from $1.75-\mathrm{GeV} / \mathrm{c}$ to $6.5-\mathrm{GeV} / \mathrm{c}$. Sov. J. Nucl. Phys., 30(805), 1979.

[34] C. Ashery et al. True Absorption and Scattering of Pions on Nuclei. Phys. Rev., C23(2173), 1981.

[35] B. W. Allardyce et al. Pion Reaction Cross Sections and Nuclear Sizes. Nucl. Phys., A209(1), 1973.

[36] H. Tanaka and M. Wilking. MiniBooNE Technical Note. 206, 2006.

[37] A. A. Aguilar-Arevalo et al. Neutrino Flux Prediction at MiniBooNE. Phys. Rev., D79(072002), 2009.

[38] M. Tzanov. MiniBooNE Technical Note. 204, 2006.

[39] M. Shaevitz. MiniBooNE Technical Note. 209, 2006.

[40] J. R. Sanford and C. L. Wang. Emperical Formulas for Particle Production in p-Be Collision Between 10 and $35 \mathrm{BeV} / \mathrm{c}$. BNL 11299, 1967.

[41] N. V. Mokhov et al. Recent Enhancements to the MARS15 Code. Radiat. Prot. Dosim., 116(99), 2005.

[42] M. G. Catanesi et al. Measurement of the Production Cross-Section of Positive Pions in the Collision of $8.9 \mathrm{GeV} / \mathrm{c}$ Protons on Beryllium. Eur. Phys. J., C52(29), 2007.

[43] F. Abe et al. Production of Neutral Strange Particles $K_{S}^{0}$ and $\Lambda_{0}$ by $12-\mathrm{GeV}$ Protons on Nuclear Targets. Phys. Rev., D36(1302), 1987.

[44] M. Shaevitz. MiniBooNE Technical Note. 188, 2006.

[45] T. Abbott et al. Measurement of Particle Production in Proton-Induced Reactions at $14.6 \mathrm{GeV} / \mathrm{c}$. Phys. Rev., D45(3906), 1992.

[46] J. V. Allaby et al. High-Energy Particle Spectra from Proton Interactions at 19.2GeV/c. CERN 70-12, 1970.

[47] D. Dekkers et al. Experimental Study of Particle Production at Small Angles in Nucleon-Nucleon Collisions at 19 and $23 \mathrm{GeV} /$ c. Phys. Rev., 137(B962), 1965.

[48] G. J. Marmer et al. Differential Production Cross Sections of Low-Momentum Particles from 12.3-BeV/c Protons on Beryllium and Copper. Phys. Rev., 179(1294), 1969. 
[49] T. Eichten et al. Particle Production in Proton Interactions in Nuclei at $24 \mathrm{GeV} / \mathrm{c}$. Nucl. Phys., B44(333), 1972.

[50] Aleshin et al. Production of K+- Mesons from Be Targets at 62-Mrad at 9.5-GeV/c Incident Proton Momenta. ITEP-80-1977, 1977.

[51] Vorontsov et al. A-Dependence Of Fragmentation Of 9.2-Gev Protons On Nuclei. ITEF-88-011, 1988.

[52] W.-M. Yao et al. The Review of Particle Physics. J. Phys., G33(1), 2006.

[53] S. Brice. MiniBooNE Technical Note. 257, 2008.

[54] D. Casper. The nuance Neutrino Simulation, and the Future. arXiv:hep-ph/0208030v1, 2001.

[55] C. H. Llewellyn Smith. Neutrino Reactions at Accelerator Energies. Phys. Rept., 3(261), 1972.

[56] R. A. Smith and E. J. Moniz. Neutrino Reactions on Nuclear Targets. Nucl. Phys, B43(605), 1972.

[57] E. J. Moniz. Nuclear Fermi Momenta from Quasielastic Electron Scattering. Phys. Rev. Lett., 26(445), 1971.

[58] R. Brun et al. GEANT3. CERN-DD/EE/84-1, 1987.

[59] R. B. Patterson. A Search for Muon Neutrino to Electron Neutrino Oscillations at $\Delta m^{2}>0.1 \mathrm{eV}^{2}$. PhD thesis, Princeton University, 2007.

[60] D. F. Measday T. Suzuki and J. P. Roalsvig. Total Nuclear Capture Rates for Negative Muons. Phys. Rev., C35(2212), 1987.

[61] C. Zeitnitz and T. A. Gabriel. The GEANT-CALOR Interface and Benchmark Calculations of ZEUS test Calorimeters. Nucl. Instrum. Meth., A349(106), 1994.

[62] J. Birks. Theory and Practice of Scintillation Counting. Pergamon Press, 1964.

[63] I. Stancu. MiniBooNE Technical Note. 50, 2002.

[64] MINUIT. CERN Program Library entry D506. http://wwwasdoc.web.cern.ch/wwwasdoc/minuit/minmain.html, @copyright 1994-1998.

[65] M. O. Wascko. Charged Current Single Pion Cross Section Measurement at MiniBooNE. arXiv:hep-ex/0602050v1, 2006.

[66] G. D’Agostini. A Multidimensional Unfolding Method Based on Bayes' Theorem. Nucl. Instrum. Meth., A362(487), 1995.

[67] G. Cowen. A Survey of Unfolding Methods for Particle Physics. Conf. Proc. Advanced Statistical Techniques in Particle Physics, March 2002.

[68] http://www-boone.fnal.gov. 
[69] D. Allasia et al. Investigation of Exclusive Channels in $\nu / \bar{\nu}$-Deuteron Charged Current Interactions. Nucl. Phys., B343(285), 1990.

[70] S. J. Barish et al. Study of Neutrino Interactions in Hydrogen and Deuterium. II. Inelastic Charged-Current Reactions. Phys. Rev, D19(2521), 1979.

[71] G. M. Radecky et al. Study of Single-Pion Production by Weak Charged Currents in Low-Energy $\nu$ d Interactions. Phys. Rev., D25(1161), 1982.

[72] T. Kitagaki et al. Charged-Current Exclusive Pion Production in NeutrinoDeuterium Interactions. Phys. Rev., D34(2554), 1986.

[73] H.J. Grabosch et al. Cross-Section Measurements Of Single Pion Production In Charged Current Neutrino and Anti-Neutrino Interactions. Z. Phys., C41(527), 1989.

[74] P. Allen et al. A Study of Single-Meson Production in Neutrino and Antineutrino Charged-Current Interactions on Protons. Nucl. Phys., B264(221), 1986.

[75] J. Bell et al. Cross-Section Measurements for the Reactions $\nu p \rightarrow \mu^{-} \pi^{+} p$ and $\nu p \rightarrow \mu^{-} K^{+} p$ at High Energies. Phys Rev. Lett., 41(1008), 1978.

[76] J. Campbell. Study of the Reaction $\nu p \rightarrow \mu^{-} \pi^{+} p$. Phys. Rev. Lett., 30(335), 1973.

[77] J. E. Amaro et al. Theoretical Study of Neutrino-Induced Coherent Pion Production Off Nuclei at T2K and MiniBooNE Energies. Advanced Statistical Techniques in Particle Physics.

[78] M. Sajjad Athar et al. Charged Current Anti-neutrino Reactions from ${ }^{12} \mathrm{C}$ at MiniBooNE Energies. Phys. Rev., D75(093003), 2007.

[79] S. K. Singh et al. Nuclear Effects in Neutrino Induced Coherent Pion Production at K2K and MiniBooNE. Phys. Rev. Lett., 96(241801), 2006.

[80] L. Alvarez-Ruso et al. Neutrino-Induced Coherent Pion Production. arXiv:0709.3019, AIP Conf. Proc., 967(201), 2007. 


\section{Appendix A}

\section{Event Displays}

In the following sections, geometric representations of several aspects of the $\mathrm{CC} \pi^{+}$ fitter are presented. Each event display shows the $\mathrm{X}$ versus $\mathrm{Z}$ view in the left column and the $\mathrm{Y}$ versus $\mathrm{Z}$ view in the right column. The top plots show the emission point of every Cherenkov and scintillation photon in the event, and the bottom plots show the vertices where one of the following took place:

(1) event creation

(2) hadronic interaction

(3) particle decay

(4) a particle created in (1)-(3) came to rest

The particles that connect the vertices are shown as straight lines.

Each display is cumulative over the entire event time window. Particles produced in the decays of stopped particles occur in later subevents (i.e. PMT hit time groupings; see Section 6.3) and are not included in the $\mathrm{CC} \pi^{+}$fits. In particular, Michel electrons produced by stopped muon decays or $\pi \rightarrow \mu \rightarrow e$ decay chains occur in every event due to the three subevent requirement, but they do not contribute to the charge or time likelihoods of the fit.

The graphical representations of the fitter show most of the fit parameters. The muon track (red line) and the kinked pion track (magenta line) give the vertex and all 
of the directions. The remaining energy parameters for both the true and fit tracks are listed on each display, along with the goodness of fit, $-\log (\mathcal{L})$. The curved black line present in some of the displays represents the location of the front faces of the PMTs in the plane that contains the interaction, and the green stars give the locations of the reconstructed Michel electrons.

\section{A.1 Truth Seeds}

To verify that a maximum in the likelihood surface exists for the correct set of reconstructed track parameters, studies have been performed where the fitter has been seeded with the true Monte Carlo variables. In this section, event displays from true seeded fits are shown for a few different event types. The initial energy of each track segment is extracted from the Monte Carlo, so $E_{\mu}, E_{\pi}$, and the energy of the downstream pion track all are set to their generated values. The kink location and the energy lost in the kink are more difficult to obtain, so these values are approximated by adding the energies for all GEANT-recorded particles created in the kink and extrapolating upstream from the energy of the pion that exits the interaction. As we will see in at least one example, this does not always account for all energy lost in the interaction (e.g. nuclear recoil is excluded).

The following types of seed / event relationships are displayed in this section:

(1) $\mathrm{A} \mathrm{CC} \pi^{+}$event with a high energy pion and a good seed (seed: Figure A.1, fit: Figure A.2);

(2) A low energy pion with a Cherenkov ring that overlaps with a high energy muon ring (seed: Figure A.3, fit: Figure A.4);

(3) An event with multiple hadronic interactions (seed: Figure A.5, fit: Figure A.6);

(4) A pion track where most of its energy is lost in the hadronic interaction (seed: Figure A.7, fit: Figure A.8); 
(5) A slightly incorrect "true" seed (seed: Figure A.9, fit: Figure A.10);

(6) A pion that does not experience a hadronic interaction (seed: Figure A.11, fit: Figure A.12); 

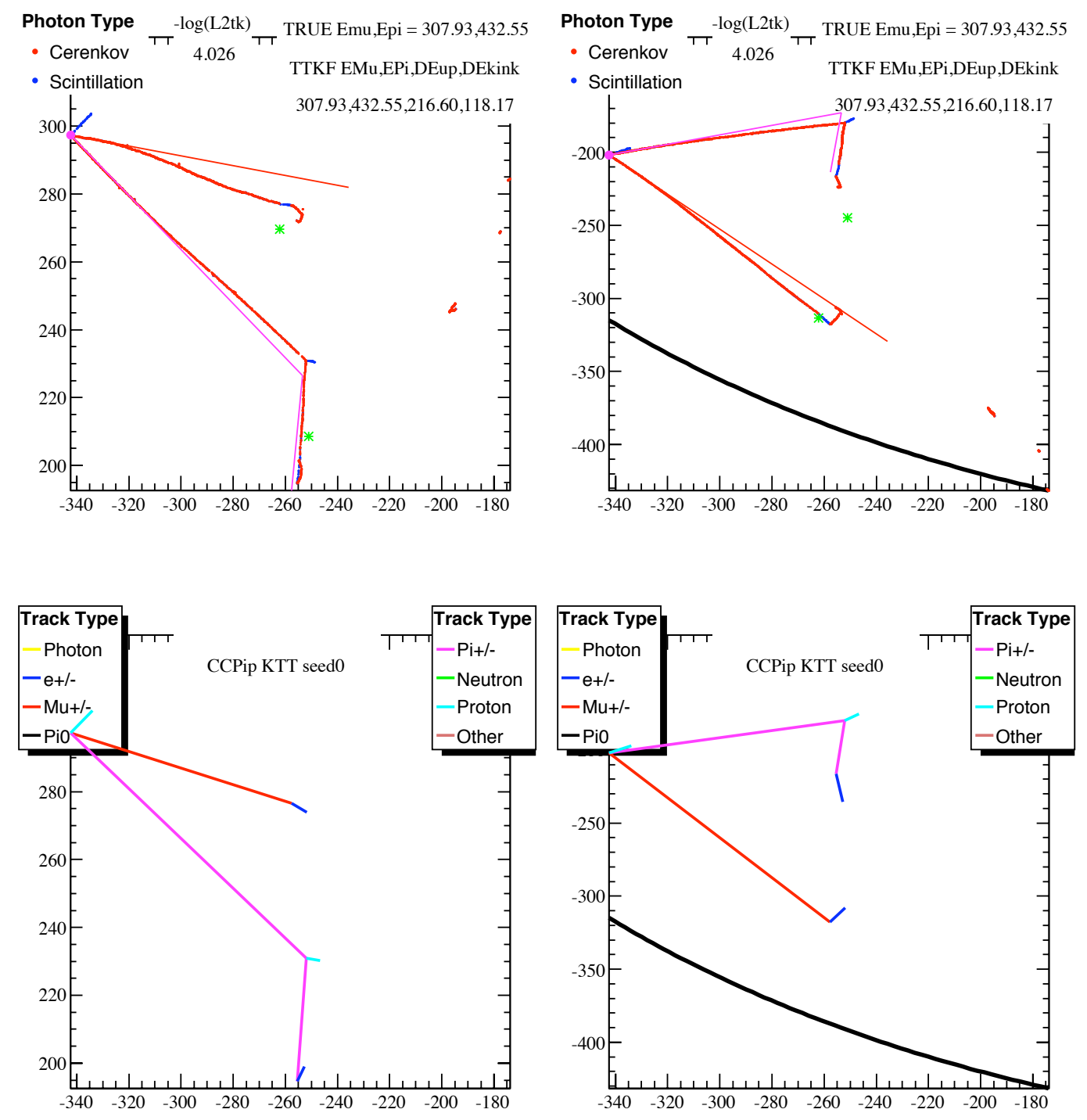

Figure A.1: The true seed for an event with a large pion energy. About half of the pion energy is lost in the upstream track segment, with about a quarter of the energy lost in both the kink and the downstream segment. The downstream segment is well above Cherenkov threshold. 

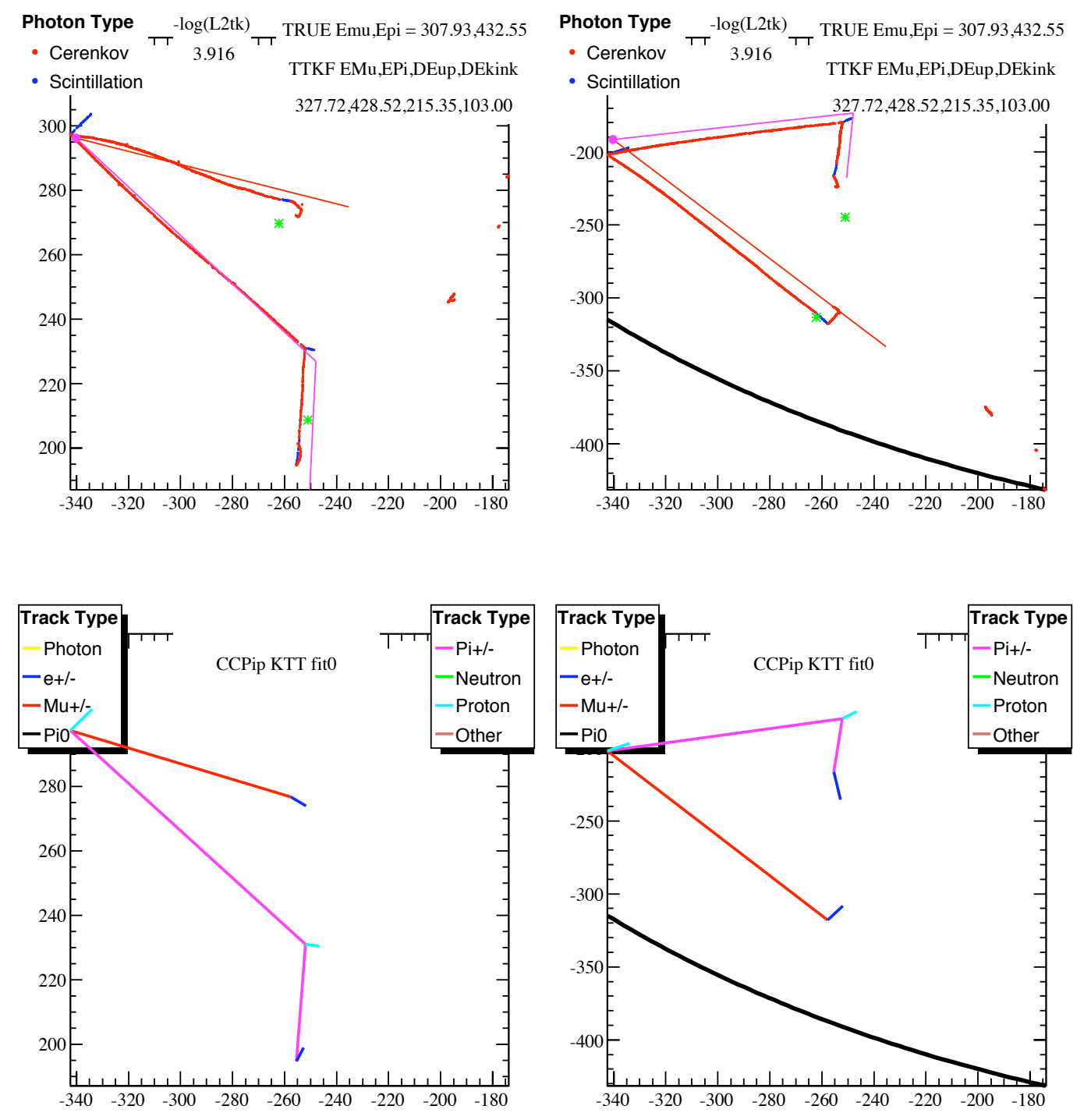

Figure A.2: A likelihood maximum clearly exists for this event. Almost no change has occurred in the track directions. A small amount of energy from the pion track (mostly the energy lost in the hadronic interaction) has been transferred to the muon track. 

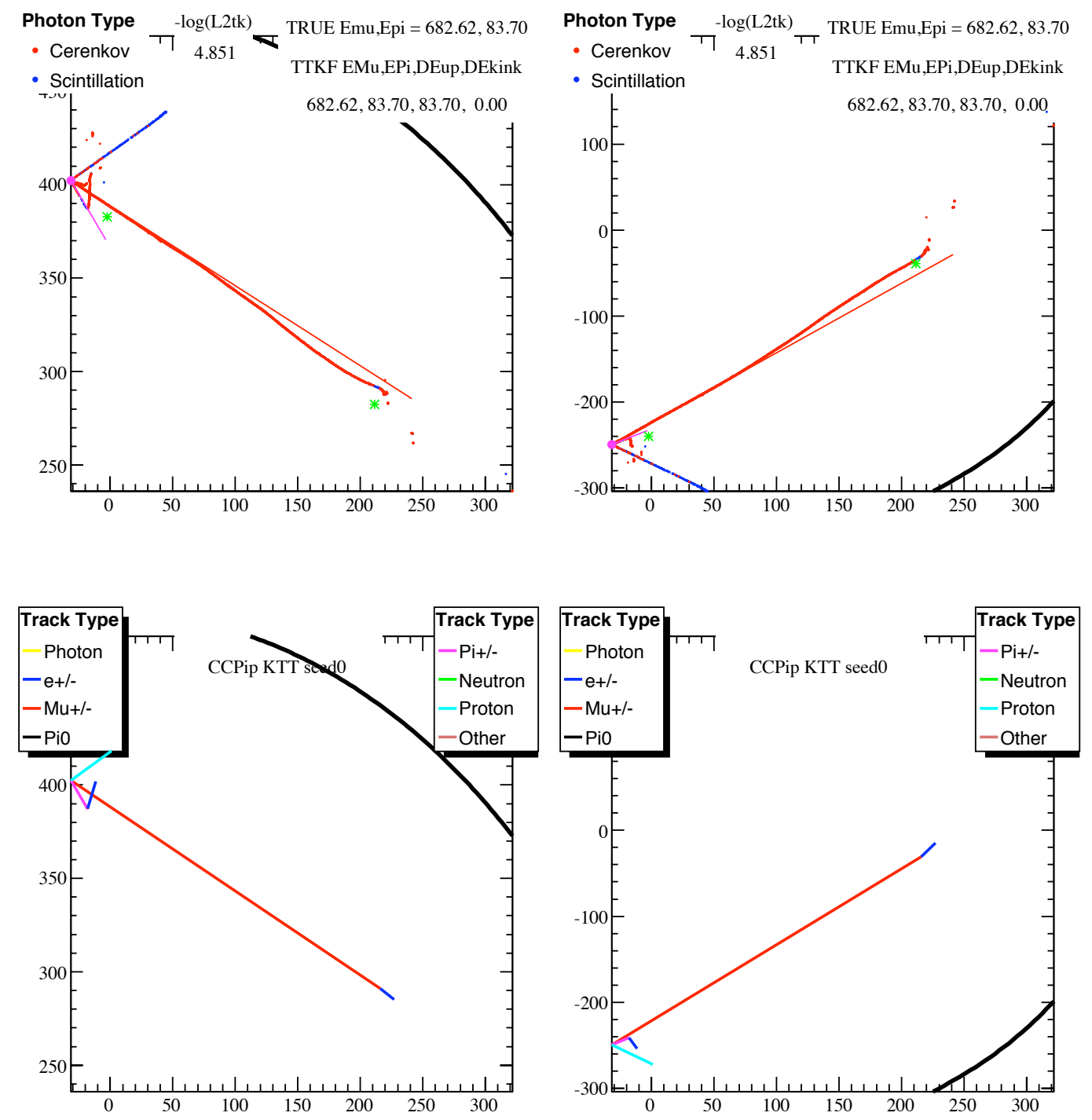

Figure A.3: A fit seed for a low energy, unkinked pion track that creates a Cherenkov ring that overlaps with the ring from a high energy muon track is shown. The Cherenkov emission angle at the upstream portion of the muon track is $~ 50$ degrees, and the angle between the two tracks is $\sim 15$ degrees. 

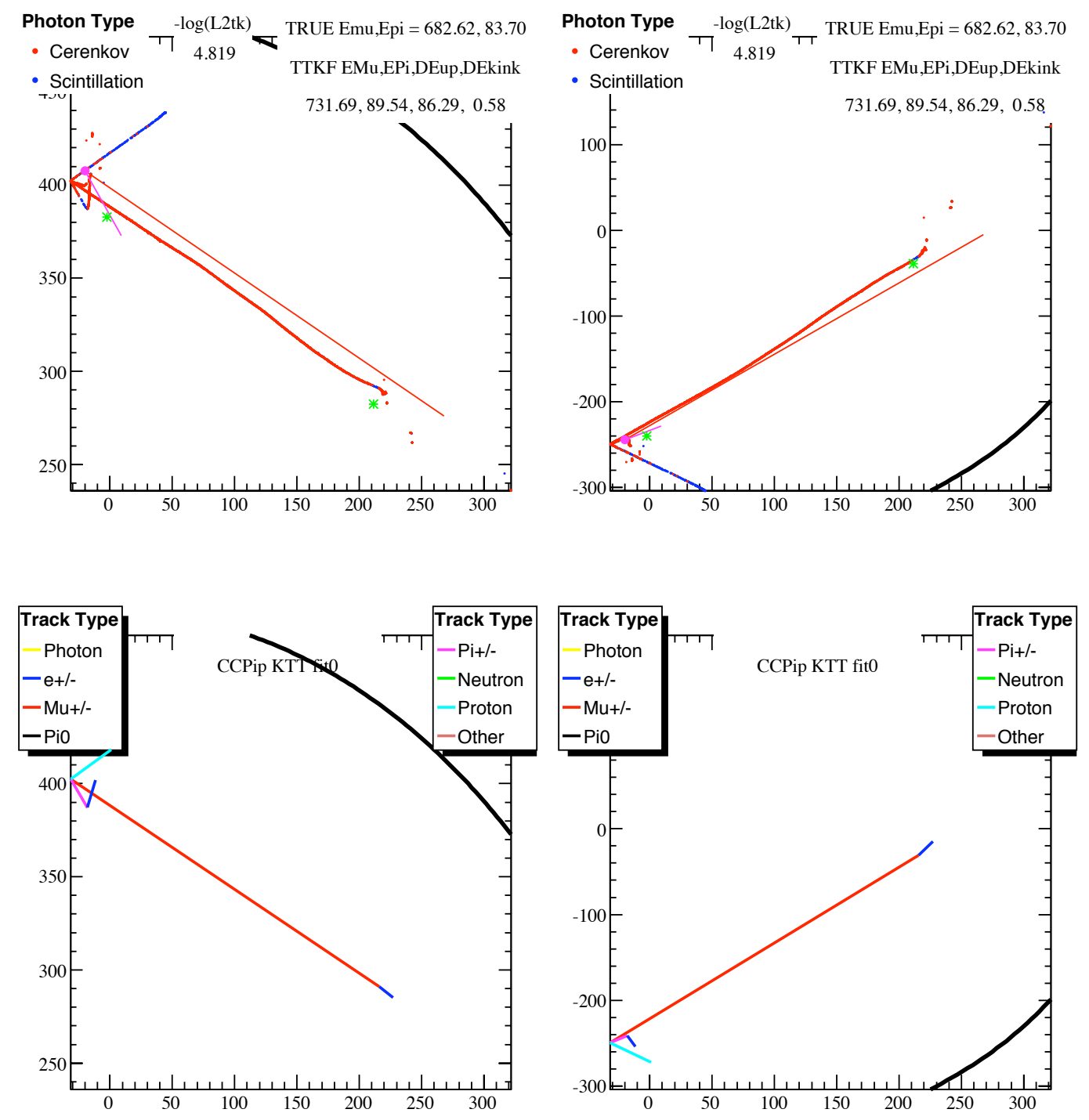

Figure A.4: Despite overlapping rings, there is still a likelihood maximum where both tracks are properly reconstructed. 

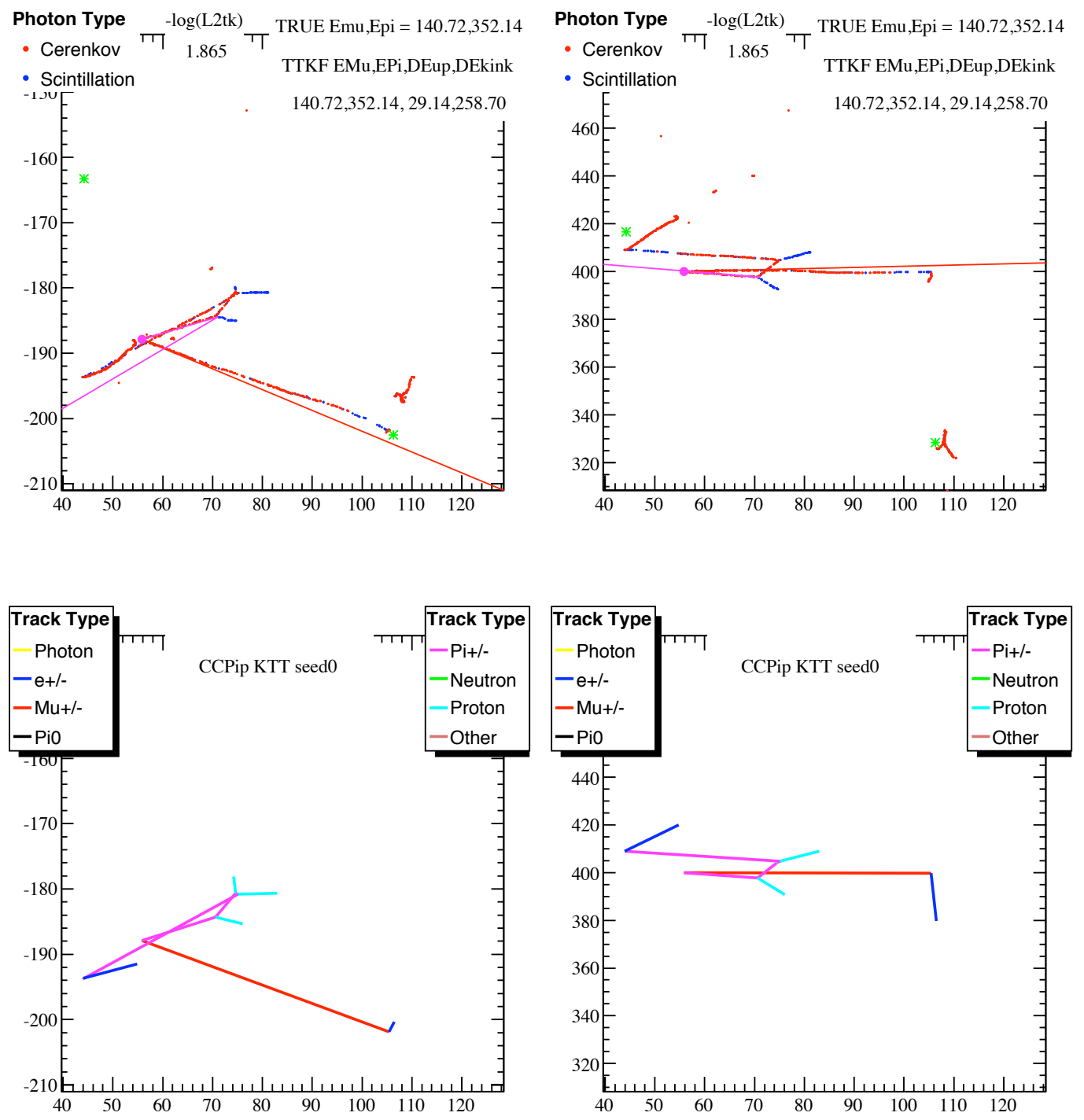

Figure A.5: A seed for an event with multiple hadronic interactions is shown. The "true" seed algorithm chooses the two longest tracks segments. The middle segment has been treated as part of the kink, which is why the "true" energy lost in the kink is so large. 

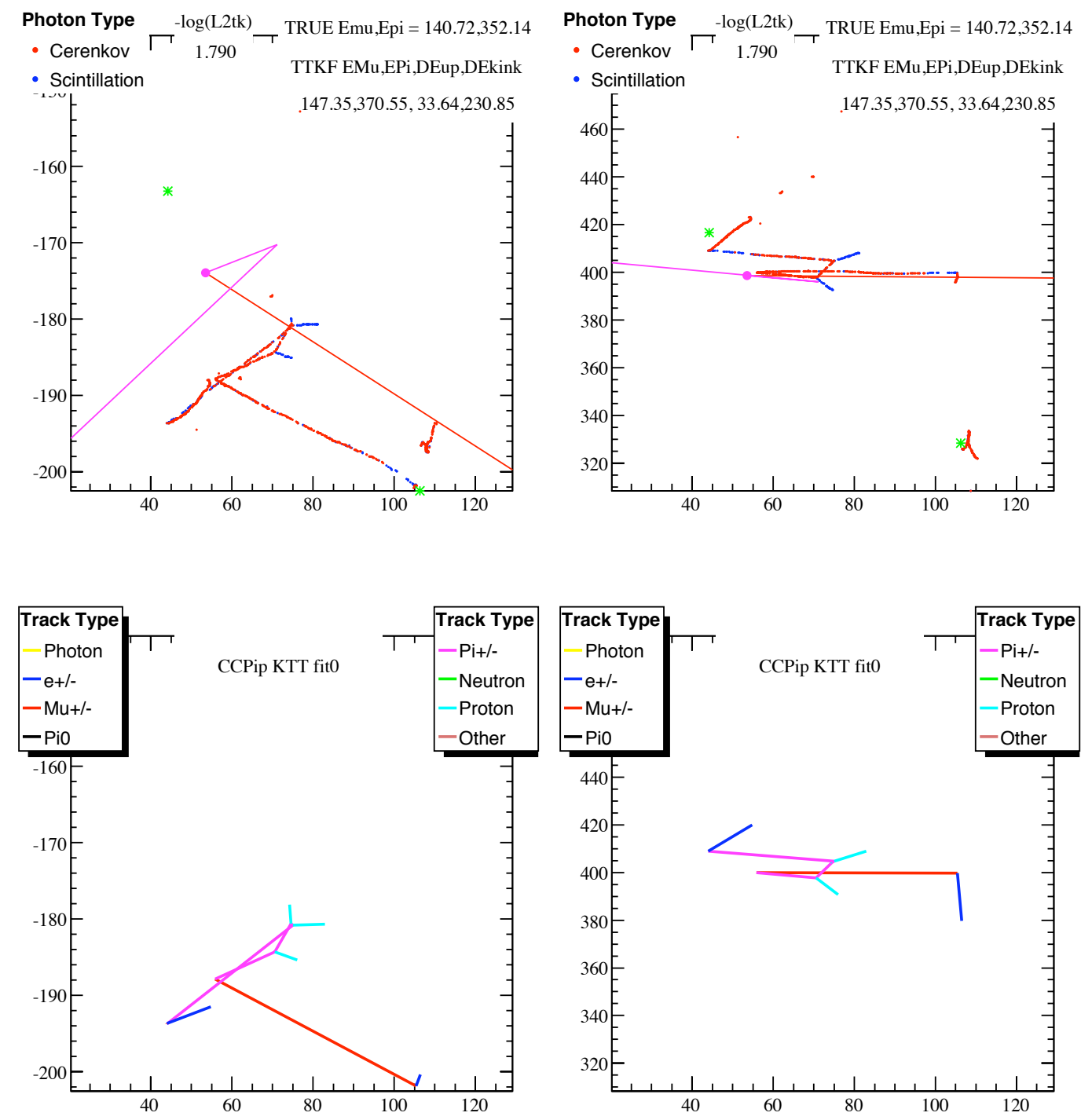

Figure A.6: The likelihood maximum was able to be properly reconstructed, in the sense that the physically interesting quantities are the initial energies and directions of each track. (Note: the reconstruction is less sensitive to the event vertex location.) 

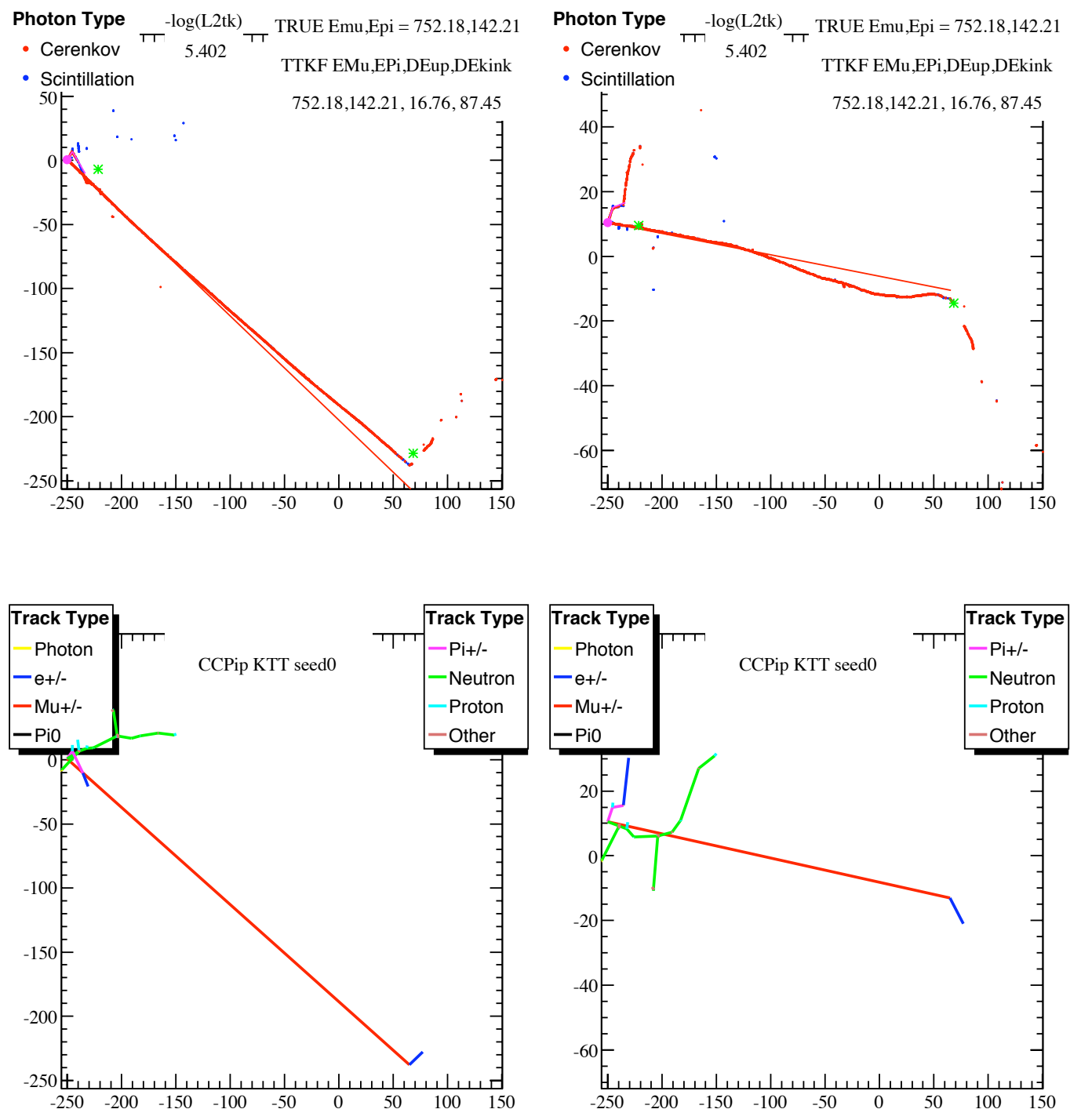

Figure A.7: More than half of the initial pion energy is lost in the hadronic interaction in this event, and the downstream segment emerges from the interaction below Cherenkov threshold. 

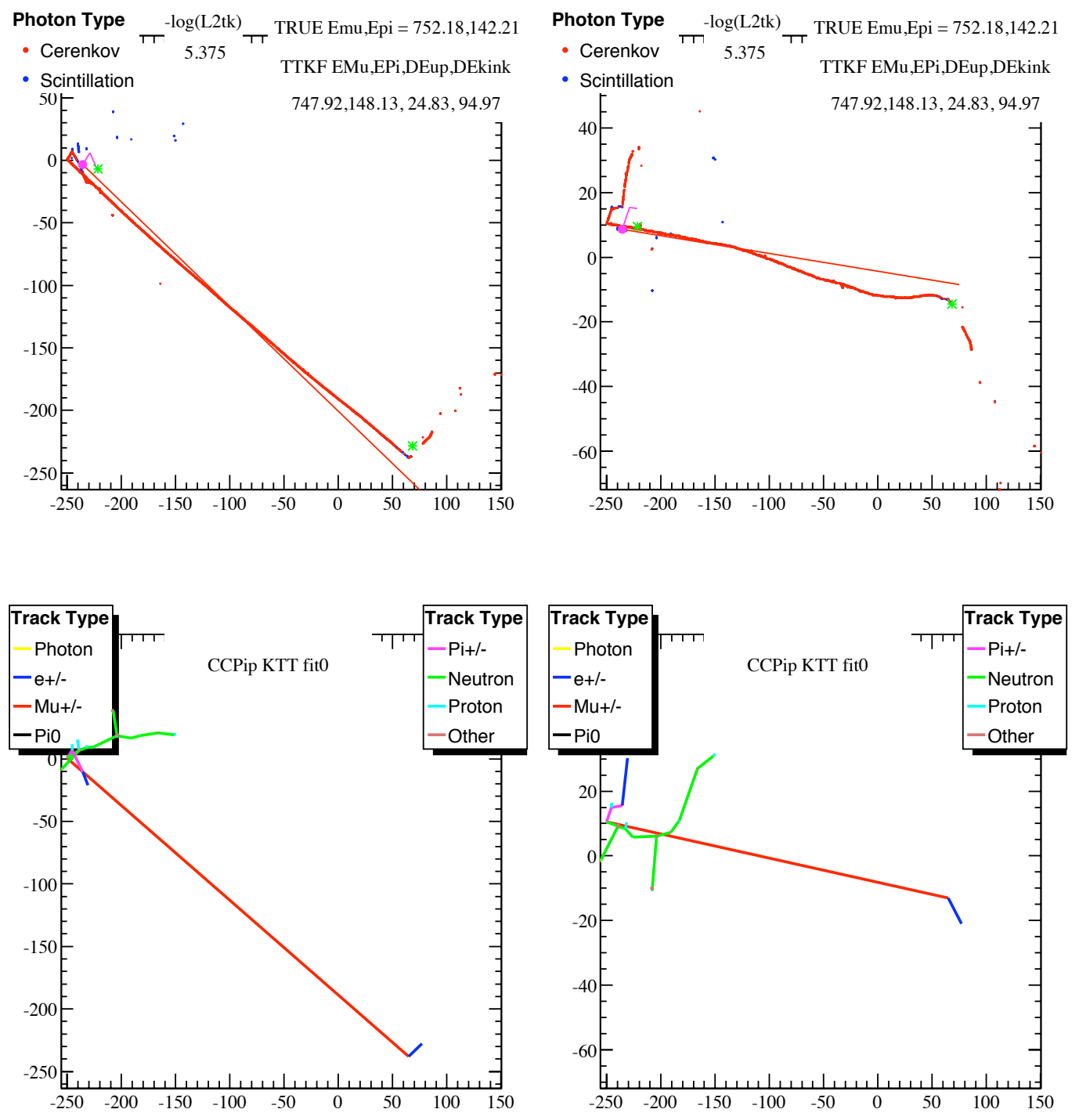

Figure A.8: The upstream pion segment is lengthened slightly, and the energy lost in the kink is increased somewhat, but the energy separation between the pion and the muon remains mostly unchanged. 

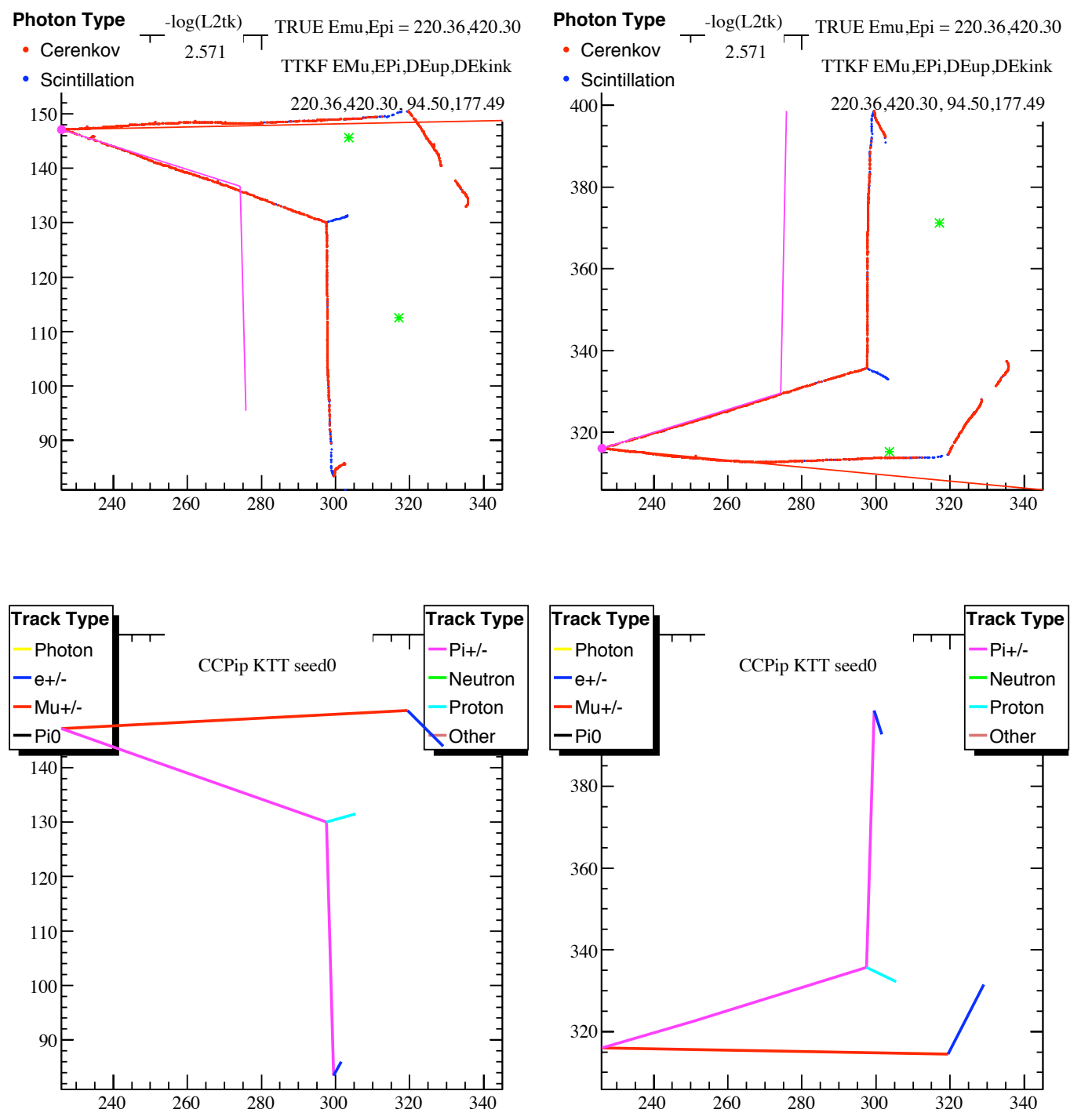

Figure A.9: This seed underestimates the energy lost in the upstream pion segment, and instead puts all of the excess energy into the hadronic interaction. 

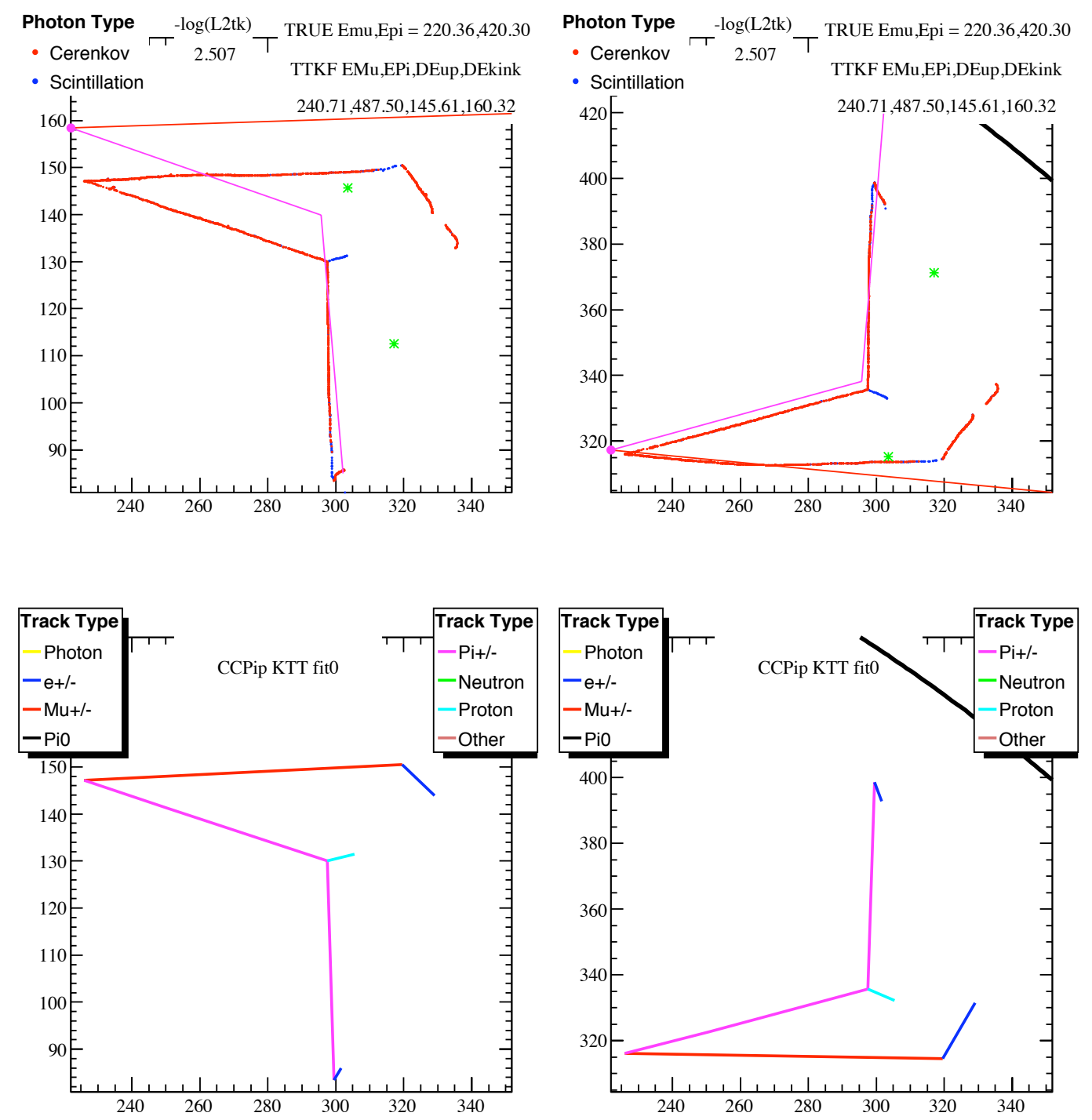

Figure A.10: The fitter has added the extra $50 \mathrm{MeV}$ required for the upstream track segment, but only $17 \mathrm{MeV}$ of that total has been taken from the hadronic energy loss. The downstream segment has also increased, largely due to a lack of constraints due to the close proximity of the tank wall, which then forces the total pion energy to be increased. 


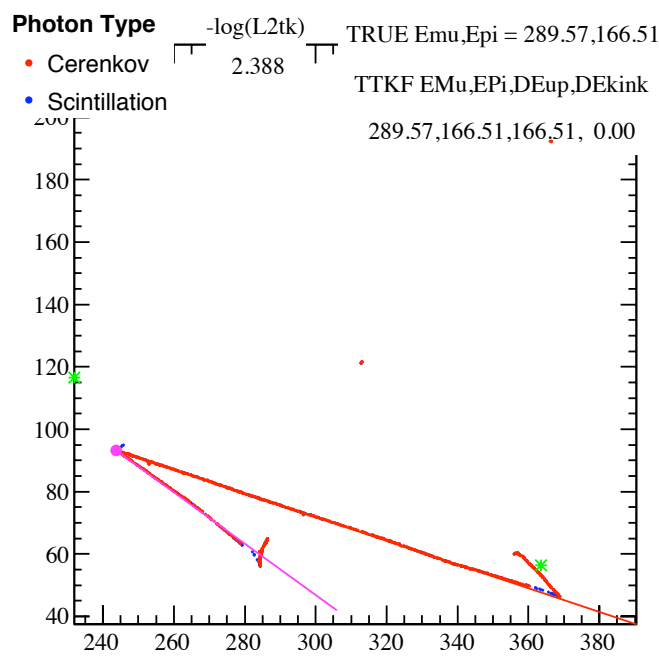

$\begin{aligned} & \text { Photon Type } \\ & \text { - Cerenkov } \\ & \text { - Scintillation }\end{aligned}$
-
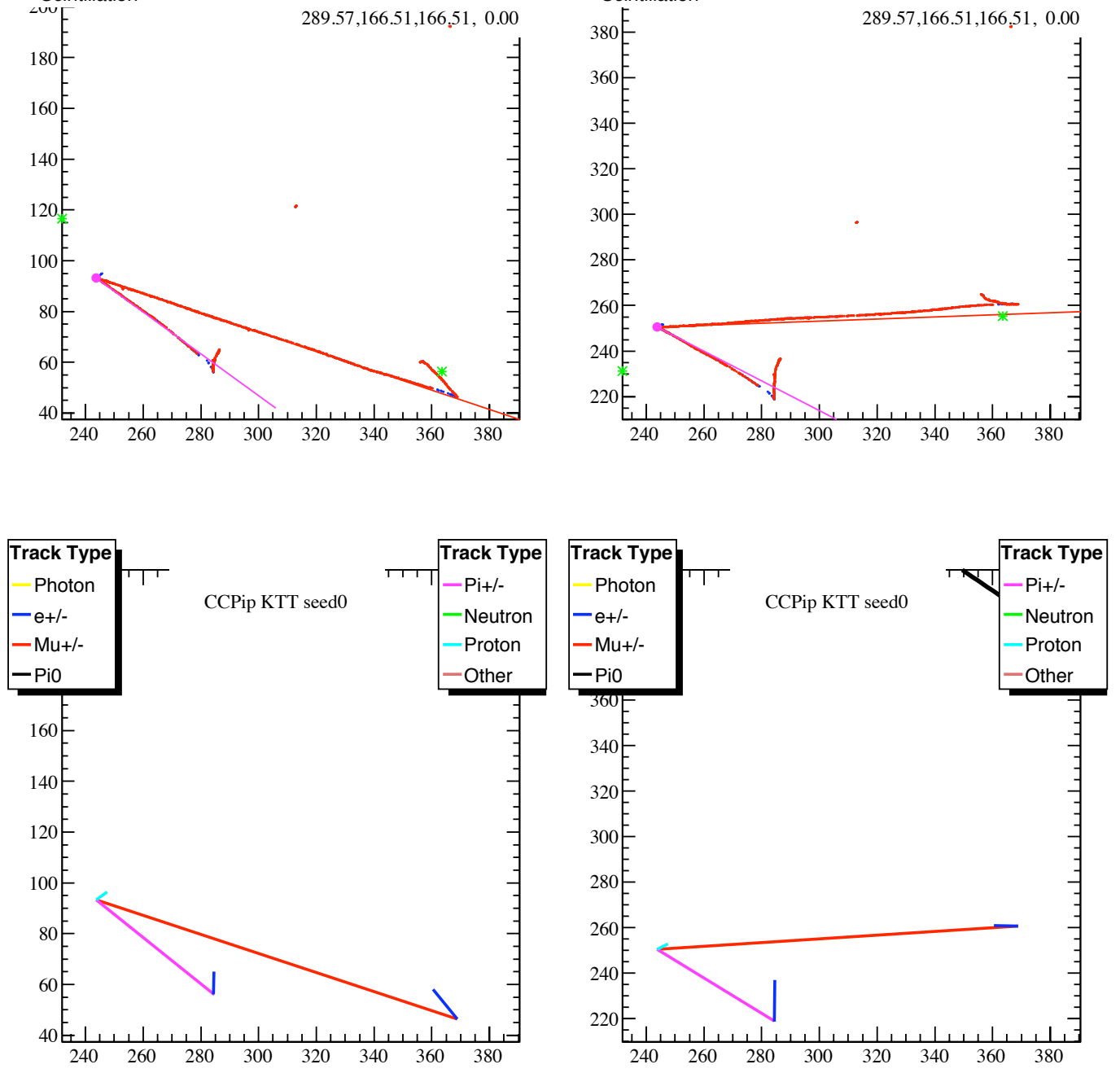

Figure A.11: This seed is for an event in which the pion does not experience a hadronic interaction. 


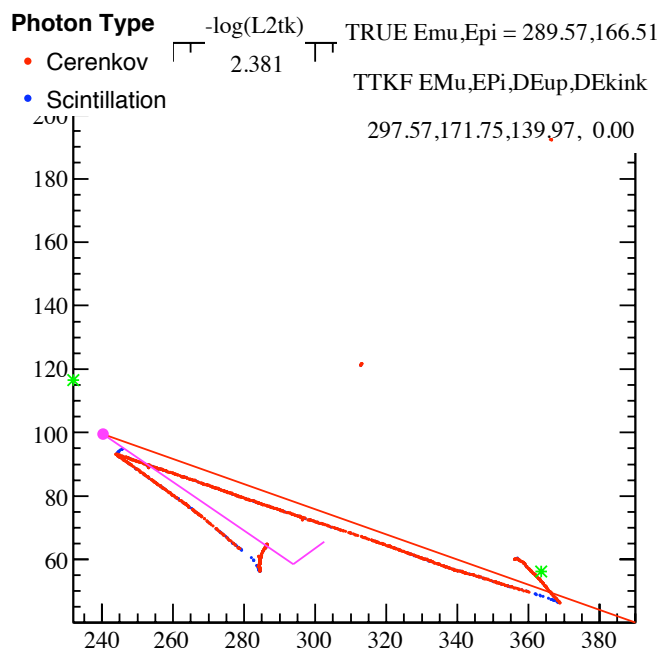
Photon Type $T^{-\log (\text { L2tk })}$ TRUE Emu,Epi $=289.57,166.51$
- Cerenkor ГT 2.381 TT
- Scintillation

$297.57,171.75,139.97,0.00$
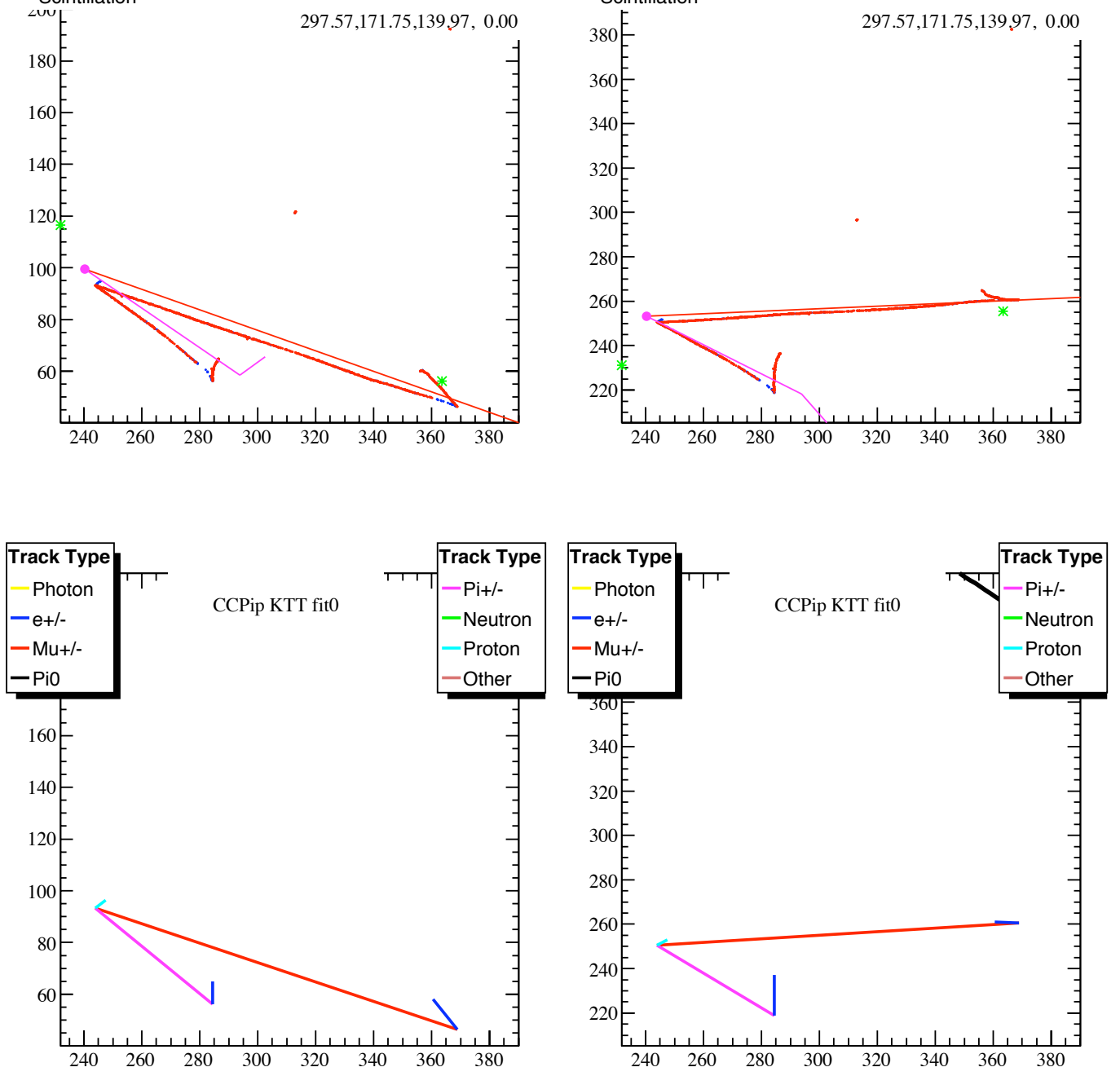

Figure A.12: A kink has been introduced to the pion trajectory, but it occurs after the track has crossed below Cherenkov threshold where there are no constraints on the track direction. 


\section{A.2 A CC $\pi^{+}$Fit Example}

A series of event displays highlighting the $\mathrm{CC} \pi^{+}$seeding and fitting process are presented in this section. A description and justification for the following sequence of events is given in Section 5.3.1. A summary of the steps and corresponding figures is given below.

(1) Figure A.13: one track fit.

(2) Figure A.14: two track likelihood scan.

(3) Figure A.15: two track fit.

(4) Figure A.16: three track likelihood scan.

(5) Figure A.17: three track fit.

(6) Figure A.18: $\mathrm{CC} \pi^{+}$seed configuration \#1

(7) Figure A.19: $\mathrm{CC} \pi^{+}$fit $\# 1(-\log (\mathcal{L})=3.112$; not accepted $)$

(8) Figure A.20: $\mathrm{CC} \pi^{+}$seed configuration \#2

(9) Figure A.21: $\mathrm{CC} \pi^{+}$fit $\# 2(-\log (\mathcal{L})=3.076$; not accepted $)$

(10) Figure A.22: $\mathrm{CC} \pi^{+}$seed configuration \#3

(11) Figure A.23: $\mathrm{CC} \pi^{+}$fit \#3 $(-\log (\mathcal{L})=2.982$; accepted $)$ 

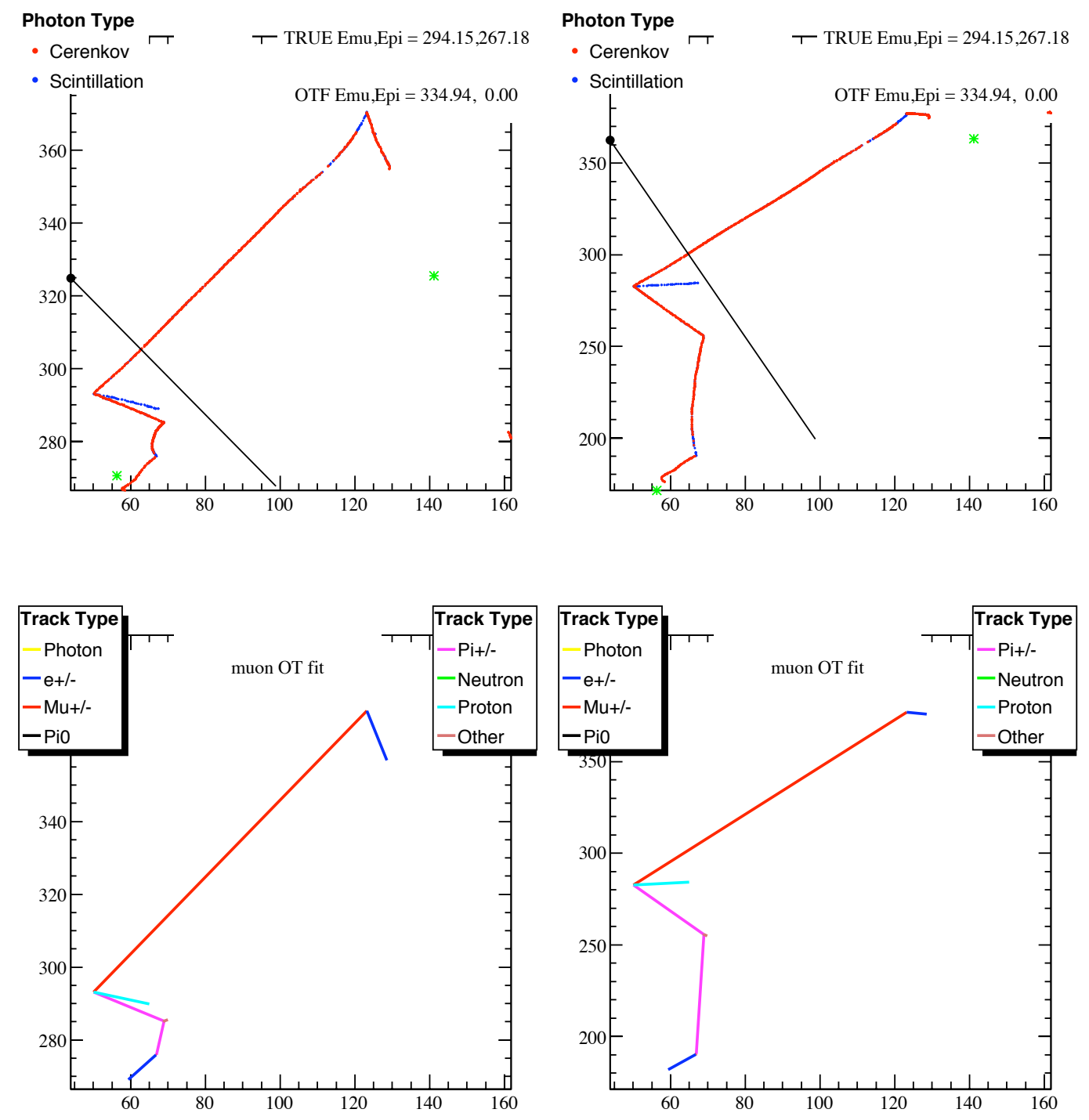

Figure A.13: $\mathrm{CC} \pi^{+}$fit/seeding step 1: the one track fitter has found the ring belonging to the upstream segment of the kinked pion track. The fit energy overestimates the pion energy in an attempt to account for the additional light created by the other two tracks in the event. 

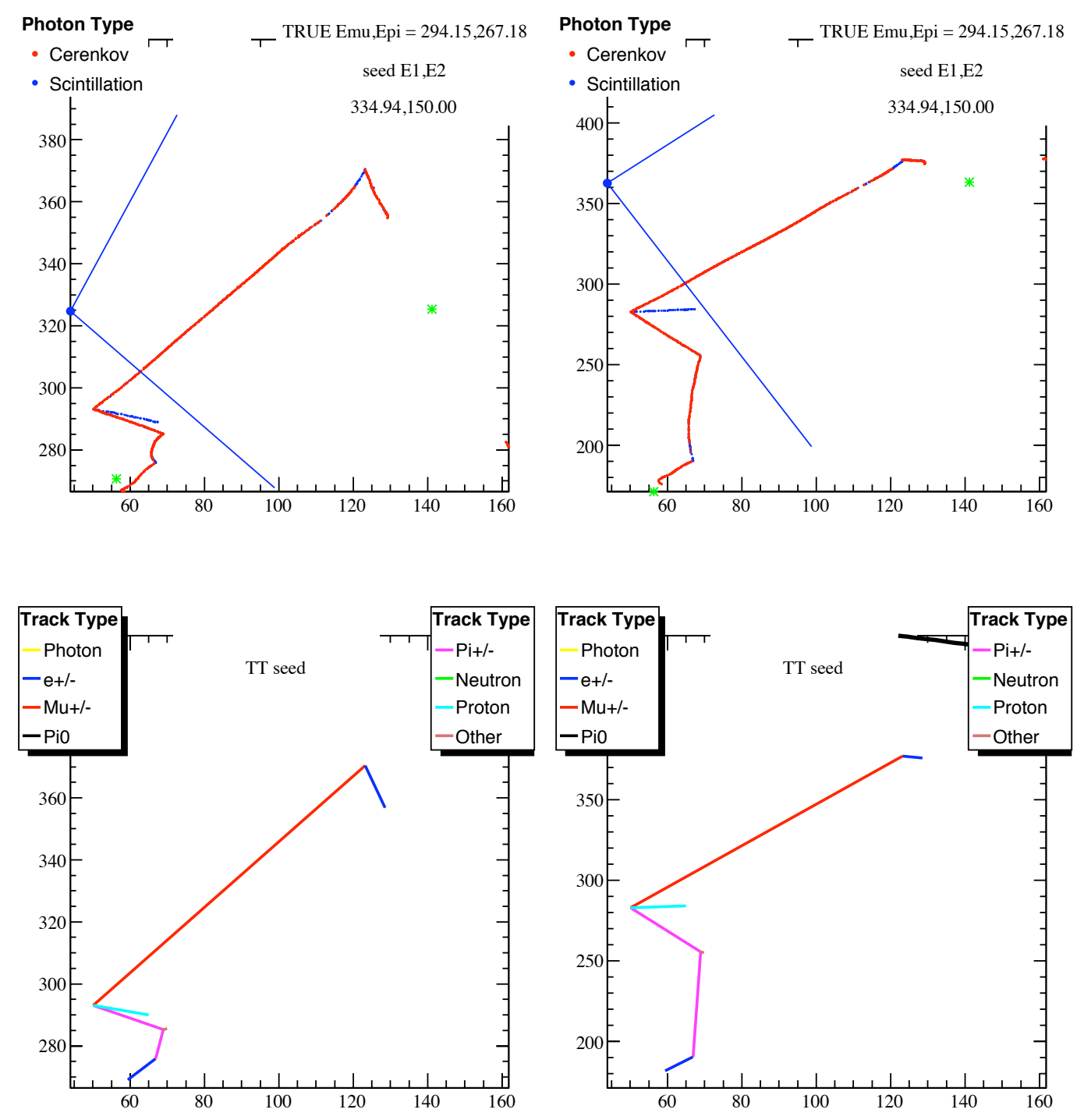

Figure A.14: $\mathrm{CC} \pi^{+}$fit/seeding step 2: the likelihood scan for the second track (keeping the result of the one track fit fixed) has found the muon. The scan was performed with a track energy of $150 \mathrm{MeV}$, although $200 \mathrm{MeV}$ is used in the final version of the fitter. Although the scan found a ring, the track direction isn't perfect. The underestimate for the track energy means that the predicted ring diameter is smaller than the actual ring diameter, so the scan has some freedom in choosing the actual track direction. 

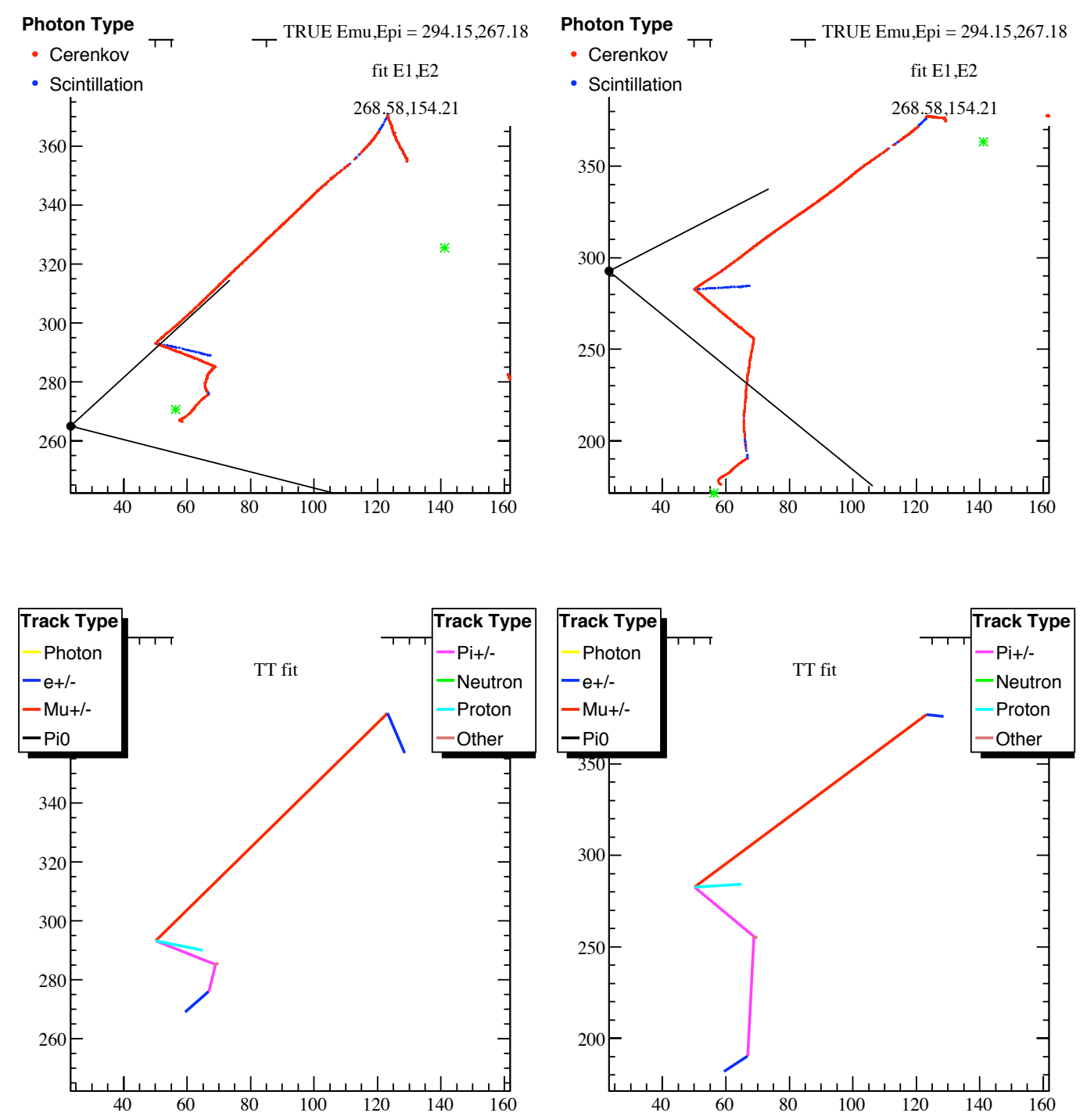

Figure A.15: $\mathrm{CC} \pi^{+}$fit/seeding step 3: the two track fit has corrected the directions of both tracks to point at the center of their respective rings. The energy overestimate from the one track fit has been greatly reduced, although the second track energy has only been slightly increased so far. 

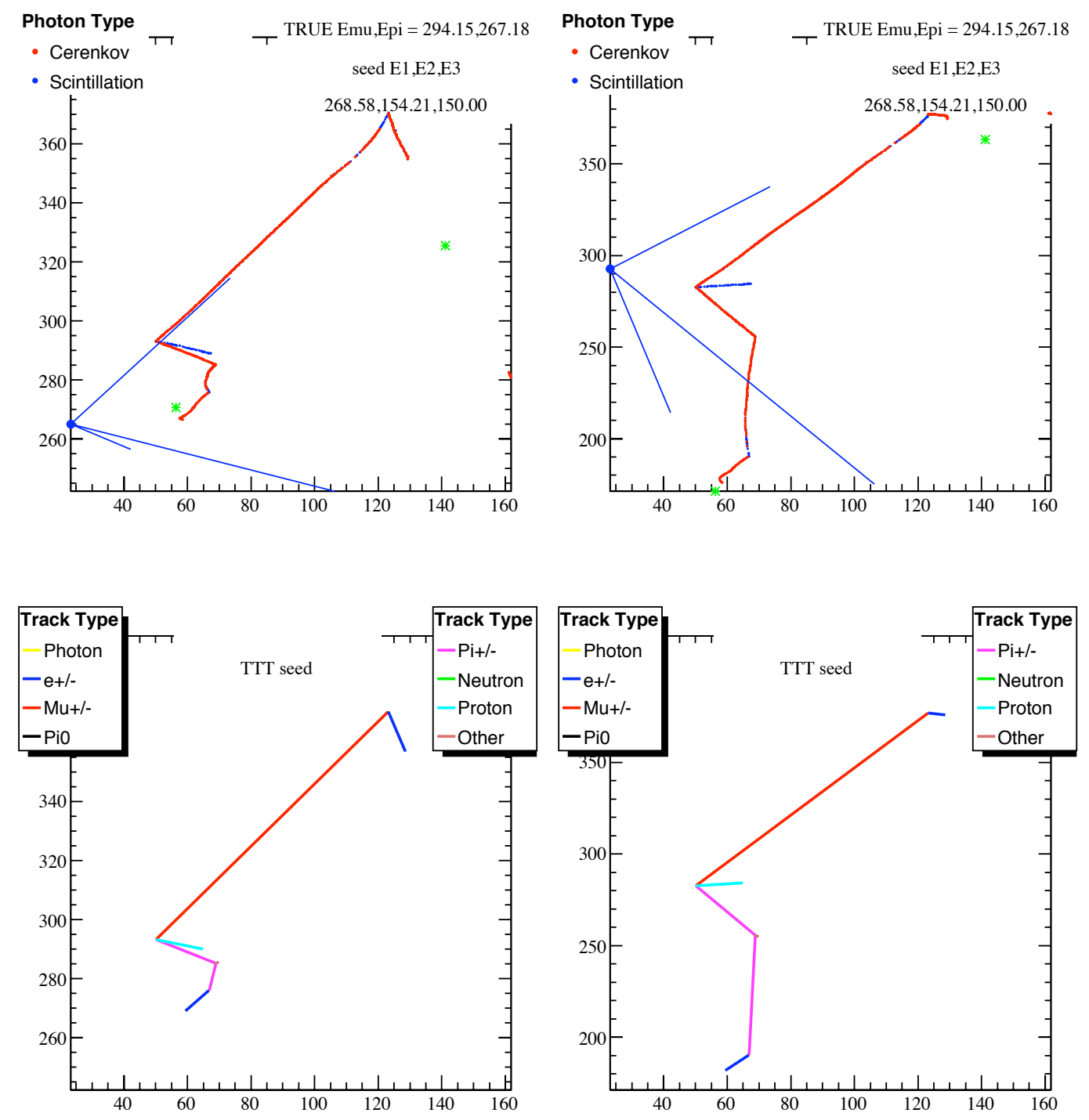

Figure A.16: $\mathrm{CC} \pi^{+}$fit/seeding step 4: the three track likelihood scan has landed near the most downstream portion of the downstream pion track. Some portion of the upstream pion ring may have pulled the scan slightly toward the upstream pion track. 

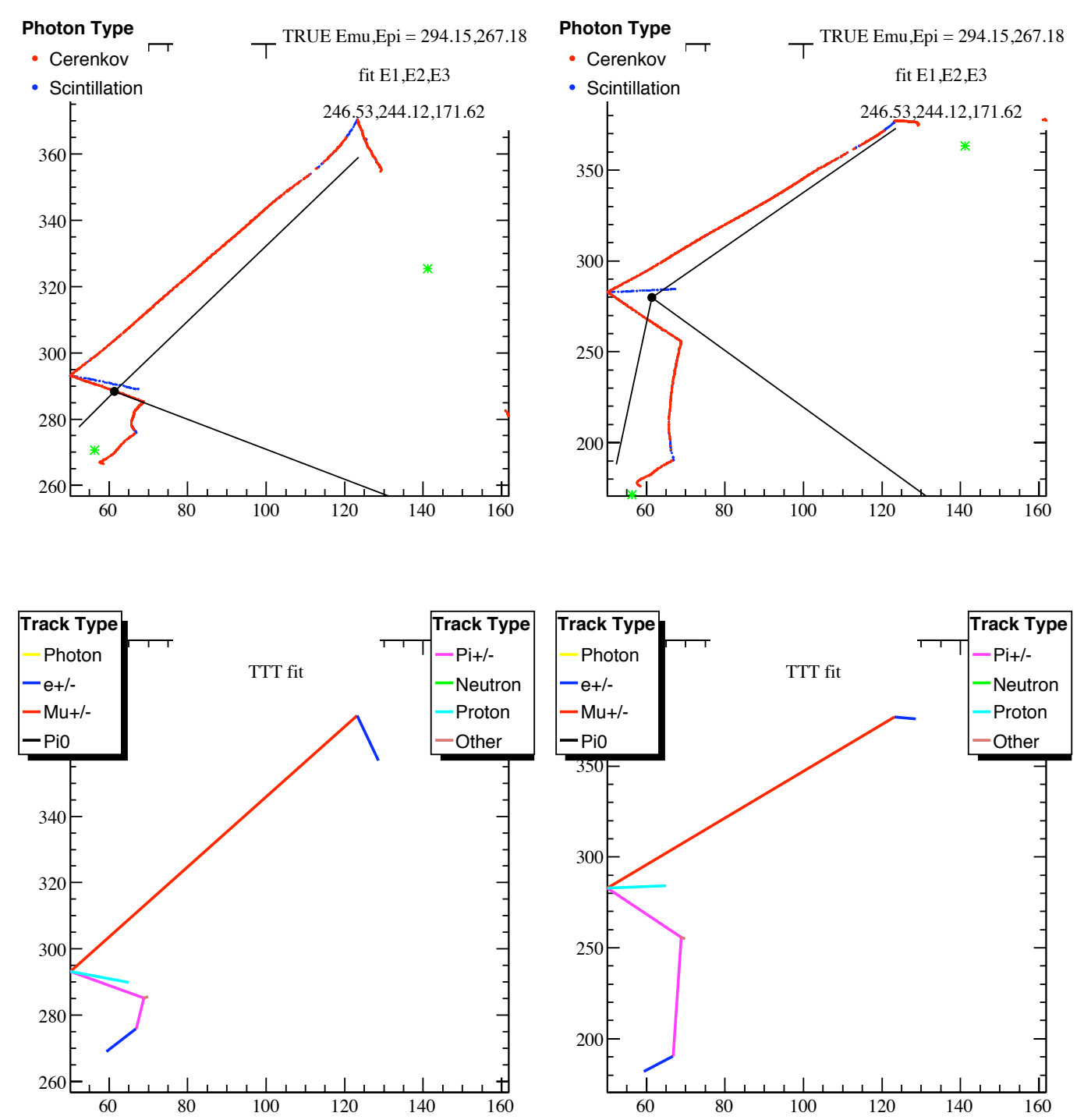

Figure A.17: $\mathrm{CC} \pi^{+}$fit/seeding step 5: the three track fit has corrected the final scan track to point in the initial direction of the downstream pion segment. The energy has also been redistributed among the three tracks to more accurately represent the size of the Cherenkov rings. The first two tracks now only slightly underestimate the true muon and pion energies. 

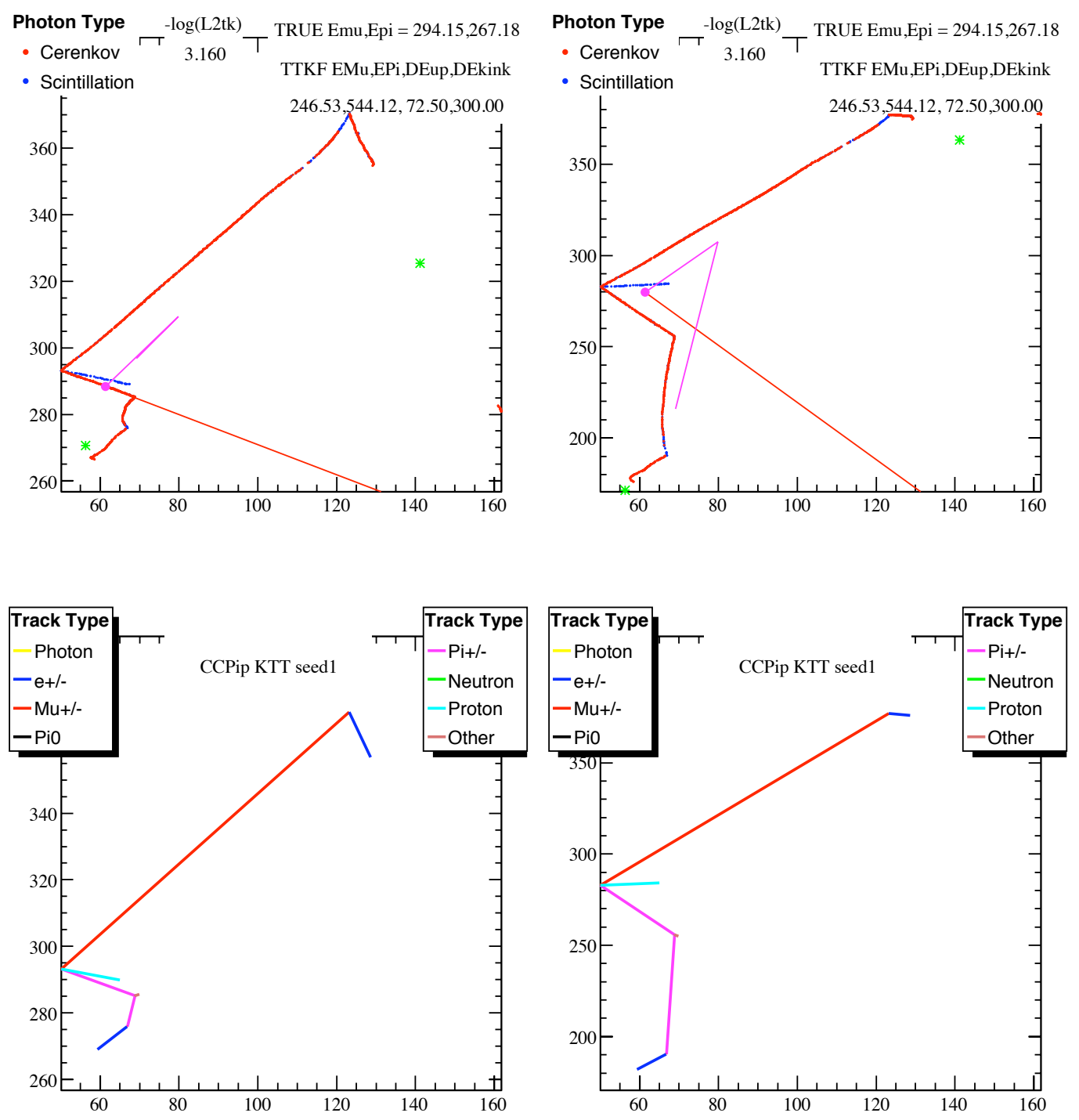

Figure A.18: $\mathrm{CC} \pi^{+}$fit/seeding step 6: the first of the three seed configurations has picked the incorrect tracks for the muon and upstream pion segments. Since the upstream pion segment does not actually point at a pion track segment, the simultaneous $\Delta E_{u p} / \Delta E_{k i n k}$ scan has incorrectly placed the energy lost in the kink at $300 \mathrm{MeV}$. 

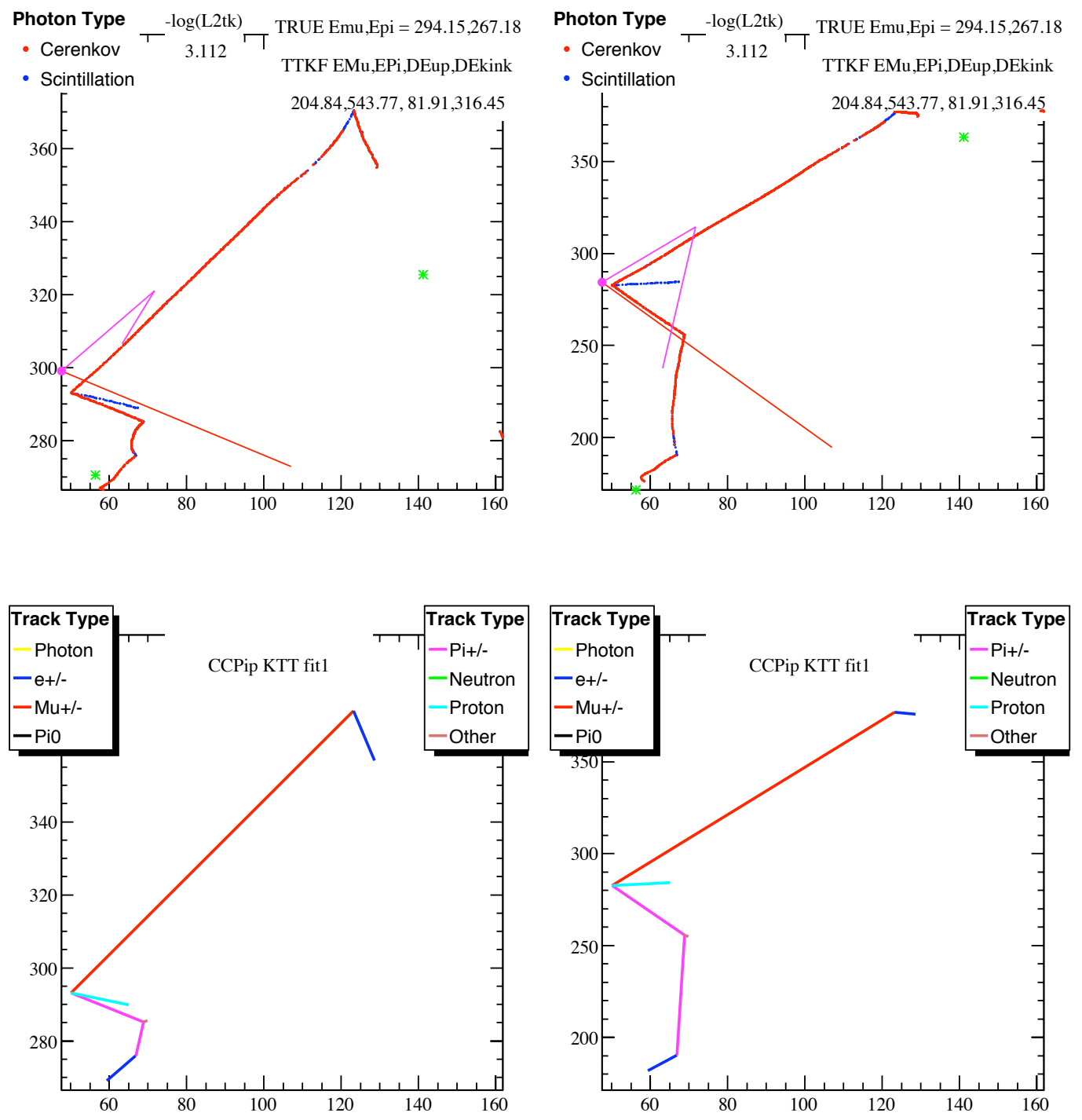

Figure A.19: $\mathrm{CC} \pi^{+}$fit/seeding step 7: the fit result for the incorrect seed in Figure A.18 is still incorrect, as expected. The fit minimum is: $-\log (\mathcal{L})=3.112$. 

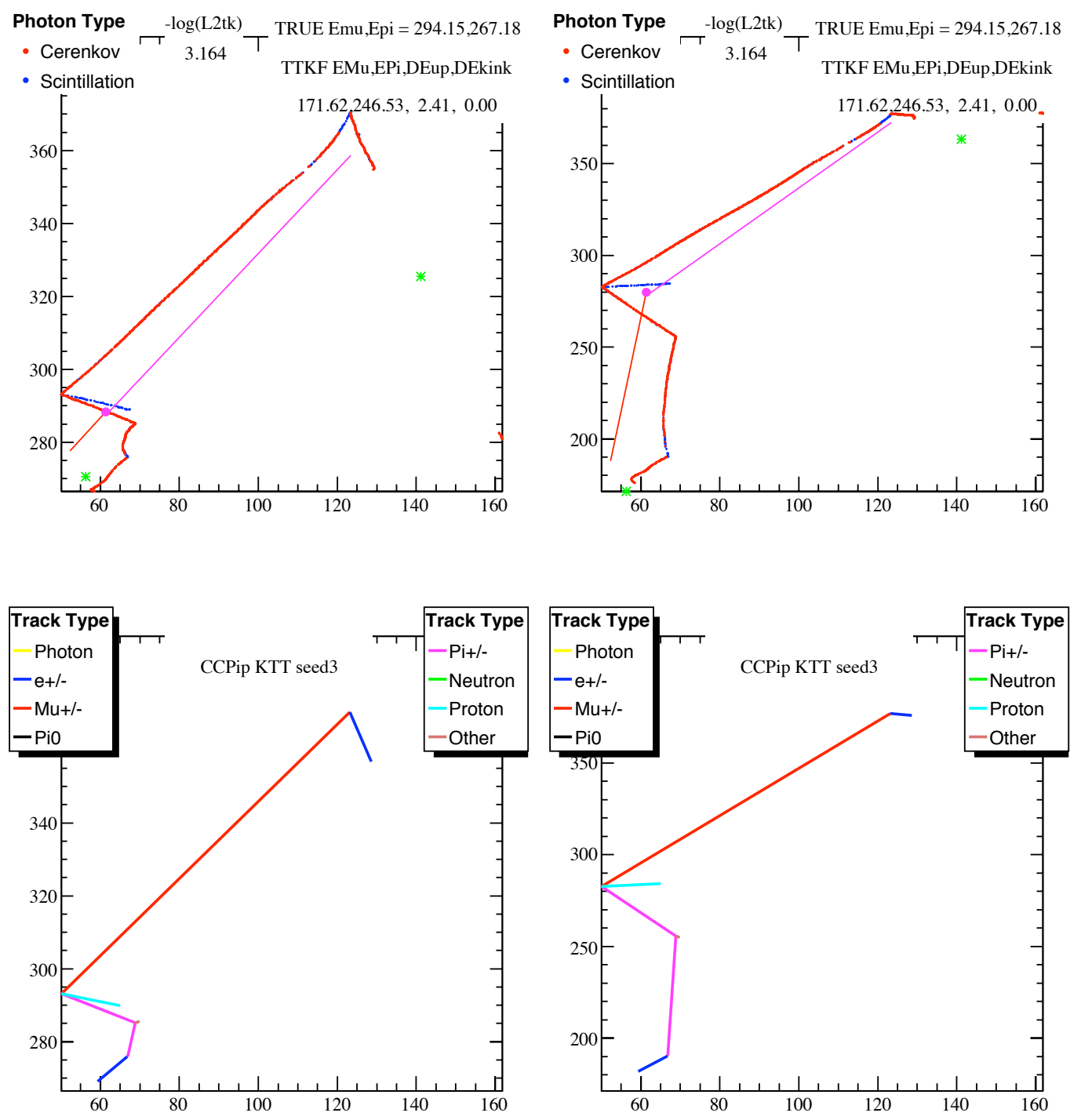

Figure A.20: $\mathrm{CC} \pi^{+}$fit/seeding step 8: the second of the three seed configurations has picked the incorrect tracks for the muon and downstream pion segments. 

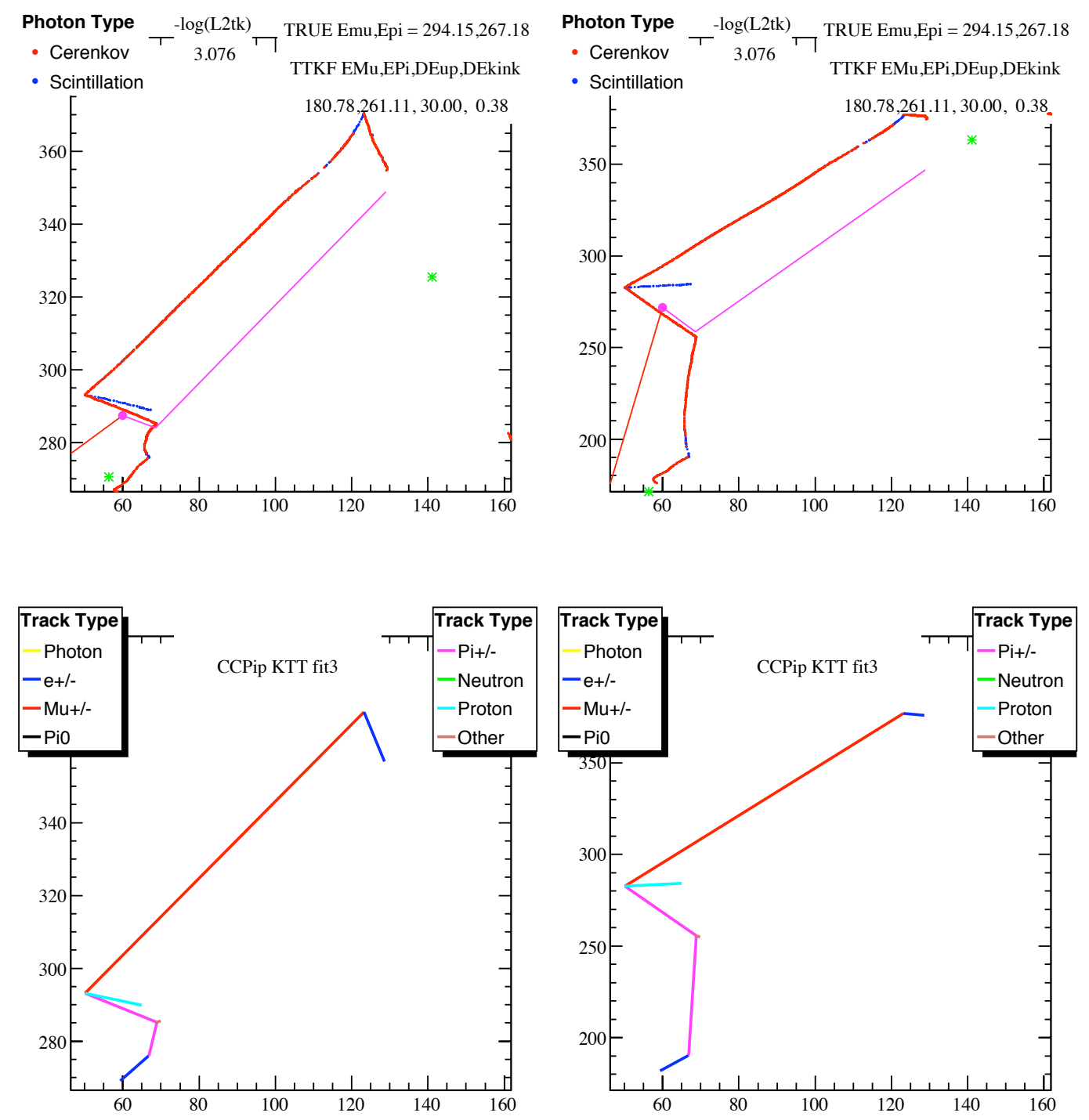

Figure A.21: $\mathrm{CC} \pi^{+}$fit/seeding step 9: the fit result for the incorrect seed in Figure A.20 is still incorrect, as expected. The fit minimum is: $-\log (\mathcal{L})=3.076$ 

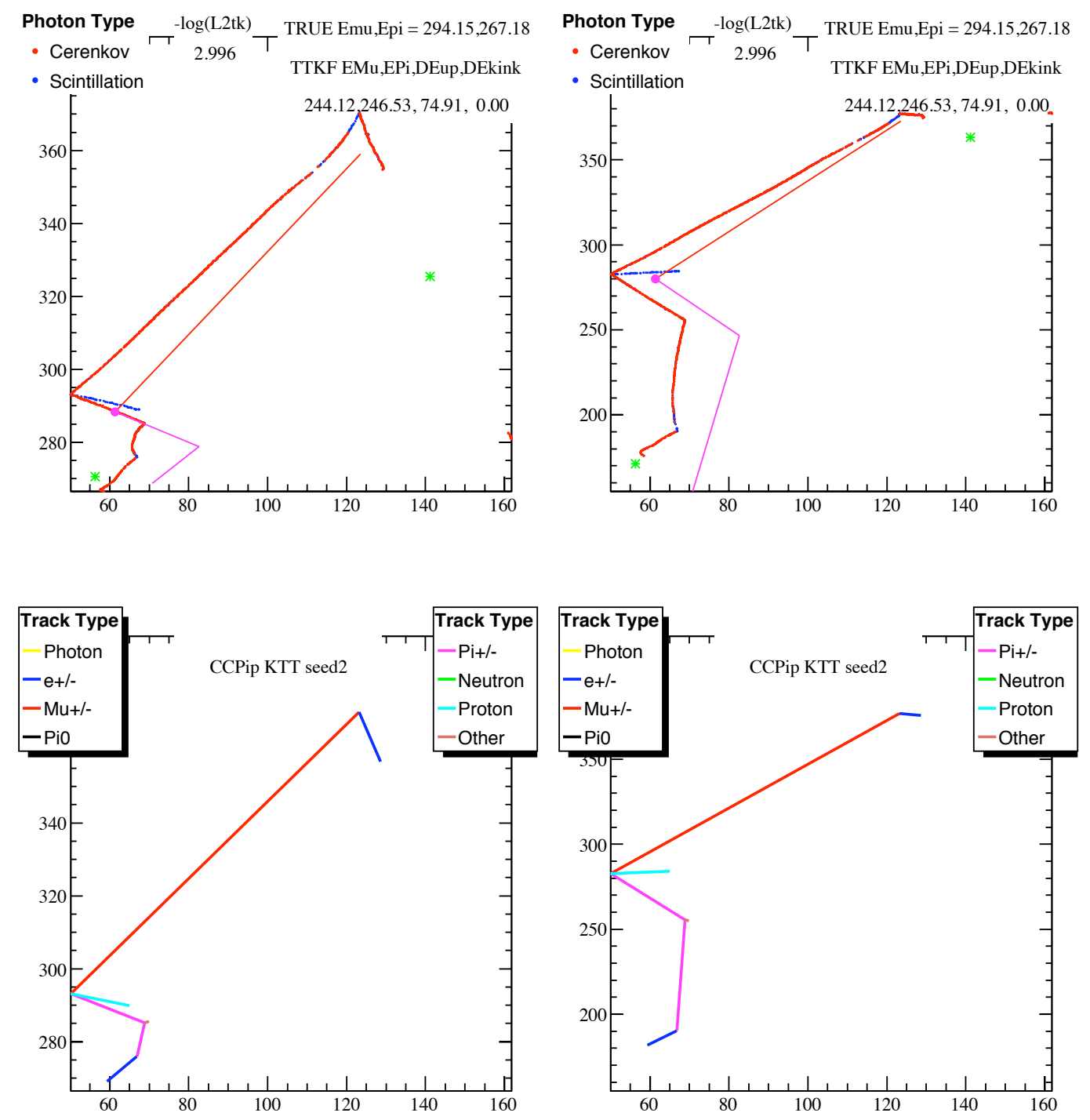

Figure A.22: $\mathrm{CC} \pi^{+}$fit/seeding step 10: the last $\mathrm{CC} \pi^{+}$seed configuration correctly assigns all three tracks. 

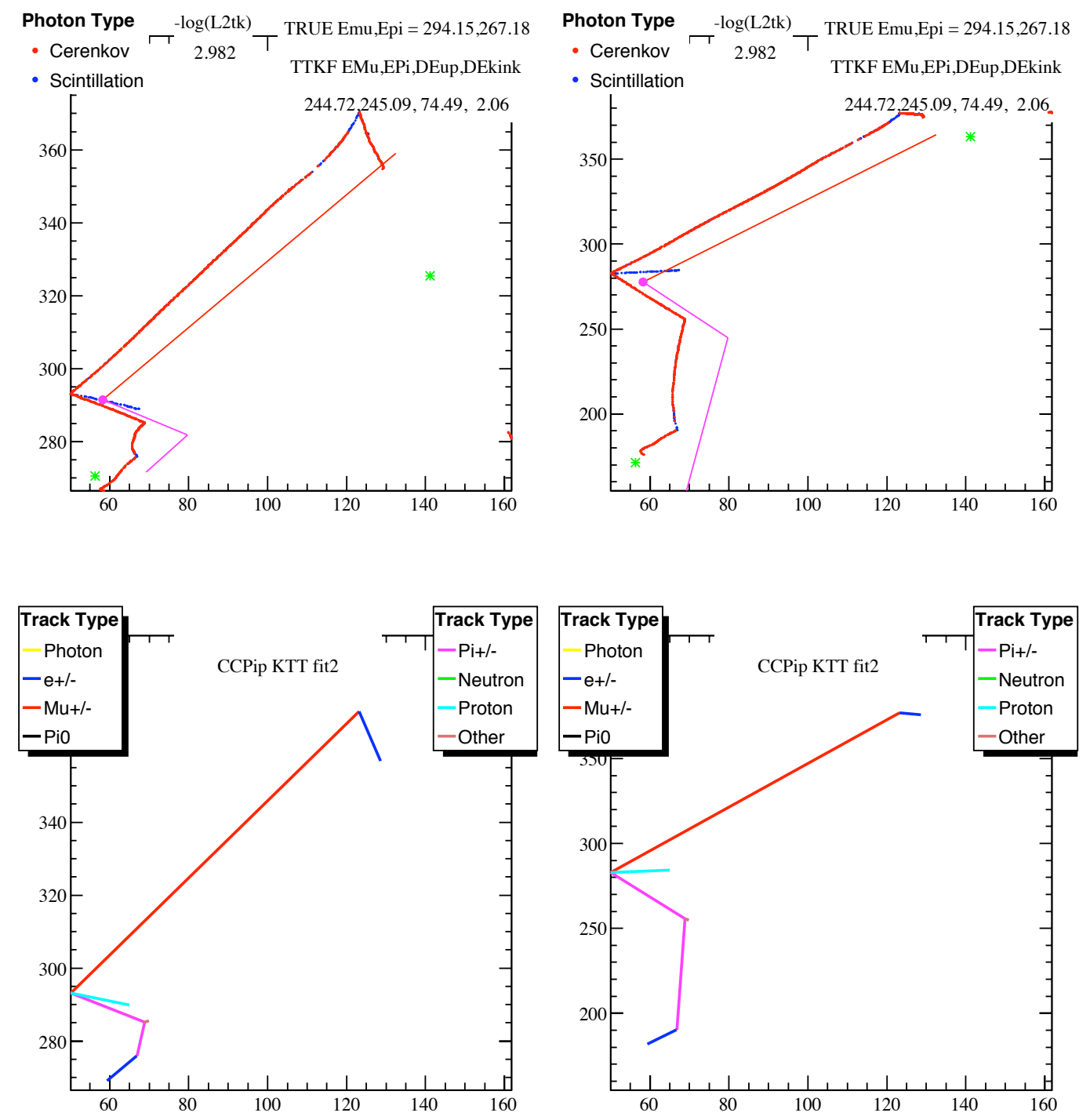

Figure A.23: $\mathrm{CC} \pi^{+}$fit/seeding step 10: the $\mathrm{CC} \pi^{+}$fit from the correct seed gives the best fit, $-\log (\mathcal{L})=2.982$. 


\section{Appendix B}

\section{Measurement Details}

Further details of the cross section measurements present in Section 6.4.8 are given in this appendix. For each measurement, signal efficiency, signal purity, and a comparison between the reconstructed data and Monte Carlo are given. For the one-dimensional measurements, the unfolding matrix is also shown. One additional data/Monte Carlo comparison, the reconstructed $\pi+N$ mass, is also presented.

Unlike the measured cross sections, the uncertainties in the reconstructed Monte Carlo are dominated by the optical model and cross section errors. The dominant effects in each of these error sources are pion absorption and charge exchange. Pion interactions in the nucleus have a very small effect on the measured cross sections because an event is only labeled as signal if a pion exits the nucleus. As described in Section 4.2.3, pion interactions that occur in the nucleus are (somewhat counter-intuitively) grouped with the optical model errors and all others are part of the cross section errors. In addition, the statistical uncertainty also contributes to the optical model errors, as described in Section 6.4.7.2. 


\section{B.1 Neutrino Energy}

- Reconstructed Data/Monte Carlo comparison: Figure B.1

- Unfolding Matrix: Figure B.2(b)

- Signal efficiency: Figure B.3(a)

- Signal purity: Figure B.3(b) 

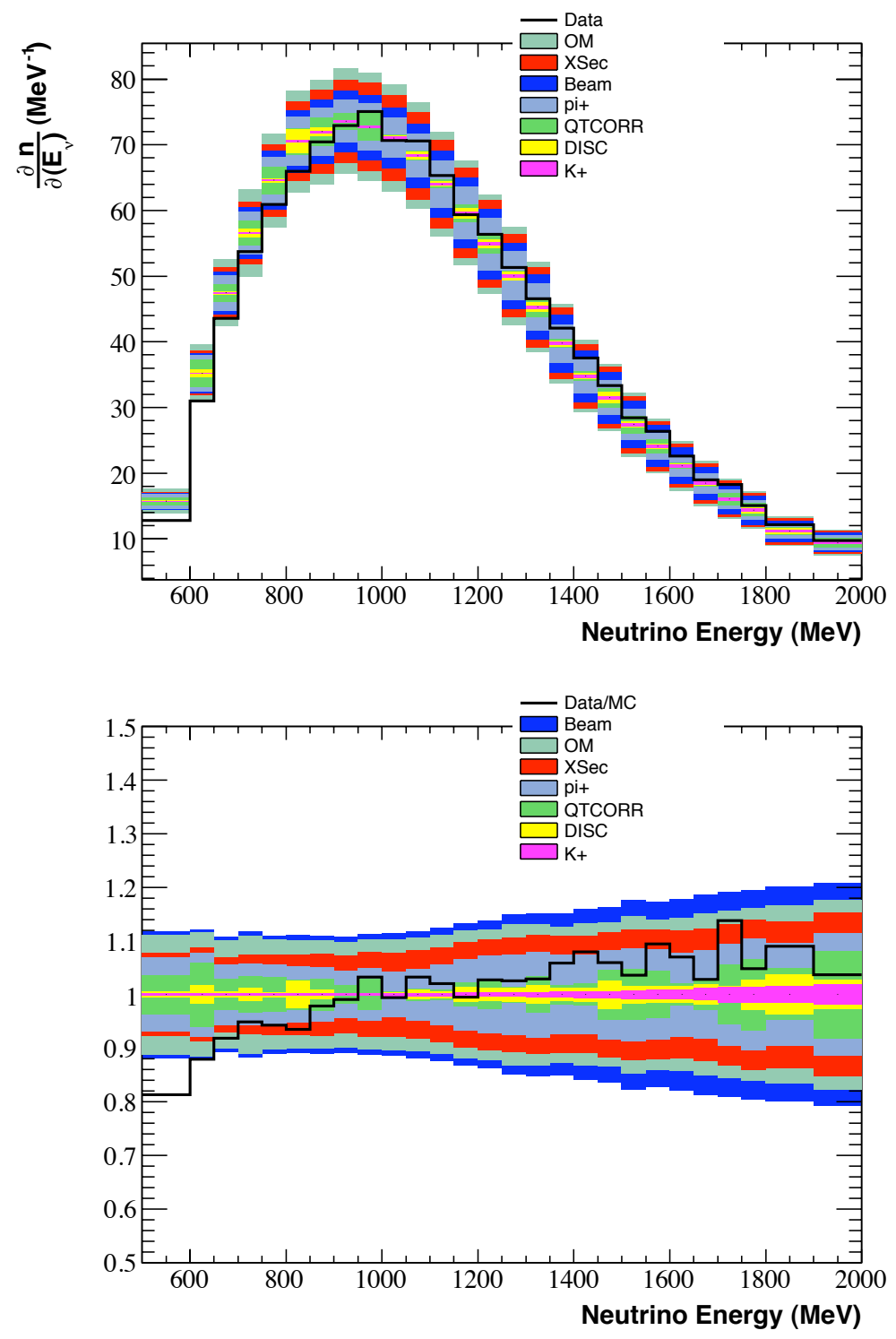

Figure B.1: A Data/Monte Carlo comparison of reconstructed neutrino energy is shown with cumulative systematic errors. The bottom plot shows the fractional errors compared with the Data/Monte Carlo ratio. 


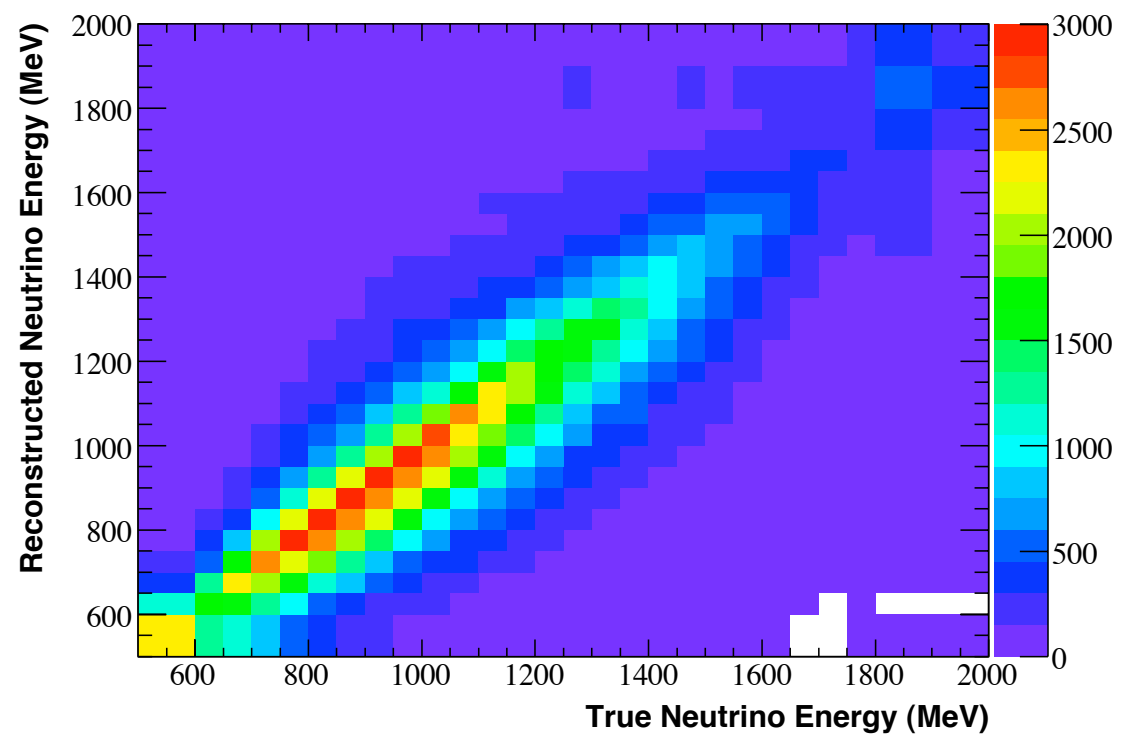

(a) Monte Carlo Event Distribution

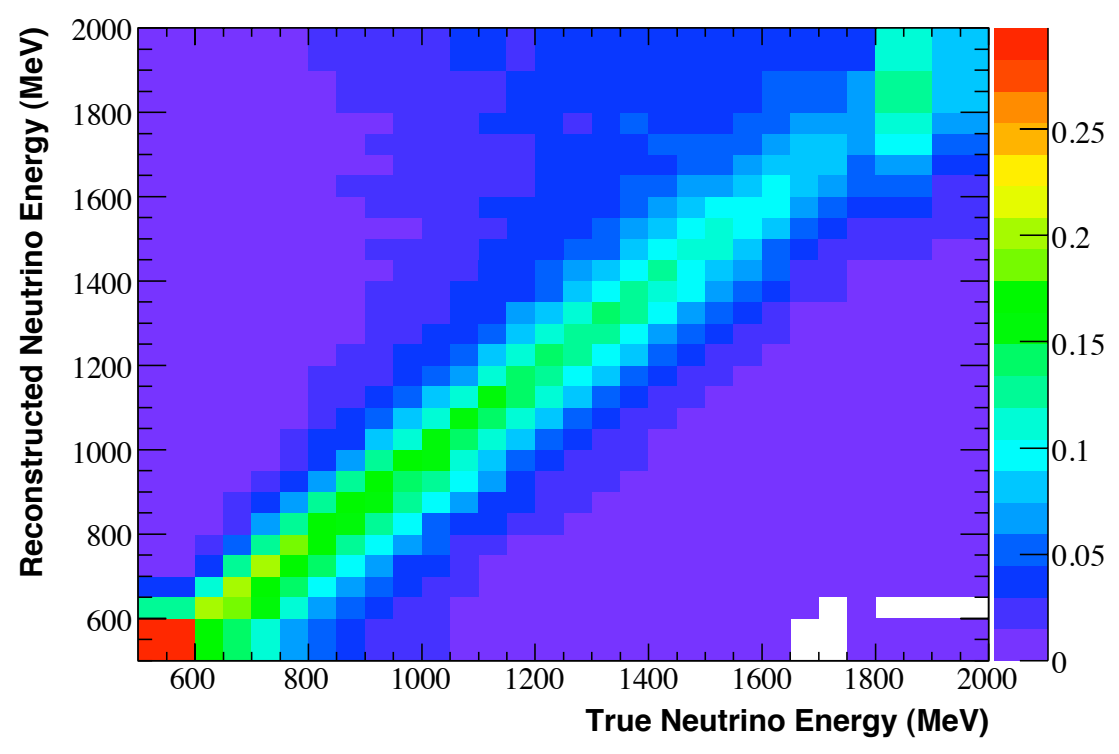

(b) Unfolding Matrix

Figure B.2: The reconstructed vs true distribution for neutrino energy is shown, along with the unfolding matrix used for the central value cross section measurement. 


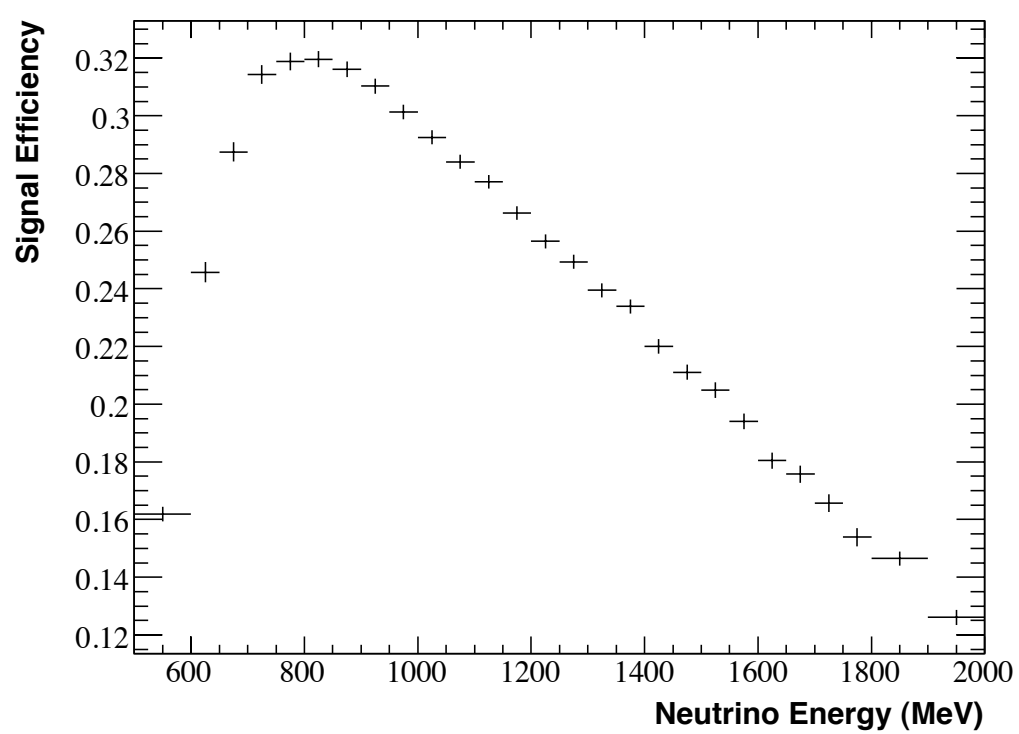

(a) Signal Efficiency

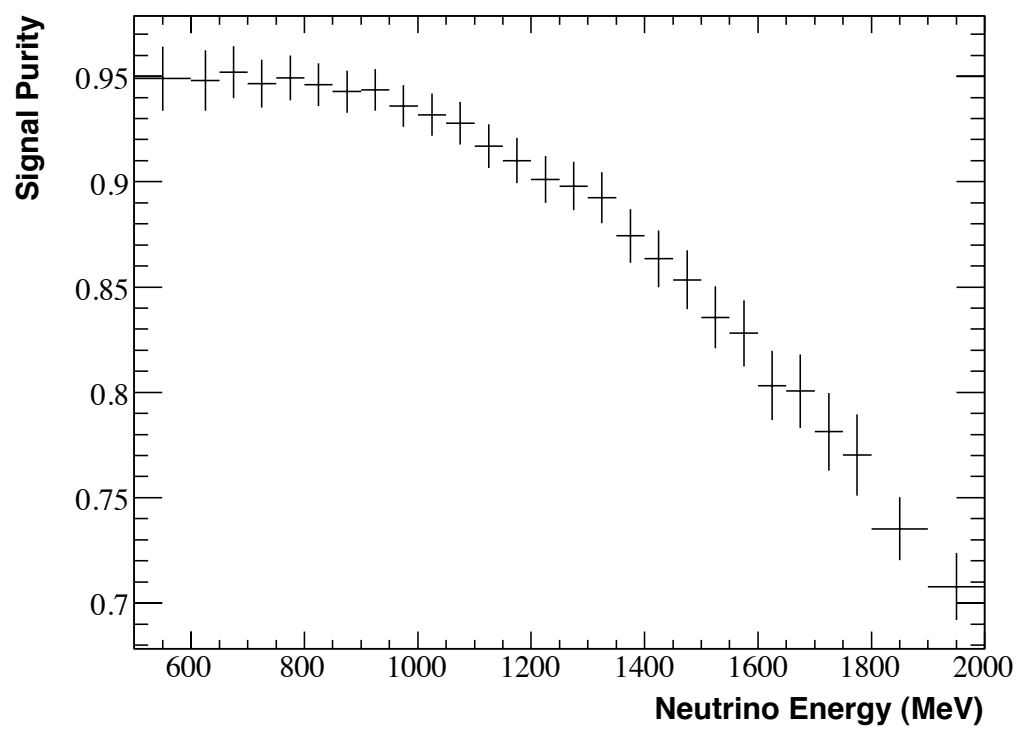

(b) Signal Purity

Figure B.3: The signal efficiency and purity is given in terms of neutrino energy. The error bars represent the Monte Carlo statistical uncertainty. 


\section{B.2 Q Squared}

- Reconstructed Data/Monte Carlo comparison: Figure B.4

- Unfolding Matrix: Figure B.5(b)

- Signal efficiency: Figure B.6(a)

- Signal purity: Figure B.6(b) 

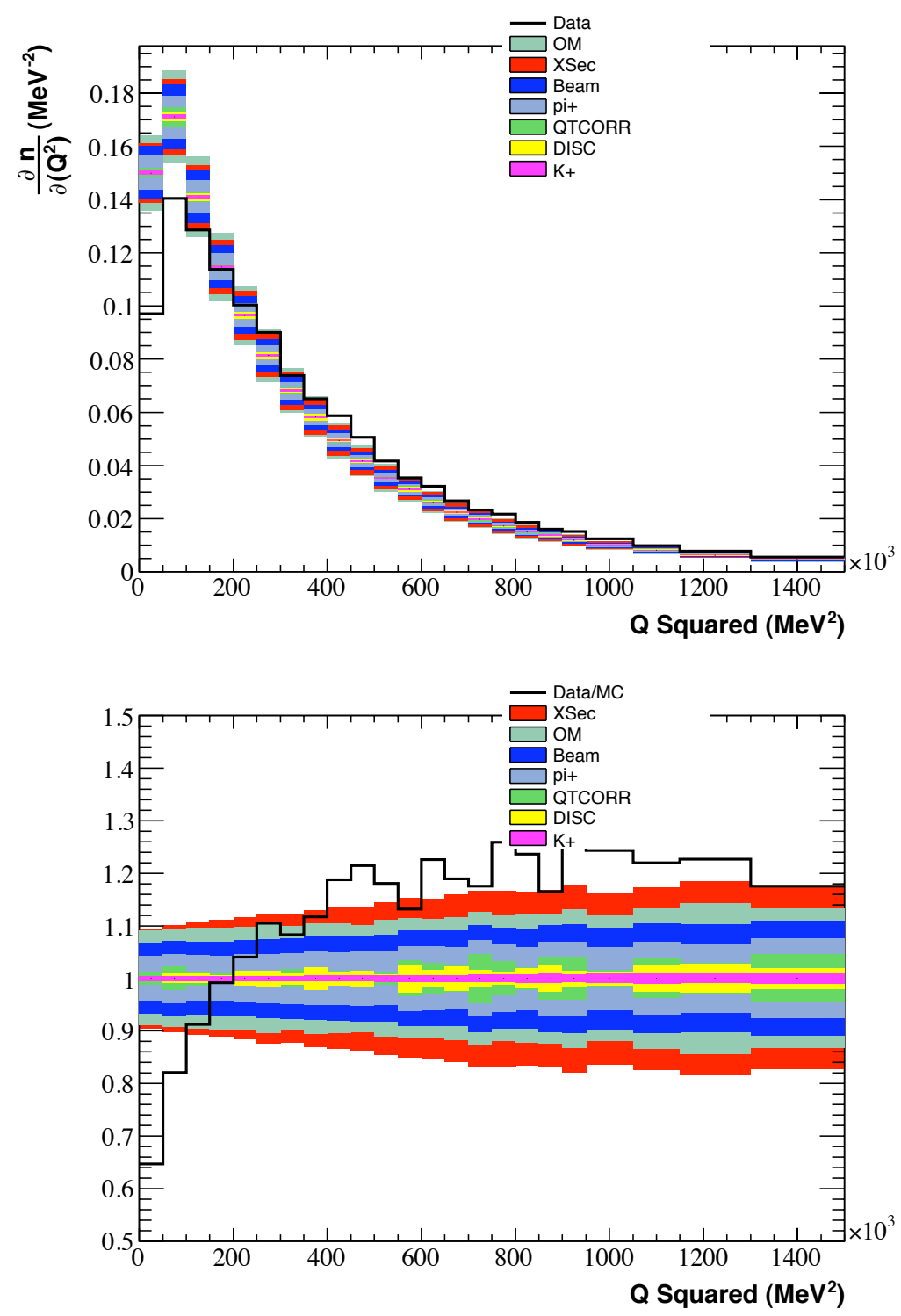

Figure B.4: A Data/Monte Carlo comparison of reconstructed $Q^{2}$ is shown with cumulative systematic errors. The bottom plot shows the fractional errors compared with the Data/Monte Carlo ratio. 


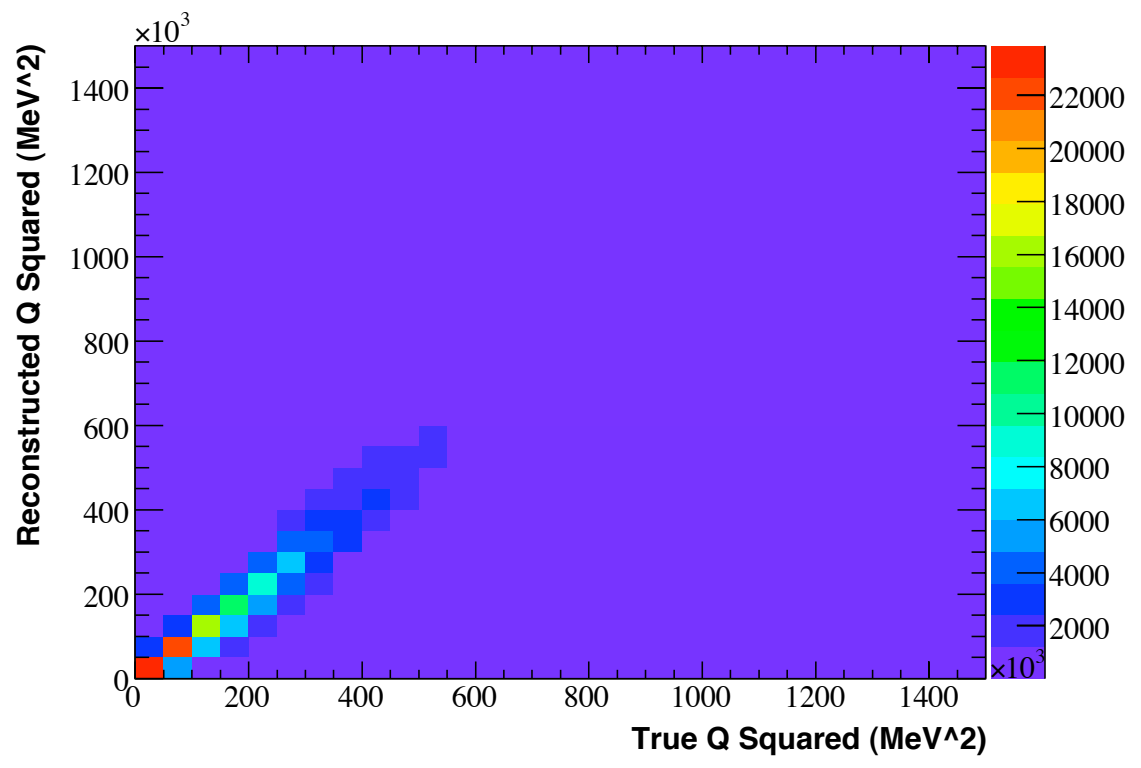

(a) Monte Carlo Event Distribution

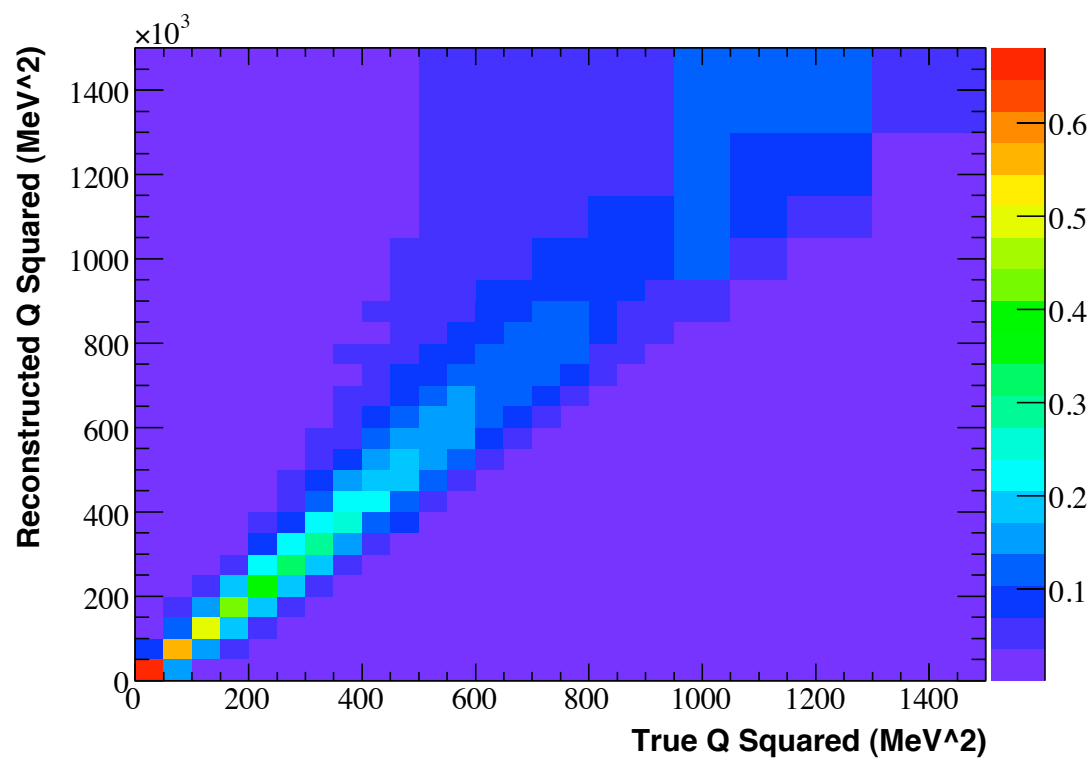

(b) Unfolding Matrix

Figure B.5: The reconstructed vs true distribution for $Q^{2}$ is shown, along with the unfolding matrix used for the central value cross section measurement. 


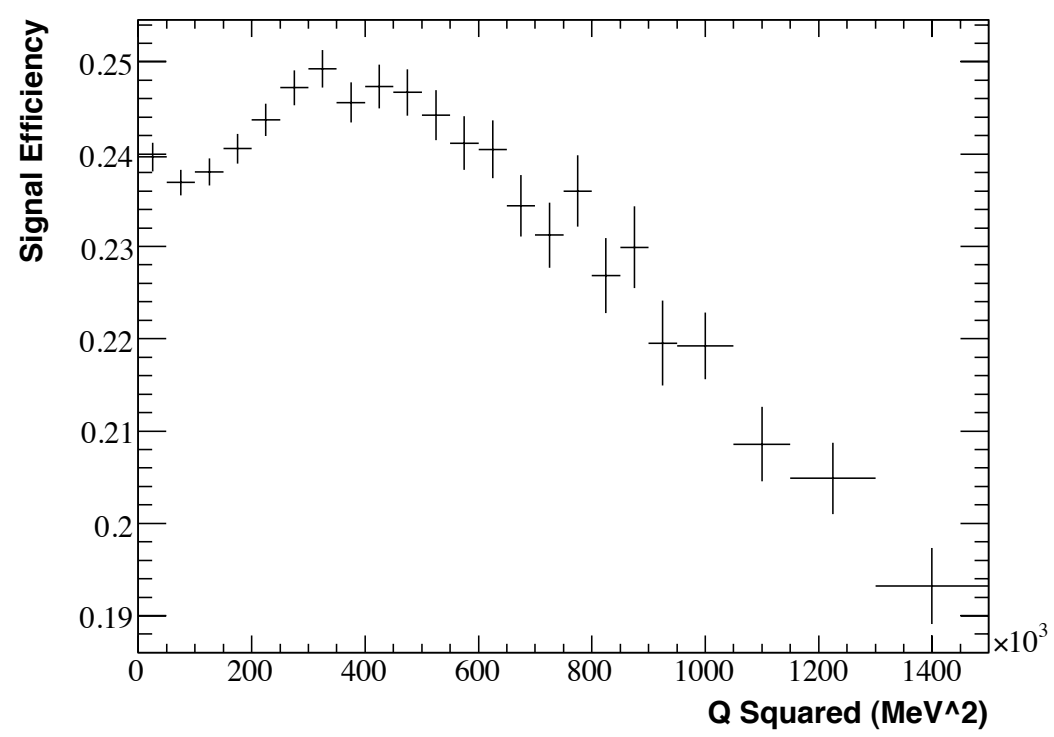

(a) Signal Efficiency

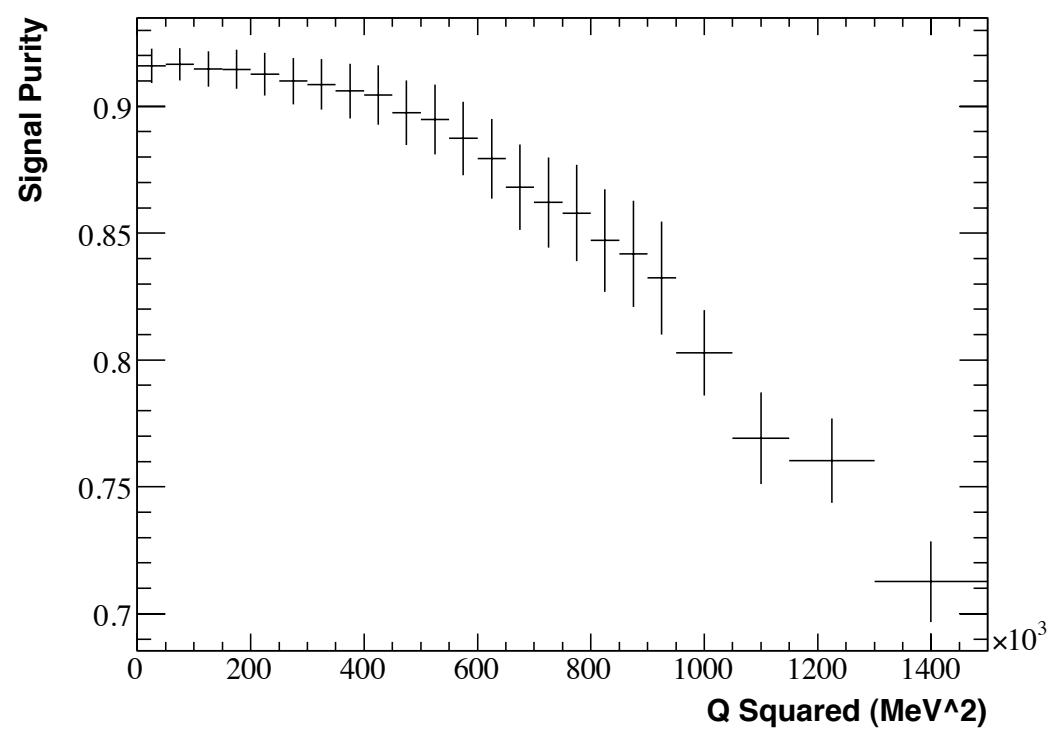

(b) Signal Purity

Figure B.6: The signal efficiency and purity is given in terms of $Q^{2}$. The error bars represent the Monte Carlo statistical uncertainty. 


\section{B.3 Muon Kinetic Energy}

- Reconstructed Data/Monte Carlo comparison: Figure B.7

- Unfolding Matrix: Figure B.8(b)

- Signal efficiency: Figure B.9(a)

- Signal purity: Figure B.9(b) 

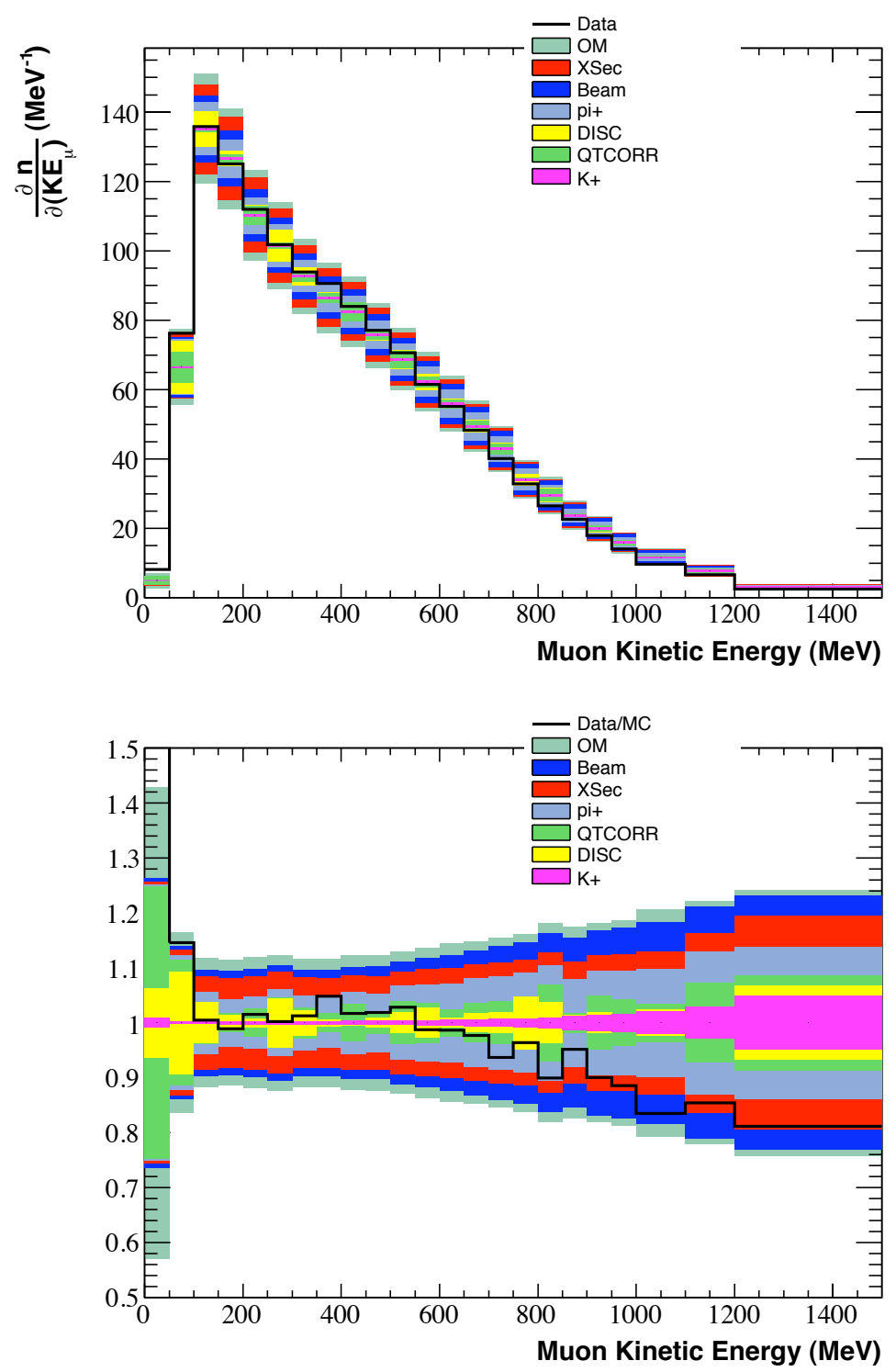

Figure B.7: A Data/Monte Carlo comparison of reconstructed muon kinetic energy is shown with cumulative systematic errors. The bottom plot shows the fractional errors compared with the Data/Monte Carlo ratio. 


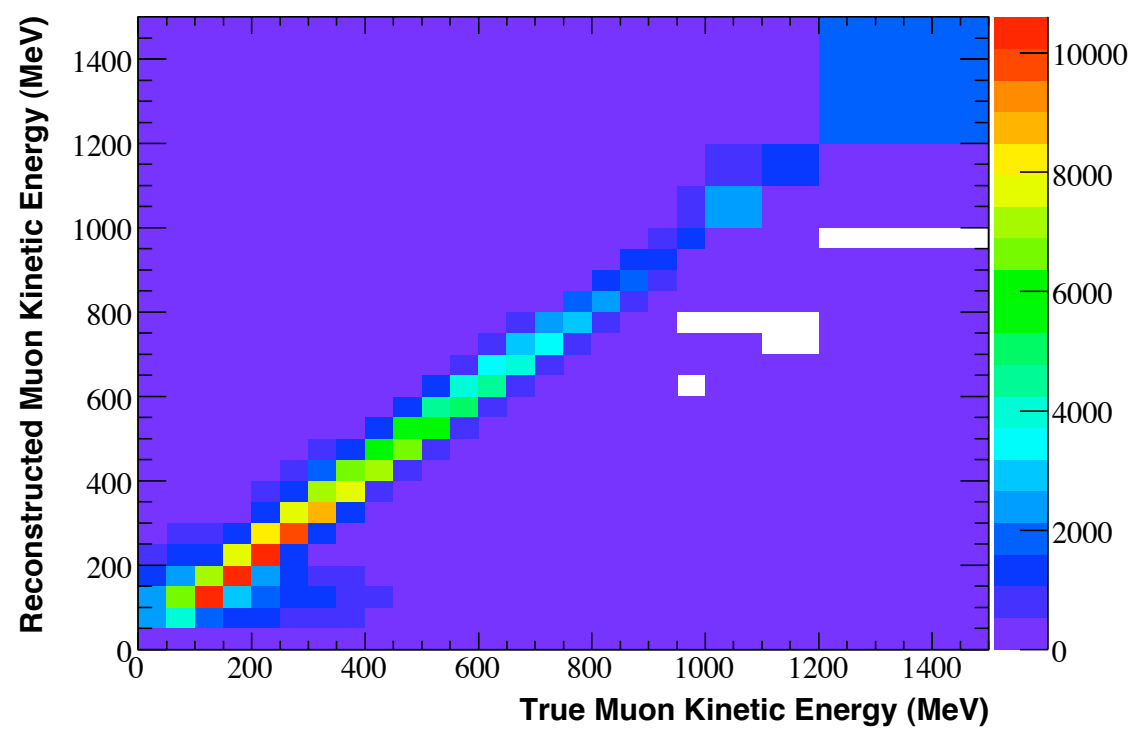

(a) Monte Carlo Event Distribution

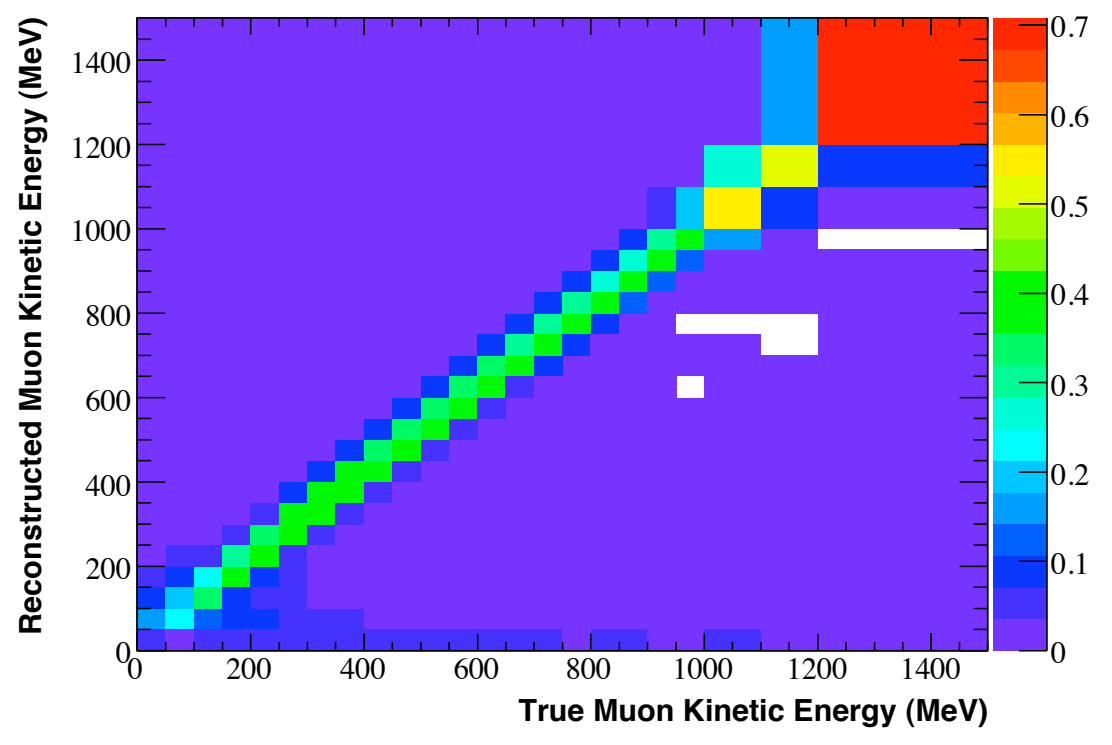

(b) Unfolding Matrix

Figure B.8: The reconstructed vs true distribution for muon kinetic energy is shown, along with the unfolding matrix used for the central value cross section measurement. 


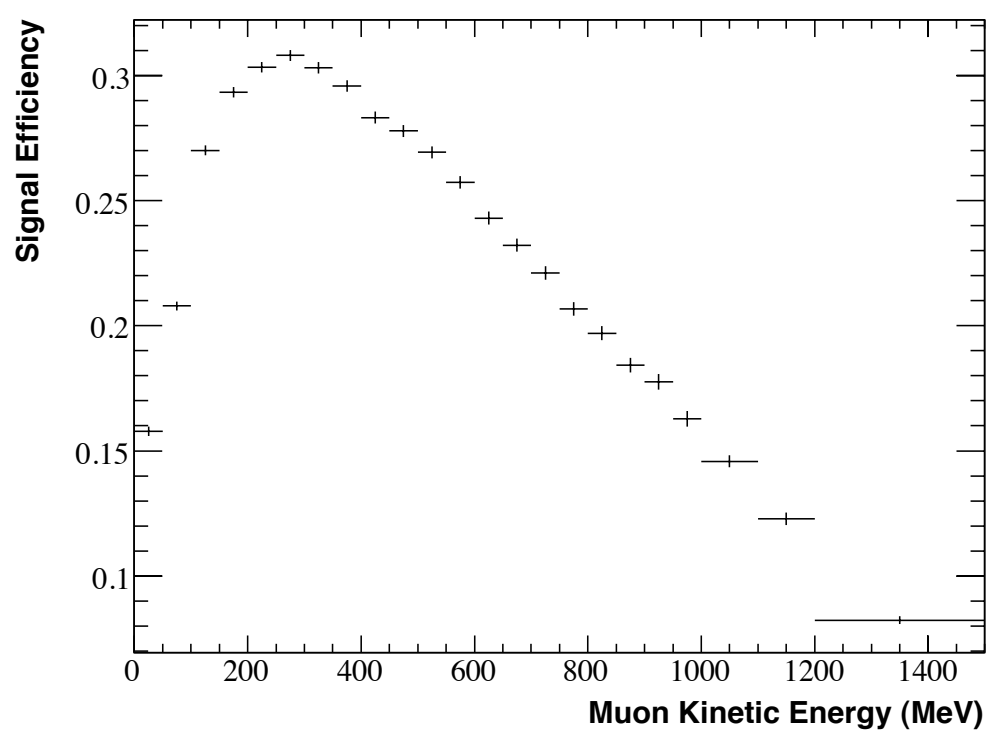

(a) Signal Efficiency

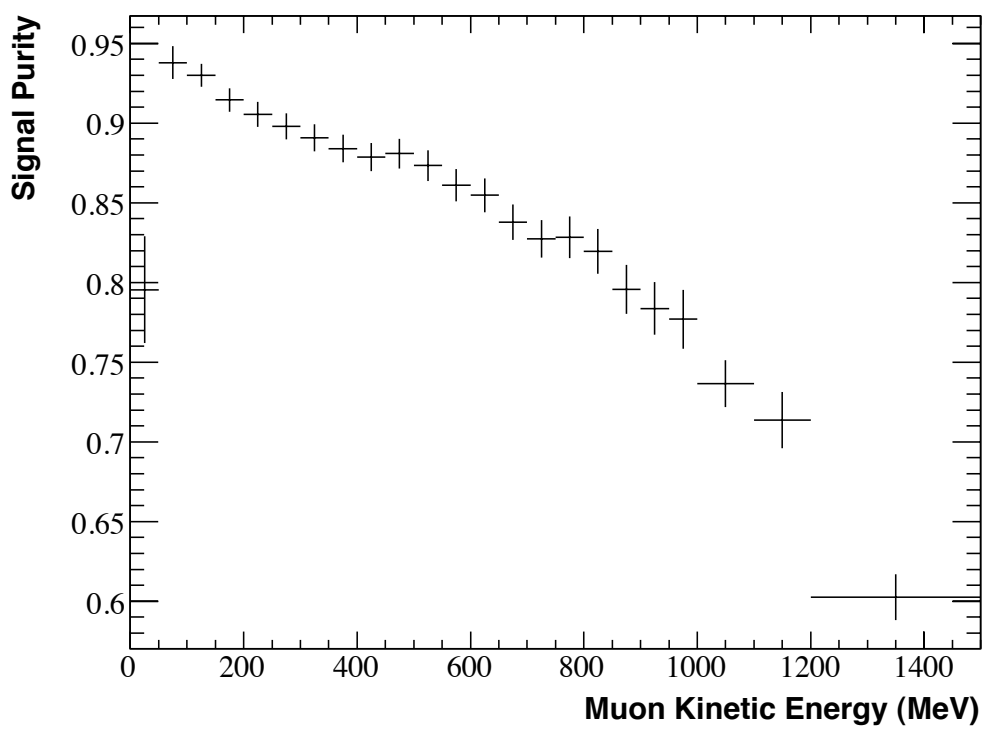

(b) Signal Purity

Figure B.9: The signal efficiency and purity is given in terms of muon kinetic energy. The error bars represent the Monte Carlo statistical uncertainty. 


\section{B.4 Muon Direction}

- Reconstructed Data/Monte Carlo comparison: Figure B.10

- Unfolding Matrix: Figure B.11(b)

- Signal efficiency: Figure B.12(a)

- Signal purity: Figure B.12(b) 

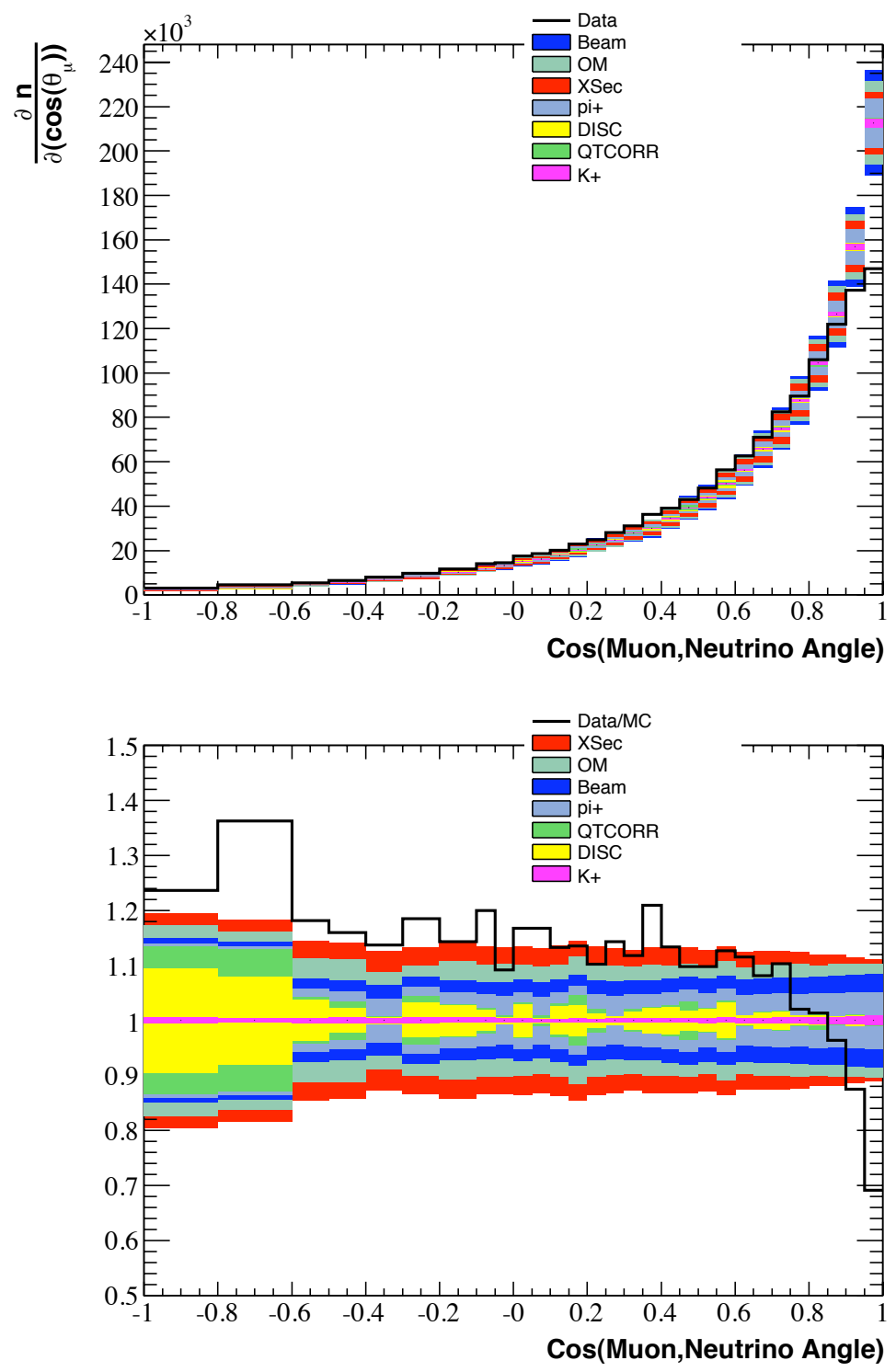

Figure B.10: A Data/Monte Carlo comparison of reconstructed cos(muon, neutrino angle) is shown with cumulative systematic errors. The bottom plot shows the fractional errors compared with the Data/Monte Carlo ratio. 


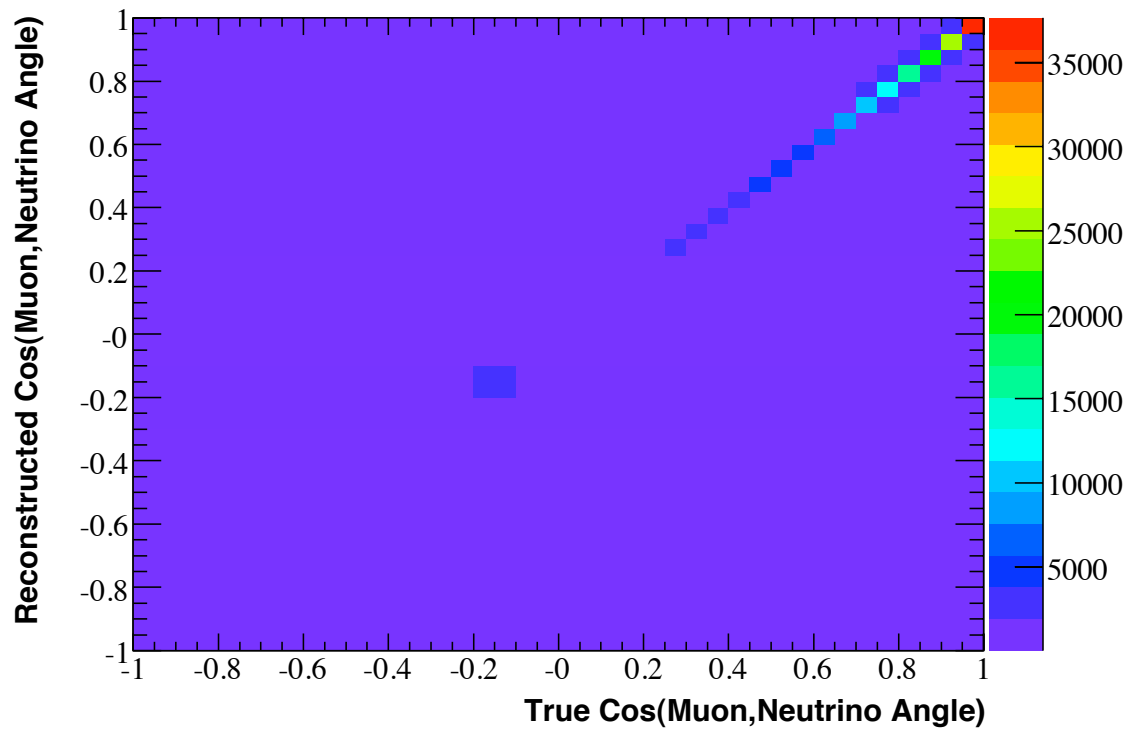

(a) Monte Carlo Event Distribution

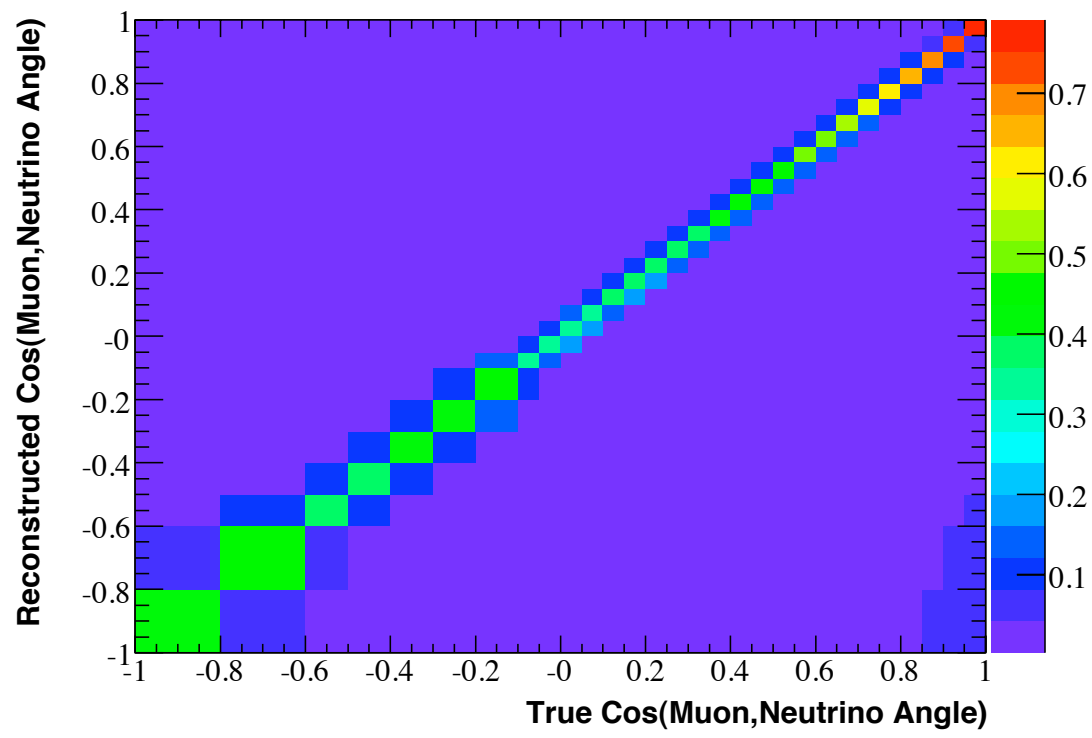

(b) Unfolding Matrix

Figure B.11: The reconstructed vs true distribution for $\cos ($ muon, neutrino angle) is shown, along with the unfolding matrix used for the central value cross section measurement. 


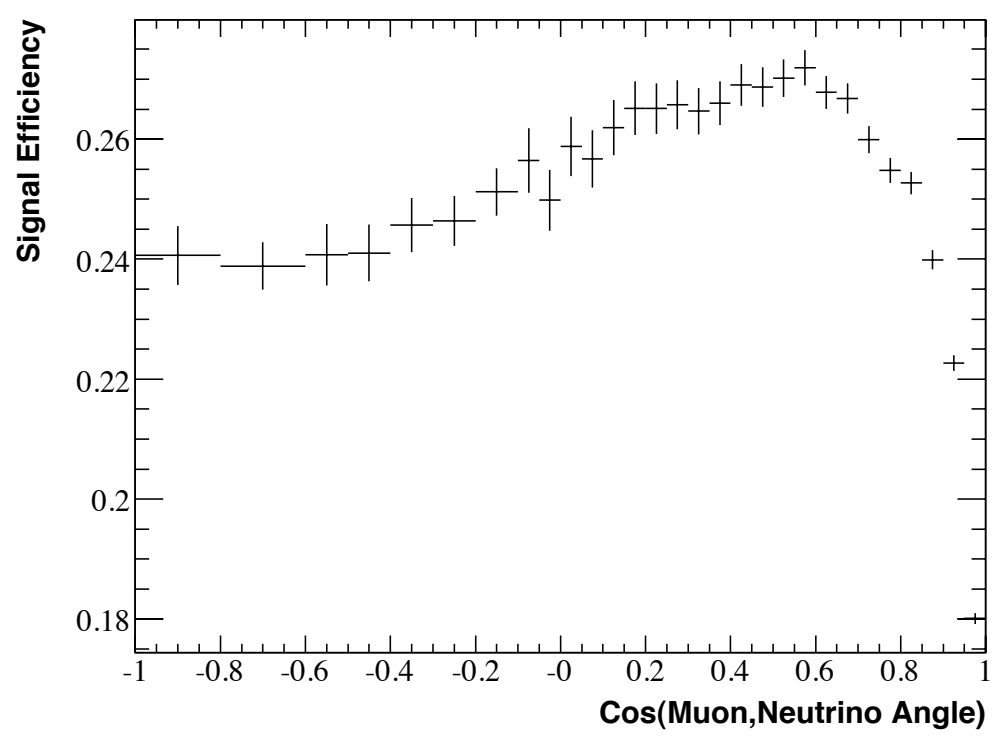

(a) Signal Efficiency

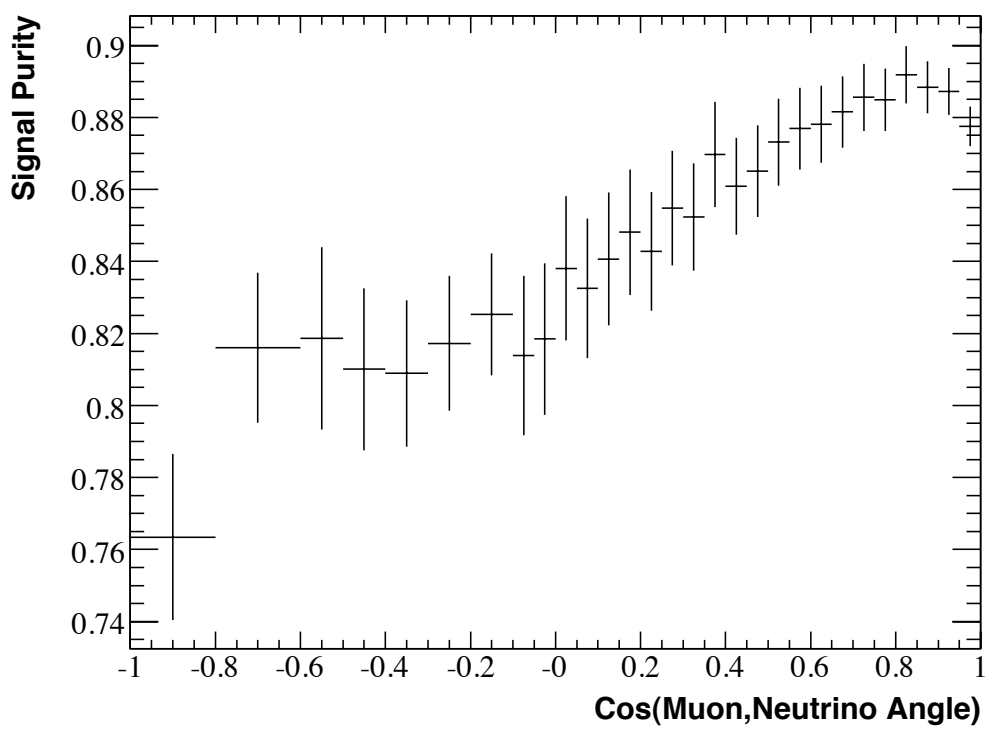

(b) Signal Purity

Figure B.12: The signal efficiency and purity is given in terms of cos(muon, neutrino angle). The error bars represent the Monte Carlo statistical uncertainty. 


\section{B.5 Pion Kinetic Energy}

- Reconstructed Data/Monte Carlo comparison: Figure B.13

- Unfolding Matrix: Figure B.14(b)

- Signal efficiency: Figure B.15(a)

- Signal purity: Figure B.15(b) 

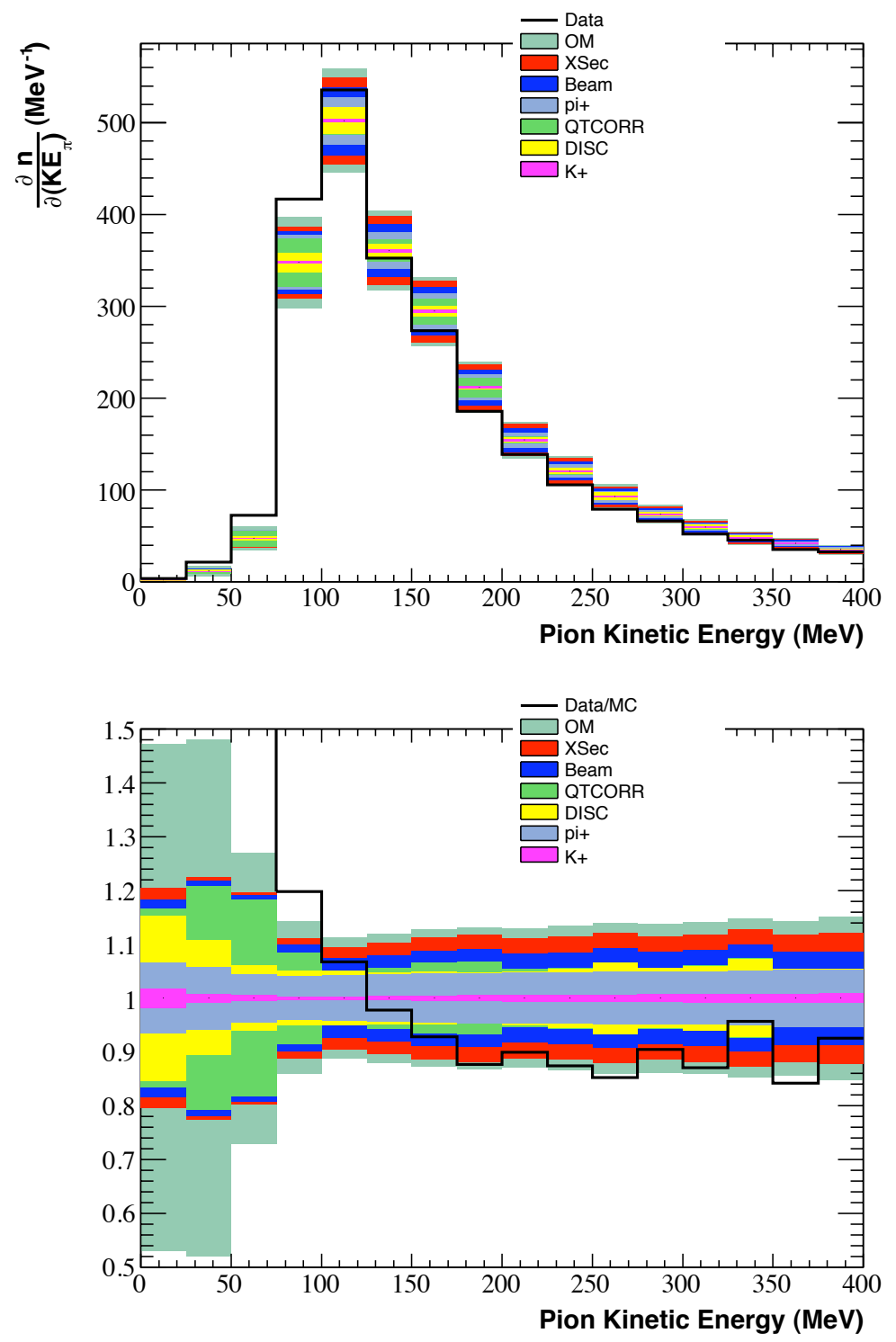

Figure B.13: A Data/Monte Carlo comparison of reconstructed pion kinetic energy is shown with cumulative systematic errors. The bottom plot shows the fractional errors compared with the Data/Monte Carlo ratio. 


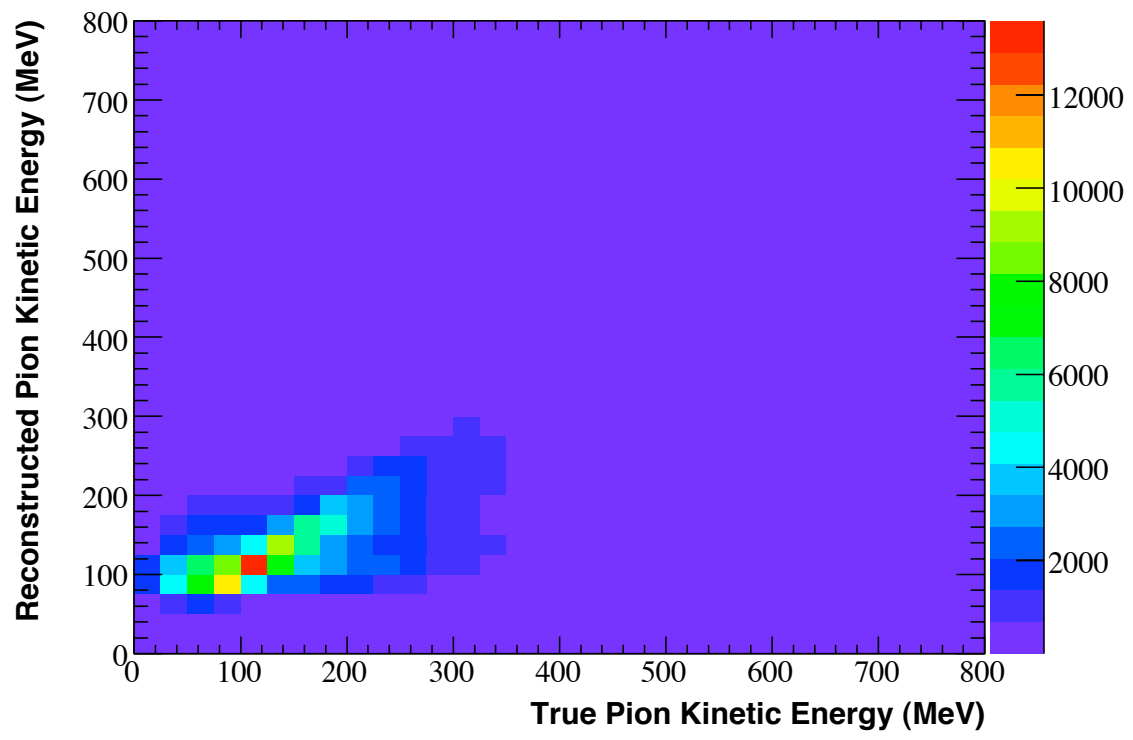

(a) Monte Carlo Event Distribution

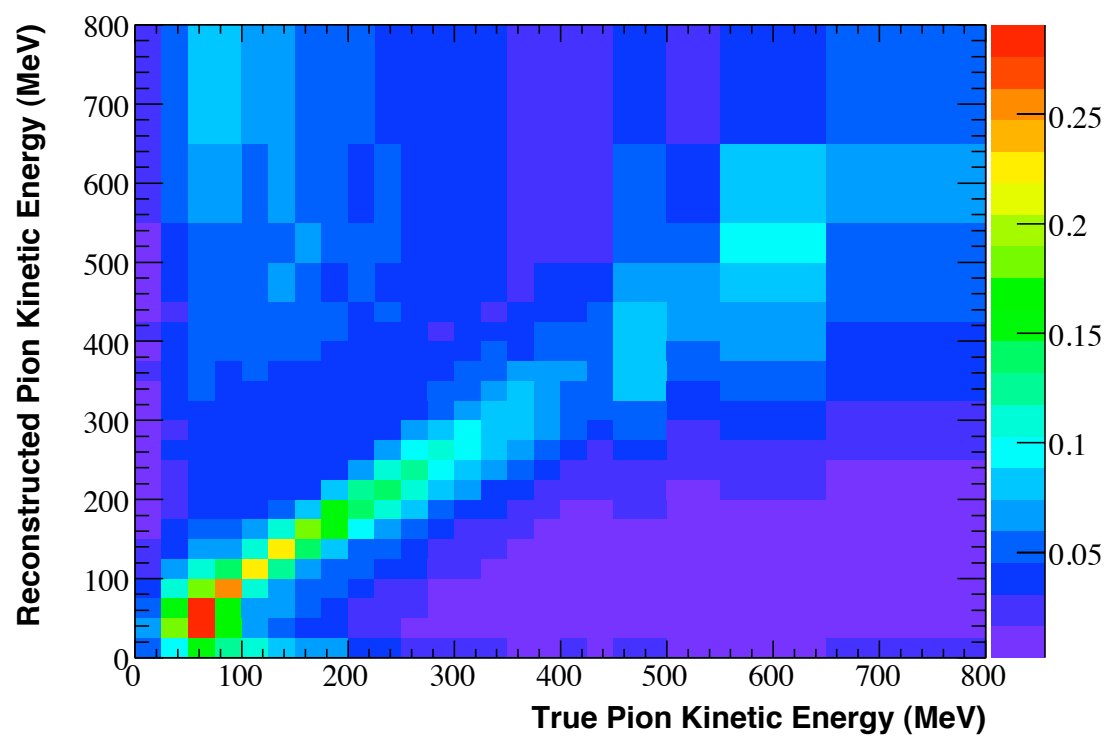

(b) Unfolding Matrix

Figure B.14: The reconstructed vs true distribution for pion kinetic energy is shown, along with the unfolding matrix used for the central value cross section measurement. 


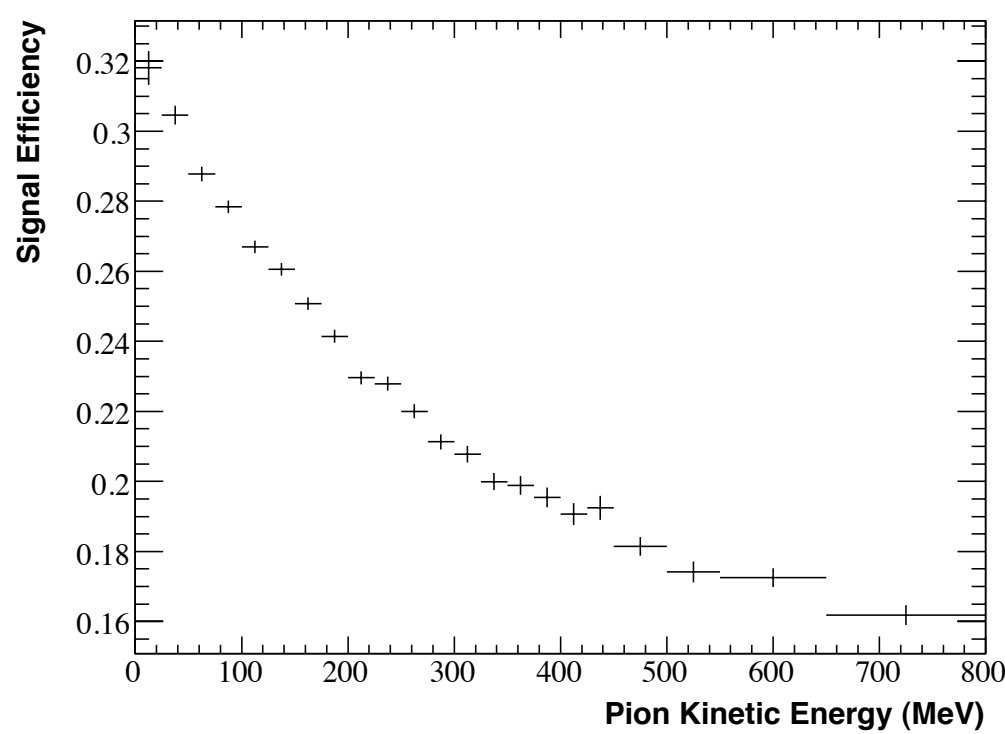

(a) Signal Efficiency

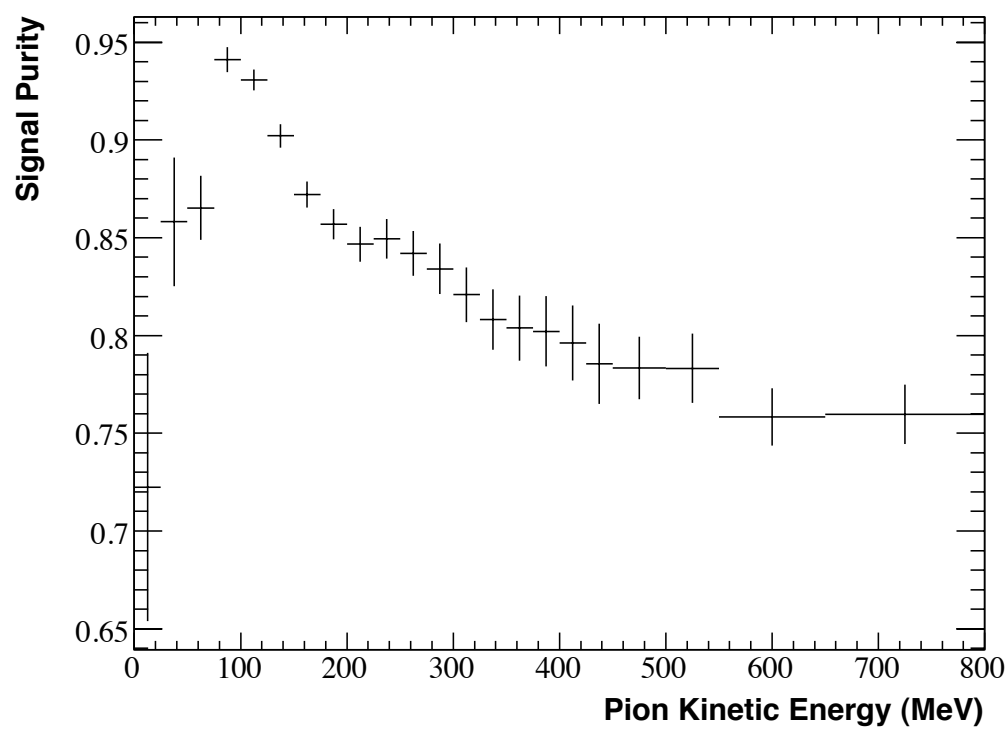

(b) Signal Purity

Figure B.15: The signal efficiency and purity is given in terms of pion kinetic energy. The error bars represent the Monte Carlo statistical uncertainty. 


\section{B.6 Pion Direction}

- Reconstructed Data/Monte Carlo comparison: Figure B.16

- Unfolding Matrix: Figure B.17(b)

- Signal efficiency: Figure B.18(a)

- Signal purity: Figure B.18(b) 

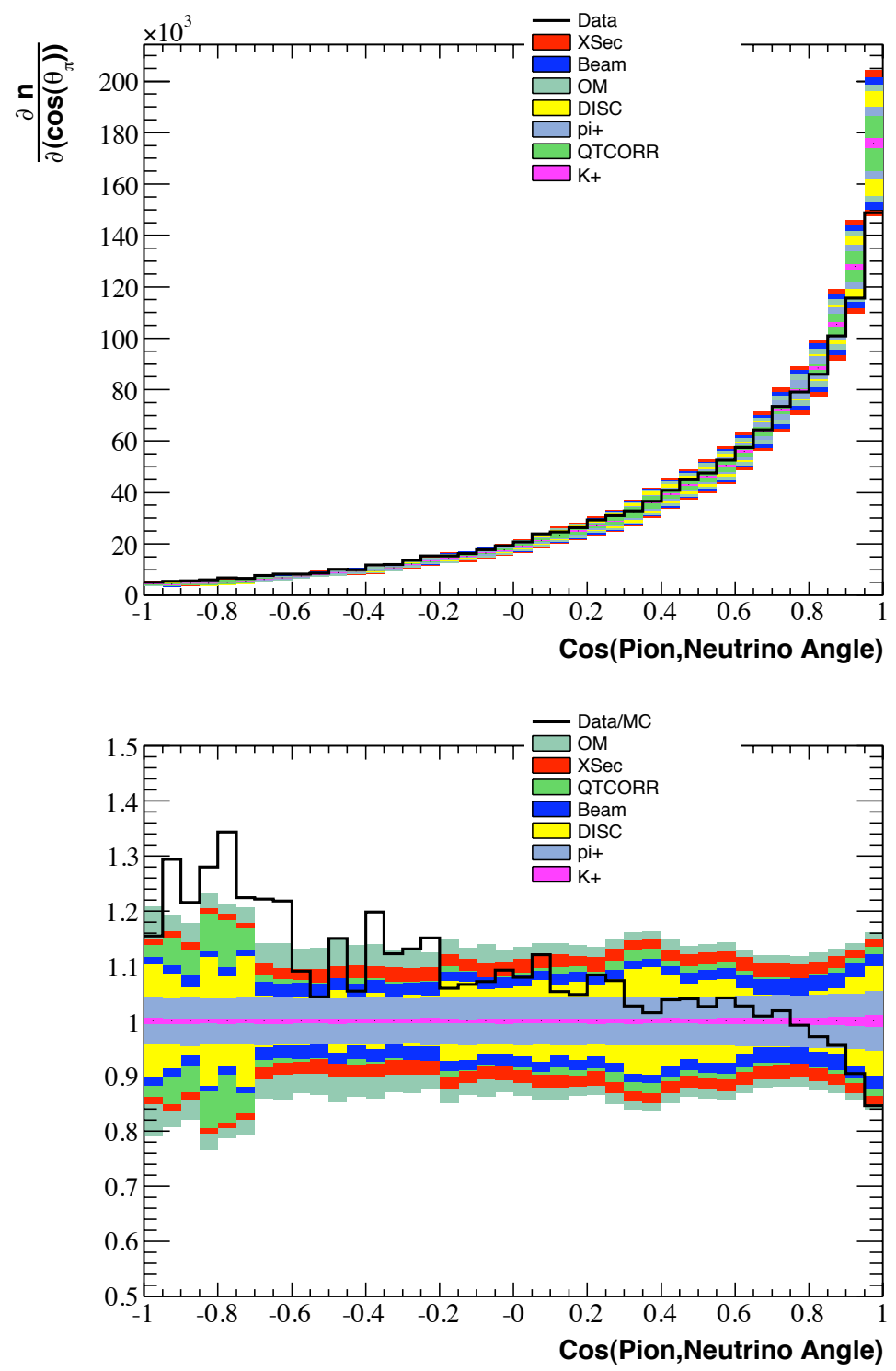

Figure B.16: A Data/Monte Carlo comparison of reconstructed cos(pion, neutrino angle) is shown with cumulative systematic errors. The bottom plot shows the fractional errors compared with the Data/Monte Carlo ratio. 


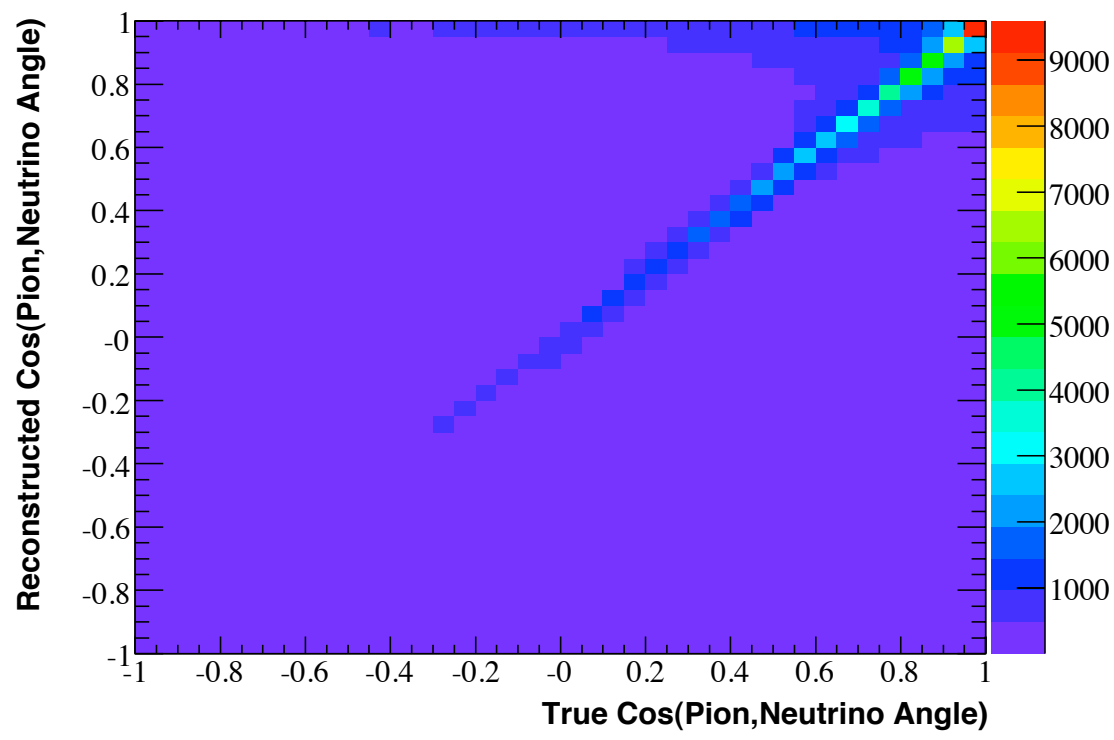

(a) Monte Carlo Event Distribution

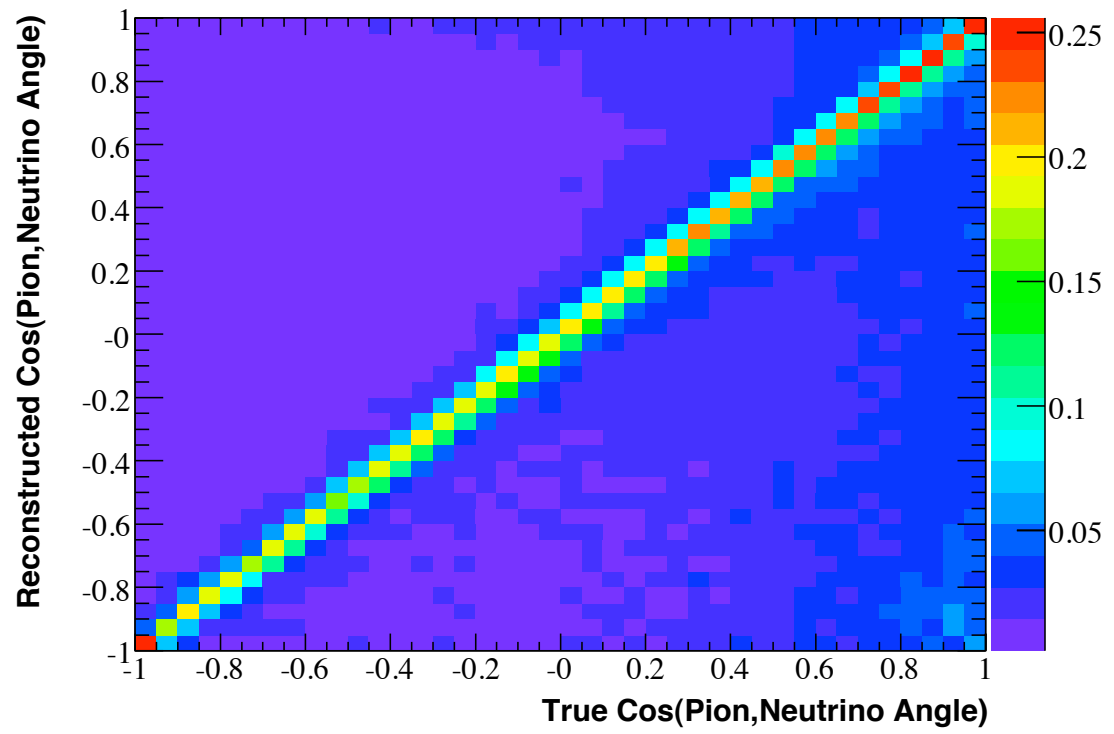

(b) Unfolding Matrix

Figure B.17: The reconstructed vs true distribution for cos(pion, neutrino angle) is shown, along with the unfolding matrix used for the central value cross section measurement. 


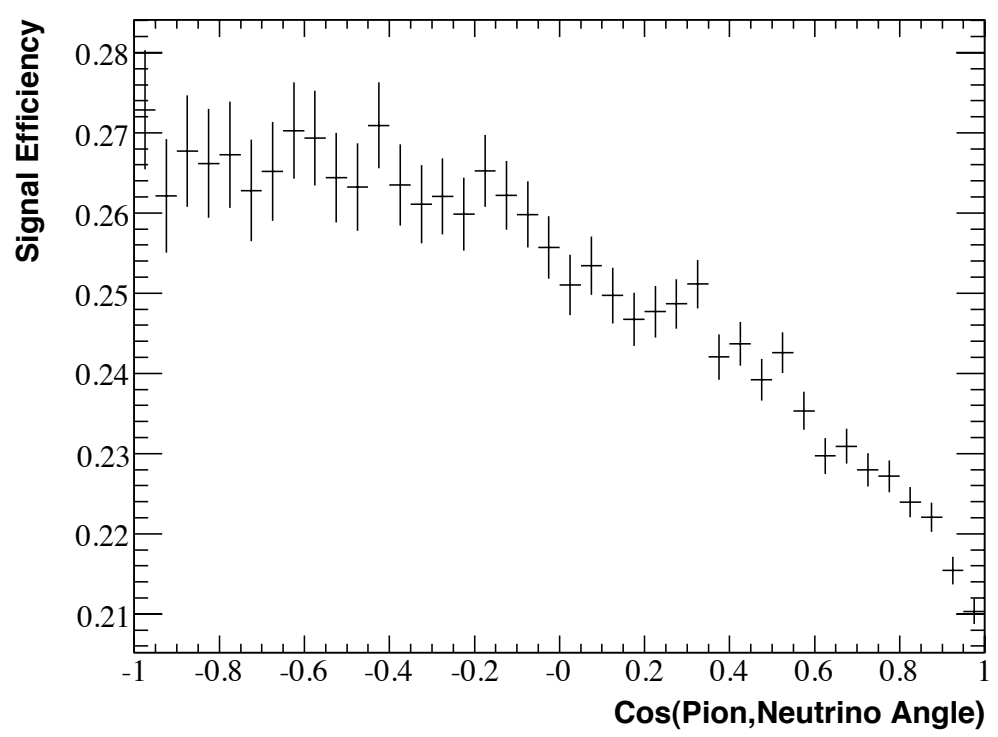

(a) Signal Efficiency

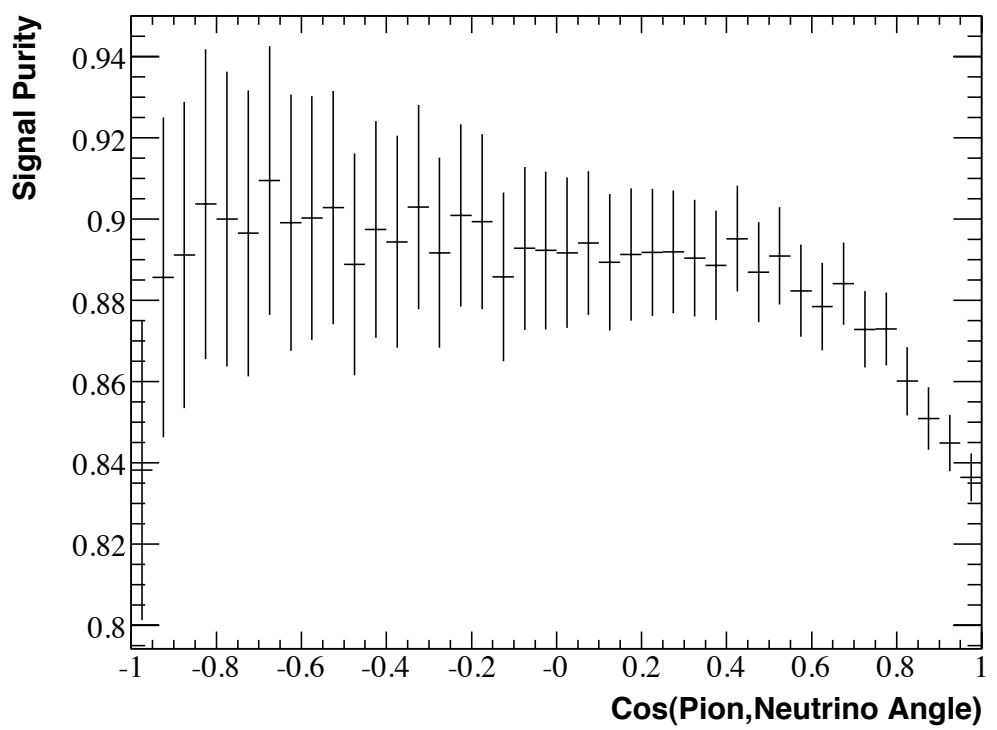

(b) Signal Purity

Figure B.18: The signal efficiency and purity is given in terms of cos(pion, neutrino angle). The error bars represent the Monte Carlo statistical uncertainty. 


\section{B.7 Pion/Nucleon Mass}

- Reconstructed Data/Monte Carlo comparison: Figure B.19
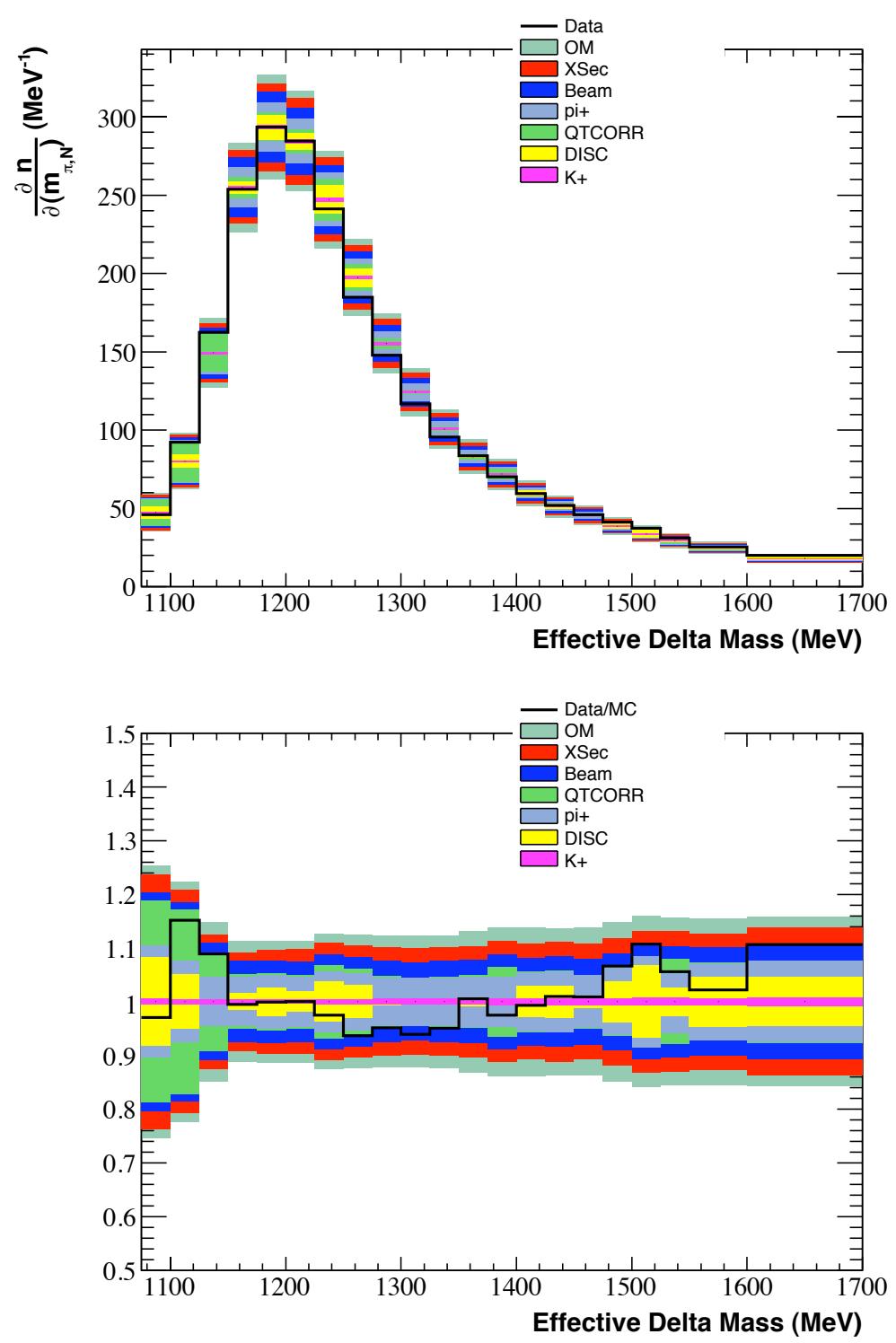

Figure B.19: A Data/Monte Carlo comparison of reconstructed $N+\pi^{+}$mass is shown with cumulative systematic errors. The bottom plot shows the fractional errors compared with the Data/Monte Carlo ratio. 


\section{B.8 Q Squared vs Neutrino Energy}

- Reconstructed Data/Monte Carlo comparison: Figure B.20

- Reconstructed Monte Carlo Fractional Errors: Figures B.22 and B.23

- Signal efficiency: Figure B.24(a)

- Signal purity: Figure B.24(c) 

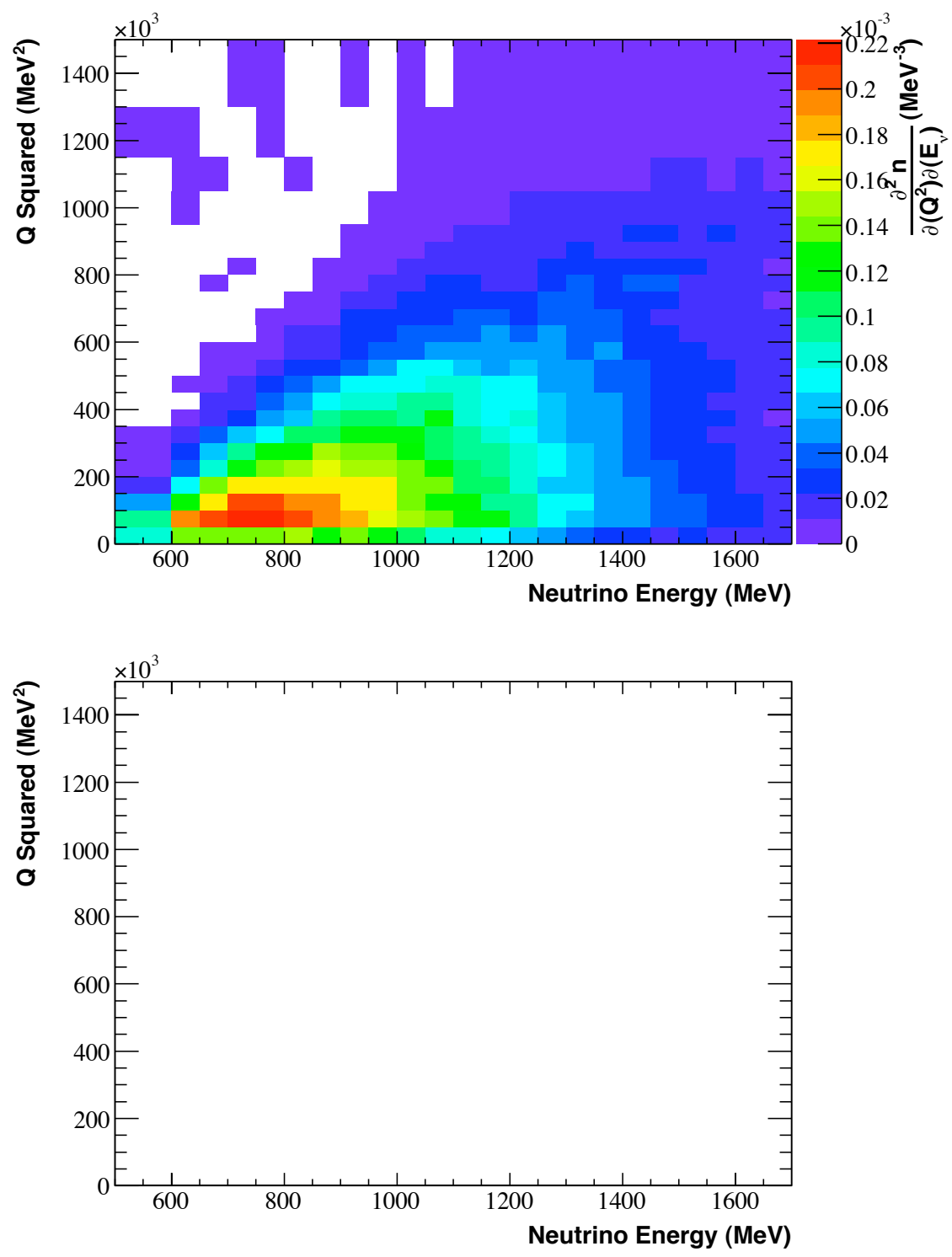

Figure B.20: The reconstructed $Q^{2}$ vs neutrino energy distribution is shown for both the data (top) and Monte Carlo (bottom). 


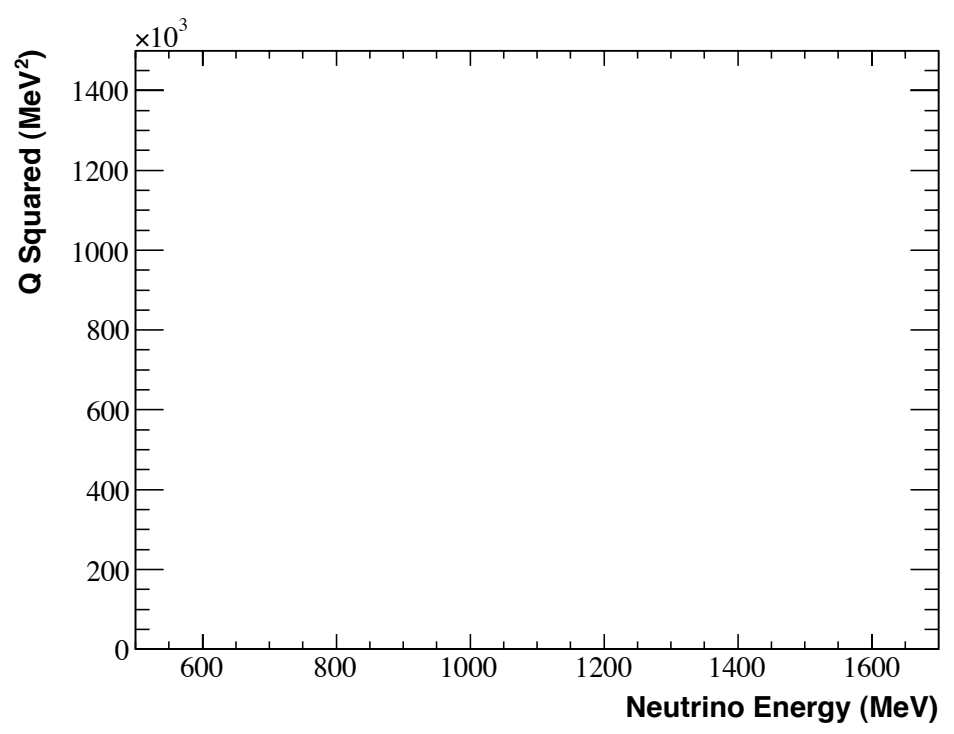

215

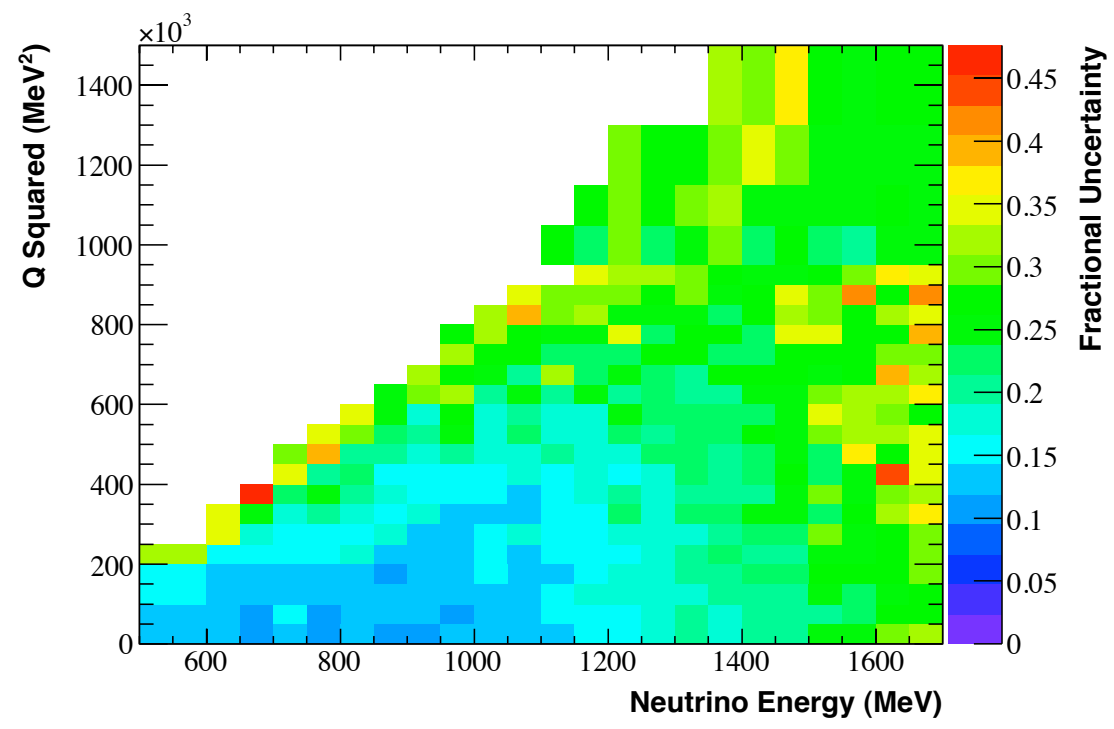

Figure B.21: The Monte Carlo reconstructed $Q^{2}$ vs energy distribution is shown (top) along with the total fractional uncertainties (bottom). 


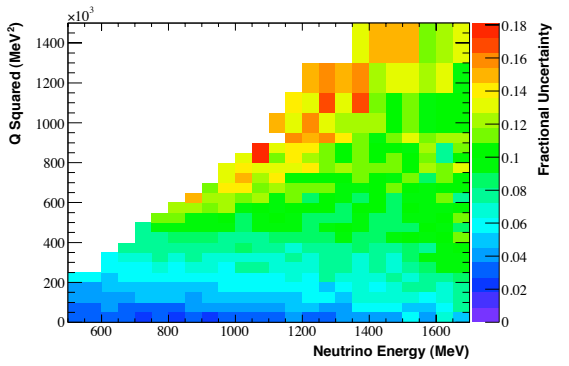

(a) The fractional systematic uncertainty from the "XSec" error source.

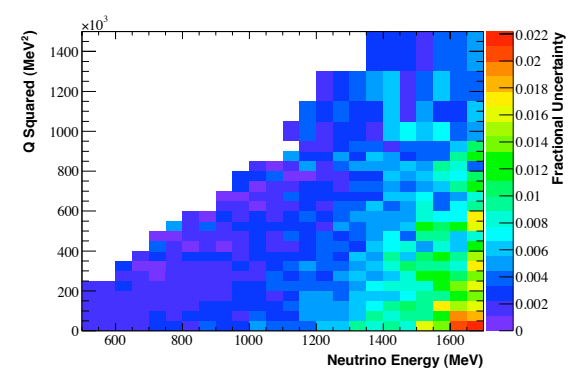

(c) The fractional systematic uncertainty from the " $\mathrm{K}^{+}$" error source.

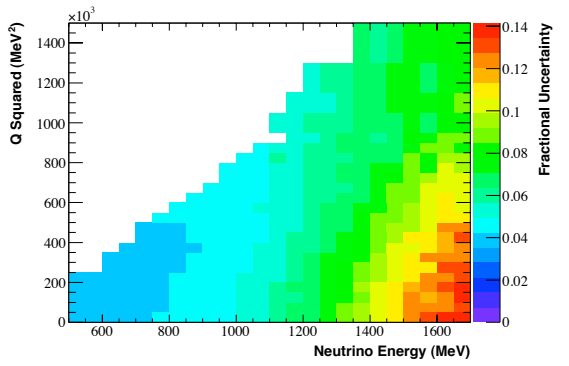

(b) The fractional systematic uncertainty from the "Beam" error source.

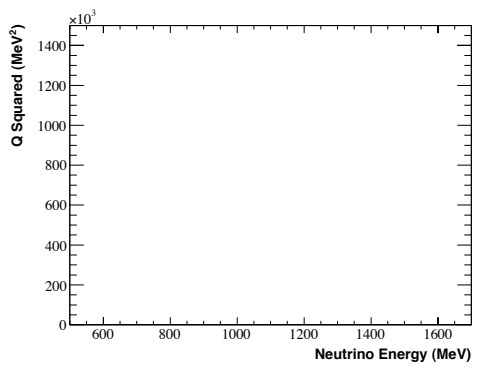

(d) Reconstructed Monte Carlo distribution

Figure B.22: The fractional systematic uncertainties on the reconstructed Monte Carlo distribution are shown for four of the error sources. The central value reconstructed Monte Carlo distribution is also shown for reference. Note that the color scales differ for each systematic error source. An explanation of each error is given in Section 6.4.7. 


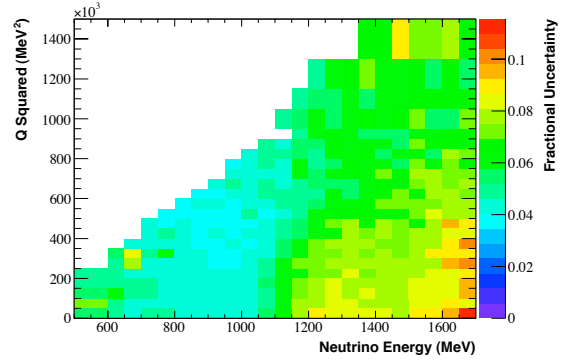

(a) The fractional systematic uncertainty from the " $\pi^{+}$" error source.

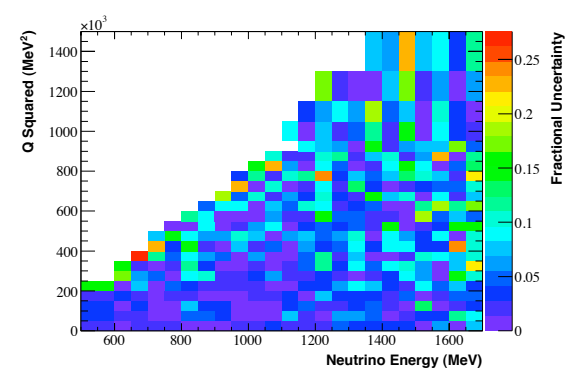

(c) The fractional systematic uncertainty from the "DISC" error source.

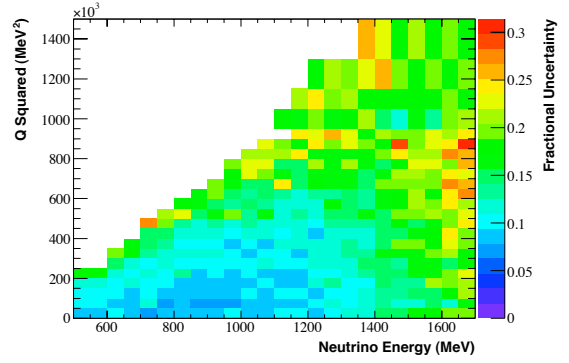

(b) The fractional systematic uncertainty from the "OM" error source.

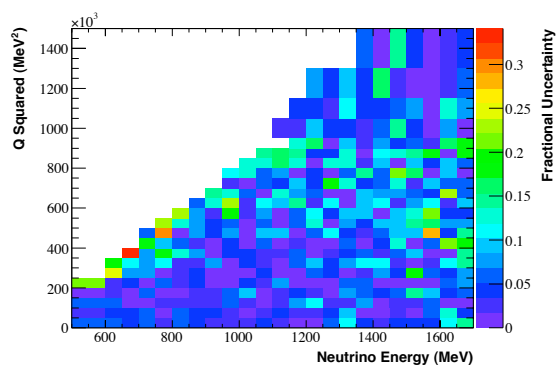

(d) The fractional systematic uncertainty from the "QTCORR" error source.

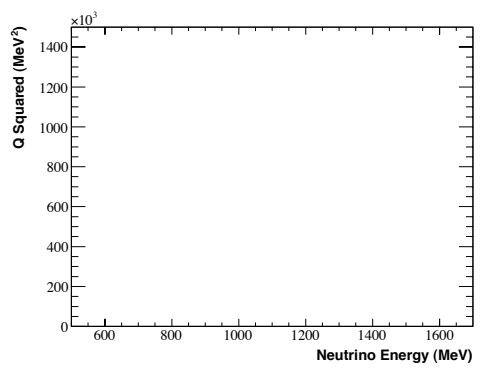

(e) Reconstructed Monte Carlo distribution

Figure B.23: The fractional systematic uncertainties on the reconstructed Monte Carlo distribution are shown for four of the error sources. The central value reconstructed Monte Carlo distribution is also shown for reference. Note that the color scales differ for each systematic error source. An explanation of each error is given in Section 6.4.7. 


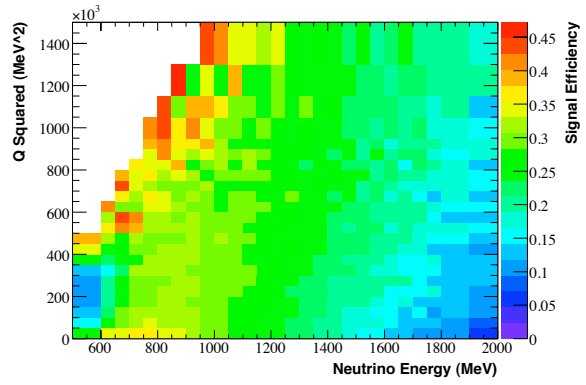

(a) Signal Efficiency

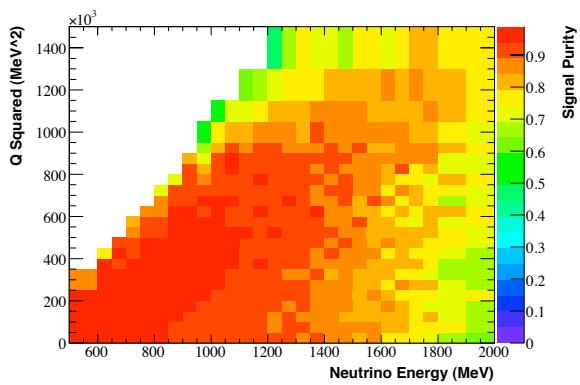

(c) Signal Purity

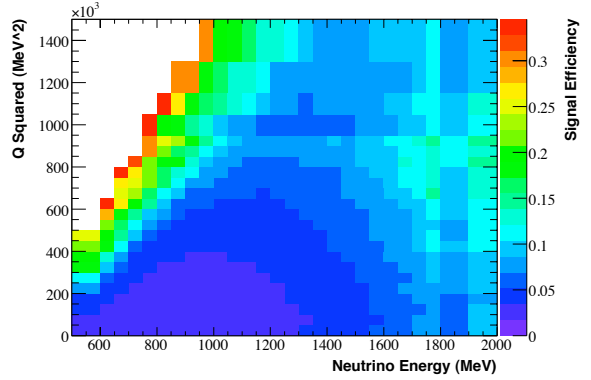

(b) Fractional Monte Carlo Statistical Error on the Signal Efficiency

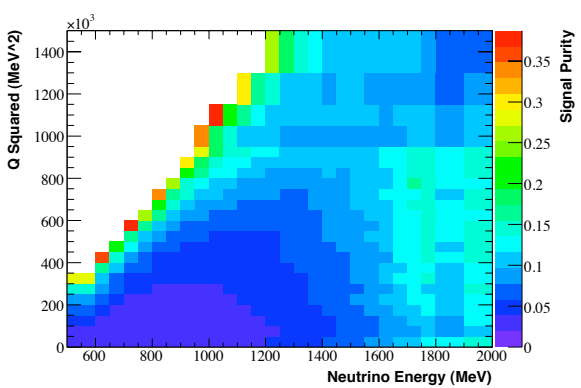

(d) Fractional Monte Carlo Statistical Error on the Signal Purity

Figure B.24: The signal efficiency and purity are shown for the $Q^{2}$ vs neutrino energy distribution. The fractional errors due to Monte Carlo statistics for each of these distributions is also given for all bins with at least 10 events in the numerator. 


\section{B.9 Muon Kinetic Energy vs Neutrino Energy}

- Reconstructed Data/Monte Carlo comparison: Figure B.25

- Reconstructed Monte Carlo Fractional Errors: Figures B.27 and B.28

- Signal efficiency: Figure B.29(a)

- Signal purity: Figure B.29(c) 

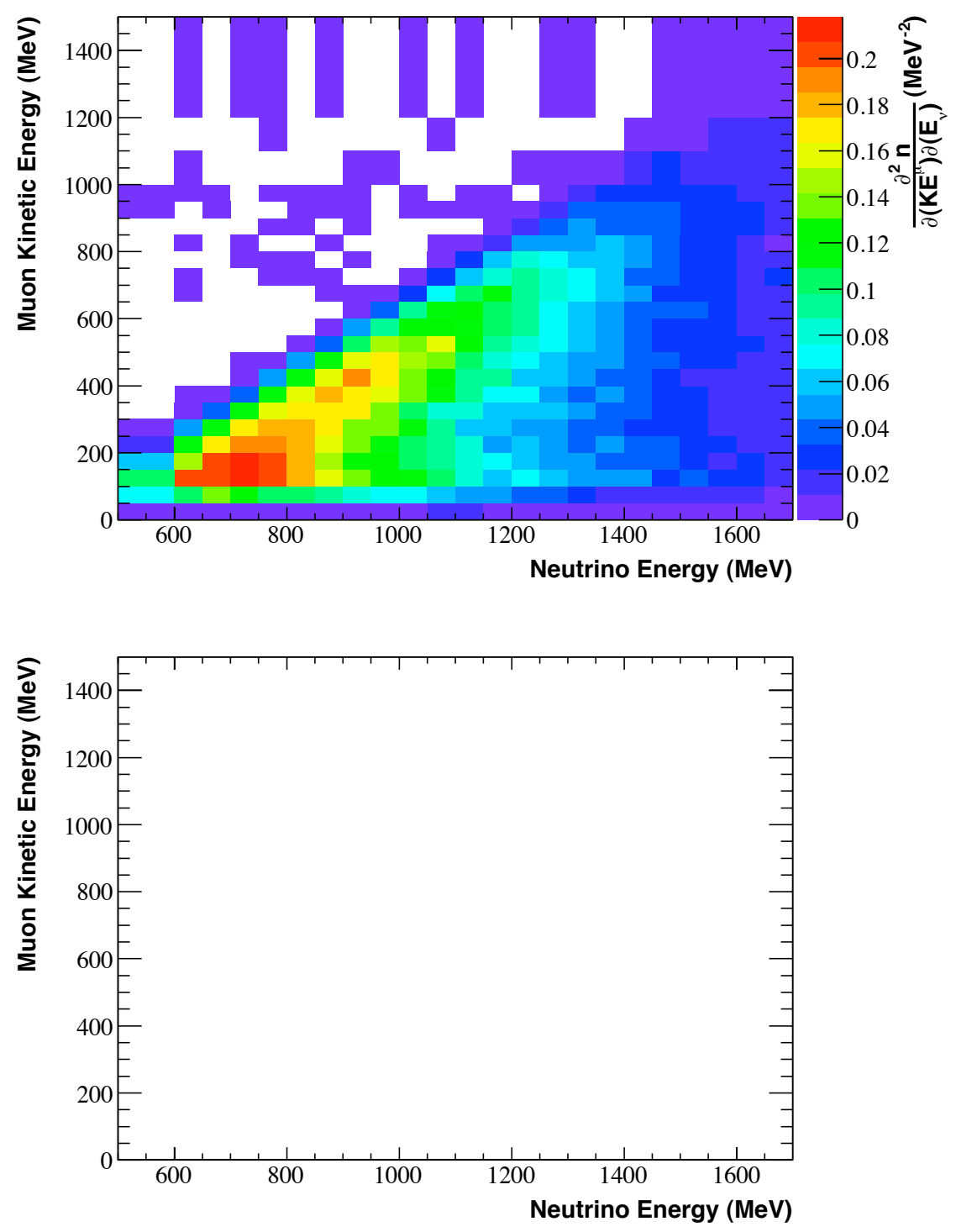

Figure B.25: The reconstructed muon kinetic energy vs neutrino energy distribution is shown for both the data (top) and Monte Carlo (bottom). 

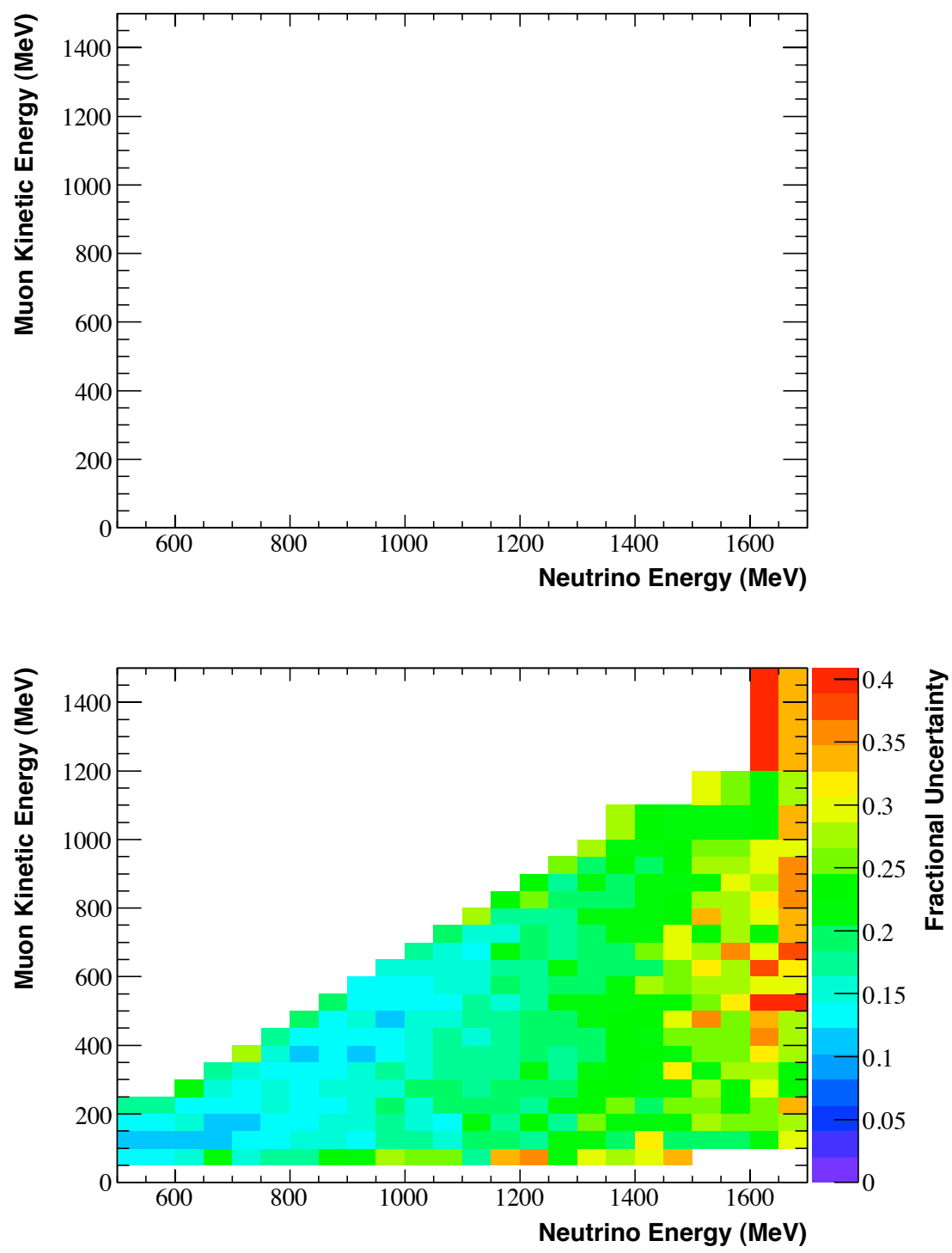

Figure B.26: The Monte Carlo reconstructed muon kinetic energy vs neutrino energy distribution is shown (top) along with the total fractional uncertainties (bottom). 


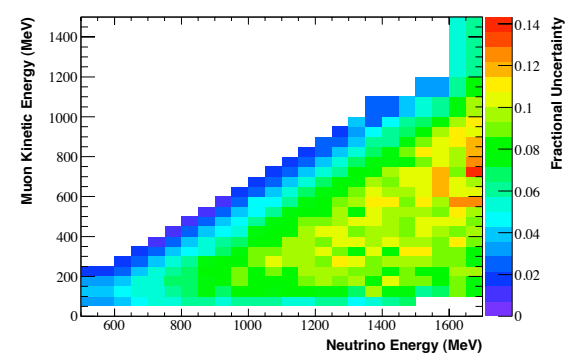

(a) The fractional systematic uncertainty from the "XSec" error source.

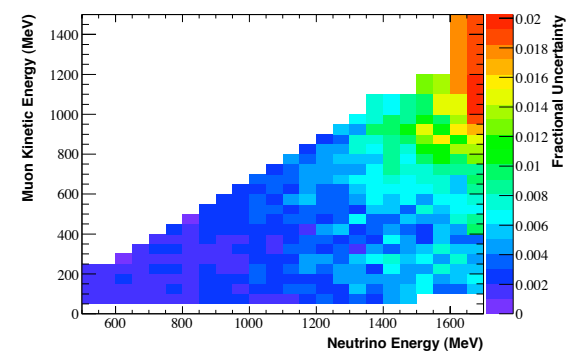

(c) The fractional systematic uncertainty from the " $\mathrm{K}^{+}$" error source.

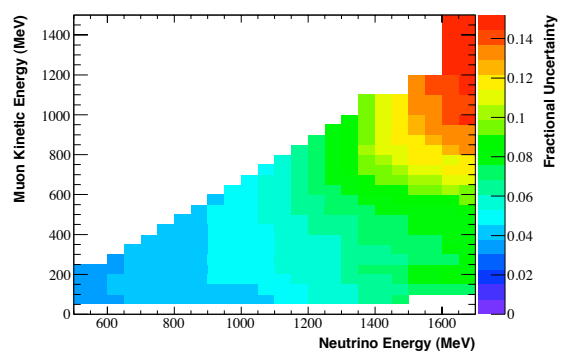

(b) The fractional systematic uncertainty from the "Beam" error source.

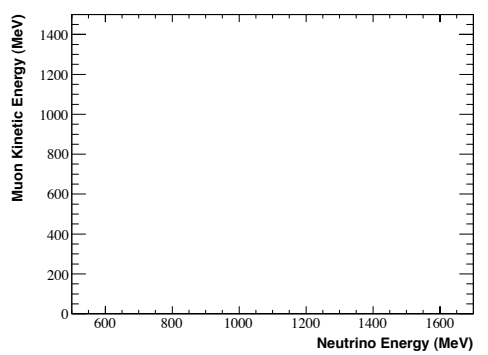

(d) Reconstructed Monte Carlo distribution

Figure B.27: The fractional systematic uncertainties on the reconstructed Monte Carlo distribution are shown for four of the error sources. The central value reconstructed Monte Carlo distribution is shown for reference. Note that the color scales differ for each systematic error source. An explanation of each error is given in Section 6.4.7. 


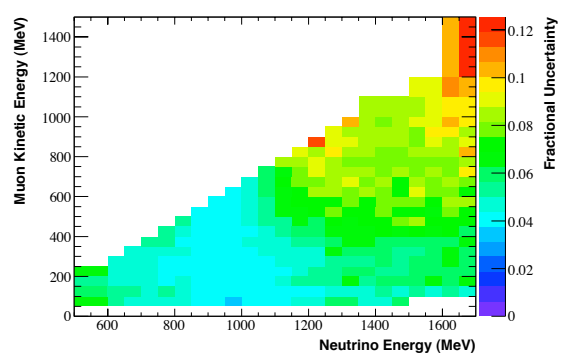

(a) The fractional systematic uncertainty from the " $\pi^{+}$" error source.

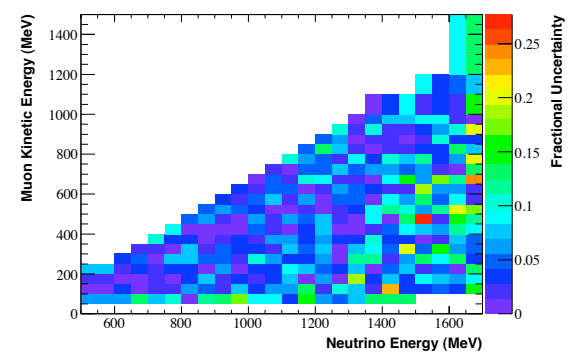

(c) The fractional systematic uncertainty from the "DISC" error source.

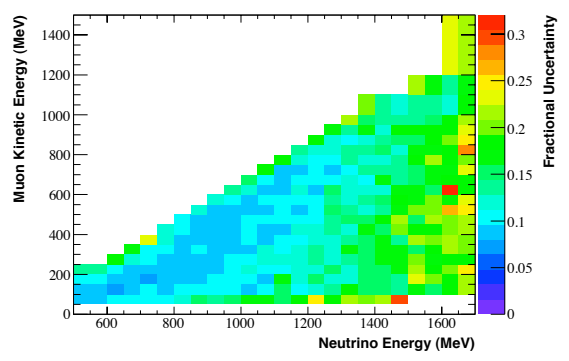

(b) The fractional systematic uncertainty from the "OM" error source.

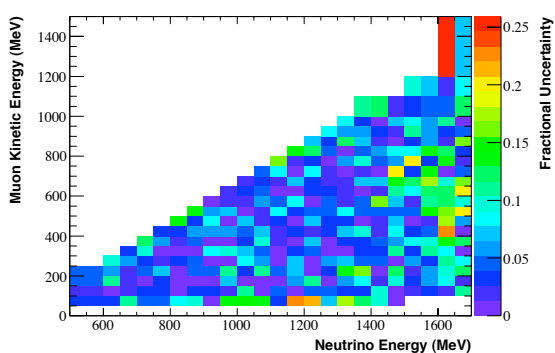

(d) The fractional systematic uncertainty from the "QTCORR" error source.

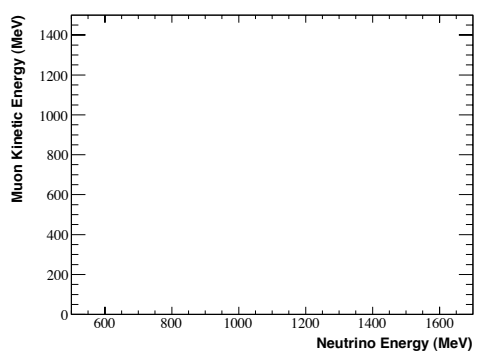

(e) Reconstructed Monte Carlo distribution

Figure B.28: The fractional systematic uncertainties on the reconstructed Monte Carlo distribution are shown for four of the error sources. The central value reconstructed Monte Carlo distribution is shown for reference. Note that the color scales differ for each systematic error source. An explanation of each error is given in Section 6.4.7. 


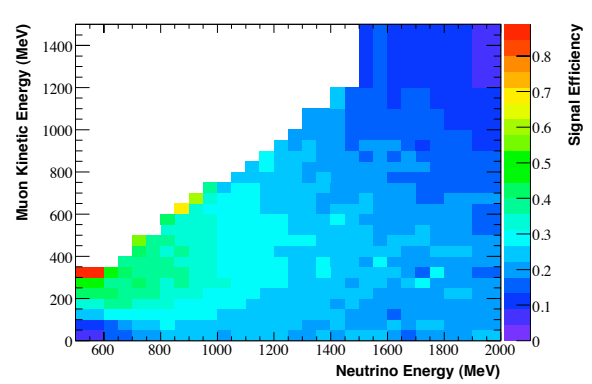

(a) Signal Efficiency

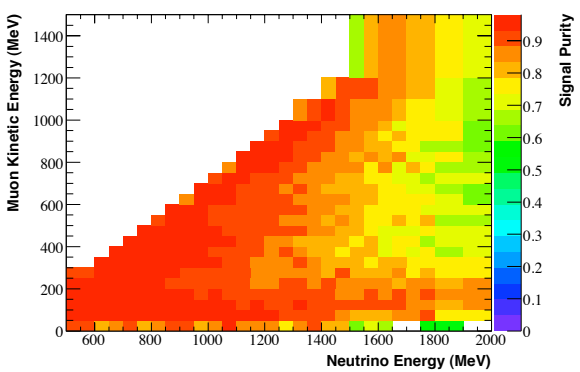

(c) Signal Purity

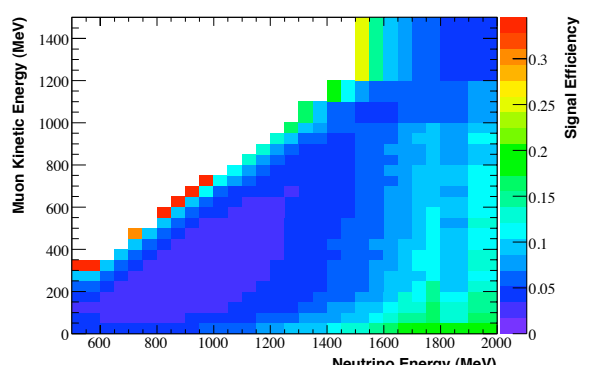

(b) Fractional Monte Carlo Statistical Error on the Signal Efficiency

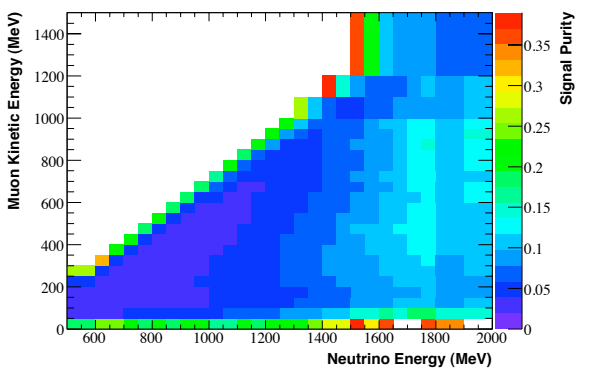

(d) Fractional Monte Carlo Statistical Error on the Signal Purity

Figure B.29: The signal efficiency and purity are shown for the muon kinetic energy vs neutrino energy distribution. The fractional errors due to Monte Carlo statistics for each of these distributions is also given for all bins with at least 10 events in the numerator. 


\section{B.10 Muon Direction vs Neutrino Energy}

- Reconstructed Data/Monte Carlo comparison: Figure B.30

- Reconstructed Monte Carlo Fractional Errors: Figures B.32 and B.33

- Signal efficiency: Figure B.34(a)

- Signal purity: Figure B.34(c) 

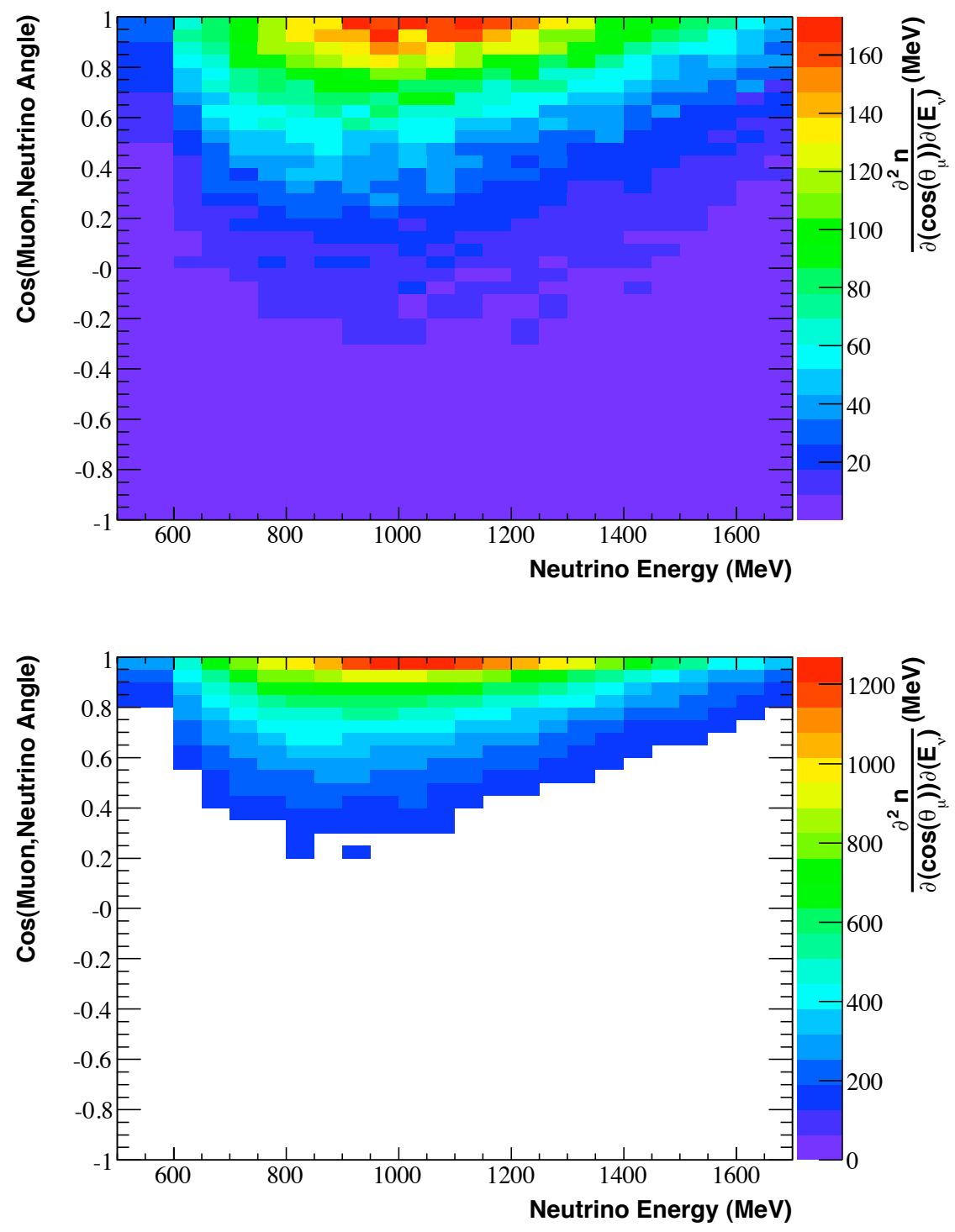

Figure B.30: The reconstructed $\cos ($ muon, neutrino angle) vs neutrino energy distribution is shown for both the data (top) and Monte Carlo (bottom). 

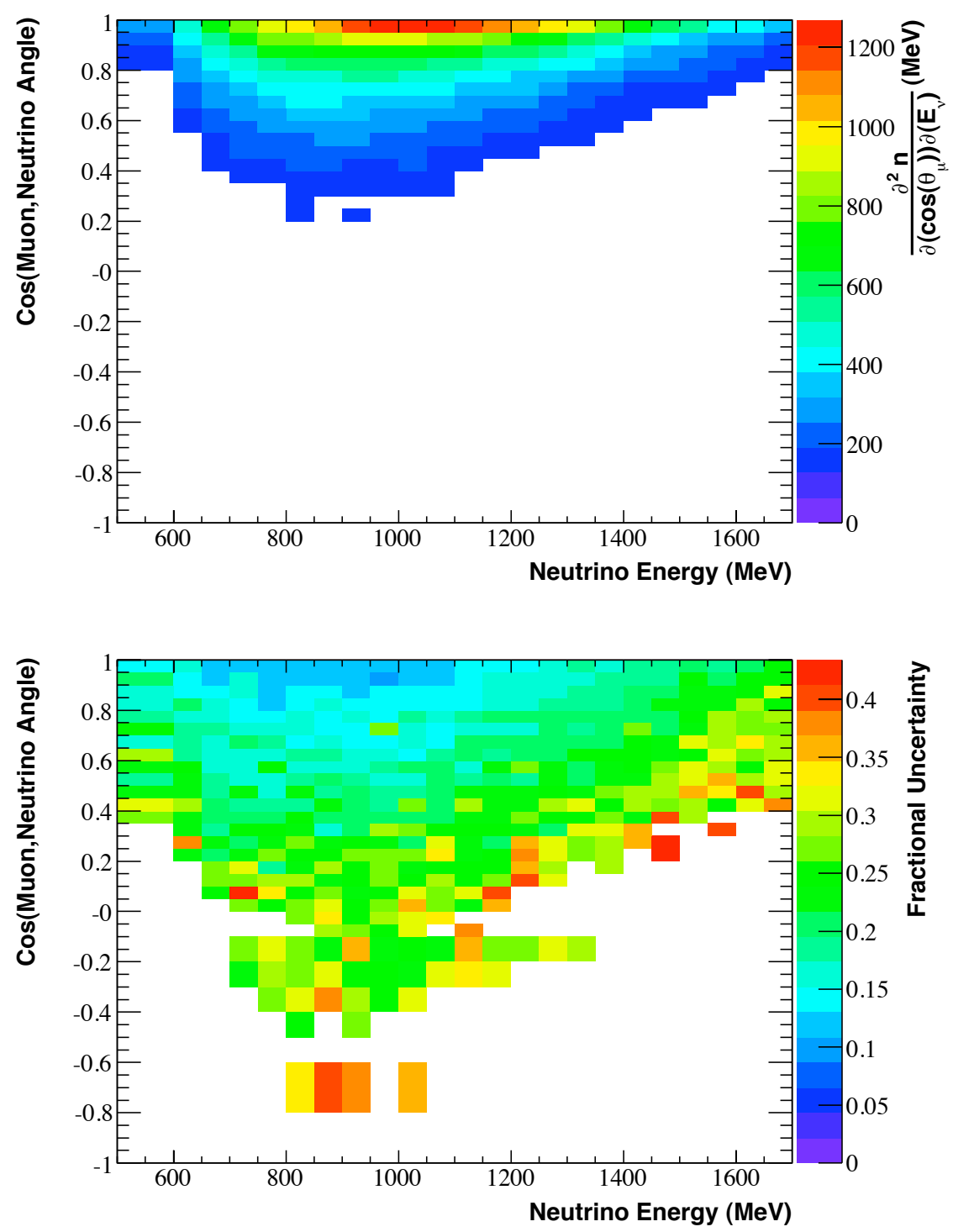

Figure B.31: The Monte Carlo reconstructed muon cos(muon, neutrino angle) vs neutrino energy distribution is shown (top) along with the total fractional uncertainties (bottom). 


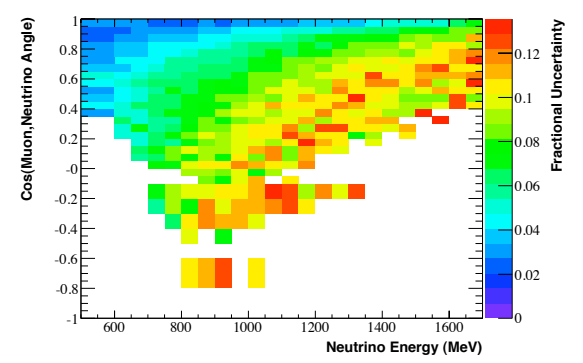

(a) The fractional systematic uncertainty from the "XSec" error source.

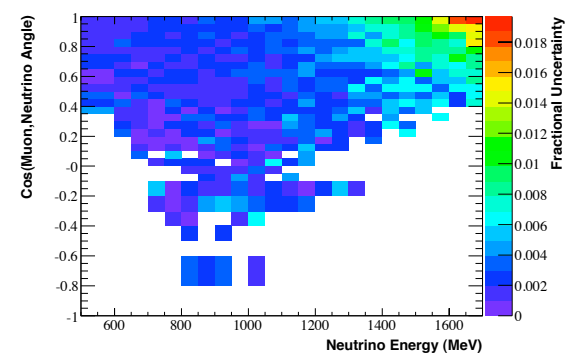

(c) The fractional systematic uncertainty from the " $\mathrm{K}^{+}$" error source.

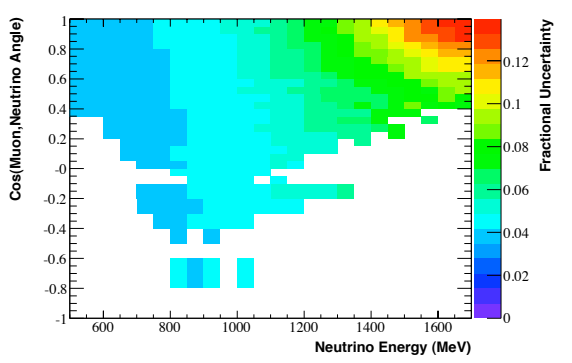

(b) The fractional systematic uncertainty from the "Beam" error source.

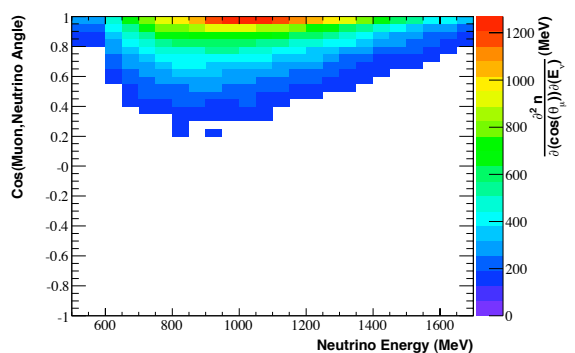

(d) Reconstructed Monte Carlo distribution

Figure B.32: The fractional systematic uncertainties on the reconstructed Monte Carlo distribution are shown for four of the error sources. The central value reconstructed Monte Carlo distribution is shown for reference. Note that the color scales differ for each systematic error source. An explanation of each error is given in Section 6.4.7. 


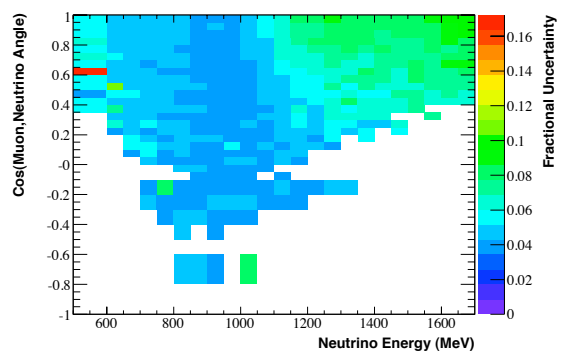

(a) The fractional systematic uncertainty from the " $\pi^{+}$" error source.

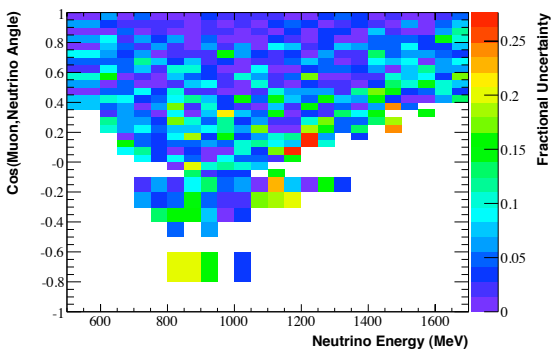

(c) The fractional systematic uncertainty from the "DISC" error source.

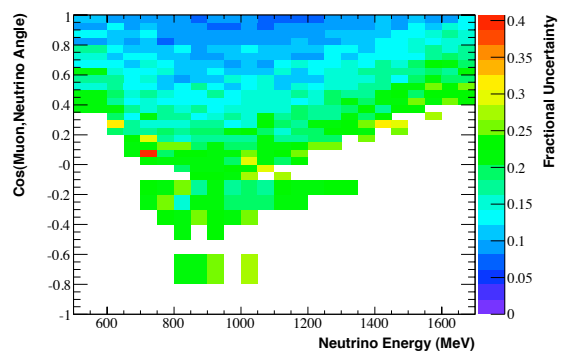

(b) The fractional systematic uncertainty from the "OM" error source.

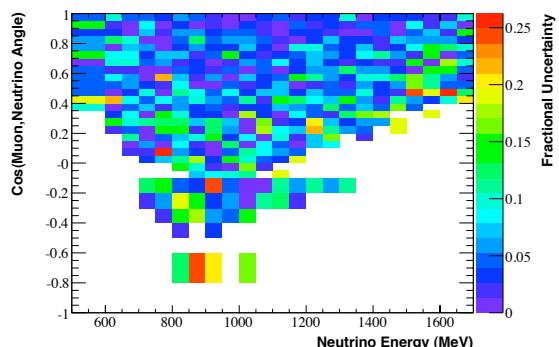

(d) The fractional systematic uncertainty from the "QTCORR" error source.

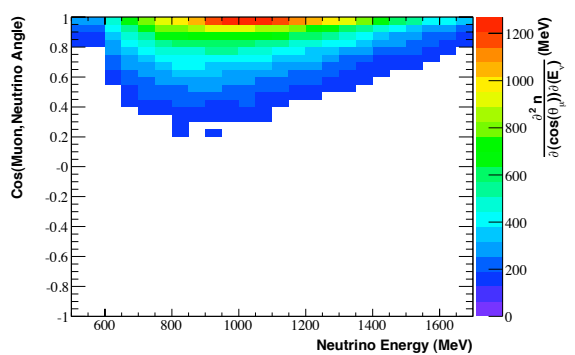

(e) Reconstructed Monte Carlo distribution

Figure B.33: The fractional systematic uncertainties on the reconstructed Monte Carlo distribution are shown for four of the error sources. The central value reconstructed Monte Carlo distribution is shown for reference. Note that the color scales differ for each systematic error source. An explanation of each error is given in Section 6.4.7. 


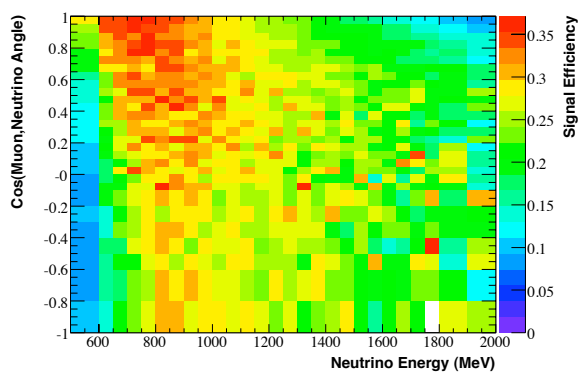

(a) Signal Efficiency

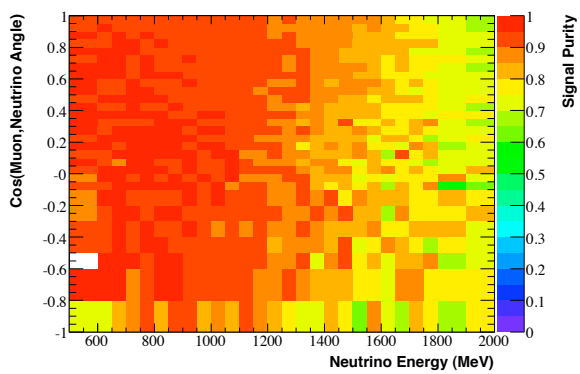

(c) Signal Purity

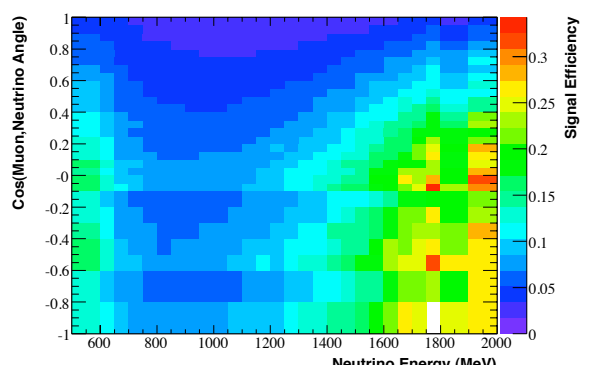

(b) Fractional Monte Carlo Statistical Error on the Signal Efficiency

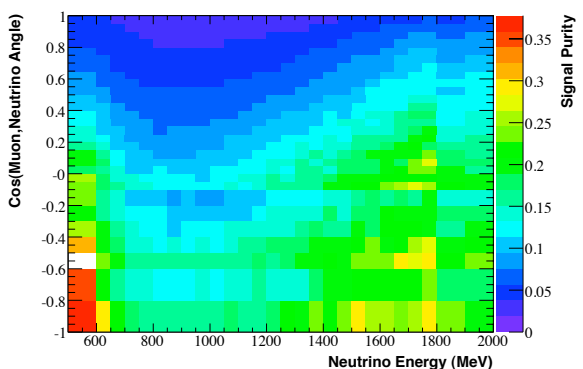

(d) Fractional Monte Carlo Statistical Error on the Signal Purity

Figure B.34: The signal efficiency and purity are shown for the muon cos(muon, neutrino angle) vs neutrino energy distribution. The fractional errors due to Monte Carlo statistics for each of these distributions is also given for all bins with at least 10 events in the numerator. 


\section{B.11 Pion Kinetic Energy vs Neutrino Energy}

- Reconstructed Data/Monte Carlo comparison: Figure B.35

- Reconstructed Monte Carlo Fractional Errors: Figures B.37 and B.38

- Signal efficiency: Figure B.39(a)

- Signal purity: Figure B.39(c) 

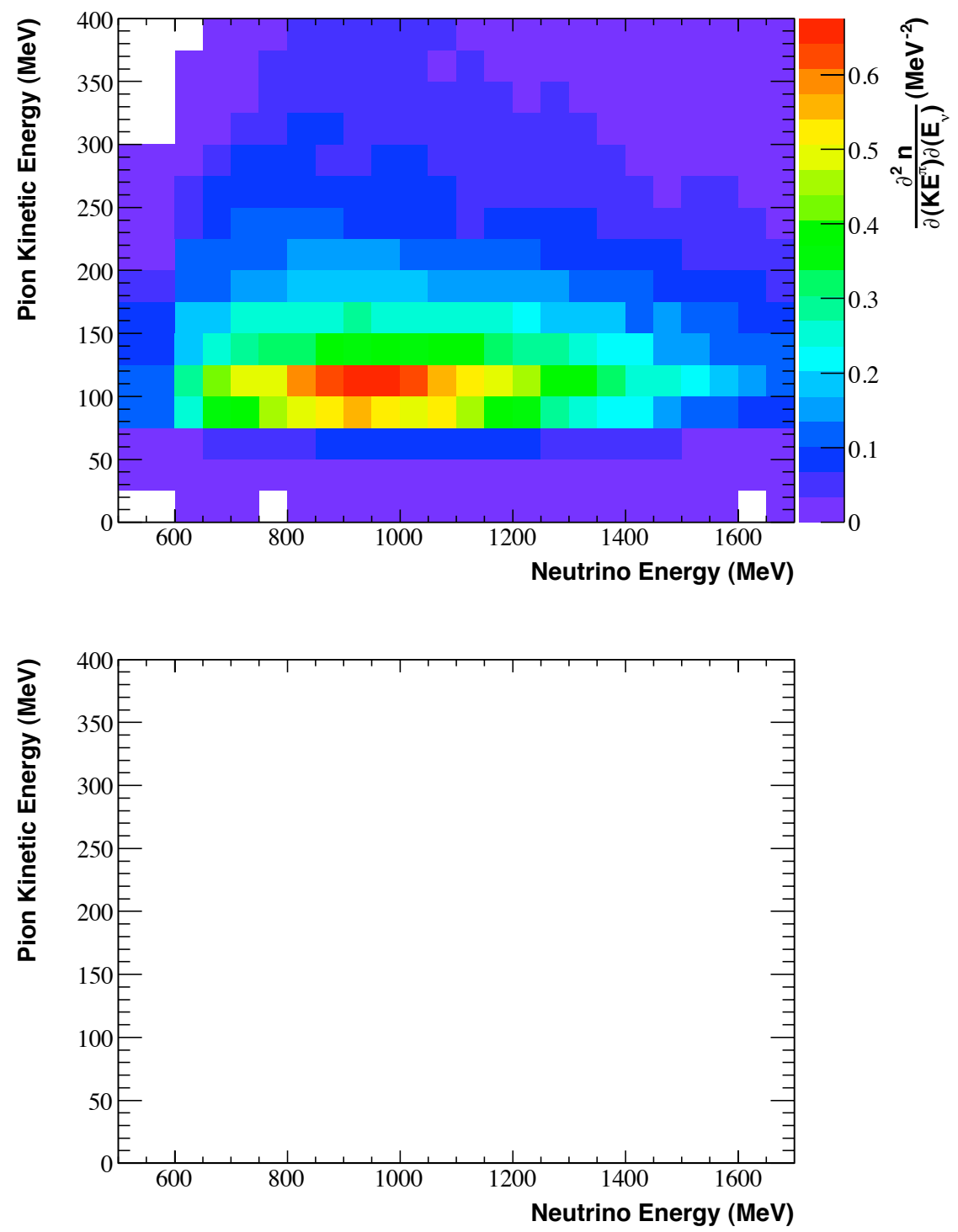

Figure B.35: The reconstructed pion kinetic energy vs neutrino energy distribution is shown for both the data (top) and Monte Carlo (bottom). 

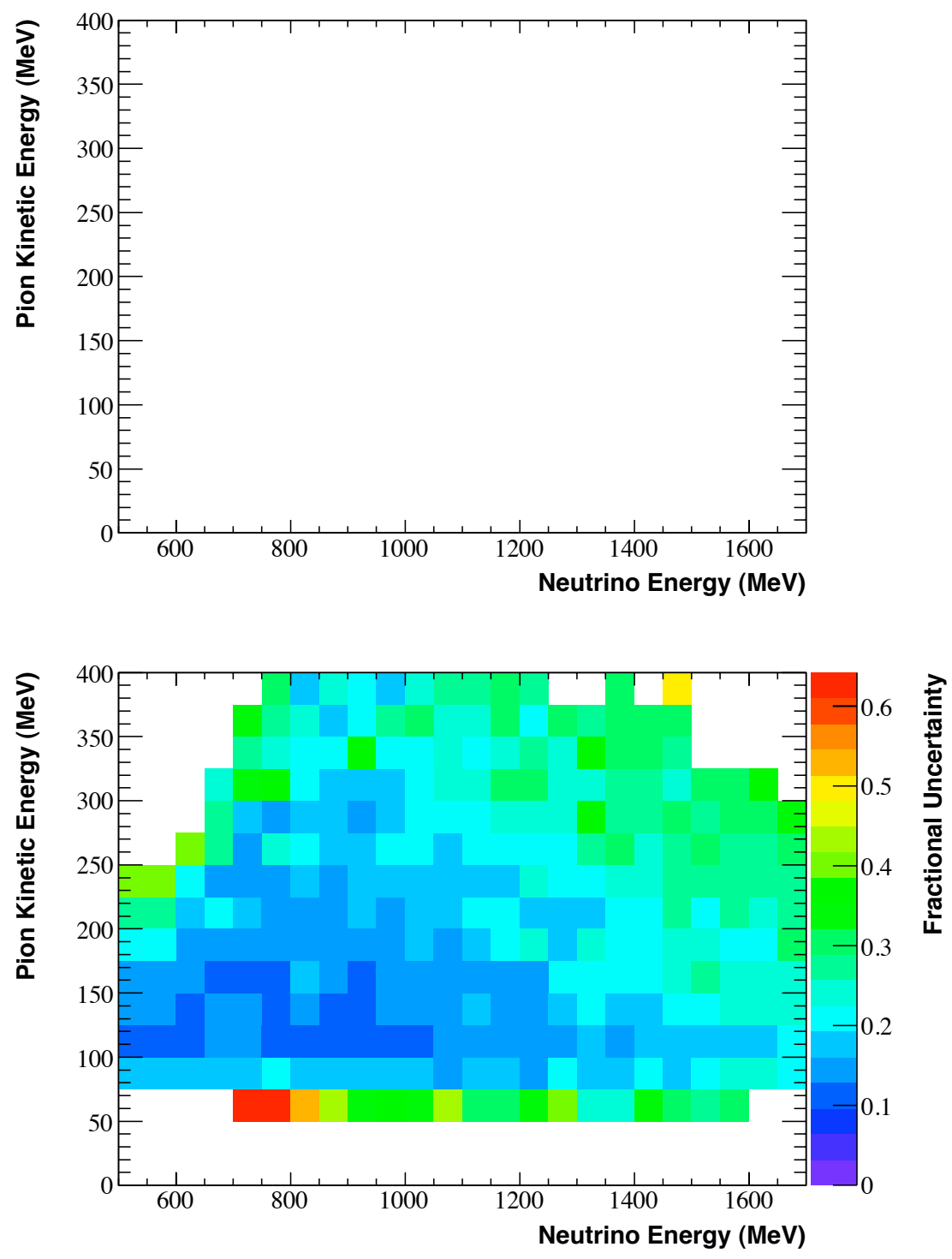

Figure B.36: The Monte Carlo reconstructed pion kinetic energy vs neutrino energy distribution is shown (top) along with the total fractional uncertainties (bottom). 


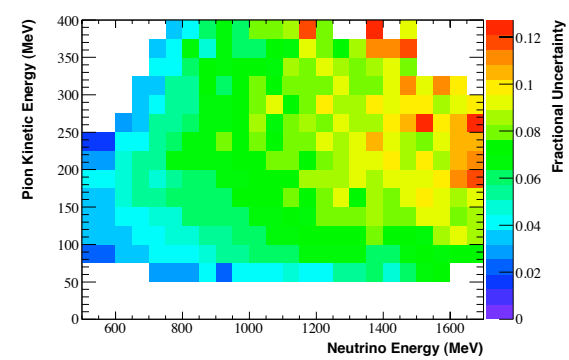

(a) The fractional systematic uncertainty from the "XSec" error source.

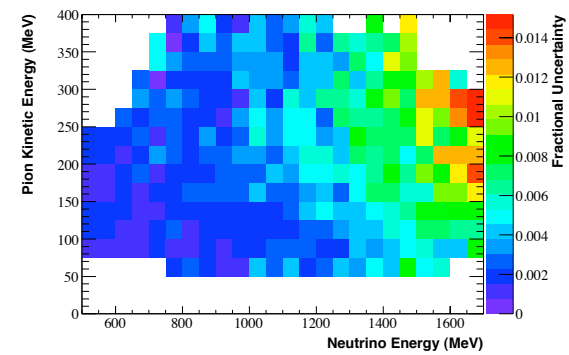

(c) The fractional systematic uncertainty from the " $\mathrm{K}^{+}$" error source.

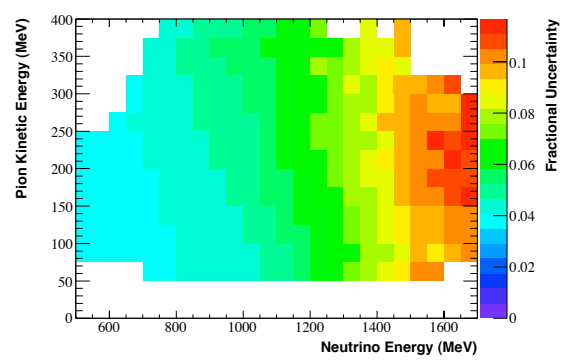

(b) The fractional systematic uncertainty from the "Beam" error source.

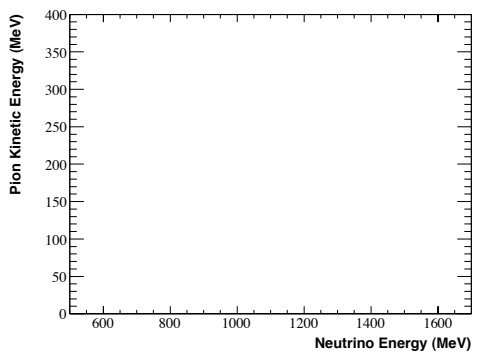

(d) Reconstructed Monte Carlo distribution

Figure B.37: The fractional systematic uncertainties on the reconstructed Monte Carlo distribution are shown for four of the error sources. The central value reconstructed Monte Carlo distribution is shown for reference. Note that the color scales differ for each systematic error source. An explanation of each error is given in Section 6.4.7. 


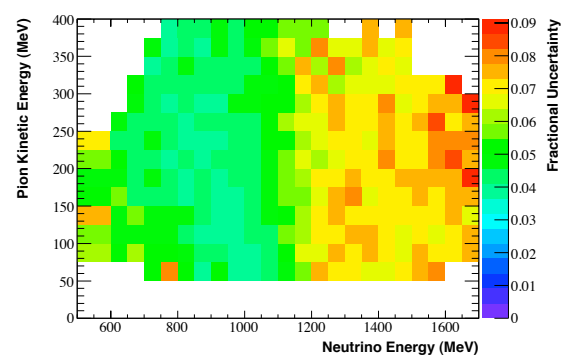

(a) The fractional systematic uncertainty from the " $\pi^{+}$" error source.

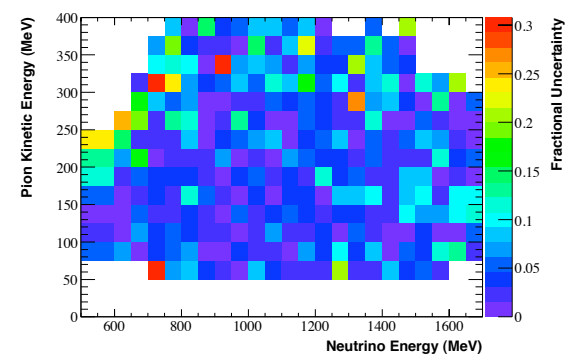

(c) The fractional systematic uncertainty from the "DISC" error source.

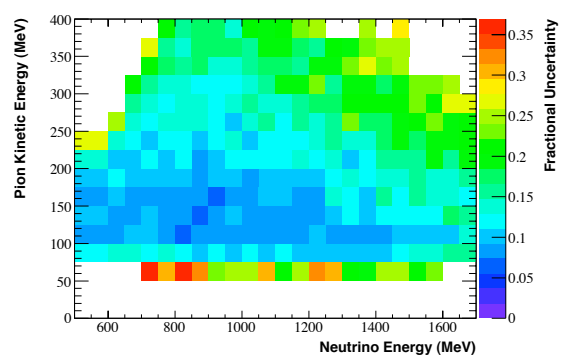

(b) The fractional systematic uncertainty from the "OM" error source.

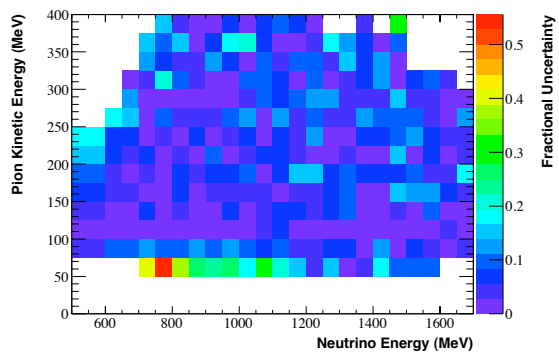

(d) The fractional systematic uncertainty from the "QTCORR" error source.

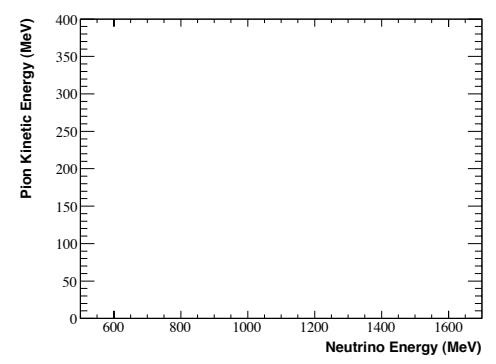

(e) Reconstructed Monte Carlo distribution

Figure B.38: The fractional systematic uncertainties on the reconstructed Monte Carlo distribution are shown for four of the error sources. The central value reconstructed Monte Carlo distribution is shown for reference. Note that the color scales differ for each systematic error source. An explanation of each error is given in Section 6.4.7. 


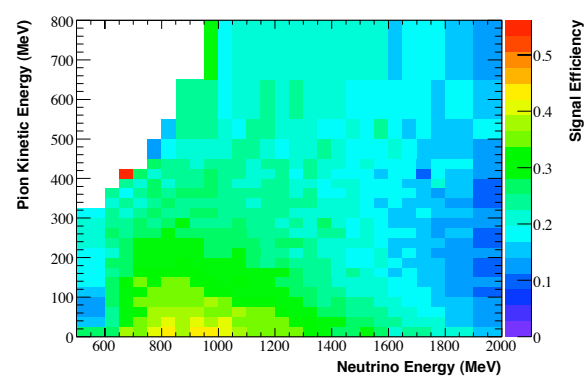

(a) Signal Efficiency

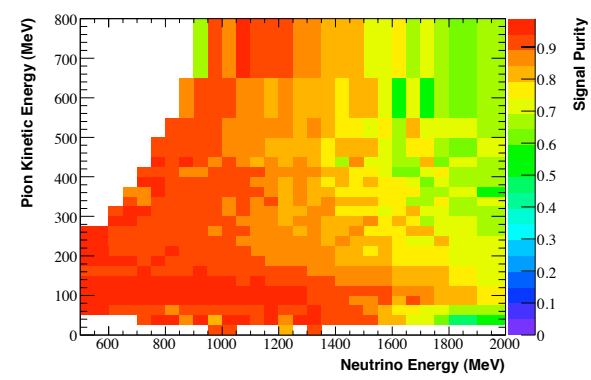

(c) Signal Purity

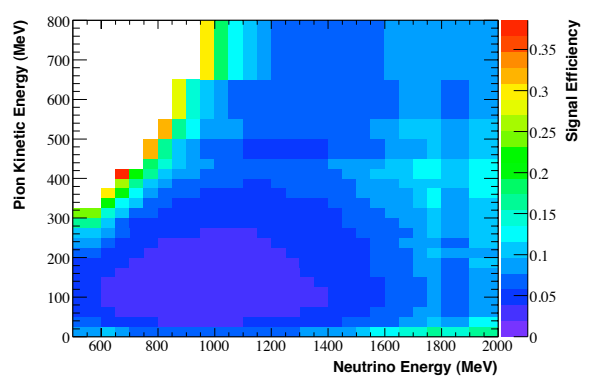

(b) Fractional Monte Carlo Statistical Error on the Signal Efficiency

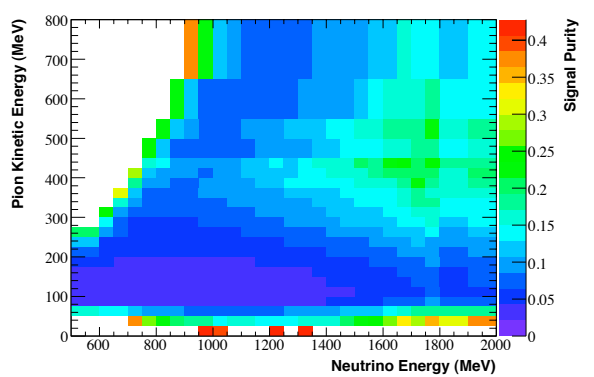

(d) Fractional Monte Carlo Statistical Error on the Signal Purity

Figure B.39: The signal efficiency and purity are shown for the pion kinetic energy vs neutrino energy distribution. The fractional errors due to Monte Carlo statistics for each of these distributions is also given for all bins with at least 10 events in the numerator. 


\section{B.12 Pion Direction vs Neutrino Energy}

- Reconstructed Data/Monte Carlo comparison: Figure B.40

- Reconstructed Monte Carlo Fractional Errors: Figures B.42 and B.43

- Signal efficiency: Figure B.44(a)

- Signal purity: Figure B.44(c) 

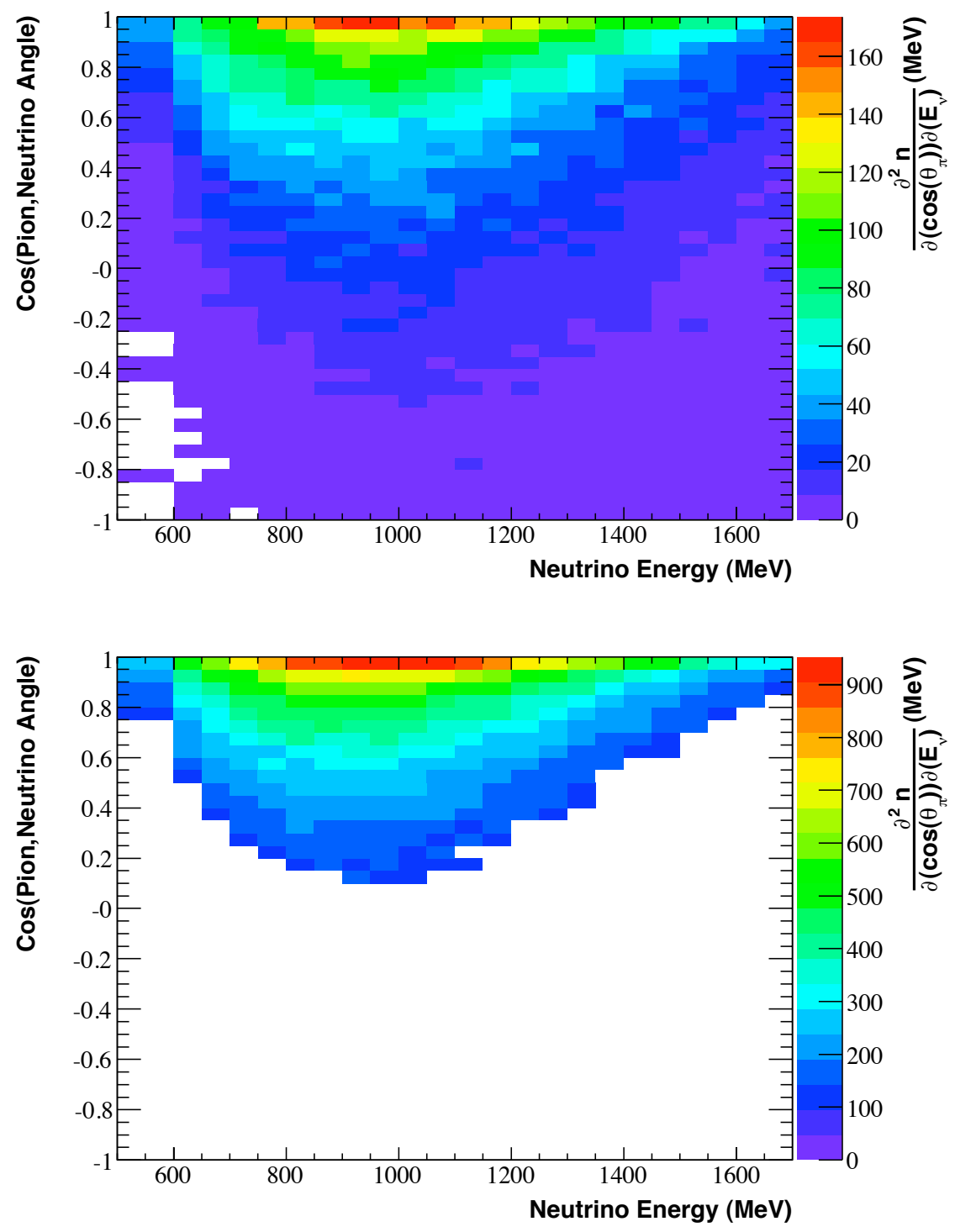

Figure B.40: The reconstructed cos(pion, neutrino angle) vs neutrino energy distribution is shown for both the data (top) and Monte Carlo (bottom). 

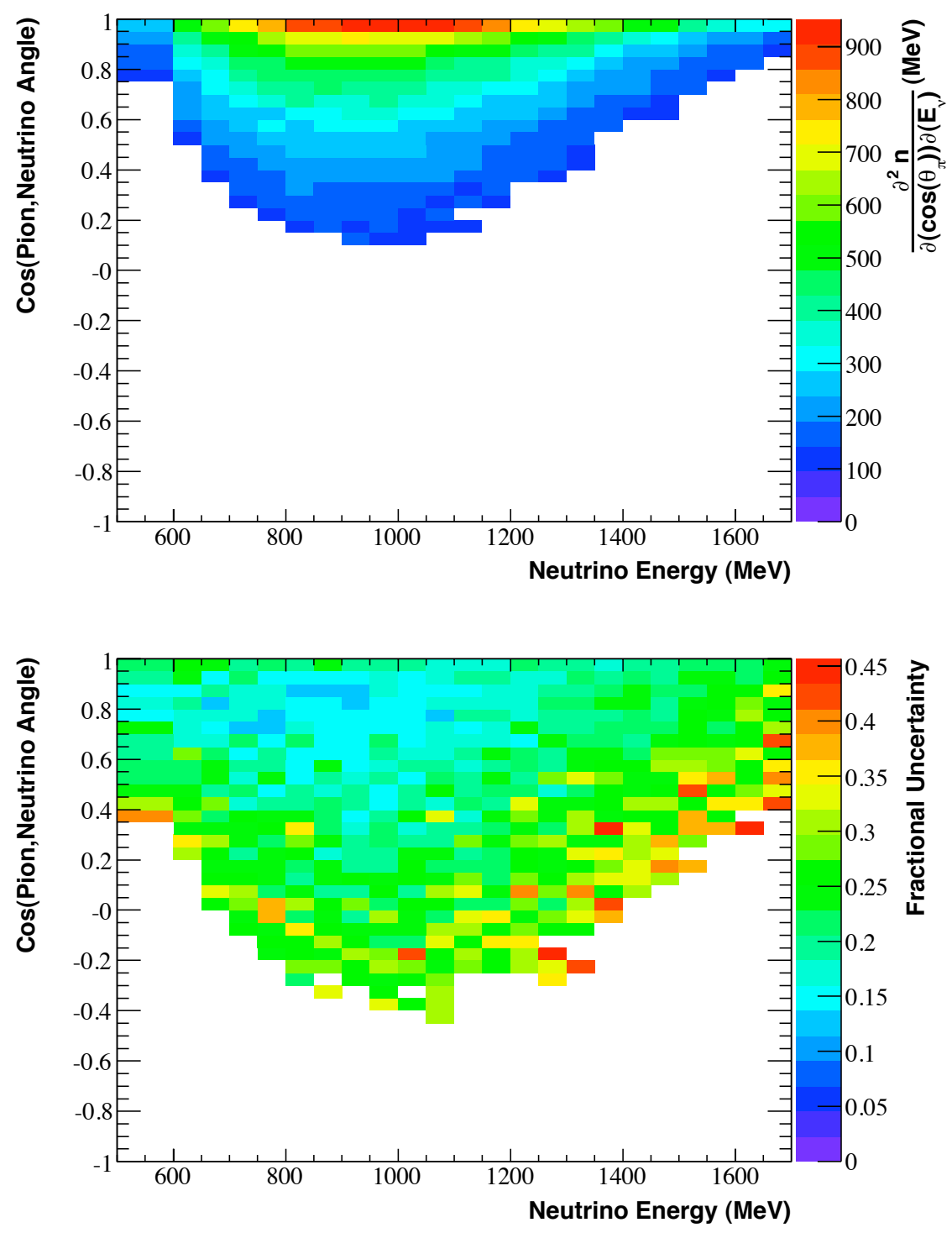

Figure B.41: The Monte Carlo reconstructed pion cos(pion, neutrino angle) vs neutrino energy distribution is shown (top) along with the total fractional uncertainties (bottom). 


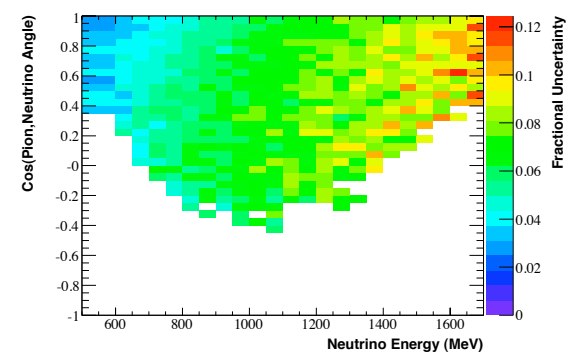

(a) The fractional systematic uncertainty from the "XSec" error source.

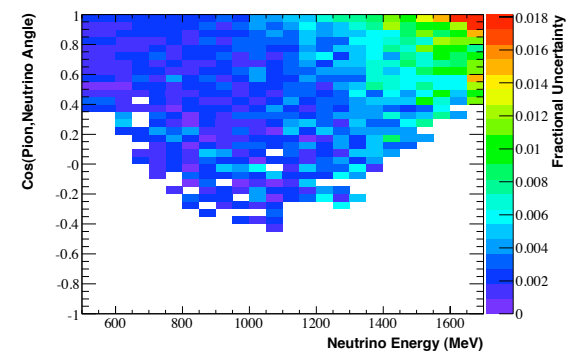

(c) The fractional systematic uncertainty from the " $\mathrm{K}^{+}$" error source.

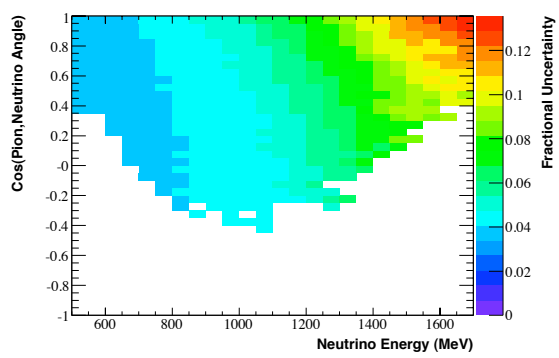

(b) The fractional systematic uncertainty from the "Beam" error source.

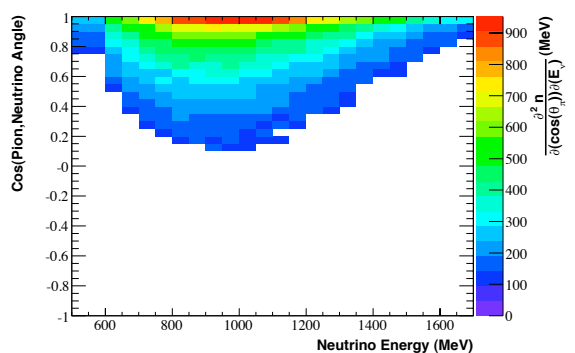

(d) Reconstructed Monte Carlo distribution

Figure B.42: The fractional systematic uncertainties on the reconstructed Monte Carlo distribution are shown for four of the error sources. The central value reconstructed Monte Carlo distribution is shown for reference. Note that the color scales differ for each systematic error source. An explanation of each error is given in Section 6.4.7. 


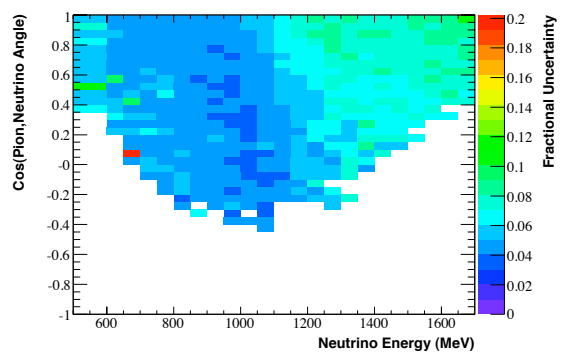

(a) The fractional systematic uncertainty from the " $\pi^{+}$" error source.

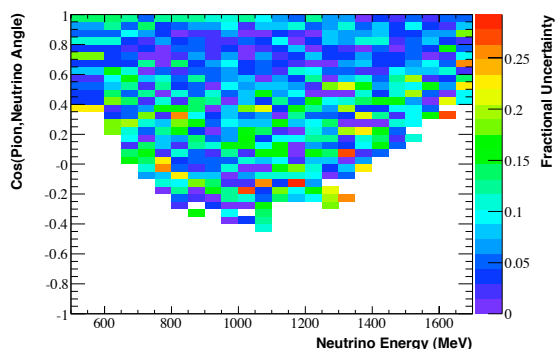

(c) The fractional systematic uncertainty from the "DISC" error source.

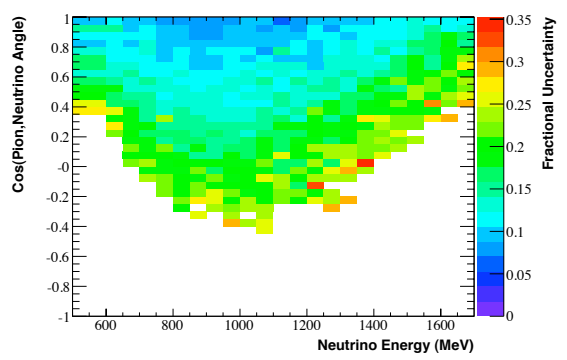

(b) The fractional systematic uncertainty from the "OM" error source.

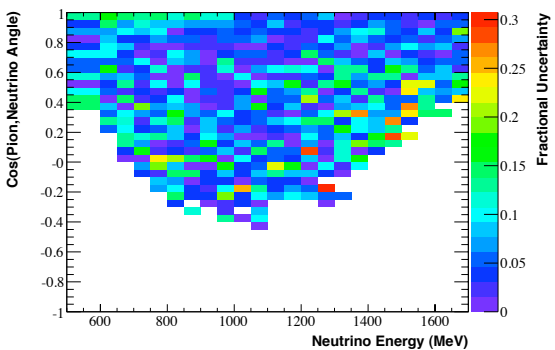

(d) The fractional systematic uncertainty from the "QTCORR" error source.

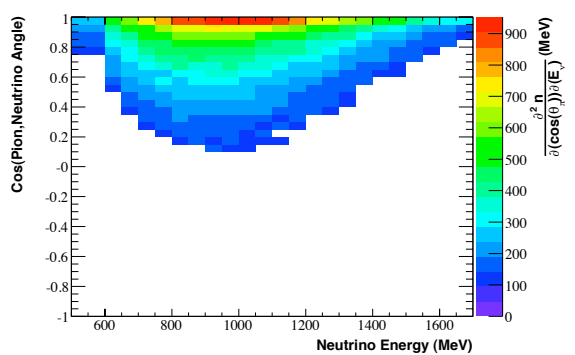

(e) Reconstructed Monte Carlo distribution

Figure B.43: The fractional systematic uncertainties on the reconstructed Monte Carlo distribution are shown for four of the error sources. The central value reconstructed Monte Carlo distribution is shown for reference. Note that the color scales differ for each systematic error source. An explanation of each error is given in Section 6.4.7. 


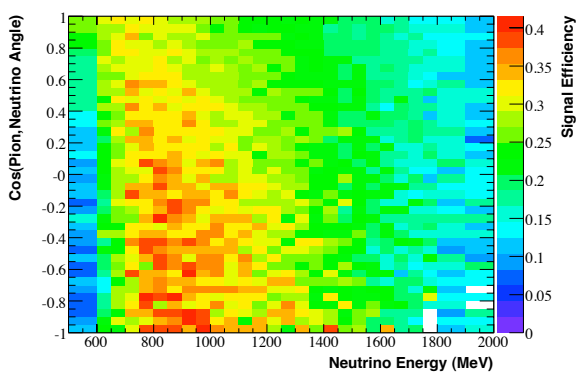

(a) Signal Efficiency

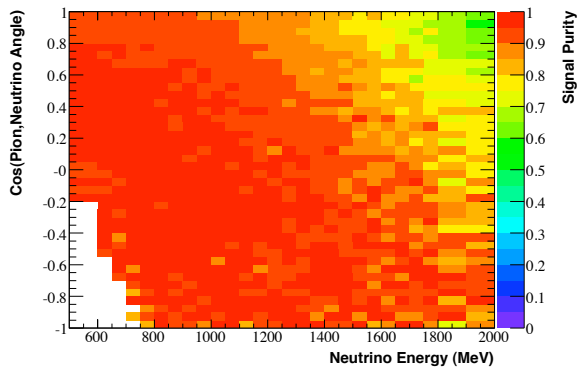

(c) Signal Purity

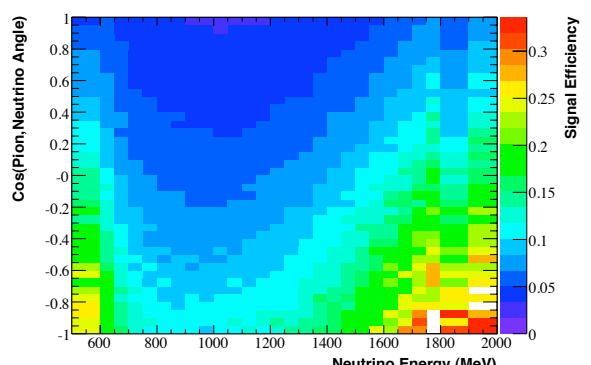

(b) Fractional Monte Carlo Statistical Error on the Signal Efficiency

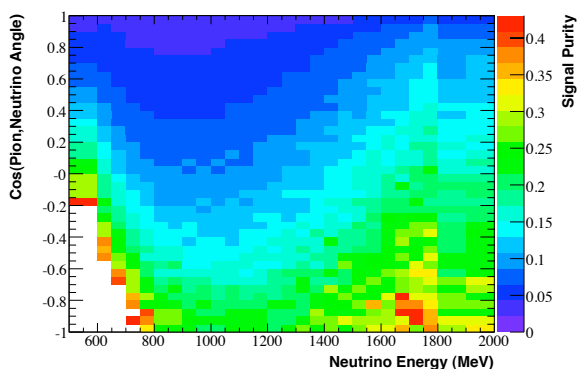

(d) Fractional Monte Carlo Statistical Error on the Signal Purity

Figure B.44: The signal efficiency and purity are shown for the pion $\cos$ (pion, neutrino angle) vs neutrino energy distribution. The fractional errors due to Monte Carlo statistics for each of these distributions is also given for all bins with at least 10 events in the numerator. 


\section{B.13 Muon Direction vs Kinetic Energy}

- Reconstructed Data/Monte Carlo comparison: Figure B.45

- Reconstructed Monte Carlo Fractional Errors: Figures B.47 and B.48

- Signal efficiency: Figure B.49(a)

- Signal purity: Figure B.49(c) 

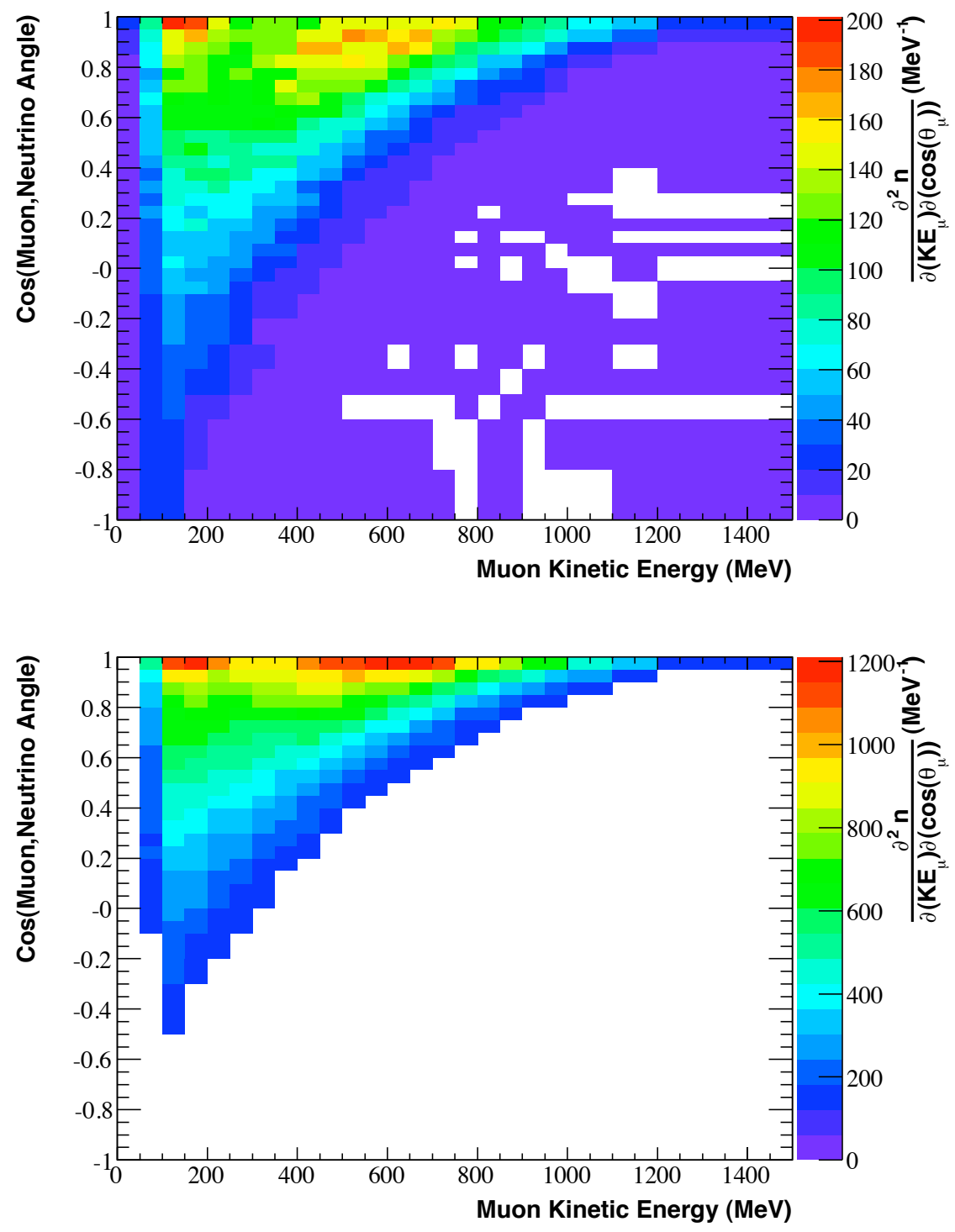

Figure B.45: The reconstructed $\cos ($ muon, neutrino angle) vs muon kinetic energy distribution is shown for both the data (top) and Monte Carlo (bottom). 

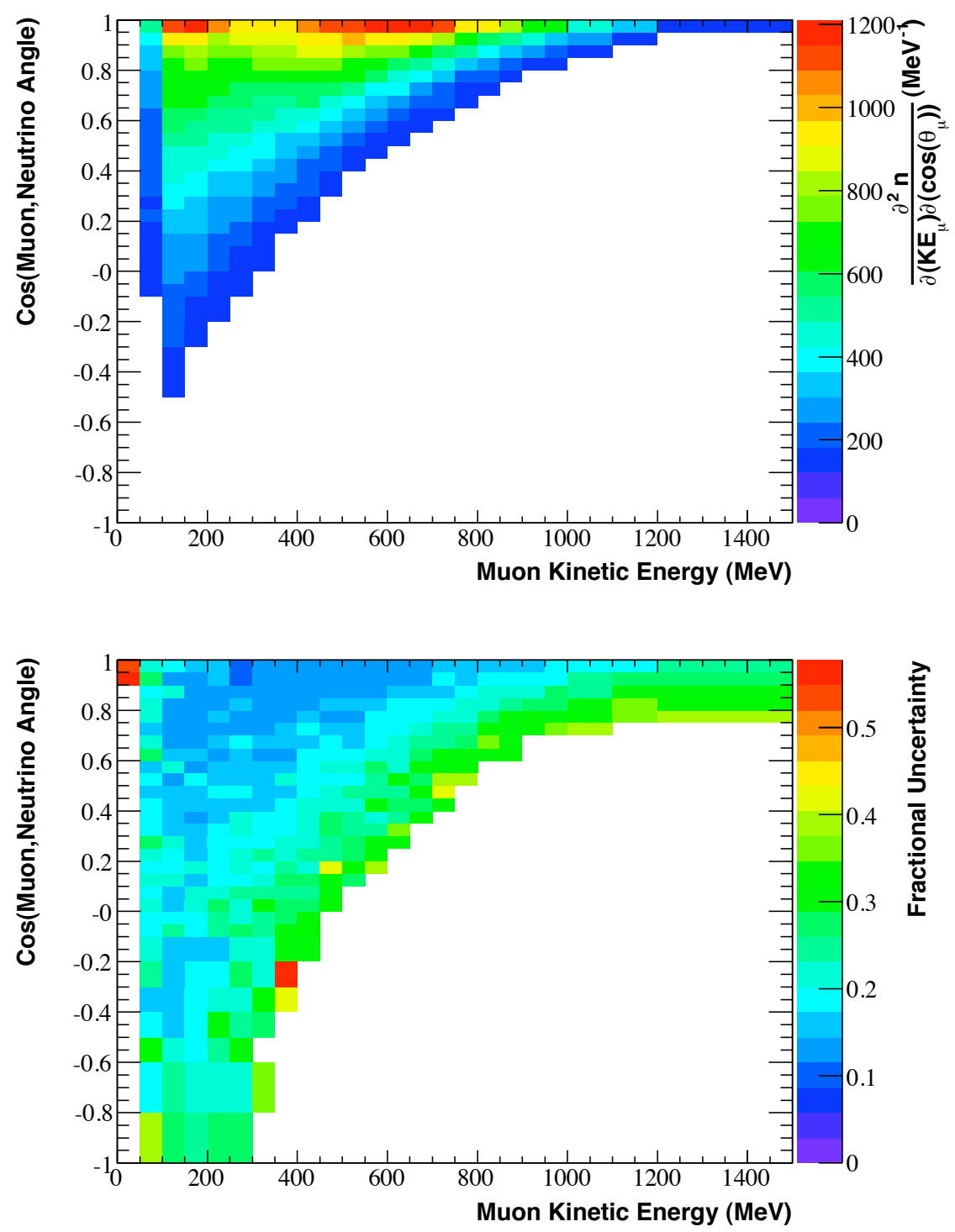

Figure B.46: The Monte Carlo reconstructed cos(muon, neutrino angle) vs muon kinetic energy distribution is shown (top) along with the total fractional uncertainties (bottom). 


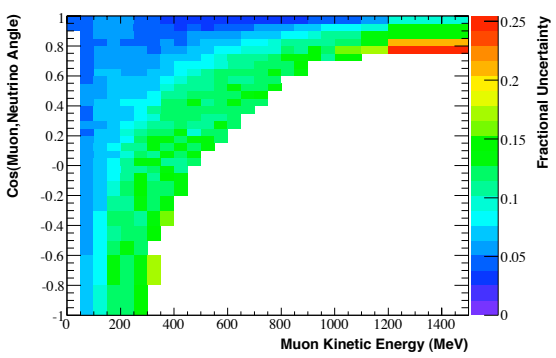

(a) The fractional systematic uncertainty from the "XSec" error source.

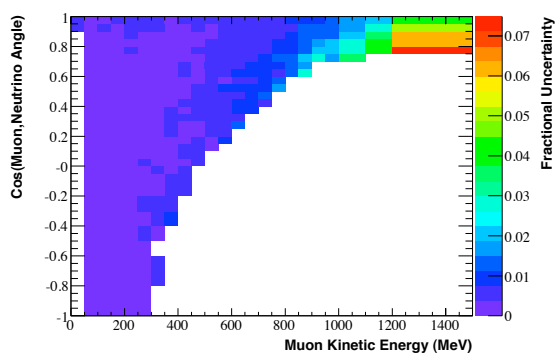

(c) The fractional systematic uncertainty from the " $\mathrm{K}^{+}$" error source.

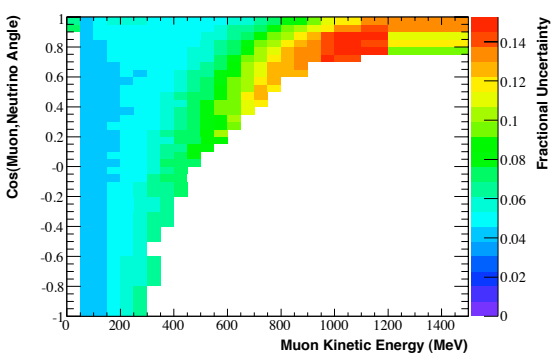

(b) The fractional systematic uncertainty from the "Beam" error source.

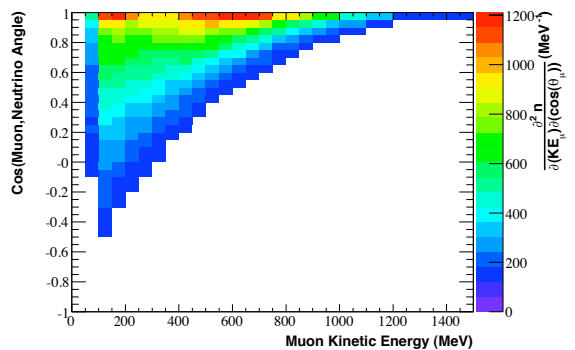

(d) Reconstructed Monte Carlo distribution

Figure B.47: The fractional systematic uncertainties on the reconstructed Monte Carlo distribution are shown for four of the error sources. The central value reconstructed Monte Carlo distribution is shown for reference. Note that the color scales differ for each systematic error source. An explanation of each error is given in Section 6.4.7. 


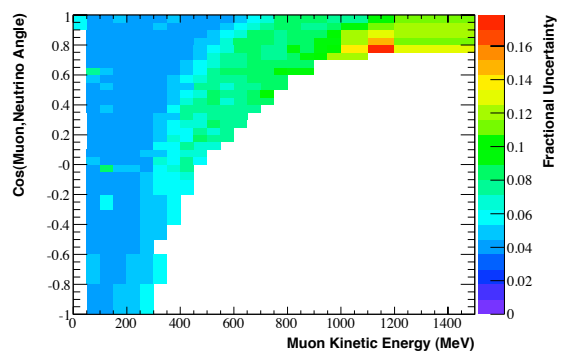

(a) The fractional systematic uncertainty from the " $\pi^{+}$" error source.

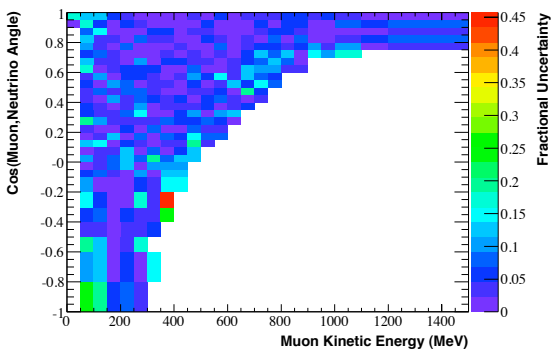

(c) The fractional systematic uncertainty from the "DISC" error source.

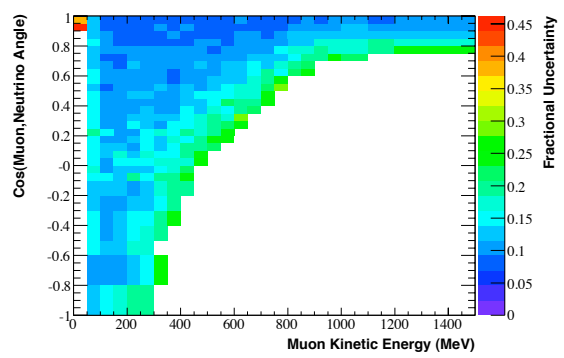

(b) The fractional systematic uncertainty from the "OM" error source.

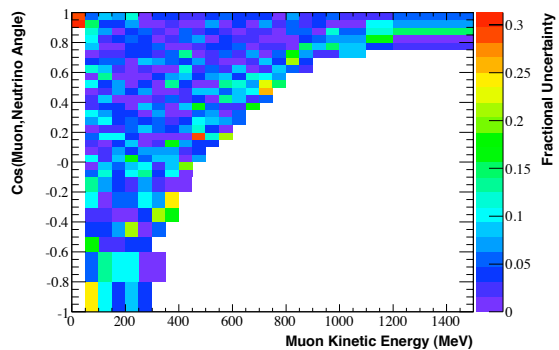

(d) The fractional systematic uncertainty from the "QTCORR" error source.

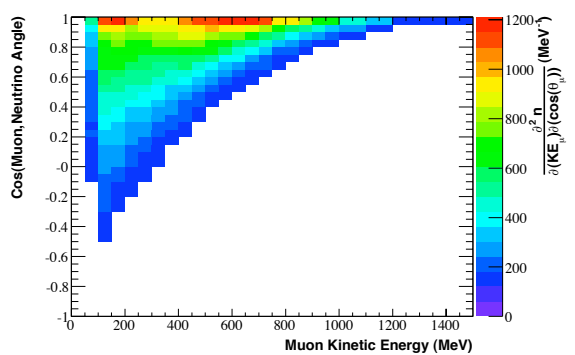

(e) Reconstructed Monte Carlo distribution

Figure B.48: The fractional systematic uncertainties on the reconstructed Monte Carlo distribution are shown for four of the error sources. The central value reconstructed Monte Carlo distribution is shown for reference. Note that the color scales differ for each systematic error source. An explanation of each error is given in Section 6.4.7. 


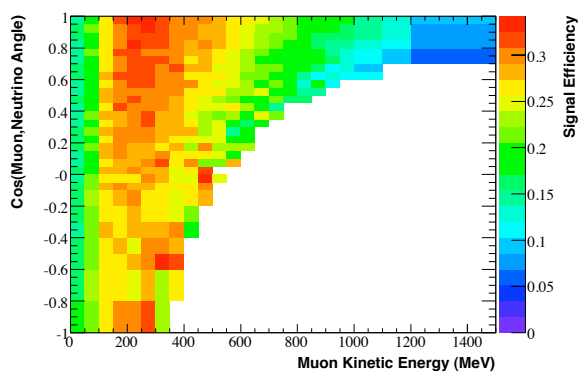

(a) Signal Efficiency

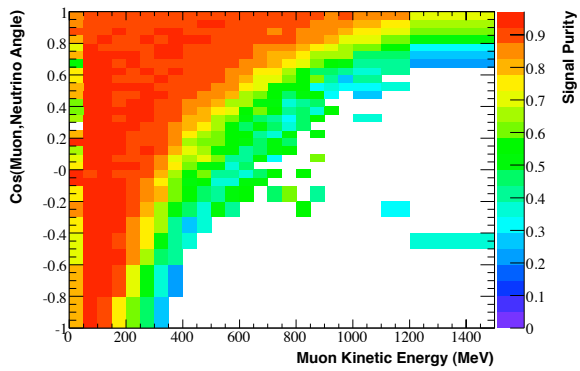

(c) Signal Purity

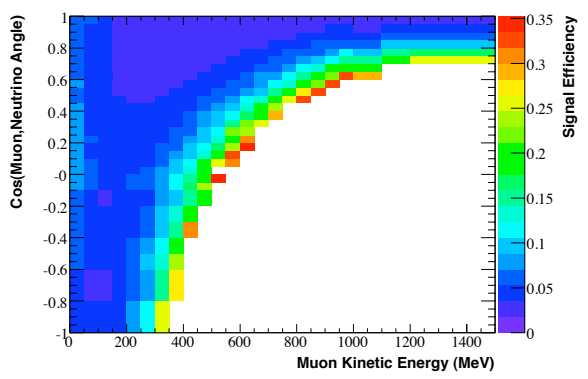

(b) Fractional Monte Carlo Statistical Error on the Signal Efficiency

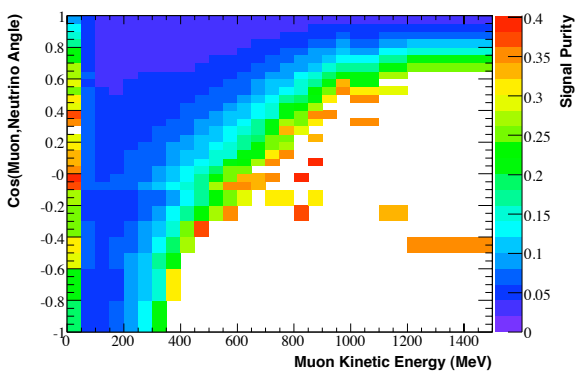

(d) Fractional Monte Carlo Statistical Error on the Signal Purity

Figure B.49: The signal efficiency and purity are shown for the $\cos$ (muon, neutrino angle) vs muon kinetic energy distribution. The fractional errors due to Monte Carlo statistics for each of these distributions is also given for all bins with at least 10 events in the numerator. 


\section{B.14 Pion Direction vs Kinetic Energy}

- Reconstructed Data/Monte Carlo comparison: Figure B.50

- Reconstructed Monte Carlo Fractional Errors: Figures B.52 and B.53

- Signal efficiency: Figure B.54(a)

- Signal purity: Figure B.54(c) 

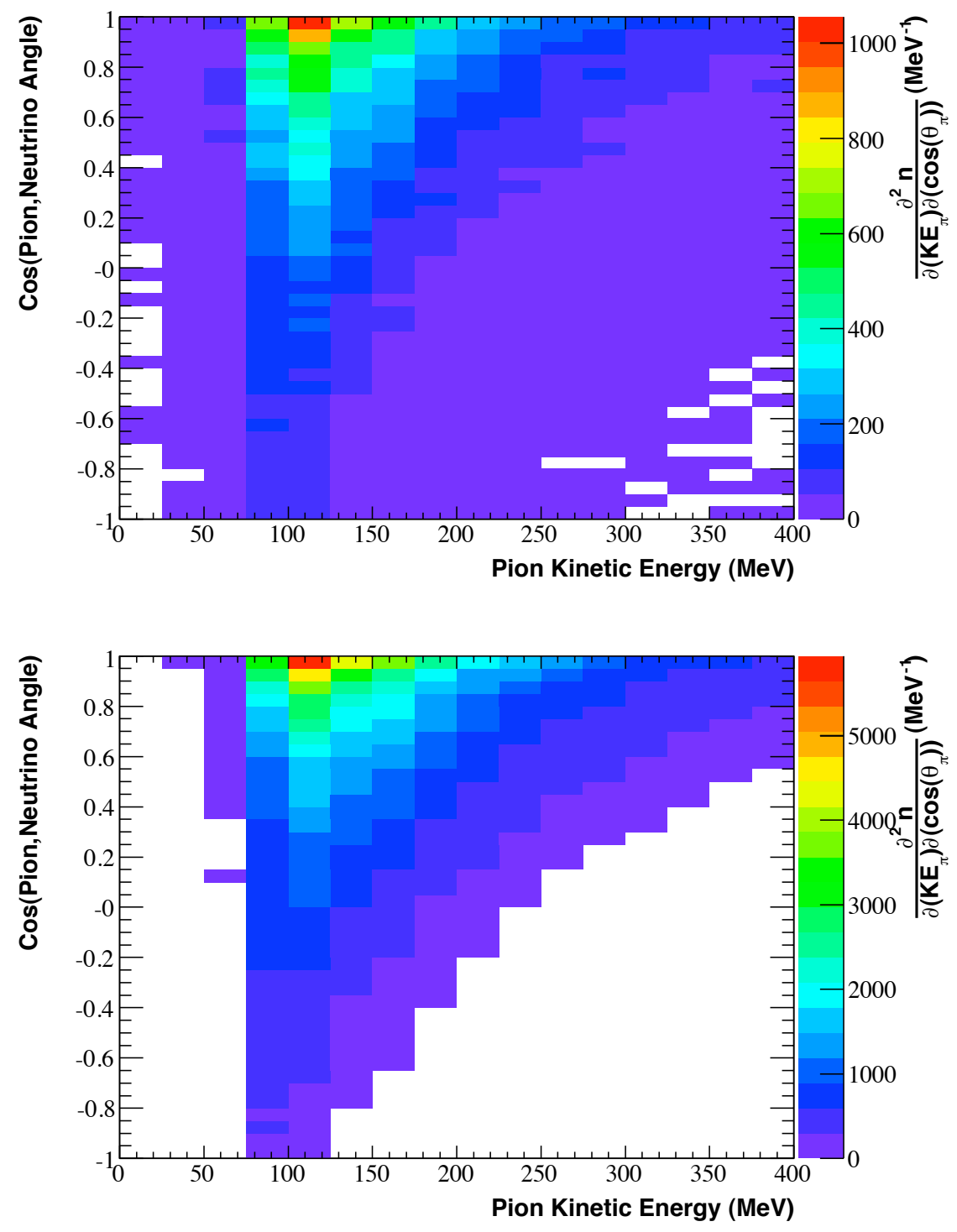

Figure B.50: The reconstructed $\cos$ (pion, neutrino angle) vs pion kinetic energy distribution is shown for both the data (top) and Monte Carlo (bottom). 

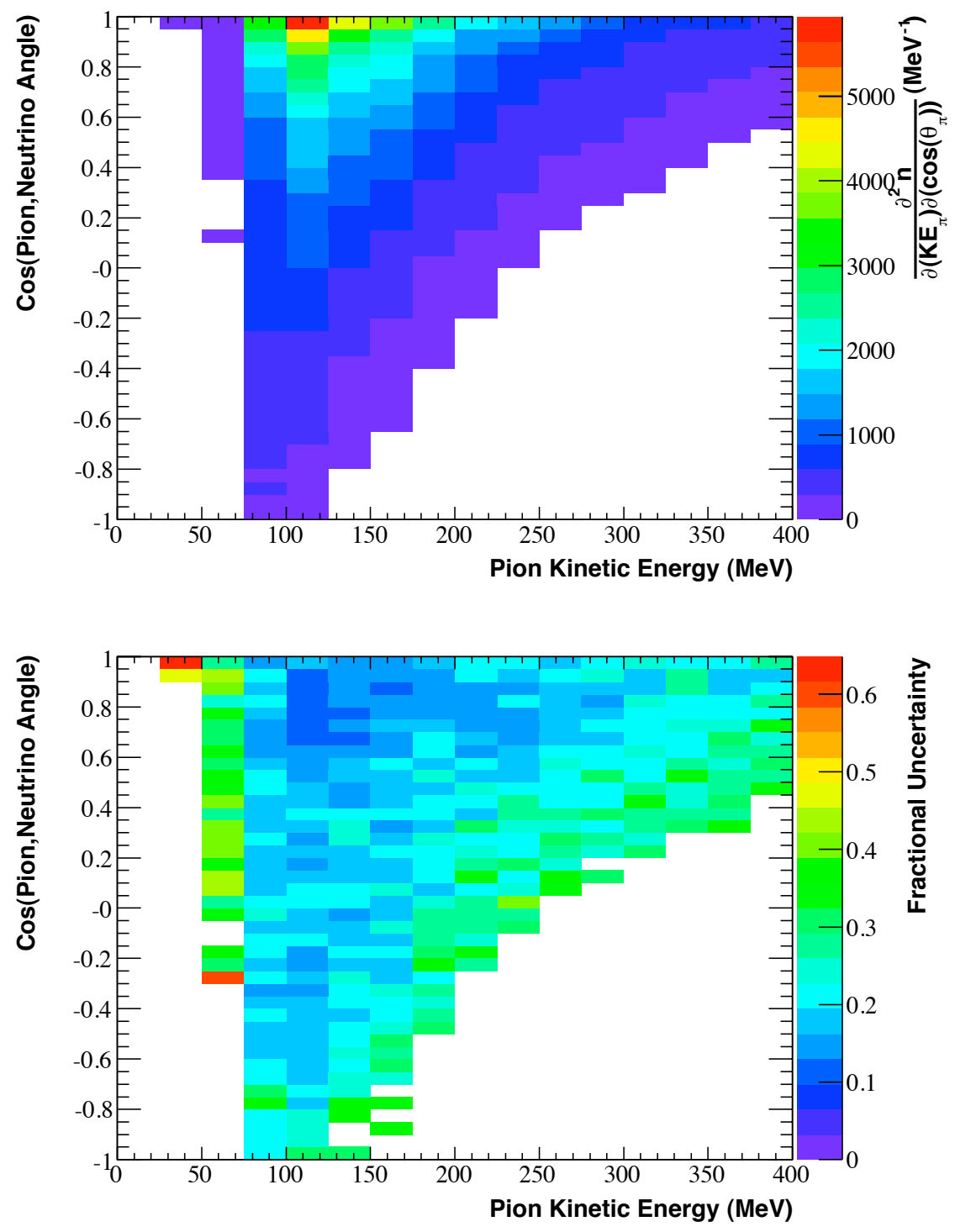

Figure B.51: The Monte Carlo reconstructed cos(pion, neutrino angle) vs pion kinetic energy distribution is shown (top) along with the total fractional uncertainties (bottom). 


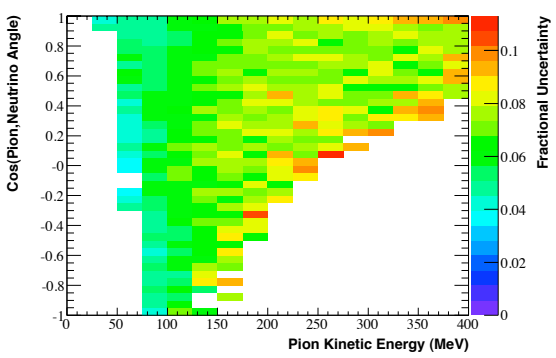

(a) The fractional systematic uncertainty from the "XSec" error source.

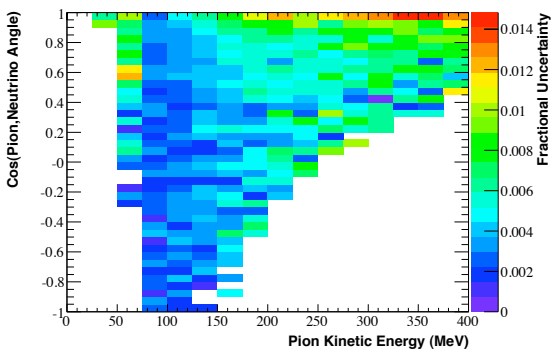

(c) The fractional systematic uncertainty from the " $\mathrm{K}^{+}$" error source.

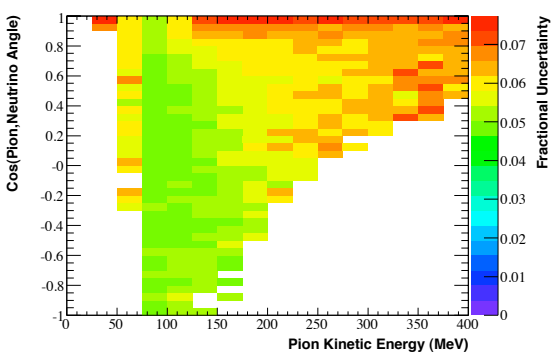

(b) The fractional systematic uncertainty from the "Beam" error source.

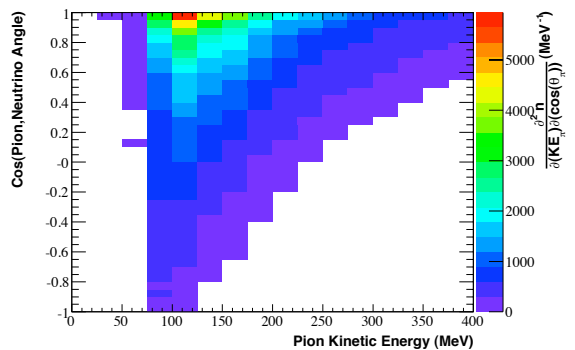

(d) Reconstructed Monte Carlo distribution

Figure B.52: The fractional systematic uncertainties on the reconstructed Monte Carlo distribution are shown for four of the error sources. The central value reconstructed Monte Carlo distribution is also shown for reference. Note that the color scales differ for each systematic error source. An explanation of each error is given in Section 6.4.7. 


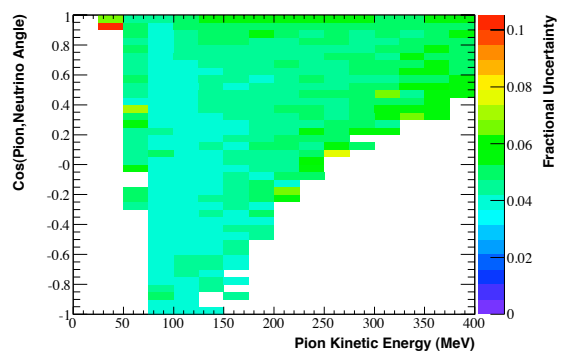

(a) The fractional systematic uncertainty from the " $\pi^{+}$" error source.

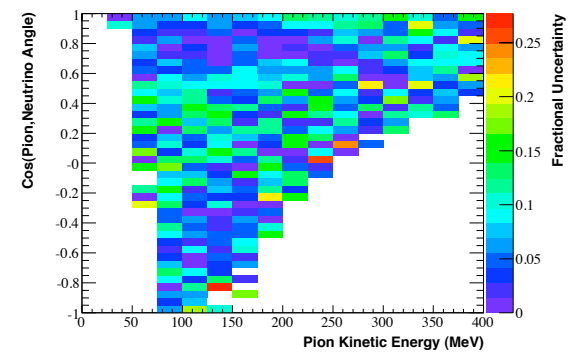

(c) The fractional systematic uncertainty from the "DISC" error source.

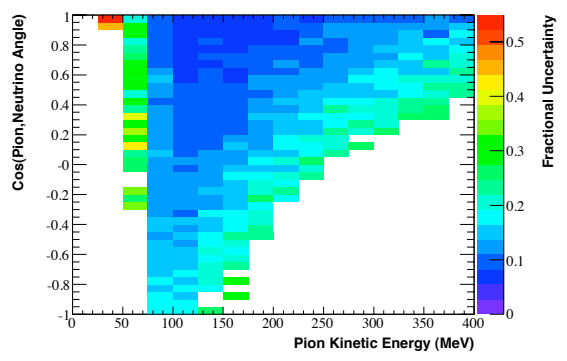

(b) The fractional systematic uncertainty from the "OM" error source.

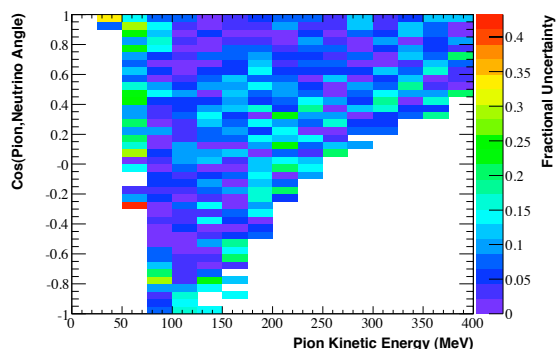

(d) The fractional systematic uncertainty from the "QTCORR" error source.

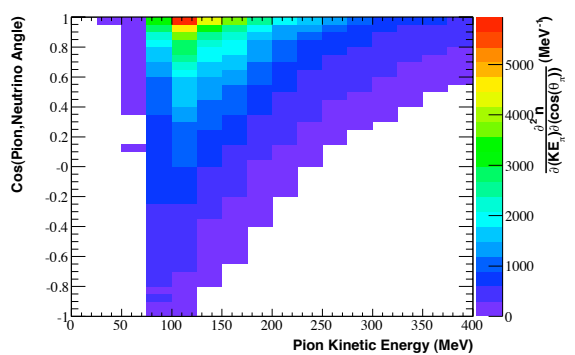

(e) Reconstructed Monte Carlo distribution

Figure B.53: The fractional systematic uncertainties on the reconstructed Monte Carlo distribution are shown for four of the error sources. The central value reconstructed Monte Carlo distribution is also shown for reference. Note that the color scales differ for each systematic error source. An explanation of each error is given in Section 6.4.7. 


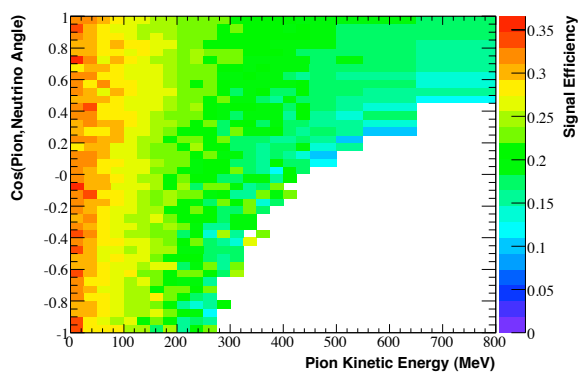

(a) Signal Efficiency

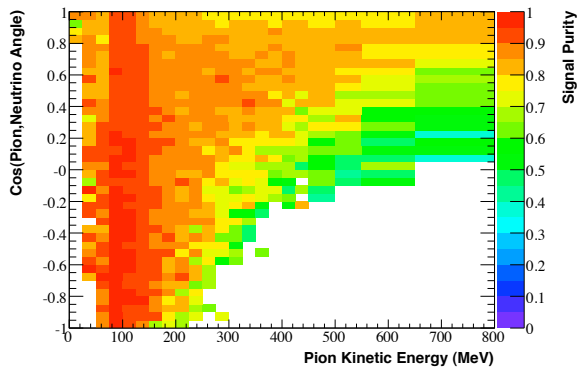

(c) Signal Purity

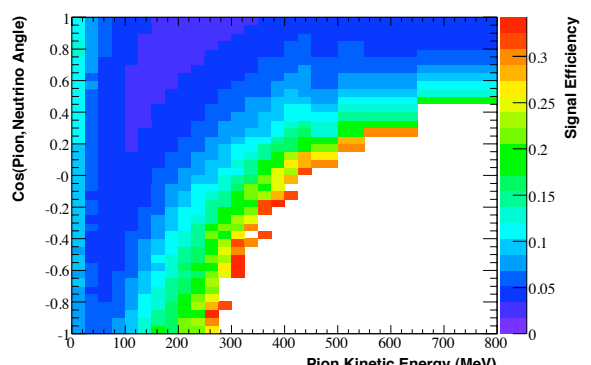

(b) Fractional Monte Carlo Statistical Error on the Signal Efficiency

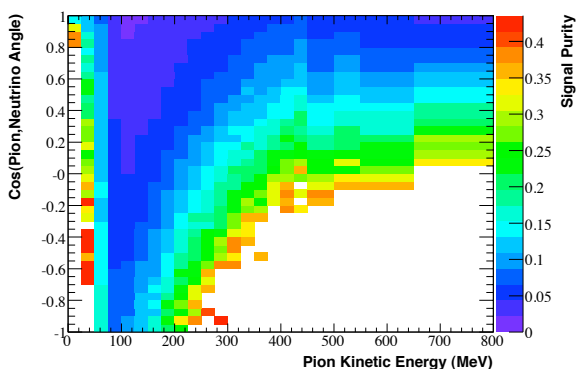

(d) Fractional Monte Carlo Statistical Error on the Signal Purity

Figure B.54: The signal efficiency and purity are shown for the pion $\cos$ (pion, neutrino angle) vs kinetic energy distribution. The fractional errors due to Monte Carlo statistics for each of these distributions is also given for all bins with at least 10 events in the numerator. 


\section{B.15 Cross Section Values}

Table B.1: The $\sigma\left(E_{\nu}\right)$ results from Figure 6.11 are given with the total uncertainty.

\begin{tabular}{|l|c|c|}
\hline bin low edge & central value $\left(/ 10^{-38} \mathrm{~cm}^{2}\right)$ & \% uncertainty \\
\hline 500 & 0.621 & 12.819 \\
\hline 600 & 1.168 & 11.576 \\
\hline 650 & 1.603 & 10.522 \\
\hline 700 & 1.996 & 10.199 \\
\hline 750 & 2.456 & 10.068 \\
\hline 800 & 2.880 & 9.957 \\
\hline 850 & 3.286 & 9.826 \\
\hline 900 & 3.757 & 10.234 \\
\hline 950 & 4.174 & 10.375 \\
\hline 1000 & 4.661 & 10.740 \\
\hline 1050 & 4.954 & 11.316 \\
\hline 1100 & 5.266 & 11.520 \\
\hline 1150 & 5.600 & 12.327 \\
\hline 1200 & 5.875 & 12.810 \\
\hline 1250 & 6.197 & 13.276 \\
\hline 1300 & 6.630 & 13.853 \\
\hline 1350 & 7.001 & 14.573 \\
\hline 1400 & 7.232 & 15.191 \\
\hline 1450 & 7.785 & 15.850 \\
\hline 1500 & 8.139 & 16.480 \\
\hline 1550 & 8.483 & 16.887 \\
\hline 1600 & 8.831 & 17.334 \\
\hline 1650 & 9.076 & 17.947 \\
\hline 1700 & 9.619 & 18.224 \\
\hline 1750 & 9.651 & 19.574 \\
\hline 1800 & 10.272 & \\
\hline 1900 & 10.737 & \\
\hline & & \\
\hline
\end{tabular}


Table B.2: The $\partial \sigma / \partial\left(Q^{2}\right)$ results from Figure 6.12 are given with the total uncertainty.

\begin{tabular}{|l|c|c|}
\hline bin low edge & central value $\left(/ 10^{-45} \mathrm{~cm}^{2} / \mathrm{MeV}^{2}\right)$ & $\%$ uncertainty \\
\hline $0.00 \mathrm{e}+00$ & 38.519 & 20.002 \\
\hline $5.00 \mathrm{e}+04$ & 56.958 & 14.916 \\
\hline $1.00 \mathrm{e}+05$ & 56.837 & 13.491 \\
\hline $1.50 \mathrm{e}+05$ & 53.352 & 12.949 \\
\hline $2.00 \mathrm{e}+05$ & 47.265 & 13.088 \\
\hline $2.50 \mathrm{e}+05$ & 42.504 & 13.366 \\
\hline $3.00 \mathrm{e}+05$ & 38.038 & 13.421 \\
\hline $3.50 \mathrm{e}+05$ & 33.204 & 13.801 \\
\hline $4.00 \mathrm{e}+05$ & 29.188 & 14.073 \\
\hline $4.50 \mathrm{e}+05$ & 25.001 & 14.365 \\
\hline $5.00 \mathrm{e}+05$ & 21.881 & 14.531 \\
\hline $5.50 \mathrm{e}+05$ & 18.851 & 14.624 \\
\hline $6.00 \mathrm{e}+05$ & 16.538 & 15.101 \\
\hline $6.50 \mathrm{e}+05$ & 14.183 & 14.928 \\
\hline $7.00 \mathrm{e}+05$ & 12.277 & 15.414 \\
\hline $7.50 \mathrm{e}+05$ & 10.576 & 15.899 \\
\hline $8.00 \mathrm{e}+05$ & 8.895 & 15.799 \\
\hline $8.50 \mathrm{e}+05$ & 7.736 & 15.812 \\
\hline $9.00 \mathrm{e}+05$ & 6.549 & 16.477 \\
\hline $9.50 \mathrm{e}+05$ & 5.323 & 16.856 \\
\hline $1.05 \mathrm{e}+06$ & 3.994 & 16.637 \\
\hline $1.15 \mathrm{e}+06$ & 2.691 & 17.320 \\
\hline $1.30 \mathrm{e}+06$ & 1.676 & \\
\hline & & 18.252 \\
\hline
\end{tabular}


Table B.3: The $\partial \sigma / \partial\left(K E_{\mu}\right)$ results from Figure 6.13 are given with the total uncertainty.

\begin{tabular}{|l|c|c|}
\hline bin low edge & central value $\left(/ 10^{-42} \mathrm{~cm}^{2} / \mathrm{MeV}\right)$ & \% uncertainty \\
\hline 0 & 24.794 & 15.043 \\
\hline 50 & 36.949 & 14.622 \\
\hline 100 & 41.325 & 14.145 \\
\hline 150 & 42.464 & 13.866 \\
\hline 200 & 41.572 & 13.825 \\
\hline 250 & 39.211 & 13.749 \\
\hline 300 & 37.265 & 13.803 \\
\hline 350 & 34.602 & 13.555 \\
\hline 400 & 31.720 & 13.610 \\
\hline 450 & 28.758 & 13.325 \\
\hline 500 & 26.119 & 13.673 \\
\hline 550 & 23.114 & 13.862 \\
\hline 600 & 20.615 & 13.995 \\
\hline 650 & 18.515 & 14.334 \\
\hline 700 & 15.970 & 14.355 \\
\hline 750 & 14.209 & 14.902 \\
\hline 800 & 12.372 & 15.381 \\
\hline 850 & 10.601 & 15.888 \\
\hline 900 & 9.219 & 16.522 \\
\hline 950 & 7.713 & 17.328 \\
\hline 1000 & 5.966 & 18.105 \\
\hline 1100 & 4.328 & 26.186 \\
\hline 1200 & 2.258 & \\
\hline & & \\
\hline
\end{tabular}


Table B.4: The $\partial \sigma / \partial\left(\cos \left(\theta_{\mu, \nu}\right)\right)$ results from Figure 6.14 are given with the total uncertainty.

\begin{tabular}{|c|c|c|}
\hline bin low edge & central value $\left(/ 10^{-39} \mathrm{~cm}^{2}\right)$ & $\%$ uncertainty \\
\hline-1.00 & 1.556 & 17.290 \\
\hline-0.80 & 2.496 & 16.926 \\
\hline-0.60 & 2.997 & 15.049 \\
\hline-0.50 & 3.446 & 14.588 \\
\hline-0.40 & 3.875 & 14.002 \\
\hline-0.30 & 4.496 & 14.317 \\
\hline-0.20 & 5.259 & 14.965 \\
\hline-0.10 & 5.802 & 14.494 \\
\hline-0.05 & 6.327 & 14.240 \\
\hline 0.00 & 6.931 & 13.983 \\
\hline 0.05 & 7.414 & 14.293 \\
\hline 0.10 & 8.063 & 13.964 \\
\hline 0.15 & 8.673 & 14.360 \\
\hline 0.20 & 9.602 & 13.839 \\
\hline 0.25 & 10.371 & 13.845 \\
\hline 0.30 & 11.594 & 13.753 \\
\hline 0.35 & 13.528 & 14.385 \\
\hline 0.40 & 14.599 & 13.839 \\
\hline 0.45 & 15.683 & 13.746 \\
\hline 0.50 & 17.844 & 13.879 \\
\hline 0.55 & 20.569 & 13.997 \\
\hline 0.60 & 23.676 & 13.715 \\
\hline 0.65 & 26.614 & 13.288 \\
\hline 0.70 & 31.753 & 13.563 \\
\hline 0.75 & 35.541 & 13.586 \\
\hline 0.80 & 42.882 & 13.307 \\
\hline 0.85 & 52.197 & 13.425 \\
\hline 0.90 & 63.302 & 14.161 \\
\hline 0.95 & 81.990 & 18.891 \\
\hline
\end{tabular}


Table B.5: The $\partial \sigma / \partial\left(K E_{\pi}\right)$ results from Figure 6.15 are given with the total uncertainty.

\begin{tabular}{|l|c|c|}
\hline bin low edge & central value $\left(/ 10^{-41} \mathrm{~cm}^{2} / \mathrm{MeV}\right)$ & \% uncertainty \\
\hline 0 & 1.791 & 12.818 \\
\hline 25 & 5.411 & 12.437 \\
\hline 50 & 8.806 & 13.150 \\
\hline 75 & 10.847 & 12.618 \\
\hline 100 & 10.904 & 11.527 \\
\hline 125 & 9.885 & 12.155 \\
\hline 150 & 8.756 & 13.079 \\
\hline 175 & 7.775 & 13.861 \\
\hline 200 & 7.128 & 14.580 \\
\hline 225 & 6.221 & 15.310 \\
\hline 250 & 5.447 & 16.084 \\
\hline 275 & 4.703 & 16.887 \\
\hline 300 & 4.038 & 17.465 \\
\hline 325 & 3.372 & 17.826 \\
\hline 350 & 2.800 & 18.055 \\
\hline 375 & 2.385 & 18.435 \\
\hline
\end{tabular}


Table B.6: The $\partial \sigma / \partial\left(\cos \left(\theta_{\pi, \nu}\right)\right)$ results from Figure 6.16 are given with the total uncertainty.

\begin{tabular}{|c|c|c|}
\hline bin low edge & central value $\left(/ 10^{-39} \mathrm{~cm}^{2}\right)$ & $\%$ uncertainty \\
\hline-1.00 & 2.877 & 12.745 \\
\hline-0.95 & 3.127 & 12.921 \\
\hline-0.90 & 3.298 & 13.495 \\
\hline-0.85 & 3.586 & 12.982 \\
\hline-0.80 & 3.797 & 13.978 \\
\hline-0.75 & 3.946 & 13.038 \\
\hline-0.70 & 4.282 & 13.079 \\
\hline-0.65 & 4.570 & 11.915 \\
\hline-0.60 & 4.738 & 12.078 \\
\hline-0.55 & 5.077 & 11.500 \\
\hline-0.50 & 5.394 & 11.459 \\
\hline-0.45 & 5.558 & 12.247 \\
\hline-0.40 & 6.117 & 12.243 \\
\hline-0.35 & 6.684 & 11.946 \\
\hline-0.30 & 7.063 & 12.141 \\
\hline-0.25 & 7.534 & 12.069 \\
\hline-0.20 & 7.979 & 11.658 \\
\hline-0.15 & 8.539 & 11.872 \\
\hline-0.10 & 8.994 & 11.727 \\
\hline-0.05 & 9.974 & 11.907 \\
\hline 0.00 & 10.756 & 11.946 \\
\hline 0.05 & 11.431 & 12.032 \\
\hline 0.10 & 12.303 & 12.405 \\
\hline 0.15 & 13.454 & 12.540 \\
\hline 0.20 & 14.364 & 12.744 \\
\hline 0.25 & 15.264 & 12.582 \\
\hline 0.30 & 16.399 & 13.136 \\
\hline 0.35 & 17.446 & 13.589 \\
\hline 0.40 & 18.867 & 13.182 \\
\hline 0.45 & 20.586 & 13.484 \\
\hline 0.50 & 22.492 & 13.566 \\
\hline 0.55 & 23.890 & 14.028 \\
\hline 0.60 & 26.112 & 13.870 \\
\hline 0.65 & 28.189 & 14.008 \\
\hline 0.70 & 30.226 & 14.102 \\
\hline 0.75 & 32.520 & 14.367 \\
\hline 0.80 & 34.819 & 14.822 \\
\hline 0.85 & 37.354 & 15.108 \\
\hline 0.90 & 39.415 & 16.496 \\
\hline 0.95 & 42.948 & 18.497 \\
\hline
\end{tabular}


Table B.7: The first of three tables detailing the $\partial \sigma\left(E_{\nu}\right) / \partial\left(Q^{2}\right)$ results from Figure 6.17 $\left(10^{-44} \mathrm{~cm}^{2} / \mathrm{MeV}^{2}\right)$.

\begin{tabular}{|l|c|c|c|c|c|c|c|c|}
\hline bin low edge & 500 & 600 & 650 & 700 & 750 & 800 & 850 & 900 \\
\hline $7.00 \mathrm{e}+05$ & & & & & & & & 0.932 \\
\hline $6.50 \mathrm{e}+05$ & & & & & & & 0.806 & 1.517 \\
\hline $6.00 \mathrm{e}+05$ & & & & & & 0.646 & 1.290 & 2.167 \\
\hline $5.50 \mathrm{e}+05$ & & & & & 0.459 & 1.158 & 1.803 & 2.327 \\
\hline $5.00 \mathrm{e}+05$ & & & & 0.353 & 0.767 & 1.787 & 2.399 & 3.150 \\
\hline $4.50 \mathrm{e}+05$ & & & & 0.683 & 1.543 & 2.353 & 3.072 & 3.886 \\
\hline $4.00 \mathrm{e}+05$ & & & 0.548 & 1.340 & 2.537 & 3.191 & 4.158 & 4.768 \\
\hline $3.50 \mathrm{e}+05$ & & & 1.150 & 2.046 & 3.198 & 4.011 & 4.777 & 5.582 \\
\hline $3.00 \mathrm{e}+05$ & & 0.993 & 1.964 & 3.159 & 4.067 & 5.099 & 5.920 & 5.970 \\
\hline $2.50 \mathrm{e}+05$ & & 1.882 & 3.081 & 4.116 & 5.271 & 5.917 & 6.591 & 6.694 \\
\hline $2.00 \mathrm{e}+05$ & 0.986 & 2.904 & 4.204 & 5.243 & 6.100 & 6.727 & 6.970 & 7.525 \\
\hline $1.50 \mathrm{e}+05$ & 1.917 & 4.096 & 5.480 & 6.357 & 7.188 & 7.488 & 7.603 & 8.328 \\
\hline $1.00 \mathrm{e}+05$ & 3.156 & 5.231 & 6.633 & 7.079 & 7.532 & 7.782 & 8.096 & 8.888 \\
\hline $5.00 \mathrm{e}+04$ & 4.375 & 6.040 & 6.601 & 7.093 & 7.429 & 7.614 & 7.789 & 8.185 \\
\hline $0.00 \mathrm{e}+00$ & 1.861 & 2.757 & 3.289 & 3.711 & 4.056 & 4.556 & 4.937 & 5.279 \\
\hline
\end{tabular}


Table B.8: The second of three tables detailing the $\partial \sigma\left(E_{\nu}\right) / \partial\left(Q^{2}\right)$ results from Figure $6.17\left(10^{-44} \mathrm{~cm}^{2} / \mathrm{MeV}^{2}\right)$.

\begin{tabular}{|l|c|c|c|c|c|c|c|c|}
\hline bin low edge & 950 & 1000 & 1050 & 1100 & 1150 & 1200 & 1250 & 1300 \\
\hline $1.15 \mathrm{e}+06$ & & & & & & 0.333 & 0.596 & 0.766 \\
\hline $1.05 \mathrm{e}+06$ & & & & & 0.595 & 0.834 & 1.046 & 1.159 \\
\hline $9.50 \mathrm{e}+05$ & & & 0.469 & 0.695 & 1.069 & 1.297 & 1.631 & 1.856 \\
\hline $9.00 \mathrm{e}+05$ & & & 0.820 & 1.229 & 1.449 & 1.879 & 1.875 & 2.260 \\
\hline $8.50 \mathrm{e}+05$ & & 0.742 & 1.121 & 1.416 & 1.676 & 2.054 & 2.499 & 2.619 \\
\hline $8.00 \mathrm{e}+05$ & 0.636 & 1.031 & 1.480 & 1.905 & 2.370 & 2.677 & 2.892 & 3.274 \\
\hline $7.50 \mathrm{e}+05$ & 1.008 & 1.586 & 2.029 & 2.316 & 2.608 & 3.046 & 3.303 & 3.372 \\
\hline $7.00 \mathrm{e}+05$ & 1.499 & 2.057 & 2.407 & 2.789 & 3.142 & 3.392 & 3.724 & 3.813 \\
\hline $6.50 \mathrm{e}+05$ & 2.064 & 2.496 & 2.876 & 3.018 & 3.844 & 3.597 & 4.008 & 4.439 \\
\hline $6.00 \mathrm{e}+05$ & 2.386 & 3.200 & 3.585 & 4.018 & 4.074 & 4.363 & 4.993 & 5.196 \\
\hline $5.50 \mathrm{e}+05$ & 2.994 & 3.669 & 4.008 & 4.406 & 4.548 & 5.049 & 4.862 & 5.385 \\
\hline $5.00 \mathrm{e}+05$ & 3.602 & 4.339 & 4.688 & 5.169 & 5.374 & 5.540 & 5.462 & 6.302 \\
\hline $4.50 \mathrm{e}+05$ & 4.602 & 5.047 & 5.526 & 5.256 & 5.798 & 6.214 & 6.092 & 6.214 \\
\hline $4.00 \mathrm{e}+05$ & 4.994 & 5.880 & 6.231 & 6.436 & 6.426 & 6.832 & 6.815 & 6.990 \\
\hline $3.50 \mathrm{e}+05$ & 6.262 & 6.569 & 6.563 & 6.818 & 7.139 & 7.488 & 7.278 & 7.870 \\
\hline $3.00 \mathrm{e}+05$ & 7.234 & 7.151 & 7.173 & 7.826 & 7.725 & 7.768 & 8.157 & 8.602 \\
\hline $2.50 \mathrm{e}+05$ & 7.425 & 7.544 & 8.027 & 7.951 & 7.791 & 8.131 & 8.616 & 8.677 \\
\hline $2.00 \mathrm{e}+05$ & 7.850 & 8.582 & 8.326 & 8.630 & 8.484 & 9.038 & 8.943 & 9.329 \\
\hline $1.50 \mathrm{e}+05$ & 8.696 & 9.166 & 8.978 & 9.307 & 9.107 & 9.353 & 9.215 & 9.497 \\
\hline $1.00 \mathrm{e}+05$ & 8.397 & 9.308 & 9.311 & 9.425 & 9.666 & 9.245 & 9.329 & 9.848 \\
\hline $5.00 \mathrm{e}+04$ & 8.117 & 8.280 & 8.509 & 8.845 & 9.052 & 8.684 & 9.115 & 8.676 \\
\hline $0.00 \mathrm{e}+00$ & 5.781 & 6.062 & 6.058 & 5.843 & 6.484 & 6.555 & 6.622 & 6.939 \\
\hline
\end{tabular}


Table B.9: The third of three tables detailing the $\partial \sigma\left(E_{\nu}\right) / \partial\left(Q^{2}\right)$ results from Figure 6.17 $\left(10^{-44} \mathrm{~cm}^{2} / \mathrm{MeV}^{2}\right)$.

\begin{tabular}{|l|c|c|c|c|c|c|c|}
\hline bin low edge & 1350 & 1400 & 1450 & 1500 & 1550 & 1600 & 1650 \\
\hline $1.30 \mathrm{e}+06$ & 0.438 & 0.606 & 0.746 & 0.984 & 1.022 & 1.199 & 1.171 \\
\hline $1.15 \mathrm{e}+06$ & 1.058 & 1.050 & 1.264 & 1.502 & 1.553 & 1.882 & 1.805 \\
\hline $1.05 \mathrm{e}+06$ & 1.375 & 1.793 & 1.899 & 2.252 & 2.496 & 2.274 & 2.592 \\
\hline $9.50 \mathrm{e}+05$ & 2.111 & 2.194 & 2.326 & 2.604 & 3.160 & 3.146 & 3.031 \\
\hline $9.00 \mathrm{e}+05$ & 2.345 & 2.721 & 3.381 & 2.946 & 3.485 & & \\
\hline $8.50 \mathrm{e}+05$ & 2.813 & 3.055 & 3.464 & 4.094 & 3.578 & 3.745 & \\
\hline $8.00 \mathrm{e}+05$ & 3.305 & 3.280 & 3.758 & 3.571 & 4.125 & 4.249 & \\
\hline $7.50 \mathrm{e}+05$ & 3.760 & 4.486 & 4.092 & 4.525 & 3.957 & 4.384 & \\
\hline $7.00 \mathrm{e}+05$ & 4.129 & 4.350 & 5.271 & 4.625 & 5.042 & 4.644 & \\
\hline $6.50 \mathrm{e}+05$ & 4.527 & 4.931 & 4.894 & 5.365 & 5.191 & 4.878 & 5.262 \\
\hline $6.00 \mathrm{e}+05$ & 5.074 & 4.805 & 5.616 & 5.856 & 5.345 & 5.990 & 5.815 \\
\hline $5.50 \mathrm{e}+05$ & 5.215 & 5.770 & 5.588 & 6.548 & 6.928 & 7.009 & 6.828 \\
\hline $5.00 \mathrm{e}+05$ & 6.082 & 6.269 & 6.604 & 5.908 & 6.977 & 7.043 & 6.844 \\
\hline $4.50 \mathrm{e}+05$ & 6.506 & 7.303 & 6.800 & 7.075 & 6.239 & 7.311 & 7.508 \\
\hline $4.00 \mathrm{e}+05$ & 7.189 & 6.774 & 7.877 & 7.358 & 8.101 & 7.682 & 8.538 \\
\hline $3.50 \mathrm{e}+05$ & 7.642 & 7.517 & 8.022 & 8.483 & 8.091 & 8.253 & 8.469 \\
\hline $3.00 \mathrm{e}+05$ & 8.659 & 8.488 & 8.425 & 8.523 & 8.910 & 8.678 & 9.805 \\
\hline $2.50 \mathrm{e}+05$ & 9.662 & 9.219 & 9.449 & 8.803 & 9.665 & 10.011 & 10.328 \\
\hline $2.00 \mathrm{e}+05$ & 9.463 & 9.367 & 9.811 & 10.191 & 10.055 & 11.370 & 11.142 \\
\hline $1.50 \mathrm{e}+05$ & 9.779 & 9.953 & 10.354 & 11.403 & 10.905 & 11.464 & 10.531 \\
\hline $1.00 \mathrm{e}+05$ & 9.600 & 10.109 & 10.819 & 9.854 & 10.978 & 10.876 & 10.345 \\
\hline $5.00 \mathrm{e}+04$ & 9.032 & 8.598 & 9.618 & 10.206 & 10.193 & 10.347 & 10.747 \\
\hline $0.00 \mathrm{e}+00$ & 7.489 & 7.162 & 7.385 & 7.918 & 8.393 & 8.392 & 8.842 \\
\hline & & & & & & &
\end{tabular}


Table B.10: The first of three tables detailing the percent uncertainty of the $\partial \sigma\left(E_{\nu}\right) / \partial\left(Q^{2}\right)$ results from Figure 6.17.

\begin{tabular}{|l|c|c|c|c|c|c|c|c|}
\hline bin low edge & 500 & 600 & 650 & 700 & 750 & 800 & 850 & 900 \\
\hline $7.00 \mathrm{e}+05$ & & & & & & & & 17.076 \\
\hline $6.50 \mathrm{e}+05$ & & & & & & & 18.670 & 19.730 \\
\hline $6.00 \mathrm{e}+05$ & & & & & & 24.816 & 22.389 & 20.757 \\
\hline $5.50 \mathrm{e}+05$ & & & & & 21.930 & 17.187 & 15.991 & 17.035 \\
\hline $5.00 \mathrm{e}+05$ & & & & 24.148 & 17.522 & 18.547 & 15.262 & 13.727 \\
\hline $4.50 \mathrm{e}+05$ & & & & 19.273 & 16.476 & 14.636 & 12.762 & 12.697 \\
\hline $4.00 \mathrm{e}+05$ & & & 18.832 & 15.072 & 15.785 & 11.566 & 11.957 & 13.192 \\
\hline $3.50 \mathrm{e}+05$ & & & 17.857 & 13.842 & 11.961 & 12.696 & 11.782 & 12.899 \\
\hline $3.00 \mathrm{e}+05$ & & 15.731 & 12.518 & 11.060 & 12.504 & 12.242 & 11.840 & 12.274 \\
\hline $2.50 \mathrm{e}+05$ & & 13.159 & 12.030 & 12.520 & 11.531 & 11.475 & 11.645 & 11.519 \\
\hline $2.00 \mathrm{e}+05$ & 14.390 & 13.563 & 11.254 & 11.258 & 11.347 & 10.819 & 10.680 & 10.892 \\
\hline $1.50 \mathrm{e}+05$ & 10.705 & 10.617 & 11.069 & 10.712 & 10.692 & 10.860 & 10.610 & 11.520 \\
\hline $1.00 \mathrm{e}+05$ & 10.406 & 11.120 & 10.751 & 10.731 & 10.842 & 11.431 & 11.396 & 11.233 \\
\hline $5.00 \mathrm{e}+04$ & 12.380 & 12.798 & 12.516 & 13.953 & 13.894 & 13.658 & 13.626 & 13.295 \\
\hline $0.00 \mathrm{e}+00$ & 20.463 & 19.097 & 19.683 & 19.353 & 18.618 & 18.143 & 17.532 & 16.648 \\
\hline
\end{tabular}


Table B.11: The second of three tables detailing the percent uncertainty of the $\partial \sigma\left(E_{\nu}\right) / \partial\left(Q^{2}\right)$ results from Figure 6.17.

\begin{tabular}{|l|c|c|c|c|c|c|c|c|}
\hline bin low edge & 950 & 1000 & 1050 & 1100 & 1150 & 1200 & 1250 & 1300 \\
\hline $1.15 \mathrm{e}+06$ & & & & & & 22.806 & 26.379 & 23.959 \\
\hline $1.05 \mathrm{e}+06$ & & & & & 24.119 & 23.494 & 28.685 & 23.828 \\
\hline $9.50 \mathrm{e}+05$ & & & 24.824 & 21.910 & 21.837 & 20.730 & 19.260 & 20.610 \\
\hline $9.00 \mathrm{e}+05$ & & & 21.933 & 22.214 & 20.136 & 23.845 & 20.378 & 19.112 \\
\hline $8.50 \mathrm{e}+05$ & & 28.602 & 21.824 & 18.064 & 20.220 & 18.063 & 21.357 & 20.409 \\
\hline $8.00 \mathrm{e}+05$ & 24.182 & 21.858 & 20.610 & 20.631 & 19.266 & 18.015 & 17.698 & 19.275 \\
\hline $7.50 \mathrm{e}+05$ & 23.584 & 19.887 & 21.405 & 18.313 & 16.142 & 18.153 & 17.168 & 18.095 \\
\hline $7.00 \mathrm{e}+05$ & 19.911 & 17.155 & 18.005 & 16.981 & 16.758 & 15.598 & 15.741 & 17.691 \\
\hline $6.50 \mathrm{e}+05$ & 18.157 & 18.974 & 16.808 & 14.970 & 15.230 & 16.686 & 16.235 & 17.935 \\
\hline $6.00 \mathrm{e}+05$ & 16.799 & 16.266 & 14.710 & 14.406 & 16.380 & 14.913 & 16.357 & 17.656 \\
\hline $5.50 \mathrm{e}+05$ & 15.227 & 14.775 & 13.565 & 14.282 & 15.224 & 16.326 & 16.013 & 16.106 \\
\hline $5.00 \mathrm{e}+05$ & 14.386 & 13.306 & 14.459 & 15.244 & 15.118 & 14.451 & 15.340 & 16.202 \\
\hline $4.50 \mathrm{e}+05$ & 13.325 & 13.188 & 13.852 & 13.855 & 14.579 & 17.523 & 15.894 & 16.627 \\
\hline $4.00 \mathrm{e}+05$ & 13.237 & 14.047 & 14.476 & 14.730 & 15.223 & 14.493 & 15.574 & 14.841 \\
\hline $3.50 \mathrm{e}+05$ & 12.533 & 13.321 & 13.810 & 13.504 & 14.768 & 14.422 & 15.065 & 15.249 \\
\hline $3.00 \mathrm{e}+05$ & 12.265 & 12.670 & 12.609 & 12.686 & 12.808 & 14.653 & 15.004 & 14.665 \\
\hline $2.50 \mathrm{e}+05$ & 11.473 & 11.760 & 11.862 & 12.518 & 13.350 & 13.379 & 13.779 & 14.920 \\
\hline $2.00 \mathrm{e}+05$ & 11.139 & 12.265 & 12.174 & 12.568 & 13.296 & 13.889 & 14.272 & 14.562 \\
\hline $1.50 \mathrm{e}+05$ & 11.480 & 11.847 & 12.139 & 12.084 & 13.661 & 13.882 & 15.069 & 14.888 \\
\hline $1.00 \mathrm{e}+05$ & 12.123 & 12.007 & 12.688 & 12.663 & 13.709 & 14.514 & 14.808 & 15.552 \\
\hline $5.00 \mathrm{e}+04$ & 13.277 & 13.305 & 13.971 & 14.023 & 15.061 & 15.477 & 17.314 & 19.337 \\
\hline $0.00 \mathrm{e}+00$ & 16.382 & 16.446 & 18.496 & 18.757 & 19.266 & 18.693 & 19.873 & 21.585 \\
\hline & & & & & & & & \\
\hline
\end{tabular}


Table B.12: The third of three tables detailing the percent uncertainty of the $\partial \sigma\left(E_{\nu}\right) / \partial\left(Q^{2}\right)$ results from Figure 6.17.

\begin{tabular}{|l|c|c|c|c|c|c|c|}
\hline bin low edge & 1350 & 1400 & 1450 & 1500 & 1550 & 1600 & 1650 \\
\hline $1.30 \mathrm{e}+06$ & 21.978 & 26.894 & 23.020 & 27.855 & 30.377 & 27.739 & 25.605 \\
\hline $1.15 \mathrm{e}+06$ & 22.942 & 22.018 & 25.809 & 21.762 & 23.289 & 22.462 & 25.960 \\
\hline $1.05 \mathrm{e}+06$ & 20.985 & 20.616 & 26.295 & 24.242 & 20.202 & 25.326 & 25.508 \\
\hline $9.50 \mathrm{e}+05$ & 21.590 & 20.685 & 20.954 & 24.246 & 20.556 & 20.645 & 21.885 \\
\hline $9.00 \mathrm{e}+05$ & 19.837 & 19.542 & 22.352 & 22.159 & 28.156 & & \\
\hline $8.50 \mathrm{e}+05$ & 18.876 & 20.632 & 21.641 & 22.364 & 27.700 & 22.379 & \\
\hline $8.00 \mathrm{e}+05$ & 19.213 & 20.695 & 20.639 & 21.638 & 25.241 & 23.963 & \\
\hline $7.50 \mathrm{e}+05$ & 19.857 & 20.739 & 20.365 & 23.444 & 22.171 & 23.923 & \\
\hline $7.00 \mathrm{e}+05$ & 17.793 & 21.951 & 18.702 & 22.167 & 21.538 & 24.283 & \\
\hline $6.50 \mathrm{e}+05$ & 19.073 & 20.071 & 19.505 & 18.770 & 21.473 & 21.802 & 25.285 \\
\hline $6.00 \mathrm{e}+05$ & 17.246 & 17.348 & 19.626 & 20.669 & 23.532 & 19.290 & 24.587 \\
\hline $5.50 \mathrm{e}+05$ & 16.426 & 17.900 & 17.785 & 21.241 & 20.952 & 21.020 & 24.467 \\
\hline $5.00 \mathrm{e}+05$ & 17.020 & 19.260 & 19.344 & 21.050 & 21.323 & 22.179 & 23.441 \\
\hline $4.50 \mathrm{e}+05$ & 16.975 & 18.650 & 17.855 & 19.759 & 20.903 & 28.385 & 24.200 \\
\hline $4.00 \mathrm{e}+05$ & 16.848 & 18.124 & 17.646 & 18.577 & 19.367 & 21.174 & 22.363 \\
\hline $3.50 \mathrm{e}+05$ & 17.583 & 15.941 & 17.705 & 19.010 & 20.218 & 20.207 & 24.460 \\
\hline $3.00 \mathrm{e}+05$ & 15.657 & 17.321 & 17.602 & 18.753 & 18.601 & 20.352 & 21.525 \\
\hline $2.50 \mathrm{e}+05$ & 16.481 & 16.327 & 17.509 & 17.685 & 18.049 & 20.139 & 21.546 \\
\hline $2.00 \mathrm{e}+05$ & 15.144 & 16.927 & 18.037 & 17.820 & 19.912 & 20.318 & 23.828 \\
\hline $1.50 \mathrm{e}+05$ & 16.305 & 16.223 & 18.273 & 17.819 & 18.631 & 20.850 & 22.687 \\
\hline $1.00 \mathrm{e}+05$ & 16.308 & 16.789 & 17.662 & 18.513 & 18.823 & 20.548 & 21.797 \\
\hline $5.00 \mathrm{e}+04$ & 19.387 & 21.157 & 20.099 & 20.615 & 20.543 & 22.107 & 23.944 \\
\hline $0.00 \mathrm{e}+00$ & 24.105 & 24.796 & 25.610 & 25.867 & 24.793 & 27.592 & 26.247 \\
\hline & & & & & & & \\
\hline
\end{tabular}


Table B.13: The first of three tables detailing the $\partial \sigma\left(E_{\nu}\right) / \partial\left(K E_{\mu}\right)$ results from Figure $6.20\left(10^{-41} \mathrm{~cm}^{2} / \mathrm{MeV}\right)$.

\begin{tabular}{|l|c|c|c|c|c|c|c|c|}
\hline bin low edge & 500 & 600 & 650 & 700 & 750 & 800 & 850 & 900 \\
\hline 550 & & & & & & & 0.509 & 1.250 \\
\hline 500 & & & & & & 0.517 & 1.291 & 2.650 \\
\hline 450 & & & & & 0.411 & 1.173 & 2.431 & 4.633 \\
\hline 400 & & & & 0.361 & 1.046 & 2.293 & 4.376 & 7.084 \\
\hline 350 & & & & 1.082 & 2.116 & 4.401 & 6.895 & 8.378 \\
\hline 300 & & 0.330 & 1.026 & 2.187 & 4.395 & 6.299 & 7.930 & 9.218 \\
\hline 250 & 0.174 & 0.960 & 2.031 & 4.007 & 6.264 & 7.921 & 8.586 & 9.411 \\
\hline 200 & 0.541 & 1.864 & 4.109 & 6.129 & 7.794 & 8.542 & 9.231 & 9.298 \\
\hline 150 & 1.293 & 3.705 & 5.805 & 7.722 & 8.623 & 9.272 & 9.255 & 8.877 \\
\hline 100 & 2.298 & 5.533 & 7.364 & 8.330 & 8.824 & 8.741 & 8.129 & 7.634 \\
\hline 50 & 4.049 & 6.906 & 7.837 & 7.581 & 7.371 & 6.575 & 5.902 & 5.402 \\
\hline 0 & 4.619 & 5.036 & 4.818 & 4.079 & 3.733 & 3.364 & 2.913 & 2.761 \\
\hline
\end{tabular}


Table B.14: The second of three tables detailing the $\partial \sigma\left(E_{\nu}\right) / \partial\left(K E_{\mu}\right)$ results from Figure $6.20\left(10^{-41} \mathrm{~cm}^{2} / \mathrm{MeV}\right)$.

\begin{tabular}{|l|c|c|c|c|c|c|c|c|}
\hline bin low edge & 950 & 1000 & 1050 & 1100 & 1150 & 1200 & 1250 & 1300 \\
\hline 950 & & & & & & & & 1.545 \\
\hline 900 & & & & & & & & 2.694 \\
\hline 850 & & & & & & 1.330 & 2.732 & 4.500 \\
\hline 800 & & & & & 1.272 & 2.607 & 5.073 & 7.500 \\
\hline 750 & & & & 1.441 & 2.788 & 4.941 & 7.215 & 8.726 \\
\hline 700 & & & 1.218 & 2.563 & 5.080 & 7.222 & 8.260 & 9.030 \\
\hline 650 & & 1.256 & 2.622 & 4.816 & 7.083 & 8.532 & 9.787 & 9.571 \\
\hline 600 & 1.226 & 2.639 & 4.966 & 7.181 & 8.828 & 9.129 & 9.004 & 8.972 \\
\hline 550 & 2.461 & 4.787 & 6.840 & 8.450 & 9.137 & 9.345 & 8.836 & 9.658 \\
\hline 500 & 4.606 & 7.248 & 8.544 & 9.405 & 9.185 & 9.172 & 8.911 & 8.836 \\
\hline 450 & 7.255 & 8.379 & 9.101 & 9.771 & 9.578 & 8.856 & 8.489 & 8.406 \\
\hline 400 & 8.753 & 9.596 & 9.791 & 9.262 & 8.707 & 8.870 & 8.711 & 8.088 \\
\hline 350 & 9.746 & 10.209 & 9.561 & 9.194 & 8.758 & 7.945 & 8.486 & 7.953 \\
\hline 300 & 9.406 & 9.917 & 9.884 & 8.718 & 9.002 & 8.040 & 7.970 & 7.719 \\
\hline 250 & 9.574 & 9.186 & 9.134 & 8.617 & 8.197 & 7.918 & 7.195 & 7.412 \\
\hline 200 & 9.346 & 9.078 & 8.572 & 7.958 & 7.738 & 7.196 & 6.500 & 6.229 \\
\hline 150 & 8.383 & 8.046 & 7.265 & 7.074 & 6.563 & 6.468 & 5.919 & 5.490 \\
\hline 100 & 6.699 & 6.737 & 6.181 & 5.702 & 5.471 & 4.836 & 4.446 & 4.143 \\
\hline 50 & 4.783 & 4.475 & 4.034 & 3.801 & 3.604 & 3.289 & 3.097 & 2.990 \\
\hline 0 & 2.583 & 2.554 & 2.463 & 2.220 & 1.955 & 1.932 & 1.848 & 1.837 \\
\hline
\end{tabular}


Table B.15: The third of three tables detailing the $\partial \sigma\left(E_{\nu}\right) / \partial\left(K E_{\mu}\right)$ results from Figure $6.20\left(10^{-41} \mathrm{~cm}^{2} / \mathrm{MeV}\right)$.

\begin{tabular}{|l|c|c|c|c|c|c|c|}
\hline bin low edge & 1350 & 1400 & 1450 & 1500 & 1550 & 1600 & 1650 \\
\hline 1200 & & & & & & & 2.123 \\
\hline 1100 & & & & 2.294 & 4.436 & 6.776 & 8.775 \\
\hline 1000 & 0.952 & 2.068 & 4.056 & 6.528 & 8.514 & 9.483 & 9.233 \\
\hline 950 & 3.048 & 5.045 & 7.303 & 8.862 & 9.693 & 9.839 & 9.499 \\
\hline 900 & 5.182 & 7.333 & 9.137 & 9.661 & 10.080 & 9.209 & 9.378 \\
\hline 850 & 7.152 & 8.763 & 9.344 & 10.488 & 9.668 & 9.052 & 8.868 \\
\hline 800 & 8.234 & 9.698 & 9.481 & 9.661 & 9.464 & 8.217 & 9.136 \\
\hline 750 & 9.600 & 9.546 & 9.525 & 8.046 & 8.281 & 8.905 & 9.057 \\
\hline 700 & 8.871 & 9.071 & 9.011 & 8.417 & 8.519 & 8.313 & 7.389 \\
\hline 650 & 9.798 & 9.033 & 8.721 & 7.838 & 7.828 & 7.155 & 7.110 \\
\hline 600 & 9.102 & 9.086 & 8.170 & 8.208 & 7.413 & 7.640 & 6.511 \\
\hline 550 & 8.592 & 7.388 & 8.586 & 7.598 & 7.937 & 6.935 & 7.209 \\
\hline 500 & 8.800 & 7.821 & 8.014 & 7.816 & 7.031 & 7.276 & 6.270 \\
\hline 450 & 7.895 & 8.599 & 7.281 & 7.636 & 7.417 & 6.176 & 5.680 \\
\hline 400 & 7.689 & 7.307 & 7.624 & 7.142 & 6.270 & 6.195 & 6.025 \\
\hline 350 & 7.203 & 6.738 & 6.856 & 7.277 & 5.849 & 6.655 & 5.821 \\
\hline 300 & 7.002 & 6.712 & 6.856 & 6.437 & 6.339 & 5.866 & 5.647 \\
\hline 250 & 6.645 & 6.334 & 6.419 & 6.054 & 5.484 & 5.113 & 5.040 \\
\hline 200 & 6.578 & 5.695 & 5.545 & 5.643 & 4.899 & 4.793 & 4.489 \\
\hline 150 & 5.209 & 4.716 & 4.924 & 4.515 & 4.238 & 4.407 & \\
\hline 100 & 4.064 & 3.549 & 3.783 & 3.413 & 3.148 & & \\
\hline 50 & 3.066 & 2.686 & 2.750 & 2.733 & & & \\
\hline 0 & 1.811 & & & & & & \\
\hline
\end{tabular}


Table B.16: The first of three tables detailing the percent uncertainty of the $\partial \sigma\left(E_{\nu}\right) / \partial\left(K E_{\mu}\right)$ results from Figure 6.20.

\begin{tabular}{|l|c|c|c|c|c|c|c|c|}
\hline bin low edge & 500 & 600 & 650 & 700 & 750 & 800 & 850 & 900 \\
\hline 550 & & & & & & & 21.814 & 14.167 \\
\hline 500 & & & & & & 20.471 & 14.496 & 11.495 \\
\hline 450 & & & & & 20.470 & 16.346 & 12.777 & 11.316 \\
\hline 400 & & & & 23.378 & 17.554 & 15.004 & 12.382 & 11.226 \\
\hline 350 & & & & 19.259 & 16.071 & 13.198 & 11.046 & 10.570 \\
\hline 300 & & 20.445 & 16.453 & 14.631 & 12.951 & 11.563 & 10.791 & 10.700 \\
\hline 250 & 22.921 & 16.256 & 13.362 & 11.856 & 11.207 & 10.957 & 9.708 & 10.744 \\
\hline 200 & 17.893 & 13.677 & 11.238 & 10.209 & 10.245 & 10.589 & 10.118 & 11.333 \\
\hline 150 & 14.647 & 12.615 & 10.944 & 10.701 & 10.553 & 10.626 & 10.715 & 11.606 \\
\hline 100 & 15.183 & 12.344 & 11.092 & 11.047 & 10.779 & 11.465 & 11.805 & 12.699 \\
\hline 50 & 14.288 & 13.429 & 12.204 & 12.529 & 13.221 & 12.716 & 13.826 & 14.785 \\
\hline 0 & 14.015 & 13.168 & 13.519 & 14.866 & 15.020 & 15.465 & 17.773 & 18.029 \\
\hline
\end{tabular}


Table B.17: The second of three tables detailing the percent uncertainty of the $\partial \sigma\left(E_{\nu}\right) / \partial\left(K E_{\mu}\right)$ results from Figure 6.20.

\begin{tabular}{|l|c|c|c|c|c|c|c|c|}
\hline bin low edge & 950 & 1000 & 1050 & 1100 & 1150 & 1200 & 1250 & 1300 \\
\hline 950 & & & & & & & & 25.088 \\
\hline 900 & & & & & & & & 18.354 \\
\hline 850 & & & & & & 23.249 & 18.368 & 17.970 \\
\hline 800 & & & & & 20.305 & 18.445 & 18.196 & 16.102 \\
\hline 750 & & & & 17.463 & 15.616 & 14.687 & 15.020 & 15.347 \\
\hline 700 & & & 18.359 & 16.167 & 14.382 & 14.381 & 15.708 & 15.824 \\
\hline 650 & & 19.331 & 16.827 & 14.576 & 14.231 & 14.214 & 14.881 & 16.170 \\
\hline 600 & 14.755 & 14.052 & 12.347 & 12.453 & 13.675 & 15.146 & 14.693 & 15.492 \\
\hline 550 & 13.964 & 13.497 & 12.701 & 13.237 & 13.651 & 15.008 & 15.235 & 16.645 \\
\hline 500 & 11.700 & 12.100 & 11.422 & 12.936 & 14.048 & 14.256 & 15.214 & 16.311 \\
\hline 450 & 11.083 & 10.784 & 11.427 & 12.443 & 13.474 & 14.240 & 14.475 & 15.080 \\
\hline 400 & 11.250 & 11.523 & 12.037 & 12.359 & 13.630 & 13.395 & 15.744 & 16.348 \\
\hline 350 & 10.856 & 11.818 & 12.294 & 13.085 & 13.309 & 13.773 & 14.766 & 16.341 \\
\hline 300 & 10.871 & 11.929 & 13.380 & 13.666 & 14.683 & 14.669 & 14.621 & 17.747 \\
\hline 250 & 10.916 & 12.299 & 12.621 & 13.547 & 13.837 & 15.218 & 15.646 & 16.479 \\
\hline 200 & 11.437 & 12.094 & 13.075 & 13.433 & 14.634 & 15.236 & 16.122 & 17.624 \\
\hline 150 & 12.224 & 12.712 & 13.813 & 13.378 & 15.318 & 15.656 & 16.746 & 17.341 \\
\hline 100 & 12.731 & 13.334 & 14.528 & 15.463 & 15.623 & 17.357 & 16.446 & 20.274 \\
\hline 50 & 14.085 & 16.201 & 16.396 & 17.926 & 18.306 & 18.851 & 20.213 & 19.264 \\
\hline 0 & 16.935 & 18.468 & 21.206 & 18.833 & 24.038 & 22.265 & 22.611 & 25.563 \\
\hline
\end{tabular}


Table B.18: The third of three tables detailing the percent uncertainty of the $\partial \sigma\left(E_{\nu}\right) / \partial\left(K E_{\mu}\right)$ results from Figure 6.20.

\begin{tabular}{|l|c|c|c|c|c|c|c|}
\hline bin low edge & 1350 & 1400 & 1450 & 1500 & 1550 & 1600 & 1650 \\
\hline 1200 & & & & & & & 24.634 \\
\hline 1100 & & & & 23.074 & 20.985 & 21.298 & 21.646 \\
\hline 1000 & 23.891 & 19.533 & 20.394 & 17.988 & 19.658 & 21.897 & 23.351 \\
\hline 950 & 19.403 & 18.425 & 19.058 & 20.047 & 20.771 & 21.509 & 26.089 \\
\hline 900 & 18.676 & 18.665 & 18.778 & 19.799 & 21.205 & 22.769 & 24.365 \\
\hline 850 & 16.558 & 17.440 & 18.099 & 19.501 & 23.183 & 22.018 & 25.582 \\
\hline 800 & 16.233 & 17.253 & 19.369 & 20.555 & 20.766 & 26.124 & 24.908 \\
\hline 750 & 16.171 & 18.548 & 18.976 & 20.266 & 20.640 & 25.908 & 28.601 \\
\hline 700 & 16.548 & 18.139 & 18.668 & 21.089 & 21.155 & 23.280 & 26.921 \\
\hline 650 & 18.164 & 18.392 & 19.941 & 20.630 & 22.547 & 21.834 & 25.720 \\
\hline 600 & 17.235 & 18.404 & 20.070 & 20.500 & 22.442 & 25.160 & 23.165 \\
\hline 550 & 17.264 & 19.325 & 20.613 & 21.459 & 23.129 & 21.487 & 24.649 \\
\hline 500 & 17.485 & 17.718 & 20.167 & 24.692 & 23.273 & 24.402 & 24.598 \\
\hline 450 & 16.319 & 18.992 & 18.969 & 21.795 & 21.075 & 21.391 & 21.341 \\
\hline 400 & 16.514 & 18.135 & 19.914 & 19.832 & 22.522 & 21.994 & 24.511 \\
\hline 350 & 18.192 & 17.801 & 17.739 & 20.870 & 20.240 & 22.989 & 24.870 \\
\hline 300 & 18.205 & 19.205 & 19.962 & 20.205 & 21.778 & 21.352 & 24.541 \\
\hline 250 & 17.029 & 19.622 & 20.661 & 19.821 & 22.183 & 24.656 & 28.373 \\
\hline 200 & 17.466 & 16.664 & 18.785 & 21.373 & 23.937 & 23.150 & 21.099 \\
\hline 150 & 20.342 & 20.105 & 19.694 & 22.805 & 23.250 & 23.892 & \\
\hline 100 & 20.105 & 22.192 & 23.041 & 25.924 & 25.396 & & \\
\hline 50 & 22.852 & 23.824 & 26.277 & 25.495 & & & \\
\hline 0 & 24.089 & & & & & & \\
\hline
\end{tabular}


Table B.19: The first of three tables detailing the $\partial \sigma\left(E_{\nu}\right) / \partial\left(\cos \left(\theta_{\mu, \nu}\right)\right)$ results from Figure $6.23\left(10^{-38} \mathrm{~cm}^{2}\right)$.

\begin{tabular}{|l|c|c|c|c|c|c|c|c|}
\hline bin low edge & 500 & 600 & 650 & 700 & 750 & 800 & 850 & 900 \\
\hline 0.95 & 0.736 & 1.353 & 1.983 & 2.548 & 3.204 & 4.194 & 5.314 & 6.289 \\
\hline 0.90 & 0.868 & 1.607 & 2.116 & 2.811 & 3.732 & 4.442 & 5.307 & 6.508 \\
\hline 0.85 & 0.811 & 1.491 & 2.058 & 2.720 & 3.315 & 4.246 & 4.925 & 6.217 \\
\hline 0.80 & 0.743 & 1.429 & 1.927 & 2.652 & 3.157 & 3.591 & 4.365 & 5.319 \\
\hline 0.75 & 0.694 & 1.266 & 1.857 & 2.131 & 2.628 & 3.401 & 3.797 & 4.794 \\
\hline 0.70 & 0.684 & 1.173 & 1.703 & 2.260 & 2.739 & 3.324 & 3.766 & 4.499 \\
\hline 0.65 & 0.591 & 1.124 & 1.676 & 2.031 & 2.639 & 2.861 & 3.264 & 3.853 \\
\hline 0.60 & 0.563 & 1.207 & 1.448 & 1.912 & 2.346 & 2.702 & 3.021 & 3.391 \\
\hline 0.55 & 0.560 & 1.095 & 1.439 & 1.743 & 2.245 & 2.506 & 2.836 & 3.059 \\
\hline 0.50 & & 0.913 & 1.379 & 1.696 & 1.859 & 2.200 & 2.515 & 2.783 \\
\hline 0.45 & & 0.900 & 1.206 & 1.578 & 1.728 & 1.911 & 2.337 & 2.461 \\
\hline 0.40 & & 0.810 & 1.036 & 1.490 & 1.722 & 1.983 & 1.943 & 2.409 \\
\hline 0.35 & & 0.720 & 1.030 & 1.273 & 1.620 & 1.986 & 2.199 & 2.294 \\
\hline 0.30 & & 0.706 & 0.989 & 1.049 & 1.480 & 1.690 & 1.976 & 1.850 \\
\hline 0.25 & & & 0.890 & 1.165 & 1.308 & 1.421 & 1.802 & 1.868 \\
\hline 0.20 & & & 0.838 & 1.024 & 1.202 & 1.389 & 1.449 & 1.612 \\
\hline 0.15 & & & 0.748 & 0.927 & 1.123 & 1.248 & 1.584 & 1.449 \\
\hline 0.10 & & & 0.772 & 0.846 & 1.130 & 1.286 & 1.361 & 1.420 \\
\hline 0.05 & & & 0.724 & 0.752 & 1.056 & 1.112 & 1.253 & 1.120 \\
\hline 0.00 & & & 0.592 & 0.768 & 0.984 & 1.143 & 1.207 & 1.282 \\
\hline-0.05 & & & 0.640 & 0.840 & 0.880 & 1.050 & 1.033 & 1.146 \\
\hline-0.10 & & & & 0.723 & 0.813 & 0.856 & 1.008 & 1.030 \\
\hline-0.20 & & 0.459 & 0.527 & 0.643 & 0.822 & 0.837 & 0.857 & 0.942 \\
\hline-0.30 & & & 0.469 & 0.505 & 0.664 & 0.807 & 0.780 & 0.873 \\
\hline-0.40 & & 0.432 & 0.489 & 0.589 & 0.653 & 0.649 & 0.744 \\
\hline-0.50 & & 0.421 & 0.452 & 0.493 & 0.581 & 0.619 & 0.669 \\
\hline-0.60 & & 0.370 & 0.429 & 0.488 & 0.553 & 0.537 & 0.526 \\
\hline-0.80 & & & 0.195 & 0.226 & 0.246 & 0.246 & 0.287 & 0.273 \\
\hline-1.00 & & & & & & & & \\
\hline & & & & & & & &
\end{tabular}


Table B.20: The second of three tables detailing the $\partial \sigma\left(E_{\nu}\right) / \partial\left(\cos \left(\theta_{\mu, \nu}\right)\right)$ results from Figure $6.23\left(10^{-38} \mathrm{~cm}^{2}\right)$.

\begin{tabular}{|l|c|c|c|c|c|c|c|c|}
\hline bin low edge & 950 & 1000 & 1050 & 1100 & 1150 & 1200 & 1250 & 1300 \\
\hline 0.95 & 7.893 & 9.056 & 10.407 & 11.558 & 13.791 & 14.901 & 16.715 & 18.160 \\
\hline 0.90 & 7.139 & 8.579 & 9.616 & 10.855 & 12.143 & 12.843 & 14.171 & 15.972 \\
\hline 0.85 & 6.542 & 7.825 & 8.589 & 9.913 & 10.571 & 11.646 & 12.665 & 13.455 \\
\hline 0.80 & 6.071 & 7.375 & 7.663 & 8.248 & 8.470 & 9.627 & 10.068 & 11.830 \\
\hline 0.75 & 5.424 & 6.022 & 6.636 & 7.272 & 7.572 & 8.445 & 8.597 & 9.785 \\
\hline 0.70 & 4.939 & 5.775 & 5.868 & 6.498 & 6.764 & 7.267 & 7.695 & 7.761 \\
\hline 0.65 & 4.630 & 4.642 & 4.922 & 5.483 & 5.598 & 5.919 & 6.307 & 6.455 \\
\hline 0.60 & 3.914 & 4.263 & 4.942 & 5.215 & 5.098 & 5.559 & 5.142 & 5.512 \\
\hline 0.55 & 3.543 & 3.959 & 3.993 & 4.097 & 4.236 & 4.221 & 4.243 & 4.728 \\
\hline 0.50 & 3.200 & 3.633 & 3.842 & 3.521 & 3.841 & 3.304 & 4.141 & 4.037 \\
\hline 0.45 & 2.836 & 2.647 & 3.202 & 3.173 & 3.088 & 3.708 & 3.290 & 3.196 \\
\hline 0.40 & 2.912 & 2.894 & 3.075 & 2.824 & 3.047 & 3.363 & 2.964 & 3.045 \\
\hline 0.35 & 2.417 & 2.519 & 2.786 & 2.691 & 2.791 & 2.727 & 2.907 & 2.788 \\
\hline 0.30 & 2.070 & 2.325 & 2.357 & 2.159 & 2.447 & 2.359 & 2.497 & 2.398 \\
\hline 0.25 & 1.864 & 1.910 & 2.047 & 2.154 & 2.286 & 1.791 & 2.176 & 1.873 \\
\hline 0.20 & 1.884 & 1.689 & 1.881 & 1.935 & 1.889 & 2.087 & 2.120 & 1.932 \\
\hline 0.15 & 1.524 & 1.696 & 1.640 & 1.894 & 1.641 & 1.551 & 1.620 & 1.830 \\
\hline 0.10 & 1.392 & 1.634 & 1.587 & 1.726 & 1.468 & 1.486 & 1.507 & 1.710 \\
\hline 0.05 & 1.343 & 1.522 & 1.405 & 1.507 & 1.513 & 1.487 & 1.390 & \\
\hline 0.00 & 1.170 & 1.532 & 1.299 & 1.201 & 1.282 & 1.156 & & \\
\hline-0.05 & 1.251 & 1.175 & 1.155 & 1.190 & 1.132 & 1.059 & & \\
\hline-0.10 & 1.052 & 1.139 & 1.184 & 1.123 & 1.003 & 1.066 & & \\
\hline-0.20 & 0.881 & 1.032 & 0.978 & 0.996 & 0.956 & 0.913 & 0.938 & 0.869 \\
\hline-0.30 & 0.838 & 0.912 & 0.830 & 0.824 & 0.838 & 0.714 & 0.708 & 0.714 \\
\hline-0.40 & 0.709 & 0.759 & 0.679 & 0.680 & 0.689 & 0.679 & 0.647 & \\
\hline-0.50 & 0.639 & 0.609 & 0.593 & 0.529 & 0.727 & 0.591 & & \\
\hline-0.60 & 0.558 & 0.561 & 0.536 & 0.514 & & & & \\
\hline-0.80 & 0.454 & 0.435 & 0.460 & 0.420 & 0.423 & 0.397 & 0.361 & 0.314 \\
\hline-1.00 & 0.293 & 0.267 & 0.247 & 0.196 & 0.235 & 0.303 & & \\
\hline
\end{tabular}


Table B.21: The third of three tables detailing the $\partial \sigma\left(E_{\nu}\right) / \partial\left(\cos \left(\theta_{\mu, \nu}\right)\right)$ results from Figure $6.23\left(10^{-38} \mathrm{~cm}^{2}\right)$.

\begin{tabular}{|l|c|c|c|c|c|c|c|}
\hline bin low edge & 1350 & 1400 & 1450 & 1500 & 1550 & 1600 & 1650 \\
\hline 0.95 & 20.684 & 21.727 & 24.874 & 27.900 & 31.365 & 32.930 & 36.202 \\
\hline 0.90 & 17.304 & 18.903 & 21.093 & 23.509 & 23.495 & 25.895 & 27.000 \\
\hline 0.85 & 15.393 & 15.876 & 16.993 & 16.632 & 18.979 & 19.791 & 21.329 \\
\hline 0.80 & 11.925 & 12.528 & 13.173 & 13.017 & 13.954 & 14.749 & 15.485 \\
\hline 0.75 & 9.697 & 9.733 & 10.479 & 10.183 & 11.242 & 11.294 & 10.970 \\
\hline 0.70 & 8.028 & 8.217 & 9.028 & 9.402 & 9.414 & 8.335 & 8.933 \\
\hline 0.65 & 6.212 & 6.331 & 6.684 & 7.701 & 6.721 & 7.342 & 6.973 \\
\hline 0.60 & 5.415 & 5.754 & 5.936 & 5.993 & 5.825 & 5.454 & 6.109 \\
\hline 0.55 & 4.859 & 5.092 & 4.840 & 4.856 & 4.868 & 5.345 & 4.412 \\
\hline 0.50 & 4.056 & 3.626 & 3.769 & 3.990 & 3.997 & 4.298 & \\
\hline 0.45 & 3.277 & 3.377 & 3.202 & 3.795 & 3.165 & & \\
\hline 0.40 & 2.978 & 2.799 & 3.113 & 3.034 & & & \\
\hline 0.35 & 2.542 & 2.827 & 2.875 & & 2.426 & & \\
\hline 0.30 & 2.307 & 2.336 & 1.943 & & & & \\
\hline 0.25 & 2.055 & 2.145 & & & & & \\
\hline 0.20 & 1.936 & 1.989 & & & & & \\
\hline 0.15 & 1.589 & 1.619 & & & & & \\
\hline 0.10 & 1.618 & & & & & & \\
\hline-0.20 & 0.851 & & & & & & \\
\hline-0.30 & 0.800 & & & & & & \\
\hline
\end{tabular}


Table B.22: The first of three tables detailing the percent uncertainty of the $\partial \sigma\left(E_{\nu}\right) / \partial\left(\cos \left(\theta_{\mu, \nu}\right)\right)$ results from Figure 6.23.

\begin{tabular}{|l|c|c|c|c|c|c|c|c|}
\hline bin low edge & 500 & 600 & 650 & 700 & 750 & 800 & 850 & 900 \\
\hline 0.95 & 25.918 & 24.595 & 23.772 & 22.510 & 20.909 & 19.174 & 17.633 & 16.622 \\
\hline 0.90 & 20.028 & 18.144 & 16.955 & 17.502 & 15.846 & 14.587 & 13.832 & 12.658 \\
\hline 0.85 & 20.724 & 17.406 & 16.195 & 15.000 & 14.145 & 13.873 & 13.503 & 12.095 \\
\hline 0.80 & 16.526 & 15.737 & 13.479 & 13.992 & 13.157 & 12.737 & 11.731 & 11.277 \\
\hline 0.75 & 15.493 & 14.457 & 13.962 & 14.648 & 12.581 & 11.951 & 11.730 & 11.177 \\
\hline 0.70 & 15.000 & 14.375 & 13.747 & 11.900 & 11.734 & 11.556 & 11.737 & 12.771 \\
\hline 0.65 & 15.428 & 14.222 & 12.964 & 11.217 & 11.603 & 10.743 & 10.680 & 11.698 \\
\hline 0.60 & 16.290 & 12.769 & 14.419 & 12.337 & 12.624 & 12.046 & 12.306 & 12.066 \\
\hline 0.55 & 15.096 & 13.321 & 13.311 & 12.530 & 13.254 & 11.485 & 13.023 & 13.288 \\
\hline 0.50 & & 14.472 & 14.179 & 12.973 & 12.772 & 11.894 & 11.679 & 13.217 \\
\hline 0.45 & & 15.042 & 12.974 & 12.288 & 12.266 & 14.173 & 12.273 & 13.262 \\
\hline 0.40 & & 15.994 & 13.105 & 12.615 & 11.601 & 13.314 & 12.892 & 14.020 \\
\hline 0.35 & & 13.434 & 14.185 & 13.755 & 14.299 & 14.556 & 14.079 & 14.013 \\
\hline 0.30 & & 17.402 & 15.385 & 15.110 & 12.340 & 13.916 & 14.510 & 16.109 \\
\hline 0.25 & & & 14.910 & 14.206 & 15.436 & 13.686 & 13.393 & 15.617 \\
\hline 0.20 & & & 14.546 & 13.870 & 13.136 & 13.496 & 14.772 & 15.788 \\
\hline 0.15 & & & 15.747 & 17.038 & 14.020 & 13.226 & 14.449 & 14.484 \\
\hline 0.10 & & & 17.541 & 14.732 & 15.404 & 15.532 & 13.716 & 16.141 \\
\hline 0.05 & & & 16.348 & 15.625 & 13.203 & 16.113 & 15.176 & 18.602 \\
\hline 0.00 & & & 17.401 & 15.764 & 18.178 & 17.372 & 17.761 & 16.177 \\
\hline-0.05 & & & 18.963 & 20.048 & 16.777 & 14.657 & 19.936 & 18.417 \\
\hline-0.10 & & & & 16.265 & 16.682 & 16.384 & 16.413 & 21.818 \\
\hline-0.20 & & 24.322 & 15.189 & 17.270 & 19.201 & 14.380 & 14.799 & 17.317 \\
\hline-0.30 & & 14.663 & 15.247 & 15.777 & 16.183 & 14.677 & 14.772 \\
\hline-0.40 & & & 15.754 & 18.690 & 16.912 & 17.134 & 13.787 & 19.794 \\
\hline-0.50 & & 19.884 & 19.082 & 21.025 & 19.029 & 20.291 & 21.595 & 16.548 \\
\hline-0.60 & & 19.708 & 27.104 & 17.329 & 20.127 & 22.905 & 19.328 \\
\hline-0.80 & & & & & & & & \\
\hline-1.00 & & & & & & \\
\hline & & & & & & & &
\end{tabular}


Table B.23: The second of three tables detailing the percent uncertainty of the $\partial \sigma\left(E_{\nu}\right) / \partial\left(\cos \left(\theta_{\mu, \nu}\right)\right)$ results from Figure 6.23.

\begin{tabular}{|l|c|c|c|c|c|c|c|c|}
\hline bin low edge & 950 & 1000 & 1050 & 1100 & 1150 & 1200 & 1250 & 1300 \\
\hline 0.95 & 16.151 & 16.118 & 16.761 & 16.191 & 16.607 & 16.269 & 17.229 & 18.398 \\
\hline 0.90 & 12.330 & 12.351 & 12.531 & 12.329 & 13.470 & 13.655 & 14.619 & 14.799 \\
\hline 0.85 & 11.898 & 11.807 & 11.847 & 12.414 & 13.103 & 13.768 & 14.035 & 14.280 \\
\hline 0.80 & 12.070 & 11.682 & 12.610 & 12.245 & 13.067 & 13.798 & 14.483 & 15.111 \\
\hline 0.75 & 11.472 & 11.658 & 11.980 & 12.395 & 13.370 & 14.210 & 15.528 & 15.365 \\
\hline 0.70 & 12.120 & 12.861 & 12.728 & 12.782 & 13.619 & 14.668 & 15.921 & 15.090 \\
\hline 0.65 & 11.201 & 12.963 & 13.057 & 13.547 & 13.663 & 14.461 & 15.400 & 16.387 \\
\hline 0.60 & 12.354 & 12.782 & 13.336 & 13.656 & 15.294 & 15.665 & 15.958 & 15.678 \\
\hline 0.55 & 13.597 & 12.983 & 13.715 & 14.394 & 15.552 & 15.352 & 15.512 & 17.001 \\
\hline 0.50 & 13.023 & 12.945 & 14.235 & 14.260 & 14.829 & 16.121 & 17.476 & 18.045 \\
\hline 0.45 & 12.313 & 13.657 & 14.201 & 13.209 & 15.860 & 15.351 & 16.510 & 17.583 \\
\hline 0.40 & 13.329 & 14.329 & 15.497 & 15.108 & 15.754 & 18.836 & 16.681 & 17.570 \\
\hline 0.35 & 14.119 & 15.303 & 16.760 & 15.957 & 19.043 & 18.453 & 18.170 & 19.584 \\
\hline 0.30 & 14.704 & 16.658 & 15.273 & 15.096 & 16.958 & 17.653 & 16.499 & 18.858 \\
\hline 0.25 & 14.207 & 15.130 & 17.120 & 17.583 & 16.668 & 20.766 & 17.763 & 21.763 \\
\hline 0.20 & 16.555 & 16.921 & 16.521 & 18.155 & 18.713 & 19.442 & 21.198 & 24.138 \\
\hline 0.15 & 16.182 & 18.681 & 18.421 & 16.923 & 18.137 & 19.027 & 20.468 & 25.423 \\
\hline 0.10 & 14.540 & 21.048 & 16.547 & 21.392 & 21.825 & 25.132 & 21.684 & 21.836 \\
\hline 0.05 & 16.397 & 16.876 & 17.583 & 17.486 & 18.642 & 23.977 & 20.916 & \\
\hline 0.00 & 17.931 & 18.538 & 16.133 & 17.559 & 18.340 & 22.732 & & \\
\hline-0.05 & 18.162 & 18.799 & 19.726 & 19.414 & 21.007 & 21.290 & & \\
\hline-0.10 & 17.657 & 18.646 & 18.793 & 20.442 & 21.021 & 22.285 & & \\
\hline-0.20 & 16.112 & 15.326 & 17.271 & 17.015 & 18.034 & 19.033 & 21.209 & 28.795 \\
\hline-0.30 & 16.215 & 15.725 & 17.593 & 17.784 & 19.288 & 18.615 & 19.384 & 22.032 \\
\hline-0.40 & 17.383 & 16.331 & 17.754 & 18.212 & 19.571 & 24.366 & 20.528 & \\
\hline-0.50 & 17.457 & 17.995 & 19.044 & 18.385 & 21.251 & 24.468 & & \\
\hline-0.60 & 16.640 & 16.978 & 24.731 & 25.479 & & & & \\
\hline-0.80 & 17.783 & 18.270 & 19.468 & 18.319 & 22.779 & 20.362 & 23.645 & 23.026 \\
\hline-1.00 & 19.871 & 21.797 & 22.559 & 20.772 & 23.566 & 27.009 & & \\
\hline & & & & & & & & \\
\hline
\end{tabular}


Table B.24: The third of three tables detailing the percent uncertainty of the $\partial \sigma\left(E_{\nu}\right) / \partial\left(\cos \left(\theta_{\mu, \nu}\right)\right)$ results from Figure 6.23.

\begin{tabular}{|l|c|c|c|c|c|c|c|}
\hline bin low edge & 1350 & 1400 & 1450 & 1500 & 1550 & 1600 & 1650 \\
\hline 0.95 & 19.333 & 20.564 & 20.338 & 19.925 & 19.862 & 21.926 & 22.661 \\
\hline 0.90 & 15.559 & 15.451 & 17.304 & 17.489 & 18.974 & 19.863 & 20.450 \\
\hline 0.85 & 15.626 & 16.435 & 17.565 & 18.054 & 18.514 & 20.024 & 21.840 \\
\hline 0.80 & 16.049 & 16.564 & 17.124 & 18.058 & 19.092 & 20.099 & 21.960 \\
\hline 0.75 & 16.446 & 18.260 & 18.028 & 19.434 & 19.198 & 19.792 & 24.192 \\
\hline 0.70 & 17.590 & 17.251 & 18.085 & 19.922 & 20.007 & 20.413 & 22.092 \\
\hline 0.65 & 15.889 & 19.634 & 18.991 & 19.574 & 20.619 & 20.264 & 21.735 \\
\hline 0.60 & 17.968 & 18.925 & 19.325 & 20.983 & 20.704 & 22.228 & 23.519 \\
\hline 0.55 & 17.130 & 18.841 & 21.573 & 20.753 & 25.525 & 22.817 & 27.837 \\
\hline 0.50 & 20.370 & 20.596 & 20.545 & 22.374 & 21.545 & 22.379 & \\
\hline 0.45 & 17.699 & 18.284 & 20.715 & 28.177 & 28.857 & & \\
\hline 0.40 & 19.524 & 25.766 & 20.831 & 24.689 & & & \\
\hline 0.35 & 19.442 & 20.856 & 22.003 & & 25.084 & & \\
\hline 0.30 & 20.626 & 18.132 & 20.453 & & & & \\
\hline 0.25 & 20.937 & 20.960 & & & & & \\
\hline 0.20 & 27.180 & 25.640 & & & & & \\
\hline 0.15 & 22.366 & 23.950 & & & & & \\
\hline 0.10 & 24.535 & & & & & & \\
\hline-0.20 & 27.136 & & & & & & \\
\hline-0.30 & 23.038 & & & & & & \\
\hline
\end{tabular}


Table B.25: The first of three tables detailing the $\partial \sigma\left(E_{\nu}\right) / \partial\left(K E_{\pi}\right)$ results from Figure $6.26\left(10^{-41} \mathrm{~cm}^{2} / \mathrm{MeV}\right)$.

\begin{tabular}{|l|c|c|c|c|c|c|c|c|}
\hline bin low edge & 500 & 600 & 650 & 700 & 750 & 800 & 850 & 900 \\
\hline 375 & & & & & & 1.590 & 1.962 & 2.439 \\
\hline 350 & & & & & 1.319 & 1.922 & 2.351 & 3.098 \\
\hline 325 & & & & & 1.965 & 2.726 & 3.398 & 4.219 \\
\hline 300 & & & & 1.691 & 2.708 & 3.807 & 4.305 & 5.023 \\
\hline 275 & & & 1.616 & 2.784 & 3.700 & 4.608 & 5.459 & 6.779 \\
\hline 250 & & 1.374 & 2.413 & 3.431 & 4.741 & 5.595 & 6.621 & 7.504 \\
\hline 225 & 0.693 & 2.392 & 3.364 & 4.435 & 5.612 & 6.670 & 7.855 & 8.666 \\
\hline 200 & 1.398 & 3.189 & 4.311 & 5.755 & 7.110 & 8.558 & 9.519 & 10.259 \\
\hline 175 & 1.928 & 3.840 & 5.241 & 6.365 & 8.374 & 9.788 & 10.226 & 11.431 \\
\hline 150 & 2.616 & 5.295 & 6.952 & 8.032 & 8.973 & 10.084 & 11.637 & 12.902 \\
\hline 125 & 3.382 & 5.741 & 7.277 & 8.761 & 10.635 & 11.282 & 12.445 & 14.847 \\
\hline 100 & 3.832 & 6.600 & 8.977 & 10.614 & 11.758 & 12.835 & 14.452 & 15.746 \\
\hline 75 & 4.272 & 7.083 & 9.075 & 10.580 & 12.336 & 13.625 & 14.848 & 16.074 \\
\hline 50 & 3.764 & 5.923 & 7.517 & 8.597 & 9.819 & 11.032 & 12.080 & 13.418 \\
\hline 25 & 2.216 & 3.533 & 4.422 & 5.079 & 5.821 & 6.681 & 7.401 & 8.001 \\
\hline 0 & 0.672 & 1.038 & 1.332 & 1.683 & 1.712 & 2.144 & 2.529 & 2.588 \\
\hline
\end{tabular}


Table B.26: The second of three tables detailing the $\partial \sigma\left(E_{\nu}\right) / \partial\left(K E_{\pi}\right)$ results from Figure $6.26\left(10^{-41} \mathrm{~cm}^{2} / \mathrm{MeV}\right)$.

\begin{tabular}{|l|c|c|c|c|c|c|c|c|}
\hline bin low edge & 950 & 1000 & 1050 & 1100 & 1150 & 1200 & 1250 & 1300 \\
\hline 375 & 2.807 & 3.729 & 4.613 & 5.119 & 5.474 & 5.555 & 6.216 & 6.772 \\
\hline 350 & 3.719 & 4.562 & 5.560 & 5.699 & 6.404 & 7.169 & 6.933 & 7.490 \\
\hline 325 & 5.355 & 5.579 & 6.245 & 7.181 & 7.165 & 7.881 & 8.053 & 9.008 \\
\hline 300 & 5.931 & 6.991 & 7.613 & 8.511 & 8.474 & 9.033 & 10.227 & 11.246 \\
\hline 275 & 7.644 & 8.140 & 8.691 & 9.044 & 9.578 & 10.836 & 10.854 & 12.039 \\
\hline 250 & 8.433 & 9.887 & 10.313 & 11.450 & 11.928 & 11.503 & 12.015 & 13.169 \\
\hline 225 & 9.765 & 11.208 & 11.442 & 12.435 & 13.175 & 12.978 & 13.662 & 14.545 \\
\hline 200 & 11.602 & 12.413 & 12.135 & 13.247 & 14.967 & 14.207 & 15.192 & 15.918 \\
\hline 175 & 12.424 & 13.333 & 13.171 & 14.999 & 15.461 & 15.540 & 15.948 & 16.968 \\
\hline 150 & 13.762 & 15.290 & 16.294 & 15.760 & 16.357 & 16.095 & 17.667 & 18.248 \\
\hline 125 & 15.868 & 17.410 & 17.552 & 17.974 & 18.422 & 19.646 & 19.715 & 21.049 \\
\hline 100 & 17.015 & 18.403 & 19.043 & 20.238 & 20.261 & 21.091 & 22.012 & 22.330 \\
\hline 75 & 16.622 & 18.101 & 19.469 & 19.059 & 19.003 & 20.611 & 20.774 & 21.976 \\
\hline 50 & 13.832 & 15.150 & 14.467 & 15.177 & 16.017 & 16.623 & 17.784 & 16.778 \\
\hline 25 & 8.898 & 8.988 & 9.980 & 9.527 & 10.241 & 10.187 & 10.257 & 11.478 \\
\hline 0 & 2.544 & 3.034 & 3.141 & 3.626 & 3.476 & 3.352 & 3.603 & 3.953 \\
\hline
\end{tabular}

Table B.27: The third of three tables detailing the $\partial \sigma\left(E_{\nu}\right) / \partial\left(K E_{\pi}\right)$ results from Figure $6.26\left(10^{-41} \mathrm{~cm}^{2} / \mathrm{MeV}\right)$.

\begin{tabular}{|l|c|c|c|c|c|c|c|}
\hline bin low edge & 1350 & 1400 & 1450 & 1500 & 1550 & 1600 & 1650 \\
\hline 375 & 7.382 & 7.715 & 8.186 & 8.737 & 9.026 & 10.002 & \\
\hline 350 & 8.476 & 8.050 & 9.688 & 9.862 & 10.554 & 9.662 & 11.594 \\
\hline 325 & 9.576 & 10.368 & 11.344 & 11.416 & 12.168 & 11.746 & 12.591 \\
\hline 300 & 11.119 & 11.076 & 11.951 & 12.491 & 14.079 & 13.195 & 15.057 \\
\hline 275 & 11.293 & 12.349 & 14.624 & 13.722 & 14.552 & 15.562 & 13.816 \\
\hline 250 & 13.083 & 13.815 & 14.339 & 15.168 & 17.079 & 16.521 & 17.585 \\
\hline 225 & 15.674 & 16.244 & 15.628 & 16.630 & 18.085 & 17.916 & 17.969 \\
\hline 200 & 16.539 & 17.021 & 18.070 & 18.233 & 18.278 & 20.475 & 19.120 \\
\hline 175 & 18.105 & 17.403 & 20.113 & 20.715 & 19.250 & 20.015 & 22.985 \\
\hline 150 & 18.693 & 20.371 & 19.131 & 20.486 & 22.697 & 23.181 & 20.613 \\
\hline 125 & 20.671 & 22.141 & 21.384 & 22.833 & 24.818 & 24.999 & 25.691 \\
\hline 100 & 22.771 & 23.218 & 25.310 & 25.909 & 25.920 & 27.105 & 27.879 \\
\hline 75 & 22.844 & 21.720 & 23.506 & 25.308 & 24.698 & 22.489 & 25.582 \\
\hline 50 & 17.516 & 17.741 & 18.878 & 19.239 & 18.985 & 19.411 & 18.558 \\
\hline 25 & 12.342 & 11.186 & 11.262 & 11.725 & 11.914 & 13.108 & 12.585 \\
\hline 0 & 3.958 & 4.244 & 4.552 & 4.126 & & & \\
\hline
\end{tabular}


Table B.28: The first of three tables detailing the percent uncertainty of the $\partial \sigma\left(E_{\nu}\right) / \partial\left(K E_{\pi}\right)$ results from Figure 6.26.

\begin{tabular}{|l|c|c|c|c|c|c|c|c|}
\hline bin low edge & 500 & 600 & 650 & 700 & 750 & 800 & 850 & 900 \\
\hline 375 & & & & & & 21.601 & 19.008 & 19.524 \\
\hline 350 & & & & & 22.864 & 19.517 & 18.002 & 18.422 \\
\hline 325 & & & & & 19.170 & 18.575 & 18.088 & 17.753 \\
\hline 300 & & & & 18.722 & 17.018 & 18.050 & 17.500 & 17.131 \\
\hline 275 & & & 19.825 & 17.251 & 17.059 & 16.780 & 15.712 & 16.843 \\
\hline 250 & & 19.728 & 18.022 & 17.248 & 15.496 & 15.066 & 14.467 & 15.569 \\
\hline 225 & 19.683 & 17.080 & 16.679 & 16.090 & 15.162 & 14.447 & 14.349 & 14.075 \\
\hline 200 & 17.621 & 16.495 & 15.726 & 15.102 & 13.683 & 13.654 & 14.153 & 13.596 \\
\hline 175 & 16.787 & 17.523 & 15.966 & 14.121 & 12.494 & 12.451 & 12.050 & 12.174 \\
\hline 150 & 15.848 & 15.065 & 13.712 & 12.793 & 12.761 & 11.722 & 11.167 & 11.748 \\
\hline 125 & 16.587 & 14.906 & 11.554 & 10.881 & 11.366 & 10.926 & 10.460 & 10.707 \\
\hline 100 & 14.389 & 12.125 & 10.495 & 9.437 & 9.654 & 9.601 & 8.720 & 9.399 \\
\hline 75 & 11.841 & 12.041 & 10.078 & 10.264 & 10.170 & 11.338 & 10.498 & 11.757 \\
\hline 50 & 13.317 & 11.947 & 11.163 & 12.061 & 12.380 & 11.530 & 12.725 & 12.421 \\
\hline 25 & 12.117 & 13.586 & 11.881 & 12.028 & 14.278 & 12.744 & 13.053 & 13.268 \\
\hline 0 & 14.695 & 20.311 & 18.489 & 16.527 & 14.231 & 17.868 & 17.317 & 19.245 \\
\hline
\end{tabular}


Table B.29: The second of three tables detailing the percent uncertainty of the $\partial \sigma\left(E_{\nu}\right) / \partial\left(K E_{\pi}\right)$ results from Figure 6.26.

\begin{tabular}{|l|c|c|c|c|c|c|c|c|}
\hline bin low edge & 950 & 1000 & 1050 & 1100 & 1150 & 1200 & 1250 & 1300 \\
\hline 375 & 17.763 & 19.581 & 18.866 & 19.561 & 20.294 & 19.485 & 20.811 & 21.421 \\
\hline 350 & 17.920 & 17.461 & 19.500 & 18.672 & 19.920 & 19.296 & 18.635 & 20.020 \\
\hline 325 & 17.508 & 17.171 & 16.985 & 18.162 & 19.657 & 19.289 & 19.797 & 18.987 \\
\hline 300 & 16.228 & 16.292 & 16.877 & 16.942 & 19.185 & 20.000 & 18.574 & 19.463 \\
\hline 275 & 15.317 & 16.145 & 17.553 & 17.207 & 19.082 & 17.663 & 17.233 & 18.093 \\
\hline 250 & 16.009 & 15.866 & 15.822 & 15.914 & 16.356 & 16.887 & 17.759 & 16.956 \\
\hline 225 & 14.344 & 14.882 & 14.690 & 14.110 & 15.508 & 15.640 & 17.081 & 16.021 \\
\hline 200 & 13.569 & 13.426 & 13.915 & 13.953 & 15.477 & 15.014 & 15.333 & 15.713 \\
\hline 175 & 11.794 & 12.682 & 13.373 & 13.562 & 13.815 & 13.590 & 14.258 & 16.099 \\
\hline 150 & 11.623 & 11.587 & 12.861 & 12.578 & 12.943 & 13.706 & 14.328 & 14.092 \\
\hline 125 & 10.290 & 10.706 & 11.859 & 11.581 & 13.003 & 12.963 & 13.704 & 14.079 \\
\hline 100 & 9.530 & 11.541 & 11.070 & 11.088 & 12.035 & 13.095 & 13.364 & 14.574 \\
\hline 75 & 12.201 & 12.083 & 13.504 & 13.193 & 13.315 & 13.710 & 14.452 & 13.813 \\
\hline 50 & 11.915 & 13.339 & 13.237 & 13.192 & 15.072 & 14.092 & 13.383 & 14.324 \\
\hline 25 & 11.858 & 13.529 & 13.486 & 14.041 & 13.686 & 13.168 & 13.524 & 14.882 \\
\hline 0 & 12.760 & 16.133 & 15.361 & 15.523 & 17.562 & 16.628 & 16.376 & 18.358 \\
\hline
\end{tabular}

Table B.30: The third of three tables detailing the percent uncertainty of the $\partial \sigma\left(E_{\nu}\right) / \partial\left(K E_{\pi}\right)$ results from Figure 6.26.

\begin{tabular}{|l|c|c|c|c|c|c|c|}
\hline bin low edge & 1350 & 1400 & 1450 & 1500 & 1550 & 1600 & 1650 \\
\hline 375 & 21.273 & 22.409 & 22.806 & 25.214 & 25.510 & 25.306 & \\
\hline 350 & 23.113 & 23.409 & 21.402 & 24.047 & 26.875 & 28.068 & 26.796 \\
\hline 325 & 21.422 & 21.154 & 21.428 & 21.699 & 21.482 & 25.873 & 24.632 \\
\hline 300 & 19.168 & 22.221 & 23.184 & 20.896 & 21.083 & 22.785 & 26.674 \\
\hline 275 & 18.455 & 18.962 & 19.311 & 22.834 & 20.254 & 23.084 & 23.972 \\
\hline 250 & 17.151 & 18.556 & 21.664 & 19.989 & 22.348 & 20.635 & 23.055 \\
\hline 225 & 16.377 & 17.409 & 20.733 & 21.791 & 17.779 & 20.748 & 21.429 \\
\hline 200 & 17.793 & 17.073 & 17.002 & 18.785 & 20.846 & 20.642 & 20.019 \\
\hline 175 & 16.065 & 16.038 & 16.825 & 18.009 & 18.383 & 21.121 & 22.375 \\
\hline 150 & 16.040 & 17.421 & 17.366 & 18.586 & 18.324 & 19.809 & 19.038 \\
\hline 125 & 15.370 & 17.486 & 16.676 & 17.071 & 18.508 & 17.853 & 21.032 \\
\hline 100 & 15.257 & 15.204 & 16.480 & 16.700 & 18.098 & 19.483 & 19.061 \\
\hline 75 & 14.922 & 15.815 & 15.817 & 17.967 & 18.163 & 18.160 & 21.134 \\
\hline 50 & 15.942 & 15.953 & 15.389 & 17.699 & 17.418 & 17.527 & 20.487 \\
\hline 25 & 14.569 & 14.991 & 16.091 & 17.293 & 17.104 & 20.353 & 19.313 \\
\hline 0 & 17.480 & 22.051 & 19.049 & 27.068 & & & \\
\hline
\end{tabular}


Table B.31: The first of three tables detailing the $\partial \sigma\left(E_{\nu}\right) / \partial\left(\cos \left(\theta_{\pi, \nu}\right)\right)$ results from Figure $6.29\left(10^{-38} \mathrm{~cm}^{2}\right)$.

\begin{tabular}{|c|c|c|c|c|c|c|c|c|}
\hline bin low edge & 500 & 600 & 650 & 700 & 750 & 800 & 850 & 900 \\
\hline 0.95 & 0.887 & 1.328 & 1.840 & 2.327 & 2.865 & 3.606 & 4.251 & 4.971 \\
\hline 0.90 & 0.685 & 1.406 & 1.842 & 2.239 & 2.824 & 3.469 & 3.755 & 4.610 \\
\hline 0.85 & 0.650 & 1.342 & 1.742 & 2.206 & 3.070 & 3.492 & 3.968 & 4.723 \\
\hline 0.80 & 0.587 & 1.113 & 1.546 & 2.008 & 2.734 & 3.300 & 3.492 & 4.364 \\
\hline 0.75 & 0.570 & 1.197 & 1.534 & 2.001 & 2.591 & 3.075 & 3.411 & 3.924 \\
\hline 0.70 & 0.563 & 1.077 & 1.503 & 1.937 & 2.584 & 3.056 & 3.737 & 3.849 \\
\hline 0.65 & 0.559 & 1.073 & 1.474 & 1.905 & 2.286 & 2.892 & 3.269 & 3.762 \\
\hline 0.60 & 0.530 & 1.033 & 1.431 & 1.801 & 2.105 & 2.631 & 2.951 & 3.449 \\
\hline 0.55 & 0.488 & 0.921 & 1.288 & 1.655 & 1.946 & 2.393 & 2.659 & 3.131 \\
\hline 0.50 & 0.479 & 0.914 & 1.336 & 1.630 & 2.050 & 2.237 & 2.665 & 2.997 \\
\hline 0.45 & 0.470 & 0.819 & 1.049 & 1.639 & 1.832 & 2.068 & 2.356 & 2.716 \\
\hline 0.40 & 0.418 & 0.812 & 1.238 & 1.285 & 1.578 & 1.935 & 2.432 & 2.414 \\
\hline 0.35 & & 0.782 & 1.037 & 1.299 & 1.545 & 1.819 & 1.993 & 2.347 \\
\hline 0.30 & & 0.673 & 0.988 & 1.109 & 1.489 & 1.873 & 2.027 & 2.039 \\
\hline 0.25 & & 0.735 & 0.960 & 1.166 & 1.330 & 1.591 & 1.848 & 2.088 \\
\hline 0.20 & & 0.773 & 0.936 & 1.074 & 1.351 & 1.461 & 1.726 & 2.018 \\
\hline 0.15 & & 0.612 & 0.822 & 1.071 & 1.178 & 1.500 & 1.749 & 1.860 \\
\hline 0.10 & & 0.577 & 0.883 & 0.972 & 1.204 & 1.368 & 1.474 & 1.687 \\
\hline 0.05 & & & 0.784 & 0.899 & 1.066 & 1.291 & 1.354 & 1.675 \\
\hline 0.00 & & & 0.708 & 0.912 & 1.097 & 1.149 & 1.258 & 1.555 \\
\hline-0.05 & & & 0.595 & 0.849 & 1.029 & 1.059 & 1.249 & 1.491 \\
\hline-0.10 & & & 0.645 & 0.776 & 0.884 & 0.989 & 1.139 & 1.319 \\
\hline-0.15 & & & 0.620 & 0.640 & 0.833 & 0.975 & 1.100 & 1.203 \\
\hline-0.20 & & & 0.602 & 0.667 & 0.803 & 0.902 & 0.981 & 1.174 \\
\hline-0.25 & & & 0.567 & 0.681 & 0.777 & 0.843 & 0.988 & 0.994 \\
\hline-0.30 & & & 0.579 & 0.642 & 0.786 & 0.704 & 0.807 & 1.079 \\
\hline-0.35 & & & & 0.555 & 0.676 & 0.739 & 0.935 & 0.901 \\
\hline-0.40 & & & & 0.530 & 0.627 & 0.725 & 0.747 & 0.866 \\
\hline-0.45 & & & & 0.391 & 0.601 & 0.617 & 0.717 & 0.769 \\
\hline-0.50 & & & & 0.480 & 0.474 & 0.610 & 0.788 & 0.776 \\
\hline-0.55 & & & & & 0.506 & 0.594 & 0.609 & 0.759 \\
\hline-0.60 & & & & 0.462 & & 0.470 & 0.553 & 0.663 \\
\hline-0.65 & & & & & 0.476 & 0.544 & 0.609 & 0.621 \\
\hline-0.70 & & & & & 0.477 & 0.418 & 0.506 & 0.580 \\
\hline-0.75 & & & & & & 0.497 & 0.542 & 0.594 \\
\hline-0.80 & & & & & 0.364 & 0.439 & 0.483 & 0.500 \\
\hline-0.85 & & & & & & & 0.495 & \\
\hline-0.90 & & & & & & 0.421 & 0.428 & 0.436 \\
\hline-0.95 & & & & & & & & 0.426 \\
\hline
\end{tabular}


Table B.32: The second of three tables detailing the $\partial \sigma\left(E_{\nu}\right) / \partial\left(\cos \left(\theta_{\pi, \nu}\right)\right)$ results from Figure $6.29\left(10^{-38} \mathrm{~cm}^{2}\right)$.

\begin{tabular}{|c|c|c|c|c|c|c|c|c|}
\hline bin low edge & 950 & 1000 & 1050 & 1100 & 1150 & 1200 & 1250 & 1300 \\
\hline 0.95 & 5.386 & 6.335 & 6.708 & 7.464 & 7.978 & 8.578 & 9.153 & 9.487 \\
\hline 0.90 & 5.426 & 6.273 & 6.528 & 7.083 & 7.901 & 8.474 & 8.418 & 9.656 \\
\hline 0.85 & 5.090 & 5.915 & 6.304 & 7.016 & 7.422 & 7.741 & 8.185 & 8.960 \\
\hline 0.80 & 5.013 & 5.745 & 6.324 & 6.441 & 6.840 & 7.710 & 8.240 & 7.999 \\
\hline 0.75 & 4.877 & 5.369 & 5.767 & 6.171 & 6.378 & 7.059 & 6.978 & 7.638 \\
\hline 0.70 & 4.496 & 4.854 & 5.188 & 5.520 & 5.852 & 6.023 & 6.599 & 7.369 \\
\hline 0.65 & 4.190 & 4.252 & 4.996 & 5.142 & 5.454 & 5.796 & 5.928 & 6.908 \\
\hline 0.60 & 3.786 & 4.431 & 4.431 & 5.036 & 5.165 & 5.245 & 5.424 & 6.334 \\
\hline 0.55 & 3.479 & 3.844 & 4.161 & 4.388 & 4.442 & 5.027 & 5.406 & 5.637 \\
\hline 0.50 & 3.222 & 3.829 & 3.726 & 4.164 & 4.308 & 4.343 & 4.816 & 5.179 \\
\hline 0.45 & 2.849 & 3.465 & 3.645 & 3.954 & 4.067 & 4.169 & 4.270 & 4.720 \\
\hline 0.40 & 2.905 & 2.953 & 3.308 & 3.287 & 3.654 & 3.732 & 3.894 & 4.173 \\
\hline 0.35 & 2.550 & 2.914 & 3.138 & 2.990 & 3.455 & 3.515 & 3.997 & 4.018 \\
\hline 0.30 & 2.644 & 2.742 & 3.015 & 3.126 & 3.029 & 3.321 & 3.532 & 3.832 \\
\hline 0.25 & 2.160 & 2.672 & 2.705 & 2.752 & 3.094 & 3.001 & 3.124 & 3.237 \\
\hline 0.20 & 2.164 & 2.400 & 2.597 & 2.782 & 2.743 & 2.541 & 2.915 & 2.967 \\
\hline 0.15 & 1.838 & 2.225 & 2.262 & 2.484 & 2.434 & 2.519 & 2.974 & 3.031 \\
\hline 0.10 & 1.787 & 1.929 & 2.051 & 2.178 & 2.510 & 2.350 & 2.464 & 2.650 \\
\hline 0.05 & 1.785 & 1.606 & 1.975 & 1.982 & 2.255 & 2.259 & 2.310 & 2.667 \\
\hline 0.00 & 1.547 & 1.670 & 1.848 & 1.777 & 2.241 & 2.065 & 2.257 & 2.344 \\
\hline-0.05 & 1.448 & 1.734 & 1.679 & 1.818 & 1.795 & 2.091 & 2.002 & 2.121 \\
\hline-0.10 & 1.389 & 1.467 & 1.533 & 1.586 & 1.527 & 1.680 & 1.660 & 1.756 \\
\hline-0.15 & 1.378 & 1.428 & 1.336 & 1.650 & 1.510 & 1.764 & 1.662 & 1.942 \\
\hline-0.20 & 1.138 & 1.430 & 1.394 & 1.350 & 1.485 & 1.623 & 1.525 & 1.715 \\
\hline-0.25 & 1.171 & 1.368 & 1.274 & 1.430 & 1.284 & 1.455 & 1.432 & 1.652 \\
\hline-0.30 & 1.194 & 1.181 & 1.196 & 1.178 & 1.575 & 1.315 & 1.670 & 1.141 \\
\hline-0.35 & 1.122 & 1.098 & 1.108 & 1.153 & 1.260 & 1.165 & 1.346 & 1.423 \\
\hline-0.40 & 0.946 & 0.919 & 1.069 & 1.078 & 1.130 & 1.154 & 1.232 & 1.394 \\
\hline-0.45 & 0.843 & 0.977 & 0.958 & 0.951 & 1.165 & 1.056 & 1.286 & 1.034 \\
\hline-0.50 & 0.847 & 0.796 & 0.857 & 1.097 & 0.934 & 1.113 & 1.029 & \\
\hline-0.55 & 0.759 & 0.783 & 0.818 & 0.873 & 0.947 & 0.895 & & \\
\hline-0.60 & 0.679 & 0.679 & 0.874 & 0.784 & 0.828 & & & \\
\hline-0.65 & 0.668 & 0.764 & 0.825 & 0.746 & 0.958 & & & \\
\hline-0.70 & 0.617 & 0.720 & 0.690 & 0.831 & 0.829 & & & \\
\hline-0.75 & 0.578 & 0.635 & 0.616 & 0.629 & & & & \\
\hline-0.80 & 0.562 & 0.642 & 0.613 & & & & & \\
\hline-0.85 & 0.528 & 0.520 & & & & & & \\
\hline-1.00 & 0.462 & & & & & & & \\
\hline
\end{tabular}


Table B.33: The third of three tables detailing the $\partial \sigma\left(E_{\nu}\right) / \partial\left(\cos \left(\theta_{\pi, \nu}\right)\right)$ results from Figure $6.29\left(10^{-38} \mathrm{~cm}^{2}\right)$.

\begin{tabular}{|l|c|c|c|c|c|c|c|}
\hline bin low edge & 1350 & 1400 & 1450 & 1500 & 1550 & 1600 & 1650 \\
\hline 0.95 & 10.482 & 11.259 & 11.522 & 12.819 & 12.595 & 13.816 & 13.895 \\
\hline 0.90 & 10.167 & 11.365 & 11.442 & 12.033 & 11.685 & 12.689 & 12.633 \\
\hline 0.85 & 9.614 & 9.773 & 10.377 & 11.595 & 11.068 & 11.429 & 12.468 \\
\hline 0.80 & 9.134 & 9.412 & 9.506 & 11.218 & 10.101 & 10.871 & 10.456 \\
\hline 0.75 & 8.179 & 8.147 & 9.430 & 9.529 & 9.380 & 10.333 & 10.111 \\
\hline 0.70 & 7.656 & 7.505 & 7.840 & 8.673 & 9.594 & 8.746 & 9.294 \\
\hline 0.65 & 6.818 & 7.049 & 7.470 & 7.691 & 8.962 & 8.560 & 8.748 \\
\hline 0.60 & 5.970 & 6.602 & 7.414 & 7.036 & 7.782 & 7.775 & 8.290 \\
\hline 0.55 & 5.976 & 5.618 & 6.734 & 6.765 & 6.719 & 7.502 & 7.634 \\
\hline 0.50 & 5.087 & 4.861 & 6.266 & 6.126 & 6.675 & 6.769 & 6.964 \\
\hline 0.45 & 4.893 & 5.238 & 5.389 & 5.575 & 6.382 & 5.899 & 5.697 \\
\hline 0.40 & 4.480 & 4.566 & 4.902 & 4.818 & 6.151 & 6.020 & 5.357 \\
\hline 0.35 & 3.919 & 3.830 & 5.127 & 4.610 & 4.608 & 5.057 & 5.049 \\
\hline 0.30 & 3.767 & 4.030 & 4.136 & 4.823 & 4.269 & 4.318 & \\
\hline 0.25 & 3.738 & 3.586 & 3.391 & 3.801 & 4.567 & 4.856 & \\
\hline 0.20 & 3.223 & 3.424 & 3.374 & 3.804 & 3.803 & & \\
\hline 0.15 & 3.319 & 3.359 & 3.535 & 3.549 & 3.656 & 3.947 & \\
\hline 0.10 & 3.149 & 3.007 & 3.016 & 2.795 & & & \\
\hline 0.05 & 2.756 & 2.699 & 2.667 & 3.169 & & & \\
\hline 0.00 & 2.461 & 2.472 & 2.801 & & & & \\
\hline-0.05 & 2.214 & 2.363 & 2.151 & & & & \\
\hline-0.10 & 1.912 & 2.200 & 2.429 & & & & \\
\hline-0.15 & 1.829 & 1.809 & & & & & \\
\hline-0.20 & 1.790 & 1.848 & 1.694 & & & & \\
\hline-0.25 & 1.637 & 1.571 & & & & & \\
\hline-0.45 & 1.180 & & & & & & \\
\hline & & & & & & & \\
\hline
\end{tabular}


Table B.34: The first of three tables detailing the percent uncertainty of the $\partial \sigma\left(E_{\nu}\right) / \partial\left(\cos \left(\theta_{\pi, \nu}\right)\right)$ results from Figure 6.29.

\begin{tabular}{|c|c|c|c|c|c|c|c|c|}
\hline bin low edge & 500 & 600 & 650 & 700 & 750 & 800 & 850 & 900 \\
\hline 0.95 & 20.800 & 21.207 & 19.474 & 19.201 & 18.895 & 18.122 & 16.699 & 16.917 \\
\hline 0.90 & 18.967 & 18.988 & 16.236 & 17.222 & 16.654 & 16.545 & 15.227 & 15.027 \\
\hline 0.85 & 19.489 & 16.300 & 15.433 & 14.651 & 13.409 & 14.128 & 14.058 & 13.898 \\
\hline 0.80 & 16.290 & 16.580 & 15.040 & 13.471 & 13.525 & 13.348 & 12.640 & 13.502 \\
\hline 0.75 & 18.086 & 14.676 & 13.157 & 13.043 & 13.151 & 12.602 & 12.690 & 12.223 \\
\hline 0.70 & 17.478 & 14.420 & 13.150 & 13.853 & 11.450 & 13.207 & 12.237 & 12.106 \\
\hline 0.65 & 16.210 & 15.517 & 13.913 & 14.038 & 12.978 & 12.215 & 12.977 & 13.077 \\
\hline 0.60 & 17.359 & 16.770 & 13.955 & 13.918 & 12.847 & 12.259 & 12.652 & 12.939 \\
\hline 0.55 & 17.660 & 14.355 & 14.581 & 14.988 & 13.483 & 11.948 & 13.246 & 14.258 \\
\hline 0.50 & 16.180 & 19.315 & 15.304 & 15.303 & 12.986 & 12.177 & 11.917 & 12.947 \\
\hline 0.45 & 16.298 & 17.041 & 13.759 & 13.166 & 14.087 & 13.851 & 12.895 & 14.462 \\
\hline 0.40 & 16.384 & 16.523 & 14.726 & 14.938 & 14.346 & 13.936 & 12.477 & 12.441 \\
\hline 0.35 & & 15.984 & 16.993 & 14.887 & 14.696 & 14.388 & 12.559 & 13.042 \\
\hline 0.30 & & 18.035 & 13.963 & 14.068 & 14.125 & 14.897 & 13.739 & 13.116 \\
\hline 0.25 & & 14.755 & 14.470 & 18.782 & 14.232 & 14.024 & 11.309 & 13.558 \\
\hline 0.20 & & 16.824 & 15.679 & 13.807 & 13.371 & 12.336 & 13.718 & 12.535 \\
\hline 0.15 & & 15.882 & 14.697 & 13.824 & 12.867 & 12.684 & 11.958 & 13.124 \\
\hline 0.10 & & 17.732 & 16.090 & 13.997 & 14.247 & 14.467 & 11.671 & 13.540 \\
\hline 0.05 & & & 14.677 & 16.455 & 13.869 & 11.261 & 12.437 & 13.194 \\
\hline 0.00 & & & 14.587 & 12.530 & 15.142 & 14.072 & 12.761 & 13.906 \\
\hline-0.05 & & & 17.156 & 13.852 & 14.964 & 12.781 & 13.815 & 12.738 \\
\hline-0.10 & & & 16.273 & 14.778 & 13.884 & 13.875 & 14.524 & 14.786 \\
\hline-0.15 & & & 17.051 & 14.128 & 13.320 & 18.161 & 12.101 & 13.920 \\
\hline-0.20 & & & 15.636 & 15.881 & 14.497 & 15.319 & 12.027 & 14.941 \\
\hline-0.25 & & & 17.653 & 15.577 & 13.628 & 14.670 & 14.420 & 15.747 \\
\hline-0.30 & & & 17.725 & 14.969 & 15.136 & 14.855 & 13.553 & 18.395 \\
\hline-0.35 & & & & 16.769 & 14.895 & 13.224 & 17.218 & 13.834 \\
\hline-0.40 & & & & 18.577 & 15.322 & 13.683 & 14.038 & 13.010 \\
\hline-0.45 & & & & 17.709 & 15.700 & 18.373 & 13.347 & 13.392 \\
\hline-0.50 & & & & 18.763 & 14.369 & 16.229 & 16.960 & 15.573 \\
\hline-0.55 & & & & & 14.330 & 15.524 & 16.303 & 16.908 \\
\hline-0.60 & & & & 16.402 & & 19.220 & 16.240 & 14.162 \\
\hline-0.65 & & & & & 16.512 & 14.449 & 16.737 & 15.484 \\
\hline-0.70 & & & & & 17.846 & 15.541 & 14.645 & 17.339 \\
\hline-0.75 & & & & & & 18.444 & 15.351 & 17.676 \\
\hline-0.80 & & & & & 16.701 & 20.162 & 16.938 & 18.860 \\
\hline-0.85 & & & & & & & 17.819 & \\
\hline-0.90 & & & & & & 20.697 & 20.327 & 19.404 \\
\hline-0.95 & & & & & & & & 21.636 \\
\hline
\end{tabular}


Table B.35: The second of three tables detailing the percent uncertainty of the $\partial \sigma\left(E_{\nu}\right) / \partial\left(\cos \left(\theta_{\pi, \nu}\right)\right)$ results from Figure 6.29.

\begin{tabular}{|c|c|c|c|c|c|c|c|c|}
\hline bin low edge & 950 & 1000 & 1050 & 1100 & 1150 & 1200 & 1250 & 1300 \\
\hline 0.95 & 16.708 & 16.332 & 16.857 & 16.282 & 16.860 & 18.255 & 19.049 & 19.484 \\
\hline 0.90 & 14.562 & 15.800 & 15.481 & 15.824 & 15.989 & 17.203 & 16.919 & 17.619 \\
\hline 0.85 & 13.181 & 13.528 & 14.148 & 14.607 & 15.282 & 15.273 & 15.891 & 16.903 \\
\hline 0.80 & 12.614 & 13.820 & 14.173 & 14.063 & 15.363 & 15.481 & 16.689 & 16.648 \\
\hline 0.75 & 12.995 & 13.384 & 13.331 & 13.425 & 15.449 & 15.760 & 15.831 & 16.972 \\
\hline 0.70 & 12.674 & 13.654 & 13.664 & 13.292 & 14.814 & 15.127 & 15.741 & 16.867 \\
\hline 0.65 & 12.728 & 12.958 & 13.413 & 14.498 & 14.998 & 15.428 & 16.220 & 15.801 \\
\hline 0.60 & 12.471 & 13.096 & 13.992 & 13.686 & 15.680 & 15.814 & 16.033 & 17.309 \\
\hline 0.55 & 12.930 & 13.163 & 14.563 & 14.060 & 14.792 & 15.766 & 15.561 & 17.585 \\
\hline 0.50 & 13.136 & 12.517 & 13.769 & 13.052 & 14.162 & 16.276 & 15.750 & 16.611 \\
\hline 0.45 & 13.590 & 11.135 & 13.097 & 14.075 & 15.425 & 15.383 & 15.358 & 16.141 \\
\hline 0.40 & 12.836 & 13.331 & 13.686 & 15.040 & 15.398 & 15.589 & 15.712 & 16.498 \\
\hline 0.35 & 13.257 & 13.460 & 14.270 & 15.470 & 15.359 & 16.076 & 15.815 & 19.169 \\
\hline 0.30 & 15.411 & 13.411 & 13.128 & 13.719 & 13.742 & 15.095 & 19.068 & 16.496 \\
\hline 0.25 & 12.943 & 12.821 & 13.778 & 13.467 & 15.147 & 16.463 & 14.505 & 15.669 \\
\hline 0.20 & 12.811 & 13.807 & 13.920 & 13.908 & 13.594 & 16.702 & 16.864 & 16.411 \\
\hline 0.15 & 15.168 & 13.892 & 13.516 & 13.479 & 13.714 & 14.988 & 16.018 & 16.652 \\
\hline 0.10 & 14.084 & 13.284 & 13.033 & 14.087 & 14.767 & 15.174 & 15.536 & 16.255 \\
\hline 0.05 & 14.049 & 11.838 & 13.075 & 13.315 & 14.339 & 15.961 & 17.466 & 17.845 \\
\hline 0.00 & 13.379 & 13.650 & 13.555 & 13.650 & 13.833 & 16.616 & 16.785 & 14.872 \\
\hline-0.05 & 12.969 & 13.273 & 13.724 & 14.026 & 14.510 & 14.720 & 17.025 & 17.504 \\
\hline-0.10 & 13.266 & 12.765 & 16.012 & 13.431 & 14.936 & 16.789 & 17.301 & 19.092 \\
\hline-0.15 & 12.327 & 13.651 & 14.994 & 15.040 & 15.394 & 15.891 & 15.679 & 19.681 \\
\hline-0.20 & 14.157 & 13.334 & 14.936 & 14.348 & 14.537 & 16.398 & 17.113 & 20.666 \\
\hline-0.25 & 15.576 & 13.688 & 14.851 & 13.932 & 13.563 & 15.138 & 16.701 & 16.304 \\
\hline-0.30 & 16.067 & 15.975 & 14.777 & 17.564 & 15.004 & 16.726 & 16.445 & 17.771 \\
\hline-0.35 & 14.161 & 16.166 & 14.137 & 16.895 & 17.659 & 16.146 & 18.010 & 20.461 \\
\hline-0.40 & 11.845 & 14.154 & 15.560 & 16.499 & 16.390 & 17.905 & 17.652 & 20.326 \\
\hline-0.45 & 14.068 & 14.059 & 14.144 & 17.359 & 22.326 & 17.681 & 16.023 & 17.035 \\
\hline-0.50 & 14.436 & 12.985 & 18.857 & 16.799 & 16.876 & 19.008 & 16.386 & \\
\hline-0.55 & 15.921 & 12.507 & 16.482 & 16.593 & 17.645 & 17.183 & & \\
\hline-0.60 & 15.674 & 16.074 & 16.322 & 17.028 & 16.049 & & & \\
\hline-0.65 & 16.079 & 16.669 & 15.592 & 17.085 & 19.528 & & & \\
\hline-0.70 & 16.237 & 17.996 & 17.720 & 22.815 & 17.828 & & & \\
\hline-0.75 & 16.446 & 19.365 & 20.507 & 21.066 & & & & \\
\hline-0.80 & 17.192 & 19.459 & 17.447 & & & & & \\
\hline-0.85 & 15.541 & 17.766 & & & & & & \\
\hline-1.00 & 17.241 & & & & & & & \\
\hline
\end{tabular}


Table B.36: The third of three tables detailing the percent uncertainty of the $\partial \sigma\left(E_{\nu}\right) / \partial\left(\cos \left(\theta_{\pi, \nu}\right)\right)$ results from Figure 6.29.

\begin{tabular}{|l|c|c|c|c|c|c|c|}
\hline bin low edge & 1350 & 1400 & 1450 & 1500 & 1550 & 1600 & 1650 \\
\hline 0.95 & 19.449 & 20.675 & 21.308 & 21.664 & 22.230 & 23.430 & 25.874 \\
\hline 0.90 & 18.559 & 19.564 & 19.746 & 22.340 & 22.613 & 22.553 & 23.706 \\
\hline 0.85 & 19.008 & 19.622 & 21.905 & 21.038 & 20.992 & 22.018 & 23.051 \\
\hline 0.80 & 18.125 & 18.817 & 19.750 & 20.603 & 25.084 & 22.420 & 22.866 \\
\hline 0.75 & 17.784 & 18.147 & 19.768 & 20.886 & 20.991 & 24.650 & 23.766 \\
\hline 0.70 & 17.518 & 19.779 & 20.218 & 19.324 & 20.030 & 22.252 & 24.979 \\
\hline 0.65 & 17.321 & 21.941 & 20.042 & 20.113 & 23.068 & 23.215 & 21.524 \\
\hline 0.60 & 18.602 & 18.469 & 19.924 & 21.135 & 21.544 & 21.800 & 24.294 \\
\hline 0.55 & 17.460 & 19.398 & 19.921 & 22.913 & 21.784 & 23.513 & 25.287 \\
\hline 0.50 & 18.136 & 18.823 & 18.259 & 22.780 & 19.299 & 23.524 & 21.837 \\
\hline 0.45 & 17.172 & 17.751 & 17.528 & 19.788 & 21.499 & 23.964 & 26.177 \\
\hline 0.40 & 17.589 & 18.312 & 22.105 & 19.757 & 19.927 & 21.450 & 22.239 \\
\hline 0.35 & 16.883 & 20.483 & 19.305 & 20.840 & 20.111 & 22.505 & 24.765 \\
\hline 0.30 & 17.900 & 18.689 & 22.360 & 21.113 & 21.235 & 23.656 & \\
\hline 0.25 & 17.219 & 19.800 & 20.124 & 18.919 & 20.543 & 25.707 & \\
\hline 0.20 & 17.490 & 21.987 & 19.504 & 21.279 & 26.401 & & \\
\hline 0.15 & 17.851 & 20.238 & 19.969 & 20.539 & 25.499 & 22.012 & \\
\hline 0.10 & 17.117 & 18.646 & 22.870 & 25.153 & & & \\
\hline 0.05 & 18.483 & 17.855 & 19.875 & 23.918 & & & \\
\hline 0.00 & 17.578 & 18.781 & 20.371 & & & & \\
\hline-0.05 & 17.337 & 19.203 & 24.658 & & & & \\
\hline-0.10 & 20.673 & 20.830 & 21.920 & & & & \\
\hline-0.15 & 16.747 & 18.932 & & & & & \\
\hline-0.20 & 19.300 & 18.760 & 21.198 & & & & \\
\hline-0.25 & 18.081 & 19.458 & & & & & \\
\hline-0.45 & 24.848 & & & & & & \\
\hline & & & & & & & \\
\hline
\end{tabular}


Table B.37: The first of three tables detailing the $\partial^{2} \sigma / \partial\left(K E_{\mu}\right) \partial\left(\cos \left(\theta_{\mu, \nu}\right)\right)$ results from Figure $6.32\left(10^{-42} \mathrm{~cm}^{2} / \mathrm{MeV}\right)$.

\begin{tabular}{|l|c|c|c|c|c|c|c|c|}
\hline bin low edge & 0 & 50 & 100 & 150 & 200 & 250 & 300 & 350 \\
\hline 0.95 & 17.798 & 13.968 & 21.164 & 26.281 & 29.365 & 34.319 & 41.483 & 45.205 \\
\hline 0.90 & 21.830 & 26.129 & 27.996 & 33.186 & 38.107 & 42.214 & 46.595 & 54.308 \\
\hline 0.85 & 21.220 & 24.725 & 30.728 & 34.559 & 37.591 & 44.224 & 50.632 & 56.669 \\
\hline 0.80 & 20.377 & 22.825 & 31.709 & 34.175 & 40.208 & 43.944 & 52.822 & 60.313 \\
\hline 0.75 & 16.694 & 25.800 & 28.230 & 35.465 & 40.796 & 45.277 & 52.258 & 55.306 \\
\hline 0.70 & 17.112 & 24.686 & 30.457 & 38.413 & 40.914 & 47.241 & 56.130 & 54.599 \\
\hline 0.65 & 16.139 & 24.829 & 28.792 & 37.334 & 41.482 & 44.970 & 48.849 & 53.554 \\
\hline 0.60 & 16.262 & 24.244 & 28.947 & 34.278 & 43.032 & 46.044 & 45.632 & 48.215 \\
\hline 0.55 & 16.129 & 23.788 & 29.173 & 34.932 & 40.749 & 44.844 & 46.791 & 40.094 \\
\hline 0.50 & 14.631 & 22.535 & 28.472 & 32.532 & 36.968 & 42.072 & 41.463 & 37.040 \\
\hline 0.45 & 14.071 & 22.583 & 28.346 & 32.322 & 36.053 & 36.238 & 36.535 & 30.855 \\
\hline 0.40 & 14.659 & 23.428 & 26.388 & 32.346 & 35.412 & 38.663 & 34.981 & 28.355 \\
\hline 0.35 & 13.849 & 21.561 & 27.477 & 36.228 & 38.156 & 34.769 & 30.227 & 27.358 \\
\hline 0.30 & 12.928 & 21.878 & 26.134 & 30.057 & 33.307 & 28.934 & 27.886 & 20.222 \\
\hline 0.25 & 13.518 & 20.991 & 25.281 & 29.108 & 30.216 & 29.203 & 21.700 & 17.033 \\
\hline 0.20 & 12.219 & 20.135 & 24.673 & 26.648 & 29.887 & 26.547 & 21.025 & 13.138 \\
\hline 0.15 & 12.216 & 21.049 & 25.488 & 26.397 & 27.735 & 23.911 & 17.709 & 9.145 \\
\hline 0.10 & 11.960 & 21.099 & 23.756 & 26.227 & 26.181 & 21.093 & 14.890 & 8.043 \\
\hline 0.05 & 12.450 & 17.837 & 23.766 & 25.152 & 25.688 & 19.400 & 12.751 & 6.365 \\
\hline 0.00 & 12.674 & 20.846 & 23.373 & 23.765 & 22.053 & 16.153 & 9.795 & 5.099 \\
\hline-0.05 & 12.150 & 19.586 & 23.252 & 24.252 & 19.009 & 13.294 & 7.181 & \\
\hline-0.10 & 11.633 & 19.000 & 22.931 & 21.054 & 17.137 & 11.106 & 5.894 & \\
\hline-0.20 & 11.676 & 18.404 & 20.568 & 20.001 & 16.109 & 9.864 & 4.876 & 2.448 \\
\hline-0.30 & 10.063 & 17.423 & 19.414 & 17.697 & 12.493 & 6.093 & 2.864 & \\
\hline-0.40 & 10.411 & 17.217 & 16.879 & 14.862 & 9.498 & 5.523 & 1.950 & \\
\hline-0.50 & 10.055 & 16.290 & 16.299 & 12.453 & 7.791 & 3.328 & & \\
\hline-0.60 & 9.228 & 14.794 & 14.556 & 10.602 & 5.742 & 2.508 & & \\
\hline-0.80 & 8.357 & 13.809 & 12.347 & 7.914 & 3.377 & 1.486 & & \\
\hline-1.00 & 5.975 & 9.696 & 7.910 & 4.108 & 1.504 & & & \\
\hline & & & & & & & & \\
\hline
\end{tabular}


Table B.38: The second of three tables detailing the $\partial^{2} \sigma / \partial\left(K E_{\mu}\right) \partial\left(\cos \left(\theta_{\mu, \nu}\right)\right)$ results from Figure $6.32\left(10^{-42} \mathrm{~cm}^{2} / \mathrm{MeV}\right)$.

\begin{tabular}{|l|c|c|c|c|c|c|c|c|}
\hline bin low edge & 400 & 450 & 500 & 550 & 600 & 650 & 700 & 750 \\
\hline 0.95 & 50.062 & 57.570 & 60.914 & 63.030 & 65.862 & 68.693 & 70.577 & 73.235 \\
\hline 0.90 & 58.905 & 64.431 & 71.462 & 70.470 & 71.978 & 72.455 & 65.354 & 64.490 \\
\hline 0.85 & 66.737 & 66.021 & 67.521 & 69.549 & 69.693 & 67.506 & 54.151 & 50.242 \\
\hline 0.80 & 59.280 & 62.355 & 64.548 & 58.949 & 54.518 & 53.013 & 44.318 & 36.734 \\
\hline 0.75 & 57.055 & 56.394 & 57.130 & 50.728 & 43.139 & 35.489 & 32.112 & 25.552 \\
\hline 0.70 & 56.319 & 52.308 & 50.791 & 41.468 & 34.152 & 27.743 & 21.773 & 14.918 \\
\hline 0.65 & 47.087 & 43.503 & 37.563 & 29.397 & 23.620 & 17.033 & 14.444 & 9.865 \\
\hline 0.60 & 49.090 & 39.284 & 29.860 & 23.715 & 16.107 & 11.381 & 7.825 & \\
\hline 0.55 & 35.430 & 32.490 & 23.624 & 16.098 & 10.471 & 6.228 & & \\
\hline 0.50 & 32.347 & 25.587 & 15.477 & 11.626 & 7.179 & & & \\
\hline 0.45 & 25.079 & 17.896 & 12.601 & 8.098 & & & & \\
\hline 0.40 & 21.937 & 14.788 & 8.701 & 5.265 & & & & \\
\hline 0.35 & 17.001 & 11.995 & 6.821 & & & & & \\
\hline 0.30 & 12.226 & 8.108 & & & & & & \\
\hline 0.25 & 10.323 & 6.184 & & & & & & \\
\hline 0.20 & 8.416 & & & & & & & \\
\hline 0.15 & 5.710 & & & & & & & \\
\hline
\end{tabular}

Table B.39: The third of three tables detailing the $\partial^{2} \sigma / \partial\left(K E_{\mu}\right) \partial\left(\cos \left(\theta_{\mu, \nu}\right)\right)$ results from Figure $6.32\left(10^{-42} \mathrm{~cm}^{2} / \mathrm{MeV}\right)$.

\begin{tabular}{|l|c|c|c|c|c|c|c|}
\hline bin low edge & 800 & 850 & 900 & 950 & 1000 & 1100 & 1200 \\
\hline 0.95 & 69.351 & 60.842 & 59.787 & 56.517 & 48.687 & 43.415 & 26.724 \\
\hline 0.90 & 58.081 & 56.950 & 49.551 & 44.935 & 37.239 & 25.639 & 11.287 \\
\hline 0.85 & 46.050 & 38.861 & 33.634 & 25.846 & 18.525 & 11.770 & 5.132 \\
\hline 0.80 & 32.725 & 23.404 & 18.083 & 14.240 & 8.540 & & \\
\hline 0.75 & 18.242 & 13.005 & 9.033 & & & & \\
\hline 0.70 & 11.700 & 8.753 & & & & & \\
\hline
\end{tabular}


Table B.40: The first of three tables detailing the percent uncertainty of the $\partial^{2} \sigma / \partial\left(K E_{\mu}\right) \partial\left(\cos \left(\theta_{\mu, \nu}\right)\right)$ results from Figure 6.32 .

\begin{tabular}{|l|c|c|c|c|c|c|c|c|}
\hline bin low edge & 0 & 50 & 100 & 150 & 200 & 250 & 300 & 350 \\
\hline 0.95 & 17.996 & 18.718 & 19.846 & 23.435 & 25.199 & 24.280 & 21.122 & 20.058 \\
\hline 0.90 & 18.488 & 17.231 & 19.710 & 19.034 & 18.817 & 18.467 & 18.316 & 17.124 \\
\hline 0.85 & 17.909 & 16.867 & 18.360 & 18.534 & 18.457 & 17.230 & 17.381 & 16.388 \\
\hline 0.80 & 17.482 & 16.954 & 16.877 & 17.489 & 16.912 & 16.223 & 14.718 & 14.893 \\
\hline 0.75 & 18.243 & 16.243 & 16.988 & 16.308 & 16.394 & 14.959 & 14.807 & 13.831 \\
\hline 0.70 & 16.620 & 16.900 & 15.300 & 15.669 & 16.043 & 14.986 & 15.124 & 16.306 \\
\hline 0.65 & 18.683 & 15.481 & 16.708 & 15.857 & 14.306 & 14.161 & 14.676 & 15.051 \\
\hline 0.60 & 18.118 & 16.341 & 17.159 & 15.934 & 13.888 & 14.057 & 15.072 & 15.161 \\
\hline 0.55 & 16.072 & 16.340 & 17.222 & 15.037 & 15.169 & 15.348 & 14.933 & 15.037 \\
\hline 0.50 & 18.106 & 16.595 & 16.371 & 16.427 & 15.400 & 14.519 & 16.506 & 15.705 \\
\hline 0.45 & 18.800 & 15.690 & 14.765 & 14.591 & 15.711 & 14.661 & 15.370 & 14.925 \\
\hline 0.40 & 17.134 & 15.441 & 15.397 & 15.260 & 14.997 & 15.660 & 16.777 & 16.408 \\
\hline 0.35 & 17.005 & 16.049 & 14.855 & 16.377 & 17.671 & 16.213 & 16.496 & 19.219 \\
\hline 0.30 & 17.577 & 15.581 & 15.303 & 15.215 & 16.631 & 15.852 & 15.645 & 16.652 \\
\hline 0.25 & 17.583 & 16.122 & 14.761 & 14.611 & 15.382 & 16.261 & 17.325 & 19.068 \\
\hline 0.20 & 17.995 & 15.677 & 16.306 & 14.944 & 15.732 & 16.648 & 17.434 & 19.007 \\
\hline 0.15 & 17.236 & 16.454 & 15.170 & 15.345 & 15.748 & 17.824 & 19.641 & 19.267 \\
\hline 0.10 & 18.402 & 17.394 & 14.911 & 15.140 & 17.172 & 18.373 & 19.498 & 19.637 \\
\hline 0.05 & 20.004 & 16.405 & 13.268 & 17.333 & 19.057 & 19.094 & 21.752 & 21.587 \\
\hline 0.00 & 17.251 & 16.275 & 16.144 & 16.010 & 19.025 & 19.172 & 19.469 & 31.547 \\
\hline-0.05 & 19.753 & 16.242 & 15.625 & 17.924 & 17.680 & 19.560 & 24.469 & \\
\hline-0.10 & 17.281 & 15.144 & 16.982 & 20.840 & 18.041 & 21.548 & 22.819 & \\
\hline-0.20 & 17.714 & 16.511 & 15.865 & 16.871 & 18.802 & 22.646 & 25.537 & 36.101 \\
\hline-0.30 & 16.755 & 15.373 & 15.790 & 17.424 & 17.918 & 20.049 & 24.975 & \\
\hline-0.40 & 16.064 & 16.165 & 15.451 & 18.329 & 18.248 & 23.338 & 31.433 & \\
\hline-0.50 & 18.915 & 17.256 & 15.380 & 19.157 & 20.566 & 22.999 & & \\
\hline-0.60 & 16.343 & 17.269 & 17.120 & 20.896 & 20.283 & 34.666 & & \\
\hline-0.80 & 14.380 & 17.883 & 20.755 & 19.489 & 20.374 & 24.729 & & \\
\hline-1.00 & 15.156 & 22.598 & 23.703 & 20.256 & 24.138 & & & \\
\hline & & & & & & & & \\
\hline
\end{tabular}


Table B.41: The second of three tables detailing the percent uncertainty of the $\partial^{2} \sigma / \partial\left(K E_{\mu}\right) \partial\left(\cos \left(\theta_{\mu, \nu}\right)\right)$ results from Figure 6.32 .

\begin{tabular}{|l|c|c|c|c|c|c|c|c|}
\hline bin low edge & 400 & 450 & 500 & 550 & 600 & 650 & 700 & 750 \\
\hline 0.95 & 19.882 & 16.437 & 17.526 & 18.597 & 17.636 & 17.591 & 15.786 & 15.183 \\
\hline 0.90 & 15.448 & 14.136 & 14.351 & 14.296 & 14.714 & 14.560 & 15.561 & 15.501 \\
\hline 0.85 & 14.792 & 13.538 & 14.274 & 13.779 & 15.396 & 15.095 & 14.269 & 14.631 \\
\hline 0.80 & 15.267 & 14.036 & 14.685 & 13.572 & 14.364 & 14.656 & 15.530 & 16.083 \\
\hline 0.75 & 14.331 & 14.227 & 14.451 & 15.039 & 16.380 & 16.548 & 16.328 & 17.562 \\
\hline 0.70 & 15.497 & 14.859 & 15.699 & 16.212 & 15.190 & 15.952 & 20.110 & 19.761 \\
\hline 0.65 & 14.746 & 15.333 & 14.723 & 15.059 & 16.197 & 15.733 & 19.057 & 23.664 \\
\hline 0.60 & 17.033 & 15.933 & 15.841 & 16.233 & 21.435 & 20.490 & 21.892 & \\
\hline 0.55 & 14.612 & 17.874 & 19.083 & 17.791 & 23.763 & 27.331 & & \\
\hline 0.50 & 16.970 & 18.656 & 18.758 & 25.079 & 22.939 & & & \\
\hline 0.45 & 17.546 & 17.822 & 17.585 & 25.201 & & & & \\
\hline 0.40 & 17.285 & 17.618 & 24.370 & 35.067 & & & & \\
\hline 0.35 & 21.757 & 23.340 & 25.452 & & & & & \\
\hline 0.30 & 21.514 & 25.719 & & & & & & \\
\hline 0.25 & 22.044 & 28.761 & & & & & & \\
\hline 0.20 & 24.499 & & & & & & & \\
\hline 0.15 & 22.600 & & & & & & & \\
\hline
\end{tabular}

Table B.42: The third of three tables detailing the percent uncertainty of the $\partial^{2} \sigma / \partial\left(K E_{\mu}\right) \partial\left(\cos \left(\theta_{\mu, \nu}\right)\right)$ results from Figure 6.32.

\begin{tabular}{|l|c|c|c|c|c|c|c|}
\hline bin low edge & 800 & 850 & 900 & 950 & 1000 & 1100 & 1200 \\
\hline 0.95 & 16.621 & 19.487 & 19.344 & 18.284 & 17.675 & 16.207 & 19.763 \\
\hline 0.90 & 14.620 & 14.986 & 14.215 & 16.774 & 17.411 & 18.902 & 25.094 \\
\hline 0.85 & 15.476 & 17.286 & 18.232 & 18.897 & 19.718 & 23.582 & 30.520 \\
\hline 0.80 & 16.468 & 17.215 & 21.763 & 24.733 & 30.434 & & \\
\hline 0.75 & 17.413 & 21.381 & 29.104 & & & & \\
\hline 0.70 & 23.359 & 30.077 & & & & & \\
\hline
\end{tabular}


Table B.43: The first of three tables detailing the $\partial^{2} \sigma / \partial\left(K E_{\pi}\right) \partial\left(\cos \left(\theta_{\pi, \nu}\right)\right)$ results from Figure $6.35\left(10^{-41} \mathrm{~cm}^{2} / \mathrm{MeV}\right)$.

\begin{tabular}{|l|c|c|c|c|c|c|}
\hline bin low edge & 0 & 25 & 50 & 75 & 100 & 125 \\
\hline 0.95 & & 1.536 & 2.674 & 3.991 & 5.145 & 6.514 \\
\hline 0.90 & & 1.391 & 2.628 & 4.475 & 5.647 & 6.998 \\
\hline 0.85 & & 1.432 & 3.036 & 4.636 & 6.605 & 7.993 \\
\hline 0.80 & & 1.776 & 3.112 & 4.554 & 6.541 & 8.347 \\
\hline 0.75 & & 1.612 & 3.346 & 5.047 & 7.108 & 9.074 \\
\hline 0.70 & 0.690 & 1.645 & 3.533 & 5.612 & 7.701 & 9.054 \\
\hline 0.65 & & 1.900 & 3.657 & 6.028 & 8.414 & 9.315 \\
\hline 0.60 & 0.720 & 1.878 & 3.759 & 6.333 & 8.312 & 9.268 \\
\hline 0.55 & & 1.956 & 3.657 & 6.214 & 8.690 & 9.210 \\
\hline 0.50 & & 2.206 & 4.427 & 7.080 & 8.725 & 8.654 \\
\hline 0.45 & & 2.053 & 4.133 & 6.806 & 8.691 & 9.177 \\
\hline 0.40 & 0.841 & 2.360 & 4.392 & 7.041 & 8.296 & 8.352 \\
\hline 0.35 & & 2.122 & 4.779 & 6.840 & 8.312 & 8.430 \\
\hline 0.30 & & 2.398 & 4.648 & 7.179 & 8.574 & 7.465 \\
\hline 0.25 & & 2.310 & 4.851 & 7.509 & 8.578 & 7.581 \\
\hline 0.20 & & 2.599 & 4.971 & 7.391 & 8.732 & 7.080 \\
\hline 0.15 & 0.809 & 2.860 & 5.417 & 7.522 & 8.113 & 7.444 \\
\hline 0.10 & 0.855 & 2.810 & 5.120 & 7.578 & 7.414 & 6.424 \\
\hline 0.05 & 0.711 & 2.737 & 5.236 & 7.009 & 7.826 & 6.160 \\
\hline 0.00 & 0.922 & 2.865 & 5.300 & 7.061 & 7.160 & 5.515 \\
\hline-0.05 & 0.987 & 2.932 & 5.200 & 7.159 & 6.782 & 5.139 \\
\hline-0.10 & 0.848 & 3.072 & 5.029 & 6.855 & 5.902 & 4.461 \\
\hline-0.15 & 0.809 & 3.124 & 5.539 & 6.947 & 5.885 & 3.681 \\
\hline-0.20 & 0.961 & 3.168 & 5.059 & 6.525 & 5.556 & 3.741 \\
\hline-0.25 & 0.998 & 3.169 & 5.223 & 6.351 & 5.064 & 3.385 \\
\hline-0.30 & 0.969 & 3.275 & 5.133 & 6.223 & 4.800 & 2.857 \\
\hline-0.35 & 0.995 & 3.202 & 5.300 & 5.909 & 4.276 & 2.672 \\
\hline-0.40 & 1.115 & 3.242 & 5.000 & 5.522 & 3.763 & 2.173 \\
\hline-0.45 & 1.179 & 3.202 & 4.786 & 4.800 & 3.110 & 1.887 \\
\hline-0.50 & 1.097 & 3.285 & 4.944 & 4.517 & 3.340 & 1.769 \\
\hline-0.55 & 1.152 & 3.377 & 4.595 & 4.451 & 2.580 & 1.645 \\
\hline-0.60 & 1.064 & 3.319 & 4.720 & 3.845 & 2.427 & 1.402 \\
\hline-0.65 & 1.117 & 3.281 & 4.418 & 3.731 & 2.261 & 1.144 \\
\hline-0.70 & 1.166 & 3.178 & 4.177 & 3.312 & 2.213 & 1.128 \\
\hline-0.75 & 1.094 & 3.243 & 3.789 & 3.039 & 1.688 & 1.036 \\
\hline-0.80 & 1.156 & 3.076 & 3.784 & 2.973 & 1.527 & 1.058 \\
\hline-0.85 & 3.059 & 3.056 & 3.562 & 2.654 & 1.469 & \\
\hline-0.90 & & 3.061 & 3.481 & 2.382 & 1.300 & \\
\hline-0.95 & 1.216 & 2.955 & 1.702 & 1.211 & \\
\hline-1.00 & & & & & \\
\hline & & & & & & \\
\hline
\end{tabular}


Table B.44: The second of three tables detailing the $\partial^{2} \sigma / \partial\left(K E_{\pi}\right) \partial\left(\cos \left(\theta_{\pi, \nu}\right)\right)$ results from Figure $6.35\left(10^{-41} \mathrm{~cm}^{2} / \mathrm{MeV}\right)$.

\begin{tabular}{|l|c|c|c|c|c|}
\hline bin low edge & 150 & 175 & 200 & 225 & 250 \\
\hline 0.95 & 7.873 & 8.947 & 10.435 & 10.970 & 10.258 \\
\hline 0.90 & 8.427 & 9.631 & 10.198 & 10.106 & 10.145 \\
\hline 0.85 & 9.578 & 9.997 & 10.829 & 10.153 & 9.420 \\
\hline 0.80 & 9.110 & 9.862 & 9.642 & 9.255 & 8.550 \\
\hline 0.75 & 9.630 & 9.100 & 9.152 & 8.695 & 7.986 \\
\hline 0.70 & 9.139 & 9.666 & 8.991 & 8.316 & 7.557 \\
\hline 0.65 & 9.265 & 8.669 & 8.360 & 7.760 & 6.636 \\
\hline 0.60 & 9.342 & 8.517 & 8.478 & 7.164 & 6.061 \\
\hline 0.55 & 9.048 & 7.832 & 7.404 & 6.215 & 5.565 \\
\hline 0.50 & 8.497 & 7.549 & 6.449 & 5.747 & 5.148 \\
\hline 0.45 & 7.545 & 6.876 & 6.034 & 5.091 & 4.216 \\
\hline 0.40 & 7.629 & 6.347 & 5.511 & 4.586 & 3.638 \\
\hline 0.35 & 7.070 & 6.014 & 5.020 & 3.847 & 3.217 \\
\hline 0.30 & 7.013 & 5.715 & 4.516 & 3.491 & 2.722 \\
\hline 0.25 & 6.425 & 5.398 & 3.837 & 2.759 & 2.467 \\
\hline 0.20 & 6.001 & 4.467 & 3.635 & 2.828 & 2.081 \\
\hline 0.15 & 5.323 & 3.899 & 2.921 & 2.065 & 1.721 \\
\hline 0.10 & 4.606 & 3.653 & 2.363 & 1.813 & 1.703 \\
\hline 0.05 & 4.384 & 2.955 & 2.333 & 1.681 & 1.432 \\
\hline 0.00 & 3.872 & 2.585 & 2.069 & 1.834 & 1.163 \\
\hline-0.05 & 3.243 & 2.258 & 1.729 & 1.428 & \\
\hline-0.10 & 3.021 & 1.879 & 1.582 & & \\
\hline-0.15 & 2.439 & 1.699 & 1.489 & & \\
\hline-0.20 & 2.233 & 1.540 & 1.239 & & \\
\hline-0.25 & 2.105 & 1.417 & & & \\
\hline-0.30 & 1.605 & 1.246 & & & \\
\hline-0.35 & 1.499 & 1.061 & & & \\
\hline-0.40 & 1.530 & & & & \\
\hline-0.45 & 1.325 & & & & \\
\hline-0.50 & 1.156 & & & & \\
\hline & & & & & \\
\hline
\end{tabular}


Table B.45: The third of three tables detailing the $\partial^{2} \sigma / \partial\left(K E_{\pi}\right) \partial\left(\cos \left(\theta_{\pi, \nu}\right)\right)$ results from Figure $6.35\left(10^{-41} \mathrm{~cm}^{2} / \mathrm{MeV}\right)$.

\begin{tabular}{|l|c|c|c|c|c|}
\hline bin low edge & 275 & 300 & 325 & 350 & 375 \\
\hline 0.95 & 10.643 & 9.606 & 8.276 & 6.901 & 6.195 \\
\hline 0.90 & 9.364 & 8.854 & 7.767 & 6.488 & 5.541 \\
\hline 0.85 & 8.728 & 7.743 & 6.851 & 5.644 & 4.855 \\
\hline 0.80 & 7.750 & 7.112 & 6.113 & 5.512 & 4.484 \\
\hline 0.75 & 7.385 & 6.491 & 5.446 & 4.412 & 4.318 \\
\hline 0.70 & 6.421 & 5.607 & 4.306 & 4.003 & 3.426 \\
\hline 0.65 & 6.026 & 4.908 & 4.296 & 3.306 & 3.052 \\
\hline 0.60 & 5.115 & 4.134 & 3.565 & 3.198 & 2.594 \\
\hline 0.55 & 4.760 & 3.630 & 3.169 & 2.454 & 2.237 \\
\hline 0.50 & 3.867 & 3.354 & 2.741 & 2.113 & 2.098 \\
\hline 0.45 & 3.390 & 2.727 & 2.384 & 2.053 & 1.726 \\
\hline 0.40 & 2.633 & 2.288 & 2.013 & 1.665 & 1.442 \\
\hline 0.35 & 2.636 & 1.993 & 1.702 & 1.543 & \\
\hline 0.30 & 2.053 & 2.203 & 1.537 & & \\
\hline 0.25 & 1.943 & 1.479 & 1.227 & & \\
\hline 0.20 & 1.610 & & & & \\
\hline 0.15 & 1.590 & & & & \\
\hline 0.10 & 1.488 & & & & \\
\hline
\end{tabular}


Table B.46: The first of three tables detailing the percent uncertainty of the $\partial^{2} \sigma / \partial\left(K E_{\pi}\right) \partial\left(\cos \left(\theta_{\pi, \nu}\right)\right)$ results from Figure 6.35 .

\begin{tabular}{|l|c|c|c|c|c|c|}
\hline bin low edge & 0 & 25 & 50 & 75 & 100 & 125 \\
\hline 0.95 & & 19.098 & 17.068 & 15.740 & 15.130 & 17.074 \\
\hline 0.90 & & 23.775 & 15.252 & 15.324 & 14.774 & 15.101 \\
\hline 0.85 & & 16.540 & 17.527 & 11.069 & 13.621 & 13.889 \\
\hline 0.80 & & 17.630 & 17.426 & 12.517 & 12.406 & 12.805 \\
\hline 0.75 & & 20.075 & 14.402 & 13.476 & 11.649 & 12.912 \\
\hline 0.70 & 20.544 & 17.261 & 13.509 & 12.711 & 12.829 & 12.698 \\
\hline 0.65 & & 16.149 & 16.057 & 13.835 & 13.657 & 13.241 \\
\hline 0.60 & 27.079 & 16.051 & 15.458 & 14.096 & 12.146 & 13.542 \\
\hline 0.55 & & 22.156 & 14.021 & 15.206 & 12.799 & 15.013 \\
\hline 0.50 & & 15.424 & 13.524 & 13.637 & 13.791 & 14.396 \\
\hline 0.45 & & 17.041 & 15.275 & 14.221 & 13.649 & 13.636 \\
\hline 0.40 & 24.671 & 15.936 & 13.865 & 13.091 & 14.465 & 14.668 \\
\hline 0.35 & & 16.360 & 14.020 & 13.460 & 13.940 & 15.743 \\
\hline 0.30 & & 14.984 & 12.993 & 13.613 & 15.030 & 15.627 \\
\hline 0.25 & & 13.195 & 11.814 & 13.845 & 13.921 & 15.423 \\
\hline 0.20 & & 14.152 & 13.914 & 13.527 & 14.739 & 14.422 \\
\hline 0.15 & 16.979 & 14.294 & 14.212 & 13.375 & 14.238 & 14.125 \\
\hline 0.10 & 20.960 & 14.935 & 14.337 & 12.754 & 14.253 & 16.484 \\
\hline 0.05 & 13.604 & 13.263 & 12.816 & 13.765 & 15.346 & 15.649 \\
\hline 0.00 & 24.008 & 14.995 & 14.962 & 13.238 & 14.414 & 14.677 \\
\hline-0.05 & 19.241 & 14.070 & 13.665 & 12.845 & 14.334 & 14.704 \\
\hline-0.10 & 17.709 & 15.715 & 11.476 & 13.680 & 13.484 & 16.292 \\
\hline-0.15 & 18.348 & 14.764 & 15.253 & 13.727 & 15.685 & 15.813 \\
\hline-0.20 & 16.851 & 12.429 & 12.587 & 14.212 & 14.450 & 15.123 \\
\hline-0.25 & 18.077 & 14.919 & 13.117 & 14.574 & 18.040 & 16.290 \\
\hline-0.30 & 19.836 & 13.843 & 13.346 & 16.043 & 15.038 & 14.457 \\
\hline-0.35 & 16.966 & 13.812 & 14.814 & 17.566 & 14.352 & 15.343 \\
\hline-0.40 & 17.018 & 13.196 & 13.549 & 15.165 & 14.898 & 17.673 \\
\hline-0.45 & 18.866 & 13.810 & 13.966 & 14.493 & 18.684 & 16.323 \\
\hline-0.50 & 14.809 & 13.065 & 13.832 & 13.469 & 14.146 & 16.727 \\
\hline-0.55 & 16.436 & 15.309 & 12.889 & 13.770 & 13.580 & 17.198 \\
\hline-0.60 & 19.470 & 13.306 & 14.508 & 14.770 & 16.957 & 17.436 \\
\hline-0.65 & 18.176 & 12.493 & 15.286 & 16.694 & 16.714 & 16.433 \\
\hline-0.70 & 15.156 & 12.263 & 15.037 & 17.161 & 20.494 & 20.825 \\
\hline-0.75 & 14.807 & 11.543 & 14.148 & 18.383 & 19.990 & 19.919 \\
\hline-0.80 & 14.759 & 12.400 & 16.590 & 21.048 & 19.793 & 25.052 \\
\hline-0.85 & 14.483 & 12.227 & 14.396 & 19.111 & 20.196 & \\
\hline-0.90 & 20.409 & 13.433 & 15.263 & 18.445 & 25.167 & \\
\hline-0.95 & 15.132 & 12.751 & 14.961 & 17.030 & 23.027 & \\
\hline-1.00 & 17.440 & 14.483 & 17.662 & 20.686 & 26.851 & \\
\hline & & & & & & \\
\hline
\end{tabular}


Table B.47: The second of three tables detailing the percent uncertainty of the $\partial^{2} \sigma / \partial\left(K E_{\pi}\right) \partial\left(\cos \left(\theta_{\pi, \nu}\right)\right)$ results from Figure 6.35 .

\begin{tabular}{|l|c|c|c|c|c|}
\hline bin low edge & 150 & 175 & 200 & 225 & 250 \\
\hline 0.95 & 17.142 & 16.913 & 16.948 & 17.222 & 18.863 \\
\hline 0.90 & 14.987 & 16.047 & 15.782 & 17.239 & 17.868 \\
\hline 0.85 & 13.209 & 13.734 & 15.297 & 16.067 & 17.078 \\
\hline 0.80 & 14.042 & 14.426 & 15.217 & 15.776 & 17.222 \\
\hline 0.75 & 13.339 & 14.984 & 16.180 & 16.071 & 17.043 \\
\hline 0.70 & 14.544 & 15.454 & 15.102 & 16.910 & 16.928 \\
\hline 0.65 & 13.572 & 15.483 & 14.624 & 15.725 & 16.093 \\
\hline 0.60 & 13.465 & 15.547 & 15.609 & 16.401 & 18.917 \\
\hline 0.55 & 14.691 & 16.061 & 18.573 & 17.768 & 18.322 \\
\hline 0.50 & 15.156 & 16.907 & 18.377 & 16.456 & 17.692 \\
\hline 0.45 & 16.064 & 16.744 & 17.545 & 17.288 & 19.448 \\
\hline 0.40 & 15.240 & 15.066 & 17.947 & 19.210 & 20.513 \\
\hline 0.35 & 15.303 & 15.274 & 18.283 & 17.978 & 19.382 \\
\hline 0.30 & 16.283 & 16.798 & 18.020 & 17.422 & 19.188 \\
\hline 0.25 & 14.909 & 16.540 & 19.075 & 18.775 & 18.791 \\
\hline 0.20 & 14.982 & 17.111 & 18.247 & 19.524 & 18.904 \\
\hline 0.15 & 15.864 & 16.394 & 18.031 & 17.435 & 21.418 \\
\hline 0.10 & 16.098 & 15.449 & 17.980 & 19.061 & 20.913 \\
\hline 0.05 & 16.433 & 15.871 & 15.697 & 20.920 & 19.639 \\
\hline 0.00 & 15.331 & 15.604 & 19.690 & 21.116 & 21.897 \\
\hline-0.05 & 15.297 & 16.087 & 18.882 & 20.257 & \\
\hline-0.10 & 17.375 & 18.762 & 19.650 & & \\
\hline-0.15 & 19.895 & 17.441 & 20.828 & & \\
\hline-0.20 & 16.283 & 18.443 & 21.728 & & \\
\hline-0.25 & 16.943 & 19.278 & & & \\
\hline-0.30 & 20.128 & 19.211 & & & \\
\hline-0.35 & 21.054 & 20.422 & & & \\
\hline-0.40 & 22.146 & & & & \\
\hline-0.45 & 20.188 & & & & \\
\hline-0.50 & 19.318 & & & & \\
\hline & & & & & \\
\hline
\end{tabular}


Table B.48: The third of three tables detailing the percent uncertainty of the $\partial^{2} \sigma / \partial\left(K E_{\pi}\right) \partial\left(\cos \left(\theta_{\pi, \nu}\right)\right)$ results from Figure 6.35 .

\begin{tabular}{|l|c|c|c|c|c|}
\hline bin low edge & 275 & 300 & 325 & 350 & 375 \\
\hline 0.95 & 20.074 & 20.251 & 21.692 & 21.603 & 22.148 \\
\hline 0.90 & 19.192 & 19.092 & 19.178 & 20.799 & 21.193 \\
\hline 0.85 & 17.830 & 19.321 & 18.438 & 18.793 & 19.840 \\
\hline 0.80 & 19.749 & 18.857 & 19.279 & 19.347 & 19.160 \\
\hline 0.75 & 17.063 & 18.930 & 18.546 & 20.084 & 19.059 \\
\hline 0.70 & 17.220 & 19.128 & 18.398 & 18.636 & 19.801 \\
\hline 0.65 & 16.810 & 17.379 & 21.202 & 19.197 & 21.029 \\
\hline 0.60 & 18.157 & 19.912 & 19.231 & 20.101 & 21.455 \\
\hline 0.55 & 17.878 & 19.300 & 19.778 & 20.640 & 21.644 \\
\hline 0.50 & 18.308 & 19.868 & 21.849 & 21.849 & 19.619 \\
\hline 0.45 & 20.392 & 19.711 & 21.216 & 20.511 & 21.486 \\
\hline 0.40 & 19.679 & 19.490 & 21.785 & 24.972 & 25.029 \\
\hline 0.35 & 21.338 & 22.529 & 20.548 & 24.372 & \\
\hline 0.30 & 20.324 & 21.019 & 23.361 & & \\
\hline 0.25 & 23.067 & 21.823 & 25.748 & & \\
\hline 0.20 & 21.936 & & & & \\
\hline 0.15 & 20.165 & & & & \\
\hline 0.10 & 27.356 & & & & \\
\hline
\end{tabular}

